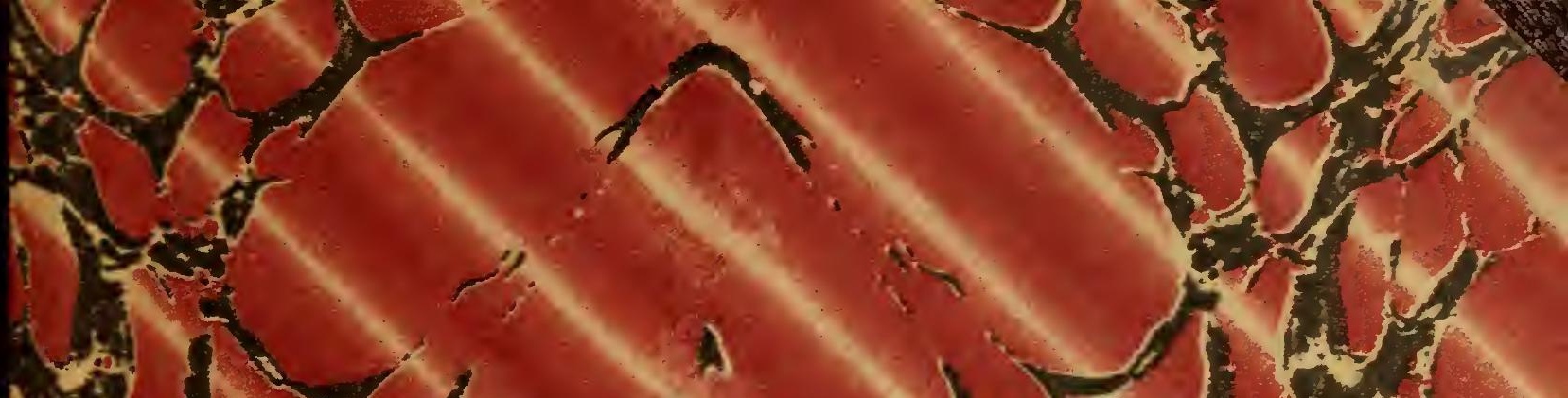

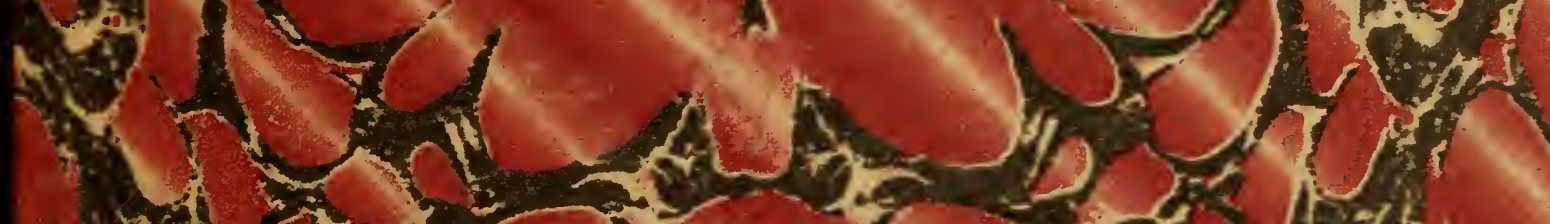

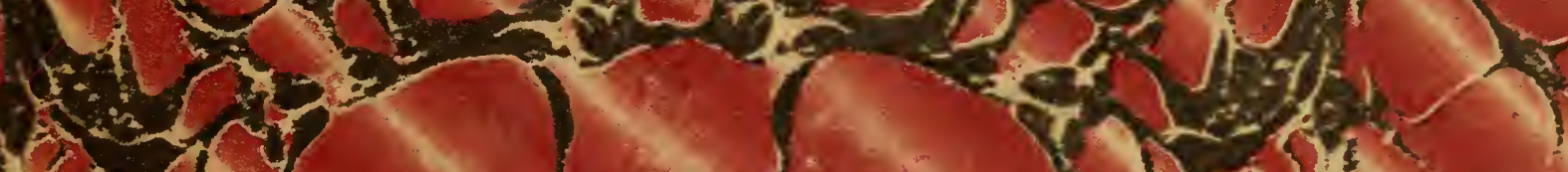
$2 x^{2}-3-14$ f $x$ 는

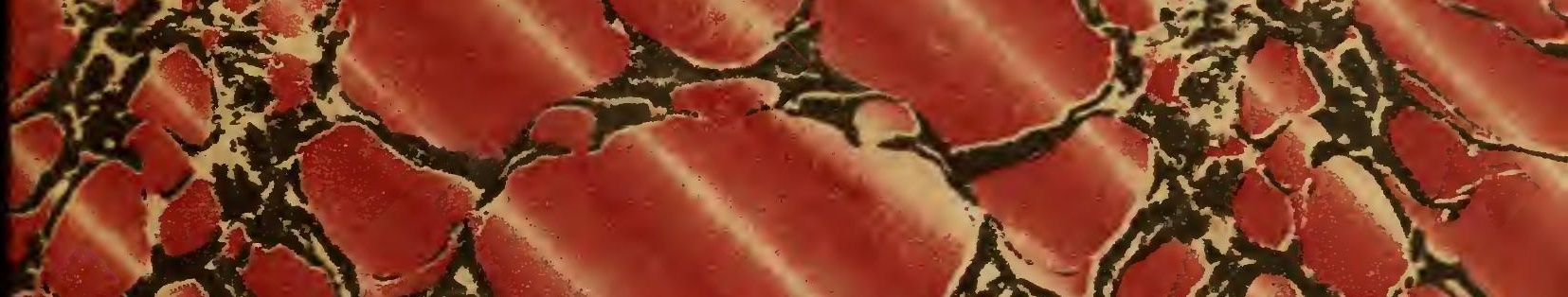

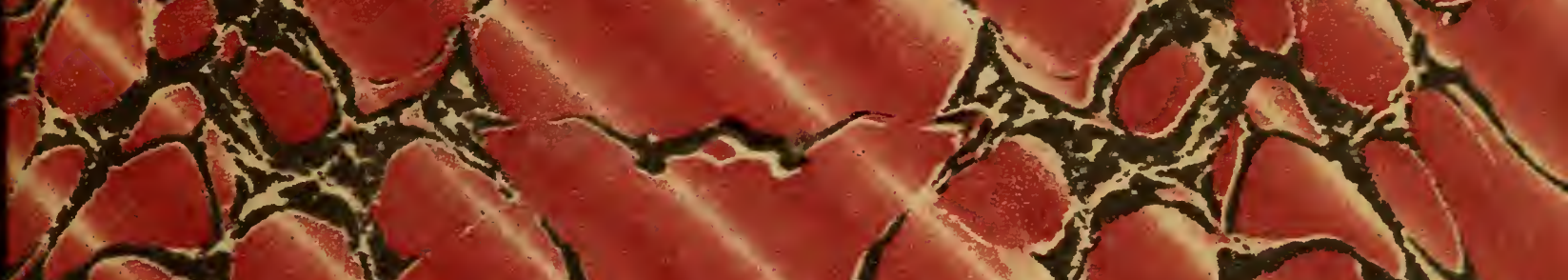

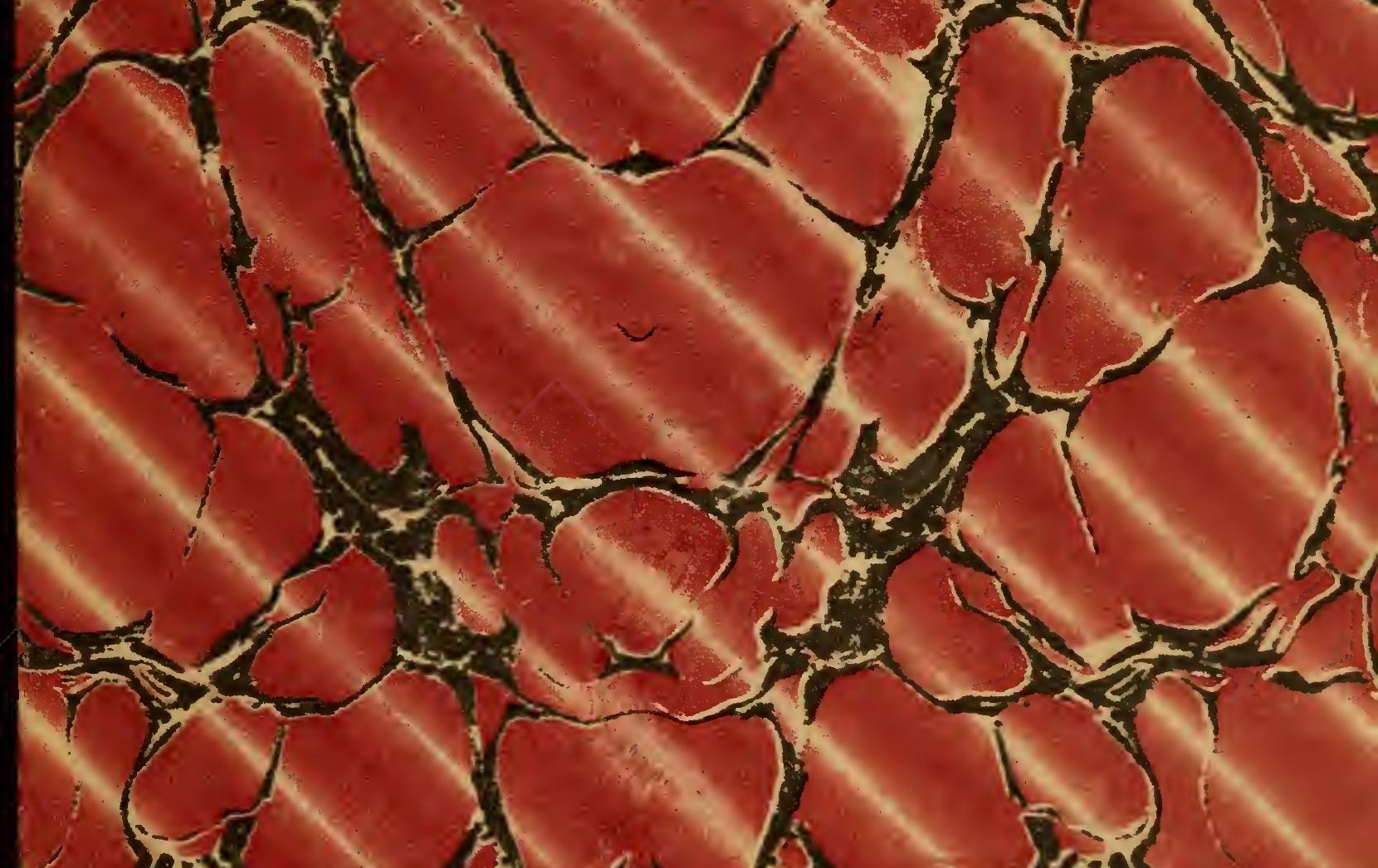
(1) 
Cooper-Hewitt Museum Library

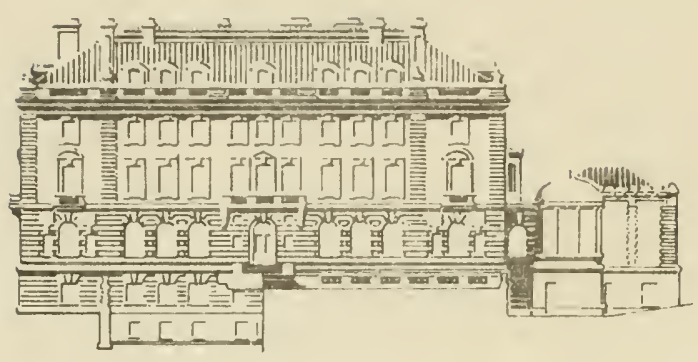

Smithsonian Institution Libraries 
$27^{50}$ 

To

MRS. POTTER PALMER,

FRESIDENT BOARD OF LADY MANAGERS

OF THE

"WOLRD'S COLUMBIAN EXPOSITION"

I HIS BOOK IS RESPECTFULLY DEDICATED BY SPECIAL PERMISSION。

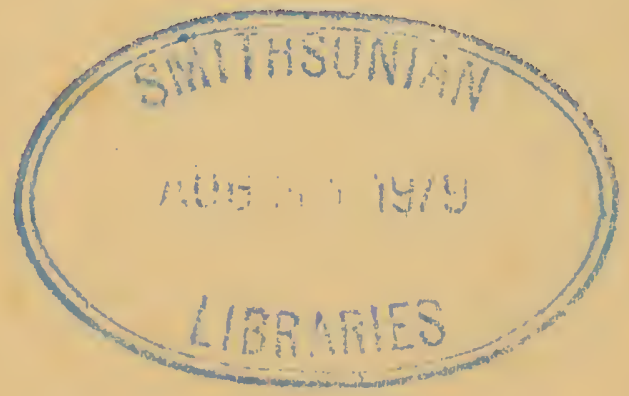




\section{PREFACE.}

The publishers of "Dainty Work for Pleasure and Profit," in issuing a work of such importance to women have considered it only just that the book should be dedicated to the most representative American woman of the day. That woman is unquestionably the President of the Board of Lady Managers of the World's Columbian Exposition, Mrs. Potter Palmer -a woman of the highest social distinction, possessed of all the gracious attributes of true womanhood, honored by the nation and beloved by alt who know her. The dedication is made by special permission. 


\section{DAINTY WORK.}

CHAPTER I.

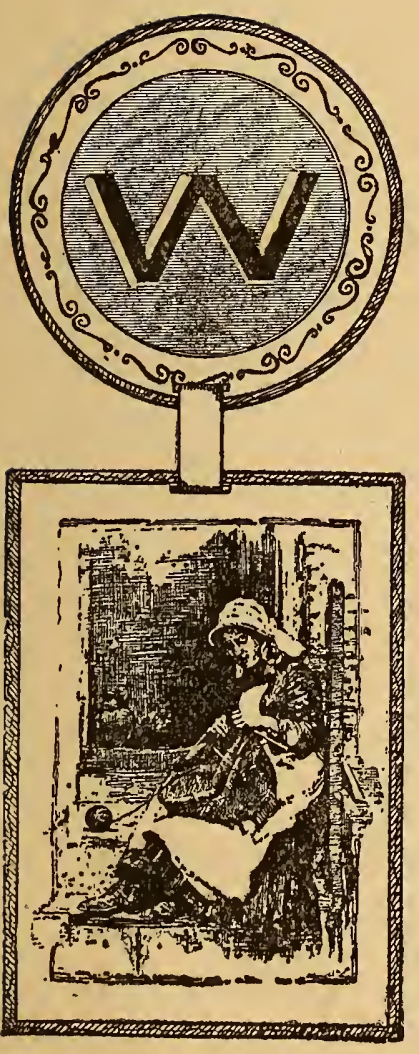

INTRODUCTION.

E HAVE tried in the following pages to inculcate a love for home beautifying; to show how every home in this broad land can be rendered beautiful, according to surrounding circumstances and the financial ability of the owners; to teach economy, appropr1ateness and harmony in interior decorations; how to make use of the homely, every-day articles, transforming them from unsightly objects into things beautiful to behold. In a word, we have tried to give instructions in all things pertaining to the homc beautiful so clear and simple that no household. however humble, need be without the refining infuences of dainty environments.

We have always recognized that the first requisite of a true home is order and cleanliness, for without these there can be no beauty, no true refinement, even though mother and daughters be able to paint like a Raphael and embroider like a Queen Matilda; without the first, the last accomplishment is worse than useless as a means of home decoration. It is better to be a good cook than a poor painter, better to be a deft and handy garment-maker than an indifferent embroideress, better to have order and system than a small knowledge of china painting, but given the former, the latter are beautiful and useful accomplishments for wife, mother, sisters and daughters. 
It is certainly the first duty of a wife and mother to make home the pleasantest and happiest spot on earth for the members of her family, and to do this requires more than order, system, immaculate cleanliness, more than the purchase of expensive carpets and pieces of heavy furniture. It requires the home-making, home-beautifying talent. It needs the exercise of an ingenious mind and nimble fingers in fashioning dainty accessories in the shape of mantel drapes, screens, wall-pockets, toilet sets, dainty table linen, cushions, photograph holders, and all the numberless odds and ends that go to make up the pretty home comfort of a room.

Taste, more than money, is needed in the decorating of American homes; oftentimes the simplest and least expensive efforts are those which give the most homelike, pleasing and restful effects. And now, dear reader, right here let me say a few words directly and individually to you. Do not wait to build a fine residence before you begin to make a home, in the only sense in which the word should ever be used. Do not let your children grow up amid uncouth and unlovely surroundings, while you are waiting for the dollars to grow. Remember, the young will seek for beauty elsewhere, if it is denied them at home. The old house may be too square or too long, too low or too high, too old fashioned, aye, even "tumble-down" may be the only word to fully express its appearance, yet it can be made a lovely home, if only the spirit of home beautifying abides in the heart of its mistress. Now, do not say you cannot afford it; you can afford it, because it is one of the necessities and not a luxury of life. With the facilities offered in these pages for learning the different kinds of needle-work, and the making up of an almost innumerable amount of fancy and pretty articles, and the present low prices of materials, there is no excuse for bare, cheerless looking rooms in any part of our country. And beside, decorative effort of some kind is a necessary part of home culture. We say effort advisedly, as the result is not so much the thing to be considered as that the spirit, wish or desire to make our homes not only orderly places of abode, but beautiful as well, should exist. And we repeat, this effort at home decoration may be costly or simple, according to individual means; but there is no home so poor that some attempt at beautifying cannot be made. An attempt with crudest results is better than none at all. If one lives in few rooms, and curtains and carpets have so far been unattainable, the first effort at 
decoration will naturally be to drape the curtainless windows and cover the bare floors. The curtains may be of cheese cloth, and covering the floors may mean to simply stain them, and, later on, to lay rugs of home manufacture; but what a change this effort to beautify will have made. Having begun the work of beautifying, how simple it seems to go a step further, and add a few pots of healthy plants to the curtained windows. Expensively embroidered cushions, table spreads, lambrequins, etc., would here be out of place, and so far from adding to the effect would be inharmonious. The embroidered spread would laugh at the homemade rugs, and the expensive cushion turn up its nose at the homely lounge.

We hear some dissenting voice cry out: "You would have the mistress of this primitive home cease her efforts at home beautifying ere she has fairly begun?" By no means! We simply wish to say that articles of mere luxury should give way to those of necessity, and that the decorations should be harmonious with the general surroundings. If the household means will allow it, we would have the mistress thereof purchase a good supply of nice firm table linen, a good supply of bedding and towels, and embroider the family initial or monogram on each piece. The constant use of dainty linen, the constant sight of a well-appointed table, are factors in the home education of every child; we hold, therefore, that embroidered household linen is a decorative necessity and within the reach of the very poor. The trouble with those who exclaim most loudly at the expense attached to fancy work, lies in the fact that articles of mere ornament are begun with only the vaguest idea on the part of the worker as to the ultimate cost; hence, discredit is brought upon the very name of fancy work. All this might be obviated if as much thought were given to the subject and the same rules governed the purchase of art materials as is given to the ordinary purchases of the household.

When women cease to make $\$$ I 5 to $\$ 20$ lambrequins for a room whose entire furnishings did not cost three times that amount, and \$10 table spreads when the income would not warrant an outlay of more than onethird as much; when they learn that a few dollars' worth of embroidery silks disposed over several articles in keeping with all the surroundings is far more effective than one elaborate piece whose very richness renders shabby what before was passable, then will "Home Decoration" occupy its rightful 
position as a necessary and useful art. And now a last word before we enter upon the subject of this work. If anything must be dispensed with in your furnishings let it be the carpet. You can do quite well in procuring a pretty effect with a few home-made rugs and an oiled floor. Ingenuity, taste and skill are to you worth dollars. If you cannot afford silk bolting at $\$ 3.50$ per yard for your doyleys and dressing case covers, use scrim at 15 cents per yard. If you cannot afford a Persian rug, or even a very good imitation of one, buy a Ross rug hook, take a piece of coffee sacking, ravel pieces of old carpet for the necessary yarn, and make one; it will cost about $\$ 1.50$, and perhaps less; if neatly done, it will look almost as well as one that sells at $\$ 15$ to $\$ 25$. And remember, first, last and all the time, that "Refined ornamentation in every room in the house, especially those of the boys and the help, and a union of effort in this kind of work, particularly in the parlor and all other family apartments, will have a wonderful effect in developing character and preserving harmony in the household."

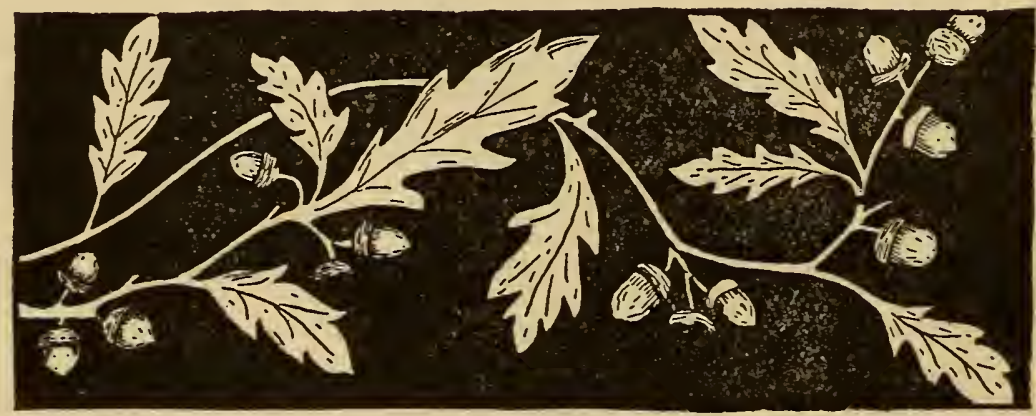




\section{CHAPTER II.}

MATERIALS.

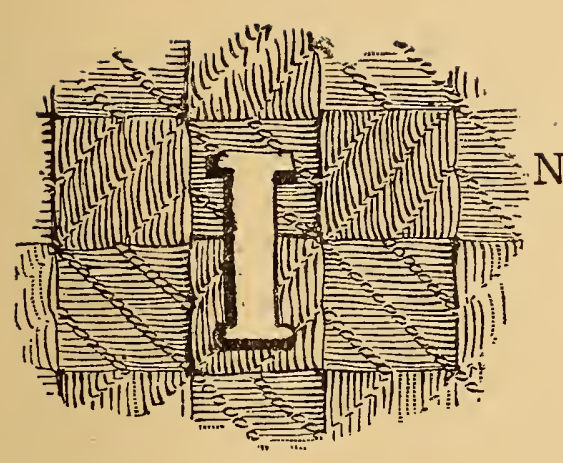

N THIS day of progressive ideas almost every kind of textile fabric is used as a background for decorative neeedle-work, from the coarse, unbleached linen crash to the finest silks and velvets, and each and every kind is appropriate, according to the use for which it is designed. We will here give the names and a brief description of the different fabrics more generally used for decorative purposes.

Linen Crash, such as is used for kitchen towels, is a fabric that offers a particularly pleasing ground for embroidery done in crewels, tapestry wools or linen embroidery threads, and can be purchased in various widths, from sixteen to thirty inches, and for to to 25 cents per yard.

Bleached Linen, of a fineness suitable for shirt fronts, is suited for doyleys, center cloths, pillow shams, toilet sets, sideboard sets and guest towels, when something very nice is desired. This kind of linen can be had in widths from twenty-four to thirty-six inches, and for various prices; according to quality, from 60 cents to $\$ I .00$ per yard being the usual price.

Domestic Linen is a firm fabric with a very light twill, and is made in widths from sixteen to eighty inches. Domestic linen is used for all the purposes for which the finer qualities are employed, and is especially suited for bedspreads, pillow shams, dresser scarfs and toilet sets that are to be embroidered with either silk or linen threads. Price per yard, from 20 cents to $\$$ I.5O.

Bolton Sheeting is a coarse, heavily twilled cotton fabric, two yards wide. It derives its name from the English town where it was first manufactured. It is sometimes called workhouse sheeting, from the fact that it is used for sheets and pillow slips in English workhouses. It is very 
effective when embroidered in colors with either silk or linen threads, and is much used for fancy bedspreads and dresser sets, beside portieres and sofa covers. It is cheap, costing but 50 cents a yard for the best quality, and it is full two yards wide.

Colored Art Linen. This fabric is a recent weave, and from the beauty and durability of the colors is destined to enter largely into the field of decorative work. Art linen is fifty inches wide, and is made in all the beautiful art shades, such as dead-leaf brown, old rose, gray blue, golden brown, leaf green, ecru, nasturtium, orange and gray. It is used for covering furniture, portieres, curtains, cushion covers and table spreads, and lends itself to the lights and shades of embroidery silks with beautiful effect. Price per yard, $\$$ I.5O.

Huck-a-buck, commonly called huck toweling, is a linen material in pure white and cream white, having threads slightly raised on the surface forming a honeycomb mesh. The mesh is large or small, close together or far apart, according to the fine or coarse quality of the huck. This fabric is usually decorated with outline in stem stitch, and background darned with silk or linen threads. The widths vary from sixteen to forty inches, and the price from 25 cents to $\$ 1.25$ per yard.

Linen Bolton Sheeting is a heavy linen fabric woven in imitation of the cotton article of that name. It is nearly as heavy as duck or canvas, which it closely resembles. It is used for the same articles as the cotton bolton; it is, of course, more expensive, costing about $\$$ I.30 per yard. It is two yards wide.

Art Satin is a cotton fabric, in white only, having a satin finished surface. It is very pretty made into table squares, tidies, center pieces, dresser scarfs and toilet sets, as the soft finish makes a good background for silk embroidery. It is forty inches wide, and can be had for 75 cents per yard.

Butchers' Linen is a very coarse linen, partly bleached, full bleached or unbleached. It is made in various widths, though the fortyinch weave is the one more commonly employed, as it cuts to better advantage in making up articles. It can be used for decorative needlework, though it is not so nice as the domestic linen. The forty-inch width usually sells for 30 cents per yard. 
Scrim is a very useful and pretty material with which to furnish a bedroom; spread, pillow scarf, dresser drape, curtains, tidies, etc., should be en suite, decorated throughout in a harmonious design. This material takes outline effectively. It is made in various widths, and costs from 15 cents to $\$ 1.00$ per yard.

Morris Cloth is a beautiful, soft diagonal twill goods, two yards wide. It comes in all the art tones, among which may be mentioned silver green, sage green, apple green and apple red. It can be trimmed with bands of harmonizing or contrasting shades of the same, and decorated with embroidered designs in couching and rope silks. This fabric is sold for $\$ 3.00$ per yard.

Frieze is a beautiful English-looking material, very rough as to surface and thick as to texture; it is used for floor cloths, table cloths, some kinds of upholstering and rugs. It should be decorated with large, bold design worked out with linen threads in rope, couching and art cord sizes, and if the piece is small, or expense is no object, silk can be introduced with beautiful effect. Price per yard, $\$ 2.50$.

Concordia Canvas is a thick and heavy cotton material in cream white, with a honeycombed surface; it can be purchased in lengths for chair backs and is designed for darning; the pattern being darned in under the honeycomb with rope silk, the effect is that of filmy lace thrown over the colored design. Price per yard, 50 cents.

Egyptian Cloth is a white, wash cotton goods, quite fine and sheer, somewhat resembling cheese cloth, but very much handsomer. It is used for summer drapery, bedroom sets and sash curtains. It is a lovely material for outlining or painting. Price per yard, 50 cents.

Turkish Crepe, a white, wash goods, particularly suitable for picture and easel drapes, curtains and bed draperies. It is soft and creamyv with a fine crepe effect, which is retained after washing. Price per yard, 50 cents.

Satin Damask is a beautiful heavy linen, with smooth, satinfinished surface. It is made in pure white, in various widths, from sixteen to seventy-two inches, and costs from 60 cents to $\$ 3.00$ per yard. It is used for nice table linen, lunch sets, fruit sets, doyleys napkins, center cloth, tray cloth and table mats. 
Gobelin Cloth is a pure white cotton fabric, eighteen inches wide. It has a decided twill, amounting almost to a rib, crosswise of the material. Price per yard, 50 cents. This is a popular fabric for toilet sets, cushion covers, table mats and centers for table covers.

Russian Crash is a fine woven linen fabric, about eighteen inches wide, in natural linen color-a pure gray. It is a beautiful background for outlined embroidery or Roman cut work. Price per yard, 50 cents.

Hollywood Drapery is a cotton fabric, made in cream, white and colors. Cream, white and some colors are made two yards wide, while a few colors come only fifty-four inches wide. The price varies from $\$ 1.25$ to $\$ 1.75$ per yard, according to width and color. The weave is very close and firm, and the surface presents an almost invisible basket effect. It is a very desirable material for table covers, plain spreads, portieres, bed spreads, toilet sets and cushion covers. It is beautiful embroidered with linen thread in conventional style, and as it needs no lining is an econom. ical material for portieres especially.

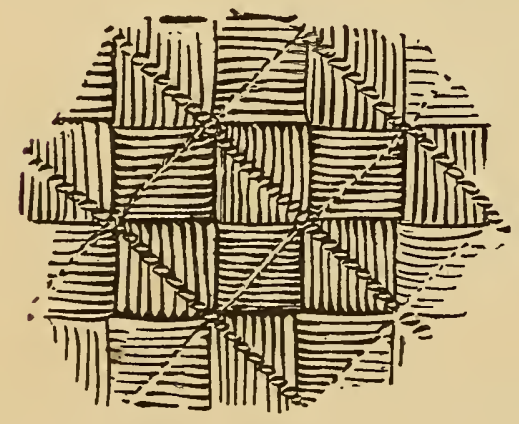

Devonshire Art Cloth is a beautiful linen fabric made of the natural flax, that is, not dyed, but comes in the beautiful flax color. It is made in squares or checks of different sizes, one of which we show here. It is a firm, closeiy woven fabric, and will be found very artistic for centers to cushions, table covers, wall panels, DEvonshiRe CLOTh. FIG. I. chair covers or sideboard scarfs. It is twc yards wide and costs $\$ 3.00$ per yard.

Bargarren Art Cloth is a fabric designed especially for "Darned" embroidery. It is made in four styles, each one having a mesh of different size and shape. The surface is honeycombed by means of the thread which is raised to form the mesh. This thread is very heavy and the weave firm, so that the largest sized embroidery linen threads can easily be darned under it. The fabric is made in pure white, cream white and colors, and is first woven eighty-eight inches wide and then shrunken to seventy-two inches, so there is no trouble with shrinkage after an article is made up. For bedspreads, dresser scarfs, toilet sets, portieres and table covers there is no 
material that offers such possibilities for economical embroideries, as large surfaces can be covered with darned and outline work at little expense. The price varies from $\$ 1.25$ to $\$ 1.60$ per yard.

Bolting Silk is a fine, transparent, gauze-like material, made of finest white silk and very strong. Millers use it for bolting a certain brand of flour, hence its name. It is made in different widths, from eighteen to forty inches wide, and is a popular fabric for dainty articles for the toilet table, for doyleys, drapes, shams, curtains, and tea cloths lined with silk. It can be washed, and is beautiful embroidered in either pure white or colors. Prices range from 75 cents to $\$ 3.00$ per yard.

Silks, plain and ribbed, are used as a background for decorative needlework, and for articles that are not exposed to much usage and consequently that will not need frequent cleaning. They will be found both serviceable and elegant. Width, from eighteen to thirty-two inches. Price, from 75 cents to $\$ 1.75$.

Velvets and Plushes are both much used for handsome pieces of embroidery, and cost, according to quality, from 75 cents to $\$ 2.25$ per yard. Width, eighteen to thirty-two inches.

Felt is a heavy cloth without any weave, and with a perfectly smooth surface, alike on both sides. It is full two yards wide, and comes in all colors. Price per yard, $\$$ r.oo.

Satin offers a beautiful background for all kinds of silk embroidery. Price per yard, from 60 cents to $\$ 1.25$, according to quality. Width, eighteen to twenty-seven inches.

Banner Silk is a twilled silk, made in two widths, namely, fortyfour and seventy-two inches. It is designed especially for society banners, but is also used for door and window draperies when expense is no object. Price per yard, $\$ 8.00$ and $\$ 10.00$.

Upholsterers' Plush and Satin. These materials closely resemble the ordinary fabrics of the same name, but are usually a heavier quality. Widths, fifty-four and seventy-two inches. Price, \$IO.00, \$I 2.00 and $\$ 5.00$ per yard, for best qualities.

Sateen is a satin-faced material with a wool back. It comes fifty-four inches wide and costs $\$ 2.50$ per yard. It is used for table scarfs and covers, portieres and panels. 
Russian Tapestry is a very heavy, all-silk material, woven with a broken twill crosswise of the fabric, and presents a crepy effect which lights up wonderfully, showing several different shades of its own color. It is woven fifty inches wide, and is very beautiful for portieres, cushions, table and bed covers and piano covers. Price per yard, $\$ 5.50$.

Basket Silk is a silk-faced material, fifty inches wide. It comes in white, ecru, old rose and gray. It is woven with basket effect on the right surface; the mesh is about as pronounced as that on medium coarse huck toweling. Basket silk is fifty inches wide, and can be had for $\$ 3.00$ per yard.

Mail Cloth is a very heavy silk-faced fabric, fifty inches wide. It has a small bird's-eye weave on the right surface and requires no lining. It comes in all the art shades, such as Indian red, maize yellow, sage green, dead-leaf brown, old blue, ecru and cream. It is a beautiful background for heavy embroidery with rope silk, couching silk and art cord. Price per yard, $\$ 3.00$.

India Silk is a thin, plain woven silk, much used for draperies, puffs, linings and trimmings for fancy articles. It is from twenty-seven to thirtysix inches wide, and can be had for from 60 to 75 cents per yard. It is very dainty embroidered in outline stitch only.

Double-faced Sateen is a very heavy goods, plain satin surface on both sides. As its name indicates, it is reversible; thus, one side will be a deep old blue, while the reverse side will be a pale old blue overcast with a silvery sheen, but both blues will be of the same line of color. Where handsome portieres or table covers are desired there could be no more beautiful fabric. No linings are ever used with this sateen. It is fifty inches wide, and can be had for $\$ 3.50$ per yard.

The above list contains most of the fabrics commonly used for embroidery work, but it is by no means a complete list, as there are the canvases used in cross-stitch, the lawns, muslins, merinos and flannel used for articles of wearing apparel, the serges, velours and brocaded silks of infinite variety that are used for furniture covers, that need no description here as they are familiar to all. 


\section{CHAPTER III.}

\section{IMPLEMENTS AND EMBROIDERY THREADS.}

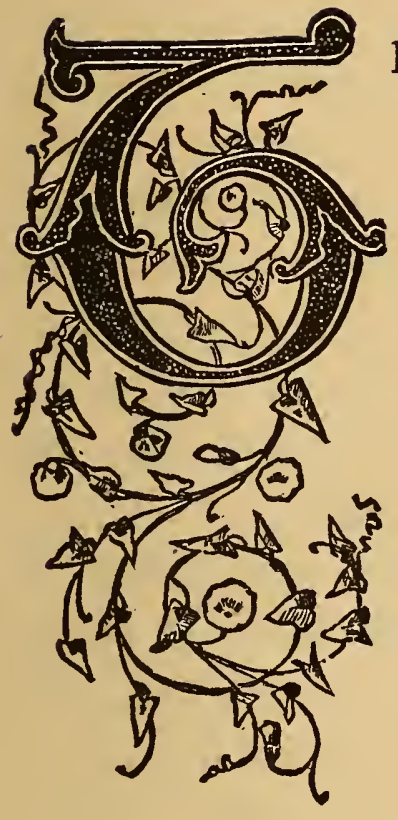

HIS IS a subject of vital importance to the needleworker, and deserves a chapter to itself. No good work can be accomplished without the proper implements to do it with, hence we will discuss them in the order of their importance. First, then, the embroiderer who expects to do a variety of work must have a frame and a pair of hoops. Now, there are embroidery frames in the market by the score, and at prices ranging from $\$ 2.50$ to $\$ 7.50$ each. We give here an illustration of one of the popular frames on a standard. It is very convenient for large pieces of work, and allows the worker the free use of both hands. This style of frame has adjustable screws and can be lowered or raised at will, and tipped to any angle desired by the worker; but these frames are expensive and would hardly be purchased by the lady who does embroidery only semi-occasionally, and so we show, in figure 3 , the frame we have used in all our own work during the last ten years. It will cost fifty cents if a carpenter makes it, and nothing at all if one is fortunate enough to possess a husband or a big brother who can be pressed into

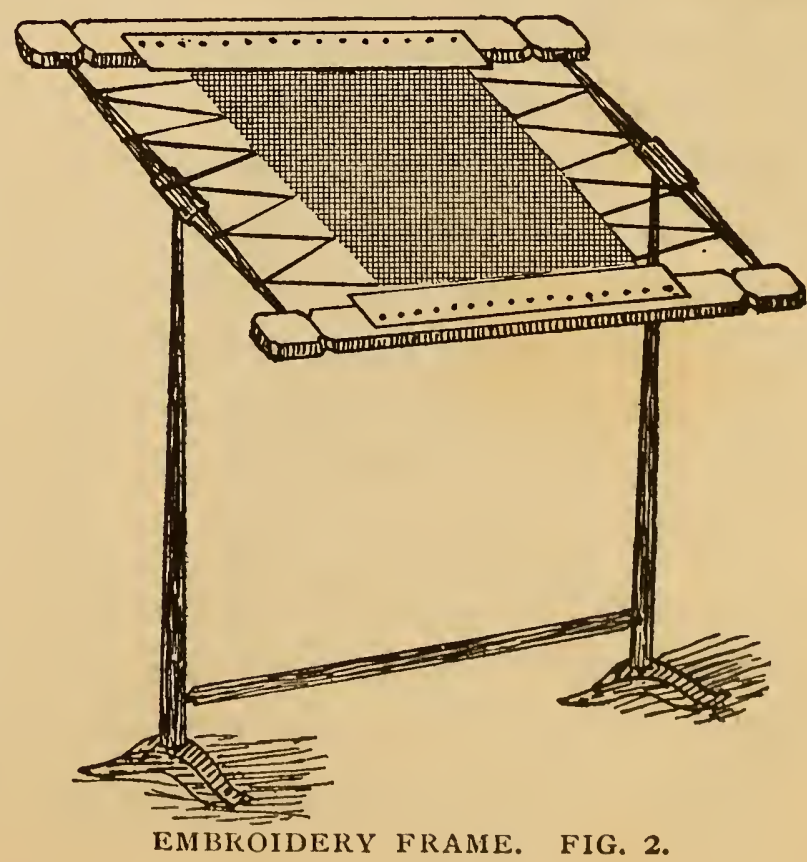


service. It is simple in the extreme, consisting of four pieces and as many wcoden pins; holes, large enough to hold the pins, are made at regular distances apart on the two side pieces, and one hole in each end of the two end pieces, after the fashion of quilt frames. This allows the work to be

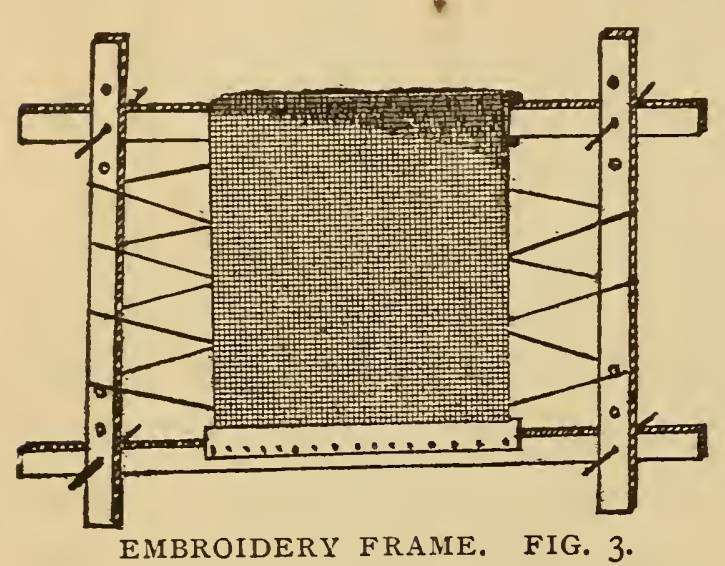
rolled out of the way as fast as finished. The end pieces of the frame need not be more than is inches long, and those for the sides 20 or 24 inches long, for convenience in handling. The top and bottom pieces of the frame should have fastened to them a double piece of bed-ticking, about two inches wide. This can be fastened to the frame by means of tacks driven quite close together in order to hold the ticking firmly in place. In putting work in the frame, sew to the ends of material a strong piece of cotton factory or tape, which is then sewn with strong thread to the ticking on the frame. It should be as tight and firm as possible, the strain being increased gradually, the sides of the material to be embroidered should then be braced to the side pieces of the frame by means of a heavy twine threaded in a coarse needle. Begin at the upper right corner by drawing the thread through the tape or cotton, and securely tie the end; the twine must then be sewn over the side piece of frame and into the side of material until the lower corner is reached, then knotted securely and cut off. The other side must be done in same manner. When the material is larger than the frame, it must be sewn on to the bars and rolled round one of them, putting tissue paper and wadding between to prevent the fabric from becoming creased. When the part in the frame is finished, it is then rolled around the opposite bar, and so on until the work is completed.

In embroidering in a frame both hands should be employed. The right hand should be used to put the needle down and the left hand to return it to the surface again. This is slow work at first, but if persevered in will amply repay the time spent in the increased speed acquired.

There are certain fabrics and kinds of embroidery that can be done over 
hoops Figure 4 shows a pair of these hoops. The work is placed smuothly over the smaller hoop, and the larger is then drawn over the whole. If the hoops do not hold the work firmly and smoothly, wind the upper hoop evenly with clean cotton cut into strips about an inch wide. It is convenient to have about three pairs of hoops of different sizes; one about four inches in diameter, for working initials, hat crowns, doyleys and other small articles; another, about six inches in diameter, for

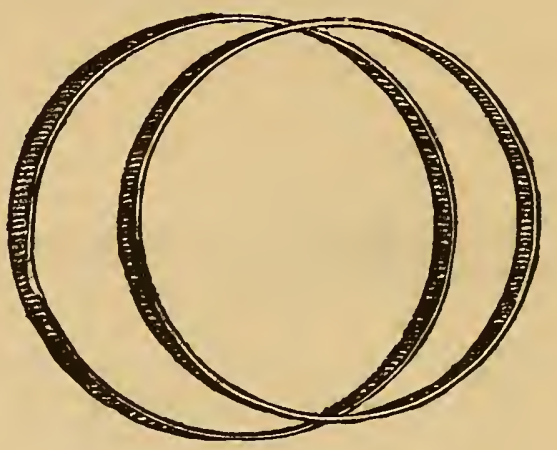

EMBROIDERY HOOPS. FIG. 6 larger work, and still another size, eight inches in diameter, for still large work, which is also a good size for doing drawn work.

The hoops and frames secured, the next item to claim attention is the matter of needles, and be sure this is a very important item in the embroiderer's outfit, as upon the use of proper needles depends much of the beauty of the work. A needle too coarse or too fine will be sure to prove a source of constant annoyance. There should be at hand a supply of needles of various sizes, both round eyed and long eyed. The kind of needle known as arrasene needles are best for carrying chenille, arrasene, ribbosene, rope silk, art silk, Bargarren linen and rope linen. They can De had in packages containing assorted sizes. The needle for working with Bargarren linen should be two sizes larger than that for carrying rope linen, and the needle for carrying wool arrasene should have a larger eye than the one employed in carrying silk arrasene or rope silk. It is better to have a needle too large than too small for such threads as Bargarren arrasene and chenille. For embroidering with etching silk, filo floss, wash twist, linen floss and embroidery cotton a round-eyed needle should be used-Millward's No. 9 for filo floss and linen floss, and No. 8 for the other threads mentioned. A long, slender needle with sharp point is better than a short, blunt needle.

A piercer and stilletto should be found in every work basket. The stilletto is used to make the holes through which the cord edging has to be taken in embroidering a pattern and for eyelet holes.

The piercer is invaluable for laying the threads in gold and silver embroidery, and in raising the work in either crewel or silk embroidery. In church 
embroidery it is used for working silk over cardboard. This little instru ment is made of steel, which is round and pointed at one end, resembling a stilletto, and flat at the other.

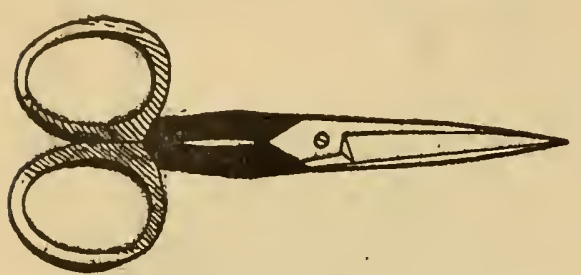

A pair of sharp-pointed scissors is an absolute necessity. Do not depend upon the family scissors or shears, if it is possible to have a pair exclusively for embroidery. The slender, sharp-pointed kind are the only ones EMBROIDERY SCISSORS. FIG. 5. suited to cutting out in Roman embroidery and applique work and for cutting ends of threads.

A pair of white sleeves to draw on over the dress sleeves and a linen apron to cover the skirt are necessary adjuncts to the toilet of the embroideress when at work, as without them the dust that naturally adheres to the dress will soil the work. For those ladies who are troubled with rough hands, a piece of fine sandpaper or pumice stone should be kept in the work basket and the fingers rubbed with it occasionally. If the hands become troublesome through perspiration, bathe them in strong alum water. We show here a model for a convenient work apron. The apron should be long enough to nearly, if not quite, cover the skirt, and the hem should be turned up on the right side to a depth of twelve inches and caught down at regular intervals from the ends, to form pockets for threads, small embroidery hoops, piercer, stilletto, and the smaller articles one uses most frequently

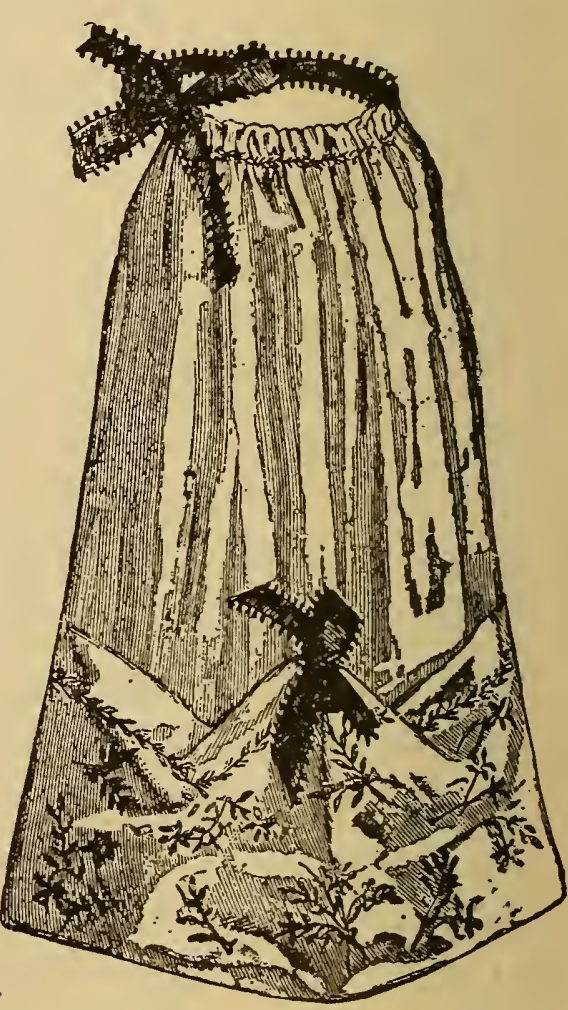

WORK APRON. FIG. 6. in embroidering. The apron can be made as ornamental as one chooses or as plain, but it is better to make it of linen, as dust will not adhere to it so readily.

So much for the implements used in embroidering. We will now consider the materials with which to embroider, and they are almost as varied as the materials to be embroidered upon. The following are in use: 
Crewels. The crewel is a strong twisted woolen yarn, not at all like eephyr, but finer and firmer. The English crewels come in lovely soft shades, which blend beautifully.

Zephyr is a soft twisted woolen yarn used on canvas worked in cross" stitch.

Tapestry Wool is an English thread, larger than the crewel, but with the same firm twist; it is used on heavy linens, tapestry cloths and friezes. It comes in dull art tones, and is much affected by the Art Societies.

Arrasenes, both silk and wool, have been very popular and are still employed in some kinds of embroidery. It resembles a very narrow piece of silk which has been ravelled out on both sides, having one thread left in the center.

Chenille is a velvety-looking round thread, made in two sizes, large and small.

Smyrnasene is a coarse, round thread, resembling chenille, but having a rough, fuzzy surface; used sometimes to work thistle leaves and begonia foliage.

Ribbosene is a narrow, crinkled ribbon, used in the needle in the same manner as arrasene. No blending of shades is possible with this mate rial. We will speak further of the method of using ribbosene under the heading of ribbon work.

Feathersene is a thread much resembling arrasene, except that the silk has been ravelled only on one edge, and the remaining threads have been left on the opposite edge instead of in the center. It is designed to sew around the outline of designs, and is not used in the needle. It is very perishable, and should not be employed upon articles that are for use rather than ornament.

Embroidery Cotton is used for French embroidery upon linens and cottons, and for initials. It comes in various colors, but red and white are the only ones recommended.

Embroidery Wash Silks, made in four sizes, as follows: Etching Silk, very fine, with a firm twist. Wash Twist, larger than etching silk, but with the same twist. Filo Floss, a soft, untwisted, fine silk. Royal Floss, a soft silk resembling filo floss, but about twice as large. Rope Silk, the largest size of silk to be used in the needle, has a slight twist. 
Couching Silk is a large, cord-like silk, but with a soft, loose twist; it is used for couching around outlines, etc.

Art Cord is a small silk cord; can be used for fancy filling stitches of certain kinds, for lacing, etc.

Real Scotch Linen Threads, made in four sizes, as follows: Bargarren Linen, the largest size made. Rope Linen, corresponding to rope silk, and the size next smaller than the Bargarren linen. Flourishing Thread No. 4, which is finer than the rope, comes next. Flourishing Thread No. 8, which is the finest of all, and corresponds to filo floss in silk, comes last.

Tyrol Cord is a twisted linen, and to be used in the same manner as couching silk.

German Cord, not art cord, is a white linen cord used for button. holing over in Roman embroidery.

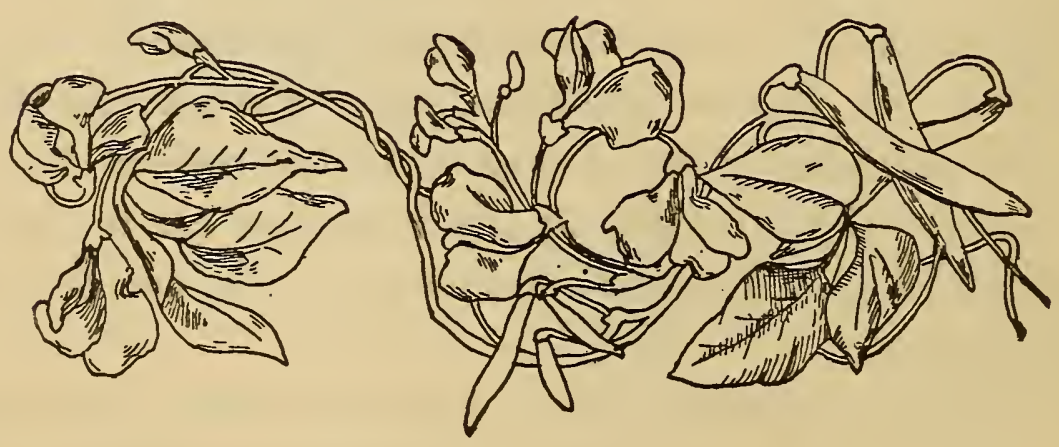


CHAPTER IV.

TRANSFERRING AND STAMPING DESIGNS.

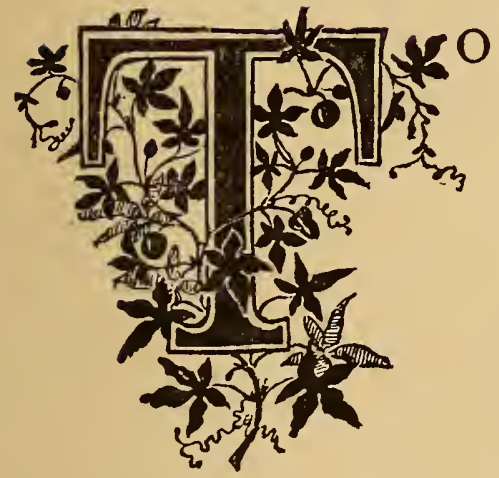

THE woman who cannot draw, the question of securing designs for embroidery is often a serious one; indeed, to some, proving an insurmountable barrier to the doing of decorative needlework.

A good design is of the utmost importance, for if the pattern be inartistic and clumsy, no amount of excellent needlework can cover the defect, and the whole will be spoiled. Perforated stamping patterns here come to our aid, and so important and remuner. ative has this industry become that the best artists are employed to furnish designs for both painting and embroidery. These designs are now furnished so cheap that the expense of patterns for embroidery has come to be a secondary consideration, and it is much better, where possible to do so, to purchase the design wanted perforated ready for stamping. However, it often happens that one wishes to use a design from a book or magazine, or even wall paper, and then it will be necessary to transfer the design to the fabric, which is done as follows:

Place over the design a sheet of tracing paper, and trace over the lines very carefully. After the design is transferred to the tracing paper, place on the table a sheet of bond paper, over this place a sheet of transfer paper, either blue or black, and over this again place the tracing paper on which you have previously traced the design, as before directed. Now, with an ivory style or a piece of wood pointed bluntly for the purpose, go over every line of the design, bearing on the lines gently and firmly. Remove the tracing and transfer papers and the design will be found transferred to the bond paper. You will now proceed to make a perforated pattern of it 
by simply pricking holes along all the lines, very close together, with a fine cambric needle. Ladies possessing a sewing machine can make this part of the process less tedious by setting into the machine the finest needle and sewing, without thread, through all the outlines of design; when finished, 'he result will be a perforated pattern as good as could have been purchased rom a regular pattern house.

The material required for transferring is: A sheet of tracing paper, costing either five or ten cents, according to size; one sheet of transfer paper, fifteen cents; these can be obtained from any art store. The transfer paper can be used any number of times until torn, when, of course, it is useless. New tracing and bond paper will be needed for each new design.

After obtaining the perforated pattern, the next thing is to stamp it. upon the material to be embroidered. And here is the rock upon which many a promising attempt has been wrecked; for if the pattern has been blurred, or unevenly joined, in case of a repeating design, then the embroideress will have need of patience, a correct eye for form and distance, and some knowledge of drawing, to enable her to remedy the defect of the stamping. Remember that a good pattern, cleanly and clearly stamped, is half the battle.

The foregoing instruction in stamping is all the knowledge at command of any dealer in fancy art supplies who advertises to do stamping; she knows no more than is here told, because there is no more to tell. Some dealers, in fact we might almost say all dealers, in art supplies make a great mystery about stamping, refusing to do it before a customer, and keeping as a profound secret the material used. This is a short-sighted policy at best, for the more women know about art embroidery, and the easier it is made for them, the more they will do, and as a natural consequence the more supplies in that line will be sold, and instead of a dealer having less trade she will probably have a hundred per cent more.

The amateur cannot expect, however, at the first or second trial to succeed in getting as good results from her efforts at stamping as a person who has made a business of it for years. There must be a certain amount uf practice to make perfect.

Many beautiful designs are frequently given in miniature, in books and magazines, that one wishes to use; but as they are too small to be practical 
we are obliged to pass them by. There is a small instrument called a pantagraph, which costs from $\$ 1.00$ to $\$ 4.00$, according to quality, by means of which any design can be reduced or enlarged to any given size. It is very simple, and not at all difficult to operate. We show here a very good

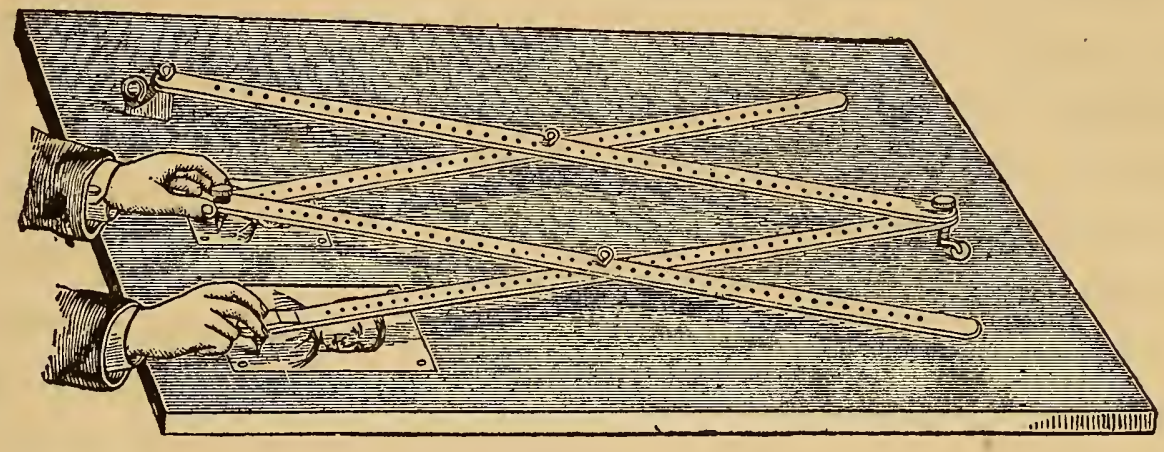

PANTAGRAPH. FIG. 7.

illustration of one set ready for working. If the design to be copied is $2 \times 5$ inches, and it is desired to make it $8 \times 20$, the pantagraph will be set to that size. In copying any design, the pattern to be copied will be placed át a given point under one pencil and a clean piece of paper under the other pencil of the pantagraph. Then, with the right hand, the pencil over the design will be moved firmly around its outlines. The corresponding pencil over the clean paper will move in unison, only on a larger or smaller scale, according as the instrument has been set. Full directions for setting and using accompany each pantagraph. These convenient aids to drawing can be purchased at any store where artists' supplies are kept. After the design has been enlarged, as directed above, the perforated pattern can be made according to instructions previously given. In the pages of this book we shall frequently give designs for different purposes, and as space forbids having them produced full working size, a pantagraph will be useful in enlarging them. Generally speaking, the designs so given will require to be enlarged five times to bring them up to working size.

\section{MATERIALS FOR STAMPING.}

The materials used for stamping are many and varied, but we have never found anything more satisfactory for general use than artists' oil paints put up in tubes-white for colored fabrics, and white with a little burnt umber mixed for white materials. There are several liquids on the market designed for the same purpose, but the great objection to them is 
their cost. The manufacture of stamping liquids is considered a trade secret, hence they cannot be made at home; and as liquids cannot be sent by mail, the cost of expressage, added to the original price, has put their use out of question with the majority of women. Liquids are more especially designed for use in stamping on white cotton or linen fabrics, and, as they dry instantly, there is no waiting for several hours before the work of cmbroidering can be commenced. Another advantage they possess over paint is the fact that the patterns need no after cleaning. Powders for stamping are now seldom used, as they will rub off before the work is half finished; yet for stamping on silk bolting, where the design is to be painted in water colors, there is nothing else that will take their place. For large designs that are to be stamped on satin, powder is best, as the oil in the paint and liquids is apt to separate and spread beyond the outlines, though in the case of paint this danger can be obviated by first placing the paint on blotting paper to absorb the oil. In case the powder is used, the design should be lightly traced over with a fine-pointed lead pencil. A good powder is made by mixing ivory black, gum demar, gum copal and gum sandarac in equal parts; pulverize fine and keep in a cool place.

A good liquid suitable for any white fabric is made by placing a teaspoonful of black or blue printer's ink in a four ounce bottle and filling the bottle with kerosene. Be careful to wipe off both sides of the pattern after each impression when using the liquid. This liquid is cheap, as any printer will cheerfully give the small amount of ink needed, and kerosene is pretty sure to be at hand, and it does beautiful stamping. A pounce for either powder or liquid is made by tying a piece of felt or flannel over a small ball of cotton wool.

\section{DIRECTIONS FOR STAMPING.}

Place the article to be stamped smoothly on the table. Next lay the design, smooth side up, on the material, being careful to adjust it exactly in the desired position before you attempt to apply the paint. Now secure the two upper corners firmly by weights-flat-irons will do nicely. Squeeze out some of the paint on a piece of glass. You are now ready to apply the paint to the pattern, which you do by means of a knife blade. Take a small amount of paint on the blade and begin at the top; rub the paint on the pattern smoothly and gently, always remembering to rub toward you and 
from the weights; always move the knife in the same direction, never rut it back and forth, as that is liable to move the pattern and cause the stamping to look blurred. Never raise the pattern entirely from the material to see how it looks, but carefully raise one corner clear from the table and look under; let fall into place again and proceed with the stamping until every detail of the pattern is transferred to the material. When all is finished, remove the pattern; have ready an old paper, place the design upon it, and pour a small quantity of benzine on a rag and rub the pattern thoroughly, then wipe with a dry cloth. Repeat the operation on the other side, and then hold the pattern to the light and observe if the perforations are all open and free from paint. If any should be still closed, add more benzine and rub until perfectly clean. Never allow a pattern to get dry before cleaning, as that would ruin it. 'Clean at once. With proper care a pattern ought to be capable of fifty impressions. Be sure it pays to take care of your designs. These directions are to be followed when using liquid, except that the pattern will not need cleaning. To stamp with powder, place the pattern on the material, rough side up, dip the pounce into the powder, shaking off the loose particles that may adhere, and rub the pounce lightly over the perforations. When the design is perfectly transferred, remove the pattern and on the stamping place a piece of tissue paper; over this press lightly a medium hot iron; this sets the powder and to a great degree prevents its rubbing off. Do not rub the iron back and forth, but lift it when removing to another part of the design. Rubbing is apt to cause the powder to run and blur.

\section{CONVENTIONAL DESIGNS.}

The words "conventional design" will frequently be used throughout the different chapters of this work, and so it will perhaps be better to explain their meaning here. To conventionalize a flower or plant means to draw the geometrical or general form of the leaf with the main details and acciderital markings omitted, thus converting the natural form into a form suitable for ornament only. The designer of ornamental work is not bound to be faithful to the minute particulars of botanic life, because he and his work are superior to the mere imitation of plant forms. 
Beautiful conventional designs can be obtained from artistic wall paper, and designs thus obtained will possess the advantage of not having become common by much use. As a general thing the designs on paper bordering lend themselves to embroidery better than those on the wall paper proper, though beautiful patterns can be obtained here, also. The border pattern should be used on curtains or other large articles, where the treatment is intended to be bold and broad. Many of these designs are also suitable for smaller articles when reduced by means of the pantagraph. To procure the design it will be necessary to purchase so much of the paper as will show a complete section of the design, which must be perforated and then repeated as many times in the stamping as will be necessary to fill the space to be decorated. Many magazines and old prints will also furnish suitable designs, as previously stated.

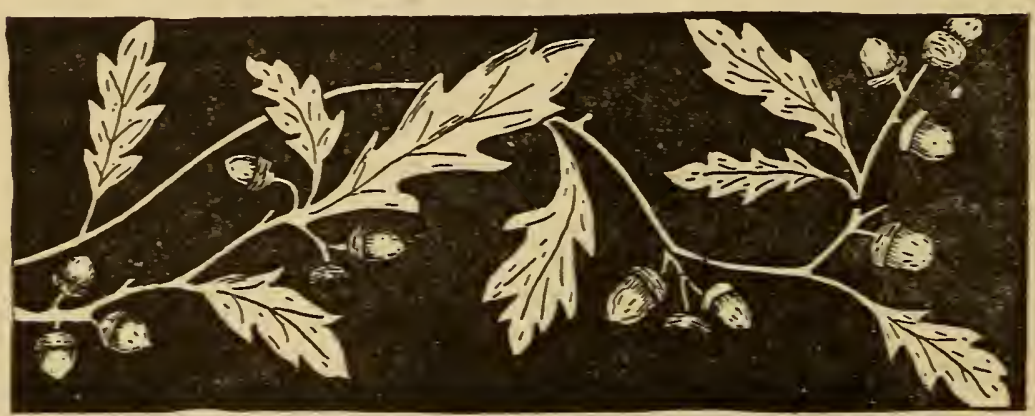




\section{CHAPTER V.}

EMBROIDERY STITCHES.

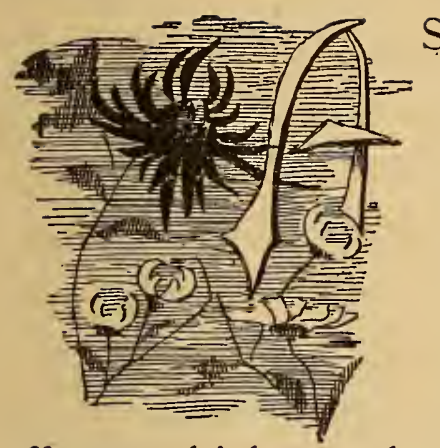

SUBJECT that occupies the attention of the beginner in embroidery, almost to the exclusion of every other idea in connection with the work, is that of stitches. This should not be the case. Supplied with the requisite materials, the first idea should be, not how to make this or that stitch, but how to combine this or that shade with some other, to produce certain artistic effects, which are clearly enough defined in the inner consciousness of the worker. So long as we are hampered with the idea of stitches, just so long will our work appear stiff and awkward. A piece of embroidery should grow and grow, without mental effort, until it has reached its final state of complete finish. The work should be but the expression of an artistic idea or conception. However, one must begin somewhere in learning needlework as well as in learning to read; and as with the latter we begin with the alphabet, learning first the letters then the combination of letters, so with the former we begin with certain fundamental stitches, which are to embroidery what the letters of the alphabet are to the reader. We use them, but we never think of them as a thing apart from the work complete, any more than we think of the letters which compose a word.

\section{STEM OR OUTLINE STITCH.}

Outline, or stem stitch, is the simplest and oldest of all the embroidery stitches, and it is also one of the most important. It is this stitch which was used on the old Bayeaux tapestry, and hundreds of years ago, in the days of the Pharoahs, it was employed in Egyptian embroidery. There is hardly any limit to the usefulness of this simple stitch, and by its use beautiful effects are easily obtained, at little expense of time and material.

There are several varieties of stem or outline stitch, and we will mention them here, as this seems the proper order in which to classify them. 
First, then, in the list is simple outline stitch, shown in figure 8 , then in figure 9 we have cording outline, close outline and twisted outline. Fig. Io

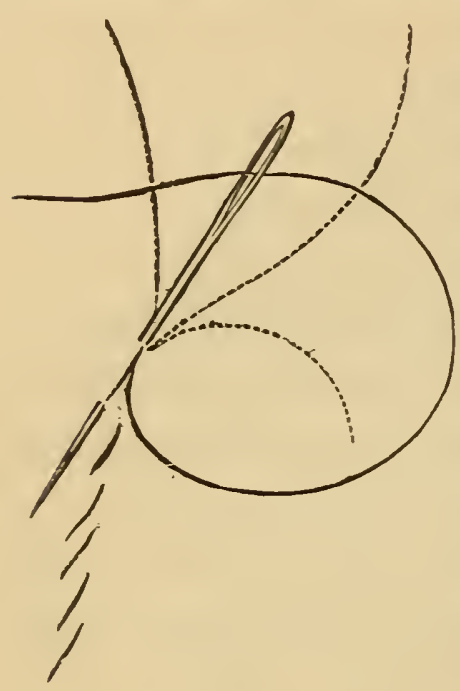
shows fancy chain outline, and figure I I double chain outline. In working outline stitch, if an even line is required, insert the needle in a straight line with the preceding stitch. See center illustration in figure 9. For the outline of leaves, where a sloping effect is desired, insert the needle at a slight angle with the preceding stitch. [See figure 8.] If a close outline, resembling a heavy cord, is desired, work the stitch over a thread; take each stitch at a slight angle, and insert needle so that its point will come to the surface about midway down the preceding stitch and close to it. The first illustration in STEM STITCH. FIG. 8. figure 9 will make this idea plain enough to be easily followed. Twisted outline is made by taking the first stitch so that the thread lies to the right of the needie; in the next stitch the needle is inserted from the opposite direction. This is repeated, alternating each time a stitch is taken; the third illustration in figure 9 shows method of working twisted outline.

Chain outline is often used to finish outlines of applique work, and for stems of foliage and stalks of flowers. Sometimes two rows are used in stalks, one a light and the other a darker shade of the same color. Single chain is made by forming a loop upon the right surface of material, through which the needle is passed upward, securing the loop. The needle is then passed down very near where it is drawn up, the left thumb holding the thread so that it cannot be drawn wholly back. Another stitch is then taken towards you,

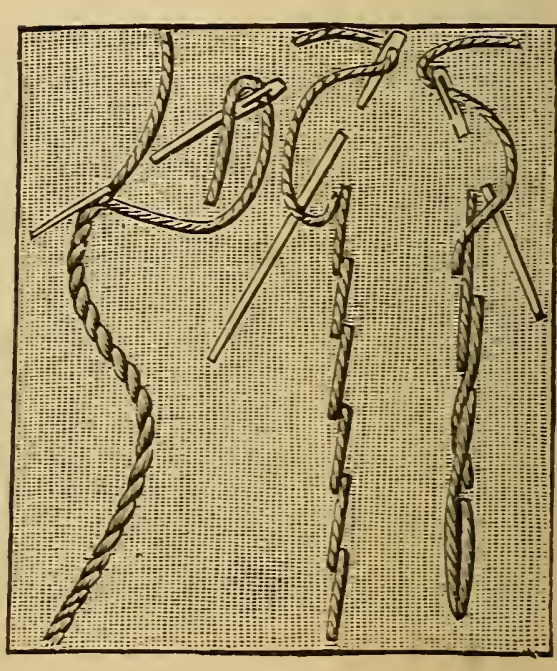

FIG. 9. inserting point of needle in preceding looped stitch; and each time a stitch is made leave loop of thread around the needle, as in taking a button hole stitch; this forms a link in the chain stitch. Double 
chain stitch is made in the same manner as single chain only the thread is twisted by the needle being inserted in a slanting direction.

Variations of single and double chain stitches are shown in figures 9 and II. The loops that form the chain in figure I I are not made in a direct

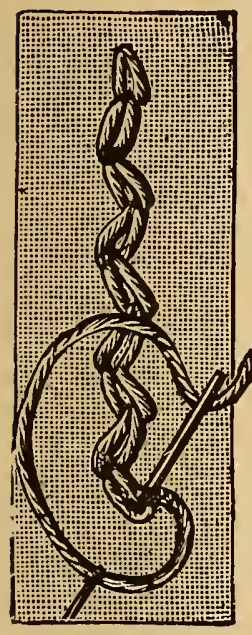

FIG. II. line, but slant, first to the right and then to the left. Figure Io shows a chain stitch in the center, with loops to the right and left. The center of this stitch is made according to directions given for chain outline, and the side loops are taken from right to left. These varieties are used to finish hems, join borders and as fancy joining stitches for rugs and crazy quilts.

In beginning the stalk of a flower, when the work is to be solid outline stitch, begin at the lower end of stalk and work upward and away from you,-see figure 8- until the junction of a leaf or some other interrupting point is met; then take the needle under to the other line and work back, continuing in this way until the stem is solid. Always work the stalk of a plant or flower lengthwise. Reaching a leaf you make use of the same stitch to work around the right side to the top, taking care that the needle is to the left of the thread as you draw it out. When the point of the leaf is gained reverse the operation by working down the left side toward the stalk again, keeping the needle to the right of the thread instead of to the left, as in going up. This method supplies the necessary serration to the edฐe.

\section{KENSINGTON STITCH.}

The next stitch to be considered here is the "Kensington," the proper name for which is "feather stitch;" but as

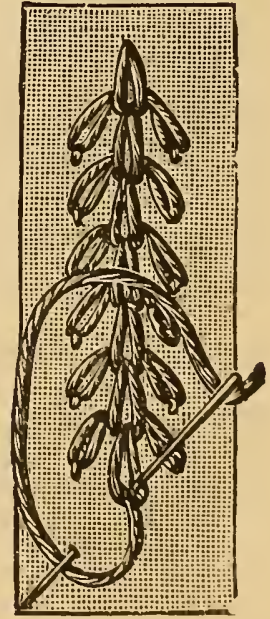

FIG. ro. the public have chosen to christen it "Kensington," from the fact that it was taught and used in the Royal Art Schools of Kensington, England, we think it better in these pages to do the same, for as "Kensington" the stitch is familiar to thousands. while as "feather" very few would recognize it. Figure 12 shows section of leaf begun in Kensington stitch. In working, care must be taken to keep the outside edge of design smooth and 
even. Do not have all of the stitches of regular lengths, but have some of them long, and some short, with odd lengths to fill unexpected spaces. Figure I3 shows portion of flower worked; the direction of stitches and mode of blending shades are here clearly indicated. Kensington effects are introduced in most of the conventional art needlework, though less solid embroidery in natural shadings in the above stitch is now done than at any time since it first became popular.

Floral designs of natural shape and size look better when worked in either solid Kensington or

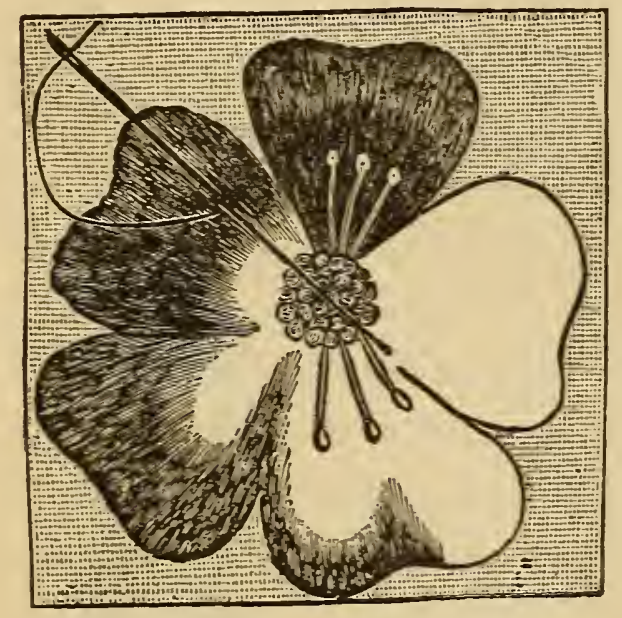

FIG. I3. long and short stitch, which is

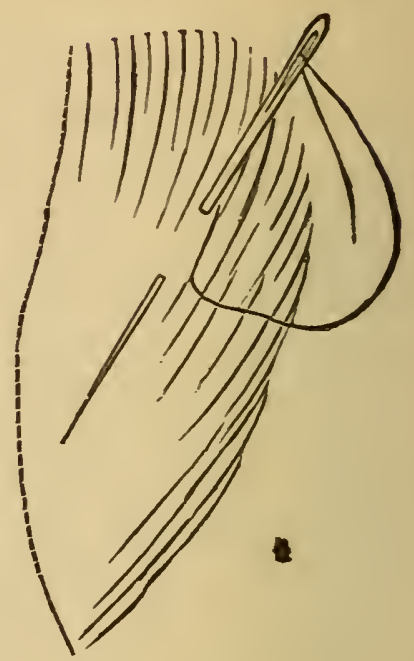

FIG. I2. but a modification of the former. All flowers having large petals and leaves are especially effective wrought in solid Kensington stitch. In a design having a number of small leaves satin stitch can be happily combined with the Kensington. In this case the small leaves only would be worked in the satin stitch. Solid Kensington is not so well adapted for working out large conventional scrolls, but should rather be confined to natural floral designs in which natural shading is desired, as indicated above.

\section{SHORT AND LONG STITCH.}

Short and long stitch is another mode of working that is largely used, and consists of the same stitch taken three times side by side, but in different lengths, keeping the outline firm and even. It is a modification of Kensington, and called by some the half-Kensington stitch. There is this difference, however, it is taken through the material, like a satin stitch, leaving as much silk on the wrong side as is shown on the right. The 
length of the longest stitch depends upon the size of leaf or petal you are working; if a wild rose petal is in process of construction the entire petal should not be worked with stitches longer than onequarter of its entire depth. Begin in center of topedge of petal, take a regular over stitch to the depth desired; take another back to top edge, but a little shorter than the first; the third is shorter still; then the three stitches are repeated, and so on. Work from center top edge of petal to the right, then go back to center and work

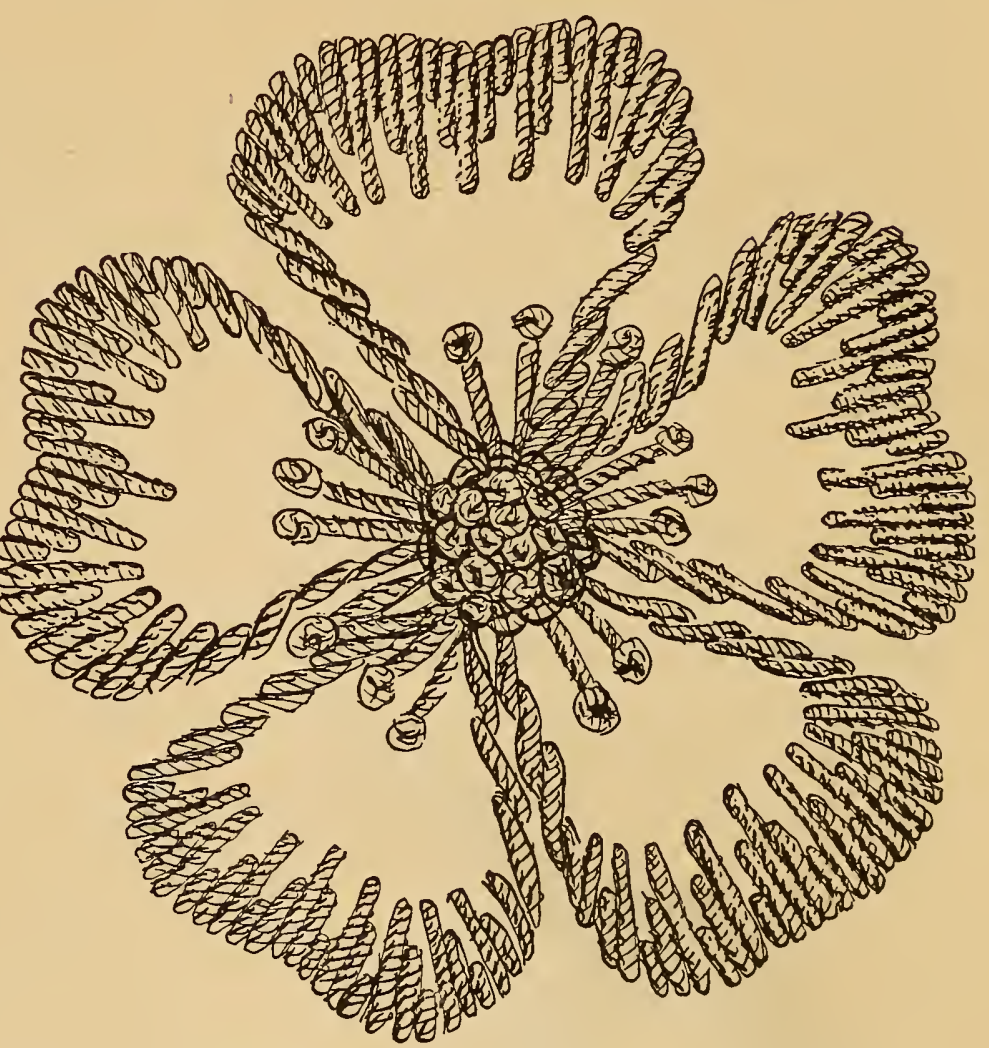

SHORT AND LONG STITCH. FIG. I4. to the left. This gives a better effect than where the work is begun at one side of petal and carried to the opposite side. The irregularity of the stitches must be made at inner part of petal or leaf; the outside edge must be kept even and smooth.

The above stitch is specially suited to thin fabrics and where a light and dainty effect is to be secured, but it is used on both light and heavy materials with good effect. With the outline stitches and the Kensington and half-Kensington stitches thoroughly mastered an almost unlimited amount and variety of decorative needlework is within your reach. In fact, the greater amount of embroidery shown at the rooms of Decorative Art Societies in this country and England is wrought with the above-mentioned stitches alone.

\section{SATIN STITCH.}

Satin stitch is that used in all the old French embroidery on silk and fiannel. It is also sometimes effectively combined with the Kensington 
stitch, especially in the case of small leaves and flowers. It is also used alone and in combination with outline and dotted stitch for initials. Satin stitch is made by passing a thread from one outline of design to the other, back and forth, as shown in Figure I5, leaving as much material on the wrong as on the right side of the work. The pattern should be first run with filling stitches, to give the work, when finished, a slightly raised and rounded effect. In working a leaf that has a strongly veined center, work from one outside

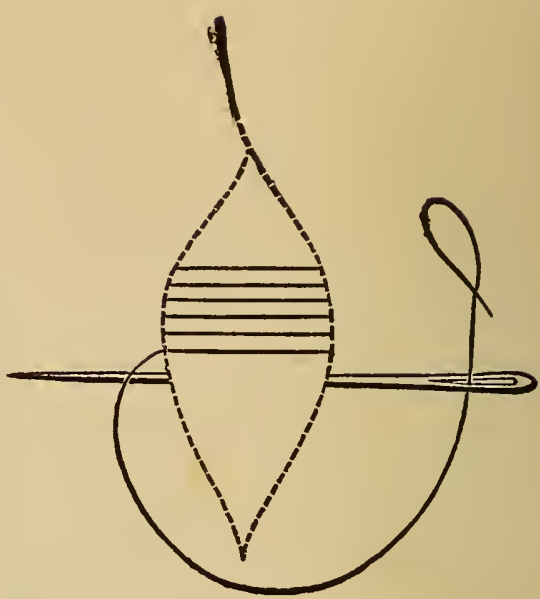

FIG. I5.

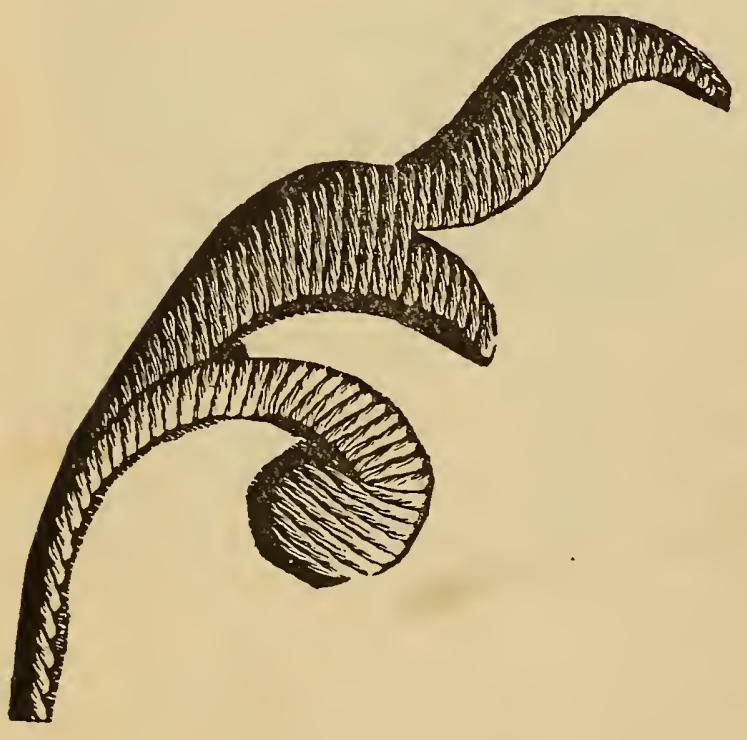

FIG. I6.

edge of leaf to center of leaf at an angle, then from the opposite outside edge to the center again. This method makes the veining sufficiently plain.

Figure 15 is designed to show the manner of taking the stitch rather than how it is to look when finished. The surface of the satin stitch should present a smooth, compact and even appearance. Figures 16 and 17 give a better idea of satin stitch when finished.

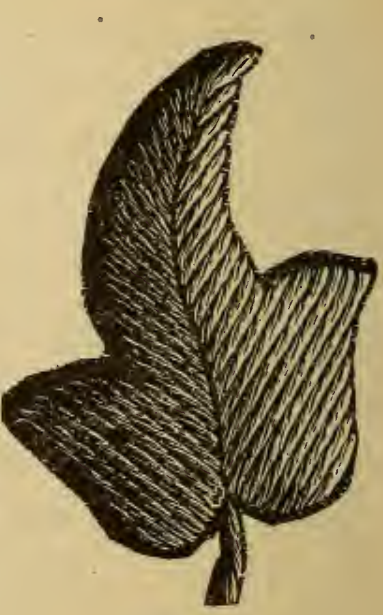

FIG. 17.

\section{BUTTONHOLE STITCH.}

This stitch is used in working scallops in French embroidery on silk, flannel and cotton fabrics, and also to finish outlines on Roman and applique embroidery.

In using buttonhole stitch to work scalloped edges, the point or scal, lop must first be carefully stamped or traced upon the material, after which 
two rows of stitches should be 1 un in to define both upper and under edge

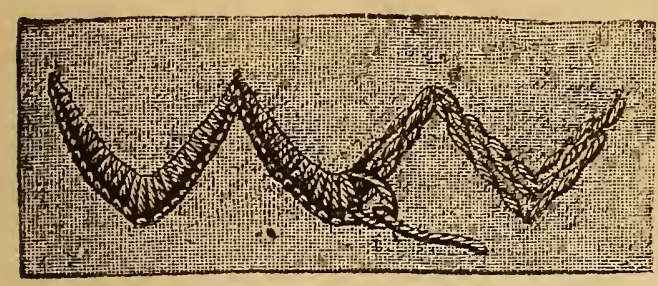

FIG. I8. of scallop. It is well to then fill in between these two rows of stitches with another row or rows, according to depth of scallop, of long loose stitches of cotton. This will give the scallop a heavy, rich and raised effect, besides adding to the durability of the edge. Figure I8 will show method of defining edge of scallop, also method of filling in between.

Figure I9 shows method of working buttonhole stitch as a simple outline. It will be seen that the needle should pass through the material just at the edge of the top thread, coming out under the lower line, while the thread is held by the left thumb, a little to the right of where the needle is

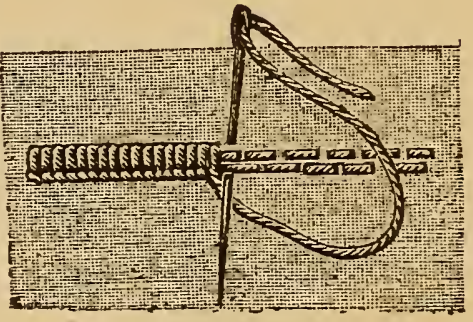

FIG. I9. to come through, so that as the needle is drawn up a loop is formed which fastens itself. In working buttonhole outline for Roman embroidery the stitch is usually worked over a German cord; and in articles where the material is to be cat away from the edge the buttonhole short and long stitch is effectively used. This has the same effect as the short and long stitch previously shown, except that the edge is buttonhole stitch. Figure I9 shows this stitch and method of working.

\section{SPOT STITCH.}

Spot stitch, like buttonhole and satin stitch, is used in French embroidery, and is made by working over and over, with regular satin stitch,

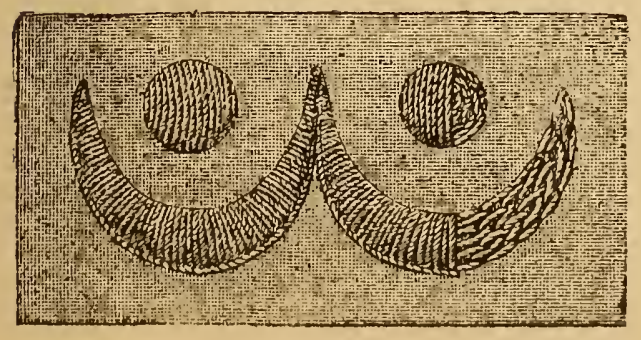

FIG. 20. the stamped circie or spot. It is better to begin in the middle of one edge of spot, and work one-half the circle, then return to the center and work the remaining portion of the circle. If the spot is to be slightly raised it will be necessary to work it over twice, in reverse directions. The unfinished spot in figure 20 will give the correct idea for working 


\section{ANTIQUE EMBROIDERY.}

This illustration gives a stitch used in heavy embroidery and, unlike

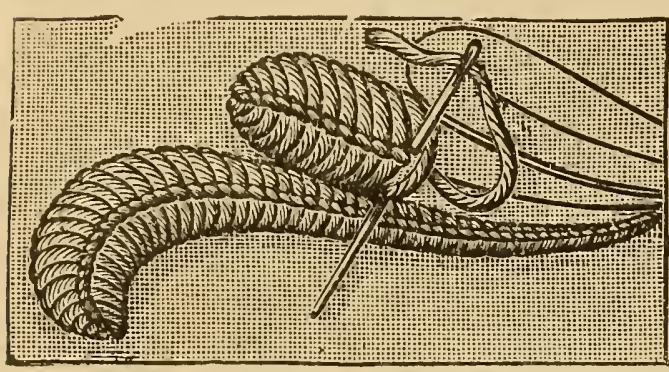

FIG. 2 I. satin stitch, the veins of the leaves are outlined while working the stitch. The cut shows very clearly the manner of forming the stitch, which is worked upon an outline of stem stitches, the vein also being defined in the same way. This work is much used in borders of a conventional design, where a figure or scroll-work is to be made more prominent than the rest of the pattern, and an outline of some fancy stitch, like couching or chain, is often placed around the figure.

\section{PERSIAN EMBROIDERY.}

Persian embroidery is a showy stitch, much resembling braiding. The material used may be either a strand of tapestry wool or three strands of rope silk threaded in the needle. It is a great addition to designs in applique, the section here shown representing a small portion of a palm leaf. This work is introducedin mantellambrequins, portieres, table covers, scarf-tidies, and in decorations of this work small gilt beads, span-

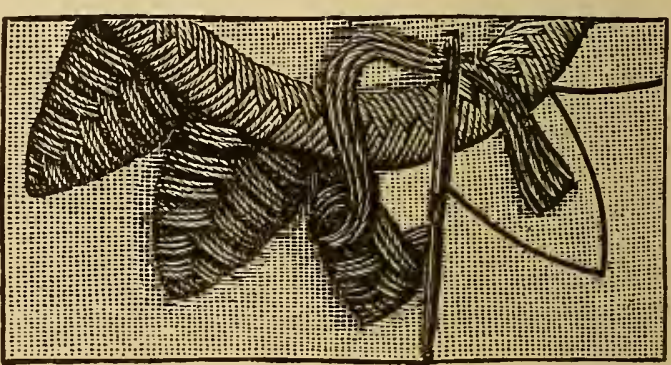

FIG. 22. gles and gold thread are very appropriate additions. A heavy cording can be made with this stitch.

\section{FRENCH KNOT.}

This stitch has the merit of great antiquity, app saring in early ecclesiastical embroideries to represent the hair of "men and asgels," as well as

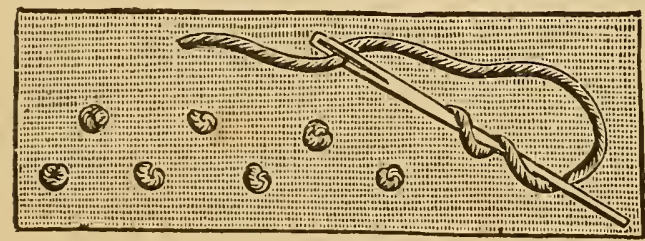

FIG. 23. in elaborate landscape specimens of the time of James I, when it came into play for the foliage of trees and shrubs; also, in some ancient Chinese embroidery executed entirely in knots skillfully disposed. Describing certain examples of the latter, a recent writer observes that the design appears to have been printed in flat, low colors on a cotton fabric, and that 
over the ground thus diversified are worked knots of silk, which have the effect, at a short distance, of a stippled drawing on a large scale. The Hesh in the figures and some other portions of the design are. worked entirely in close knot. To-day French knot is of greatest importance in fine work; it is almost always used in combination, seldom alone. As it is such an important member of our family of stitches it should be thoroughly and carefully practiced. Our illustration shows how it should look when finished rather than the mode of working it. The following will be readily understood: Bring the needle to the surface of the material from the wrong side; bring it up in the exact spot where the knot is to be when finished. Take the needle in the right hand, hold the thread firmly in the left in a straight line from you, then twist the thread around the needle three times, now put the needle down exactly through the place where it was brought up, still keeping hold of the thread in left hand until all the slack thread has been drawn through, then release the thread and pull through with the right hand simultaneously. This completes the knot, which should present a compact and smooth appearance. This stitch is used for filling center of flowers, for underlaying raised work, for golden rod, thistles and cat-tails, on wash goods and also in initials.

\section{COUCHING STITCHES.}

Couching stitch is very simple, but its uses are many and varied, and it is quite as important in our alphabet of embroidery as the letter "a" in

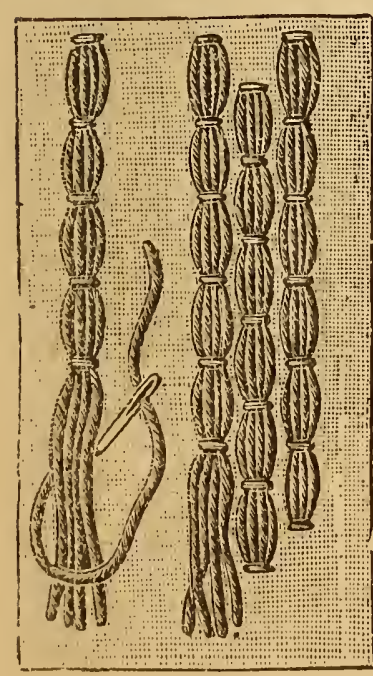

FIG. 25. the alphabet of our language, and must be learned. While very simple, it is yet one of the stitches that require our utmost care in the making, as all its beauty depends upon the neatness with which it is wrought. The simplest use to which this stitch is put is to couch, or fasten down, Tyrol cord, art cord or couching silk, to form outline of design-see figure 24 ; also, to couch down gold thread on church work,

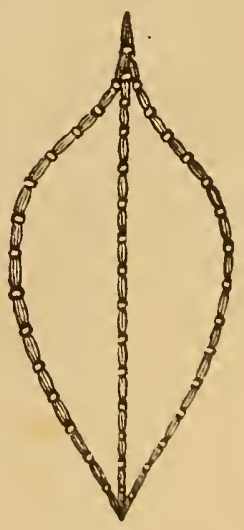

FIG. 24 . and to form patterns by means of laying straight lines of silk and couching over them at intervals with silk of another color or shade. The couching can be done straight 
over the laid threads; that is, the needle will be brought up on one sidc of the thread to be fastened down, and put down exactly opposite on the other side of thread. By this means the thread is secured to surface of material; but the fastening or couching stitch must be placed straight over the thread to be so fastened, as a little slant to one side or the other would ruin the effect. Figure 25 shows the idea we wish to convey. Another method of fastening the couching silk, now so popular, is to use etching silk or wash twist a shade lighter, and with it form couching stitches diagonally across the couching silk, taking care to place a stitch into every twist of the silk you are couching down. The effect is beautiful.

\section{HEMSTITCHING.}

Hemstitching is at once the most elegant and useful accomplishment of the needle worker. The ends of towels, sheets and pillow slips, as well as articles of mere ornament are, or should be, finished with a row of hem stitching. This is not one of the things that can be left to personal choice, for custom has decreed that the refined housekeeper shall at least have the hems of her best table and bed linen hemstitched, even if they are guilt-

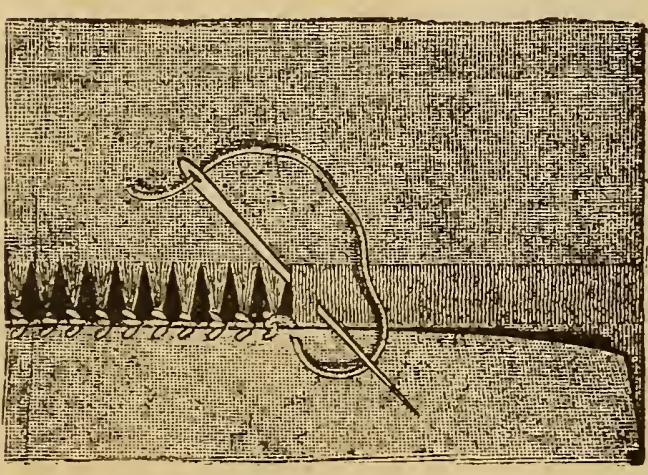

FIG. 26. less of every other form of art needlework. There are many methods of hemstitching, but of the many we find the following gives most satisfactory results: Measure the depth you wish the hem, and then at the point where the edge of hem will come when turned and basted, pull two or three threads; turn the hem, and beginning at the left edge of work pass the needle around two or three of the threads, having the point of the needle to the left, then reverse the needle so that it will point to the right, and catch the edge of hem; repeat this until the hem is completed. If both edges of the drawn threads are to be hemstitched, repeat the operation on the opposite edge. Figure 26 shows method of wrorking, and as it is very simple can be easily fo'lowed. 


\section{SPANISH LAID WORK.}

This style of work is very beautiful for working borders to center clotk for stand, for scarf ends and for cushion covers, and is an application of couching stitches. Figure 27 shows the manner of working border $i_{i}$ Spanish laid work. For this purpose either a plain border or large scallop

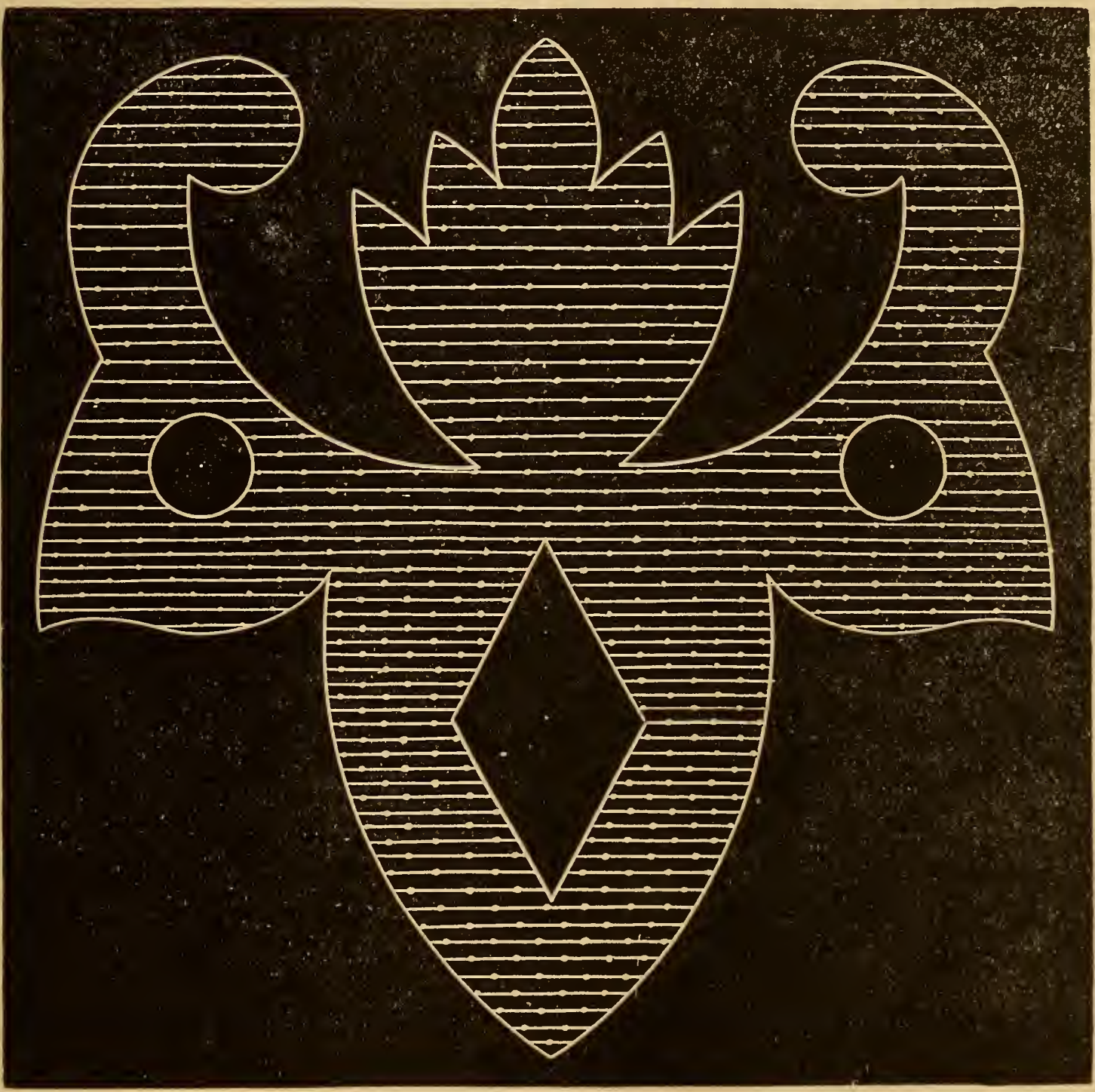

SPANISH LAID WORK. FIG. 27

is used. For our purpose we have selected the scalloped edge. The scallop is first filled with either silk or linen threads, laid straight across from one outline to the outline opposite, being careful to carry them across in a perfectly straight line; place the threads so close together that the surface of material does not show, yet be careful not to crowd them, one upon 
another. If the work is being done upon a white linen or cotton wash fabric, then for the straight lines use white Bargarren art linen. Thread an arrasene needle with a strand of the Bargarren art thread, bring the needle

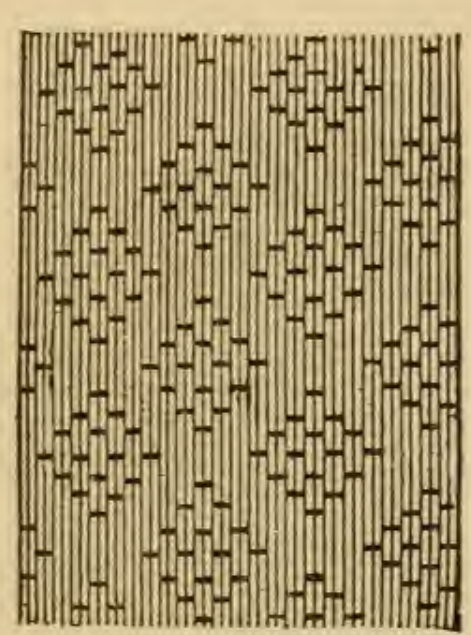

FIG. 28. up to the surface at the right side of scallop, clos to the point. Carry across and put the needle down through the opposite outline; bring needle up on same side, about one-thirty-second of an inch from where it was passed down, and carry it across to the right outline and put down; repeat this until the surface is filled; this will leave all the threads on the right side, and none on the wrong, except the tiny stitch made on the outlines by putting the needle up and down.

After all the lines are evenly and perfectly laid over the surface of pattern, thread a No. \& round-eyed needle with etching silk; begin at the point of scallop and couch one stitch over the first laid line, exactly in the center; then over the next laid line couch two stitches. Be careful to observe that no two couching stitches come exactly opposite each other, but come in alternate spaces. The couching thread may be either white or any color preferred. If fancy couching stitches

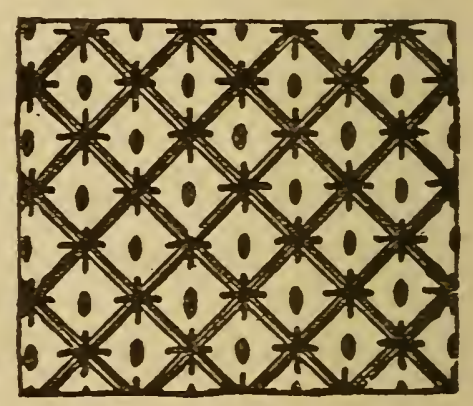

FIG. 29.

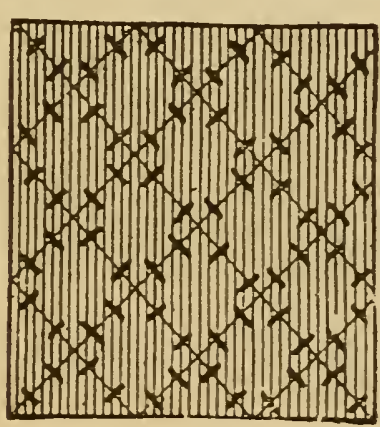

FIG. 30. should be preferred to those shown in figure 27 figures 28, 29 and 30 will offer a pleasing variety. The threads that are to be couched over should in all cases be larger than that used for the couching. The outline of scallop should then be finished with a buttonhole stitch worked over a German cord; and the diamond and oval spaces should be filled with either wheel stitch or spider web; both these stitches being also used in drawn work they will be found fully described in the chapter devoted to that subject. The material should be cut away from back of the wheel or spider web. 


\section{PLUSH STITCH.}

Plush stitch is used for raising portions of certain designs above the surface of material and is employed principally in working raised cat-tail sumac, goldenrod, coxcomb and flowers of a similar nature. A foundation is first made for the plush stitch by means of French knots made of zephyr or yarn, which are worked over the portion of flower to be raised; they should be placed quite close together, and yet should not be crowded; of course in placing the knots care must be taken to follow and preserve the outline of design perfectly. For making the plush stitch, rope silk or filo floss should be used. Cut the skeins of silk into equal lengths, according to the height you desire to raise the flower; after the silk is cut, place between each French knot two of the strands, crossed like an X, sew them firmly down to foundation and proceed to fill in each space between the knots. If the flower requires to be very thick and velvety, instead of crossing two lengths of the cut skein, double the lengths, using four instead of two pieces between each French knot. After the flower is completed whisk the silk out with a brush broom and clip off evenly. Plush stitch is used in raised embroidery only.

\section{FILLING STITCHES.}

Filling stitches, so called because they are used to fill in the surface of flower petals and foliage and the surface of conventional designs, which have their outlines worked with short and long stitch, outline, or buttonhole short and long stitch. This method of embroidery belongs to the old Saxon days, and in fact used to be called Saxon embroidery. The stitches used for filling are numerous and we will give a slight description of those most commonly employed, with a few illustrations which will be of assistance in copying them.

\section{Honey-comb and Brick Stitches.}

First on the list of filling stitches in point of general utility is the honey-comb, and while this stitch cannot be called strictly a filling stitch, from the fact that it is often used to cover backgrounds on which a design is worked in relief, yet it is more commonly used as a filling for petals and leaves and for circles and wide scrolls in conventional designs, and is 
therefore entitled tc a place in this classification. Honey-comb stitch is shown in detail in figure 32 , while figure 33 shows the stitch applied to filling pansy petal, after the edge has first been cutlined with the button-

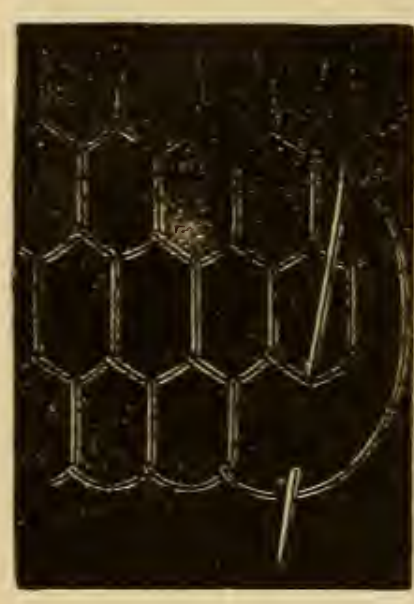

FIG. 32 .

hole stitch, each petal being treated in the same manner. The method of working this stitch will be seen from figure

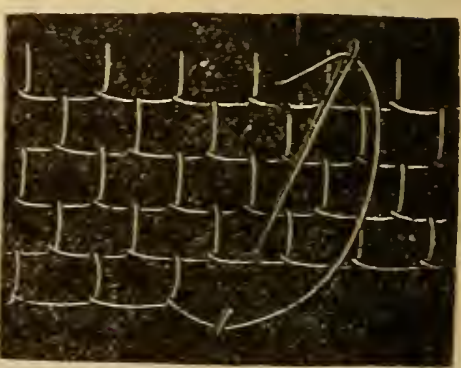

FIG. 3 I. 32. It is first, a row of buttonhole stitches placed at regular intervals apart; the second row is taken into the bottom of the first row, and so on. Pulling each stitch down in the center as it is made, is what gives the honey-comb effect. It is very effective when used for filling surface of large leaves.

Brick stitch is made in the same manner as the honey-comb, except that the threads are not pulled down in the center, as the stitch is completed, but the thread is allowed to remain straight, showing a ver. tical line over a horizontal. Figure 31 will show at once how the stitch should look when finished and in process of construction. Brick stitch can be employed for any of the purposes for which the honey-comb is suitable.

Brick and honey-comb stitches are also frequently used to cover the design in Roman embroidery and add much to the richness of the work. For this purpose the filo floss or Royal floss silk can

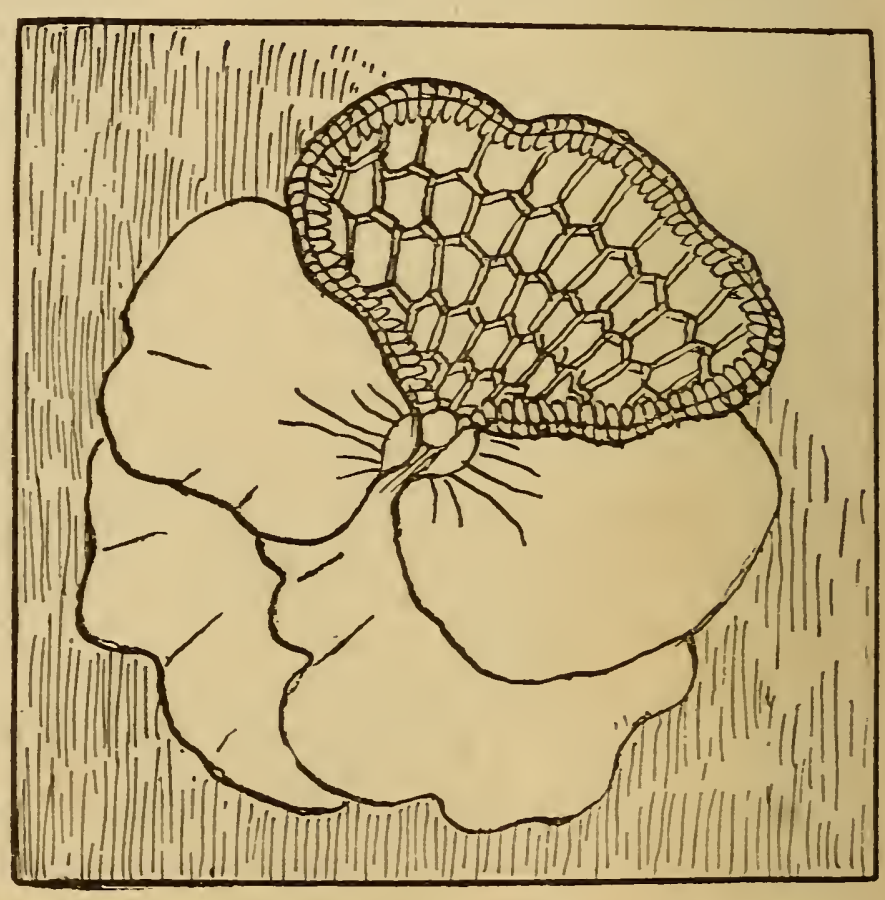

HONEY-COMB STITCH. FIG. 33. be employed, especially if a light, soft effect is sought, though where it is desired to have the work stand out in relief etching silk should•be used. 
Simple Star, Daisy Star, and Crowsfeet.

The three stitches named above are used for filling purposes, and as they are easily made and require but a small amount of material, are much used where large surfaces are to be covered.

Figures 34,35 and 36 will show clearly the mode of working. The simple star, figure 34 , is made by starting from a center and carrying out

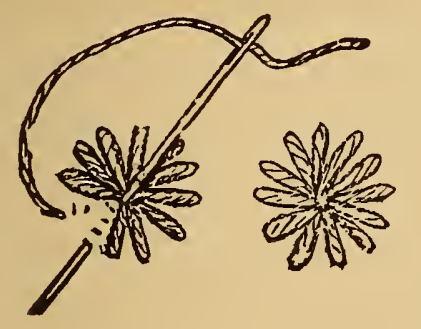

SIMPLE STAR. FIG. 34 .

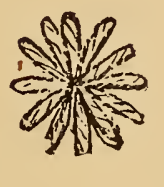

DAISY STAR. FIG. 35 .

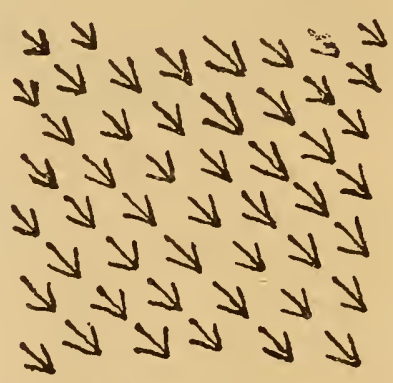

CROWSFEET. FIG. 36 .

from it six or seven radiating lines, bringing the point of needle back to center each time a stitch is taken. The daisy star, figure 35 , is made by forming four daisy loops as shown in illustration and then throwing out between each of these loops a single stitch, shorter than the looped stitch. A daisy stitch is simply a buttonhole stitch taken with a long narrow loop and fastened at the loop end. In making the crowsfeet, figure 36 , the center line is made first and then a shorter one on each side at a slight angle.

\section{Grouped Star Stitches.}

The two group stitches here shown are modifications of star stitches, and as the illustration shows the manner of placing them we will simply say

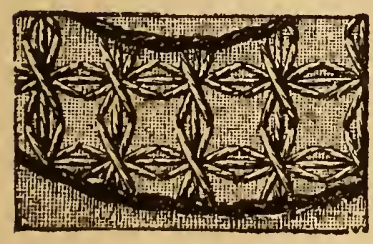

FIG. 37 . here that the two figures, 37 and 38 can be effectively used to fill space between outlines of scrolls and for small surfaces; also, for stems and stalks of conventionalized foliage, where khe space between the two

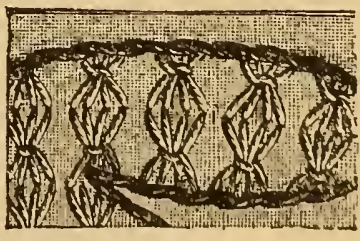

F1G. 38 . outlines is so great as to require something more than the outline stitch.

\section{Darning Stitches.}

Darning applied to decorative ncedlework produces beautiful results, and as the work is simple, quickly accomplished and little trying to the eye. 
sight, it is a popular method of embroidering. Darning stitches are used as a means of producing a decorative background for embroidery done in outline or short and long stitch; they are also used to fill the petals and

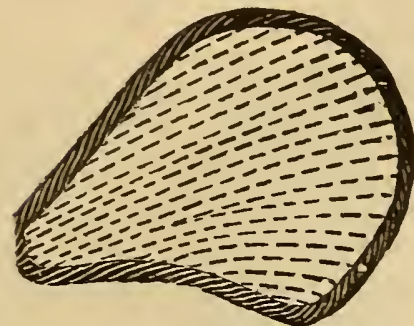

FIG. 39. foliage of designs. In figure 39 we show a leaf filled in with darning; the outline of leaf being treated with outline stitch, which throws the darned work into relief. The ordinary darning or running stitch is here used, care being observed to take just as little of the material on the needle as possible and to keep the stitches even in length. Be sure to alternate the stitches; that is, bring a stitch showing the silk or linen thread opposite a stitch formed of the material that was taken up on the needle in running or darning in the preceding row. In darning, follow the general curve of leaf or petal; when the petal forms a smaller point at the base, where it joins the calyx, the effect of shading is secured by the rows of darning coming closer together. This stitch is also used to darn background of cushions and other articles made of huck-a-buck, concordia canvas and Bargarren art cloth. See figure 40. The length of the stitch can be suited to the worker's fancy and the nature of the material.

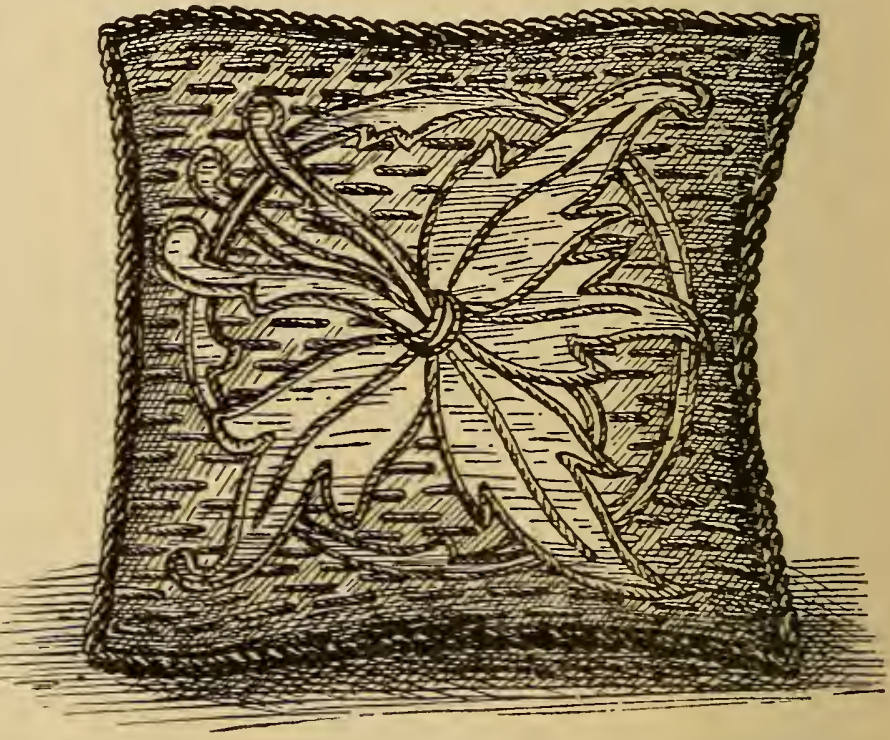

FIG. 40.

Where the entire background is darned, the design is first worked in either short and long stitch or outline.

The effect of darning leaves, stems and flowers with one strand of filo floss on bolting silk, and the whole design qutlined with the same silk, must be seen to be appreciated; anything more dainty and fairy-like cannot be conceived. For this style of work two shades of each color used will be needed, one for the darning and one for the outline stitch that is used to outline all petals, leaves and stems. Use the darker of the tw.. shades for outlining. 


\section{Janina Stitches.}

Different effects can be obtained with this stitch, as will be seen by referring to figures $4 \mathrm{I}$ and 42 . Figure $4 \mathrm{I}$ shows the stitch taken across the space to be filled from edge to edge; while figure 42 shows the stitch taken from each outline to the center of space; this presents a lighter and more open effect. Janina stitch is suitably used on sheer material like bolting silk, muslin and lawn. As will be seen, this stitch is worked entirely on the surface, except where the short back stitch occurs along the outline. To work: begin at top of leaf and pull up the needle from the wrong side, exactly in the center of the point; carry the thread to the right side of leaf nearly opposite to where it came up, and in the exact outline take a tiny backstitch, cross the thread to the left outline and in an exact

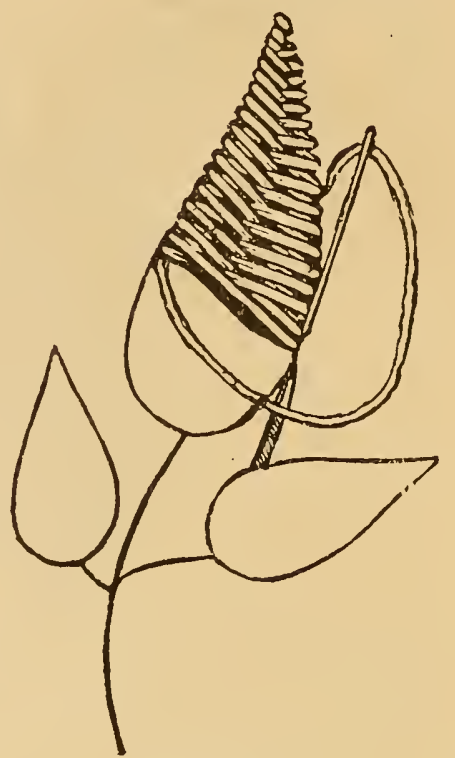

FIG. 4 I. line with the first stitch, take another backstitch, cross to the right edge again and repeat, taking care to pull the thread up through the hole

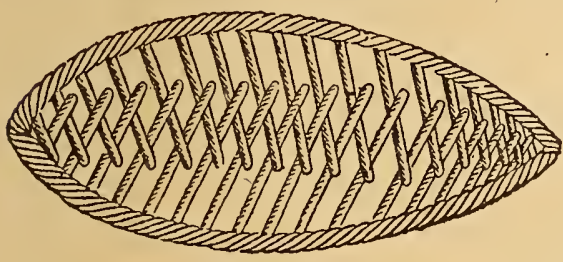

FIG. 42 . made in making the preceding stitch on that side of leaf; repeat this crossing from right to left, until the base of the leaf is reached when the thread must be fastened invisibly at the side. To work figure 42 the same method is employed except that each stitch is carried from the side to the center.

\section{Single and Double Basket Stitch.}

To work double basket stitch, see figure 44, lay straight lines across the surface of material, from one outline to the one opposite, at regular distances apart; with silk a shade lighter, but of the same color, begin at the top of leaf and over the first straight line work two basket stitches at an equal distance apart as follows: put the needle up through the material at the bottom of the first line, carry the thread across the second line and put the necdle down close to the cross line; repeat this three times, placing the threads close together, this constitutes onc basket stitch. Now, between the two 
basket stitches, worked over the first and second lines, carry another over the second and third, then over the third and fourth, and so on until space is

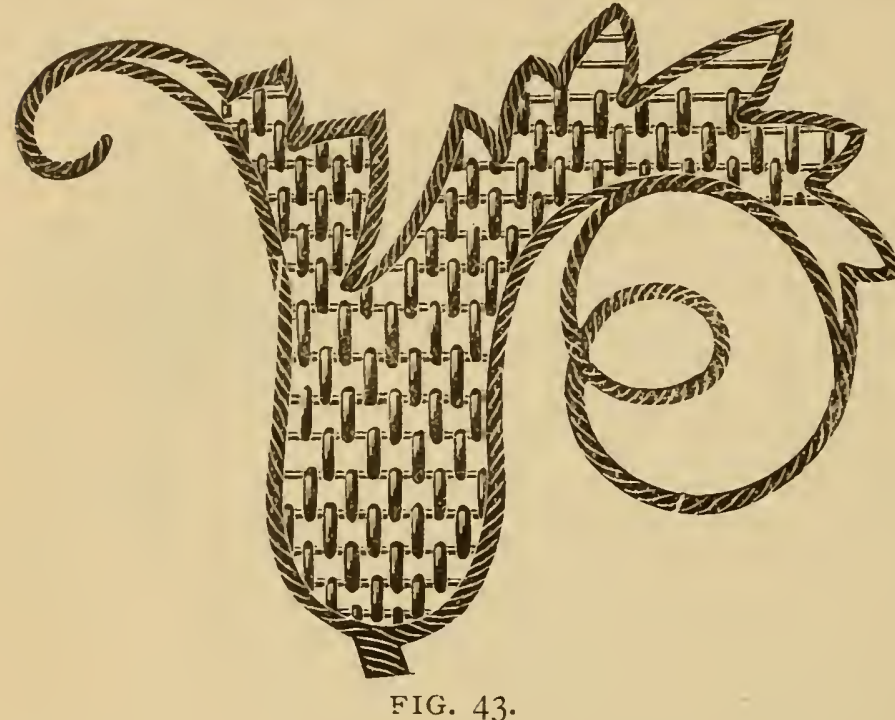

filled. The outline of leaf may be finished with heavy outline stitch, and the leaf be also veined down the center, but this is a matter of fancy. Figure 43 shows a conventionalized - lily worked over in single basket stitch, which is wrought in the same manner as above described except that instead of carrying over the lines three threads close together a single thread is used, and the outline is finished by a row of art cord sewed down with invisible stitches.

Close Basket Stitch.

This stitch is rich and effective when applied to large unbroken spacss

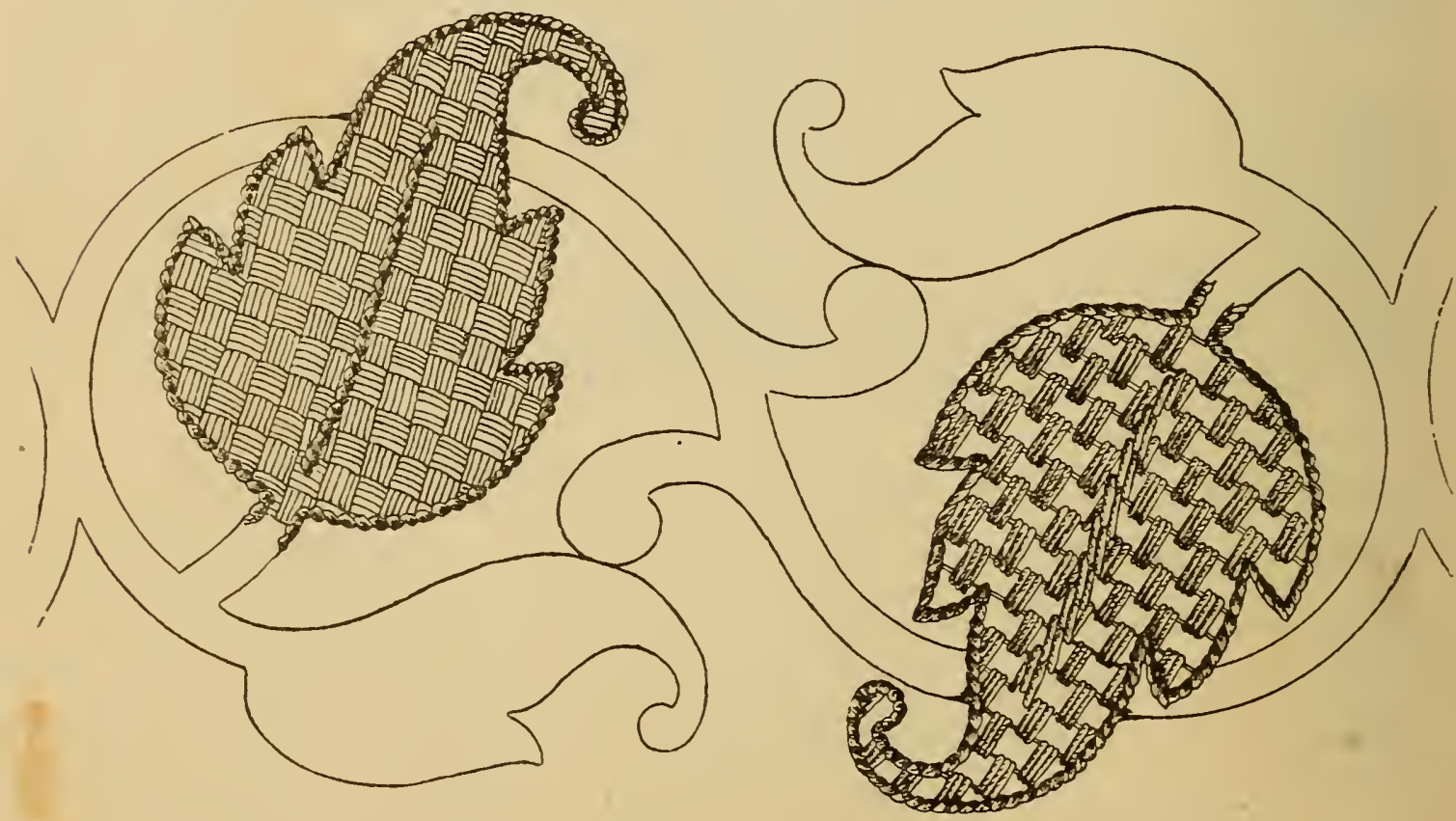

CLOSE BASKET STITCH. FIG. 44.

DOUBLE BASKET STITCH.

namely: ovals, broad or long pointed leaves without many serations, and portions of conventionalized designs that need accentuation by a heavy 
nassed effect of color. The materials for working close basket stitch depend upon the background; if that be of heavy fabric, then rope silk and art cord will be most suitable; if, however, it is desired to ornament portions Jf design on linen or light satin or silk, then wash twist will answer the purpose better. At all events the silk used should have a slight twist. To work: first lay the darker of the two shades of rope silk evenly and close together from one edge of outline to the other, back and forth; be sure that the threads lie evenly, side by side. With the next lighter shade of silk weave over and under, in basket darning, crossing four strands of silk each time. The weaving is done with four strands of silk, one at a time.

Figure 44 shows double basket and close basket stitch applied to a scroll design. The leaf to the left shows close basket, the leaf to the right shows double basket. In this design the outlines are finished with art cord and the center of leaves are finished with outline stitches or art cord sewed down with invisible stitches. Figure 44 shows both methods.

\section{D'Alencon Bars.}

The above-named stitch is one much used for filling in leaves and retais which are long and pointed, and for star-shaped flowers it is espeually suitable. The method of working is as follows: Along the two sides ci petal or leaf work a row of loose buttonhole stitches; as the beauty of the work will depend upon the regularity of these stitches, great care should be exercised in this part of the work. The D'Alencon bars are now worked into the buttonhole stitches by passing the thread four times over and under each buttonhole stitch.

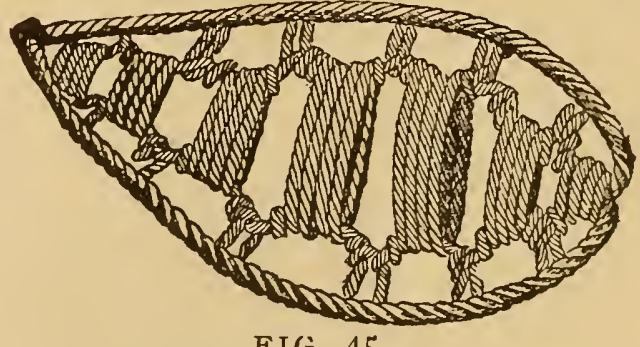

FIG. 45 .

'T he length of the bars is regulated by the space between the two opposite edges. When the thread has been passed over and under for the fourth time, making four bars, it is completed by making a tight buttonhole stitch, and the thread is then passed through to the next loose buttonhole stitch. Figure 45 shows D'Alencon bars applied to a leaf.

\section{SPIDER WHEEL.}

For filling portion of design in Roman embroidery the spider wheel here shown is beautiful, and can be quickly accomplished. In working, 
cross the threads six times from the edge of space to be filled, and at equa! distances apart. These form the spokes to the wheel. When the six threads are in, the needle will be in margin of design. To reach the center

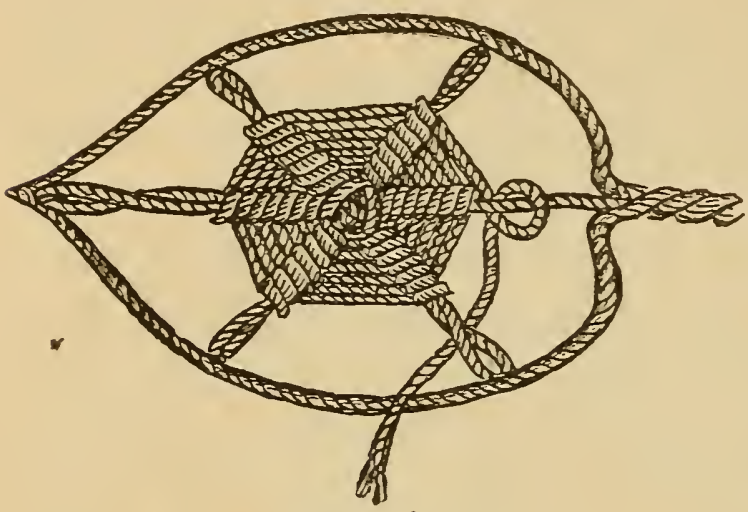

FIG. 46.

twist the needle on one of the threads forming the spokes. To work pass the needle under one spoke, and then pass around again loosely in the reverse direction; pass the needle over the second spoke and pass around in a reverse direction loosely; then pass needle under the third spoke, and repeat the operation until the six threads or spokes have been worked, and then repeat as many rows as is desired. The cloth back of this wheel should be cut out, and for this reason the edge should be first worked with buttonhole stitch. Figure 46 shows method of working.

\section{Point de Reprise.}

This stitch is admirably suited for filling large leaves and portions of conventional designs. A background for this stitch is first prepared by filling the space to be worked with threads placed parallel and crosswise from one outline of design to the other, to form triangular spaces. Thread the needle with rather a long thread, and on each triangle formed by the laced threads weave over and under, from one side of the triangle to the other, until the base of triangle is reached, then pass the thread on the wrong side to the next triangle, and repeat. Figure 47 gives an excellent

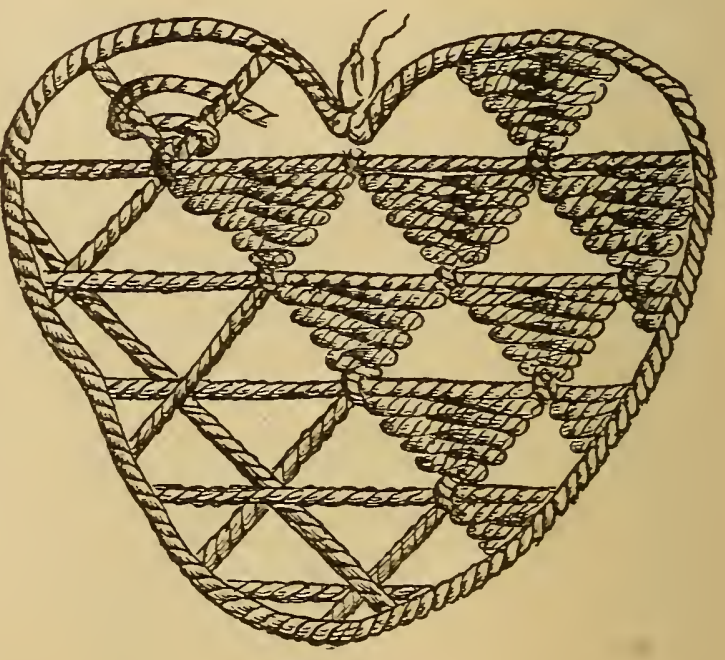

FIG. 47 . idea of the mode of working, as well as the result when completed. This stitch, as well as the rosette and spider wheel, look best on heavy material and done with art cord. 


\section{Rosette Stitch.}

The above stitch is the most effective filling stitch we have, and suitable for all the purposes for which point de reprise is used. This stitch also looks best done in art cord. Rosette stitch is made as follows:

Mark off the background to be filled into square spaces, a little less than an inch apart, by carrying the silk from one outline of the design to the opposite outline and crossing these threads, at equal distances, from the opposite outlines. Fasten the lines to the foundation at the point of intersection by couching down with a fine thread over both lines; this will give firmness to the work when it is finished. Thread the needle with the same cord or silk as has been used in laying the lines; bring the needle up from the back of the material, and on the left of one of the intersecting lines, close to the couching stitch. Pass the needle under the line, throw the thread over to form a loop, and pass the needle under the same line again and from the same side as at first, and through the loop

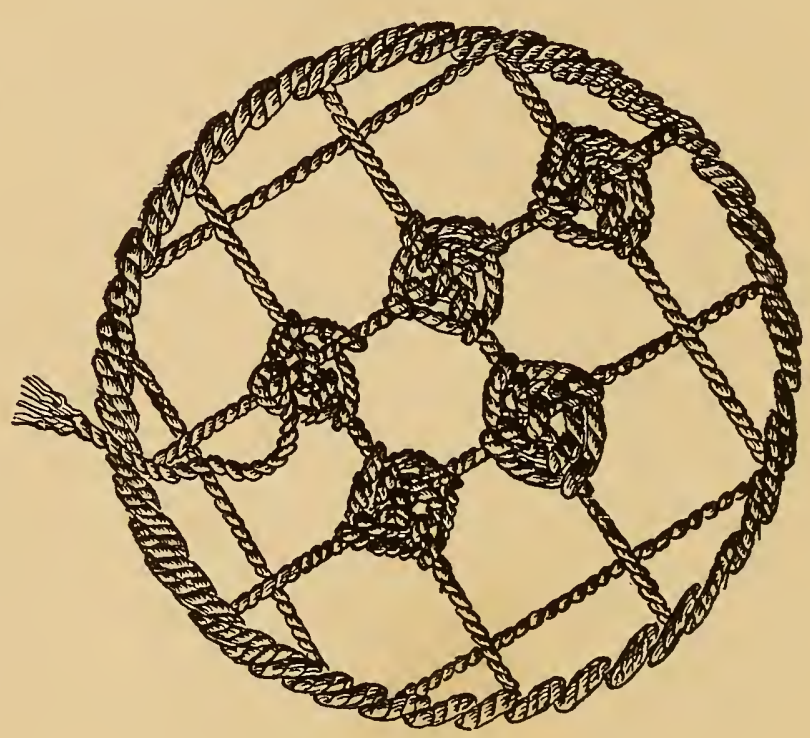

FIG. 48. formed in throwing the thread over; this forms a buttonhole stitch over the line, which must be drawn tight and close to the couching stitch that fastens the lines, at their point of intersection, to the material. Pass the needle under the next line and on to the third, where the same operation is to be repeated. The rosette has now a buttonhole stitch worked over two of the opposite intersecting lines; the two remaining lines must be treated in the same manner. This finished, the rosette has been worked once around; to complete the stitch it must be worked twice around, forming two raised or buttonhole stitches on each of the intersecting lines. When the stitch is finished, pass the needle through the stitch to the under side of the material, and on to the next intersecting lines. Work all the lines that cross each other in the same manner, and finish the design by outlining with art cord or rope silk. 
The foregoing pages on "Stitches" contain all that is necessary for the most advanced worker. None of the embroidery stitches are difficult in the execution, but all require extreme care and neatness in working, and a certain perception of the fitness of things on the part of the needlewoman. so that each stitch used shail express the worker's thought and meaning in the best and clearest manner possible. A knowledge of the relation of different stitches to different forms of work may be had from a careful studv of the following chapter, viz.: "Application of Stitches."

There are many different lace stitches which can be adapted for embroidery, with excellent effect--especially for filling stitches; but as a rule they are too intricate in detail to suit the patience or lack of patience of the modern worker.

In closing the subject of stitches we will make one more plea for the outline stitch. From time immemorial the most artistic work has been wrought by its use alone, and indeed all the wonderful and beautiful Egyptian embroideries were outlined or etched. There is scarcely a limit to the number of artistic effects that can be obtained by its use. Then, too, its simplicity is so in its favor, and indeed forms one of its chief claims to the needleworker's consideration. Outline stitches, then, should be practised diligently, until they can be made to express every variety of feeing. The least bit of carelessness will be fatal to the beauty and harmony of the design, and awful havoc can be wrought on an angel's face by a stitch too short or too long in forming nose or lips.

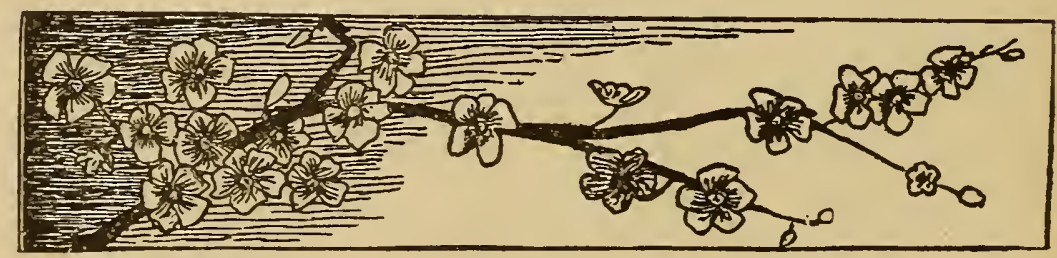




\section{CHAPTER VI.}

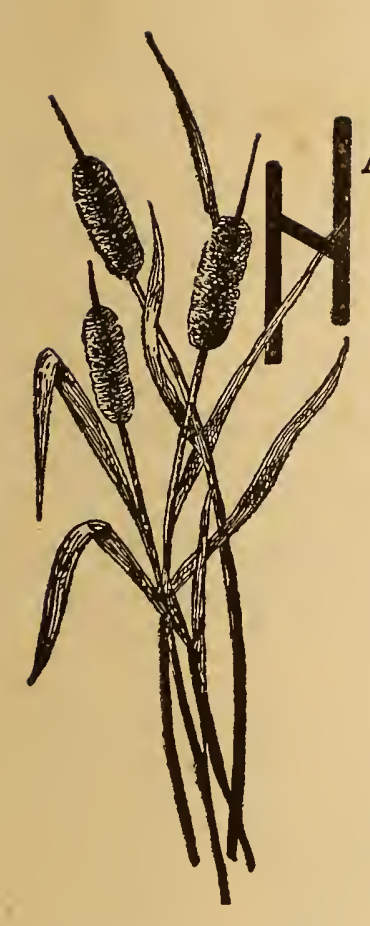

APPLICATION OF STITCHES.

AVING given descriptions and illustrations of all the essential stitches used in embroidery the first question we are sure to be asked is:

"How shall we know when and where to use the different stitches on different designs?"

This is a far-reaching question, and one that would require pages of illustrated matter to answer in detail. The best we can do is to give, in general terms, the places where certain stitches can be most appropriately used. In embroidery one must have ideas of one's own, else the work will be, more or less, copies of oftentimes poor originals. In Kensington embroidery, satin stitch is often employed in certain portions of designs, notably in the case of long, pointed leaves and in the tiny petals of the forgetme-not, and other flowers of the same description; again, in working cat-tails, thistles and golden rod, the flowers will usually be worked in plush stitch, while the leaves may be wrought in Kensington stitch.

The most daring combinations of stitches are allowable, provided the result justifies the means employed, and a harmonious ensemble of form, light and shade is secured.

The stem or outline stitch enters into almost all forms of embroidery, either in the formation of stems or outline of leaves. The short and long stitch is used in conjunction with stem and darning stitch, and so on through the catalogue of stitches.

Filling stitches are used most appropriately on wash materials and for filling conventionalized designs used on table cloths, doyleys, table runners, 
tray and carving cloths, fancy towels, pillow shams, dresser scarfs, toilet articles and for filling portions of designs in Roman embroidery. For these purposes the filling stitches are usually made with either etching silk or Real Scotch linen floss, No. 4, though for some stitches, notably janina, and for darning on fine sheer fabric, filo floss or Royal floss is preferable, while for French knots, where used in prominent portion of design, rope silk or rope linen can be appropriately used. Darning stitch can be used to form a background to throw design into relief; for darning a dado or frieze for curtain or portiere. Any conventional design wrought out with any suitable filling, stitch, or with short and long, outline, or buttonhole short and v.lg stitches can be used in connection. A combination of filling stitches is perfectly proper and very effective on the same piece of work. Thus in fic -es 49 and 5 I we have a combination of several filling stitches.

( "ching is frequently combined with satin stitch, and in fact enters largely $n$ all forms of flat embroidery, especially in ecclesiastical work, and the $C^{\prime}$ 'ination is not limited to any one stitch, as it is used with buttonhole, $\mathrm{s}_{1}$ ind long and darning stitches, and on monograms and initials.

For embro. underclothing, infants' trosseaux, cotton, flannel and cashmere, Frenc imbroidery is almost exclusively used. By French embroidery we mean that class of work done with a combination of satin, couching, buttonhole, spot, eyelet, dot or seed stitches and hem stitches. Many ladies embroider their initial on handkerchiefs, napkins, pillow slips, sheets, etc., and finish every hem with hemstitching in pure white embroidery cotton. It is a dainty fancy, and will give an air of refinement to the most commonplace surroundings. For this purpose spot, seed, satin and outline stitches are more commonly used. For embroidery on silk, satin, velvet or cloth the filling știtches are not so appropriately used. Short and long stitches, darning stitches, couching, applique, solid Kensington and outline are here in better taste. Sometimes one sees beautiful specimens of French embroidery on these fabrics, which of course is always in good taste, but the amount of work required to complete a design cione in satin stitch, every thread of which must be laid "on honor," is a task not often undertaken in these days of hurry and scramble, where, if the need of a piano cover is discovered on Mofday morning, Saturday 
night finds the cover supplied, decorated with needlework. In the days of Edward the Confessor a fair dame thought nothing of applying herself diligently and patiently to a single piece of embroidery through the months of an entire year. But such work is preserved as priceless heirlooms in private families and public museums, while we are obliged to confess that very little of our modern embroidery is wrought with such perfection of detaii.

In the matter of filling stitches the utmost latitude is allowed as to choice, and we show here a few designs filled in with stitches which have been described in the previous chapter. Our arrangement is not at all arbitrary but merely given as suggestive for pretty and artistic combinations.

\section{COMBINATION OF SPOT, AND SINGLE DAISY LOOP.}

Figure 49 shows section of conventional design filled with single daisy loop, cross stitch, spot stitch, diamond couching with French knot in

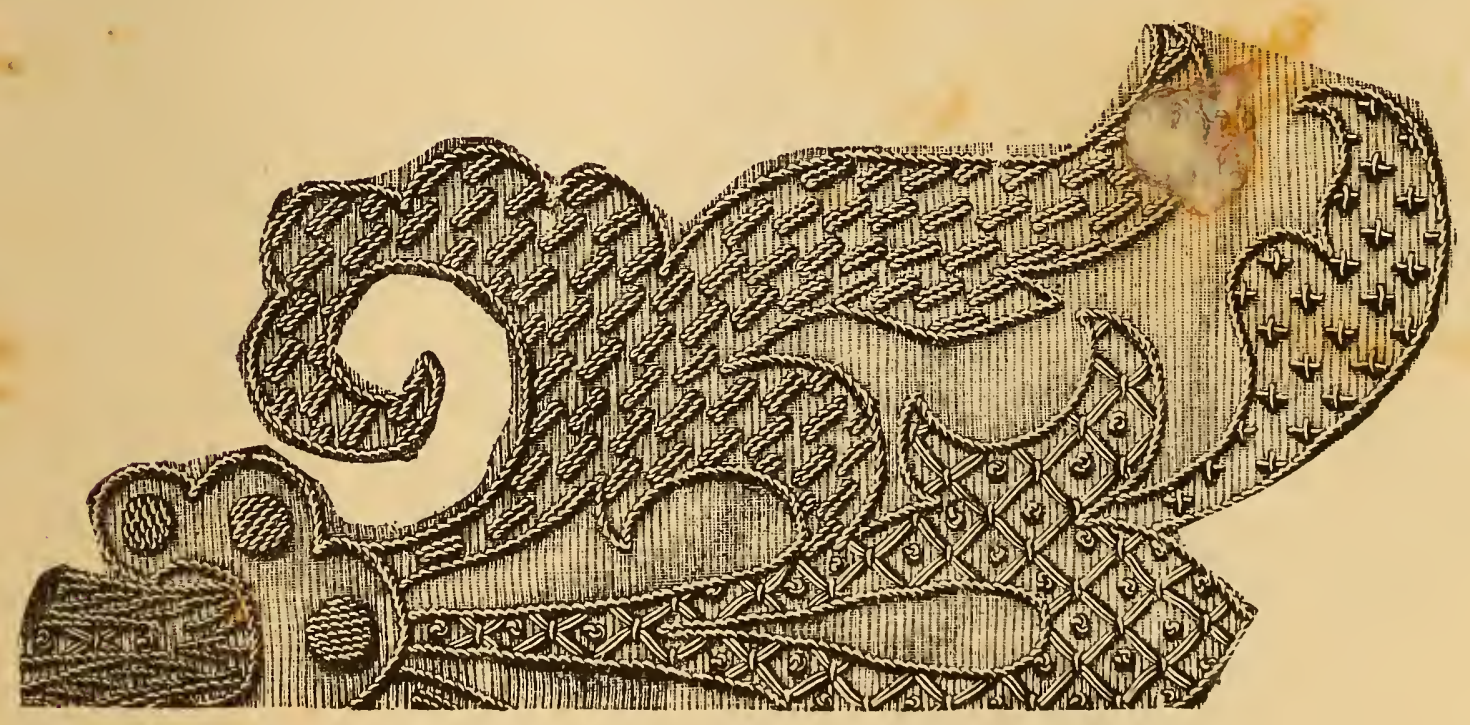

FIG. 49.

center and outlined edges. This method of applying these filling stitches will be found quite simple, and as they require but little time and material will be found economical as well as effective. 


\section{LEAF BORDER SHOWING COMBINATION OF STITCHES.}

The design shown here is a section of border of leaves for lunch cloth. The cdges of leaves are finished with buttonhole stitch over German cord, which can be clearly seen. The surface of upper leaf is then filled with star

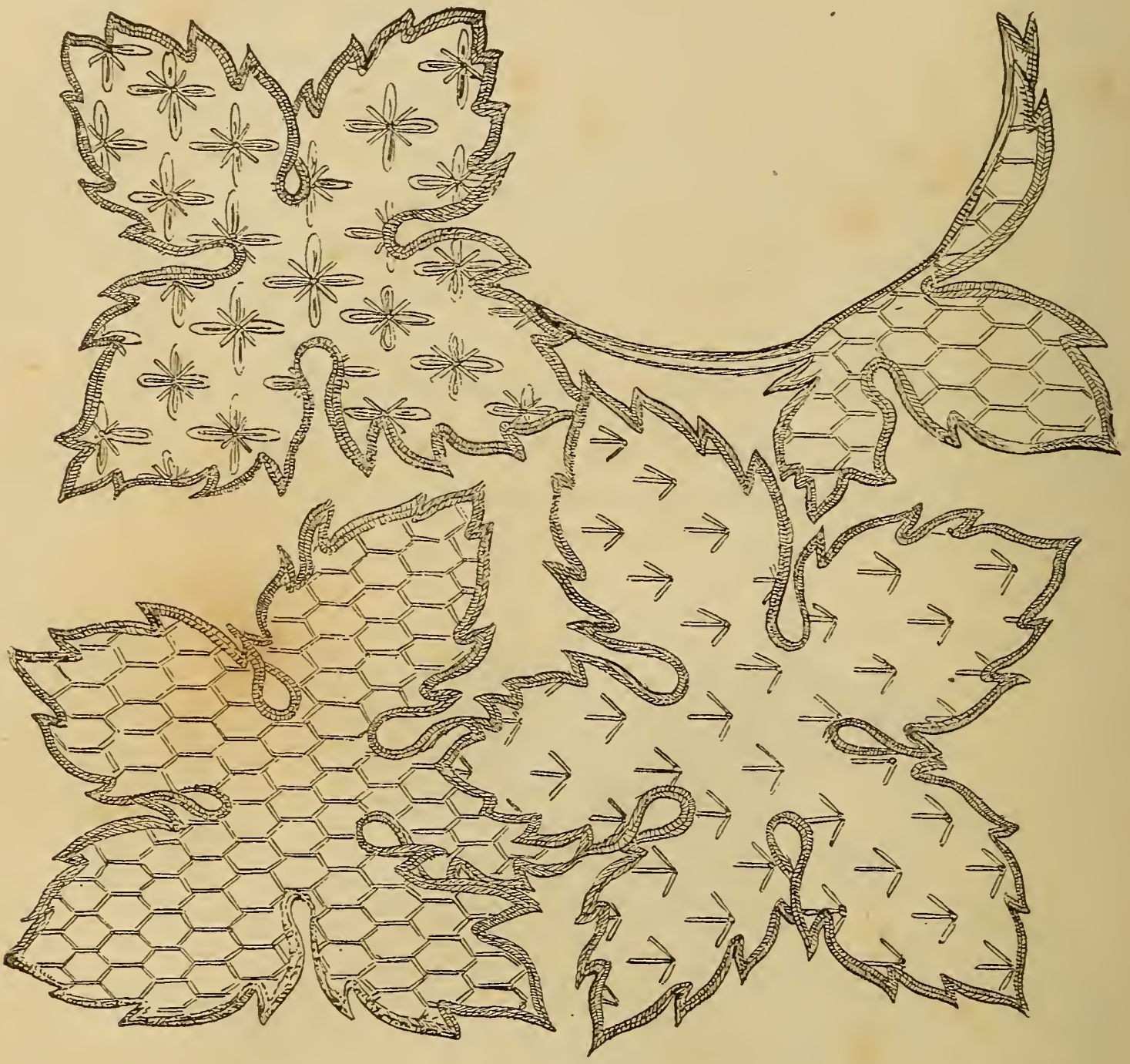

LEAF BORDER SHOWING COMBINATION OF BUTTONHOLE, HONEY-COMB, DAISY STAR AND CROWSFEET STITCHES. FIG. 50.

stitch. The leaf at the right of corner leaf is filled with crowsfeet stitch, while the leaf at left uf corner is filled with honey-comb stitch. This method of filling can be repeated tiıroughout the entire design of which our illustration shows but a section and other filling stitches not shown here can be 
used in connection, but the ones here shown are the simplest and most popular of the filling stitches, and are used on all conventional designs where solid work is not intended-indeed, they may be used on a design having solid embroidery on some of its parts.

\section{FILLING STITCH FOR APPLIQUE.}

Figure $5 \mathrm{I}$ is especially suited for applique work. The leaf and vine is first stamped on velvet which is then mucilaged to a foundation of silk or cloth, after which the outlines of pattern are finely run to a founda tion with a silk thread. The velvet is then cut away close to the stamped lines, and the edges are finished with a heavy silk cord couched down; the center of the leaves are then worked with coral stitch in arasene or chenille. Any other of the filling stitches could have been used here with equally good effect, providing silk thread had been used in place of

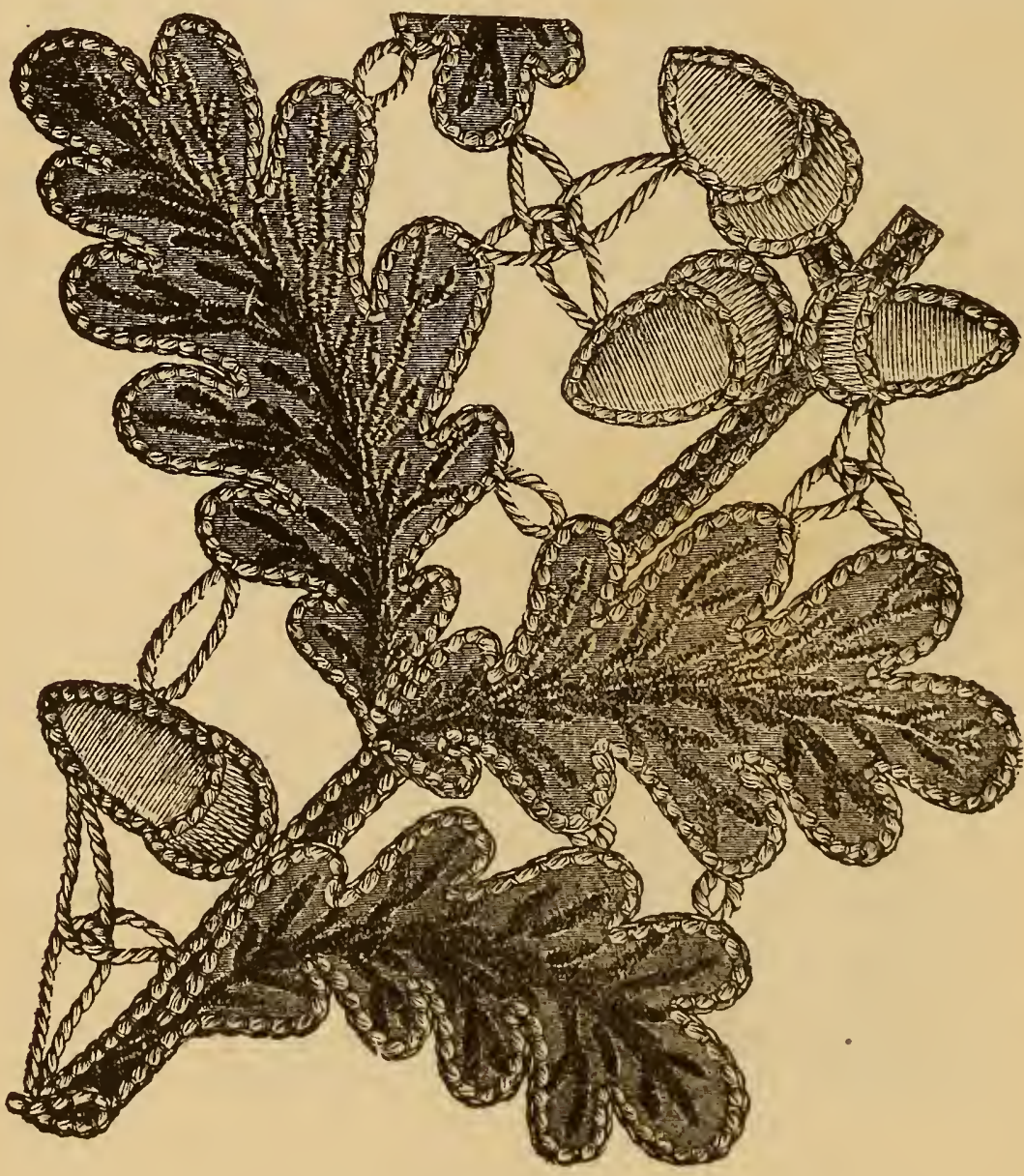

FIG. 5 I. arrasene or chenille, as these two last-named materials do not lend themselves to filling stitches with good effect.

The design here given is, of course, only a portion of complete vine, but sufficient is shown to clcarly explain mode of working. As to colors. 
one must use a little judgment and taste in the selection. If the leaves are of dark green velvet the silk used should be of a much lighter shade of the same, while if the leaves and vine be cut from ecru or cream colored velvet the couching and rope silk used may be in all the various tints of autumnal foliage, yellow, brown, green, olives, terra cotta, and so on through the list. There is no limit to the changes that can be rung on such a pattern.

In the matter of fancy filling stitches the utmost latitude is allowed as to choice.

\section{APPLICATION OF KENSINGTON STITCH.}

Kensington stitch may be appropriately used for working out any floral design, no matter where the finished work is to be used. It is also

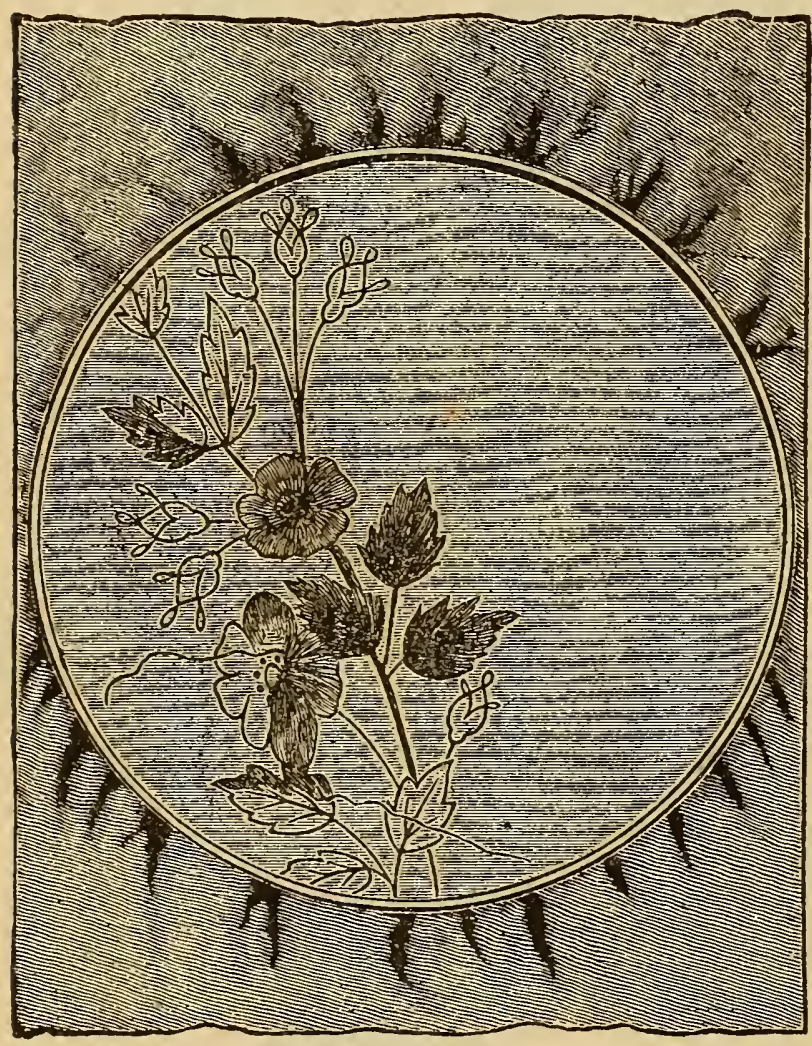

FIG. 52 . sometimes used for working animals, human figures, and landscape details, such as rocks or hills, where a massed effect of light and shade is desired.. For ornamenting articles of dress, such as dresses, wrappers, jackets, etc., it is especially beautiful, and ladies who can embroider in either Kensington or satin stitch, can indulge in many a beautifully ornamented garment that would be beyond the reach of their more wealthily endowed sisters who are without this accomplishment. Figure 52 shows design suitable for Kensington stitch stretched in the hoops ready for embroidering. When embroidering dress collars, vest fronts, etc., with this stitch, the material should be first stiffened by backing it with crinoline.

\section{APPLICATION OF SHORT AND LONG STITCH.}

Figure 53 shows a very appropriate application of short and long stitch and is only one of hundreds of similar instances where this stitch can be 
effectively applied. In general, short and long stitch can be used whereever Kensington stitch would be appropriate, and also in many instances where the latter stitch would be too heavy, notably, in portions of conventional designs where something a little more elaborate than simple outline stitch is desired. Figure 53 shows a chair cushion, flower-shaped, made of white lawn. The surface of the lawn is first tinted a pale rose color with water colors or Paris tints, after which the edges of the outer petals are worked to the depth of an inch with buttonhole short and long stitch; the inner petals are worked in the same manner but with the simple short and long stitch. A pale rose-pink

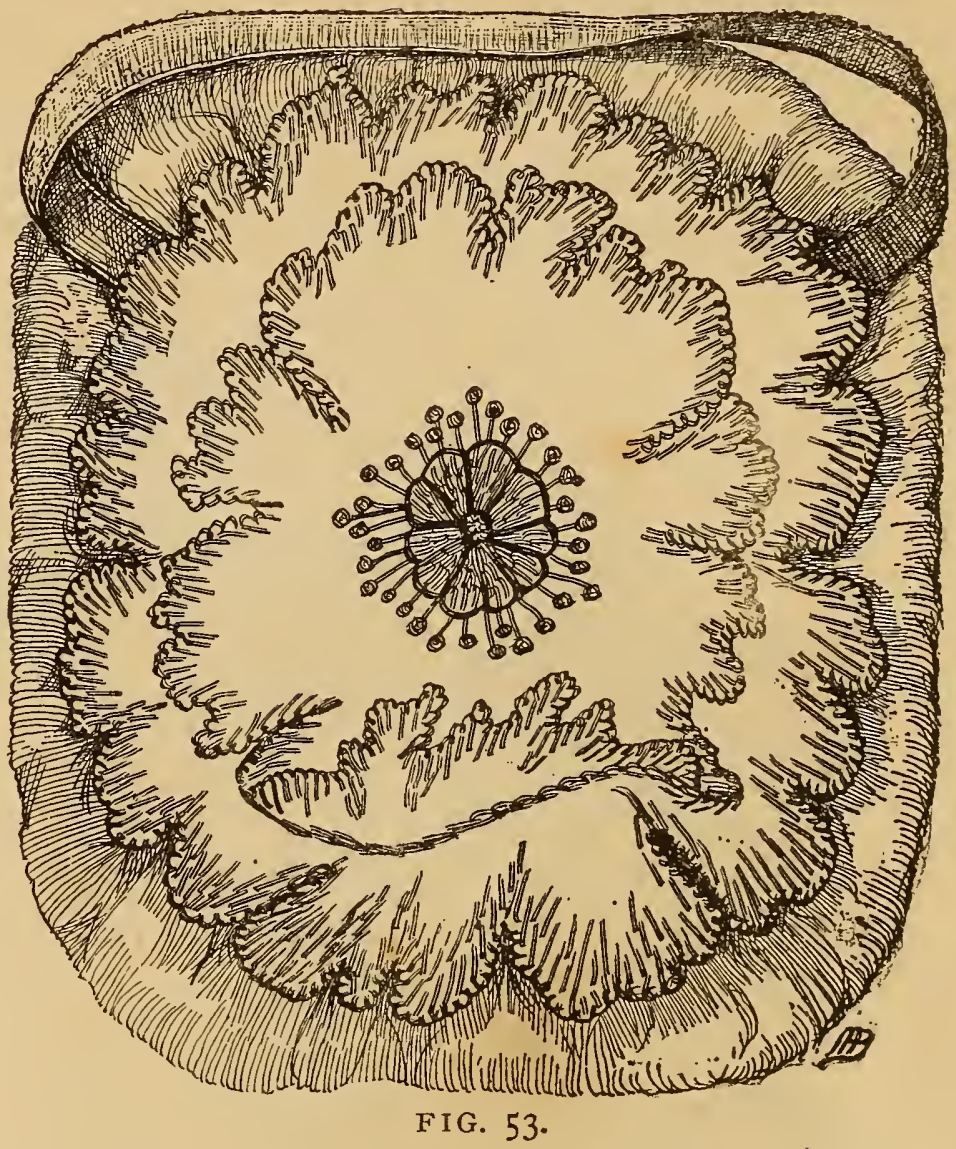
rope silk should be used for this part of the work. The center of the flower may be worked with pale green filo floss in satin stitch. To make up the cushion, after the flower-shaped center has been finished: Cut a circle out of cotton wool, using a breakfast plate as a guide to cut the circle by. The wool circle should be at least two inches thick. Place this circle in the center of a square of deep olive green surah silk, fold over the corners of the silk to the center of the circle, and fasten in tiny plaits to the wool, and close enough to the edge of the circle to form a generous puffing around it; the cushion is now ready for the flower-shaped cover which must be fastened in the center of the circle and on the side where the silk is folded over. Suspend the cushion to a chair by means of a pink satin ribbon fastened on with invisible stitches, just under the edge of the cover. The shapes for the center cover of these pretty head-rests for chairs 
may be cut from a variety of designs-large single peonies, sunflowers and single roses, slightly conventionalized -can all be made effective. In adapting a sunflower, the embroidery will look best done with some one of the shades of yellow.

\section{. SHADING CONVENTIONAL DESIGNS.}

In strictly conventional designs there is, properly speaking, no such thing as shading - a combination of colors that are effectively contrasting

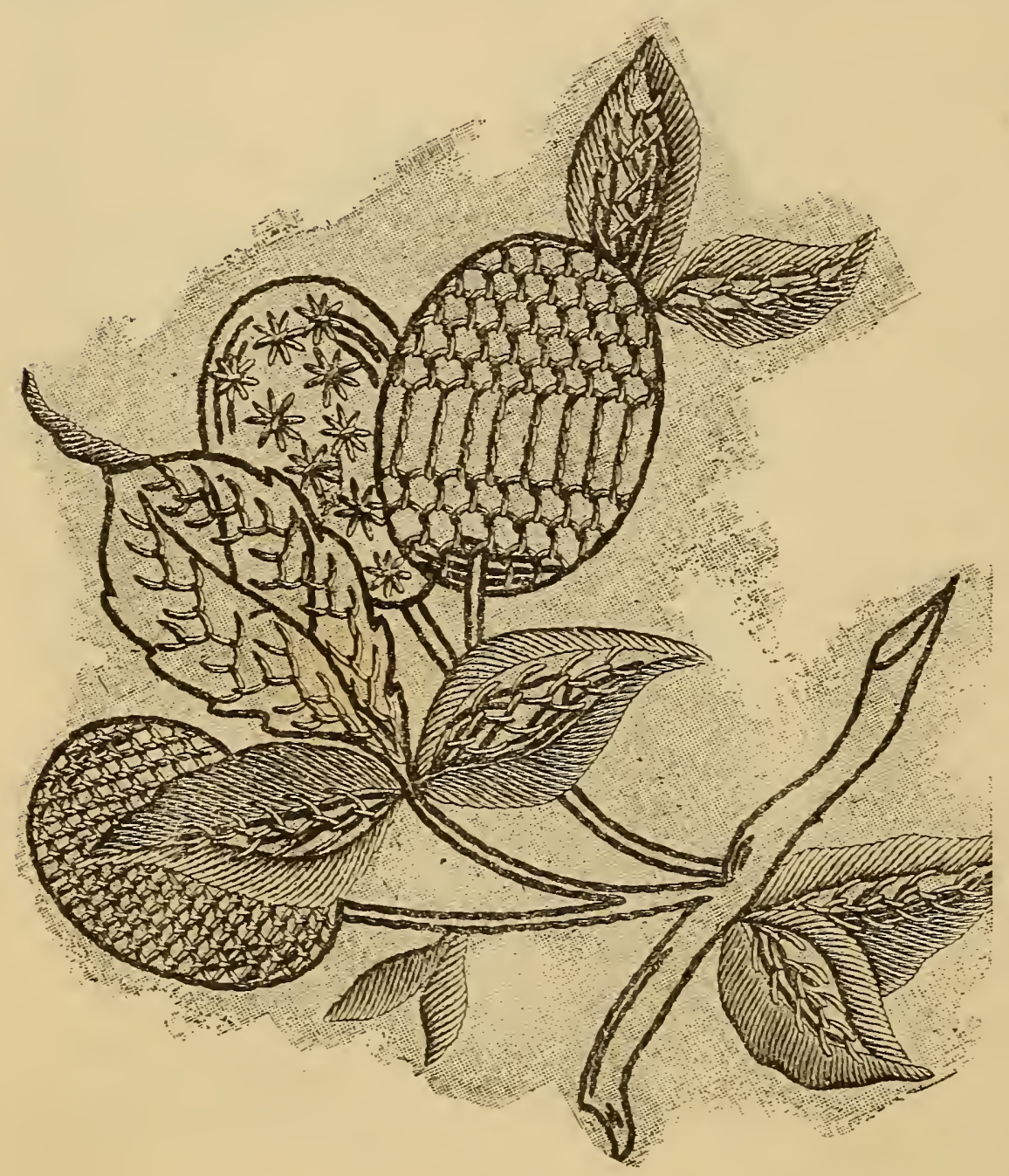

FIG. 54 .

or harmonious is the aim of the worker. The popular fad in coloring for the past few years has been what is known to the trade as one line coloring; that is, suppose the material being embroidered to be a medium light terra cotta, two or three shades of rope silk of the same color, but a shade or two darker, would be used in the embroidery.

The effect is certainly quiet and artistic to a degree, and if portions of design are outlined with gold thread the effect is heightened. Again, certain colors or combinations are effective as green and white, blue and white, brown, red, ecru, etc., care being taken to use the high colors, as red, to heighten the effect of the whole, depending on the quieter colors for the body of the work. 
Figure 54" shows a conventional design in filled outline, and we suggest the following treatment, which if carried out neatly will produce a beautiful result:

This design of fruit, with foliage, is suited for table linen, either scattered over a large surface, as a table cloth, or used in corners of mats or napkins. The outlines are all first treated in stem stitch with etching silk, and the spaces afterwards filled in with the fancy stitches, as shown, in the same silk. These stitches have been previously described, so we will confine our remarks to the colors to be used. On table linen ladies incline to pure white or the lightest, daintiest tints of a color. We suggest several combinations, any one of which would be beautiful and correct.

First combination, pure white for stems, leaves and outlines, and for filling stitches a pale silvery gray.

Second combination, outline entire design with white and fill in with a pale blue.

Third combination, outline in stem and short and long stitch with pale green, and fill in with cream white.

Fourth combination, outline stems and leaves with pale gold; outline and fill in first cherry in the cluster with a shade of gold one degree deeper than that used for stems and leaves; outline and fill in the next cherry with a still deeper shade of gold, and for the third use a shade deeper yet.

This will give a scheme of color in yellow gold, ranging from the faintest tint to a medium deep yellow. This method of coloring gives the effect of elaborate shading, and is beautiful.

The foregoing illustrations and suggestions will give the reader a general idea of the proper use and combination of embroidery stitches, and will serve as a stimulus to the inventive worker, who will not be satisfied to blindly follow our models, but will seek to mark out new lines of work for herself. 


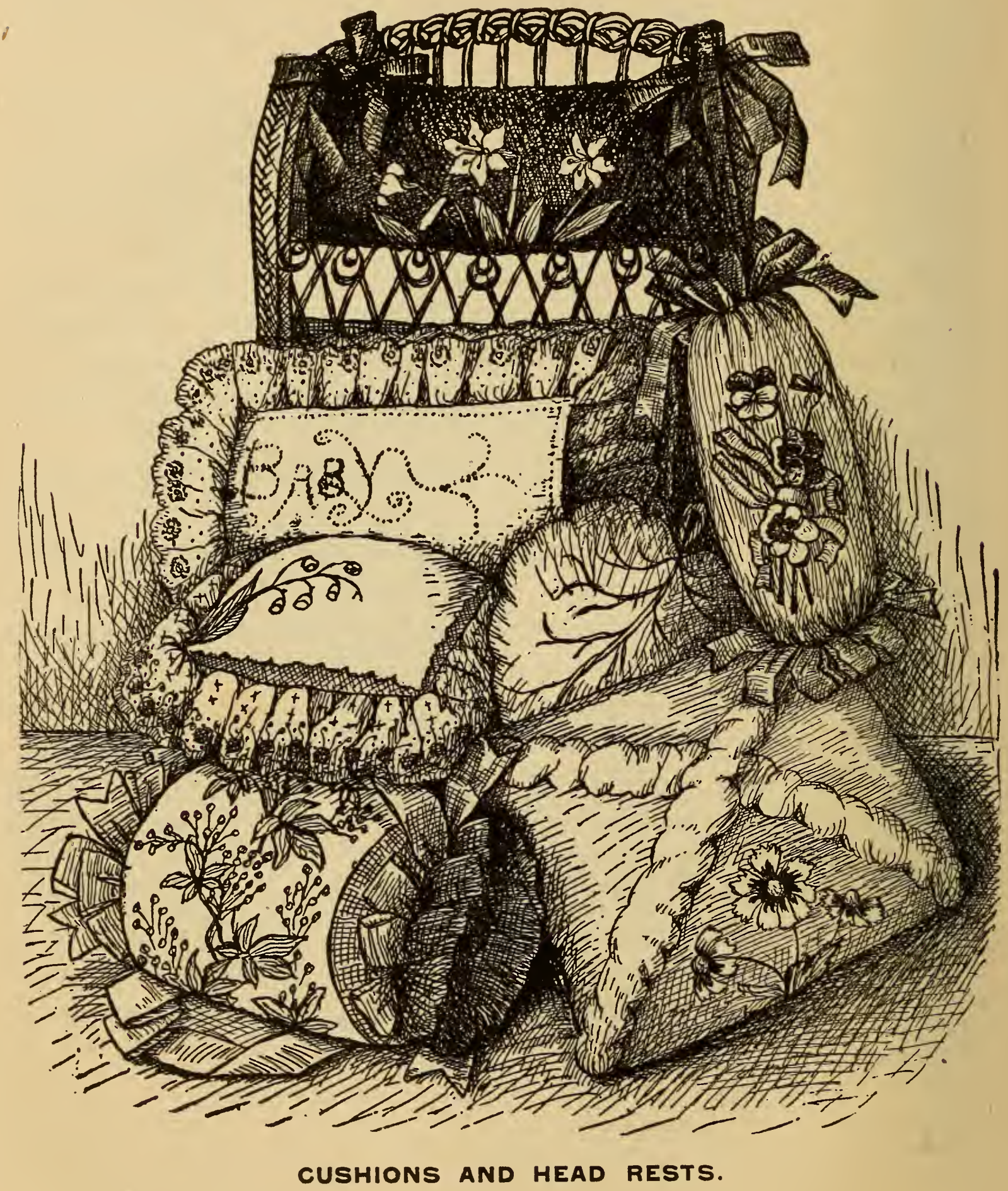




\section{CHAPTER VII.}

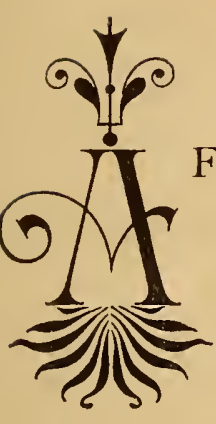

SHADING.

FTER stitches, shading is the subject that naturally claims attention, and to the amateur embroideress it is usually a stumbling block of pretty large dimensions; however, there is no reason why this part of the work should present more difficulties than the formation of the stitches. We have for several years taught a system of shading which we propose to present here, and as it is designed for the benefit of amateurs, the expert needleworker will pardon us if we are a little prolix on the subject. The woman who has an artist's eye for color needs no instruction in shading; but, unfortunately, she is sadly in the minority, and our efforts in this chapter must be devoted to her less fortunate sisters.

Reduced to a system, shading is shorn of half its difficulties. In the first place, there is a proper way to purchase embroidery threads, which, when followed, gives satisfactory and certain results.

Embroidery threads are dyed in several different shades of each color, and as each manufacturer has his own method of putting up and numbering his silks, it will be necessary for us to select one brand of silk and refer to it exclusively throughout the different lessons given in these pages. For convenience we will select the shade card* and numbers of Belding Brothers \& Co. There are other manufacturers of embroidery threads whose outputs possess equal merit in regard to quality, fast dyes and artistic shades, and in selecting one brand of silk from which to illustrate our lessons, we do not wish to be understood as discriminating against others. Arrasenes, chenilles and linen threads are made and numbered in the same manner as the silks, so that the rule for selecting and using one will do for all.

*Note.-We have arranged with Messrs. Belding Bros. \& Co., that upon receipt of ro cents they will mail to readers of "Dainty Work" a complete color card of embroidery silks, properly grouped and numbered. Attached to same is an extended shading table indicating most appropriate colorings to select for specific work. Mention "Dainty Work" and address, (enclusing ro cents) Belding Bros. \& Co., Silk Manufacturers, Fifth Ave. and Quincy St., Chicago, Ill. 


\section{SELECTING SHADES.}

To begin, we will explain a little about the colors and shades of silk to be purchased for embroidery purposes from our shade card. Each color is made in several different shades, but the number of shades any given color is made in varies. For conveniénce in reference, we will give here a small table of the colors, together with the number of shades in each color.

\section{TABLE OF COLORS.*}

The numbers of the colors are given inclusive, thus I20I to I 204 means that both numbers are to be included.

White, three shades, I200 to I202; Cardinal Red, ten shades, 1204 to I2 I3; Flame Red, seven shades, I340 to I346; Cherry Red, six shades, I288 to I293; Old Pink, five shades, I 220 to I 224; Rose Pink, five shades, I 335 to I339; Bronze Green, six shades, I2I 4 to I2I9; Olive Green, six shades, I248 to I253; Apple Green, four shades, I240 to I243; Grass Green, five shades, I 320 to I324; Sage Green, four shades, I280 to I283. Lily Green, five shades, I 375 to I379; Silver Green, five shades, 1330 to I334; Peacock Green, five shades I272 to I276; Old Blue, five shades, I225 to I229; Gray Blue, five shades, I235 to I239; Blue, nine shades, I3 I I to I3I9; Baby Blue, six shades, I 354 to I359; Peacock Blue, five shades, I365 to I369; Yellows, from pale canary to deep orange, twelve shades, I260 to I27I; Wood Brown, six shades, I294 to I299; Red Brown, three shades, I277 to I279; Bronze Brown, six shades, I305 to I3IO; Golden Brown, seven shades, I347 to I353; Old Rose, five shades, 1335 to I339; Dark Terra Cotta, five shades, I230 to I234; Light Terra Cotta, six shades, I254 to I259; Silver Gray, four shades, I 244 to I247; Lavender, four shades, I284 to I287; Heliotrope, five shades, I300 to I304; Fawn Brown, five shades, I360 to I364.

The above group of colors includes all the shades manufactured by any silk house, and from them must be selected the colors needed for any given piece of work. Thus, blue flowers must be selected from the different lines blue; all red flowers from the lines of red; all pink flowers from the lines of pink; and all purple flowers from the lines of purple.

From the list of colors given, it will be seen that there are different varieties of the same color; thus, there are blues and blues, green and greens; now, the different lines of the same color are rarely combined in the same 
leaf or petal, and such combination should never be attempted by any woman less than an artist. It is safest to confine yourself to one line of a given color; in that line you have a number of shades from light to dark, any one of which will combine with any other shade of the line.

As we have before hinted, any rule for shading is half mastered when the worker knows how to purchase or select materials. It may seem a simple thing to go into a shop and select silks for a rose spray, a poppy, or so simple a flower as a buttercup, but not one lady in twenty can accomplish the feat with any comfort to the clerk or satisfaction to herself.

In shading there are three points to be considered. First, the color of "the flower to be worked. Second, is it light, medium or dark in tone. Third, what kind of silk to work it in. These points are best decided in the quiet seclusion of home. A heterogeneous mass of silk in hopeless confusion, an anxious clerk, and a crowd of ladies clamorous for an opportunity to pull and haul the same box of silk into still worse disorder-in the vain endeavor to match silk purchased, perhaps, months before-is not condu. cive to the mental equipoise necessary for the work in hand; therefore, we repeat, do as much of the selecting at home as possible.

After you have fully decided upon the materials needed, the next difficulty to be encountered is the procuring proper shading in small stores in country towns. You are almost certain to be obliged to select from a ragged, broken and incomplete assortment, difficult for even an experienced worker to select from, and quite impossible for the amateur. When at all possible, go to a store where a large and complete line of silk is carried. If you have several colors to select, make up your mind what particular line of each color you wish to use; if green, decide whether it shall be olive green or grass green; if pink, whether it shall be old pink or rose pink, and having decided this point, ask the clerk to show you one line of color at a time. Insist on having the entire line of shades in that color placed before you. By referring to the list of colors and shades given at the beginning of this chapter, you will at once know if all the shades are before you, and if any are missing, which ones. Place them in order on the counter before you, beginning with the lightest shade first, then the next deeper, and so on, until the last shade is reached. Now, from the colors before you select as many shades as your pattern will require; thus, if you are shading wild 
roses, you will call for the line of rose pinks, in which there are five shades; you will at once see that you do not require the entire line, but only the first three shades. If you are shading morning glories of a reddish pink, you will call for the line of cherry red, which has six shades to select from, and you will require all or part, according to the size of the design. In a pattern having five or six flowers the entire line of this shade can be used effectively, while if there are but two or three flowers the first three shades of the line would be sufficient.

In working red poppies the line of flame reds should be used. This line has seven shades, and if the pattern shows several poppies the entire line can be brought into requisition, but not necessarily all in one flower. The lighter shades of the line will be used for the high lights and the outer edge of petals, then two shades next deeper will be blended in to work the body of flower, and for the markings at base of petals the deepest shade of the line should be used. Some varieties of red poppies show purplish markings at the base of petals, but these should not be attempted in embroidery. There are limitations in art needlework, and this is one of them. What would present a beautiful effect of light and shade done with either oil or water colors, would be but a patchy affair if attempted with the needle and the colors at our command in embroidery threads.

But to return to our purchasing. Having selected your first line of colors, require the clerk to put away all the silks you have been selecting from, and proceed to the next color; repeat this until you have completed your purchases. This will save the clerk a great deal of time and trouble, and spare yourself annoyance and uncertainty, and when you are through with your shopping you can return home with the consciousness that you have purchased just the right thing. This method of selecting embroidery threads leaves nothing to accident or whim, and thus will be avoided inharmonious and ignorant shading.

\section{PROPER METHOD OF SHADING.}

Having selected our embroidery silks, the next thing in order will be to use them correctly. In shading flowers and foliage Nature is not to be followed in her entirety, for we cannot successfully copy lights and shades and eccentric markings: we must depend rather upon a continuity of sur. 
face that is shaded from light to dark in the same color and with gradual blending. We must, of course, consider the position of the flower or foliage; thus, in shading most flowers presenting a full front view-see figure 55the rule is to begin at the tip of the highest petal, and with the lightest shade of silk used work toward the center, blending with it, gradually, two or three deeper shades of the same color, using the deepest in the very center of petal. Morning glories, nasturtiums, trumpetflower, orchids, in fact, most bell-shaped flowers, offer an exception to this rule, as the heart or cup of the flower is often of the lightest tint; in some varieties of

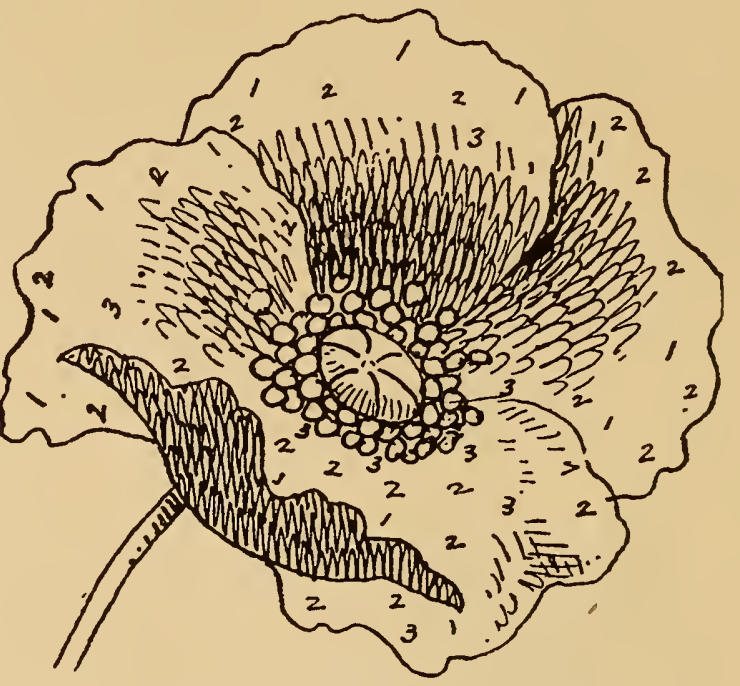

FIG. 55 . morning glories the throat or cup will be pure white, while the outer edges of petals will be the most vivid pink or purple. In figure 55 the numbers

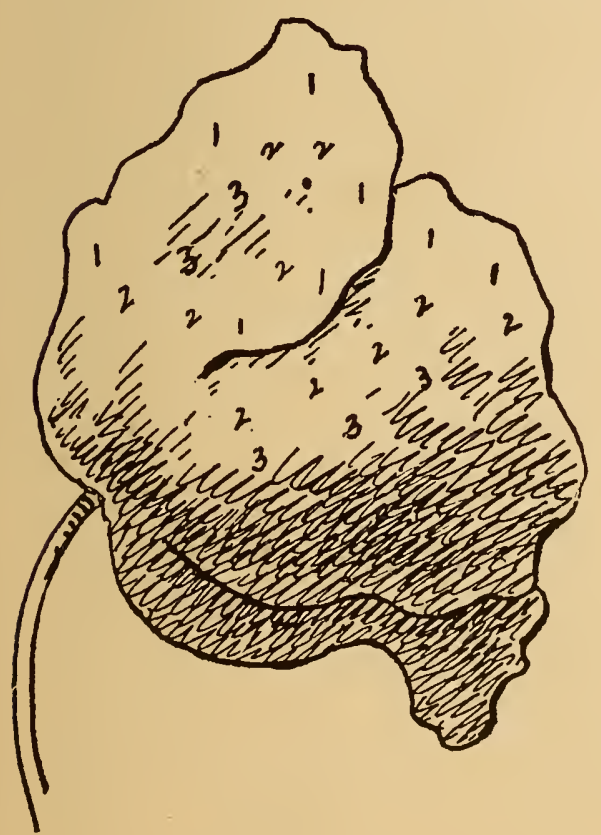

FIG. 56.

I, 2, and 3 indicate the position of the different shades of silk used. I indicates the lightest shade, 2 the next deeper and 3 the third deepest shade used, while the portion of flower heavily shaded, but without numbers, indicate silk one and two shades deeper yet than is indicated by the numbers I, 2 and 3 .

In a flower presenting a drooping side view-see figure 56-the lightest shade of the color used will be disposed on that portion of the flower where the light would naturally strike first, which, in case of the flower in question, would be on the upper petals, shading gradually darker toward the base of flower from right to left. The figures $1,2,3$ indicate the relative positions of shades as given in figure 55 , and the heavily shaded lower petals indicate the deepest shade of the color used in the entire flower. In work- 
ing, begin at the point where the lightest shade is to be used and gradually blend in the deeper shades, working always toward that point of the petals where the shadows lie, which in this case will be on the edges of those petals drooping lowest and nearest the worker.

In a flower presenting a full side view, the lightest shade will, of course, be used on the outer edges of the petals, which will be shaded deeper, gradually, toward that point where the flower joins the stalk, and the shading will be deeper on that side where the shadow falls; this tends to give the flower that rounded effect, that appearance of standing out and

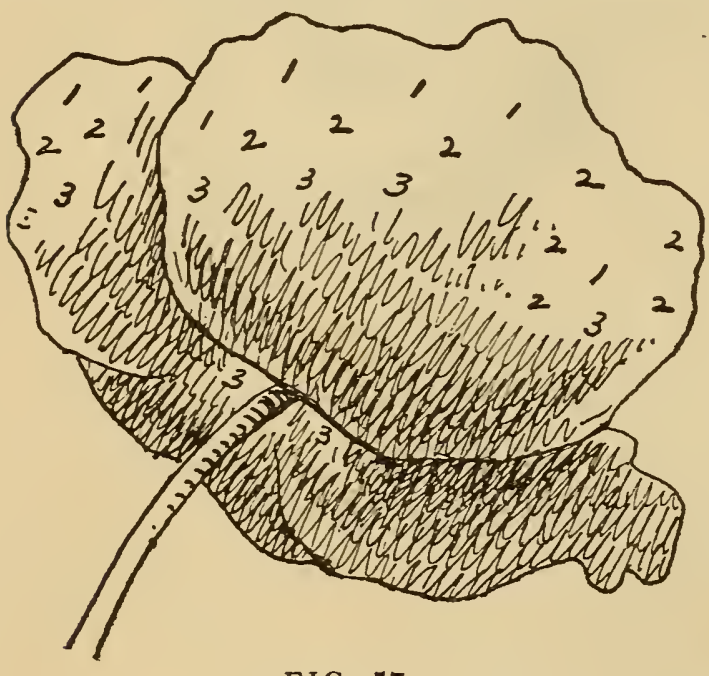

FIG. 57. away from the material. See figure 57. In this flower the figures I, 2, 3 indicating the lightest shades are placed at the top edge of petals and also the deepest of these three shades is indicated at the base of flower.

In shading foliage no cut and dried rule can be applied. The general rule of working with the lightest shade that portion of leaf or stalk which the light strikes first, must, of course, be followed, but the combination of shades to produce a mass of foliage must depend upon the arrangement of design, the nature of the foliage and the color of the background. The upper portion of foliage, the tips of leaves, the young leaves, and the wrong side of turned leaves should be wrought out with the lighter shades of the colors used, while that portion of the leaf joining the stalk, and leaves that are overshadowed by other parts of the foliage should be shaded gradually deeper until the deepest tone used in the design has been reached. We have a great variety of greens to draw upon for shading foliage, and thus natural shading can usually be obtained. In shading the foliage of poppies, carnations, geraniums, thistles, and some varieties of the lily family, the silver greens should be employed, while for the foliage of water, tiger and Easter lilies, nasturtiums, morning glories, and plants of the same coloring. the lily greens will produce natural shading; for chrysanthemums, some 
varieties of geraniums, fuschias and a few varieties of rose leaves the sage greens should be used.

In shading autumn leaves and foliage-such as oak, maple, mountain ash, Virginia creeper, begonia, sumac, etc., we have at our command all the greens, browns, olives, bronzes, flame reds and orange yellows, and with these can be wrought out a very dream of autumnal glory.

For shading grasses and ferns, apple and grass greens are usually employed, and where the background will permit, a little bronze green and olive brown may be added to represent ripened grass and ferns. For rose foliage all or any of the greens may be used; and for any foliage mentioned above, where the background will not permit the use of natural shading, the olive and bronze greens may be substituted.

The stalks of plants are better represented with either wood or red brown, though in case of young and tender stalks the lightest shade of green used in the foliage may be employed.

Having discussed natural shading, which may also be cailed popular shading as well, we come to art or conventional shading, which is quite another thing. Here we may work a rose in blue, or any other color our fancy may dictate, provided the design has been slightly conventionalized. This style of shading is used in the societies of decorative art, and among people whose taste has been cultivated in this direction. The amateur embroideress will find it hard to reconcile her ideas of the eternal fitness of things with a flower wrought out in colors never dreamed of by Nature, in connection with that particular plant, at least. However, time and contact with artistic work will modify this taste for flowers au natural, both as to form and color, and gradually will she incline to subdued tints and shades for her art needlework. There is a distinct tendency to so conventionalize our old familiar friends of the flower garden that we find it hard to recognize them in their new dress and colorings; but one must admit that a frieze of slightly conventionalized oak leaves and acorns across the bottom of an ivory colored portiere, wrought in an applique of golden browns, through several shades and tones of that color, would present a far richer effect than a frieze of flowers embroidered in natural form and coloring.

Again, with this conventional shading we can have our decorative work in distinct harmony with the furnishings of the room in which it is to be 
displayed; and the most important point in its favor is the fact tha embroideries wrought in dull art tones are less tiring to the eye, richer in effect and more refining in influence than those done with the natural shading, which in garden and field may be a delight, but which when transferred to the sitting-room, and given a setting never dreamed of in the economy of nature, becomes garrish, loud and incongruous. For those who love color, and cling to a rose that is red, or a tulip that is yellow, in all places and under all conditions, there is a modified conventional shading, which, while lying close to the natural is yet brought into subjection to surrounding objects; for instance, if a curtain is to be decorated with yellow tulips, instead of selecting for a model a tulip of bright yellow, work out the design in dull yellows, with a hint of brown in them-the golden brown of our shade card used throughout the entire flower will give the desired effect. If a red, red rose is desired, instead of using the bright and glaring reds, go down to the bottom of the line of reds and select the three darkest shades. The effect of this shading is rich in the extreme, and does not offend the taste of those people who cannot become reconciled to a blue rose.

In conventional shading there may be no attempt at shading from dark to light, or the reverse; the entire design may appropriately be wrought with one color; or with equal propriety all the different shades in one color may be employed-and foliage, stalks, flowers, may all be worked with the same shade.

In the pages of this book, when indicating the coloring and shading for specific articles we shall refer to the silks by their numbers and names as given in the table of colors at the beginning of this subject.

While it is absolutely impossible to teach all the subtle gradations of artistic shading in any written article, yet the rules and suggestions here given are so clearly marked, the limitations of our colors so well defined, that the merest novice ought not to go astray; and, given an eye for the harmony of colors, she should be able to obtain all the artistic results of which the materials are capable. Without this eye for harmony she may yet obtain beautiful and correct results, but in less variety, by simply following out our suggestions in either natural or conventional coloring. 


\section{CHAPTER VIII.}

ART OR FLAT EMBROIDERY.

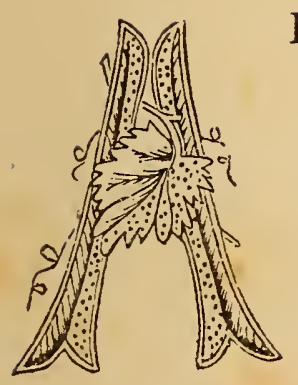

LL EMBROIDERIES are divided into two classes, namely, art or flat embroidery and raised novelty embroidery. The former includes all decorative needlework that is applied directly to the fabric that is to serve as a background, with either crewel, tapestry wool, silk, linen, cotton, or gold threads. Those embroideries wrought out with arrasene, chenille, smyrnasene, ribbosene and featherasene have been relegated to the ranks of raised novelty embroidery.

Art and raised embroidery have each a place in our decorative economy, and will be considered separately; yet, at the start, it is well to bear in mind that art or flat embroidery is to decorative art work what bread and butter is to our diet, and bears the same relation to raised embroidery that our dinner does to the dessert; so in decorative efforts let us have a good supply of the bread and butter of embroidery and a moderate amount of dessert in the shape of raised work, otherwise the effect will be bizarre and monotonous. That there can be too much of a good thing is an old saying, and nowhere is it truer than here. So do not let us go to the extreme of raised double and single roses, snowballs and thistles to the exclusion of higher forms of art work.

We confess that raised embroidery has a fascination hard to resist; it grows unde: our hands so rapidly; the effect is so striking; it fills up so quickly, with such a small comparative outlay of labor; it is so pretty! Granted, but still we must not have too much of it; like every other good thing it has its drawbacks-fashion is fickle, dust clings lovingly to its luxurious folds and obstinately refuses to "move on," and last, but by no means least, it is more expensive than its legitimate sister, flat or art em6 
broidery, and will not wear so well-in short, will not be "a thing of beauty" and a joy forever."

Under the head of art embroidery will be considered Roman embroidery, applique, outline, solid Kensington, and those embroideries wrought out with combination of filling stitches applied to articles of use or ornament.

The groundwork of all embroidery has been thoroughly laid in chapters V. VI and VII, which teach the method of making and applying the stitches and the proper shading of designs. The province of this chapter will be to make practical application of those lessons given in previous chapters, and also to give more complete detail of subjects treated therein whenever necessary.

Art embroidery is suitable for decorating all and every article that one may wish to embellish with needlework; the same, however, cannot be said of raised embroidery, whose fieid of usefulness is indeed limited; but more of this in its proper order.

\section{ROMAN EMBROIDERY.}

Roman embroidery is used upon articles of ornament or use, either alone or in combination with other styles of decorative needlework. As we wish to refer to it in future pages, we will here describe the method of working.

This form of embroidery is very ancient and never goes out of style; when nicely done, a piece of Roman embroidery is worthy to be handed down as an heirloom from one generation to another. It is most generallyapplied to table linen, articles of bedroom linen, curtains and cushion covers on white wash fabrics; but it is also applied to colored materials, either silks, velvets or cloths, and is known to some people as cut work; however, as Roman Embroidery is the name by which it is most popularly known, we will refer to it by that name.

To illustrate our lesson on Roman embroidery we will make use of a design suitable for tablespread border, the full working size being $28 \times 29$ inches. See figure 58 , on next page.

After stamping the design on the material, according to instructions previously given, begin the work by putting in the lines that connect the different portions of design; they are called lace lines. For this part of the 
work etching silk or wash twist should be used, unless linen threads are preferred, in which case Scotch linen floss, No. 8, will be required. In putting in the lace lines, begin at one edge and carry the thread across to the opposite edge of design,
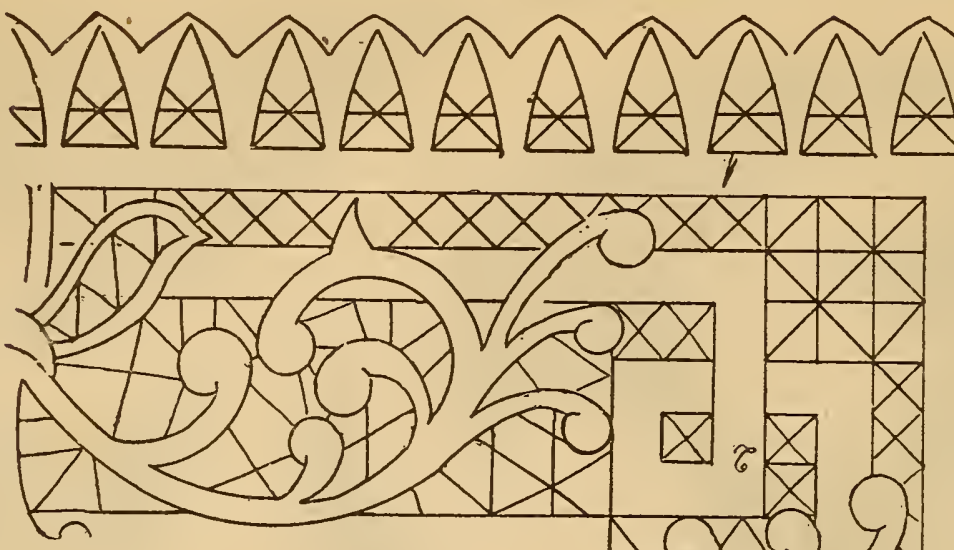
and then twist the thread back upon the line just carried across; this will give firmness to the threads and prevent the work from pulling out of shape when the material back of the lace lines is cut away. Having brought the needle back to its starting point, take a few running stitches along the edge of design until the starting point of the next lace line is reached, when the same operation of carrying across and twisting back must be
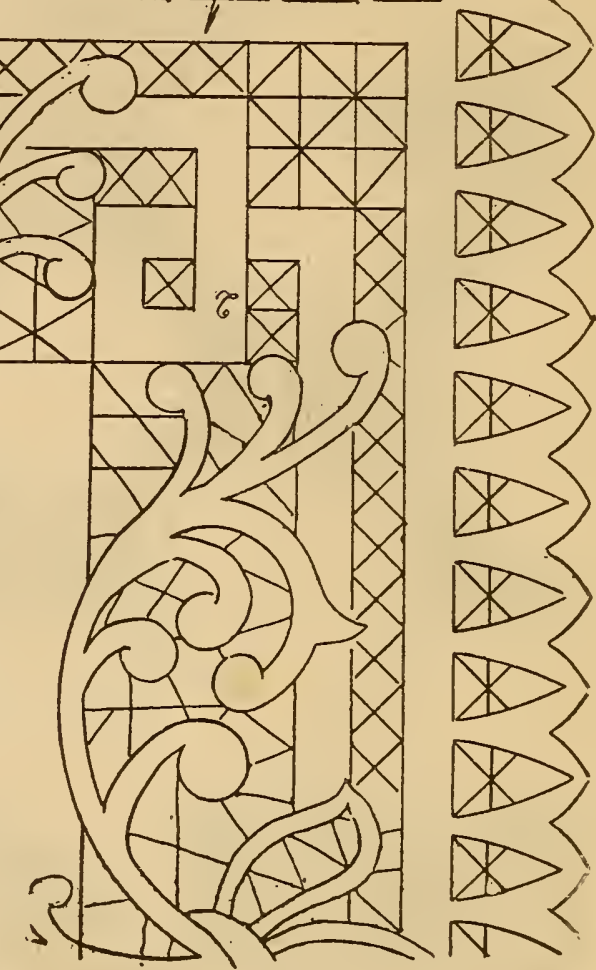

FIG. 58. repeated. Figure 59 shows section of design with the lace lines in. Now

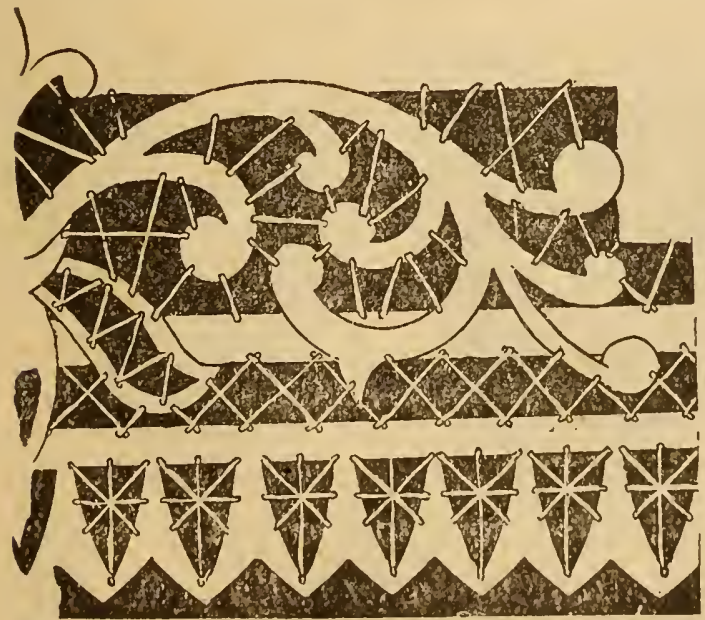

FIG. 59. around all the outlines of design, where the lines begin and end, work a buttonhole stitch over German cord, No. 1. This not only adds to the beauty of the design, but is necessary, as otherwise the cutting away of the material back of the connecting or lace lines would allow the work to pull apart. Figure 60 shows section of the design enlarged to working size and having outlines finished with buttonhole stitch.

After the lace lines are in and the edge buttonholed, the next step is to cut away the material back of the lines. This must be done with great 
DAINTY IVORK FOR PLEASURE AND PROFIT.
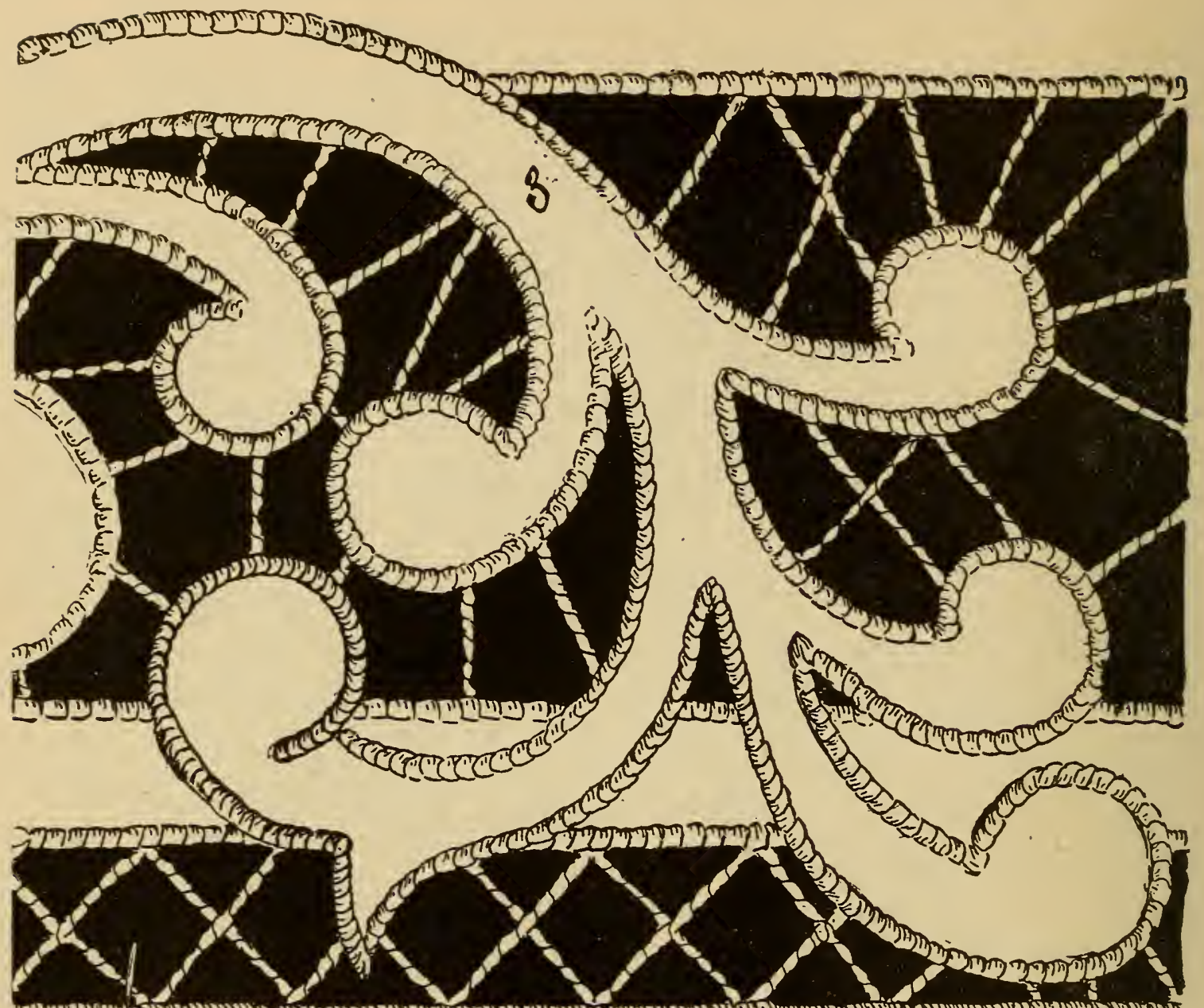

200

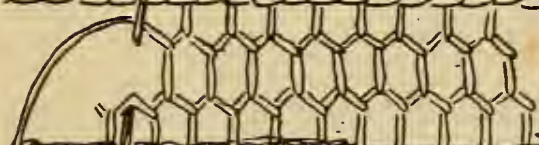

(
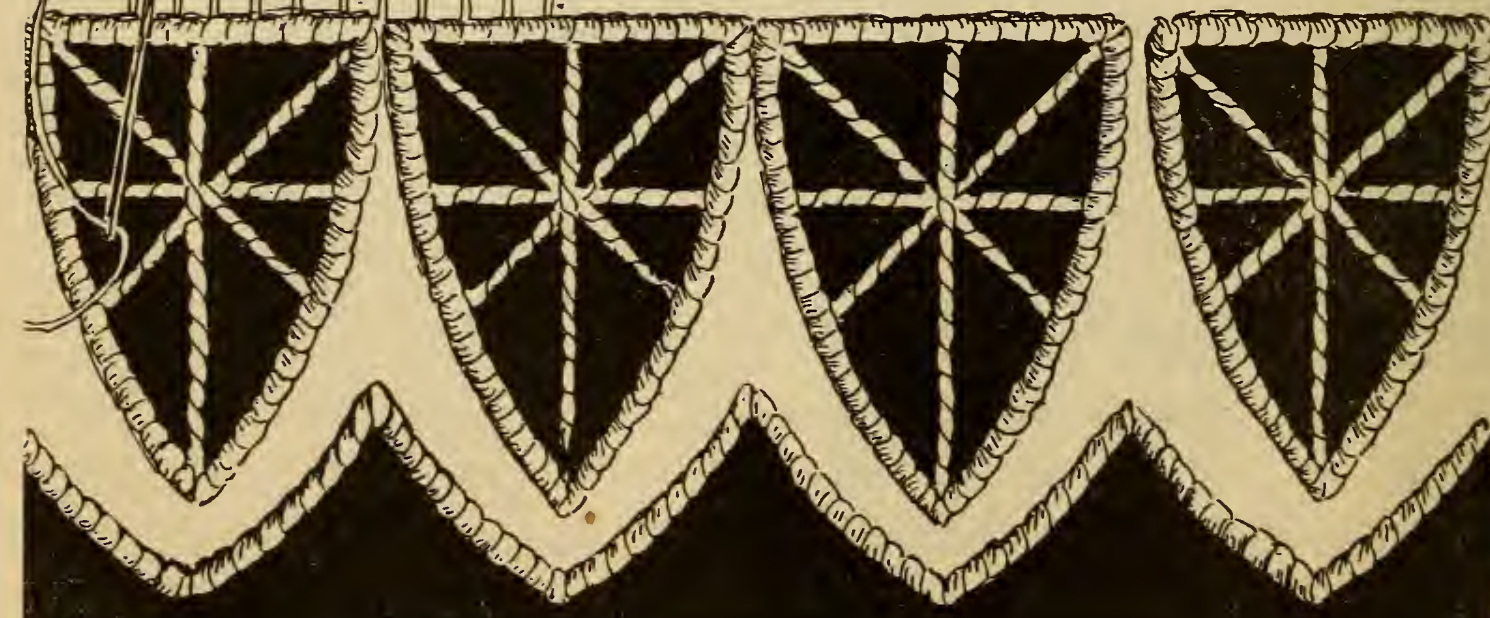

(1)
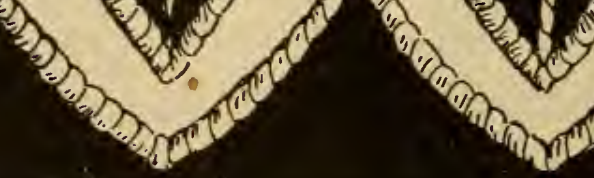
care, so as not to cut either the buttonholc edge or one of the connecting lines.

This completes the Roman embroidery proper, but, in later days, some embellishments have been added to the design. They consist of fancy filling stitches on certain parts of the material that is not cut away. Figure 60 shows a honey-comb stitch applied to a portion of the design, and the spaces marked $\mathrm{I}$ and 2 on figure 58 can be similarly treated; also the scroll portion of design, marked 3 on figure 60 , can be filled with some other stitch, thus giving variety to the design.

There is a style of Roman embroidery much used where the edges are finished with a buttonhole short and long stitch worked on the material without the strengthening aid of the German cord. For some purposes this form of the work is very pretty, but it is not in the least durable and should not be used on articles that are to receive much wear or that are to be frequently laundered.

To summarize: In making Roman embroidery-

First. Stamp design and put in the lace lines, being careful to twist back on each thread carried across. This twisting is done by simply winding the needle around the lace lines until the opposite margin is reached.

Second. Work all outlines of design with buttonhole stitch over German cord.

Third. Fill spaces of design with such filling stitches as are preferred

Fourth. Cut away material back of the lace lines, cutting close to the edge of the buttonhole stitch, taking care not to cut the buttonhole edge or one of the lace lines. The above instructions contain all there is of mys tery in the modus operandi of working Roman embroidery.

The expense of making Roman embroidery is, comparatively speaking, light. It can be done in white or colored threads, but in any case very little material suffices to work out quite an elaborate design.

Many ladies combine floral embroidery with the Roman. Thus the ends of towels, scarfs, center cloths, etc., will be finished with a Roman embroidered border, and the surface of material above the border will be embroidered solidly or with filling stitches, the combination of the twc styles of work being beautiful. 


\section{PRACTICAL EMBROIDERIES.}

Embroideries done on wash fabrics with washable embroidery threads will at once commend themselves to the wise and economical housekeeper, and dining and bedroom inens offer a field for decorative effort in which the home-loving woman never tires of working.

Table cloths, lunch sets, sideboard scarfs, doyleys, carving cloths, vegetable mats, tray cloths, pillow shams, scarfs, bedspreads, bureau and commode scarfs, splashers, tidies, toilet sets for dressing table, all should be made with a view to cleanliness, and this cannot be accomplished unless the articles are made from and embroidered with materials that will bear repeated laundering.

The articles mentioned above should all be decorated with art embroidery, and whether the design selected be conventional or natural, any of the stitches, except plush stitch, shown in Chapter V, can be employed in working. The embroidery threads to be used are a matter of taste, to a certain extent; for very fine linen or sheer fabrics, filo floss or Royal floss will be found to give good results, while for buttonhole stitch, outline stitch and some of the filling stitches, etching silk will be found better adapted. In case one wishes to use Scotch linen embroidery threads instead of silks, Scotch linen floss, No. 8 or No. 4, will be required. Gold thread is sometimes effectively employed on white wash goods, couched down as an outline, but it should never be employed on articles that are to be commonly used, and therefore frequently laundered.

Wash twist, rope sill, rope linen and Bargarren linen are all used appropriately on articles of heavy cotton and linen fabrics, whether for dining or bedrooms, though the threads first mentioned are more commonly employed upon table linen and toilet sets.

\section{USE SIMPLE DESIGNS AND STITCHES.}

Where time and expense are to be considered in decorating articles of use, the different varieties of outline stitch will be found effective and beautiful, and we say, at the risk of repeating, it is better to have several articles simply decorated with needlework than to possess one piece of marvelous and intricate workmanship, which from its very elegance is "too nice for use," and which is kept from the light of day, securely wrapped in a sheet 
or towel, and brought out only on rare occasions to be displayed with pride, and then rewrapped and retired to seclusion until, on some other occasion, it is again brought forth to delight the gaze of a favored friend-but used! perish the thought!

A bedspread, a pair of pillow shams, a toilet set, a splasher, a bureau and commode scarf, a stand cover made of good domestic linen, and embroidered with wash silk or linen threads in simple outline stitch, and all immaculately clean, are certainly within the reach of the poor and busy; and what an air of dainty home comfort they will lend to the poorest bedchamber. . Who would stop to notice that the bedstead and bureau were of commonest pine-in a word, that the room was poorly and plainly furnished. All that would appeal to the senses would be the air of sweet cleanliness, the dainty womanly touches that can make the poorest spot on earth breathe of home, rest and comfort.

If a little womanly care will do so much for the sleeping apartment, what may not be expected from an equal amount of care expended upon the dining-room, ever the true keynote of the whole home life.

Quiet elegance can be imparted to the plainest appointed table by clean and shining glass and china, which may be but common delf, and proper, though cheap, table linen in cleanly order.

Every dining-room should possess a sufficient amount of table napery to last from one wash day to the riext, and an extra set for emergencies. In the days of our great-grandmothers plain dinner napkins and damask linen table cloths alone were understood when dining-room linen was mentioned. Now the number of pieces of linen are seemingly limited only by the number of dishes on the table.

In purchasing table linen, where essentials must first be considered, one will naturally select first the everyday table cloths, which, for economy's sake, should be of good firm texture. Next, one fine damask cloth for "best." After these, napkins, and of these there should be a plentiful supply. Having secured the necessary linen, one can let her fancy have frec range through the list of modern table ?inen, including lunch cloths, ccnter pieces and table runners, tray and carving cloths, vegetable mats, fruit anch finger bowl doyleys, mats for oil and vinegar cruets, mats for water scrvice, rioyleys for fish, corn, baked potatoes and hot rolls; butter and cheese- 
plate mats; lunch sets, consisting of center piece, cup doyleys and plate doyleys, with individual doyleys for the different dishes, such as salads, cold meats, etc. These lunch sets are generally used without a table cloth, in which case the dining table should have a handsome polish.

\section{LIST OF TABLE LINEN.}

For the benefit of such of our readers as are unfamiliar with the names

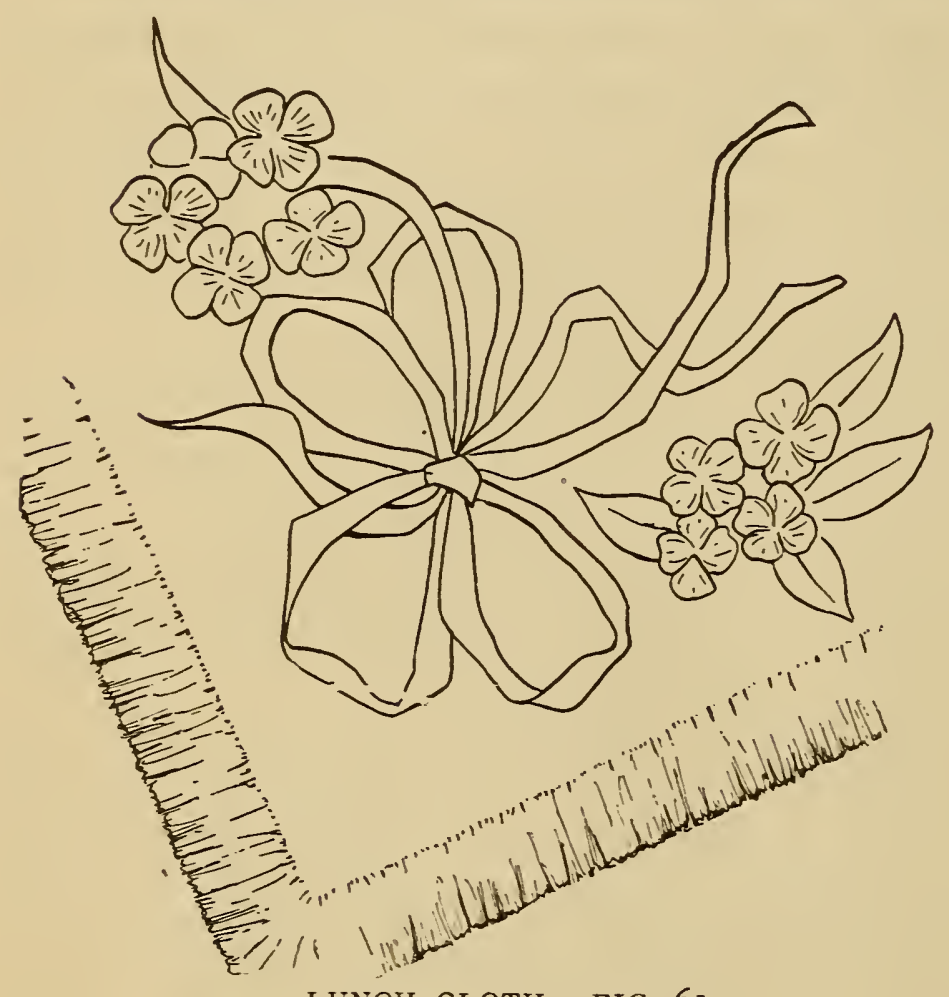

LUNCH CLOTH. FIG. 6I. and uses of the different pieces of table linen, we will here append a small descriptive list, which we trust will be of practical help.

Table Cloth.-A cloth for covering the table before the dishes are placed for the meal.

Lunch Cloth.-A cloth especially designed for luncheons, either family luncheons or those given to a number of friends. The lunch cloths are usually not as large as the ordinary table cloth, and are decorated with needlework in the corners or around the borders. Where an evening entertainment, such as a card party, is given, refreshments are generally served on small tables, at which not more than four people are seated; in such cases the lunch cluths are smaller, being not over a yard square, and the needlework is so placed as to appear upon the top of the table. Our illustration shows section of a lunch cloth made of plain satin damask and embroidered with conventional design. The ribbon portion of the pattern is one and a half inches wide, worked with simple darning stitch. See figure 6I. The edges of ribbon are then outlined. The flowers are worked in solid Kensington stitch, ir 
pure white, with a tiny French knot of pale yellow in the center of each. The ribbon is worked with pale lily green. Royal floss is used to work this design, which is repeated on each of the four corners.

Napkin.-A square of linen cloth used at table for wiping the mouth and fingers. The sizes of napkins vary, dinner napkins generally being larger than those used for breakfast or tea. The decoration on napkins usually consists of the initial or monogram of the owner, worked with white embroidery cotton or linen in the corner or center. These initials are generally worked with satin stitch, though open spaces can be most effectively filled with dot stitch or open eylet-holes.

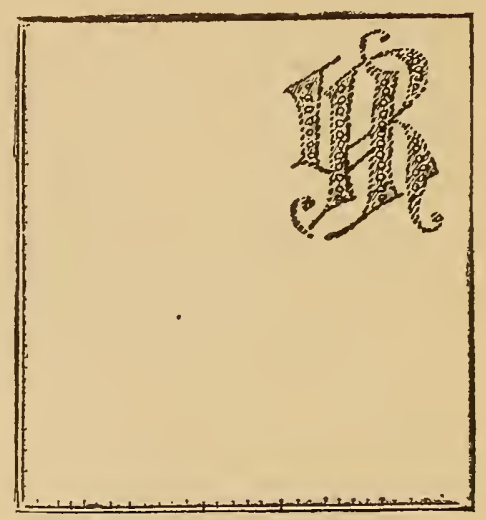

NAPKIN. FIG. 62.

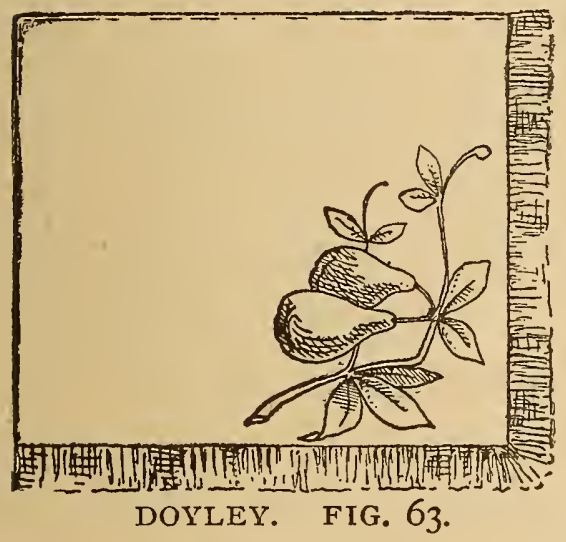

Doyley.-A small napkin used with fruit and wine and also for placing under cups, and wherever a small napkin would be required. Doyleys of a larger size are also made for placing under cheese, hot potatoes, hot roils, corn on the ear, fish and butter. These doyleys should be made of good, damask linen, with the edge fringed or hemstitched. A suitable design is usually embroidered in the corner, around the border or scattered over the entire surface.

Carving Cloth.-A piece of linen to be placed under meat, fish and game platters in order to protect the table cloth when carving. The size depends upon the size of platters, and as many carving cloths will be needed as different sizes of

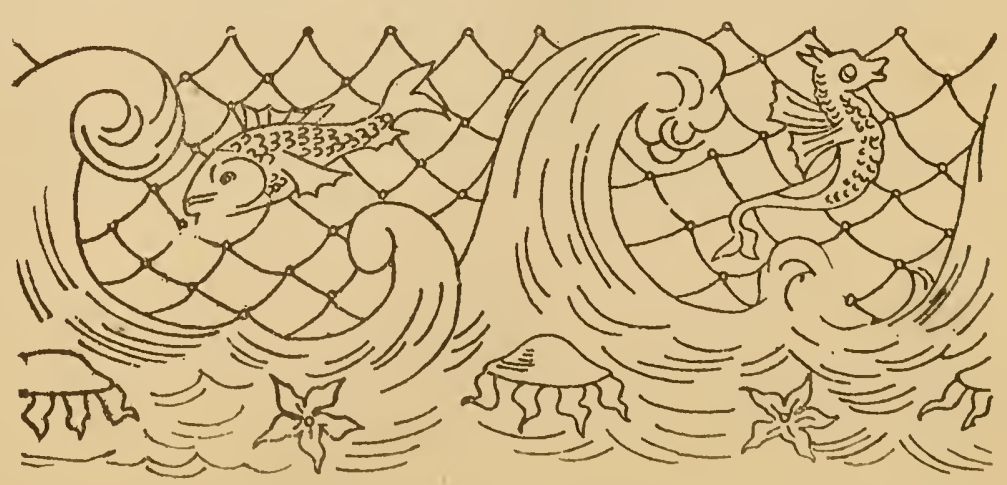

FIG. 64 .

platters are used. The cloth should come out beyond the edge of the platter at least three or four inches. The cdge can be hemstitched or fringed, 
as described for tray cloth. The design may be floral or conventional. We here show; in figure 64 , a very pretty pattern for the $=$ nd of a fish cloth. It can be enlarged to correct working size by means of the pantagraph. The design should be five times its present size. The water lines should be worked with old blue etching silk, using two shades, a light and a dark; the fish net should be worked with a light tan; the star and jelly fish with pale old rose, and the fish and dragon in the net with a combination of all the shades here given. Simple outline stitch will be all that is necessary for working the entire design.

Table Runner.-A long scarf of linen, satin damask, silk or bolting silk, having ends or sides decorated with needlework. This runiner is to be placed down the exact center of the table, and should reach within ten inches of either end. the width of the runner depends upon the widt.l of table, but from tiventy to twenty-seven inches is the usual size. A table runner is used for the same purpose as is a center cloth. The relishes, flowers, fruit and such ornamental dishes as are to remain upon the table throughout the meal are to be placed upon it.

Tray Cloth.-A piece of linen usually $18 \times 27$ inches, according to

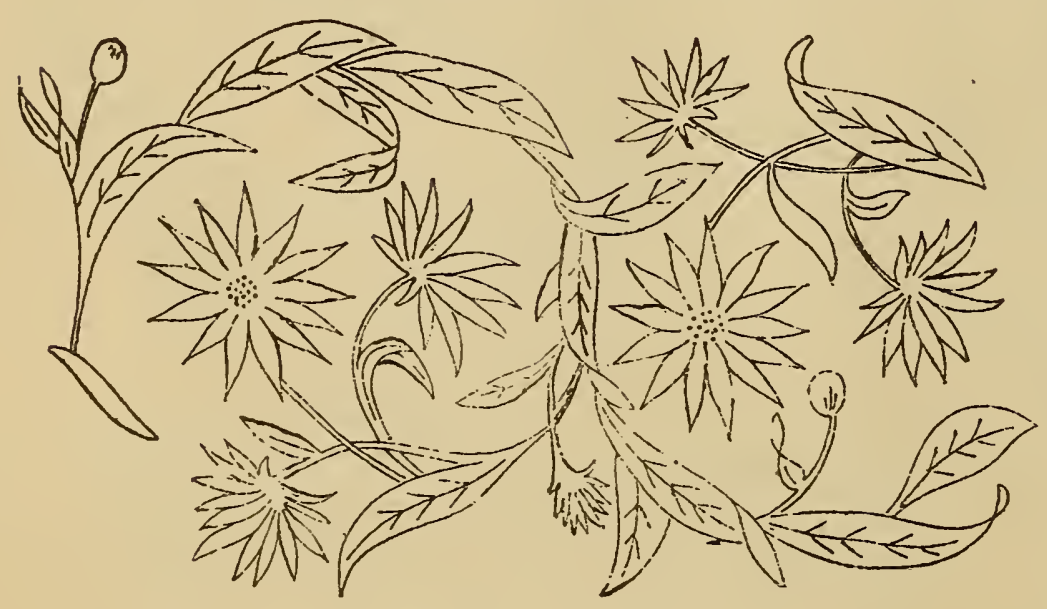

FIG. 65. the size of tablc, to be placcd beneath the tea service. The edges are either finished with hemstitching or fringe, made by raveling out the material to the requircd depth. The decoration should consist of a simpic design placed across the ends -sce figure $6 \vec{j}$-or in the four corners and outlined or worked with short and long stitch. Years ago it was considered the proper thing to spread a common hand towel on the tea tray or beneath the tea service placed upon the table, but now the dainty tray cloths, decorated appropriately for the purpose : upercedes the old style. 
Center Cloth.-A square or oblong piece of linen, silk or bolting sill, used in center of dinner table, on which is generally placed the firuit and flower picce and those dishes that are merely ornamental. These center cloths can be very elaborately ornamented, as they are in most families used on occasions of ceremony only. The needlework may be solid, or outlined with silk or linen embroidery thread. The designs most used are floral, with natural shading; sometimes the entire cloth is covered with an all-over design of flowers, worked solidly; sometimes with a floral scroll around the borders in short and long stitches. Our illustration, figure 66, shows a center cloth of satin damask, 27 iaches square, with a conventional floral design, suitable for either solid Kensington or short and long stitches. The colors used for working our model were, cream white for the outer edges of leaves and petals; into this was blended, with short and long stitches, the $t$ wo lightest shades of golden brown. The effect of blending one row of stitches into nnother is almost equal to solid Kensington stitch and not nearly as much work. The outside row

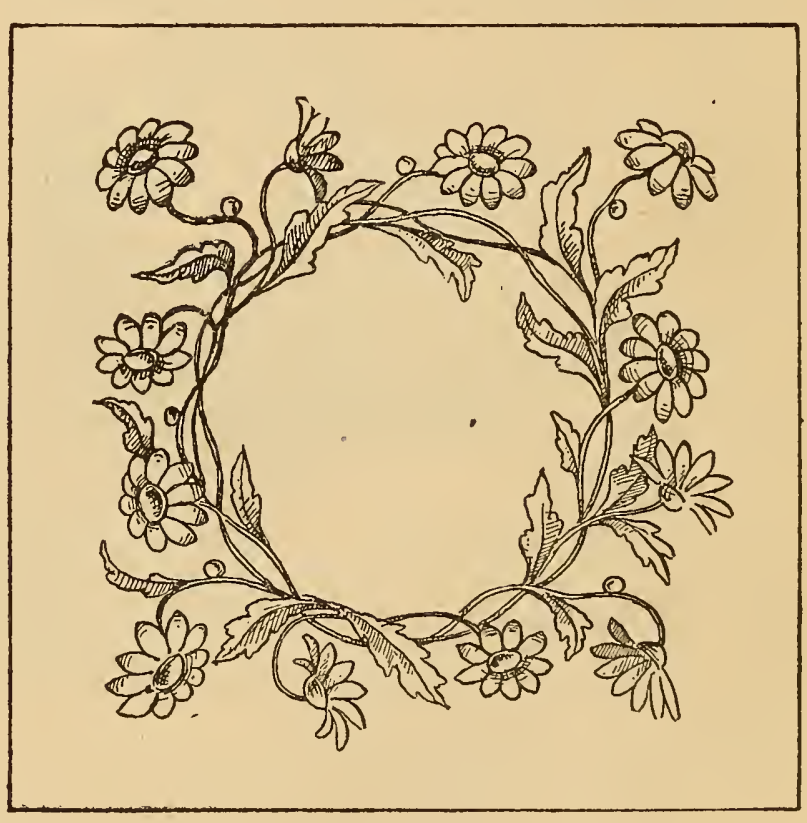

FIG. 66. of stitches may be finished with the buttonhole edge when it is desired to cut ' away the material from the edges.

Lunch Sets.-A certain number of cup and plate doyleys, napkins and center cloth, designed and worked en suite. These sets are to be used on a polished table and without a table cloth. The center cloth is made after the directions given for that article above. The cup doyleys are made from a portion of the design used in the center cloth, while the plate doyleys have a design similar to but smaller than that used on the center cloth. These lunch sets, as their name indicates, are used for formal luncicons. and consist of as many cup and plate doyleys as there are per- 
sons to be served, a center cloth and mats or doyleys for salad dishes, etc. The favorite fabric for making these sets is satin damask, and they may be $\mathrm{cm}$ -

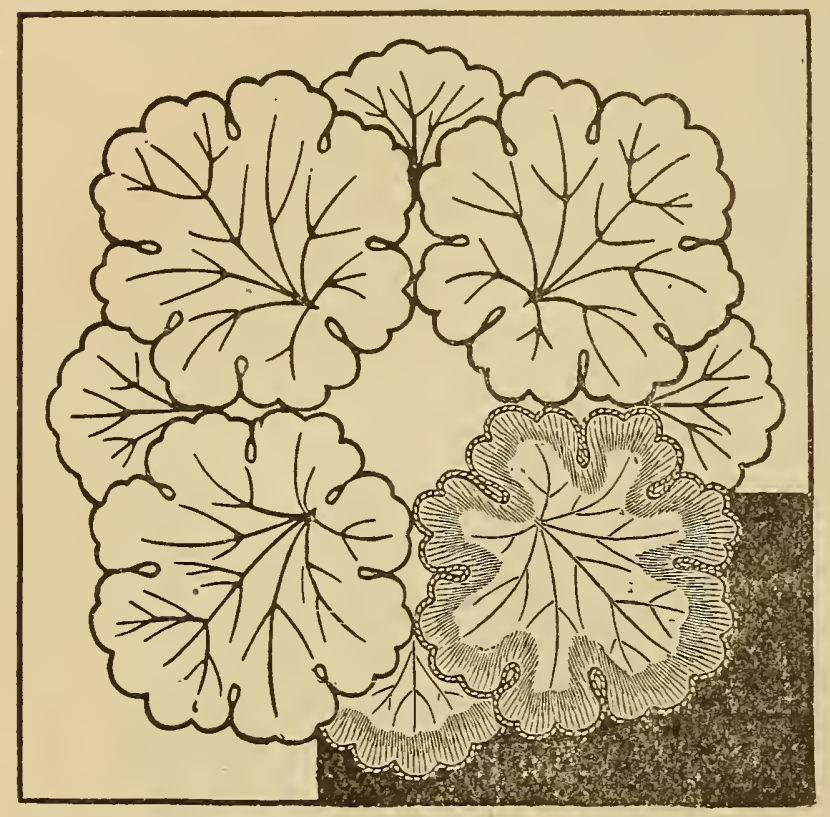

FIG. 67. broidered with either silk or linen threads. Figures 67 and 68 show center piece and cup doyley of a geranium lunch set. The plate doyley is a circle having a row of small geranium leaves arranged around it in the form of a wreath. The center piece of this particular set is but 18 inches across, and the cup doyley but nine inches. It will be seen from the illustrations that the set is worked with short and long stitches with buttonhole edge, the veins being worked with outline stitch. The three lightest shades of lily green will be required for this purpose; use the lightest shade on outside edge of leaves, and blend into it the next deeper shade, using the deepest of the three shades selected for the veins. The material is cut away from the edge of all the pieces. These lunch sets are made in different styles. A very appropriate design for lunch set where oysters are served is a row of oyster shells arranged around a circle, like a wreath. We shall give in another chapter other designs for lunch sets.

Cruet Mats.-Pieces of linen or other material for placing under oil and vinegar cruets, generally made in the form of a

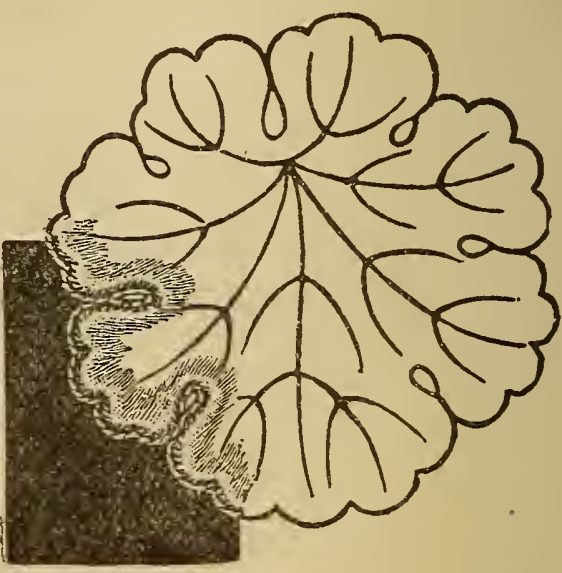

FIG. 68. leaf or flower; good domestic linen will do nicely for this purpose. The edges should be buttonholed over a German cord with silk or linen threads, and inside this a row of short and long stitches will give a rich effect. The 
veins in the centers can be worked in feather or outline stitch. Figure 69 shows a very good illustration of our meaning. A set of mats of the same design, only in varying sizes, is convenient, as the larger ones can be used for water pitcher, pickle and salad dishes, and the smaller ones for oil and vinegar cruets.

Vegetable Mats are used to place under vegetable dishes. They should be large enough to extend a little beyond the edge of the dishes used, and may be square, oblong or round in shape. Bargarren art cloth or linen canvas may be used in

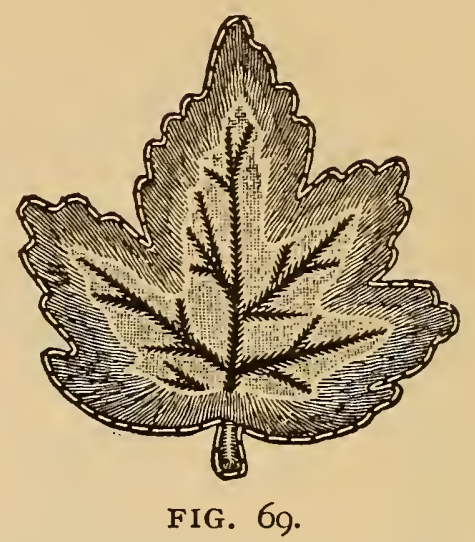
their construction. Vegetable mats should not be very elaborately decorated, a little ornamental work on the edges being all that is necessary. If Bar-

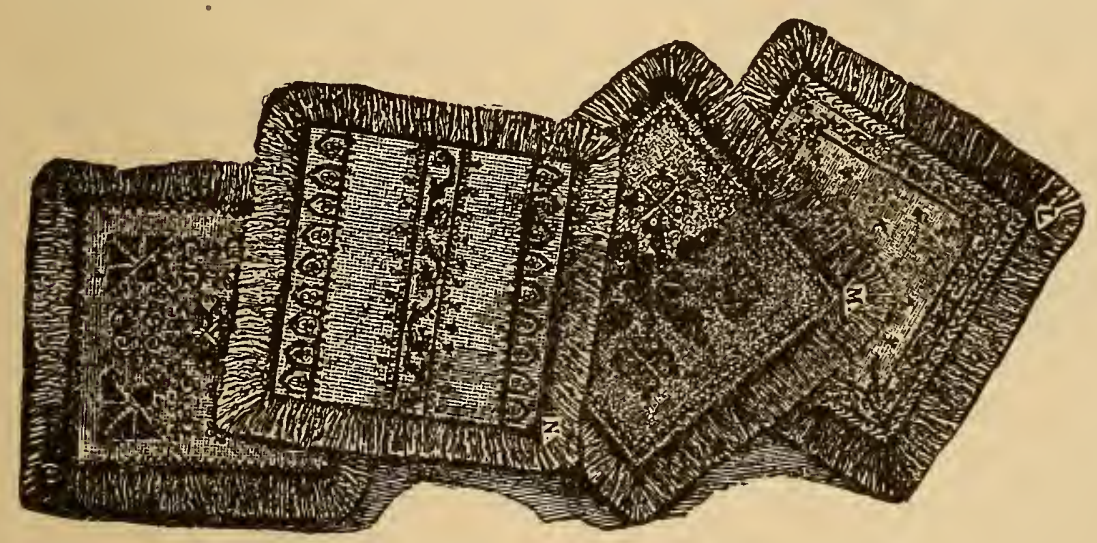

FIG. 70. garren art cloth is used a pretty border can be darned in the meshes of the fabric with Bargarren linen thread and the edge further finished by fringing the material to a suitable depth; two inches

will be deep enough. Figure 70 shows a set of these mats.

Tea or Coffee Cozy.-A covering to put over the tea or coffee pot to keep its contents warm and prevent the aroma from escaping. Figure 7I shows a very handsome cozy designed for the Chicago Society of Decorative Art. The model was made of fine white linen, embroidered solidly with white Scotch linen floss No. 4, and laced with white silk art cord over a puffing of deep golden yellow China silk. By this arrangement the covering of the cozy can be unlaced and removed for cleaning when soiled. The cozy should be made to fit the tca or coffee pot, and a good way to get the dimensions is to measure the height and width of the tea coffee pot and allow an extra two inches each way. Then out of stiff paper cut a piece the right size and round off the upper corners, making a half- 
circle, or nearly so. Now cut an inner lining of sateen and an outer lining of China silk and an interlining of wadding. Place the wadding between

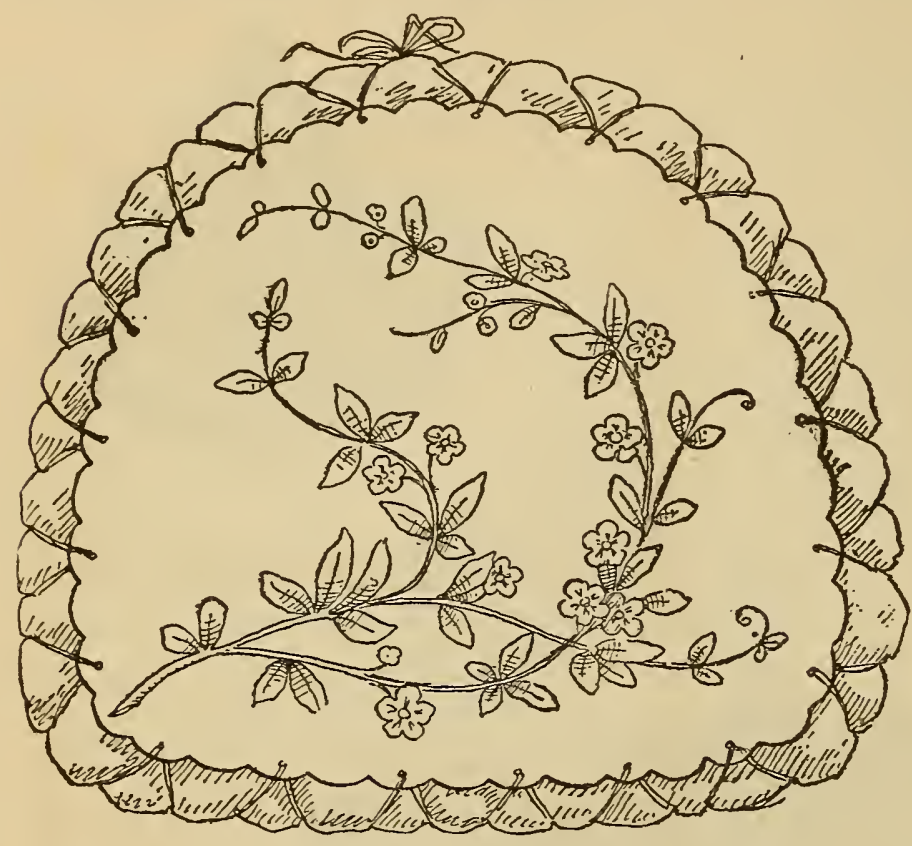

COFFEE COZY. FIG. 7I. the two linings and baste into place. Duplicate this and join the two half circles together on the sides and upper edges, leaving a space open at the bottom, like a pocket; this is to allow the covering to be slipped over the coffee pot. The outside covering should be cut out of white linen, two sizes smaller than the foundation. The edges should be scalloped and worked with buttonhole stitch, and any design preferred worked over the two pieces. Lace these embroidered pieces over the foundation by means of eyelet holes worked into each scallop, through which art cord is laced back and forth. We think that with the help afforded by the illustration no difficulty will be encountered in duplicating. The material and colors are matters to be decided by personal preference.

Mat for Water Service.-A mat to place on tray or table, for the water. pitcher and glasses to rest on. It should be made of thick material or a heavy lining should be added. Bargarren art cloth or huckabuck answers excellently for this purpose. The mats are usually cut in the form of a large leaf, haring the edges finished with buttonhole stitch

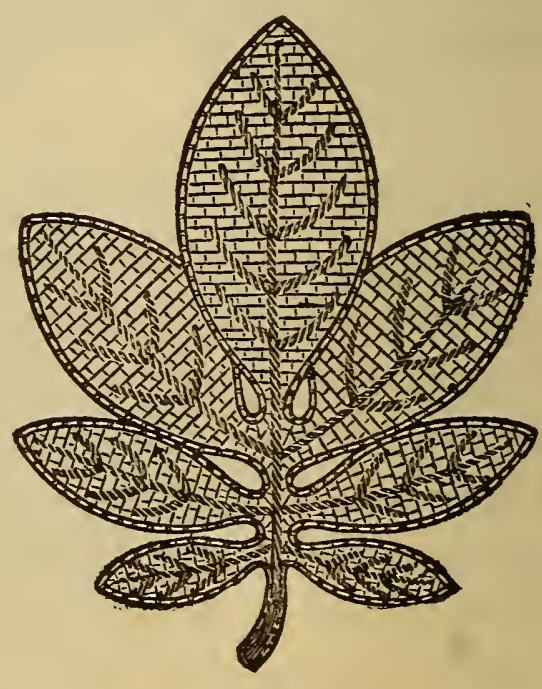

FIG. 72. taken over German cord. The surface of the mat is then darned with Bargarren'art thread through each mesh of the fabric. Any color that will harmo nize with the other decorations can be used. Figure 72 shows a $\mathrm{it}^{2}$ 
made after this idea. Other styles of decoration can be used, as it is a matter of individual fancy.

Sideboard Scarf.-A scarf of linen just long enough to reach across the sideboard, from one end to the other; or, if preferred, it can be cut long enough to allow the ends to hang over nine or ten inches, and wide enough to just cover the shelf. This scarf may be embroidered on the ends and over the front edge. Outline, short and long or solid Kensington stitch can be used with equal effect, employing any of the wash silks or linen threads preferred. Figure 73 shows a scarf having the ends ornamented with Roman embroidery. The sides and ends should be finished with hemstitch.

The illustrations given in this list of table linen are intended to illustrate the

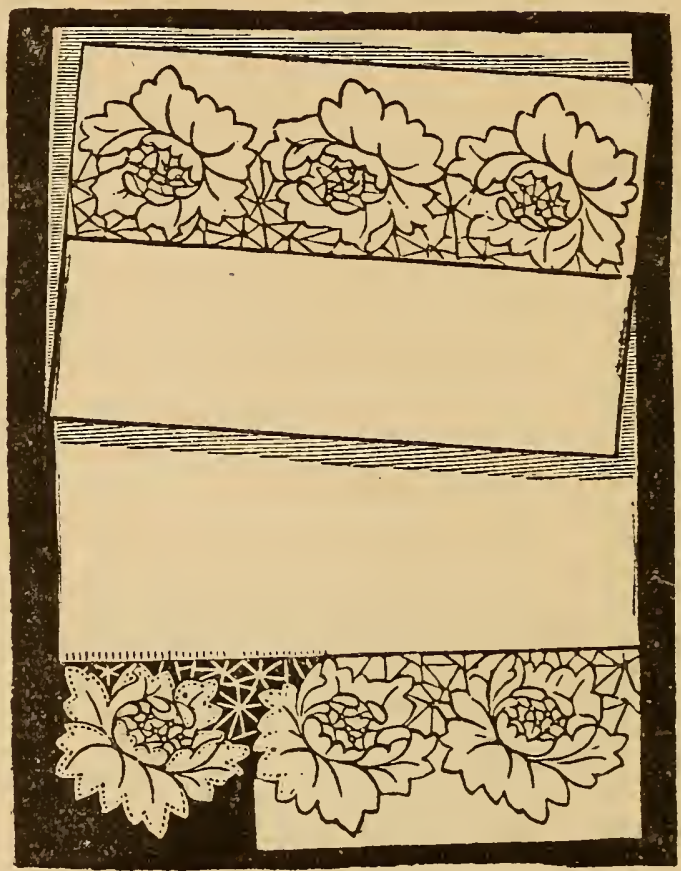

FIG. 73 . definitions only, and must not be considered as covering the field. The subject will be treated more fully in other pages of this work.

\section{EMBROIDERED BED-ROOM FURNISHINGS.}

In furnishing the sleeping apartments for members of the family a supply of articles, made ornamental by more or less needlework, should be included in the list of necessary articles.

At least two sets of bureau and commode scarfs, toilet mats, cushion covers, pillow shams, bedspreads and stand covers should be prepared for each room. The guest room should also have two sets, a little more ornamental than those used everyday. There are some people who advocate abolishing the "best" in every department of the household, but humiliation of spirit is sure to be the fate of the luckless wight who listens to such ill-judged advice. The unexpected always happens to the unprepared, and che woman who has no company china, glass, table linen, bed linen and toilet articles laid away for emergencies, is seldom prepared for the advent of the unexpected or hastily announced guest. 
The everyday articles should be of as good quality as can be afforded ror common usage, but we may well spare a fcw extra dollars for a "best" with which to do honor to those to whom we extend our hospitality.

The woman who declares that what is good enough for her is good enough for anybody, is rarely an ideal hostess. She is quite likely to be wanting in that spirit of self-sacrifice that delights to confer honor and comfort upon the guest beneath her roof; that spirit of pleasure in giving up the "best" to dear ones which is the loveliest attribute of the true "home mother."

In selecting reserve supplies it is not necessary to go to extreme expense, for there should not be a too marked disparity between the "best" and the everyday supplies, but those reserved for occasional use may well be of as fine quality as the income will warrant. They should be kept in perfect order, and those articles that are to be ornamented with needlework should be more elaborately embroidered than those used commonly.

There are many little articles of bed-room furnishing that depend, for their beauty, upon the good taste that presides over their ornamentation, rather than upon expensive materials. Following, will be found an illustrated list of articles that can be made at home for sleeping rooms that will suggest to the ingenious woman work for many an otherwise idle hour.

\section{ARTICLES FOR USE IN SLEEPING ROOMS.}

Bed Spread.-A cover to spread over the bed during the day. Bed spreads are made of many different fabrics, but those made of white wash materials are always in the best taste. Bargarren art cloth, hollywood drapery, linen bolton sheeting and bolting silk are much used for the purpose, and any one of these fabrics forms an excellent background for embroidery done with either silk or linen threads. The size and shape of bed spreads vary. Some are made long enough to come up over the pillows, in place of pillow shams; some are made just the size of the top of the bed; others come from the bottom of the bed to the lower edge of the pillows, and fall over the side board of the bedstead. The shams used with this latter style of spread are made in one piece, and reach across the top of bed, over the pillows. The full page illustration of draped beds shows a sham of this kind. The decoration may be an all-over design, or a cor- 
ner piece with a handsome center, or a border around the sides and across the ends.

Pillow Shams.-Pieces of cotton, linen or other wash fabrics, usually from $27 \times 27$ to $36 \times 36$ inches square. These shams are placed at the head of the bed, over the pillows. They can be made as ornamental as desired, or as simple. The hems are generally finished with hemstitching, and the center ornamented with design; sometimes a monogram or initial is added.

Pillow Scarf.-A long scarf, preferably of fine linen or bolting silk, though other materials can be used. This scarf should be from one and a halt to three yards long and 27 to 36 inches wide. The ends can be fringed out, or hemmed and a row of drawn work added above the hem. Above this should be embroidered a design of flowers, done solidly in fine silk or linen threads. The sides should also be hemmed. Tie the scarf in the middle with a large bow of ribbon and suspend on a hook placed in the top of head board; the ends of the scarf fall over the pillows, making a very graceful bed trimming.

\section{Commode and Dresser} Scarfs.-A strip of linen or other white wash fabric, just as wide as the top of dresser or commode and long enough to fall easily over the ends. The designs and style of work may be done to suit the worker's fancy. The ends may be hemmed, fringed or trimmed with lace. Figure 74 shows a white linen scarf hav-

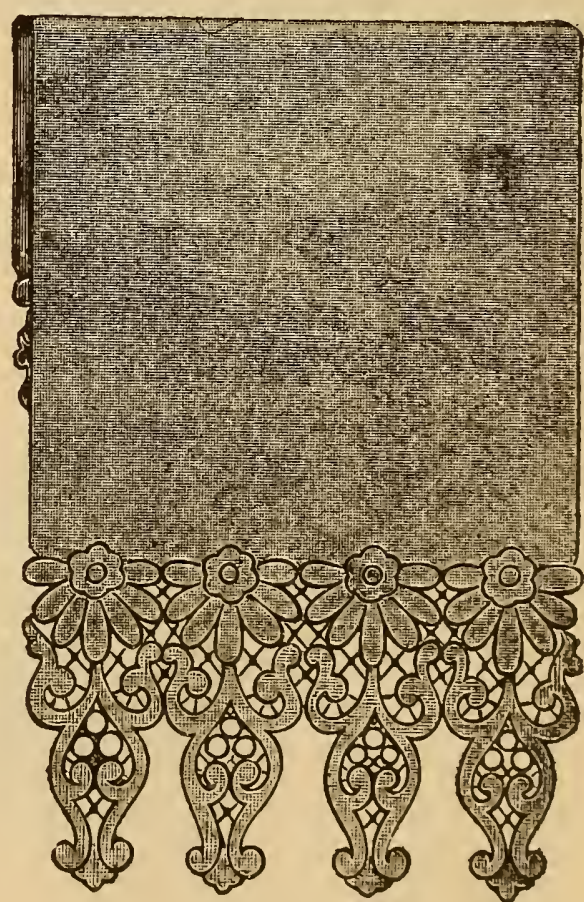

FIG. 74 . ing the ends finished in Roman embroidery with white etching silk.

Splashers.-A piece of linen or other material used to protect the wall back of the commode when the wash bowl and pitcher are used on it. The edges are stronger if finished with a hem, though some ladies prefer a short fringe, made by raveling out the matcrial. The splasher should be embroidered in outline, as more elaborate work would here seem out 
of place. The material for outlining splashers may be linen floss, No. 8, etching silk, wash twist or embroidery cotton, and some ladies who wish to be sure of a color that will stand boiling, ravel out the blue stripes in bed ticking and use the thread thus obtained.

Toilet Sets-Úsually consist of one mat for cushion to rest on, one

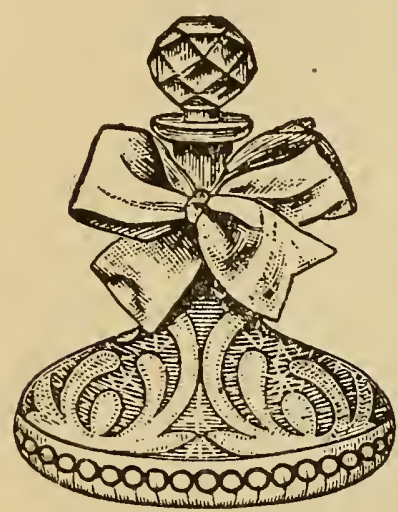

FIG. 75 . large and small cushion and a pair of covered bottles. These articles can be made as ornamental as one chooses. Figures 75,76 and 77 show large cushion and pair of bottles covered with white bolting silk, worked in Roman embroidery designs with white etching silk. The cushion and bottles are first covered with

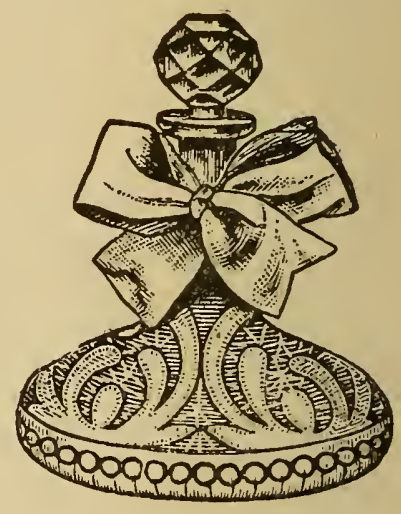

FIG. 76 . pale blue silk, over which the bolting silk cover is stretched.

Other models than those here shown can be used for toilet sets, and, the covcrings may be made of plain lawn, bolting silk, or even fine shirt linen and decorated with embroidered designs done in outline or solid Kensington stitches. Oftentimes the dressing case is furnished with two or ihree small and fancifully - s h a p ed cushions, beside

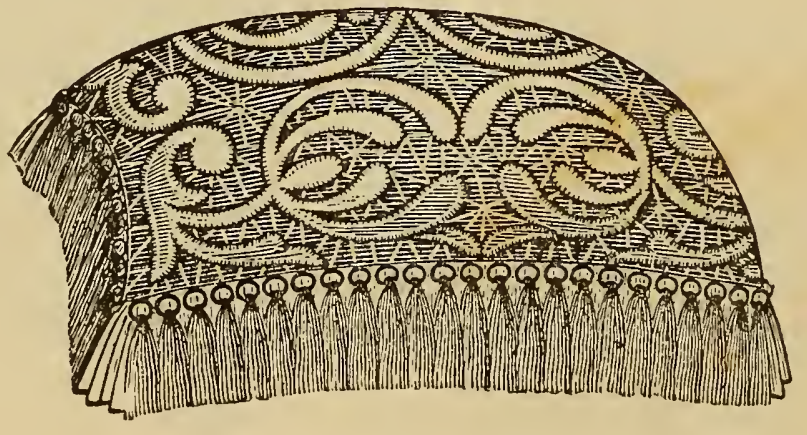

FIG. 77 . sa m e material and designs are carried throughout the entire toilet set. It is more satisfactory to purchase the $\mathrm{cush}$ ions stuffed ready the larger one. In this case the for the covering, and as the charge for even good sized cushions is very moderate it hardly pays to bother with this part of the work. Where it is desired to make the cushion at home a few hints may be helpful. First, bran makes the best possible filling, and the cushion should be stuffed with it until no more can be packed in. A blunt piece of iron or wood is a great help in pushing the bran into the corners. 


\section{OUTLINE AND COMBINATION EMBROIDERIES.}

So much effective work can be accomplished by means of outline stitches, used alone and in combination with simple short and long stitch and buttonhole outline, that we feel the subject cannot be too fully discussed.

There are so many of our sisterhood who have neither time, eyesight nor means to indulge in intricate and elaborate needlework, and who, not having the least conception of the beauty and easy accomplishment of outline embroideries, settle the matter by ignoring the subject of dccorative needlework in toto, that we feel we shall have failed in accomplishing the purpose of this book, as set forth in the introduction, did we not fully and clearly set before the readers all the possibilities of the simpler forms of 'decorative needlework.

\section{PILLOW SHAMS.}

As an illustration of what can be accomplished in this direction with little time and money we will give an example of outline, and outline combined with other stitches, applied to articles of household use.

A pair of pillow shams of bleached cotton factory are quite good enough for everyday service, and will cost, ready stamped, with rcal Scotch linen floss No. 8 for working in outline, 40 cents. If the design selected be pretty and graceful and the work neatly done, the result will delight the heart of the most fastidious housekeeper. The model we have in mind as we write consisted of a wide scroll through the center of sham, on which were the words, "Sweet Dreams." Springing from top and bottom of the scroll was a graceful arrangement of eight full-blown poppies, two buds, eight leaves, and stems. Thus it will be seen the design was quite elaborate. Figure 78 shows

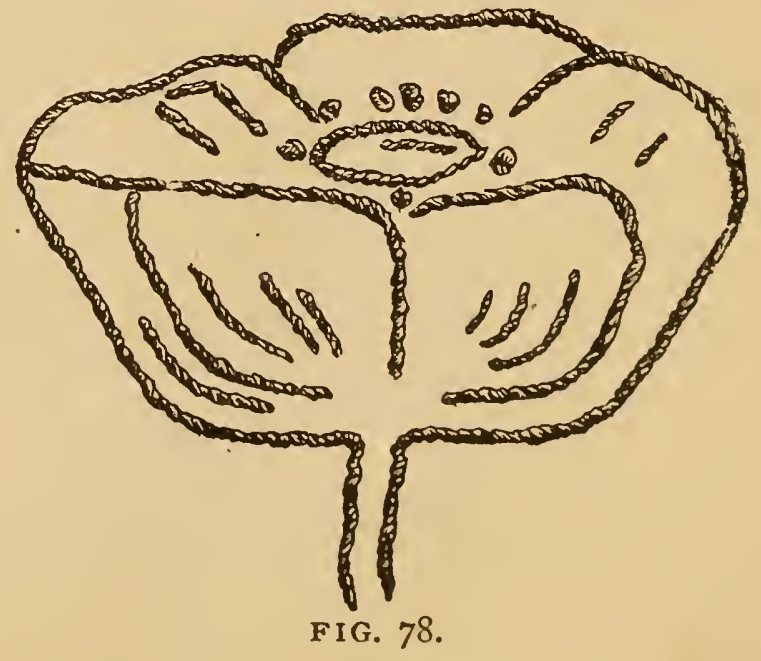
one poppy done in simple outline, and the whole design was so worked.

If more elaborate work be desired the design can be wrought out with pointed buttonhole short and long stitch for the poppies, and simple 
short and long stitch for the leaves, with outline stitch for the stems. The additicnal cost would be about 25 cents. Figure 79 shows the pointed short and long stitch, which is made by taking five stitches as follows: one short stitch, which for convenience we will call No. I; then a stitch a little

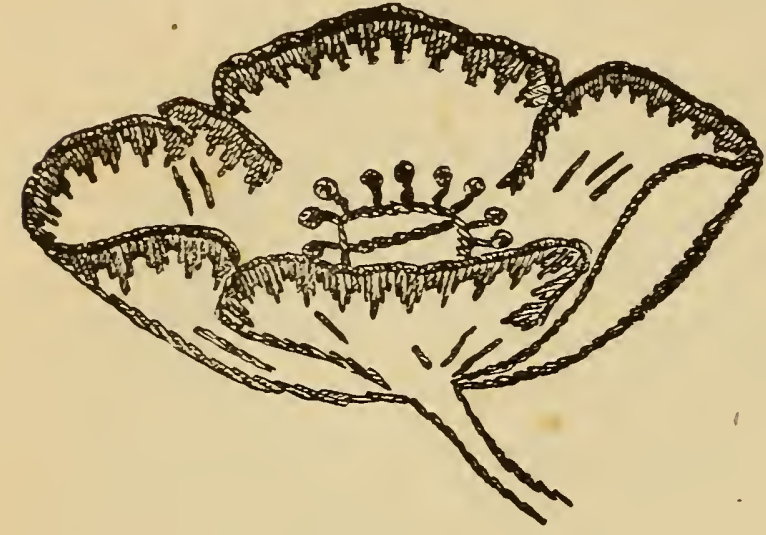

F1G. 79. longer, which we will call No. 2, and another stitch still longer, which will be No. 3. Having worked these three stitches in the order named, side by side, and from left to right. repeat No. 2 on the right of No. 3, and No. I on the right of No. 2, thus forming a point of five stitches, the longest of which is in the middle. Repeat the point around the outline of design. In taking the short and long stitches hold the thread the same as for making a buttonhole; this forms the edge. The middle stitch being the longest determines the depth to which the leaf or petal will be worked, and the length of this stitch must be determined by the size of the design.

Figure 80 , as we have said, shows a leaf of poppy worked with simple short and long stitch, which has been fully described in the chapter on stitches.

The shams can also be effectively worked by using all three stitches in the same design; for instance, work some of the poppies with the pointed short and long stitch, as shown in figure 79, and others with the outline stitch, as shown in figure 78 ; while for the leaves, work some in outline

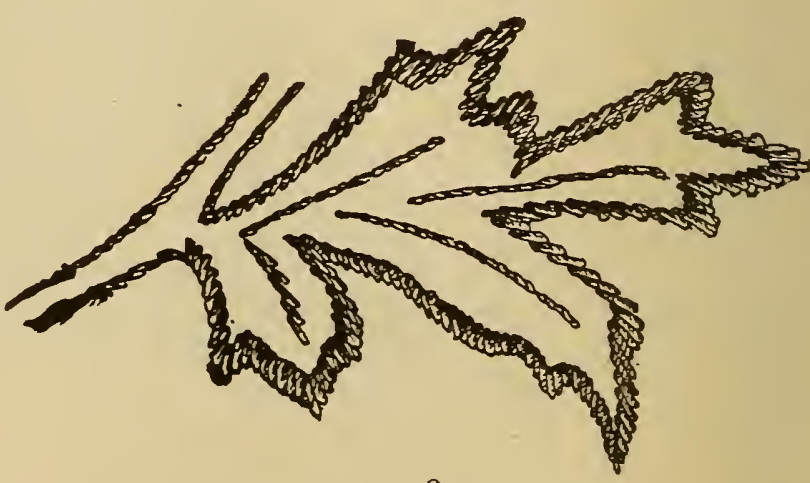

FIG. 80 . only and others with the simple short and long stitch, as shown in figure 80, and of course work the stems in outline. One shade of silk or linen can be used for all the work. The letters on the scroll, as well as the outlines of the scroll itself, should be worked out with the outline stitch, or if a more elaborate effect is desired satin stitch may be substituted. 


\section{SPLASHERS.}

The two designs shown in figure 8I are excellently adapted for splashers, and for either of them the simple outline stitch alone will be quite enough, for from the nature of the pattern the effect will be elaborate. However, if time and inclination permit, a few fancy filling stitches can be added to certain portions of the design on the right. For instance, the crescents can be filled with plain darning stitch, the petals of the star-shaped flower can be filled with honey-comb stitch and the triangle-shaped spaces with crowsfeet, while the outlines of these and the balance of design can be outlined. In case this plan of working is followed two shades can be effectively employed, as white and yellow, green and white or blue and white, or two shades of one color.

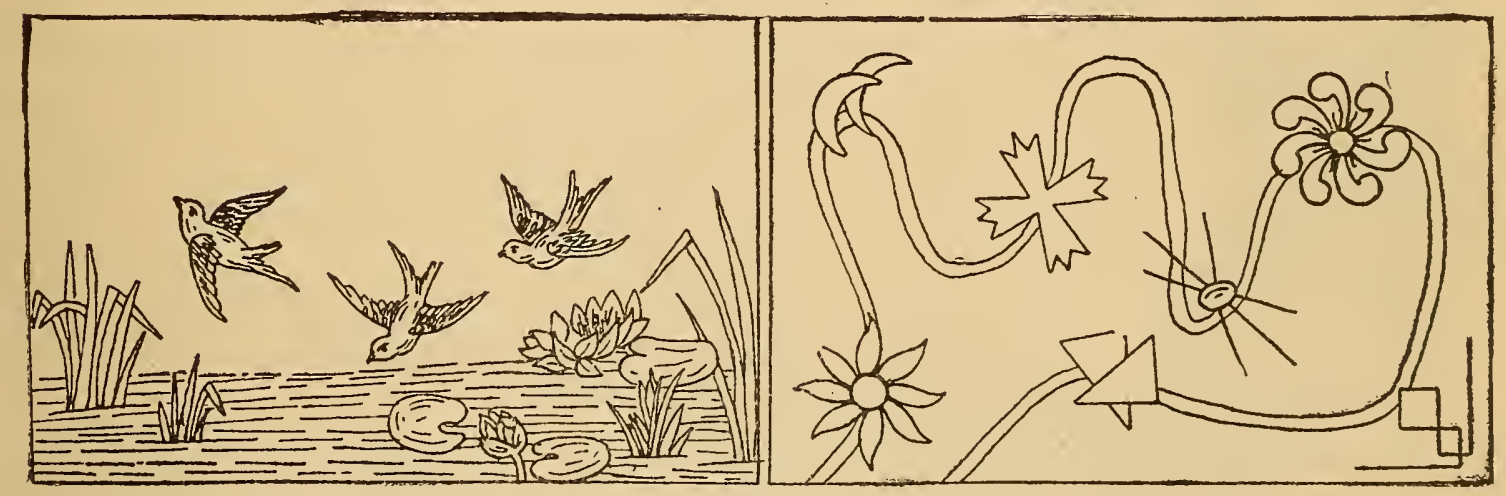

FIG. 8I.

For the design on the left - the flight of birds-outline stitch alone will be far more effective and appropriate. The birds can be outlined with three shades of fawn brown; that is; work one bird in one shade, the second bird in another shade, and the third bird in still a deeper shade of the same color. The water lines can be worked with an old blue, one shade; while for the lilies use cream white, and for the lily pads and flag stalks use two or three of the shades used in worling the birds. Of course any other scheme of coloring can be followed out. We merely offer the above as a suggestion, for in fact the entire pattern could be correctly worked with only one shade if it were so desired. The amount of material necessary for these splashers will be two picces of butcher's linen or brown crash, $27 \times 36$ inches. Hem the edge with hemstitching. For the design on the left will be required, of Scotch floss No. S, one skein of each of the colors men. 
tioned in this connection. For the design on the right, three skeins of the color used for the outline and one skein for the fancy stitches will be sufficient.

\section{BORDER FOR LUNCH CLOTH.}

This border is equally suitable for lunch cloth or center cloth, and worked out as indicated in the design here shown will be at once beautiful and inexpensive. For the cloth a good quality domestic linen or satin

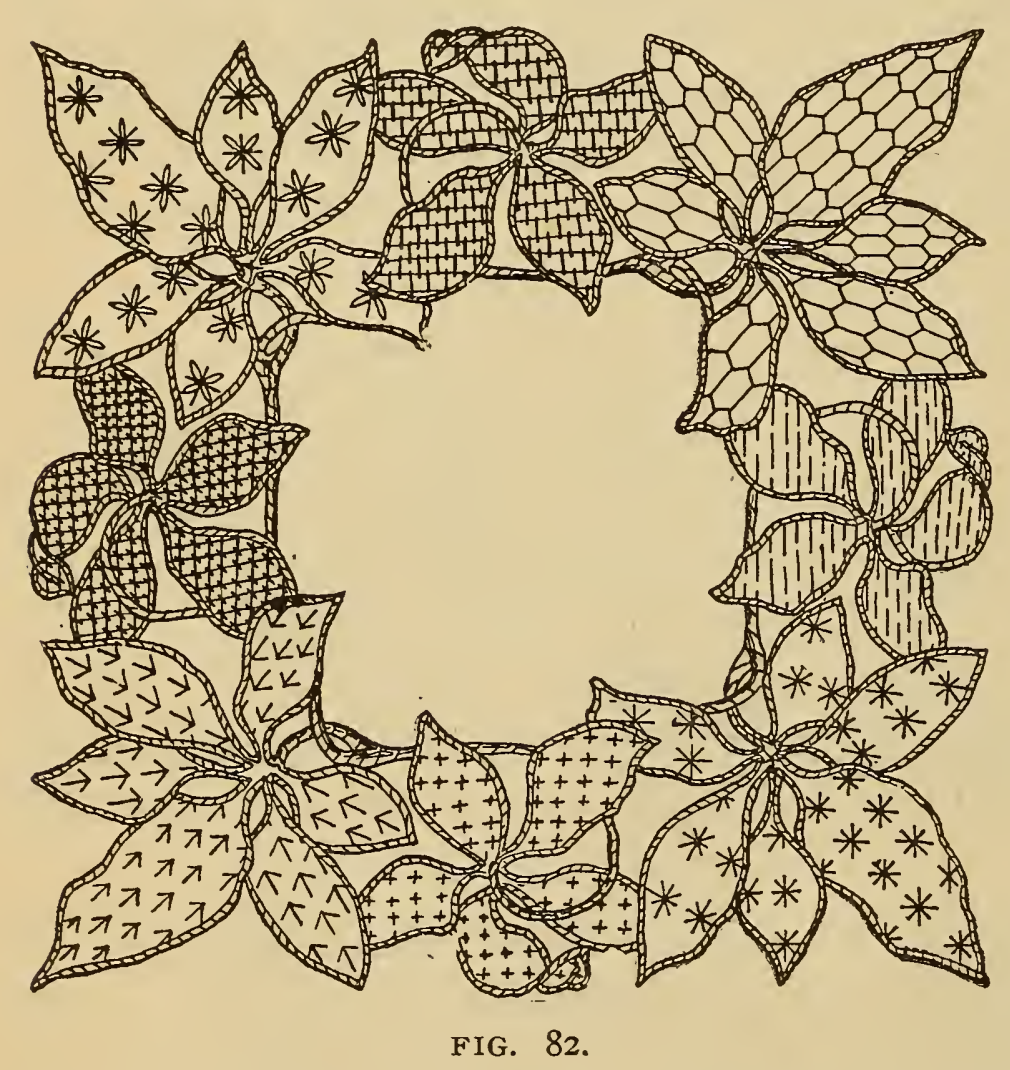
damask should be used. All the leaves and connecting outlines should be worked with buttonhole stitch over German cord, No. I, with either Scotch linen floss or etching silk, and the surface of the leaves filled in with the combination of filling stitches, as shown in design, or with any one of them, as preferred, though we think the effect is richer when the combination is used. The stitches here shown are honey-comb, single basket, daisy star, diamond couching, crowsfeet, cross stitch, star and darning, all of which will be found described in the chapter on stitches. The amount of material for making the cloth will depend upon the size of the cloth, from 27 to 54 inches square being the usual sizes. For the first-mentioned size five skeins German cord, No. I, eighteen skeins wash twist, for working buttonhole stitch over the cord, and two dozen skeins etching silk for the filling stitches, will be required. For the last-mentioned size double the amount will be needed.

If this design is desired as a border for a large cloth cover for large table the leaves will look exceedingly well filled with point d'reprise, 
rosette stitches and d'Alencon bars, worked with art cord and the outlines finished with rope silk in outline stitch.

The method of working the three stitches mentioned will be found in Chapter V.

\section{SCARFS.}

Figure 83 shows designs for three scarfs, suitable for either sideboard or dressing case. Butcher's lincn, huckabuck, domestic linen or satin damask can be used for the scarfs, though of course the last-named fabric is the most expensive. Two yards of $4 \mathrm{O}$-inch butcher's or domestic linen will make two scarfs, costing, according to the quality of linen, 80 cents or one

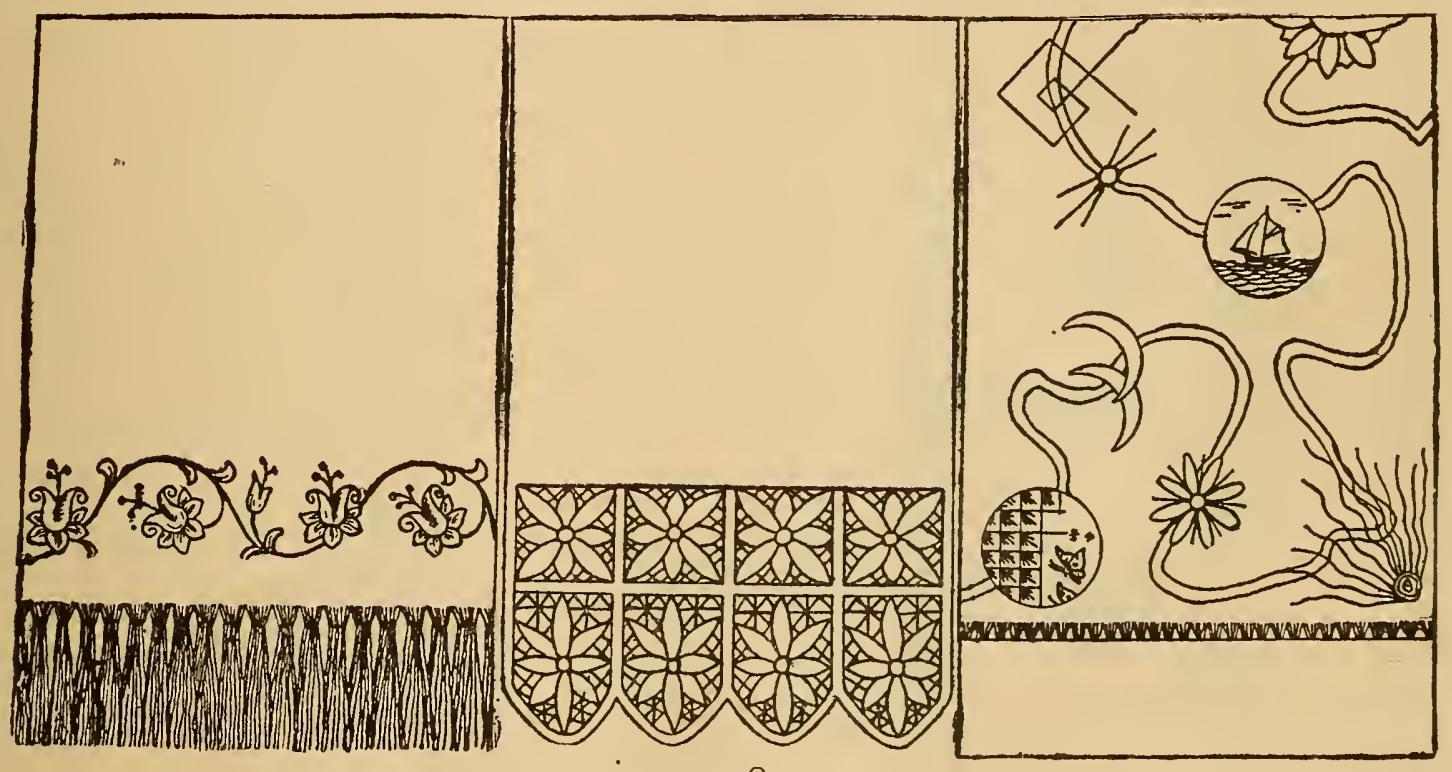

FIG. 83 .

dollar for the pair. The sides should be finished with a narrow hem hemstitched down, and the ends with either a fringe formed by raveling out the linen to the depth of nine inches, or with torchon lace sewed on, or with a hemstitched hem, with a row or two of drawn work above. The designs on all three of these scarfs are purely conventional. The one on the scarf at the left is a very graceful scroll, which will look exceedingly well outlined, and having the surface of design filled in with open basket stitch. Another effective treatment would be to outline the sitems, leaves and stamens, and fill all the rest of design with short and long stitch. To work the design in the manner indicated last will require six skeins Real Scotch linen floss, or eighteen skeins Royal floss silk; while for the treatment first mentioned, about half the amount named would suffice. 
The center scarf is a beautiful design in Roman embroidery, and should be worked according to instructions given for that style of work at the beginning of this chapter. The design in question is very lacy and light, owing to the great number of lace or connecting lines. The material for working this scarf may be either silk or linen; if the formcr is used two dozen skeins wash twist, six skeins of German cord, No. I, and one dozen skeins etching silk will be required. Use the wash twist for the lace lines and for buttonholing over the German cord around all the outlines, and use

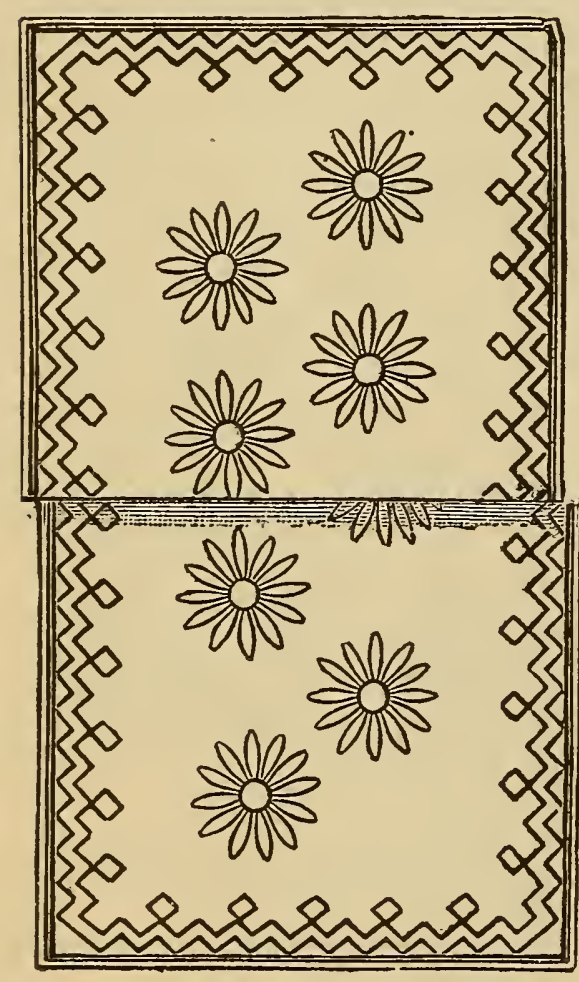

FIG. 84. the etching silk for filling in all the starshaped figures with fancy filling stitches-of which there may be a variety, or only one throughout entire design, as preferred. Honey-comb, brick, star, crowsfeet and" darning will all combine effectively. For the very center of each star figure crochet over a brass ring, large enough to cover the circle in the center, with the wash tivist, sew firmly to the foundation, exactly over the stamped circle, with a thread of the silk used to crochet with, after which cut the cloth away from the back. In a design having so many lace lines care must be observed to twist all the threads forming those lines, according to directions given for that part of the work in the lesson on Roman embroidery.

The scarf on the right, in figure 83 , is a very pretty all-over pattern, and particularly appropriate for a sideboard. Work in outline stitcl alone, and use not more than three colors or shades-and one only will be very beautiful. For this design two dozen skeins etching silk or one dozen skeins Scotch linen floss, No. 8, will be required.

Figure 84 shows a scarf finished around the edges with fancy stitches and having the surface from end to end covered with a daisy pattern. This scarf can be used as a cover for side board, runner for dinner table or as a dressing case scarf; etching silk should be used for the stitches around the edges and filo floss for the design over the surface; two shades of pink, blue or brown will be effective. 
DOYLEYS.

There are many different methods of decorating doyleys, chief among which are the all-over and the Dresden designs. The former style is well represented by the two illustrations shown in figures 85 and 86 . The two

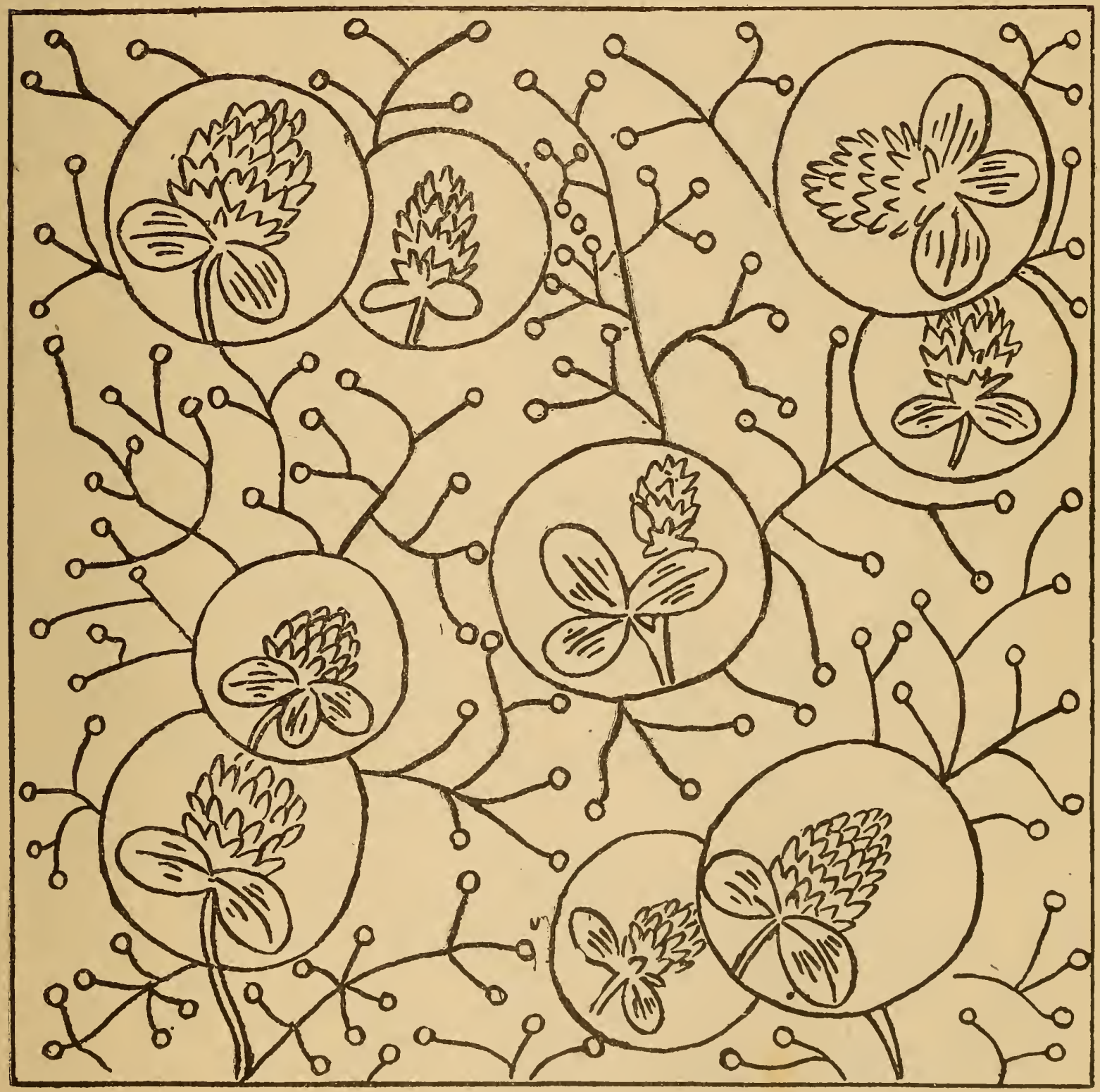

DOYLEY. FIG. 85 .

designs are given full working size and can be transferred to the material according to the directions given in Chapter IV. The edges of doyleys are usually finished with a very narrow hemstitched hem. To work figure 85 will require four skeins of filo floss, as follows: Shades Nos. I202, I240, I242 and I260; as will be seen from the table of shading, these colors 
are pure white, pale green, dark green and pale yellow. Use the white for the clover heads, the pale yellow for outlining the circles, the dark green for the clover leaves and the pale green for working the dotted

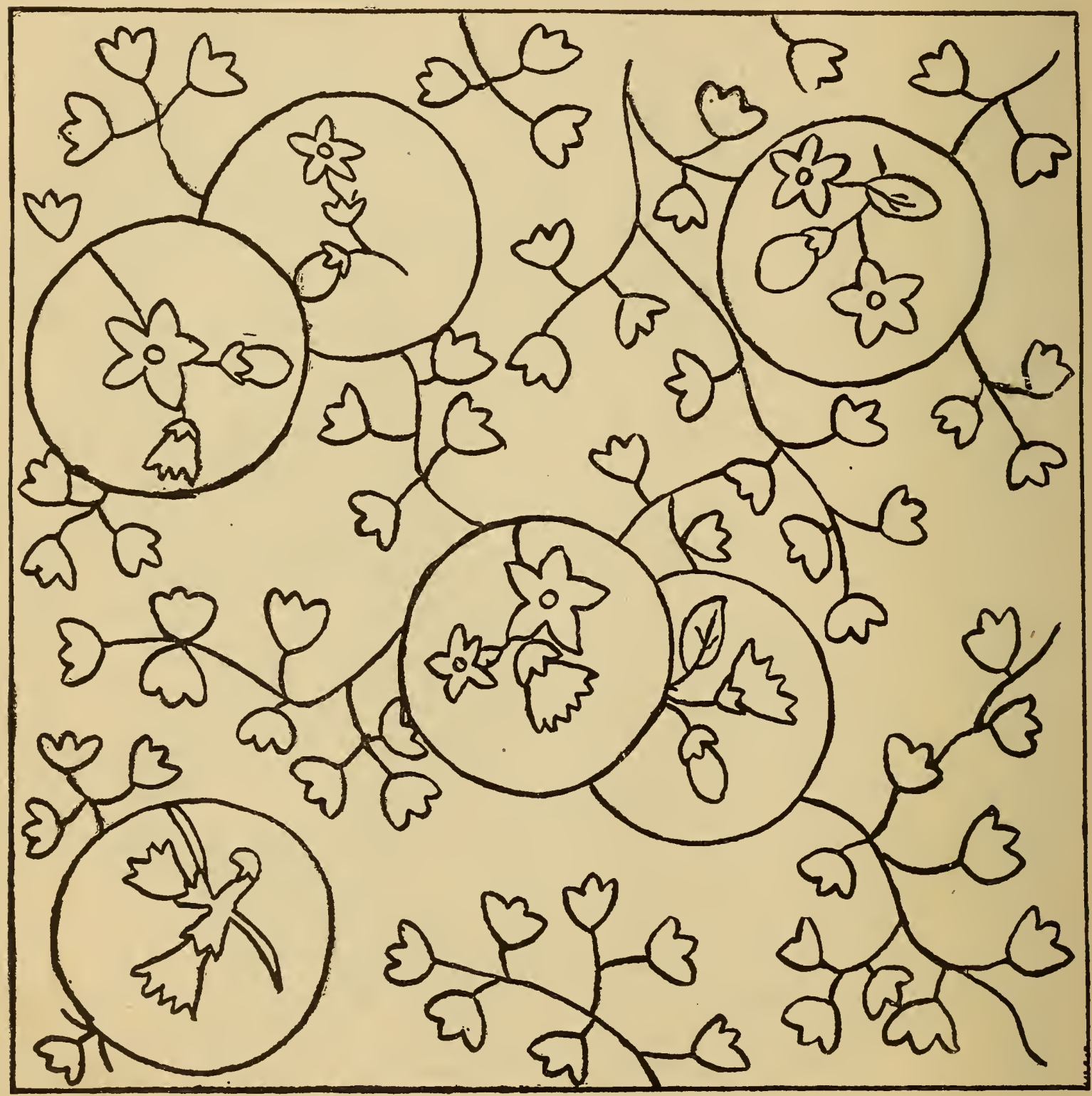

DOYLEY. FIG. 86.

vines that connect the circles; or, a very good effect can be produced by using both shades of green in this portion of the design, outlining the vines with the deeper shade and working the dots with the lighter shade of green. The stitches necessary will be outline for vines, circles, clover heads and 
leaves, and spot stitch for working the dots at the end of the vines. The four skeins of silk necessary to be purchased in order to secure the shades mentioned will not be used in working this design once, in fact but a few threads from each skein will be needed; but the same colors can be employed for working figure 86, using the white for the snowdrop and flower, the pale yellow for the center of flower, also to outline circle; use the two shades of green for working the foliage of snowdrop and the maiden hair ferns that connect the circles. As will be seen from the example given above a variety of designs can be wrought out with the same colors, and it is well, in selecting patterns, to bear this in mind, as it saves purchasing so many more skeins of silk or linen than are actually needed for the work in hand.

There are many new and beautiful designs for small fruit and finger bowl doyleys that are designed to be worked in solid Kensington stitch. As doyleys for the purposes named above are rarely over four to five inches square it follows that the designs for them must be of a light and dainty character, and where it is wished to use large sized flowers for this purpose, they must of necessity be drawn in miniature.

Figure 87 shows a sct of six doyleys ornamented with flowers in miniature, designed for solid Kensington stitch. The designs for this set were drawn expressly for this book and are among the most artistic floral arrangements we have ever seen.

The materials for these doyleys may be bolting silk, linen lawn, or fine shirt linen. The edges may be hemstitched or fringed, but the former mode of finishing is the one most commonly employed.

No. I. Narcissus. The arrangement here shown is particularly light and graceful and will require, four shades of filo floss for working in Kensington stitch. Pure white, No. I200; green, two shades, Nos. I240, I24I; yellow, 1460. Work all the narcissus petals and the throat of the flower with No. I200. The cup in the center should be worked with the yellow, and the calyx with the lightest shade of green. The leaves should be worked with both shades of green, using the darkest shade in the shaded and lower portion of leaf.

No. 2. Morning Glories. For this design will be required of filo floss green, Nos. I240, I24I, I242; pink, Nos. I288, I289, 1290; by comparing these numbers with those indicated for the narcissus doyley, it will be seen 
that but one additional shade of green is required, namely I242. Use the last named shade for the veins, stems and shaded portion of leaves; for the tendrils and calyx use the lightest shade. The throat of the morning glory, inside and outside, should be worked with the palest shade of pink and be lightly streaked with the shade next deeper. The edge of the petal should be worked with the two deepest shades; for the buds use No. I290.

No. 3. Peach Blossoms. For this design pale pink, white, pale yellow and a shade of brown filo floss will be required. Work the blossoms over in

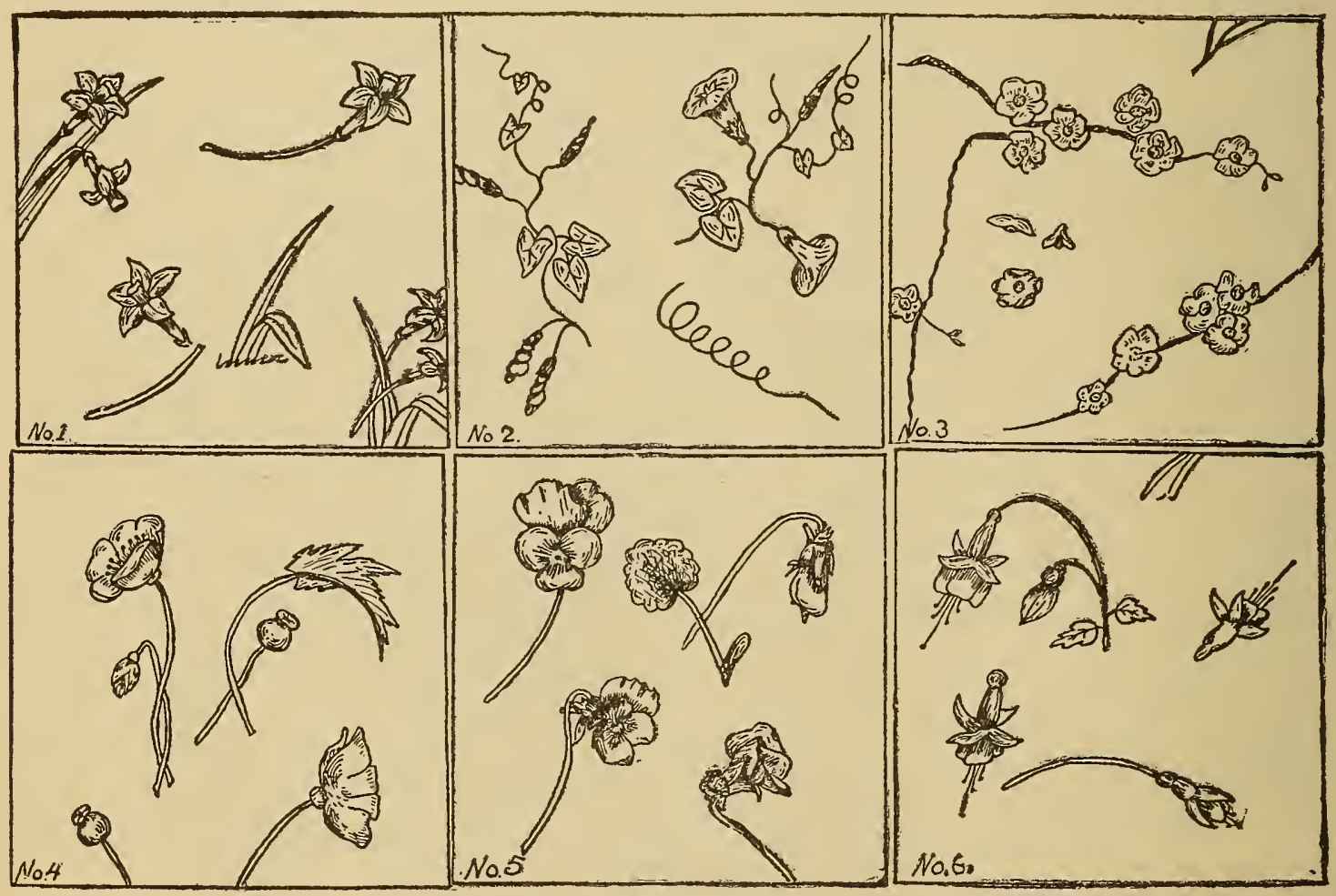

FIG. 87 .

solid Kensington stitch with the white filo floss, then with the pale pink, No. I288, work over this lightly in the same stitch; fill the center with a French knot of pale yellow, No. I260. For the leaves and the young stems use the pale green, No. 1240, and for the branch use the brown, No. I296. For this design only one additional color will be needed, namely. No. 1296 , All the others having been used in doyleys No. I and 2.

No. 4. Poppies. If it is decided to work the poppies in either pink or yellow only two additional shades will be necessary, Nos. I26I and I229, as those used in the preceding doyleys will give correct shading. For large 
design of poppies, wrought out with elaborate detail, we should prefer the line of silver green for the foliage; but in this design there is so small a surface of green that those shades used for the narcissus and morning glories will answer very well. In working the foliage use the lightest shade of green for the turned, leaf, and the darkest shade, or No. 1242, for the under side of the same leaf; for the stem and bottom portions of seed pod use the brown, shade No. I296; for the top of seed pod use the lightest shade of green, No. I260; if a red poppy is preferred then additional shades will be required, namely, Nos. I340, I34 I, I342. For the disposition of the shades in the poppies see illustrations in the Chapter on Shading.

No. 5. Pansies. A design of pansies is sure to appeal to the heart of every lover of flowers, for of all flowers it seems to be the one that is universally held dear, either for the sake of its almost intelligent beauty or for the sake of old memories and associations. At all events it is certainly the flower of the rich and poor alike. In shading pansies it is more satisfactory to select a cluster and shade them as near like the original as the silk thread will permit. To give a rule for shading pansies would be like giving a cut and dried receipt for making human faces and we fancy the result would be almost the same in either case, an absolute failure; however, as there are some ladies who insist on having a receipt or rule for everything they do, we will try to give a simple shading that can be easily followed. But, ah! if the reader loves the pansy, how tame and spiritless will seem the result. When embroidering this flower we always feel as though it has a sentient existence, and we are therefore in honor bound to do it justice; to give expression to its inmost thought and feeling-for who can look at the lovely and expressive face of a pansy and doubt for a moment that it docs think and feel.

For a deep purple pansy, four shades of purple filo floss, Nos: I 300 , I 301, I 302 and I3O4 will be required; for the eye or spot in the center, a pale yellow may be used, No. I260 will answer, and for the stem and calyx Nos. I24I and I242. Begin at the outer edge of the top petal and with the lightest shade of purple work to the center, blending in gradually the deeper shades. For the lower or under pețals, the rule may be reversed, beginning at the edge with the deepest shade and blending in to the center with the lightest shades. 
No. 6. Fuchsias. This design is a very graceful arrangement of that somewhat difficult flower-difficult both as to the setting of stitches and the

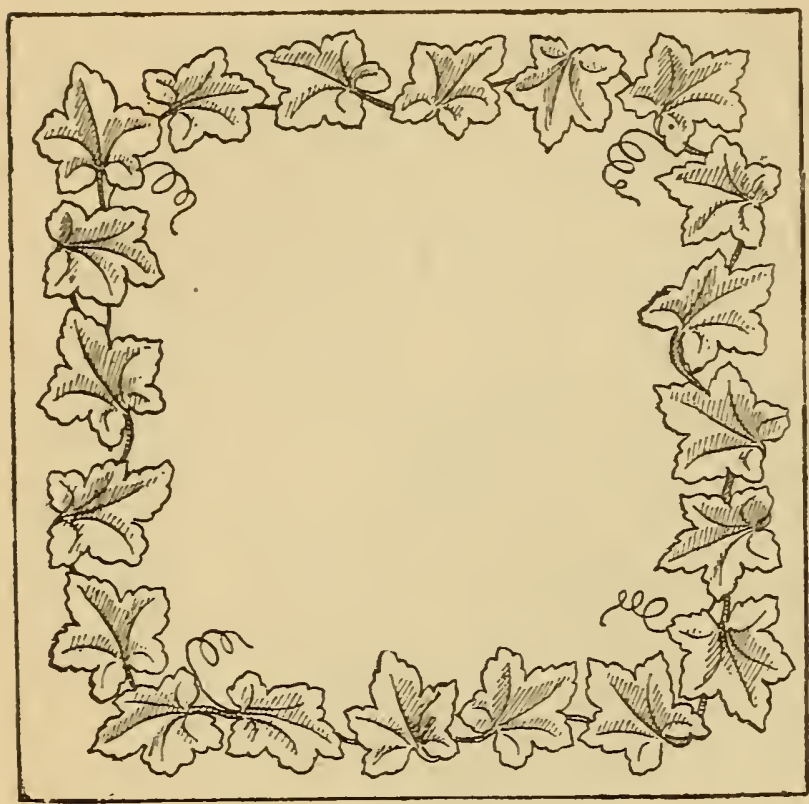

FIG. 88. coloring. The best effects are obtained by commencing at the edge of lower petals and working upwards toward the calyx. The colorings of this flower are many and varied, from combinations of pink and white to red and purple. The natural flower is the best guide for shading, as we might here indicate colors which the reader has not chanced to see duplicated in the natural flower. yet which has been taken by us direct from the hand of Dame Nature herself. In shading a purple and red fuchsia the under portion of the flower should be a deep red, best copied in shade No. I 209 or I2I2, while for the upper portion, No. I 304 or 1374 will come as near the correct coloring as our materials will allow. The sepals and stamens should be a purplish white; for the stems and foliage, shades Nos. I 240 and 1243 may be used. Fuchsias can also be worked in satin stitch with good effect.

Doyleys are frequently worked with a combination of satin, spot and seed stitches and French knots. When these stitches are used the doyleys are generally made of fine lawn or

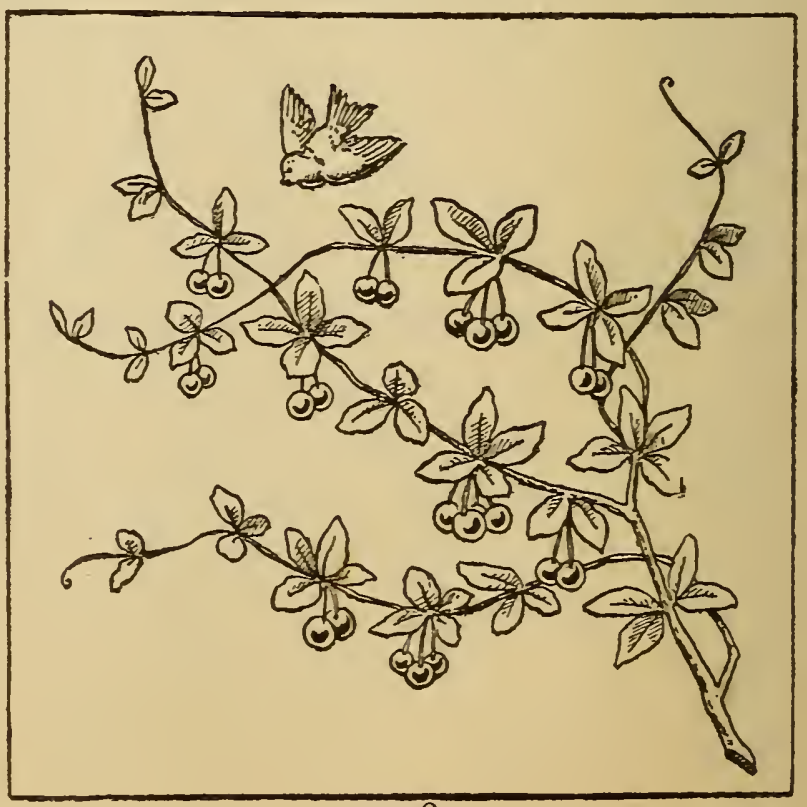

FIG. 89. shirt linen, worked with white embroidery cotton, linen floss or silk floss. Figure 88 shows a small square fruit doyley, with a leaf design suitable 
for this kind of work. The stems are worked with heavy outline stitch, the lower half of each leaf is worked in satin stitch taken at an angle, of about 45 degrees as shown in figure 17 , on page 32 . The surface of that part of the leaf is first raised, by taking running stitches lengthwise with two or three threads of linen floss threaded in a large-eyed needle; after this filling is in, work the satin stitch smoothly and firmly over it. The upper half of the leaf may be male very effective by working the edge to the depth of one half-inch with satin stitch, following the outline of the leaf, and filling in the balance of the surface with either French knots, spot or seed stitches; or, the upper portion of some of the leaves in the design may have spot stitch, some seed stitch, while others may be filled with French knots. Indeed, this combination is very effective where there are several leaves in the design. Figure 89 shows a style of decoration that is equally effective worked out with solid Kensington stitch or satin stitch, in pale lily green Royal floss; or, pure white may be used with equally artistic results. The two figures here presented may well form part of a set of six or twelve doyleys, each one of which may have a different pattern. If the designs shown in figures 88 and 89 are used they should be enlarged four times.

\section{SCROLL BORDER FOR CENTER CLOTH.}

The scroll border shown in Figure 90 should be enlarged ten times its present dimensions to give the correct working size. This pattern is susceptible of different modes of treatment, and we will indicate two, either of which will give satisfaction to the worker.

Treatment No. I. Stamp the design on a piece of white satin damask. To embroider: Use Royal floss and work the outlines of every portion of design with short and long stitches, with buttonhole edge, in cream white. Then, with a light shade of golden brown, No. 1347, blend into the first row of stitches another row of plain short and long stitch, and into this again another row, using a deeper shade of the brown, No. I348. Work the leaves and petals with this blended short and long stitch into at least two-thirds their depth. For the leaves use a deep golden brown, shade No. I35I, and work the stems in solid Kensington stitch with shade No. 1353. Fill the center of the flower with French knots, worked close 
together, with No. I35I. After the embroidery has been finished cut away all the material from the edges, following the outlines of the pattern. Be careful not to cut the buttonhole edge. To blend one row of short and long stitches into another, work between the stitches in the first row, not crowding or working solidly as for Kensington, but fill in lightly, allowing the background to show through the stitches, or rather between them.

Treatment No. 2. Outline all the edges of design with German cord,

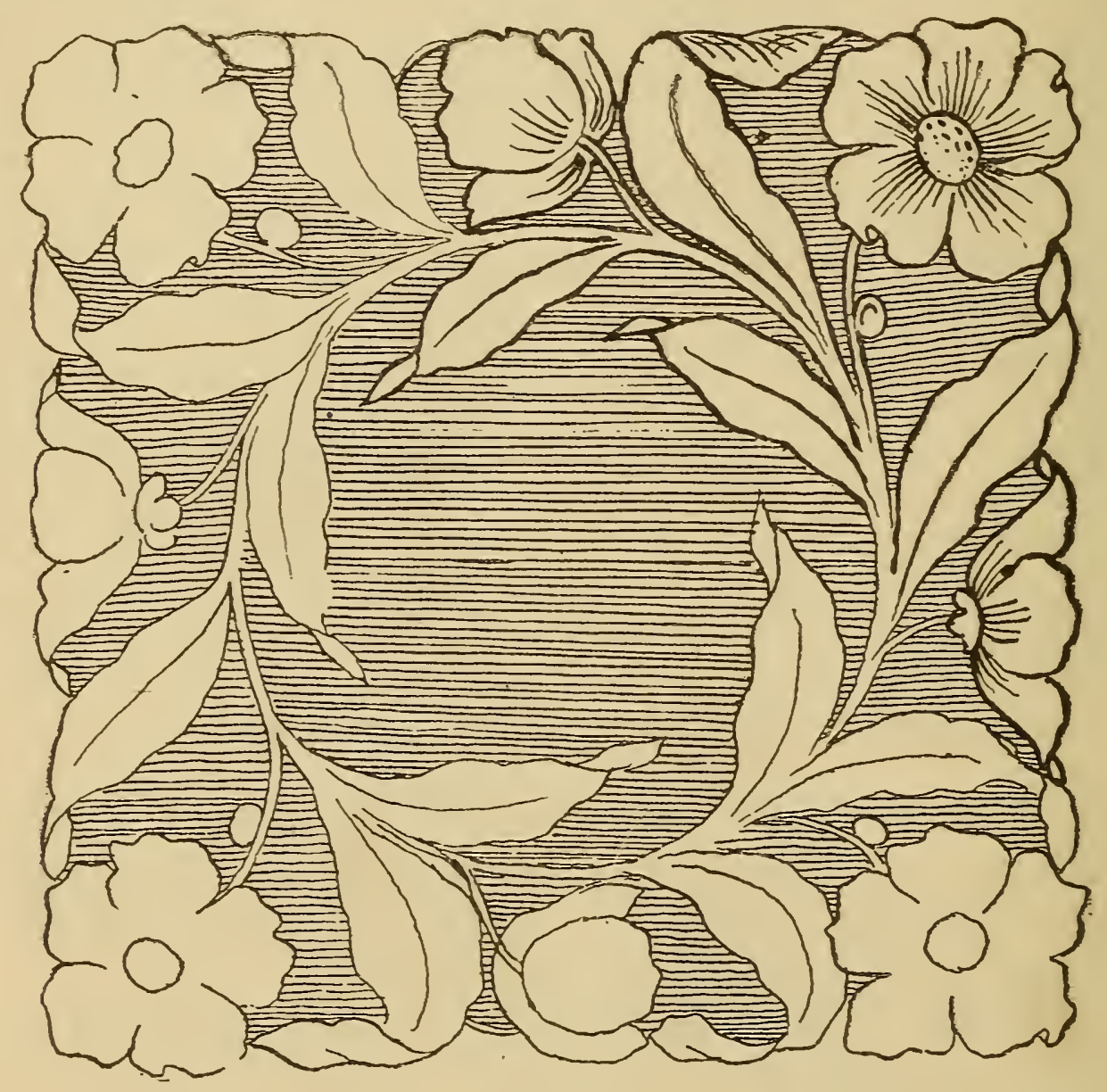

FIG. 90.

No. I, worked over with buttonhole stitch in pale gold etching silk, No. I260. Fill in the petals of the flowers with a matting of cream white filo or Royal floss, using three or four strands in the needle, and working from one.edge of petal to the opposite edge, after the manner of laying threads in Spanish laid work. When the matting or foundation has been laid solidly, allowing nothing of the fabric to be seen through, work over it a couching of gold thread, using the pattern shown in figure 30 , on page 38 
Fill the leaves with honey-comb stitch, worked with the cream white etching silk and vein in outline stitch with the gold cord.

There are other combinations of stitches and colors that can be used with equally good effect and which will suggest themselves to the worker.

\section{FLORAL CENTER CLOTH.}

The center cloth shown in figure $9 \mathrm{r}$ is designed for Kensington effects, and will look best wrought out on satin damask or bolting silk, with filo or

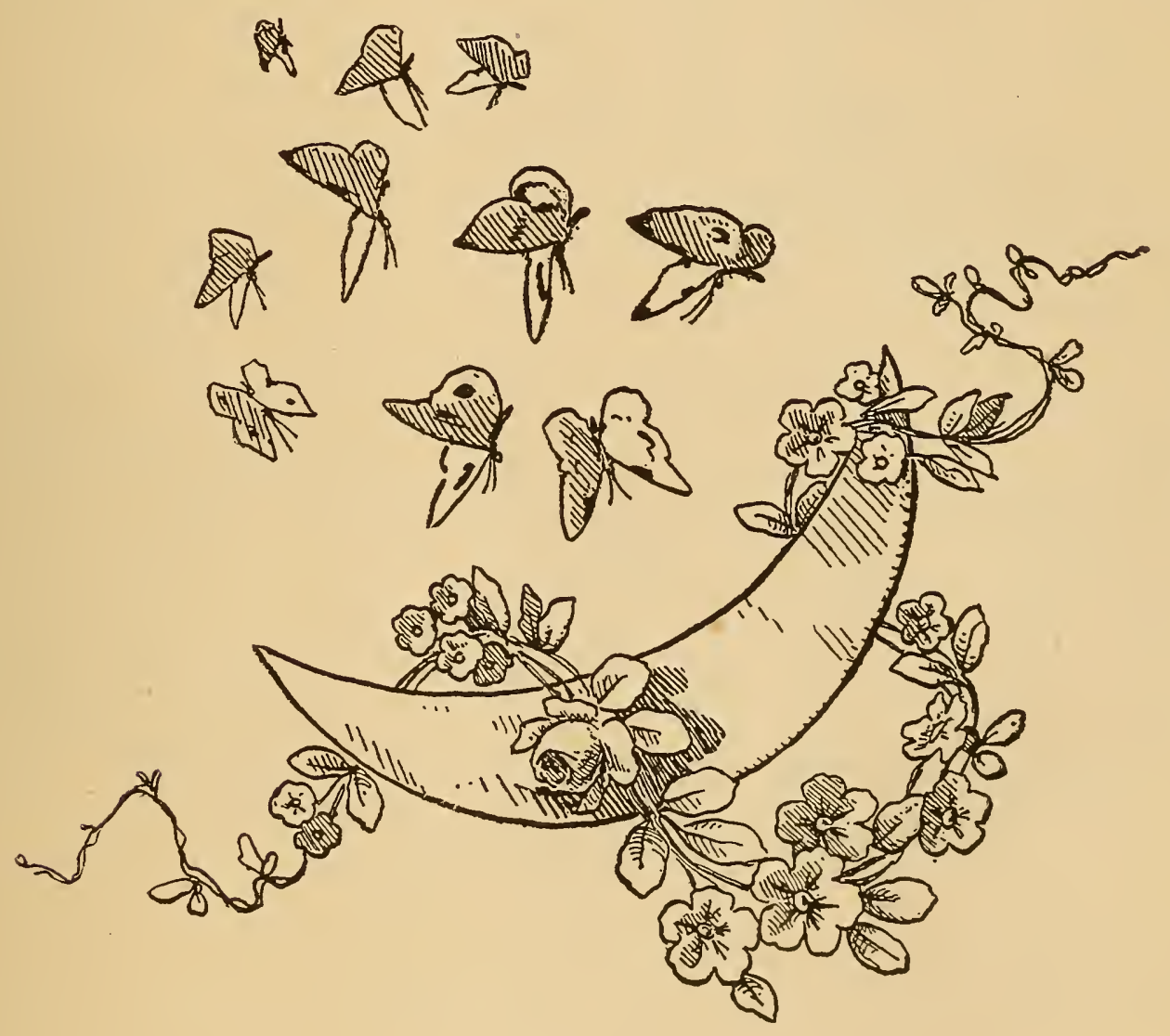

FIG. 9I.

Royal floss. The colors may be nearly or quite true to natur or they may be slightly conventionalized. Pale gold for the crescent, three shades olive green and three shades pale pink for the floral design around the crescent, and a combination of blue and gold, pink and brown and green and orange harmoniouslyblended, for the butterflies, will produce an artistic effect. The orange should be used very sparingly, and may be omitted altogether if desired, though a very little of it judiciously used, gives character to the shading. 


\section{BOOK COVERS.}

For many centuries, ladies of rank and wealth have delighted to work with rare and dainty device covers for missals, prayer books and choice rolumes. Many of these covers have been preserved as treasures of

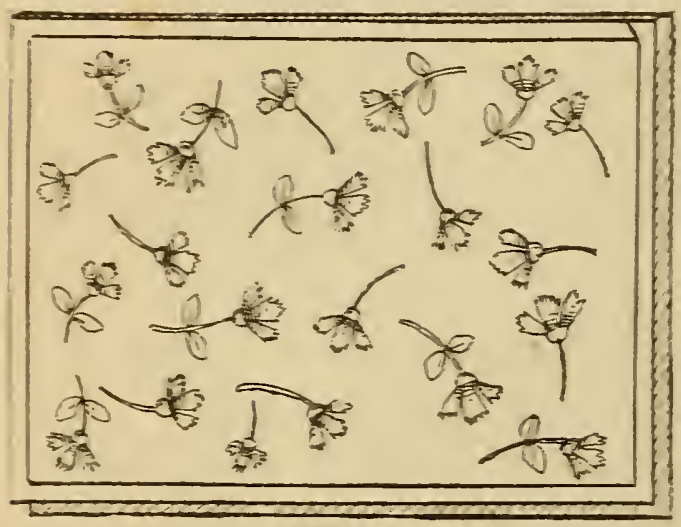

FIG. 92. decorative art. In the Eighteenth century the art of decorating covers fell into disuse; but it has again been revived, not indeed on so elaborate a scale as formerly, as the covers embroidered in these degenerate days are intended for practical use rather than as specimens of elaborate workmanship, many of them being used to cover paper-covered novels and account books, and also books whose handsome and expensive bindings need protection. Figures 92 and 93 show two designs which are at once simple and pretty. Figure 92 is a Dresden design worked out with pink and white filo floss on white duck or canvas, while figure 93 shows a conrentional design treated with brown rope linens on white canvas in outline stitch and French knots. There are many other designs that can be adapted for this purpose, floral or otherwise, but the two here given will serve as an indication of what may be accomplished

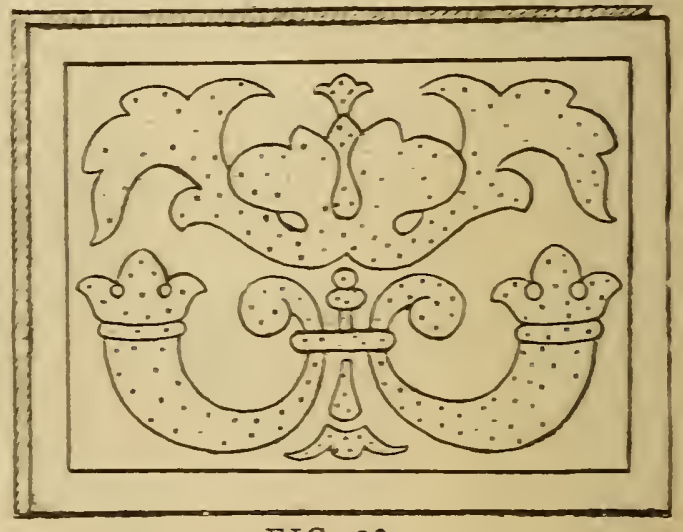

FIG. 93 . in this direction. These book covers make cheap and always acceptable wedding or christmas gifts. For putting covers on see Chapter on "Dainty Trifles."

\section{ROUND CUSHIONS.}

Cushions are or should be suggestive of restful comfort, and to. that end should be stuffed loosely yet with sufficient filling to be soft, and the outer covering should be of a material meant for usage rather than ornament. Cretonne, gobelin cloth, figured silk, art linen and tapestry linen are all suitable fabrics as they can be laundered without injury; the decoration should be flat and done with thread that will stand both wear and cleansing. 
The shapes in which cushions have been, are, and ever will be made are many and varied. Some are large and some are small, while they may be round, square, oblong, high or flat as fancy suggests. In the different pages of this book will be found further suggestions and illustrations of this subject, but in this place we wish to show a model suited for art embroidery which in its turn, will suggest other suitable forms of ornamentation.

Our model, figure 94, shows a round cushion having a puff of wood brown India silk around its edges. This puff is applied to the cushion

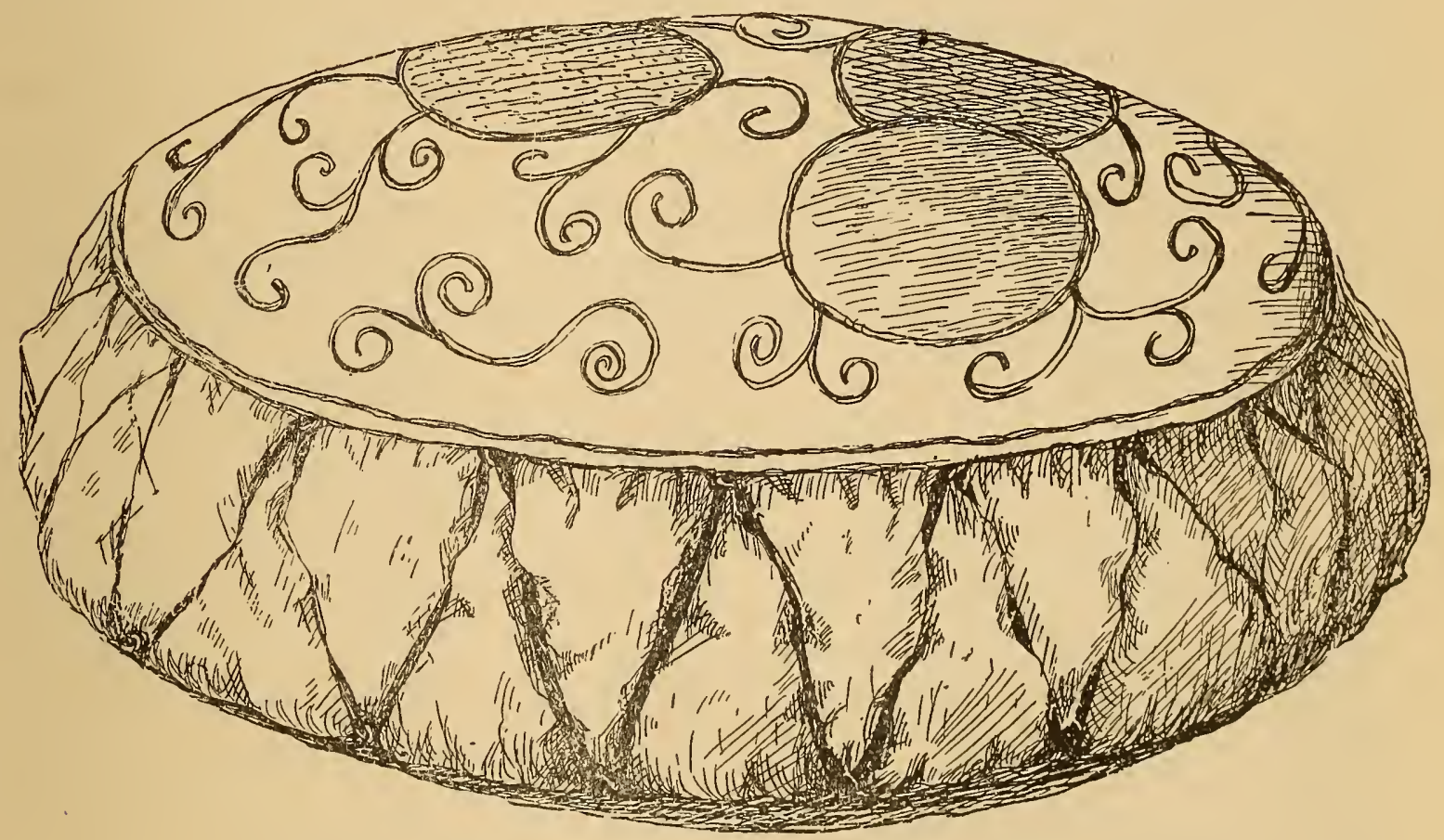

FIG. 94.

proper and is not connected with the cover, which consists of two circular pieces of cream white gobelin cloth, about I 8 inches in diameter. Only one of these circular pieces is to be ornamented, on it draw three smal1 disks, using the top of a large-sized goblet for a pattern; then, with a pencil, draw in the connecting scrolls. Or the pattern here given can bc enlarged by means of a pantagraph.

To work: Outline the disks with old blue couching silk No. 1227; couch this down with a single thread of etching silk two or three shades lighter, shace No. I225 will answer very well. Over the surface of the disks lay rope silk, shacle No. 1226, solidly from one outline to the outline upposite, and couch down with geld thread as shown in ficure 25 on piage 
35. Work the scrolls with rope silk in heavy outline stitch with shade No. I228. Finish the edges of both circular pieces with a strong buttonhole edge and either work eyelet holes at regular distances apart on the edges of both of these pieces, so that they will be exactly in line with each other when the two covers are placed one over the other, or crochet small brass rings over with golden brown etching silk and sew them to the edges in place of the eyelet holes. Place the plain circle on the bottom of cushion and the embroidered one on the top and lace them together through the eyelet holes or rings over the puffings of silk on the side, by means of a heavy silk cord of a color several shades lighter than that used in the puffing. When the cushion cover is soiled it may be unlaced, removed and laundered.

Any other scheme of harmonizing colors may be used in place of the one suggested. In fact, the models given in a work of this kind should serve as a stimulant to the inventive genius of the worker rather than to be slavishly followed.

\section{TABLE COVERS.}

Covers for dining tables, where the dining-room is used for a sittingroom as well, is a question which may prove of interest. Covers for this

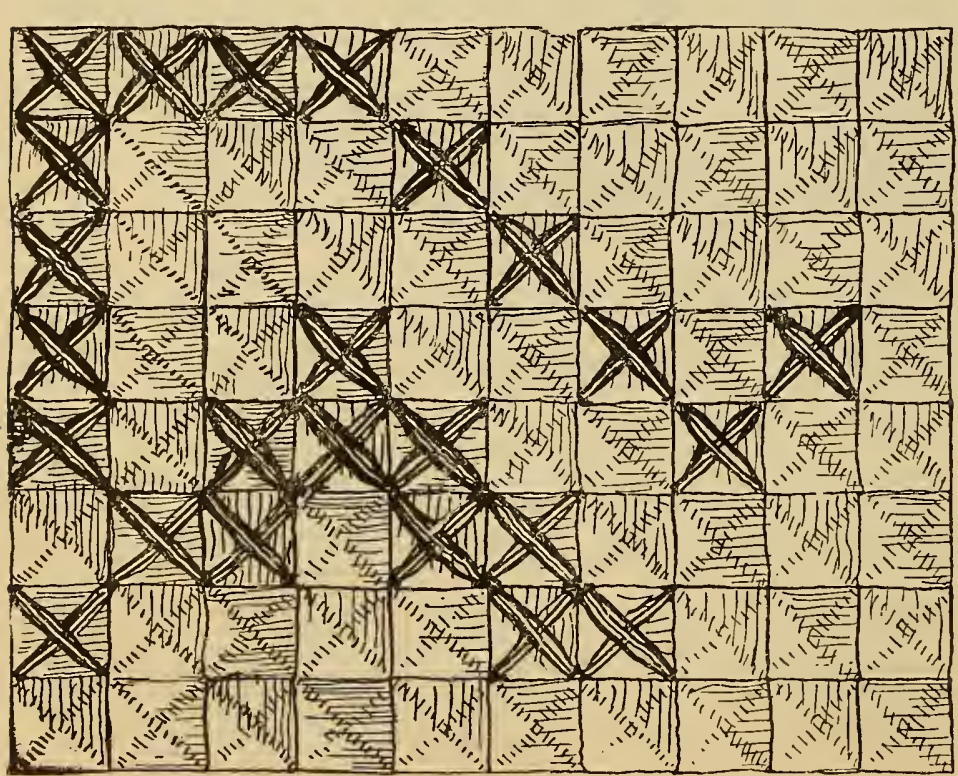

FIG. 95, DETAIL OF FIG. 96. purpose should always be in keeping with the other appointments of the room. If the furniture is handsome polished oak then some color that contrasts or harmonizes with that wood should be chosen. The same is true of other woods, as cherry, walnut or mahogany. If the woodwork and furniture be polished oak, then old blue, olive green, amber, dark terra cotta, Nile green, sage or lilly greens, tan and wood browns or old rose shades will be effective and artistic. If the furniture and woodwork 
of the room be black walnut then deep crimson, light old gold, rose coior, light terra cotta or pale blue will be appropriate. The quality of the material will depend, as we said before, upon the purse of the owner or the

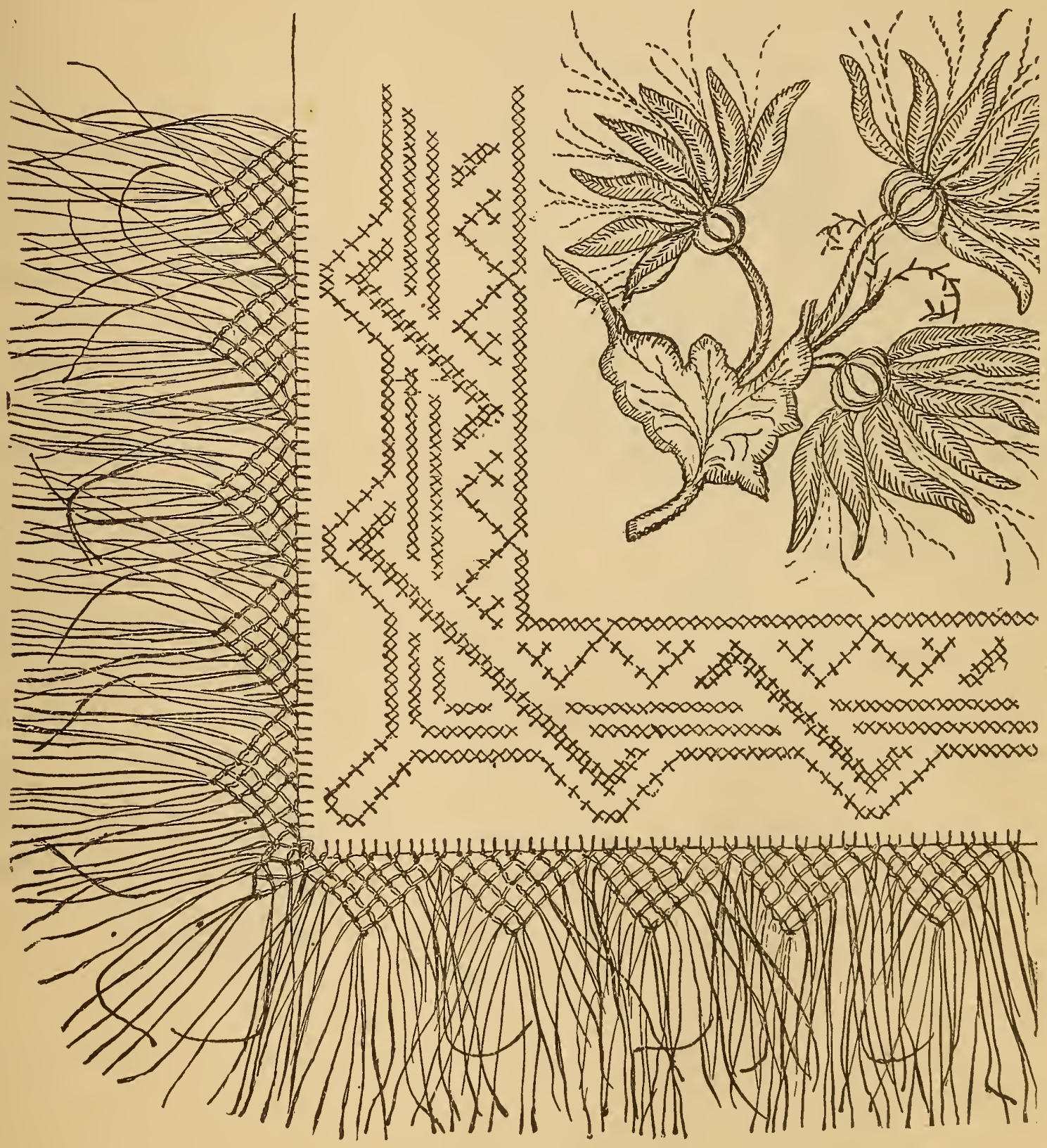

FIG. 96.

furnishings of the room. Felt, satteen, Morris cloth, basket silk, mail cloth and Devonshire art cloth are all elegant and expensive, while artistic and beautiful effects may be obtained by using hollywood drapery, Bargarren art cloth, or tapestry linen, at half the price of the former fabrics. The 
designs for decorating these spreads may be conventional or floral, but in either case the shading should be slightly conventionalized. Broad leaves, flowers and scrolls look best worked out with the coarse embroidery threads, while the filling stitches, such as double basket, close basket, rosette, point de reprise and D'Alencon bars, are all appropriate for filling portions of the design, which may have all its outlines finished in outline stitch with

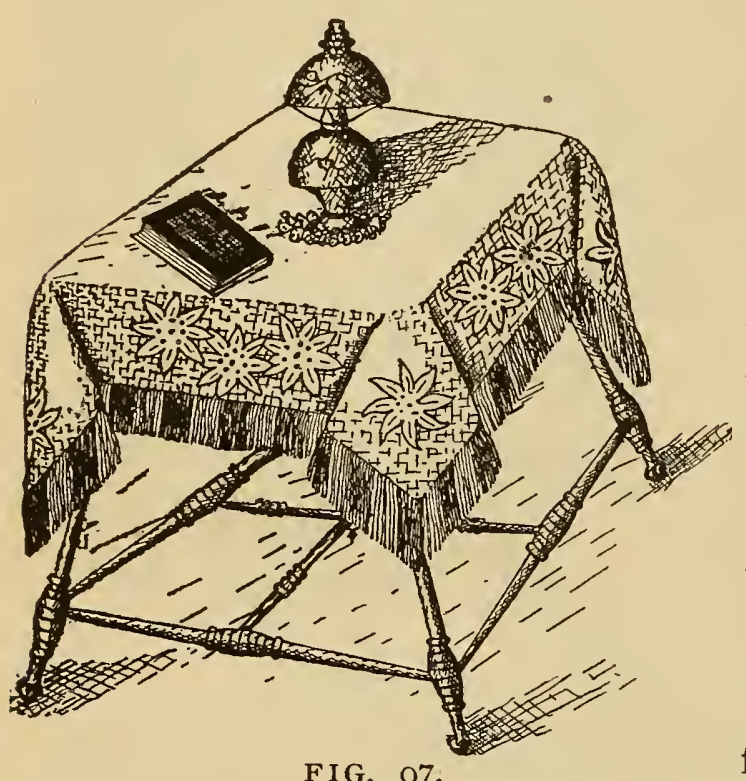
rope silk. Figure 96 shows a corner of table spread made of Devonshire art cloth. The border is worked in cross stitch with Bargarren art thread; the flower is a conventionalized chrysanthemum. Figure 95 shows section of border in detail; each stitch is crossed over one square or mesh of the good. as will be seen from the illustration; therefore it will be necessary only to count the meshes, estimating one mesh for each time the thread is crossed in figure 95 , to work out the design here given. The fringe is made of the Bargarren art thread tied in the edge and and knotted, knotting with four threads each time. The chrysanthemums are worked out with the Bargarren art thread, with short and long stitches, in three shades of wood brown, and the border with four shades of the same color. The two darker shades are also used in the fringe. This is a very effective design and can be worked out with little

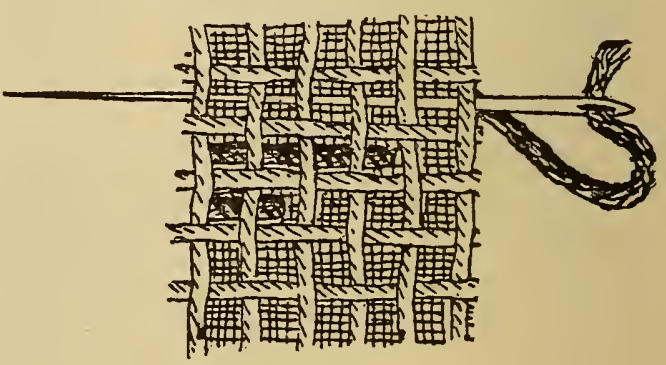
FIG. 98. expense for material. Cross stitch works up rapidly on a mesh of the size here shown, and will therefore commend itself to ladies whose time has to be considered. For the exact design, working size, enlarge with a pantagraph five times. In the Chapter on "Berlin Stitches" will be found designs that will make a beautiful border for this material.

Bargarren art cloth is an artistic and inexpensive fabric for table covers. Our model, figure 97, shows a cream white Bargarren art cloth cover. 
The border was first stamped with star shaped flowers, in groups, at regular. distances apart. See figure 97. These were outlined with Bargarren art thread, in cream white, after which the cover was darned from the bottom edge around the four sides, to a depth of 18 inches, with the palest shade of lily green Bargarren art thread. Figure 99 shows the star shaped flower outlined and the border darned, in detail. Figure 98 shows a section of the material with the needle darned through the meshes. These illustrations show the method of darning Bargarren art cloth, huck-a-buck and congress canvas, as well as the effect

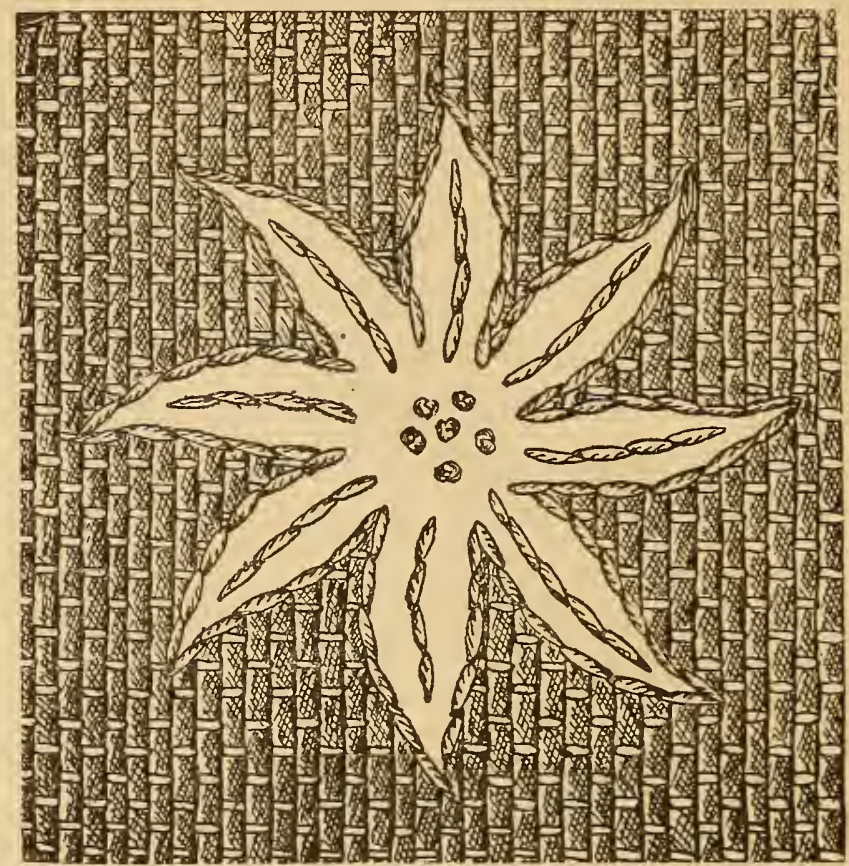

FIG. 99. when the work is finished. If one has a smooth material that will not admit darning the background, an equally good effect can be obtained by couching down two threads of Bargarren linen instead of darning them in.

\section{CURTAINS AND PORTIERES.}

Curtains are generally understood when window draperies are intended, and portieres when the hanging or drapery is designed for doors, either double or single. Door and window draperies can be made at home at much less expense than is possible when they are purchased ready made, provided some member of the family is equal to their ornamentation. The material best suited for window draperies depends upon the use of the room in which they are to serve, the general style and cost of the other furnishings, and somewhat upon the taste of the owner. Swiss muslin, bolting silk, India silk, Brussels net, Madras muslin, cretonne, Gobelin cloth, dotted Swiss, for light draperies; mail cloth, satteen, tapestry lincn, art linen, Hollywood drapery, Devonshire art cloth, Morris cloth, bolton sheeting, where heavy drape $r$ are desired, are all suitable. The ormamentation should be simple or elaborate, according to the position the curtains are to occupy. If draperies for bedroom windows are desired, then 
scrim, dotted muslin, bolton sheeting or India silk may be appropriately used, and their decoration should be of a simple character. For sittingroom windows the same materials may be employed, but the ornamentation should be of a different character. For use in parlor and library the material employed should be richer than that used for other portions of the house, and the ornamentation more elaborate. In fact a sliding scale of elegance may be adopted, with excellent results, beginning with the simplest for the sleeping and living rooms, and progressing gradually until the parlor is reached. Beware of making the fatal mistake of having the hangings for doors or windows more expensive than the other furnishings.

\section{SCREENS.}

Screens, large and small, are most convenient possessions for any housekeeper. They may be ornamental, for bedroom, parlor, library or

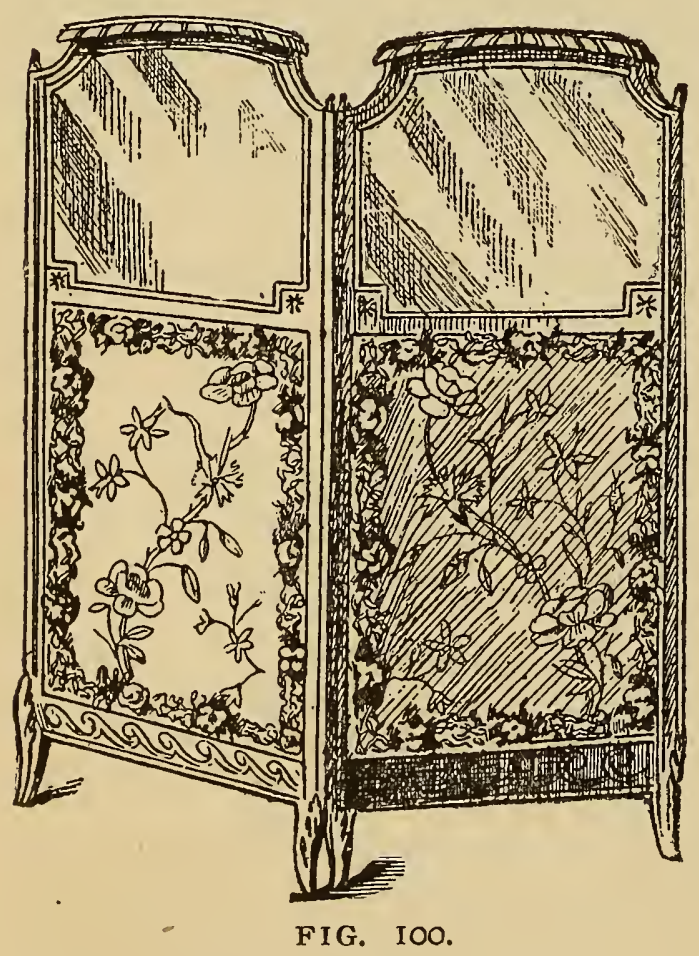
dining room; or they may be designed strictly for utility - strength, rather than beauty, being the desideratum. These screens are desirable for verandah service, to protect one from draughts, the too ardent rays of the sun, or the too curious gaze of the passer-by.

A prettily decorated screen is particularly filling, and will often supply just the bit of color and furnishing needed to brighten an otherwise dark and bare-looking corner; but there should always be some apparent use for a screen. It should not be set up in a corner, like a statuette, to be admired; it is not primarily an article of ornament and there should be some reason for its presence; so, in disposing a screen let it serve a purpose, either to conceal an open grate in summer; tc protect the occupant of some favorite chair from the heat of an open fire in winter; to half conceal or half expose a pretty cabinet filled with bric-abrac, or even to enclose a cozy nook holding a tiny table, an easy chair and a few choice books. 
In making and decorating a screen we should consider the room in which it is to be placed; even the especial position in that room it is to occupy. Parlor screens are generally more ornamental than useful, and therefore may well be smaller, lighter, and in every way more daintily ornamented than those screens designed for use in any other part of the house.

The screen shown in figure 100 is a pretty model for parlor corner screen. The foundation is of white wood, painted robin's-egg blue with English enamel. The upper portion of each panel is filled with a small rose-colored glass panel. The lower portion of the screen is filled with robin's-egg blue satin panels. Around each panel is a border of roses, carnations, foliage, etc., in miniature, while over the surface of the panels the same flowers are arranged in graceful sprays ir natural size. The border should be worked with satin stitch, in Royal floss, and the center of the panels with Kensington stitch, in filo floss. The colors required for working the design as shown in the model will bc, pale pink, three shades; cream white; sage green, three shades. After the design has been stamped upon the satin panels they should be lined with crinoline. Both sides of this screen can be made equally ornamental by working two pieces of satin for each panel instead of one, not necessarily with the same design, however.

\section{APPLIQUE EMBROIDERIES.}

This style of decorative needlework is of ancient origin, being first employed for church work. There are several styles of applique, but the one most commonly employed is where the design is applied to the foundation, having the edges finished with buttonhole stitch, or a cord couched down along the outlines. Another method also much used is to stamp the design on the foundation, which has previously been lined with some contrasting color. In this case the edges of the design are worked with buttonhole stitch or with the couched cord, after which the material is cut away from the edges of the design. The appearance is that of inlaid work.

One of the most popular appliques is that of fine lawn or muslin upon net. A pair of pillow shams and a spread to match, of net, decorated with a conventional floral design, can be made up at a merely nominal cxpense. Stamp the design upon the muslin, after which tack the muslin over the 
net; run with fine stitches all the outlines of the design, thus fastening them to the net, then with fine embroidery cotton work all the outlines with fine, light, buttonhole stitch. In the centers of flowers wheels may be worked, while leaves may be filled with tiny seed stitches and stems worked over with outline stitch. After this has been done, all the muslin should be cut away from the net except the part comprising the design. After the mus-

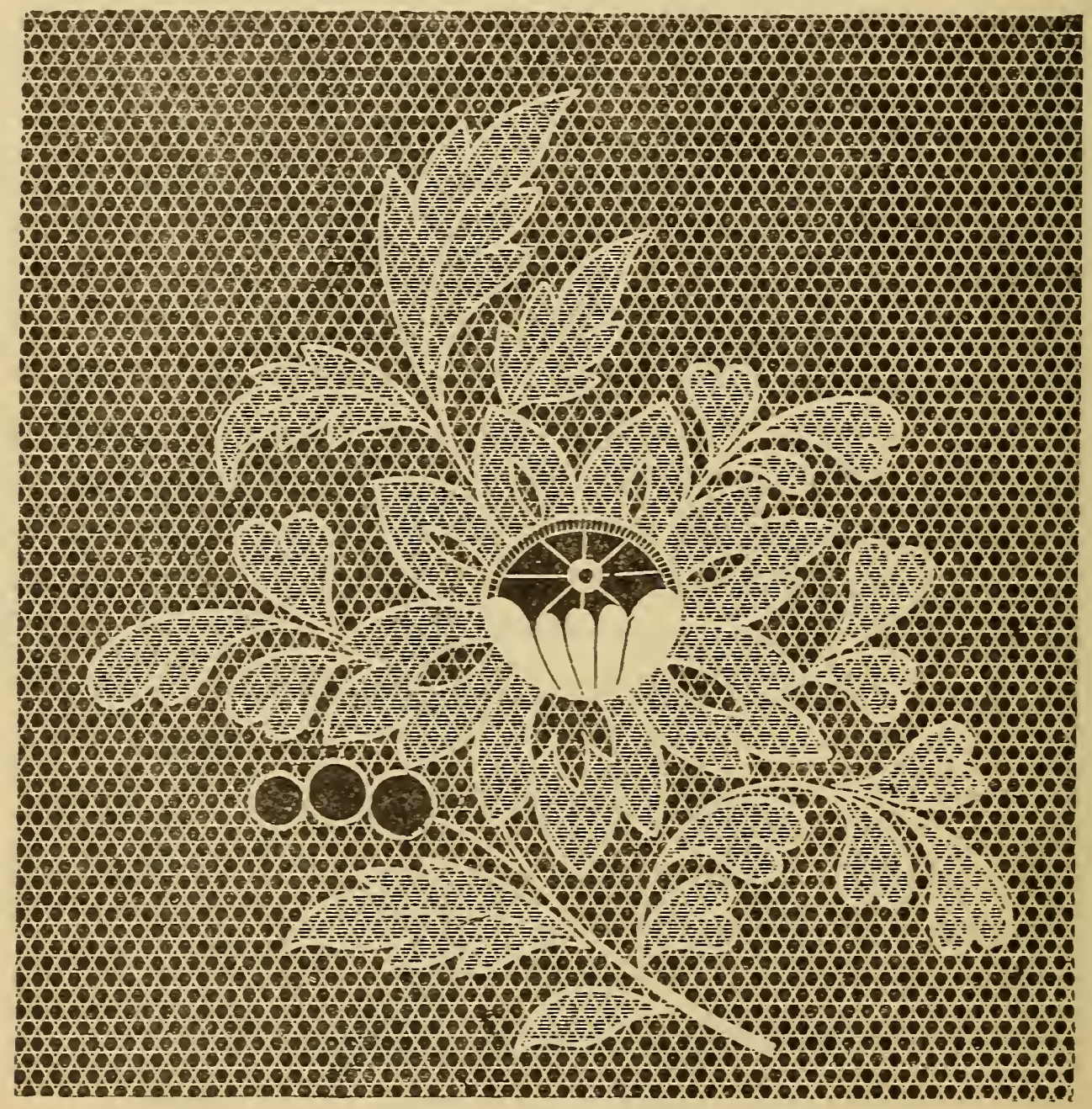

APPLIQUE ON NET. FIG. IOI.

lin has been cut away, sprays and scrolls may be darned directly into the net with the cotton. These sprays should always start from some portion of the muslin design and appear to be a part of it. Figure Ior will illus. trate our meaning, and also give a good idea of the effect of net-applique when finished. The edges of spread and shams should be finished with buttonholed scallops. 


\section{EMBROIDERED ARTICLES OF DRESS.}

There are other uses for ornamental needlework, aside from that used on household decorations; namely, ornamenting articles of apparel. Daintily embroidered handkerchiefs, collars and cuffs, dressing sacques and the numberless elegant trifles of the wardrobe so dear to the heart of the refined woman, so utterly impossible to the refined poor woman, unless she herself can embellish them with embroidery, are within the reach of all who desire them and are wiliing to give the time necessary for their production. Fine linen handkerchiefs with hemstitched hems can be purchased for a trifle at any of the large stores; an initial or monogrim worked in the corner with white embroidery cotton or linen floss will change a simple article into one of luxury. Again, from a piece of Indian mull or linen lawn four inches

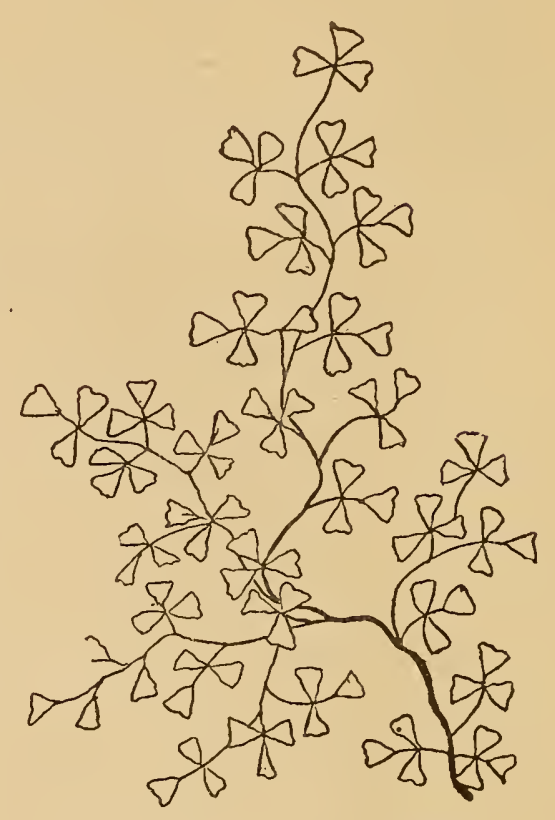

HANDKERCHIEF CORNER. FIG. IO2. square and a strip of the same material three inches wide and 24 inches long can bc produced a handkerchief that could not be purchased for $\$ 3.50$.
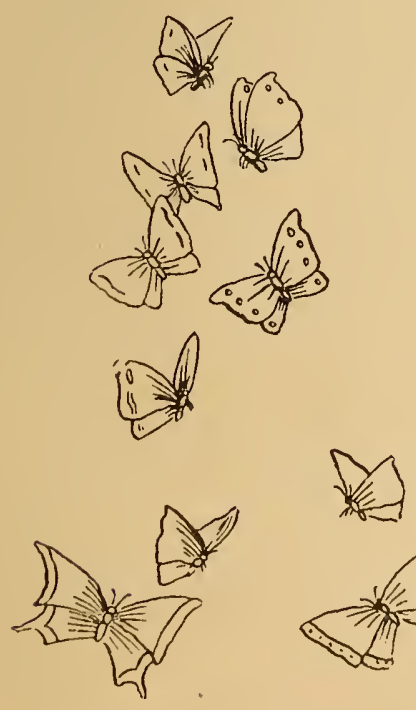

DESIGN FOR PARASOL. FIG. IO3.

With fine embroidery cotton work a tiny scallop in buttonhole stitch along one edge of the three-inch strip, then over the surface of the strip work seed or small spot stitches with the same material; in the center of the square piece work an initial inside a half-wreath of forget-me-nots, or a monogram without the wreath. This part of the work should be done with satin and spot stitches combined, using the white cmbroidery cotton for the purpose. After the needlework is finished, gather the strip of lawn by rolling the edge lightly and taking an overhand running stitch; put this strip on the square with an over-hand stitch also. The above method indicates but one of many ways in which elegant handkerchiefs can be btained at little cost to the owner. It is a pretty fancy to buy a dozen 
plain hemstitched handkerchiefs and work each one differently, with monogram or initial. Handkerchiefs intended as gifts to gentlemen can be worked elaborately or not, as desired. For instance, if the gentleman is addicted to lawn tennis, a ball and a pair of rackets worked in outline stitch will prove acceptable, while for something "extra nice," no decoration can surpass a handsome monogram worked in satin stitch.

\section{Embroidered Dresses.}

Given ten yards of fine cashmere (either colored or black) and a bunch of Royal or filo floss, the artistic embroideress will turn out a Parisian robe "that will turn all womankind green with envy and admiration." We remember, not long ago, the high-priced emporiums were importing Parisian embroidered robes for the modest price of $\$ 50, \$ 75$ and $\$$ IOO each. An ingenious friend of the writer soon came out with an exact duplicate of one of the costly affairs, and as her income was known to be more than slender, it was a matter of wonder until she explained that the dress cost her \$IO, and proudly added, "I did it myself."

Many of the Roman embroidery designs can be adapted for dress

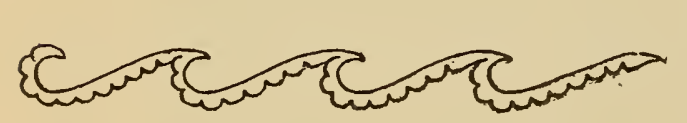

EDGE FOR COLLAR AND CUFFS. FIG. IO4. trimmings, notably those having the bottom edge vandyked. The embroidery should be done with a silk thread, either one shade lighter or darker than the foundation. Thus a dark brown cashmere or cloth may be worked with a golden brown or a wood brown, as nearest matches the cashmere, but not with the exact shade, as the contrast would not be sufficient to bring out the beauty of the work. In adapting a Roman design for a dress skirt the pattern should be at least i 2 to 18 inches deep, and on the vest front, collar and cuffs a modification of the same pattern should be carried out. In working, follow all the outline of design with a small cord, fastening it down if there is any difficulty in holding it in position; then with Royal floss, one strand, work over this cord in heavy satin stitch, being sure to place the stitches close and even. Do not take the stitch beyond the edge of the cord on either side. This method will slightly raise the prominent portions of the design. The lace or connecting lines should be worked in outline stitch on the material, from one outline to the opposite out- 
line of the design, and with the same silk. If the design is one showing many plain spaces the effect can be enhanced by filling them with Janina, rosette, point de reprise or honey-comb stitch. The effect of this method of treat-

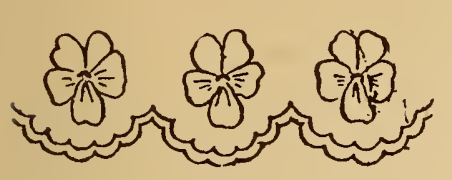

EDGE FOR DRESSING

JACKET. FIg IO5. ing designs is extremely rich, and requires no more than mechanical skill, as the work is not shaded but done with one color throughout. Of course the beauty of the work will depend upon the exactness with which the stitches are

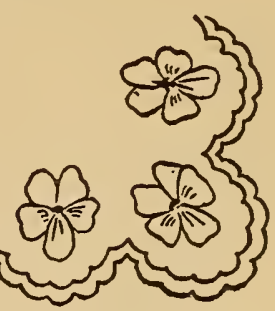

CORNER FOR taken and the general smoothness of the work. In using DRESSING JACKET floral designs for street dresses it is not good taste to do the FIG. I06. work in natural colors; thus if a deep navy blue dress is to be embroidered with a band of buttercups across the bottom and scattered flowers across the front, the colors for working them should be either a shade of navy blue or a golden brown. In this case a little shading would be admissible, say about three to four shades of the line of golden brown filo floss for the entire design. The stitch used for this style of pattern may well be the solid Kensington. For neglige jackets, to be worn during the morning hours over a dark skirt, nothing could be more dressy or serviceable than a black cashmere or satin jacket, cut after a pretty model and embroidered down the front edges, around the bottom and across the collar and cuffs with a graceful arrangement of dainty wheat and poppies, or roses, worked out in natural shades with Kensington stitch in filo floss. Again, it may be desired to renovate the bodice of a plain cashmere dress without much expense. A nicely fitting collar, with the edges finished with a tiny scallop, embroidered with buttonhole stitch in the same shade of silk as the dress material and the edge of cuffs and the bottom of bodice treated in the same manner

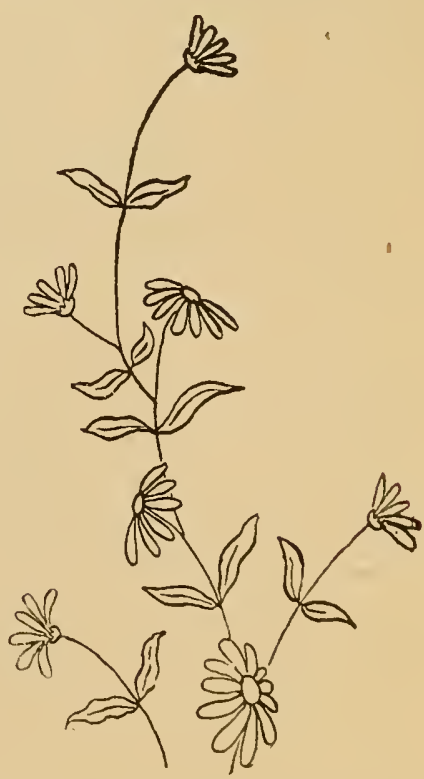

DESIGN FOR PARASOL. FIG. 107. will give an appearance of quiet elegance that could not be obtained from other trimming at five times the cost. Fans, parasols, bonnct crowns, all offer excellent opportunities for the needlewoman's skill. A plain black 
silk parasol, of a good quality, with a graceful spray of flowers embroidered upon one section, in colored silks, will make a very dressy promenade article, while fan covers of silk can be made, using a fan one already has in

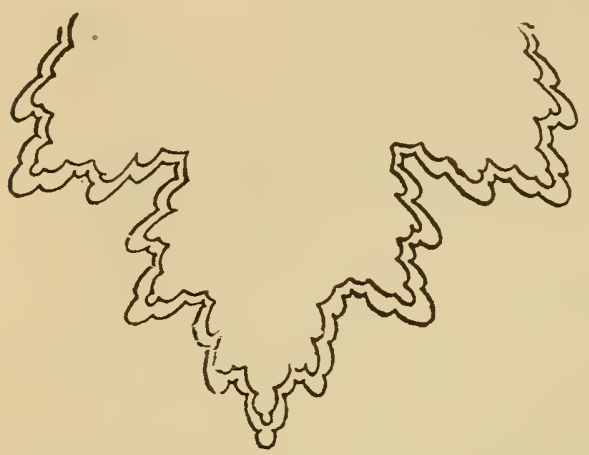

CWRNER FOR CREPE LJSSE RUFFLES. FIG. 108. possession as a pattern. Cut the silk a trifle larger than the fan it is to cover, to allow for making. The embroidery should be of a light and delicate character; heavy or massive effects will be entirely out of place for this purpose.

Double and triple crepe lisse frills for neck and sleeve trimmings, having the edge worked in scalloped buttonhole stitch and scattered seed or spot stitches above the scallops, sell in the large stores for $\$ 2.50$ to $\$ 3.50$ per yard. These frills can be made at home for one-quarter of the store price. Figures 108 and Iog show a suitable scallop for this

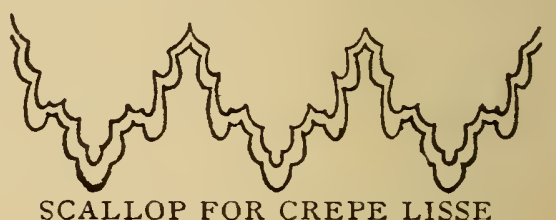
SCALLOP FOR CREPE LISSE RUFFLES. FIG. IOQ. purpose. Filo floss, a shade lighter than the crepe, is generally used.

\section{Embroidery for the Children's Wardrobe.}

The children must not be forgotten, and many are the dainty garments that can be made for the little ones by loving fingers. Infants' jackets, dressing gowns, robes, cloaks, bonnets, shawls and skirts may all be rendered elegant by much or little handwork, as the judgment of the worker decides. Embroidery for infant's clothing is almost always done with satin stitch combined with spot and seed stitch, and buttonhole stitch for finishing the edges.

Embroidered flannel skirtings can now be purchased so cheap that it hardly pays to spend the time to embroider

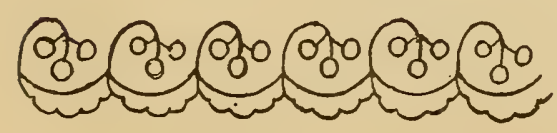

SCALLOP FOR FLANNEL. FIG. IIO. them at home, but cheap as they are they still are beyond the reach of the woman who can barely eke out enough to purchase the plain flannel. A few cents' worth of white embroidery silk, a knowledge of embroidery and time enough for the work will enable such a woman to prepare for her darling many a dainty gar- 
ment otherwise impossible. In such cases it is better to select a simple pattern and do the work well than to hurry over a large and elaborate pattern. Figure I IO shows a pretty design for edging flannel garments.

Strips of white flannel, of different widths, from one inch to two inches, stamped with a small scallop pattern and a design of small circles suitable for spot stitch will be found convenient pick-up work. The embroidery may be done with white, blue or pink embroidery silk. These strips may be used to finish the necks, sleeves, front edges of jackets, dresses, skirts and dressing gowns for the little ones we love so well to deck with our best. Many a mother's work basket is never without a little roll of flannel strips, either embroidered or in process of completion. One dozen skeins of Royal floss will embroider five yards of these strips. Another pretty decoration for flannel or cashmere dresses, jackets, etc., consists of working over the surface of material with spot stitch or French knots, using either white or colored silks for the purpose.

Specific designs for certain purposes it is impossible to give in the pages of a work of this kind. The best that can

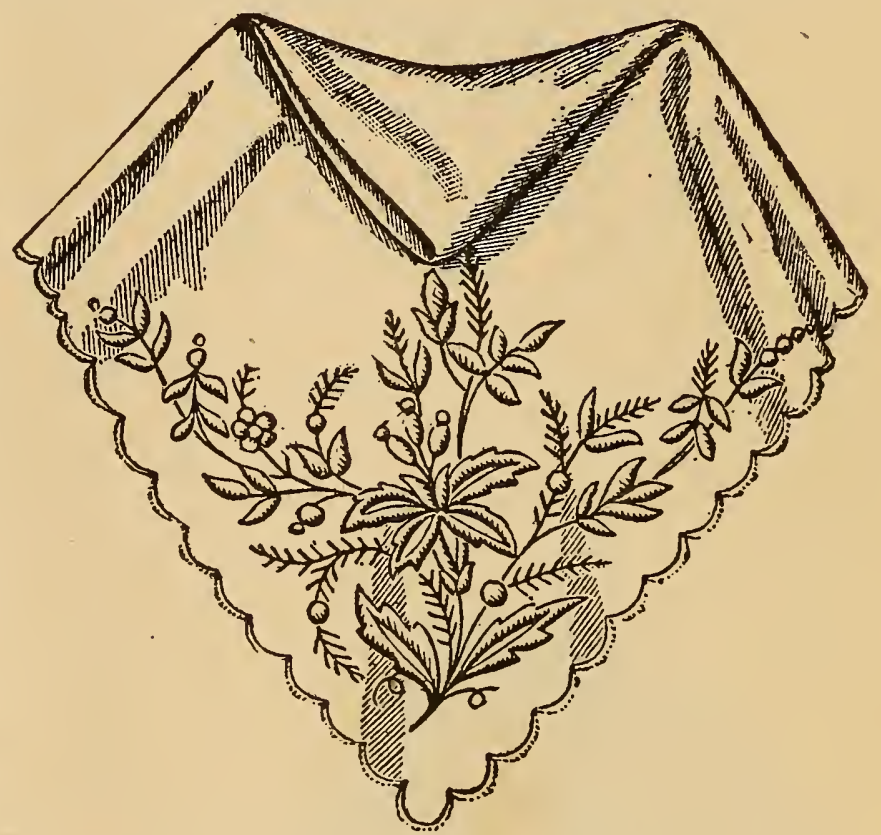

DESIGN FOR INFANTS' SHAWL IN SATIN STITCH. FIG. III. be done is to give occasionally an illustration that will be suitable for certain purposes. It is an excellent plan to have in one's possession a variety of perforated patterns, suitable for various purposes; two or three sizes of scallops, several floral arrangements, etc., should be included, and as these designs are inexpensive, nearly all ladies who embroider can purchase a good assortment. Again, magazines and periodicals often contain excellent designs, and these can be transferred, according to instructions given in Chapter IV. The different scallop designs shown in this chapter are suitable for many other purposes beside those designated. 
II4 DAINTY WORK FOR PLEASURE AND PROFIT.
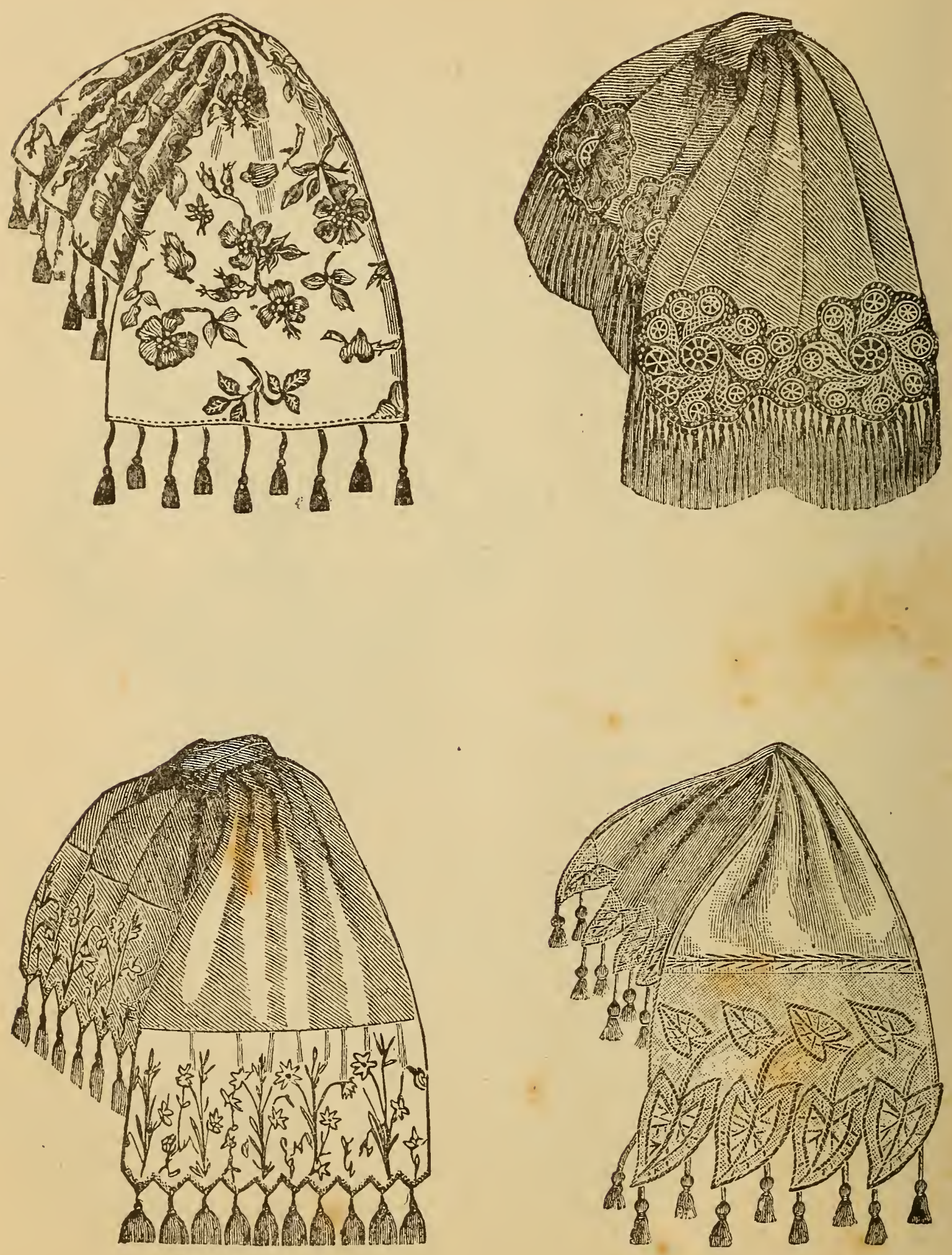

DRAPERIES FOR PICTURES, EASELS AND MANTELS. 


\section{CHAPTER IX.}

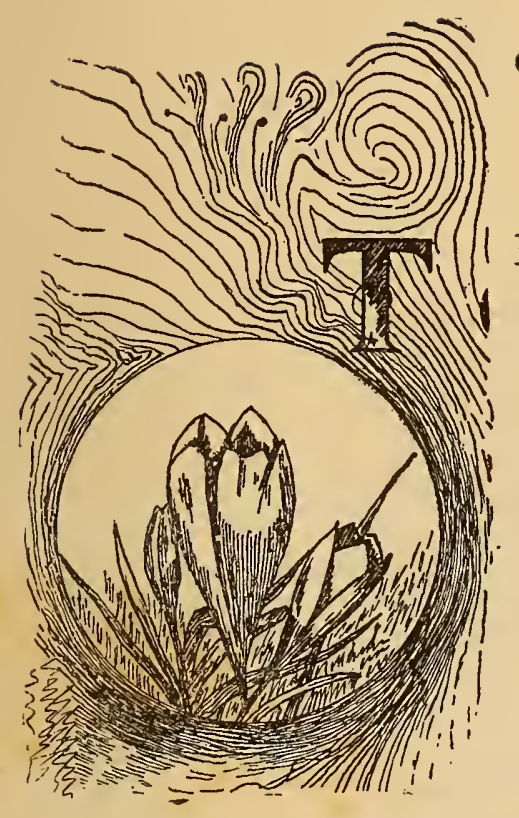

CHURCH AND BULLION EMBROIDERIES.

HE EMBROIDERIES for ecclesiastical purposes form a branch of decorative needlework quite distinct from that applied to articles of dress and household ornamentation. In the former no shading is required and the work is more set in its character, allowing less scope for individuality. The stitches employed are couching stitches of every variety, both raised and flat, long stitch, bullion knot and satin stitch. The material employed for church embroideries must be of the very best, and the workmanship must show no amateur touches; in fact, we may as well say here, in this connection, that church embroidery requires the most skilled workmanship, and should never be attempted by the amateur embroideress. The stitches required for this style of work depend entirely for their effect upon the exactness with which they are placed, and this exactness can be attained only by diligent practice. There is room for ornamental needlework in the houses of worship of all Christian denominations. Aside from altar frontals and altar curtains there are smaller articles that can be made by ladies, either privately or in the sewing societies usually connected with church work. Among the latter may be mentioned book marks, sermon cases, burses, alms bag, mats for collection plates and flower stands, and banners for the Sunday-school, especially' ior F.aster scrvices.

There has been very little written on the subject of church embroideries that can be considered authoritative, and the author of "Yainty Work" has been at considerable trouble to prepare something that should 
be practical, correct and within the comprehension of the average embroideress, and she wishes here to acknowledge the assistance received from Caulfield and Seward, London, England, who in I881 published the only practical article on ecclesiastical embroideries to be found in the English language.

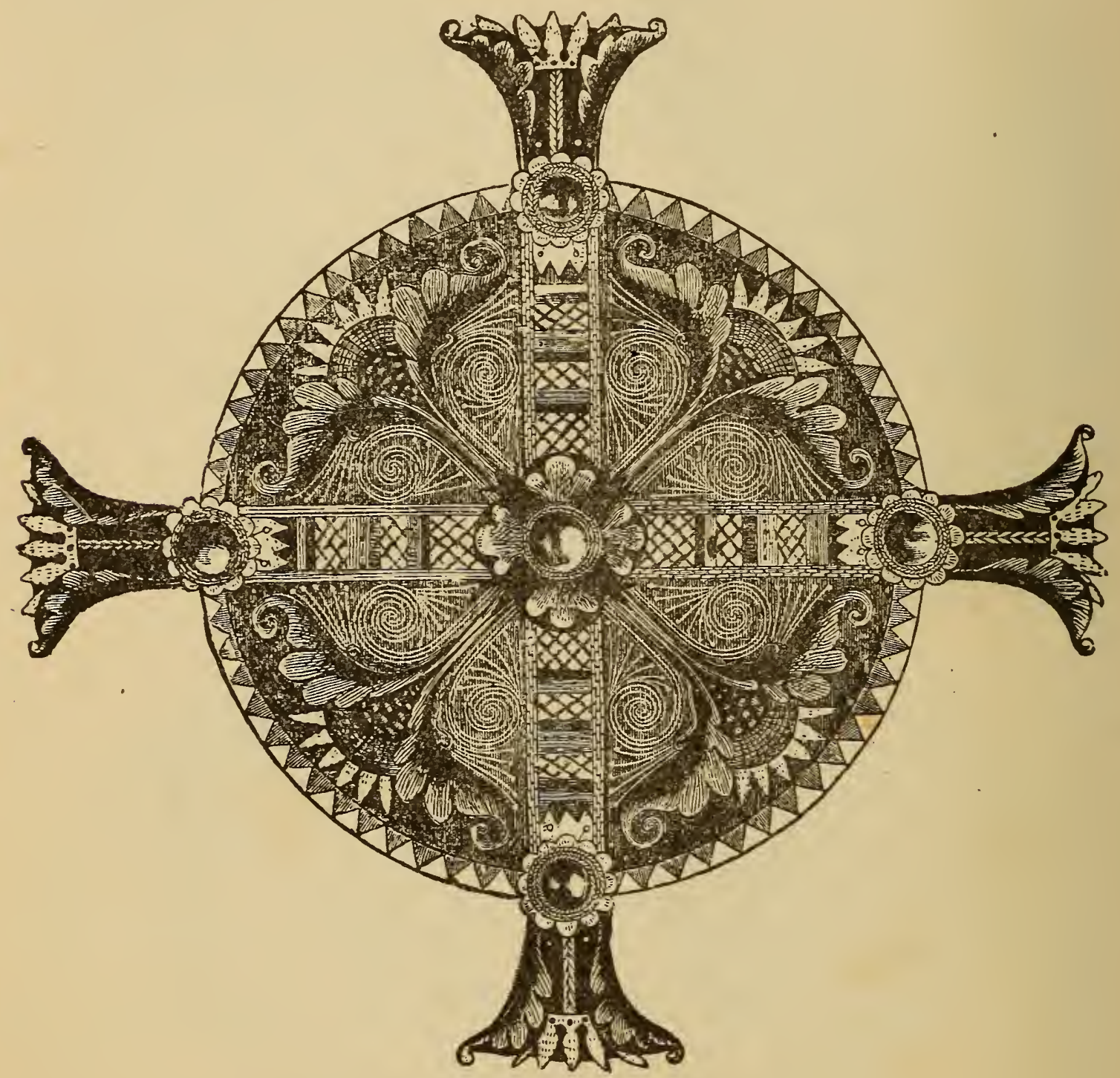

CROSS FOR ALTAR FRONT. FIG. II2.

We will give here a list of the materials used in ecclesiastical embroideries, premising that each article must be the best to be obtained: Gold thread, passing (this is a thread of gold and silk, mixed), filo floss, purse silk, knitting silk, sewing silk, silver thread, colored art cords, spangles and bullion. Of these materials floss is the most used, as it can be split and 
subdivided to the smallest strand, and is used for working over the main part of design in long stitch. Purse silk is used for the parts requiring strength, and frequently for couchings; knitting silk, gold and silver thread and passing for ornamental sprays; spangles and bullion for ornamenting the raised parts of the design; gold and silver cord for finishing design. The foundation for these embroideries should be the best velvet, plush, rep silk or broadcloth.

For doing the embroidery there will be required frames of several sizes, square frames are best for church embroideries; strong, unbleached linen, that has been boiled to take out the stiffness, upon which the design is worked, after which it is cut out and applied to the foundation; piercer, an instrument invaluable in placing the floss and gold threads in position; stiletto, for puncturing holes; round-eyed needles of different sizes, and carpet needles Nos. 2, 9 and Io.

The hands of the embroideress must be kept smooth, and for this purpose a piece of pumice stone should be kept in the work basket. Ladies whose hands are continually moist should not attempt church embroideries, as the moisture ruins the gold and silver threads.

\section{STITCHES USED IN ECCLESIASTICAL EMBROIDERIES.}

In ecclesiastical or church embroideries the following stitches are used: Couching, raised and flat, and of every variety of pattern; long stitch, satin stitch, French knots, bullion knot, spot stitch, chain and outline stitches. Satin stitch, spot stitch, chain stitch, outline stitch, French knots and flat couching stitch have been described in detail in Chapter V; but in this connection it seems advisable to give the different varieties of couchings in greater detail, as they form the distinctive features of all church needlework.

Couching stitches are divided into two classes, namely, raised couching and flat couching; in each class there are several varieties. Flat couchings are formed of threads laid smoothly upon a foundation, and caught to it with small stitches, brought up from the back of the work and then returned to the back again. Raised couchings are formed by laying the same kind of threads upon the foundation, but over whipcord that has been previously arranged upon it in a set clesign. The raised lines are secured in the same manner as employed in flat couching. The names given to the different varieties of couchings are taken from the direction of the 
securing stitches, and are Basket, Battlemented, Brick, Broad, Diagonal, Diamond, Diaper, Plain, Shell, Spider, Vandyke, Wheel, Wavy. The manner of working them differs only in the patterns formed by the securing stitches and in the direction of the whipcord in the raised designs.

The manner of working flat couching has been described, in Chapter V, therefore we will consider raised couching more particularly in this connection. To work raised couchings, sew to the linen foundation a number of strands of whipcord, in straight or waved lines, or they may be formed into a set pattern; over these lines of whipcord lay gold thread or several

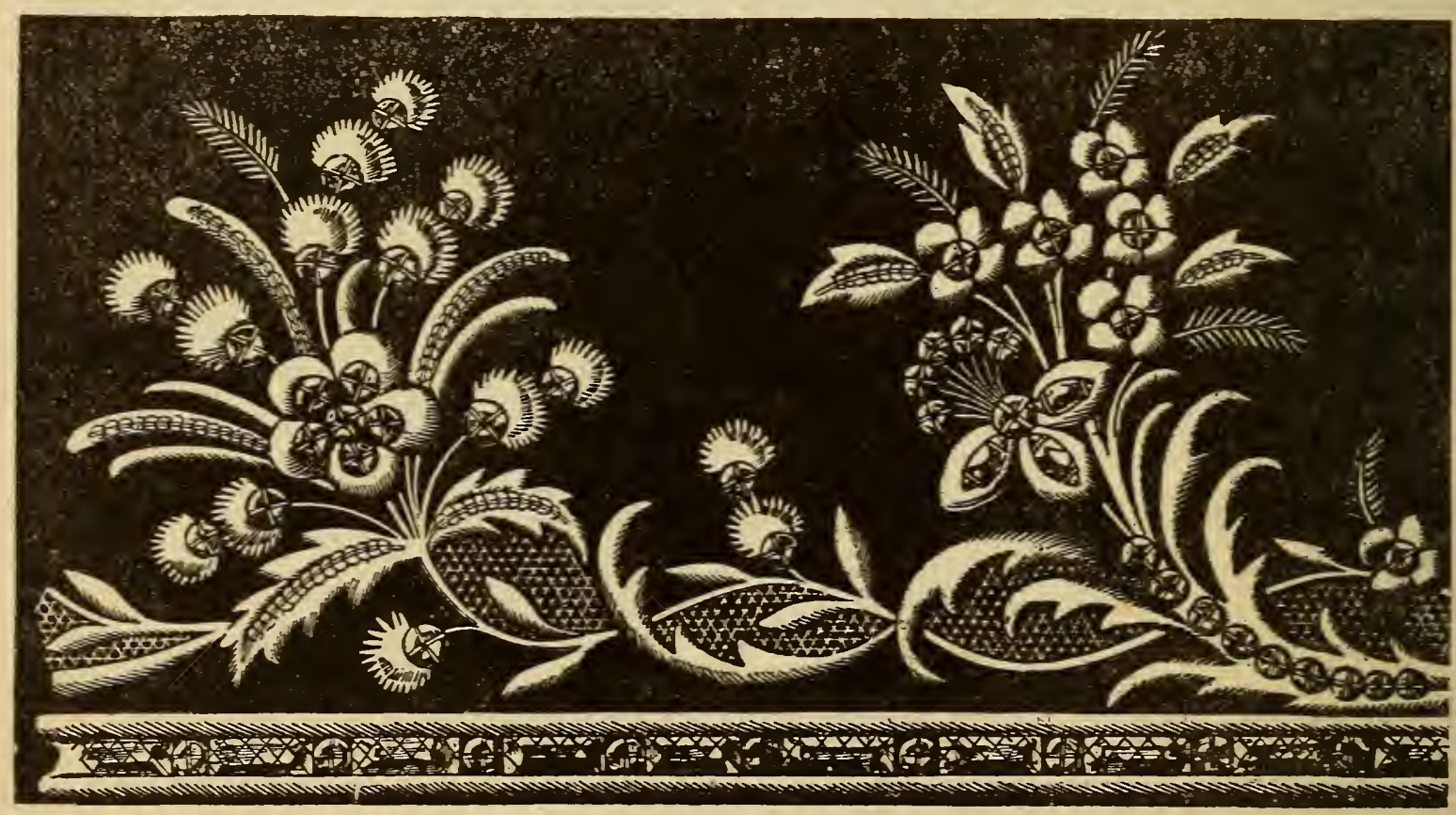

DIESIGN FOR GOLD AND SILVER THREAD. FIG. II3.

strands of filo floss. Secure this with a stitch brought from the back of the work and returned there, on each side of the raised part formed by the whipcord that is underneath the gold thread or filo floss; work a continuous line of securing or couching stitches on each side of each strand of the whipcord, so as to distinctly outline it. In the intervals between these raised parts work couching stitches, up and down, as in flat couchings, forming them into any pattern desired, without reference to the raised designs. When couching in various designs, hold the laid threads in one liand, and regulate them with that hand or with the piercer, and bring up the couching stitches with the other hand, and do not change the hands 
until the work is finished. Outline the couched design with a cord or cords of silk or gold.

The raised couchings are more frequently used for backgrounds, while the flat variety is better adapted for the centers of the various devises used as powderings or scattered all-over designs over a large surface on the centers of altar frontals and on embroidered vestments.

Basket Raised Couching.-Figure II4 shows raised basket couching. To work: Lay upon the foundation perpendicular lines of whipcord and sew them firmly into position. Take four threads of purse silk, or four strands of filo floss, or the same number of gold threads, and stitch them down with purse silk of the same color, brought through from the back of the material and returned to it. Place the securing or couching stitches between every second strand of the whipcord; form the next line with four threads laid over the cords and stitche

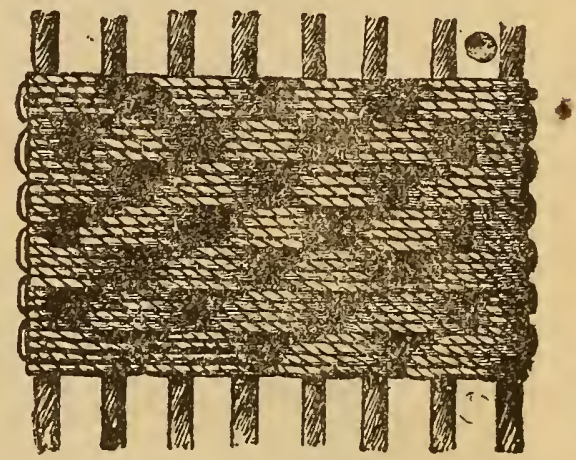

FIG. II4. down as before. To prevent the lines of securing stitches coming directly beneath each other, the first line of stitches must secure the floss or gold thread over one cord only, the rest nver two cords. Repeat these two lines until the desired space is filled in.

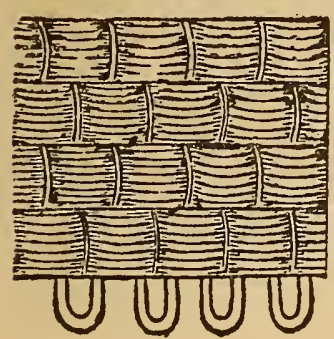

FIG. II5.

Figure II 5 shows another variety of raised basket couching, which is worked as follows: Sew down the whipcord, and over it the floss or gold thread, as described in the preceding figure, but over these again, lay short lines of fine gold thread or purse silk. Bring these short lines from the back, by puncturing a hole through the foundation with the stilletto for them to pass through; return them in the same manner.

Spider Couching.-A raise couching. To work: Upon a linen foundation fasten down short pieces of whipcord, cut of equal length and arranged like the spokes of a wheel or the chief threads of a spider's web. Fill in the whole of the foundation to be treated in this manner with the whipcord so arranged, placing the wheels they form as near together as possible. Place lines of filo floss over the whipcord, beginning at the cen- 
ter of the wheel, and couch it down. When the floss has been couched once around, repeat row after row until the outer ends of the whipcord spokes have been reached. Place the couching stitches in lines on each side of the lines of cord, so that the shape of each wheel or web may be clearly defined.

Vandyked Couching.-A rasea couching formed with lines of whipcord laid on the linen foundation in the shape of vandykes. Over the lines of whipcord lay filo floss, secured with couching stitches taken in such a manner as to outline each piece of whipcord.

Wavy Couching.-Figure il6 shows raised wavy couching. To work: Arrange upon a linen foundation curved lines of whipcord; place over them purse silk, two strands at a time, and secure as shown in broad

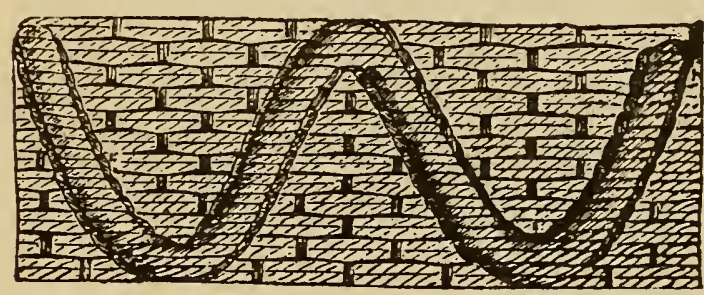

FIG. II6. couching, omitting the stitches whenever the raised part formed by the cord underneath is approached. When the broad couching is finished, lay a thread of gold or silk cord on each side of the waved line and couch it down with a fine sewing silk; or, work the lines on each side of the raised part with a continuous line of couching stitches.

Battlemented Couching.-Figure II shows a flat couching, with the securing threads arranged as battlemented lines. To work: Lay the filo floss in diagonal lines across the foundation, then work the securing stitches so as to form the design shown in the illustration. The above pattern is effective as a background for large spaces, or for filling in unbroken surfaces of a set

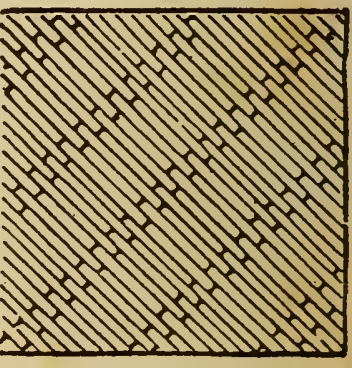

FIG. I I 7. design. It can be varied by placing the securing stitches at different distances.

Brick Flat Couching.-Figures i i 8 and I 9 show two methods of

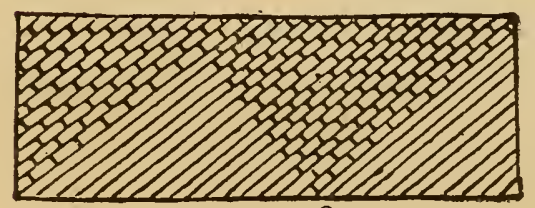

FIG. II8. working brick flat couching. Towork the design shown in figure i 8 , lay down lines of filo floss in a diagonal direction, and secure them with stitches from the back. Pass each stitch over two lines of floss, and work it in at an even distance from the stitch pre- 
ceding it, to the end of the pattern. Work the next line of securing stitches over two laid lines of the floss, but be careful not to have the sccuring stitches fall directly under those in the preceding row, but place them so that they will come in the spaces between. These securing stitches are not taken over the entire laid surface, but are arranged to form vandykes. Figure I I 9 shows the same stitch differently worked, as follows: Lay down two threads of purse silk, catch them down with a stitch from the back, also of purse silk, and placed at regular distances apart along the line; work the second

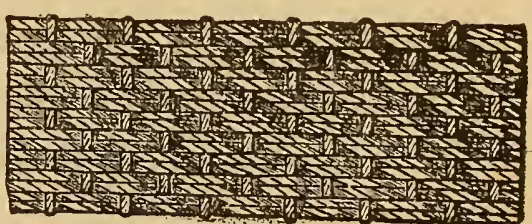

FIG. IIg. line like the first, but place the securing stitches in it so that they will fall between those taken in the previous row.

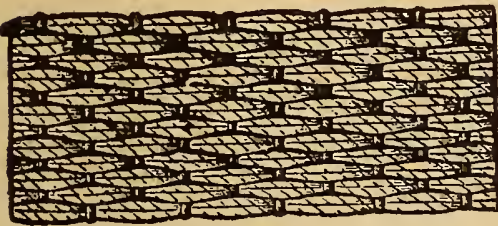

FIG. 120.

Broad Couching:-Figure I20 shows another variety of couching. It is worked like the brick flat couching previously described, but the securing stitches slightly draw the foundation floss together as they stitch it down. This gives to the work an entirely different effect.

Diamond Couching.-Figure I2I shows a flat diamond couching, worked as follows: Lay down lines of filo floss to cover the desired surface, and above them lay lines of purse silk or gold thread, singly, but in a diagonal direction, and at equal distances apart. Secure each single line with a securing stitch brought from the back. Lay all the lines in one direction first, and secure them; after which lay the lines that cross them; wherever the lines meet and form one of the points of a diamond, work in a pearl or spangle at the point of junction.

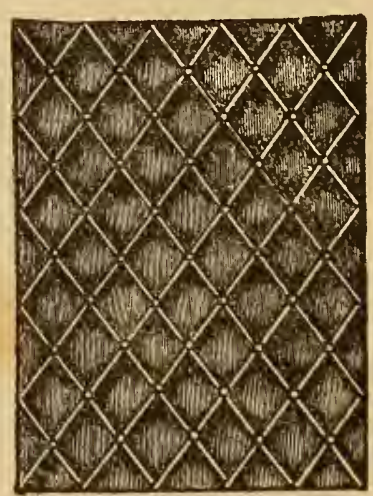

FIG. I2I.

Diaper Couching:-This is the same as plain couching, but the securing stitches are worked so as to form zigzag lines, diamonds and crosses.

Plain Flat Couching - -Figure 122 shows flat couching, which has also been described in Chapter V. Lay down filo floss evenly over the foundation, and secure with couching stitches brought from the back. Take the securing or couching stitches over two threads of the floss and return 
to the back again. Arrange the couching stitches so as to form straight or curved lines or diamonds across the space covered. See page 38 , figure 28.

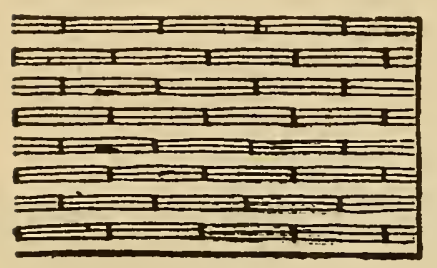

FIG. I22. In figure 122 the couched lines are not placed close together, but allow the material upon which they are laid to. show between them; when so arranged they must be placed directly upon the foundation and not upon the linen which is afterward appliqued upon the silk or velvet foundation. Figure 123 shows still another variety of plain couching. To work: Lay down perpendicular lines of filo floss, arranged close together, then, wide apart and horizontal, place single lines of purse silk or gold thread,

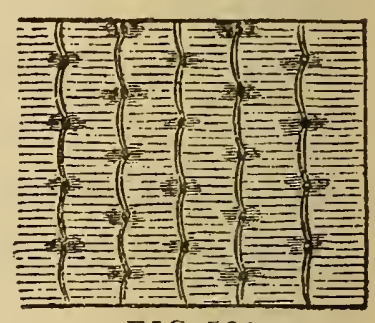
FIG. I23. and secure them at equal distances by a couching stitch. Wherever a couching stitch is taken work in a spangle or bead.

Shell Gouching is a flat couching in which the securing stitches are arranged in half curves, and bear some resemblance to the shape of a scallop shell.

The preceding comprise all the important varieties of couching, though the possibilities of new patterns and combinations are almost numberless, and the earnest worker will not be satisfied with being a mere copyist, but will never willingly stop short of the creative point. The illustrations given will form a good basis for further developments.

Long Stitch is a name given to satin stitch when worked across the design without any under padding.

Bullion Knot.-This stitch is used in church embroideries, and forms a raised roll laid along the surface of the material. To work: Secure

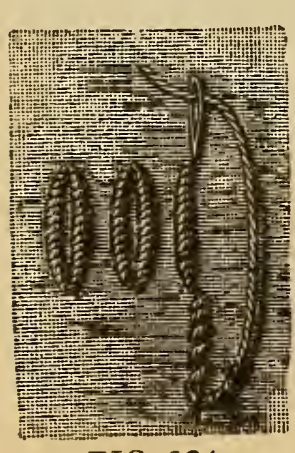

FIG. I24. the thread at the back of the work and bring it through to the front. Put the needle into the material and bring it out so that the point is close to the thread, and take up from one-quarter to one-half inch of material on the needle, according to the length desired for the knot. Wind the thread around the point of the needle from ten to twelve times-see figure 124-hold the needle down with the left thumb and wind with the right hand. Still holding the needle down, pull it through the material; pull up the thread to where 
the needle was inserted, and allow the knot to lie evenly along the surface; put the thread through to the back at this place, and repeat for a second bullion knot. In the illustration two bullion knots are arranged as an oval, but they can be laid down upon the material as single knots, or in any other device.

Chain Stitch.-This stitch was an important factor in ecclesiastical embroidery of the fourteenth century, and is again coming into use for this class of work. To make: Bring the needle from back of the material up in the line to be embroidered; put the needle down close to the place at which it came out, but on the right side; repeat; hold the thread down with the left thumb, and bring the needle out upon the line but one-eighth of an inch below where it was inserted, and over the thread held down; repeat until the pattern is finished.

\section{Outline Stitch.--See Chapter V.}

In ecclesiasticalembroideries the designs, if small, should be clearly stamped on the unbleached linen previously spoken of, after which the linen must be stretched firmly in a square frame large enough to take the entire design; or, if the pattern is too large to be easily managed in this way, the design may be stamped on the linen in sections, worked, and then cut out and applied to the foundation, piece by piece. In either case the entire design should be stamped on the real foundation as well as upon the linen, in order that the worker may the better see where and how to apply the cut-out design. After the design has been worked on the linen and then cult out and applied to the foundation, the edges should be finished with

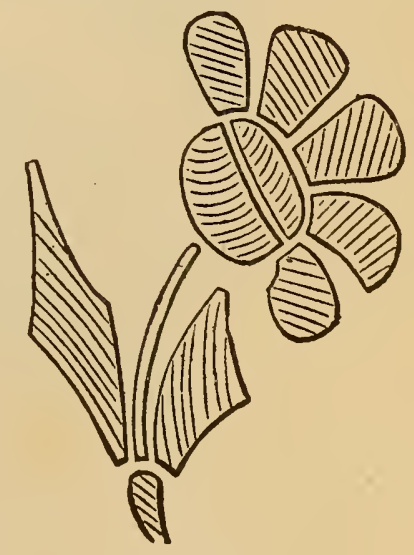

FIG. I25. one or two cords couched around the outlines. We here show working details of a design intended for ecclesiastical purposes. See figure 125 .

"In all church designs commence the work with raised couchings or with the laying down of gold threads. These lines of gold thread are usually worked so as to follow the wave of the part they are ornamenting. Thus, the flower shown in figure 125 would be entirely wrought with lines of gold or silver threads, placed as the shading of the pattern indicates. Figure $\mathrm{I} 25$ is much reduced from the natural size; an ornament so small as 
it is represented rarely has threads laid down. Wavy lines of gold are more used than straight ones, and these are shown in the working detail in figure 126 and are managed thus: Through a hole made in the foundation linen bring to the front of the work from the back two pieces of gold twist of equal size and thickness, and make a bend or curve in them by curling them once around the stilletto after which lay them on the foundation with the curve still in them, and catch them down with the holding thread thrown across both at once. Lay the gold twist so curved between each

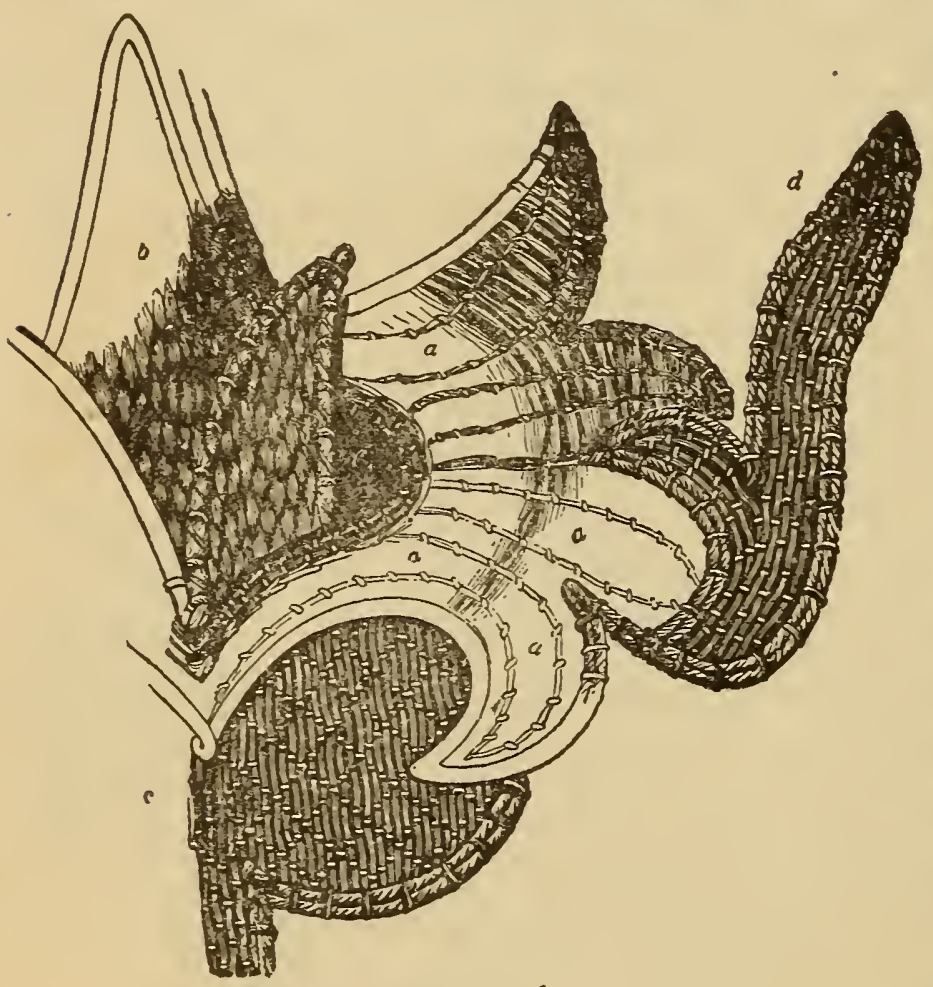

FIG. I26. securing stitch [see figure I26] on the space it is to cover, in an upright direction, then turn and bring it down, turn again and bring it up; and so on until the space i filled. These lines need not be laid close together, but with a space between them equal to one line in width, and this space may be filled in with a line laid afterward, the gold twist lying flatter on the surface when so arranged than when laid down in consecutive lines. Turn the twist wherever possible; but in many places this cannot be done and it must be cut and fastened at the back and again commenced. When angles and curves are being laid, it is a task of dexterity and patience to lay the lines and turn them so as to fill the spaces with the fewest breaks. The fastening threads should be bright-colored purse twist.

In figure 126 these fastening threads are shown worked in two ways. In the space marked $c$ they are arranged so as to form open diamonds, while in the long narrow space marked $d$ every other fastening thread forms part of a straight line arranged across the work. An illustration of the two ways of using filo floss is also given in this working detail. In $a$ it 
is laid in flat lines across the surface of the foundation and caught down with lines of purse twist of a contrasting color to the floss and laid in a contrary direction. These are fastened above the floss silk by catching stitches of silk brought from the back of the work and returned there. The lines of purse silk are laid over the floss silk at nearly equal distances from each other, and are intended to imitate the veinings of a leaf, the threads that catch them down being of a silk matching them in color or of a contrasting shade. The space marked $b$ shows the mode of working the floss silk when it is passed through the foundation and not laid upon the surface. It is a long stitch, but worked so that each stitch is placed in a slanting direction, and does not follow the preceding one with the regularity of a straight line. The long stitch is more fully illustrated in figure I27, where it is shown considerably enlarged. The small space is filled with black silk lines, which are secured by three lines, two of gold thread and one of silk. The border to the detail is formed of two lines of thick silk cord of harmonizing colors, both caught down with the same stitch. The sin-

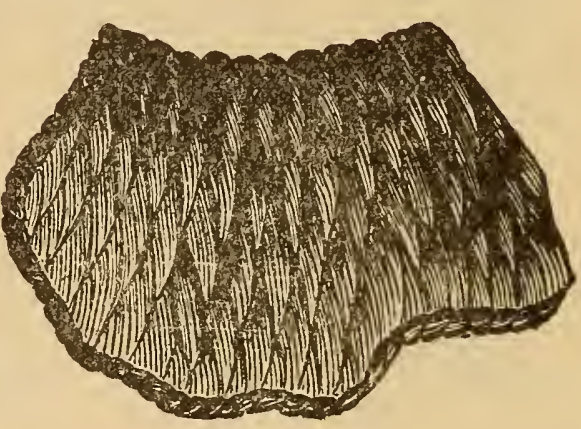

FIG. I27. gle cord that surrounds the piece of work between $e$ and $b$ is a silk cord around which a fine gold thread has been twisted, and which is couched down with a silk thread. This working detail will be an extremely useful piece for a beginner to try her hand upon, as it combines several of the stitches that are used in ecclesiastical embroidery."

Another design that is also simple is shown in figure 130 on page I28. This design is much reduced from the working size and is suitable for the ends of scarf for flower stand. The main portions of the pattern are laid in with floss couched in various patterns, as brick, flat, diamond and basket. The stems are laid in with gold thread couched down with red purse silk; all the design except the five branches at the top, are first worked upon a linen foundation, which is then cut out and applied to the article to be decorated. The outer edge of the pattern is then finished with two cordsa gold cord and a cord of black silk couched close to it. The five branching fibers are worked with gold thread and spangles upon the material. To secure this design enlarge with a pantagraph four or five times accord- 
ing to the position it is to occupy, or it can be used in connection with some other design, as powderings over a surface, in which case it may be worked on a smaller scale than where it is used alone as a set design.

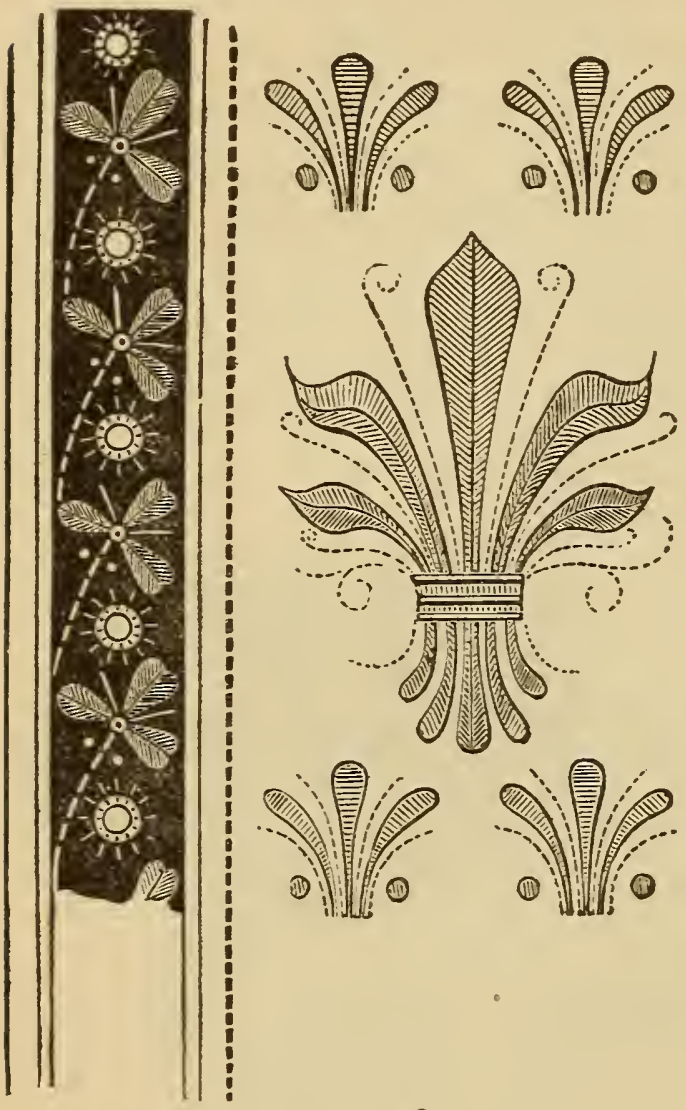

FIG. I28.

Figure 128 shows a reduced design for border and powderings, suitable for altar frontals or altar curtains. Work the large fleur-de-lis in long stitch with three distinct shades of green floss, and edge with blue purse silk. Fill the band in the center of the fleur-de-lis with crimson floss laid in perpendicular lines secured with gold thread; edge with black knitting silk. The various tendrils or sprays springing from the powderings are made of gold thread laid in lines and caught down with crimson purse silk. These lines of gold thread should have a line of floss laid close to them, and following their outlines; this is not shown in the illustration, but it should always be used when gold thread is laid as an unornamented spray. Work the small half fleur-de-lis figures in long stitch with floss. Their colorings are alternately crimson and green; the crimson shading to pink, the green from dark to light. Surround them with black knitting silk, and with branching fibers of gold thread and floss. The small circles are formed of spangles, four to each circle, caught down with green or red purse silk. Work the border upon a band of rep silk of a darker color than is used for the body of the curtain or frontal. The wheels or stars on the border should be worked with gold thread or yellow purse silk, caught down with black silk. The leaves are worked with shades of blue in long stitch, and the stems and tendrils are formed of gold cord. Add small spangles where shown in the design.

The three shades of blue, green and rose color used should be perfectly distinct from each other, and not selected as in ordinary embroidery, so 
that one shade blends imperceptibly into the other; for although harmonizing, each shade must be distinct from the one above and below it.

Figure I29 is another valuable working detail. It should be carried out as follows: Lay gold cord or thread in waved lines, as at A A A A, and catch these down with even rows of purse silk. Lay down the head of the seed pod with gold thread, as at A, but the lines must here be straight. Fill the stalk, в в B, with green floss of three distinct shades; work in long stitch. The leaves are of the same, except the veins, E E, which are of yellow floss; D D, representing the soft hairs on the stalk is done with green floss; the center of seed pod, $\mathrm{k}$, work with two shades of pinky red floss, in upright lines and surround with a silk cord; $\mathrm{H} \mathrm{H}$, work with silver thread twisted around it and caught down with pale blue silk; in the inside of the cord place a narrow black cord, and catch it down with black

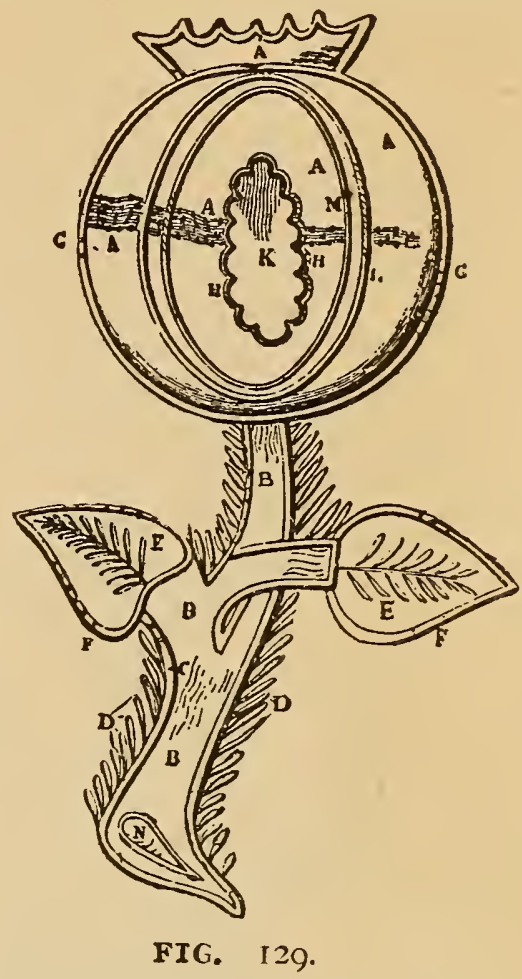
thread. The two succeeding oval cords laid upon the gold cord are of yellow silk, one thick, $L$, and one small, $M$, but both cords have a silver twist around them. The outside cord, $\mathrm{G}$, is also yellow, but thicker than either of the others. It is caught down with black.

The center cross for altar frontal shown in figure II 2 , on page I 6 , is more elaborate than any design we have given, and requires excellent workmanship. This design differs from those shown in figures 125,128 and 129 in that some of the parts forming it are worked directly on to the silk foundation, while other parts are appliqued on to velvet of a different color than either the foundation or the colors used in other parts of the design. The stitches on the cross should be worked upon white silk, the circle upon which the cross rests should be of deep crimson silk, upon which the floriated ornaments are to be appliqued. Work the boss forming the center of the cross, and containing the center jewel, on to a linen foundation and applique it on to deep blue, velvet, which in turn must be placed on the white silk foundation. 
The cross is shown as it would be in the embroidery frame; when re moved and applied to the curtain or frontal, rays of gold thread or yellow silk surround its outside circle, and branching fibers proceed from the four limbs, with spangles carried up each side of them. The five bosses are

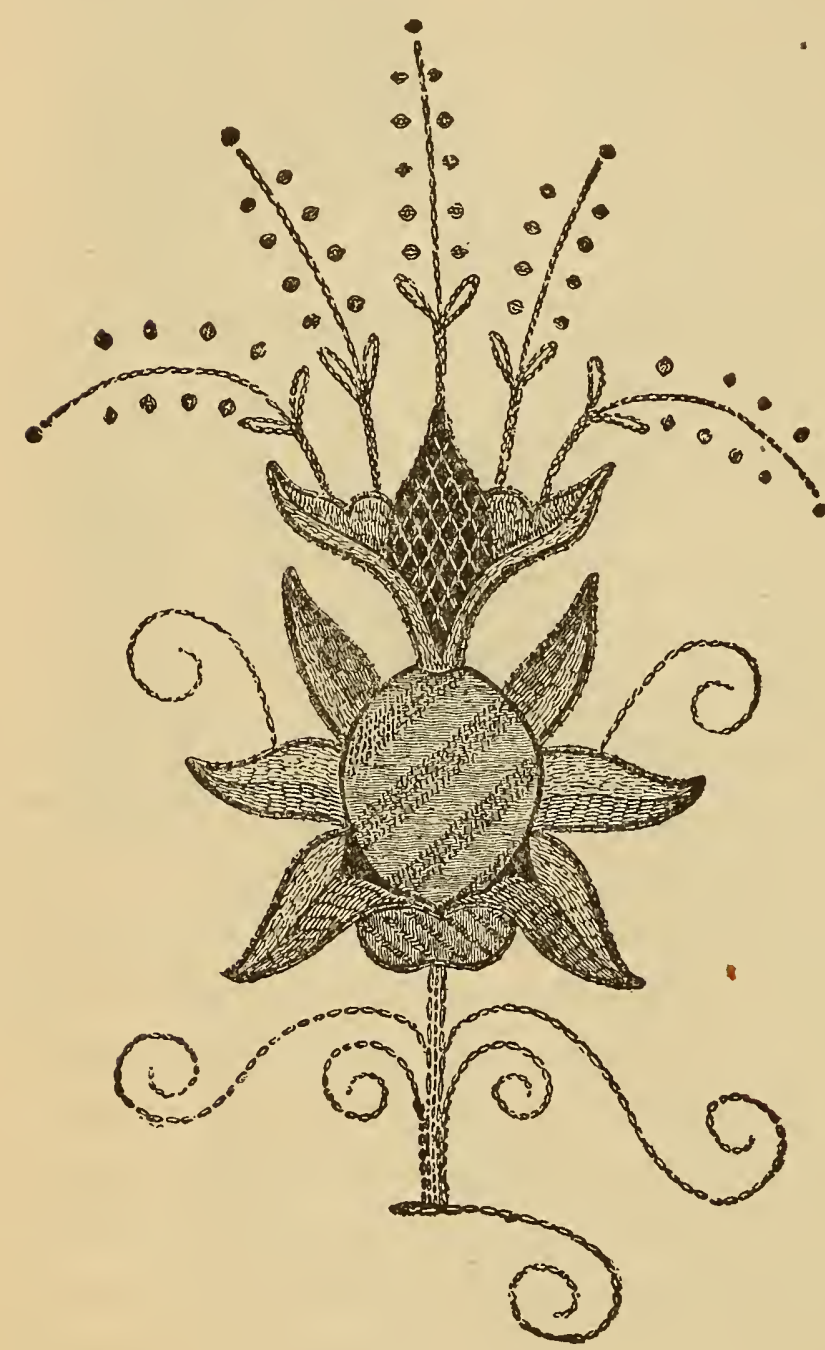

FIG. I30. made of jewels. Surround each jewel with gold thread and with rays of green floss, shading to light green. Form the body of the cross, as previously stated. with white silk. Ornament the cross with couchings of gold thread, laid in diamond pattern and caught down with spangles and red sewing silk; also some portions of the cross may be ornamented with straight lines of floss. Arrange the outside lines in brick couching with yellow silk or gold thread.

The ends of the cross that appear beyond the circle cover only partially with embroidery; the foundation of blue velvet, the same color as that used around the center boss, leave visible. The crowns finishing the ends make of gold thread laid upon the velvet, as also the thick line from which they proceed. Work the leaves in crimson silk, shading to pink. The floriated ornaments that proceed from the cross are first worked upon a linen foundation and then appliqued upon the crimson silk circle enclosing the arms of the cross. The outside leaves of these ornaments are embroidered in long stitch with three shades of green floss. Fill in the lower part of the space they enclose with crimson silk worked in long stitch, and ornament with knots or spot stitch formed of gold-colored purse silk. Above this lay lines of gold thread, and catch 
them down with crimson silk. The points which finish the ornament should be finished with pale blue silk in long stitch. Carry pale blue cords around the edges of the ornament to cover the stitches connecting them to the silk foundation. The scrolls that fill in the rounds form of lines of gold thread caught down with blue silk, and terminate with spangles.

The designs so far given illustrate the various ways of using filo floss in flat church embroidery. Thus, it is either laid down in even lines of one shade of color, and kept in position with gold or silk cord placed in devices over it, or it is worked in long stitch with three shades of color. These shades are distinct from each other, and are worked with the lightest shade uppermost; they never blend together, but they match in tint. If contrasts are used, such as pink and blue upon the same leaf, they are divided either by a line of black knitting silk or gold thread. In ecclesiastical embroidery no regard is paid to copying any design in its natural colors; the designs are never intended as realistic, but as conventional ornaments, and blue, lilac, crimson and yellow are used about leaves and other floral ornaments as well as green; though in examining specimens of old work it will be seen that green and gold are more used about the powderings and borderings than brighter hues, which are found in all their glory in the picture centers. The faces of figures are worked in satin stitch with one or two shades of flesh colors. The shade and contour of the features are managed by the direction given to the stitches, which follow the lines that would indicate them in an engraving. Chain stitch will undoubtedly soon be employed again for working faces in the modern as it was in the ancient work.

One of the richest and most popular stitches for couching background is the spider wheel, described in "Stitches for Ecclesiastical Embroideries." The wheels are usually made about an inch in size, and when the whipcord which forms the raised spokes of the wheel is covered with fine gold thread, couched down on each side of each line of cord, in such a manner as to outline each spoke of the wheel, the effect is indeed rich and beautiful.

Upon rich fabrics open couchings are frequently laid for borders. These are made of diagonal lines of gold caught down with crosses of colored silk, and the center of the diamond spaces are filled in with spangles, beads or French knots. 


\section{CHURCH WORK ON CARDBOARD FOUNDATION.}

Cardboard foundations forming this variety of work are used for sacred monograms and emblems, and are invaluable when clear, distinct and raised work is desired. This work is employed in church furnishings only for

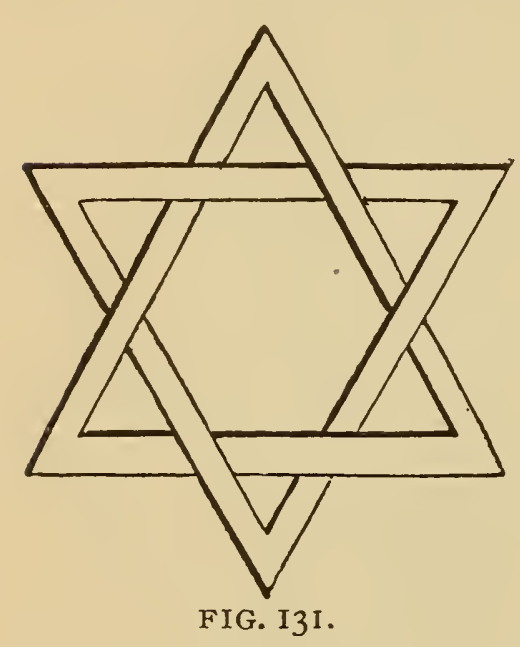
such minor details as the emblems on stoles, burses, alms bags, mats, book-markers and sermon cases, it being considered too severe in outline and too mechanical of execution for altar frontals and the vestments of the church. Being worked with silk of one shade of color throughout, and over rigid outlines, it requires no artistic taste in the execution, but it must be arranged with precision or it will entirely fail in effect. The designs selected should be simple, clear in outline and correct as to ecclesiastical form. The usual designs are the Latin cross, the initials of our Savior, triangles, circles and other unfloriated devices. Some of these are shown in figures I3I, I32, I33, I34, in their plain cardboard foundations. Figure I3I, the triangle, is an emblem of of the Trinity, as is also figure I32, the circle. Figure I33, the Latin cross combined with anchor and circle, is an emblem of atonement and patience, and figure I34, the Greek cross surrounded by triangle and trefoil combined, is a symbol of the Godhead. The manner of working is as follows:

Select the design and trace it upon thin Bristol board. Color the design yellow and cut it out carefully, leaving little supports, that are called stays, to any part of the letter or emblem that is too fine to support itself before it is caught down in its position. The stays in the designs

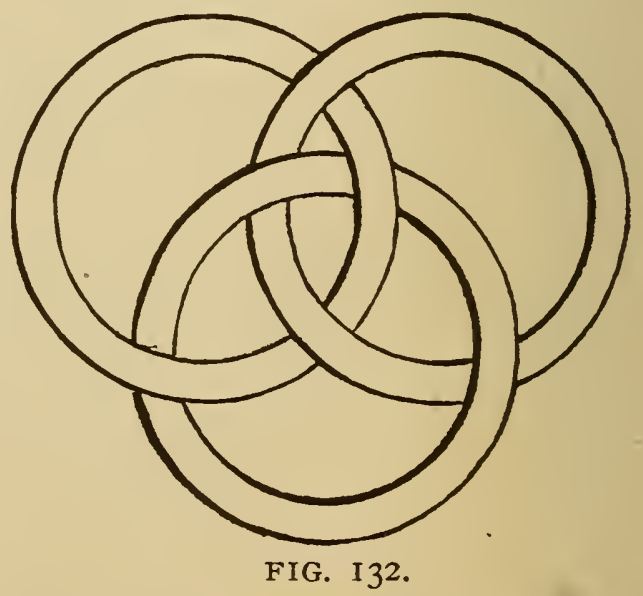
given would be required only to keep the extremities of the Greek cross, figure 134, in position. A piece of gray holland, sold expressly for this purpose, is then tightly framed, and the material to be embroidered secured to it. If velvet or a large piece of plush, paste it down, if silk sew on with 
great care, and sew round the center when the emblem is arranged. Put the design cut from the cardboard into position and carefully tack it to the foundation, and as soon as every part is secured cut away any stays that have been left. The embroidery is now commenced, and is done either with yellow purse silk or with gold twist of short lengths. The manner of working is shown in figure I36. Bring the needle up from the back of the frame on the left hand side, and pass the thread over the cardboard, the point of the piercer being used to lay it flat; insert the needle on the right side in a line parallel to the point where it came up. This operation requires great nicety. The thread or silk must be kept evenly twisted, and each line laid down with great regularity, as the entire work will be ruined with one irregular stitch. When the cardboard is covered, outline the design with a couched line of

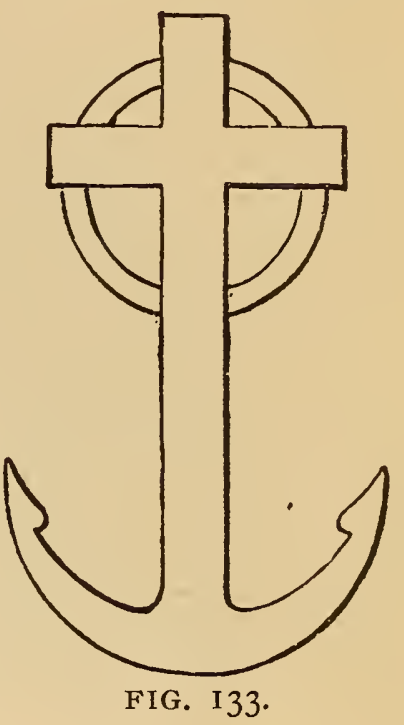
blue or red cord or gold thread, as shown in figure I35. This couched line will take away any unevenness of outline that may have been made in the working. The material is then cut away from the frame and the holland from round the edge of the embroidery at the back. Figure 136 is an illustration of a single letter worked in this manner. The exact shape and size

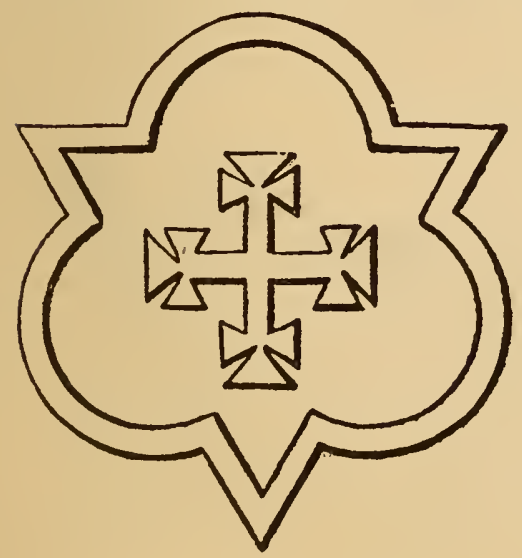

FIG. I34. is first cut out in cardboard and laid on the foundation and carefully sewed to it, the arm of the " $r$ " requiring very delicate adjustment. Lay a line of carpet thread down the center of the letter and fasten it, and cover all the cardboard over with lines of gold thread or yellow purse silk. Figure 135 shows the manner of working interlaced letters and adding the couched line around them. Interlaced letters are cut in one piece and no stays are required. They are fastened to the foundation and covered as described with the gold thread. No shading is necessary in this style of work, though a variation in coloring devices is permissible. Thus, in figure I37, "I. H. C." placed upon a cross, which can be used for a sermon case, book-mark or 
alms bag, is worked as follows: The cross is worked in gold puise silk or gold thread, and couched round with a line of black silk. The letters "I. H. C." are worked in crimson silk and couched round with pale blue silk. The entire design should be placed upon a background of green or

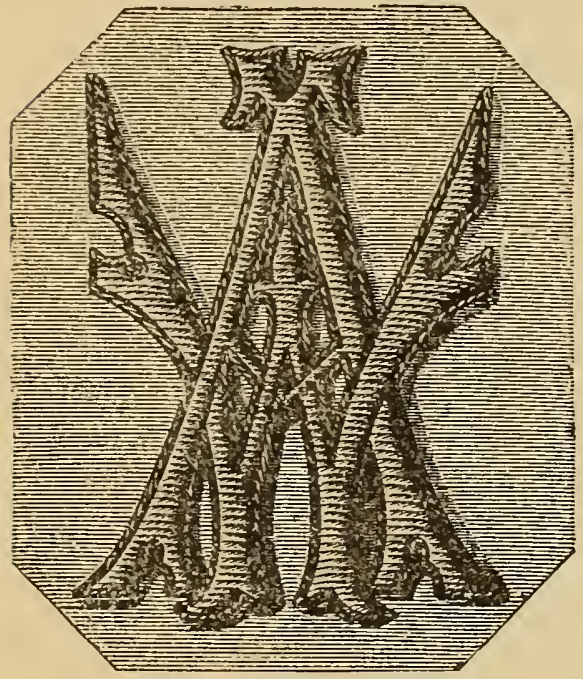

FIG. 135 . blue velvet. The "I. H. S." of figure I39 is arranged for a banner. The I should be in gold silk, the $\mathrm{S}$ in blue and the $\mathrm{H}$ in red. All the letters should be outlined with a couching of black silk. The letters are to be placed upon a white silk banner. The wreath should be worked in satin stitch, flat, without under padding.

These small pieces of church embroideries will be very welcome, as ladies can easily do them at home. For book-marks a thick corded ribbon should be used, one and one-quarter yards long and from one to three inches wide. The ribbon to be embroidered should be tacked to the framed holland, and the design put on at its lowest part six inches from the end, so as to allow five inches or more for turning up at the back to hide the lining. Both ends should be embroidered, one design on the right and one on the wrong side of the ribbon, otherwise the work will not fall right when the marker is folded for use. The marker should be finished with heavy gold fringe. Alms bags are made in two shapes, one, a regular bag, hung upon a ring or hoop of brass, and made of a straight piece of velvet eleven inches wide and nine deep, joined, gathered and sewn into a circular velvet bottom, stiffened with cardboard; the other, the ordinary hand bag, nine inches in length, six in width, with a front lap six inches long, upon which the motto or emblem is embroidered. The bag is lined with white silk, but any part that shows is covered with colored velvet sur-

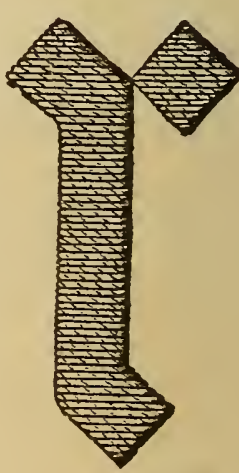
FIG. I36. rounded with an ornamental cord of gold and silk. The upper part of the alms bag is either curved or pointed. Alms mats are made to fit the plate, and the monogram ornamenting them worked so as to be contained in a square. The ornament upon a stole consists of Greek crosses worked with 
gold silk. Sermon cases are made of velvet and lined with silk, and should be covered over a cardboard foundation. The burse is used to keep the corporal and smaller eucharistic linen in, and is a pocket made of silk over a cardboard foundation and ornamented with needlework, and in shape is a square of from ten to eleven inches. The colors of these ornaments vary with those used upon the altar, which are as follows:

White for festivals of our Lord, the Virgin, Saints, and for Easter; red for Martyrs, Ash Wednesday and last three days of Holy Week and Whitsuntide; blue for week days after Trinity, and indifferently used with green on urdinary Sundays; yel low for Feast of Confession; violet, brown and gray for Advent, Lent and Vigils. black for Good Friday.

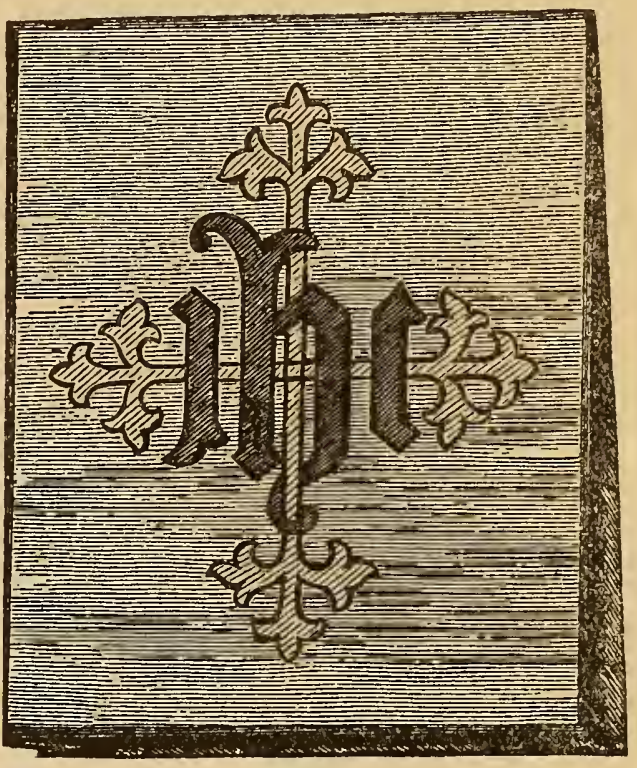

FIG. I37.

Figure I 38 shows a very pretty border, snitable for flower stand cover. It can be easily worked in gold thread. For the scroll line lay down a gold thread and secure it at intervals of one-sixteenth of an inch with red purse silk. Cut the floral portion of design out of cardboard and paste upon the foundation and work over with gold thread, as previously described.

The lines enclosing the scroll device may be worked with three shades

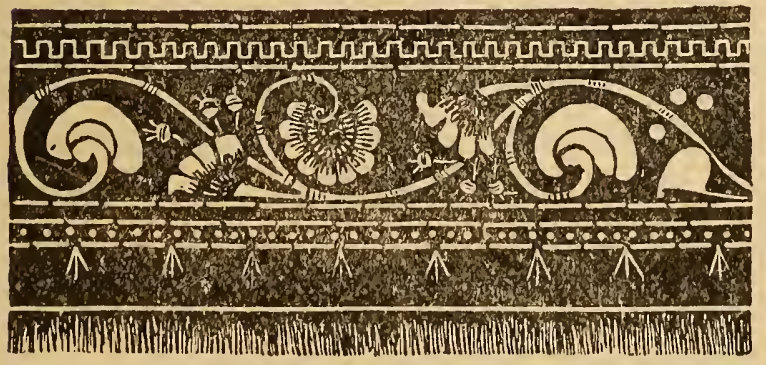

DESIGN FOR BORDER. FIG. 138 . of green and three shades of red purse silk. Couch down the straight lines and work the small pattern between with outline and spot stitches.

Figure II3, page II 8 , is also a very suitable design for either gold or silver thread or bullion, and will look particularly well wrought out on either blue, green or crimson velvet. The leaves may be treated with diamond couching in gold thread; the flowers may be worked with the same material over a cardboard foundation 


\section{I3. WANT WORK FOR PLEASURE AND PROFIT.}

\section{BULLION EMBROIDERY.}

Bullion work is sometimes used for church embroideries in place of the purse silk and gold thread over cardboard, and the treatment is the same, as concerns the designs and the laying of the threads, except that the bullion is not used in the needle, but is cut in lengths the exact size of the width of design to be covered. Thread the needle with a yellow silk, waxed; bring it up from the back of the material, close to the cardboard foundation; thread one of the cut lengths of bullion on the point of the

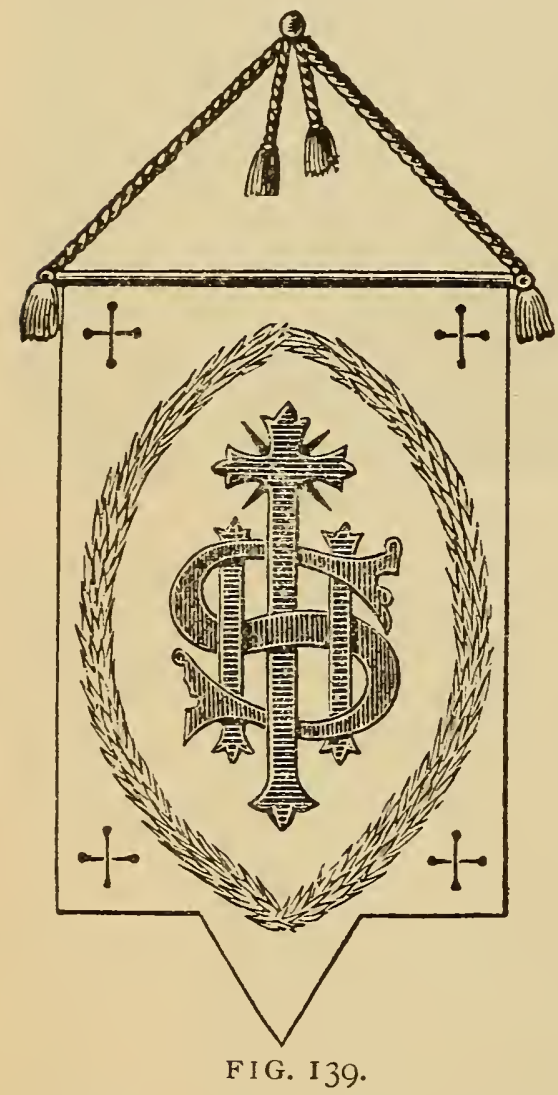
needle and put the needle down on the oppo. site side and pull through to the back. This operation brings the bullion into position, and if the length has been correctly measured the bullion will lie smooth and even across the cardboard; but if it has been cut toc long or too short, the work must be done over again, as a single poor stitch will ruin the entire work. After the cardboard has been covered, the design should be outlined with bullion of a different variety, or with other colored cords. Bullion embroidery is also used to ornament the regalias and banners of different societies, and for heraldic devices. The designs for these purposes may be secured from medals, etc., in possession of the society for which the work is to be made. Perforated patterns can be made for stamping the different parts of the design. For collars and other society vestments the cardboard foundation is pasted directly on the velvet forming the collars and covered with the bullion. Ivy leaves, arranged as a vine, is a favorite design for collars. The leaves are cut from cardboard. Sometimes one thickness and sometimes two thicknesses of the cardboard is used for a leaf. After the leaves of the pattern have been placed in position the bullion is worked over them, beginning at one edge of a leaf and ending the bullion in the center. One side of a leaf finished, the reverse side is then worked, and a thread of another shade of bullion is carried down the center to form the veining. 
After all the leaves are worked, the stems and tendrils of the vine are to be worked directly on the velvet, without any cardboard foundation. When outline stitch is desired for this purpose the bullion should be cut just the length of a stitch, and each time an outline stitch is taken with the threaded needle a piece of bullion should be threaded on the needle.

In making shields, eagles and other large devices, the foundation is cut out of cardboard, and some portion of the design will have four or five thicknesses of cardboard, one over the other, and each succeeding piece a size smaller than the first. This gives a more raised and rounded effect to the work.

In making banners, a piece of stout holland should be tacked in a frame, and the entire design should be stamped upon it and worked. The same design should also be stamped upon the silk composing the banner, and this

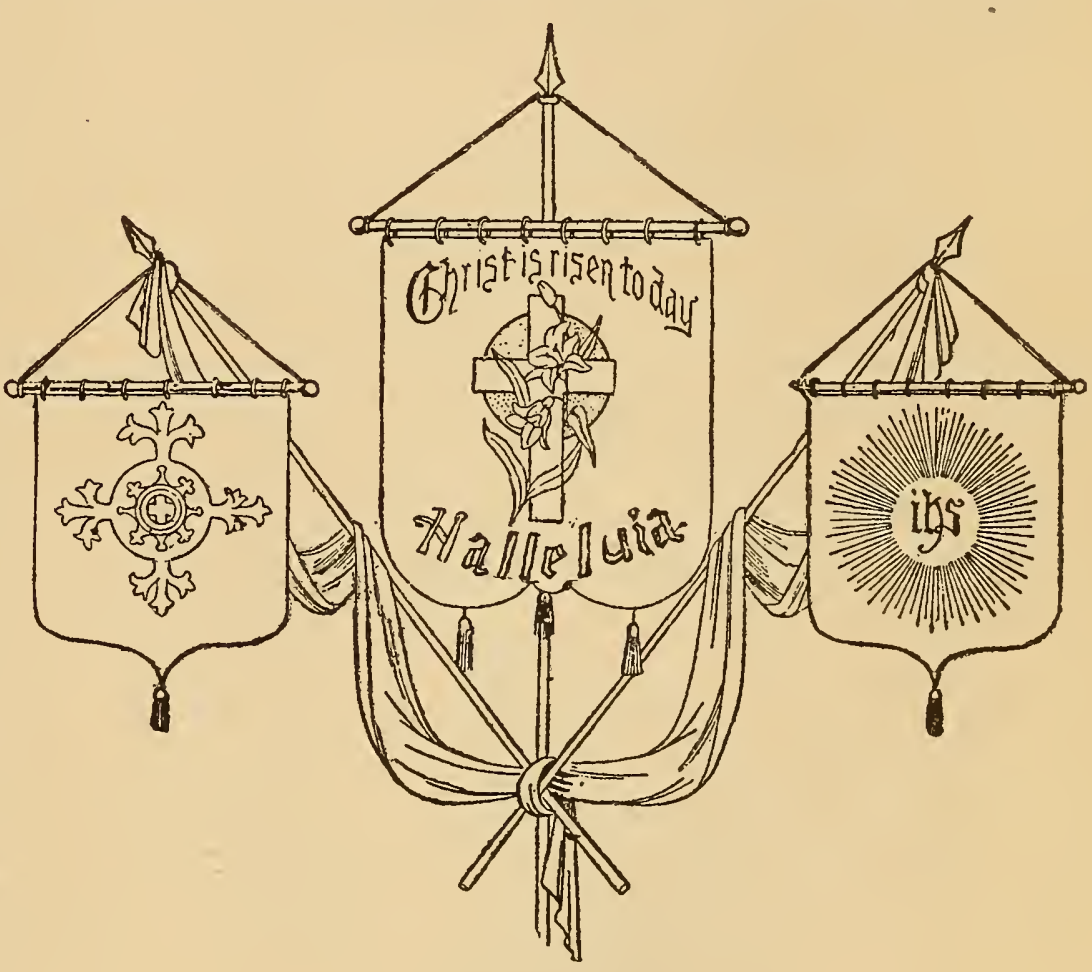

FIG. I40. should be stretched in another frame. As fast as one part of the ornamentation is finished it may be cut out of the holland and tacked into posi tion upon the banner and finished with a couched silk or gold cord.

The mottoes upon banners look best made of bullion over a cardboard foundation. Though amateurs sometimes embroider them with gold thread upon the silk, the effect is not nearly as rich and heavy. There are several kinds and sizes of bullion, and introducing a variety into the same piece of work relieves the monotony of a design worked in one color.

The trade terms for the different patterns of bullion are, checked, smooth, and spiral, and they come in bright gold and burnished gold. The 
same is true of silver bullion, which is made in the same patterns as the gold. Where the cardboard foundation is pasted directly on to the silk or velvet foundation, Chase's liquid glue is used for the purpose.

In finishing banners, an interlining of crinoline should be put in; the sides should be finished with a flat band of gold lace and the ends with heavy bullion fringe. When hung on a pole, the top should be finished with gold cord and heavy bullion tassels. Figure I 40 shows an Easter banner. It consists of three banners suspended from a standard. The foundation-of the three banners is white corded silk. The cross on the center

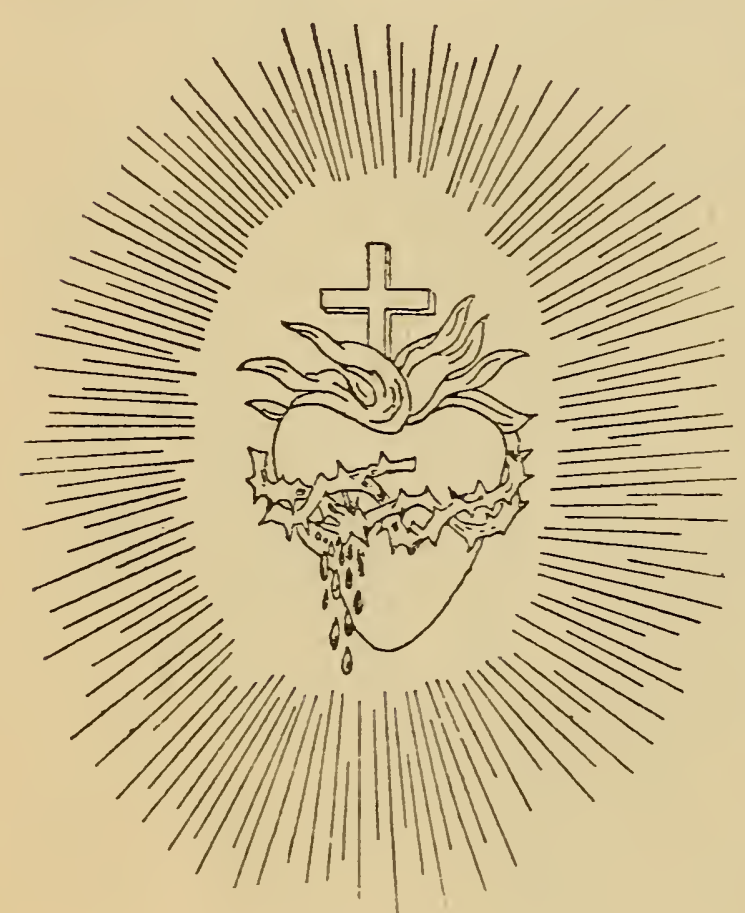

FIG. I4I. banner is made of blue silk filled in with basket couching of gold bullion. The lily decorating the cross is made of silver bullion worked over cardboard.

The cross and circle on the small banner to the left is made of couchings of three varieties of gold bullion, checked, smooth and spiral, couched directly on to the white silk foundation with blue purse silk. The letters on the banner on the right are worked with silver bullion on a blue silk foundation. Thelecters are worked over a cardboard foundation, as previously described. The scarf of silk draping the standard should be white China silk edged with gold bullion.

The Bleeding Heart is an emblem peculiar to the Church of Rome The colors for working are three shades of red, three of deep green, and for the rays surrounding it gold bullion. Figure I4I is a very good working model for this emblem, but it should be enlarged five times the given size.

Figure I 42 shows emblem suited for small banners. Work the passion Howers in three shades of purple purse silk. Couch around the edges a cord of black knitting silk. For the leaves use three shades of green. For the letters use purple, crimson and green silk. The letters should be made 
over cardboard; the wreath should be worked on holland and appliqued to the foundation.

The illustrations and directions here given will certainly be a valuabie assistarce to the experienced worker, and we trust that the explanations will be sufficiently clear to be of service to the beginner.

In parting, a word of advice to the embroideress who is andbitious to undertake church or heraldic devices, may be "the right word in the right placc." It is this, then: Practice diligently and patiently the different couching stitches explained in this chapter. Stretch a piece of material in your embroidery frame, and with gold thread and different kinds of silk threads work the couching stitches, and while they may seem simple in the reading, you will be astonished at the skill required to lay the threads evenly in position and to couch over them in the various patterns shown.

There is no royal road to this skill. Nothing short of faithful practice, and patience

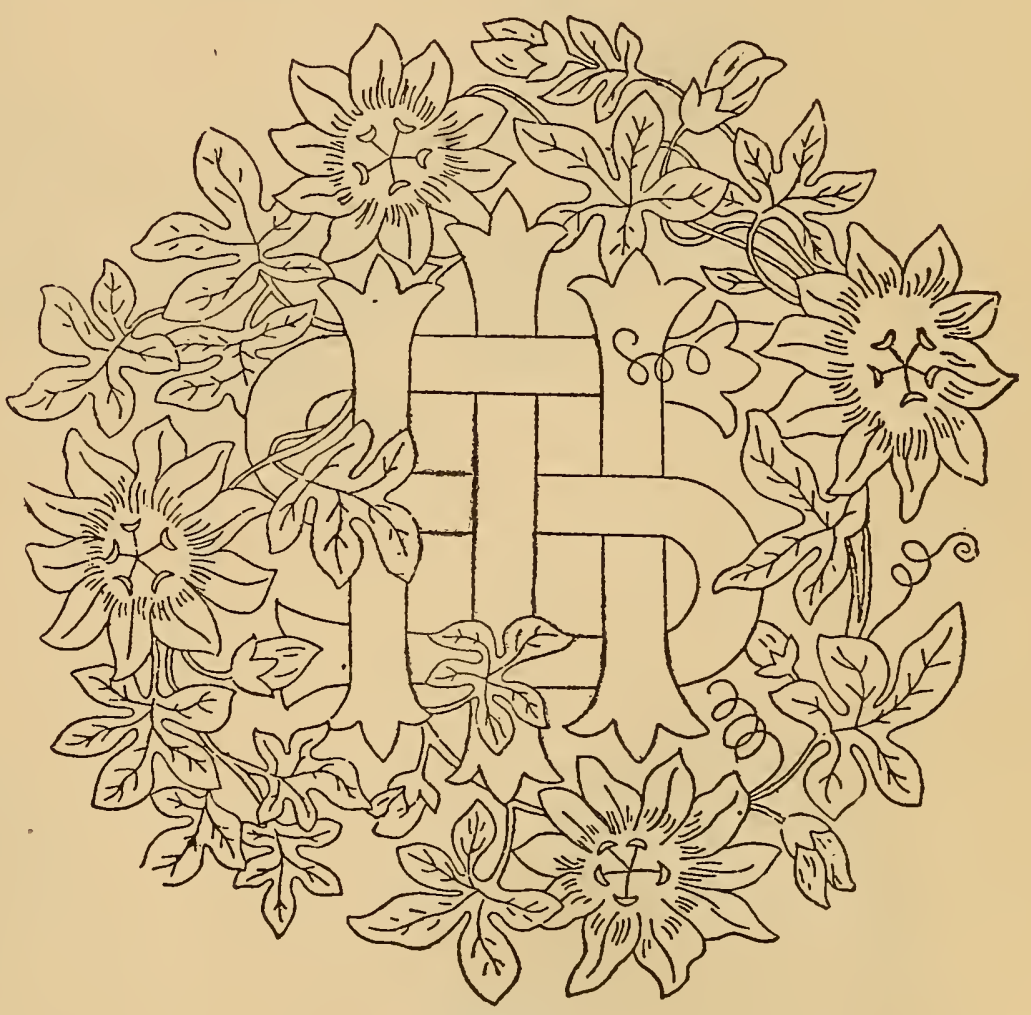

FIG. I42. unlimited will carry the worker to the goal of success. Next in importance is the long stitch. This should also be practiced until the threads can be at once placed at the exact angle desired, and in even, regular lines. 
138 DAINTY WORK FOR PLEASURE AND PROFIT.
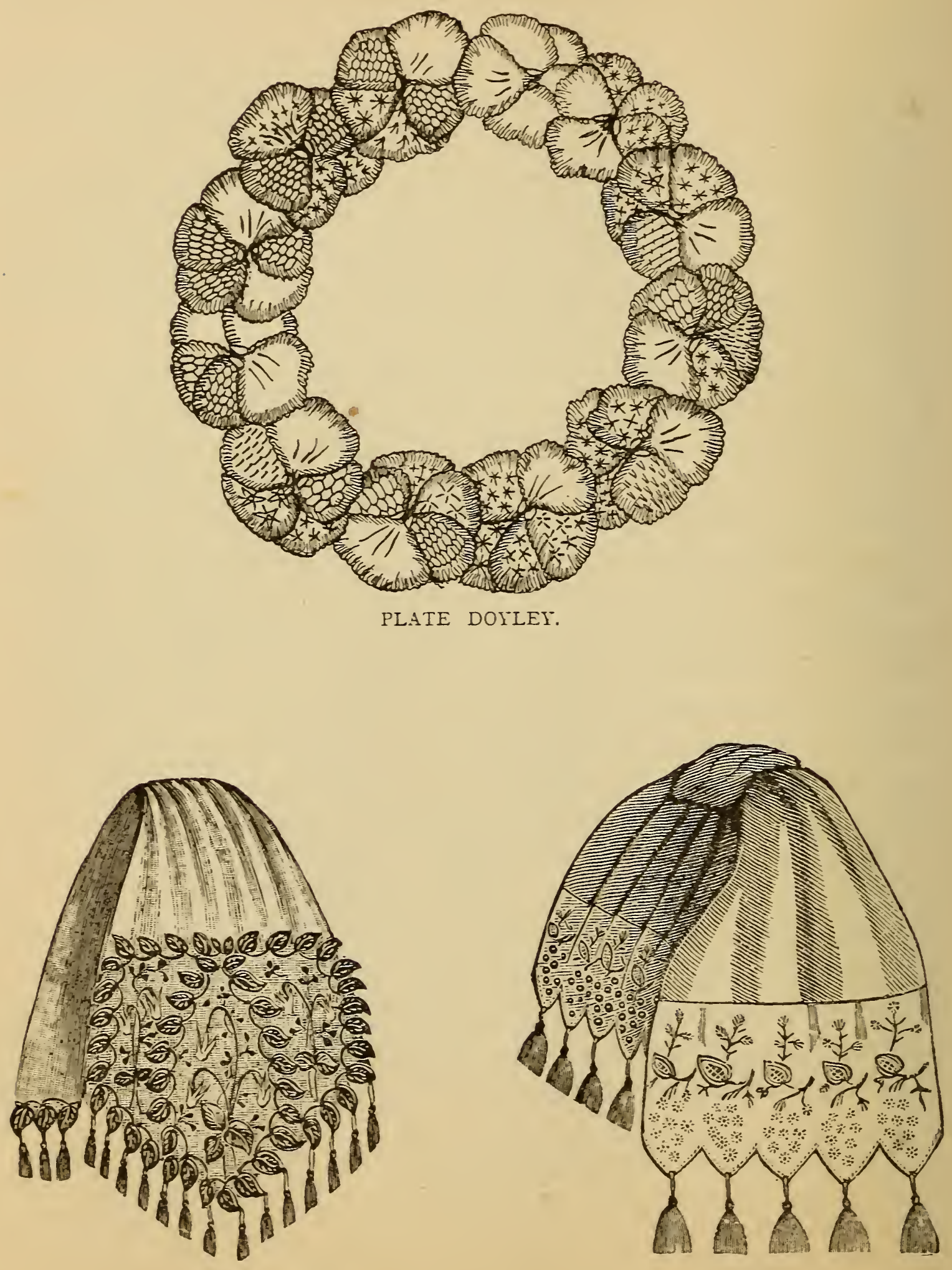

DRAPE.

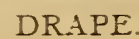




\section{CHAPTER X.}

BERLIN EMBROIDERY.

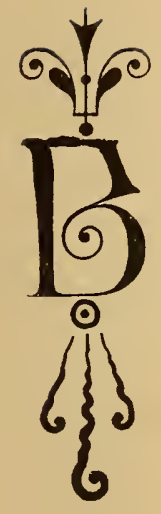

ERLIN EMBROIDERY was, anciently, as much in vogue as is the flat or art embroidery of the present day. In its original form, that is, wrought on canvas with wool or silk, it is still used for overhangings for mantels, borders for table covers and curtains, and for furniture coverings. But the modern use of Berlin work has necessitated some modifications in the method of working. Several of the stitc'ves formerly used exclusively in the manufacture of Berlin work are admirably adapted for use on plain and checked fabrics, namely cross stitch, tent stitch and a combination of the two, also satin and long crosis. Perhaps the most popular form of this work is that wrought out with simple cross stitch on checked or squaremeshed fabrics, such as Bargarren art cloth, huck-a-buck, Devonsinire art cloth, checked lawns, scrim and gingham. On these fabrics beautiful geometric, floral or mythological designs can be worked with linen or silk threads, and most beautiful results obtained for a trifling outlay of time and money.

The work may be done with one color, or with a series of shades of any given color, as three or four shades of yellow, from light to dark.

Bedroom and dining-room curtains made of scrim, and having a border of cross stitch done with filo floss or linen floss, can be easily accomplished, as the coarser qualities of scrim are quite equal to the regular Berlin canvas for the purpose. Beside curtains, toilet sets, tidies and towel ends may be worked to match.

Figure 143 shows a border of dragons that is suitable for use on curtains, bedspreads, shams, stand and dresser covers, all embroidered to 
match. Provided the materials used do not show a check or mesh, Berlin canvas can be basted into position on the different articles, after which the design can be worked on the canvas. When completed, cut the thrcads of

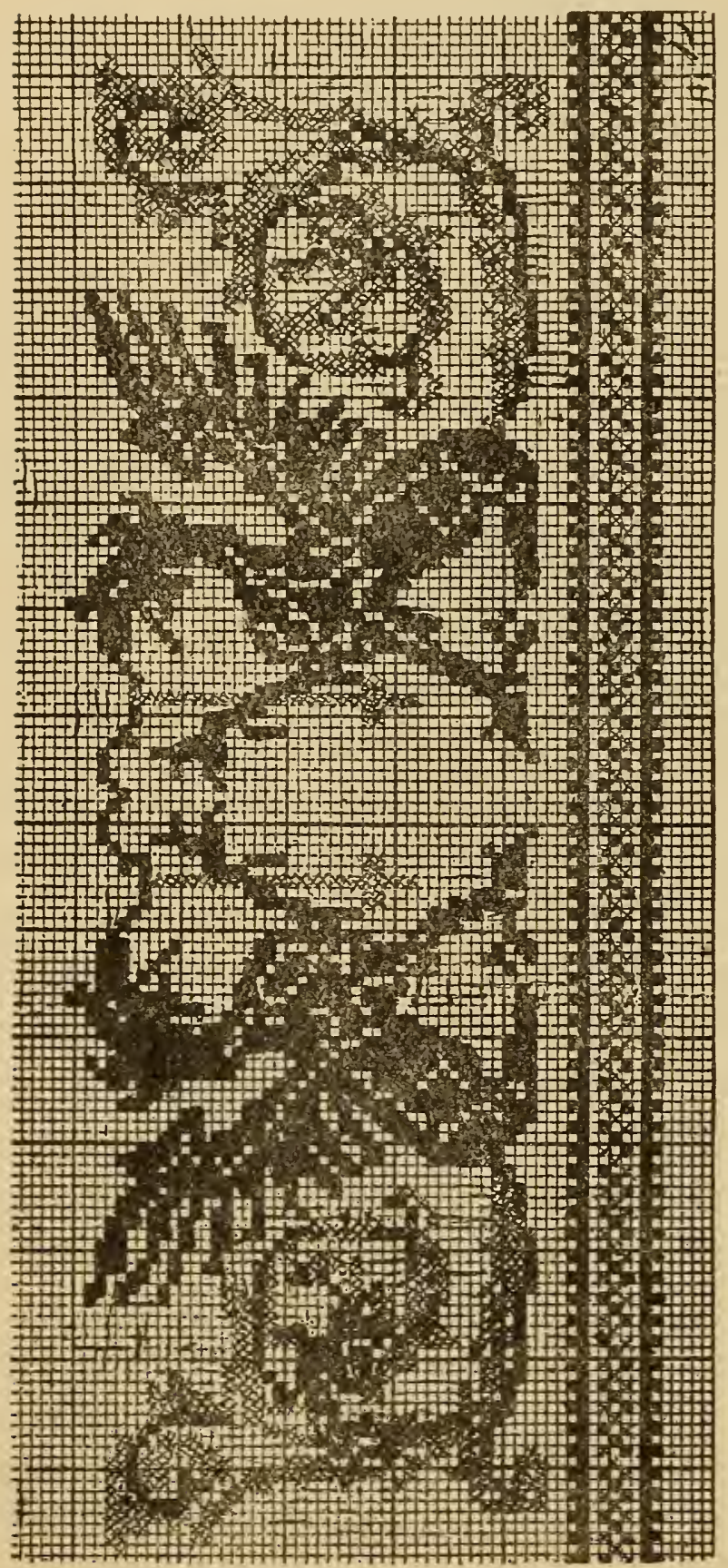

DRAGON BORDER. FIG. I43. the canvas and pull them out, leaving the work on the surface of the inaterial. Indeed, this method is always employed whenever a pattern is used that requires to be counted on a small square or check, or whenever the surface of the material to be embroidered is smooth.

The colors for working the border of dragons may be selected to harmonize with other furnishings of the room in which it is to be used. An artistic combination would be as follows: Work the bodies, heads and claws in deep yellow filo floss; the heads and tail in two shades of lily green filo floss, the lightest shade being indicated by the square crosses in the stitches shown in the illustration and the darkest by the black stitches. Work the ribbor hanging from the dragon's mouth in deep crimson filo floss. For the border: Work the straight lines with the lightest shade of lily green filo floss, and work the center stitches, alternately, with the deepest shadc of lily green and crimson filo floss. The pattern can be repeated, as the tails of the dragons join. When it is repeated, the center of the design should show the two dragons facing each other, as given in the illustration This design is particularly suitable for mantel hangings for library or study 
When Berlin work is used to decorate coverings for furniture, the pat. iern should be formed of rope silk or filo floss, and the grounding stitches done with Berlin wool. Patterns for this purpose should be conventional, and the colors used dull, dead shades for the design and black or brown

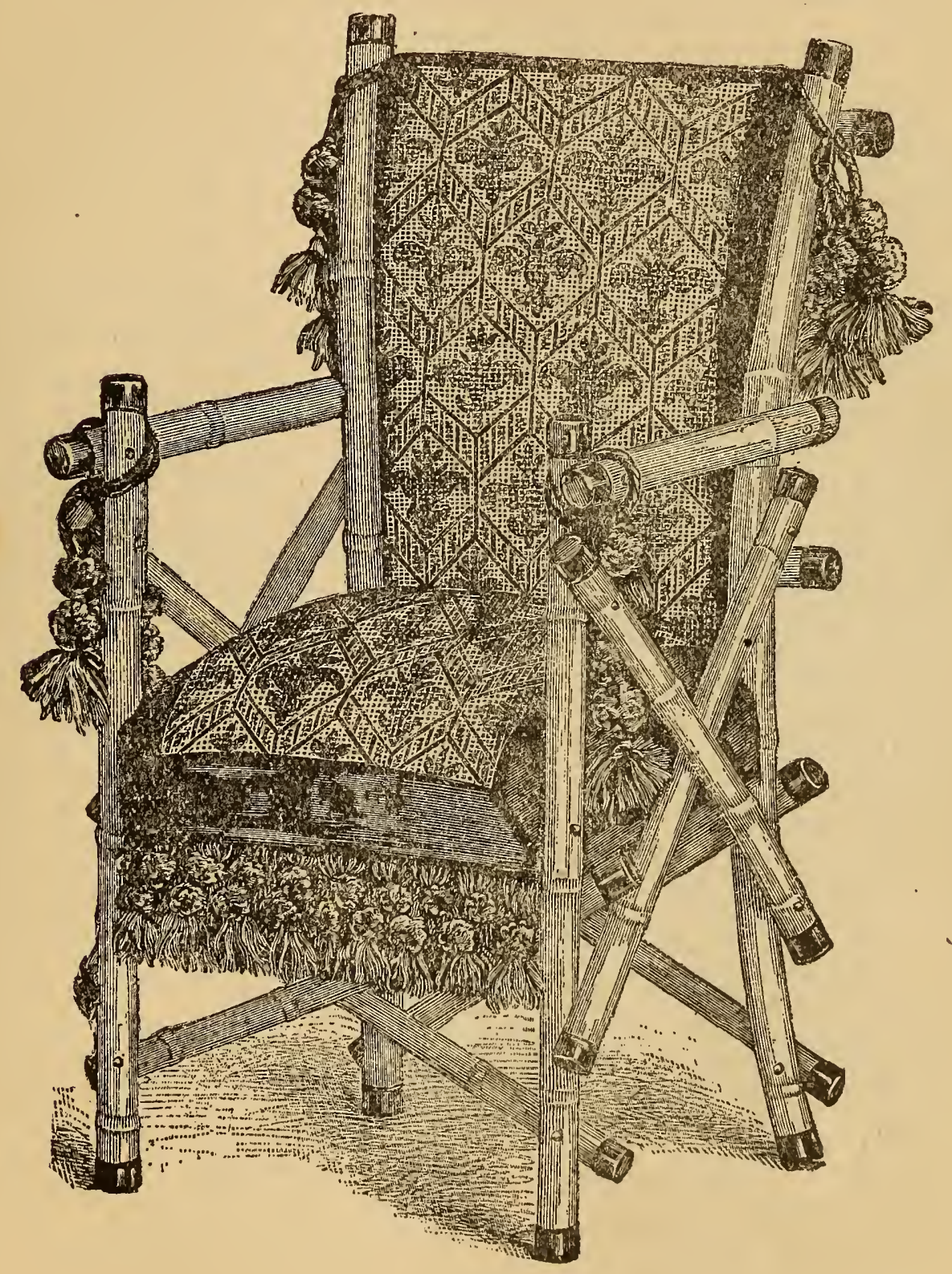

FIG. I44.

wool for the grounding. Figure's 144 and 145 show a chair upholstered " this work: and the detail of pattern with the proper colorings. As will be seen, very little grounding will be necessary. The design is worked in cross stitch, and the grounding is done with Irish stitch. If it is desired to work 
the design upon a fabric instead of canvas, Deronshire art cloth will be at once suitable and artistic. After the work of embroiclery is finished, it wil! be well to have a regular upholsterer put it on the article to be covered. The cost for this part of the work will be but a trifle, and the result will be sure to justify the extra expense. For recovering odd pieces of dilapidated furniture no work is at once so durable and inexpensive, providing the em-

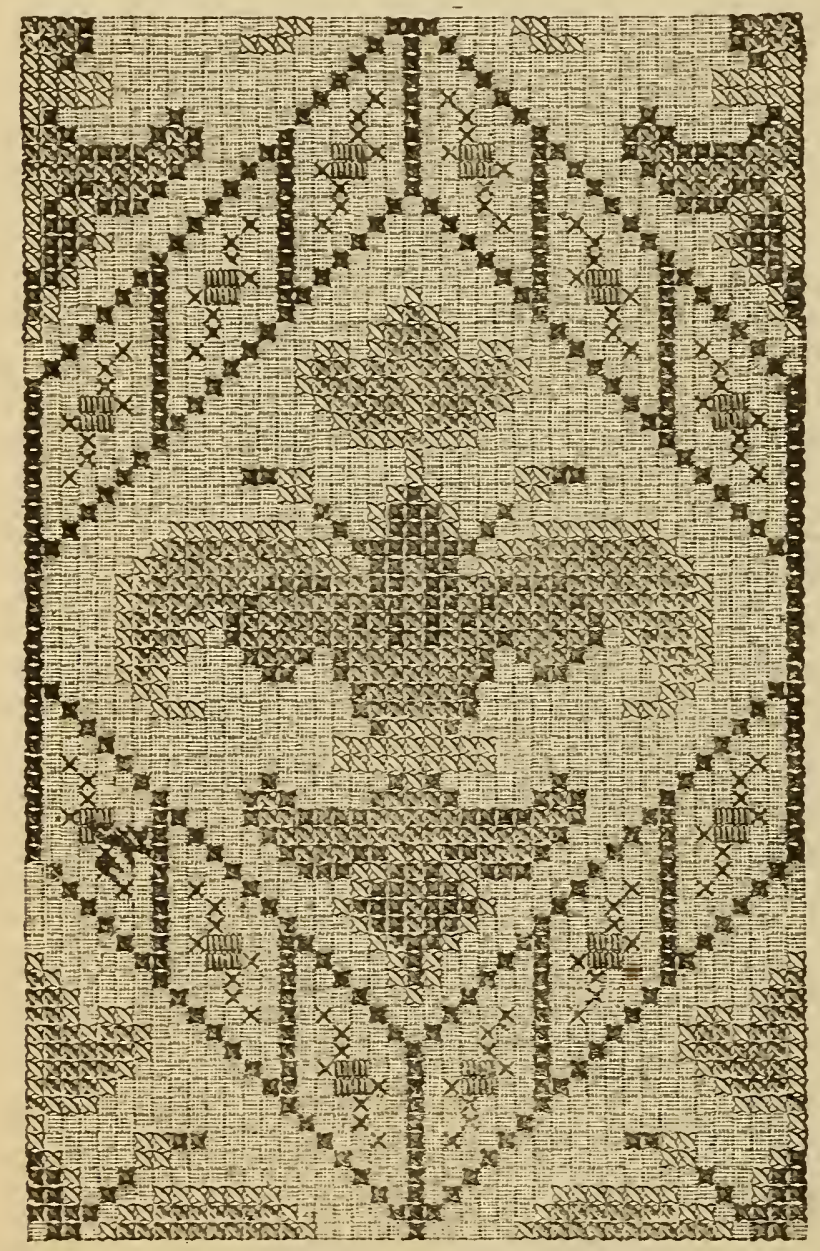

Dark olive. $\times \times$ Medinm shade. nam Light olive. Dark red or; blue. 35 Middle shade of red or blue. ars light red $0^{-}$

DETAIL OF FIG. I44. FIG. 145 . broidery is done at home.

The genuine Berlin embroidery is always worked on canvas, of which there are three styles used, namely; Berlin canvas, Penelope canvas and Java canvas. The latter is a small, square-meshed woolen canvas, which is made in white and colors. The design is first worked in cross stitch or tent stitch with silk, after which the background of canvas is filled completely with grounding stitch done with Berlin wool or crewels. Following we give a list of the principal stitches employed, also a few of the combinations.

A very little practice will suffice to render the learner familiar with the stitches and combinations shown here. The materials generaily used for forming the stitches are Beriin wool, German wool, creweils, zephyr, purse, knitting, etching and rope silks, also linen threads and working cottons. The wools are used for filling in backgrounds, the silks, smaller size linen threads and working cottons are used to work the designs. Linen and cotton threads are only suitable for work. ing patterns on meshed fabrics. 
The stitches used formerly for Berlin work were cross, cushion, satin, tapestry and tent, but these have been added to in late years; and now include German, Irish, plush, leviathan-single, double and treble--and fancy stitches combined from the above.

Back Stitch.-This stitch is made like the ordinary back stitch taken in plain sewing.

Cross Stitch.-This is the principal stitch used in Berlin work. It is used not only for working with wool on canvas, but for embroidering with any material that will thread, upon cloth, silk, satin and velvet. It was much used in the Egyptian embroideries. This stitch can be worked in a

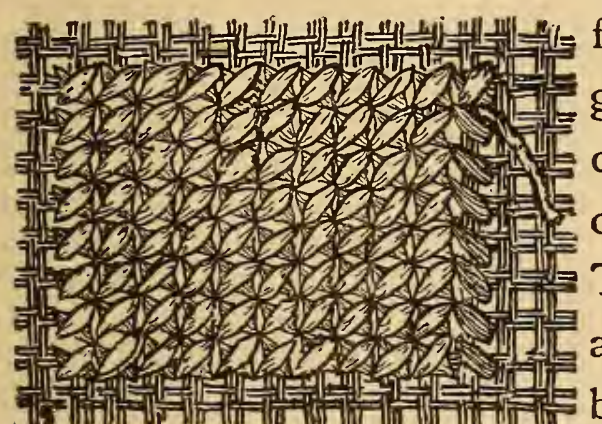
frame or upon the hand. That done in a frame generally looks the best. Cross stitch is a double stitch taken over two threads, the object being always to form a perfect square. The thread is taken in a slanting direction across this square from left to right, the needle CROSS STITCH. FIG. 146 . put in at the upper right-hand corner, brought up at the lower right-hand corner, and crossed back to the upper left-hanc: corner. When grounding in cross stitch, work the first part of the stitch in rows along the canvas, and cross it when returning. When using the stitch for a pattern, finish each stitch at once and commence from the bottom on the left-hand side.

Long Cross Stitch.-This is a variation of cross stitch, the two stitches. forming it not making a perfect square but a long stitch crossed. This is managed by taking the silk or wool over a greater number of threads in height than in width-four threads in height to two in width being the correct proportion, as shown in figure 147. This stitch is suitable for geometrical patterns, and can be varied, as shown in figure $\mathrm{I} 54$ by silk cross stitches taken over the two long cross ones; this variety of the stitch is particularly suitable for filling large

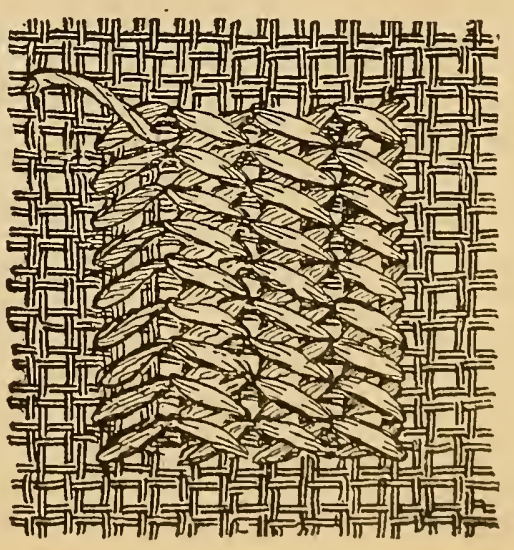

FI r. 147 . spaces in grounding, as it covers the surface rapidly and effectively. 
Persian Cross Stitch.-The first half of the stitch is a long stitch taken over six horizontal threads in a slanting direction and two in height; the second is the last half of cross stitch, over the two center threads of the long stitch, from right to left. See figure 148 .

Cushion Stitch.-One of the ancient names given to cro:s stitch.

Damask Stitch is a variety of long stitch. It is taken over four horizontal threads of canvas, or two stitches, in a slanting direction and over two upright threads. The variety is that all the remaining secend lines of damask stitch are taken over the two

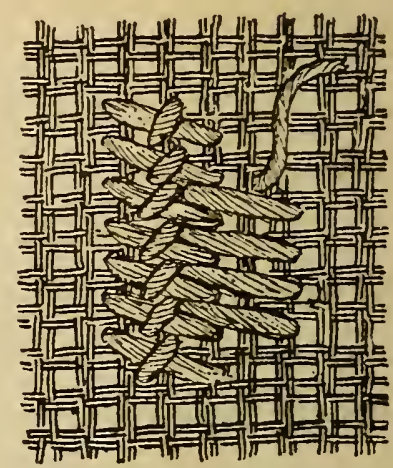

PERSIAN CROSS STITCH. FIG. I 48 . lower threads of the upper line, and two new threads, instead of all the threads being new:

German Stitch.-This is a stitch formed from a tapestry and tent stitch, being worked alternately in a diagonal line across the canvas. The tapestry passes over four threads, the tent over two-see figure I49. In the

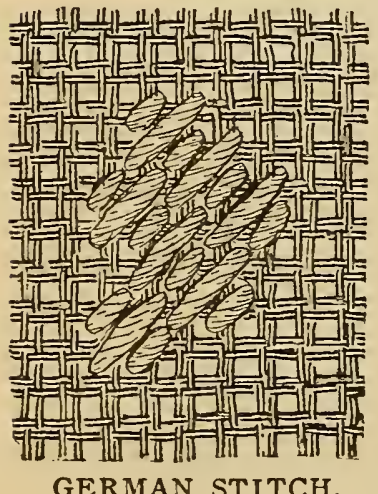

GERMAN STITCH.

FIG. I49.

succeeding line the tent is placed under the tapestry and the tapestry under the tent, but so that the canvas shows. This stitch is suitable for borders where the foundation can be left exposed, but is rarely used for patterns. See figure I 49 .

\section{Tapestry Stitch} is used in embroidery as well as in Berlin work. As will be seen from

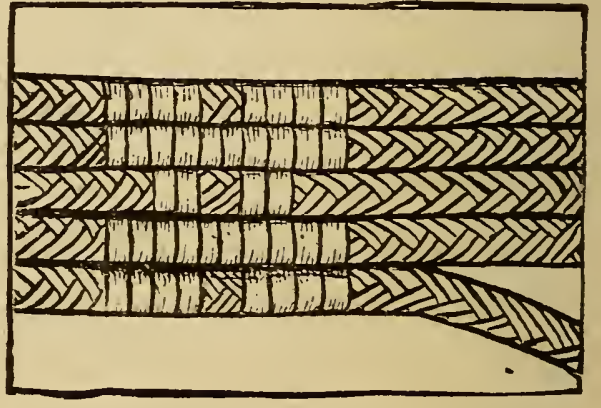

TAPESTRY STITCH. P:G. I 50. figure I5O, the stitch is raised from the canvas by means of a padding of braid. This padding is a great improvement to this stitch, as otherwise it lies quite flat, not being crossed. It is worked over two horizontal threads, and into every space left by the upright ones.

Irish Stitch is used for grounding or for patterns formed with shades of color in vandyke crossing. Irish stitch is a long stitch taken over five or more threads of canvas in an upright direction, and should be worked on fine canvas. Its only peculiarity consists in being alternately started from 
the last row of canvas and from the third. This allows the stitch to end in one line where the center of the next line comes, and gives a pleasing variety to ordinary groundings.

Leviathan Stitch.-A modern Berlin stitch, sometimes called "railway," because it is considered to cover the canvas quickly. It requires to be worked on large sized or leviathan canvas. To work: Four squares must be taken for one stitch, and a cross stitch made into the four corners of the square. The wool is then carried across the center of the stitch from top to bottom, and then from left to right, so that it passes throush all the outside holes of the square forming the stitch. It is worked all together, and each stitch is made as to crossings exactly the same, or an even appearance to the whole will not be given.

Varieties of leviathan are formed by working over six or cight threals in height and as many in width. These require a doubie crossing at top and side for the sixth thread, and a double crossing and a straight stitch top and side for the eighth. These varieties are called double leviathan and treble leviathan. See figure I5I.

Double Leviathan Stitch.-Work over eight square threads, or four square stitches. Make a cross stitch into the four corners of the square, then a long cross stitch to fill in the holes on each side of the cross stitch, and lastly an upright cross into the middle stitches in length and width of square. Fill in all the square in the same order or the uniformity of the pattern will be destroyed, and put a single long stitch between each square, to fill up the part of the canvas that is left bare. See figure I5I. When commencing a new line of stitches on the canvas make a half stitch to begin, so that the center of the second

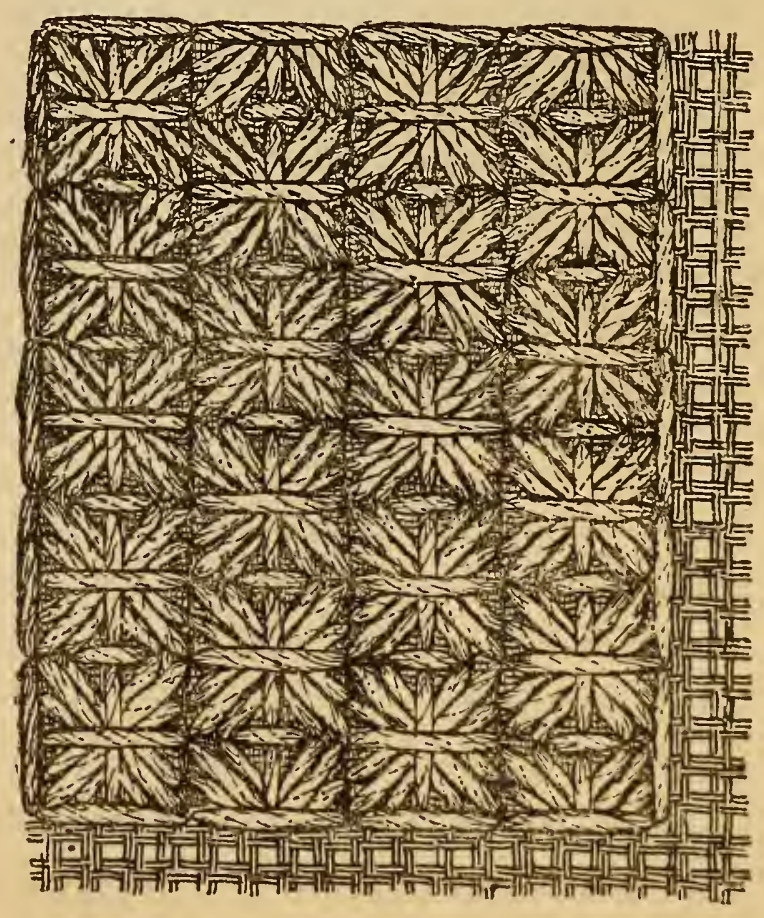

DOUBLE LEVIATHAN STITCH. FIG. I5I. line of stitches does not come under the center of the first line. Com. 
mence with a half stitch at each alternate row. This stitch should be worked upon leviathan canvas, and it is not suitable for groundings, and should only be used for geometrical designs.

Treble Leviathan Stitch.- To work this stitch a square of eight threads of four stitches is required. The work starts from the center and is taken to one of the corners, passing over four upright threads and four horizontal ones in a slanting direction. The next two stitches are placed one on each side of the first, crossing over four horizontal and two upright threads, and vice versa, all finishing in center hole. The four corners are thus worked, and the stitch is completed by taking a cross stitch over the center hole and one in the center of each side of the square. When repeating this stitch the outside crosses are worked only in every alternate square of eight, as there is not room to make one at every stitch. The crosses should be worked with a contrasting shade.

Long Stitch.-See satin stitch.

Tent Stitch.--To work this stitch requires finer canvas than that used for cross stitch, the silk or wool being laid on the canvas once instead

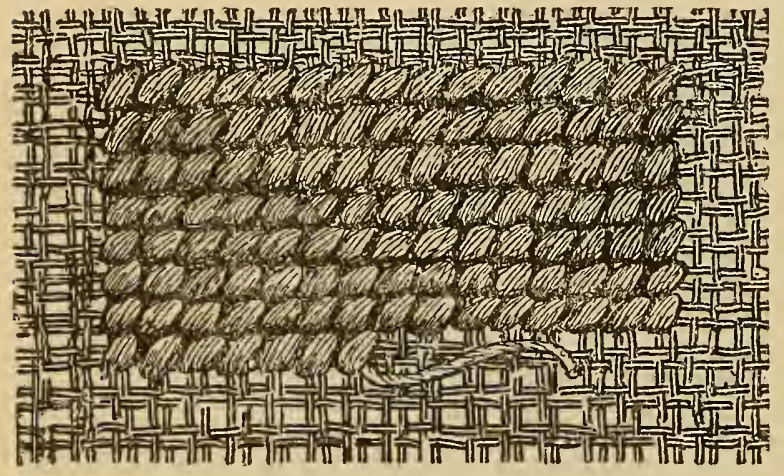
of twice., Tent stitch is shown in figure 152 and is simply the first half of cross stitch, the silk or wool passing over one or two threads of canvas in a diagonal direction.

Raised Stitch.-This stitch is used for raised wool work, and can be worked to any height by using TENT STITCH. FIG. I52. various sized meshes, and then cut and combed until the wool attains the softness of velvet pile. To make: Use a No. 4 knitting needle. The first stitch is a tent stitch. Bring the needle up where the stitch began, push the knitting needle over the tent stitch and make a gobelin stitch over it, putting the needle in two threads above the place where it came out. It is now at the bottom of the next stitch to be made, which is a tent stitch. Repeat the process described above.

The work is commenced from the bottom, and the knitting needle left in lowest row until the row above it is completed; this prevents any dragging of the wool. Cut and comb out all the loops when the work is completed. 
Satin Stitch in squares.--The squares are made over six threads in length and breadth and filled by unequal length satin stitch. The direction of the stitches are altered in (ach alternate square.

As will be seen, satin stitch is susceptible of many variations. Working in alternate colors will give a beautiful effect of shading, and is most effective where the design is geometric in character, and is worked out with black silk. See figure 153 .

Figure I54 shows a combination of long cross stitch and cross stitch. This design is well adapted for filling backgrounds; for working, see detail of

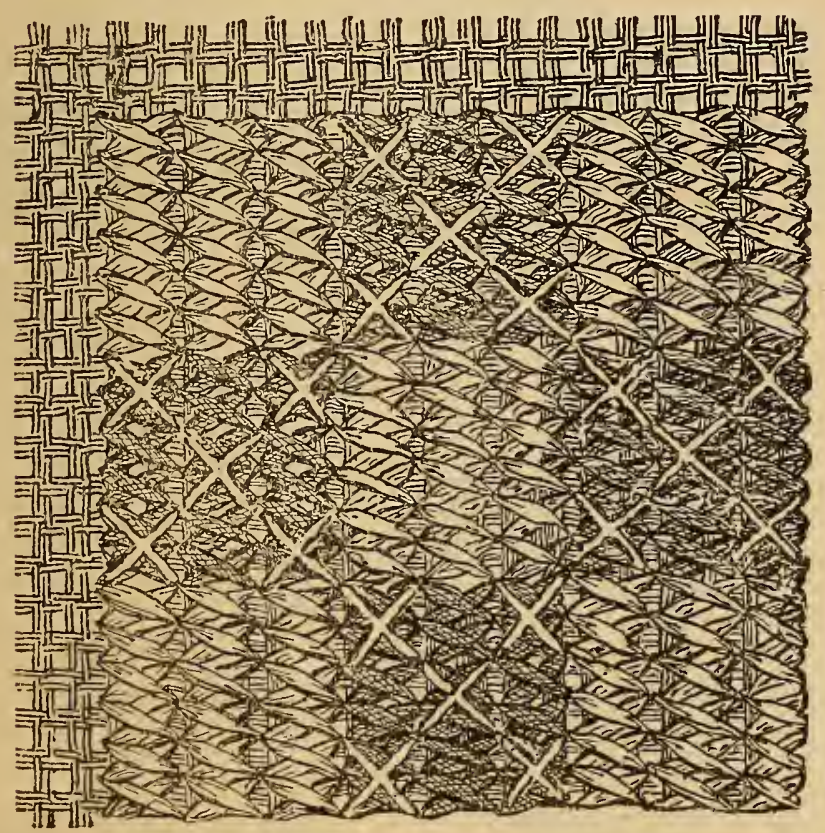

CROSS AND LONG CROSS STITCHES. FIG. I54.

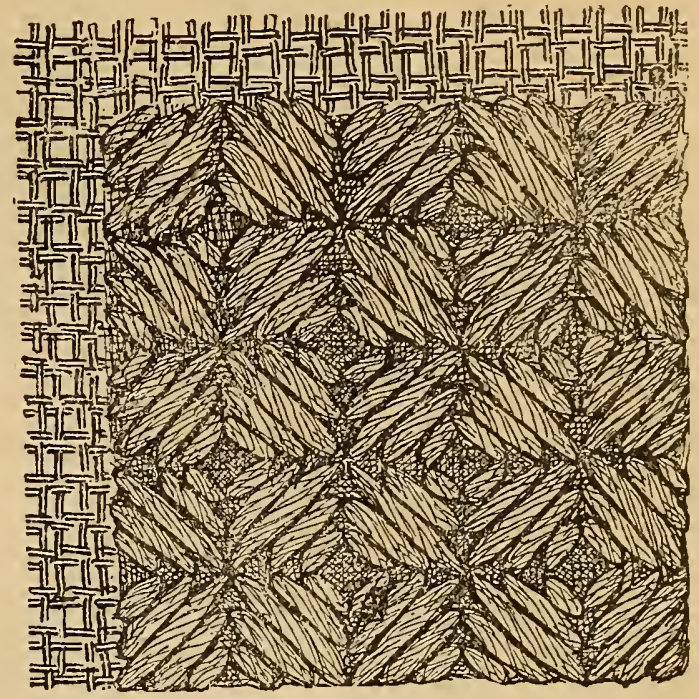

SATIN STITCH IN SQUARES. FIG. I53. long cross. After the long cross stitches have been made with the Berlin wool or crewels-using for the purpose two contrasting shades of a dark color-work over them in sections, forming squares, cross stitches of bright colored filo floss, three strands.

In the preceding pages of this chapter we have tried to give those stitches and combinations that are more commonly employed in Berlin work, either on canvas or meshed fabrics. Limited space forbids the giving of detailed working designs and we have therefore, given only sufficient to illustrate the different stitches and their applications. There is much scope in this direction for the exercising of personal ingenuity, as the different combinations that can be made with the stitches here illustrated are almost unlimited. 

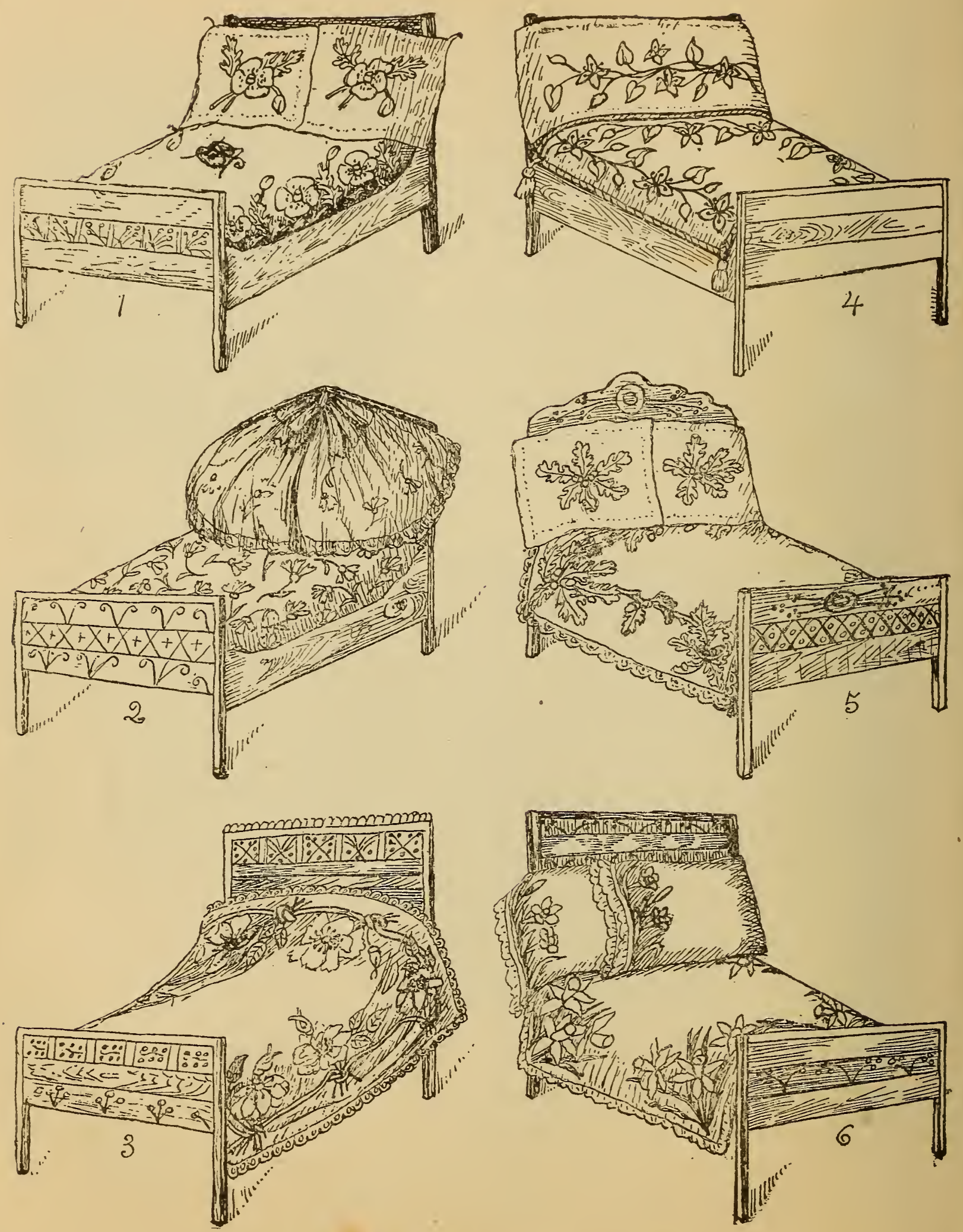

BEDSPREADS AND PILLOW SHAMS. 


\section{CHAPTER XI.}

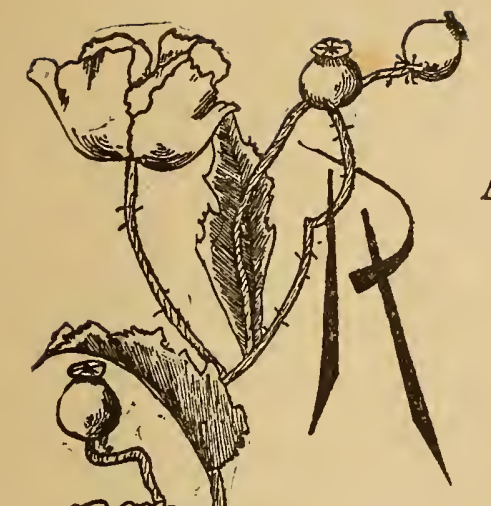

RAISED EMBROIDERY.

AISED EMBROIDERY is a subject that will interest all lovers of decorative needlework, and we have purposely delayed its discusssion until the higher forms of art needlework had been thoroughly mastered. It is a style of decoration that should be indulged in very sparingly, as too much of it in a room will cheapen rather than enhance the effect of the entire furnishing, while if prudently and sparingly used it will give a pleasing variety to the other decorative work.

Raised embroidery was not designed for its utility, evidently, as to use it means to ruin it. Therefore do not employ it to decorate cushion covers, tidies, or, in fact, any article where the embroidery will be subject to the least wear, but reserve it for the embellishment of mirror frames, panels for the walls, over-mantel curtains, easel pieces, table bannerscreens, and for the outer covering of handkerchief and glove sachets. This style of embroidery must never be skimped as to materia! nor slighted in the workmanship if satisfactory results are desired.

There are several flowers and fruits that are especially adapted for raised embroidery, and we propose in the present chapter to select those designs that are most effective when finished, and that can be used for the greatest variety of purposes, as in the pages of a work of this kind it will be quite impossible to give a specific lesson on all the fruits and flowers that can be used for this style of work.

Ribbon work, sometimes called "roccoco," is one of the oldest forms of raised embroidery, and was much used in the last century; it has had. from time to time, a revival of popularity in this. At first the ribbon was 


\section{I50 DAINTY WORK FOR PLEASURE AND PROFIT.}



RIBBON WORK-SEE PAGE 151. 
worked on the material without any effort at raising it; a narrow ribbon threaded in a long-eyed needle being used for this purpose. From this to single and double roses was but a step, after which followed raised cat-tails, golden rod, coxcomb, sumac, snowball, fruits and varieties of the lily family. We will discuss first the various forms of

\section{RIBBON WORK.}

The earlier ribbon designs were worked out with a narrow China ribbon about one-half inch in width. This ribbon was dyed with beautiful shades in art tones, as old blue, dead rose, sage green, etc. Some of the ribbons were shaded from light on one edge to dark on the other, and thus formed effective shadings.

The designs for working the ribbon were of the smaller flowers or of the larger ones drawn in miniature and arranged as Kensington designs are now made. Also Louis XVI designs-arrangement of flowers in various devices-were designed on satin bed coverings and door hangings in detached groups or in continuous garlands around the borders, with clusters powdered over the center.

To work: For the leaves, thread a long-eyed needle with a green ribbon, bring it up from the back of the material, at the point of the leaf, and put it through to the back again at the base of the leaf; this, in case of long-pointed leaves, and also for the petals of star-shaped flowers. Where the leaf is broad, the ribbon is worked in satin stitch, taking the stitch from one edge to the center of leaf at an angle; the reverse side being worked in the same manner, forms a vein in the center. For double flowers in miniature, as asters, roses, etc., shaded ribbon was used. This was gathered fuli on the darker edge, which was sewed on the design, beginning in the center and sewing around toward the outer edge, until the design was filled in. For very small single flowers the ribbon was threaded in the needle, as for the leaves, and a single stitch taken from top to bottom of petal. The centers of flowers were put in with filo floss in French knot. Page I 50 shows a design for this style of embroidery full working size. It is suitable for toilet cushion covers, or it may be repeated for borders, or clusters may be detached and used a powderings.

This form of ribbon work is indulged in by the wealthy only, as the ribbon is a foreign manufacture, and not imported into this country, except 
by the decorative art societies, which make a specialty of keeping ribbons and designs for this work. A cushion with Louis XVI design worked in the manner here described is in the possession of the Chicago Society of Decorative Art and valued at $\$ 50$. On the other hand, if one has friends in Paris, the ribbon can be there purchased for a few cents per yard, and good designs can be traced from prints of decorations of the Louis XVI period. The ribbon work above described is the highest form of raised work indulged in even at the present day. As the designs for it are of a light and graceful character and the tints of the ribbon exceedingly delicate, it follows that the background on which it is to be applied should also be of a light or medium tone of color, and satin or silk offers by far the best surface.

\section{SINGLE RIBBON ROSES.}

Branching sprays of large single roses and buds are suitable for ribbon work, and may be applied to screens or mantel hangings with good effect. The amount of material necessary for working out a design will, of course, depend upon the number of roses, buds and leaves in the arrangement. For the purposes of this lesson we will suppose a branch of roses consisting of three full-blown and three half-blown roses, four buds and thirty-three leaves. This design will require three and one-half yards No. 16 satin ribbon, divided as follows: One yard deep rose pink; one-half yard rose pink one shade lighter than that first selected; and one-half yard two shades lighter still. One yard deep rose red ribbon; one-half yard a shade lighter; and onehalf yard two shades lighter than the shade first selected. The shades of color must be in exact lines of gradation, from light to dark. This combination of blush roses and deep red roses on a dark blue velvet background is exceedingly pleasing. Beside the ribbon and the material for the background there will be required a long-eyed and a common needle; three skeins olive green filo floss; three dozen skeins No. 2 imported chenille, and a square frame.

As regards materials for ribbon roses, opinion differs, some teachers preferring satin in the piece, while others use nothing but the satin ribbon. We have always employed the latter for three very important reasons: First, closer shading can be obtained; second, the ribbon has more body and can be manipulated with greater ease; and, lastly, there are no raw 
edges to be cared for. No two persons ever secure the same results with the same materials, no matter how closely a rule may be followed; more or less of a worker's individuality is certain to creep into the work, and rib. bon seems to offer the best medium for the expression of this individuality. Stamp the design upon the material, and after the paint has thoroughly dried, stretch it firmly in the frame. Do not pin the fabric to the frame but sew it with strong thread.

In all raised work the leaves or foliage should be worked first, and the flowers added last, otherwise the raised portion would be in the way and would become crushed and soiled. At least twelve shades of chenille will be required for effective shading, as follows: Five shades of olive green, from light to dark; five shades of wood brown, shading from ecru to a deep, rich brown, and two shades of red brown.

On ribbon work the foliage should be striking and effective, rich and varied. One can hardly go astray on shading rose leaves vividly, as nature has set us an example of the utmost latitude. Each leaf should contain at least three different shades. These need not necessarily be three shades of one color in direct line of ascent or descent, as, for instance the first, second and third shades of the olive greens or the wood browns; in fact, this form of shading would give unbroken and monotonous effects if carried out through all the design - a result to be avoided, although an occasional leaf worked after this rule helps to lend variety to the whole. All that is arbitrary in this form of shading is that the outside of each leaf shall be worked with the lightest shade used, or if shaded from the tip down, the base must be darkest, the tip lightest; or, again, if opposite sides of a leaf are to be differently shaded, the side which the light strikes first must be worked with the lightest shades, while the under side should be dark.

In using chenille, follow the outline of the leaf closely, and do not make the stitch of regular lengths; preserve such a slant as will not crowd the stitches, and do not leave too many open spaces to be filled in with the succeeding shades.

After the foliage has been worked, protect it from the dust by sewing: over it fine tissue paper, leaving exposed only that portion of material where the roses and buds are to be placed. 
154 DAINTY WORK FOR PLEASURE AND PROFIT.

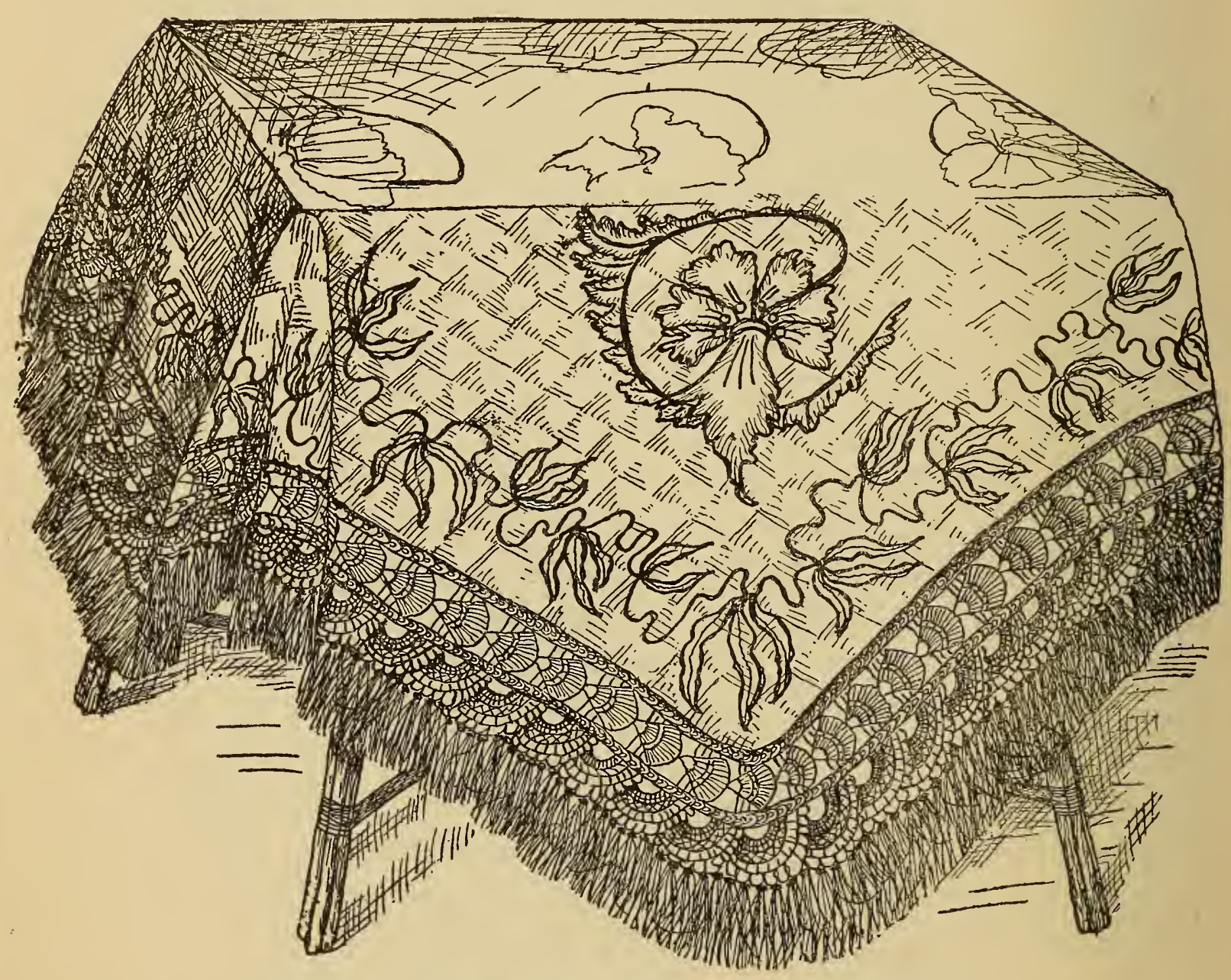

LIBRARY TABLE COVER WITH CROCHETED FRINGE. 
To make the roses: Cut two pieces of the lightest shade of u u pink ribbon two inches in length; two pieces of the shade next deeper, and one piece of the deepest shade, all two inches long. These are to form the five petals of the rose. Thread a common sewing needle with strong thread, and sew one end of each piece of ribbon in three small plaits and fasten firmly. With the same kind of thread gather the other end of the pieces of ribbon, draw together tightly and fasten.

Begin with the lightest petals first. Fasten the gathered end of a petal to the outer edge of the highest petal of a rose in the design. Sew it firmly to the material on the wrong side of the ribbon, then turn the petal over (like the flounce of a dress when sewed on the wrong side and allowed to fall over, showing no fastening stitches), and bring the plaited end to the center of rose and fasten on the right side. The mechanical part of the petal is now finished, and it rests with the worker how much individuality is put into the flower when completed. Lay one or two tiny plaits on each side of the outer edge of petal and fasten; this will give a rounded fullness to the ribbon, which fullness can be crushed into close resemblance to a crumpled rose leaf by a few judicious and gentle touches; allow the ribbon to dispose itself gracefully, and catch it here and there with a stitch to hold in place; turn the edges under slightly, and carry the thread on wrong side to the center of rose, where a few little gathered stitches on each side of the petal, close to center, will be an improvement. For the second petal proceed as for the first, catching the two petals together at the top with a blind stitch, being careful to avoid a strained or forced appearance. The two upper petals should be of the lightest shades, the three under petals of the two deepest shades. When the petals are finished fill in the center with. French knots, using three strands of green filo floss. Thread the sewing needle with a single strand of pale yellow filo floss; begin at center of petal and carry three long light stitches upon it, the longest stitch in the center and a shorter one on each side. Repeat these stitches upon each petal. This completes the rose.

The buds are formed in the same manner as the rose petals. For very large buds, a piece of ribbon one and a half or two inches long will be required, while for small buds three-quarters of an inch will be sufficient. Plait one end and gather the other, as described for the rose petals. Fasten 
the gathered end to the top of bud, fold over and fasten the plaited end to the center of the bud. This method leaves a graceful fullness, which can be manipulated easily by picking and punching into position with the needle, and caught here and there with an invisible stitch to hold in place. Carry the needle to the right of bud at the base, and take three small running stitches in the edge of the ribbon, always with the needle point towards the top of the bud. After the running stitches are in, draw the needle back to base of bud and fasten. Repeat the same operations on the left side; this will bring the ribbon into minute folds. With the lightest shade of olive green chenille work the calyx of bud, carrying a few long stitches well upon the bud itself; do not pull them, but allow each stitch to lie lightly in place. Follow the markings of pattern on each side and at the top of bud with single stitches of chenille.

The half-blown roses are manipulated in the same manner, except that three pieces of the deepest shades of ribbon, two inches long, will be required. Place these in position so as to cover the design. Little pulling or pricking into position with the needle will be necessary, and where the rose joins the stem it should be heavily worked with the second shade of olive green chenille. Follow markings of pattern, and do not be afraid to carry a few graceful, light stitches well upon the rose itself, starting from the base.

\section{DOUBLE RIBBON ROSES.}

Double ribbon roses are suitable for decorating many articles, and they can be easily made if the directions here given are carefully followed. Each rose will require three shades of ribbon, divided into twenty pieces, as follows: Five pieces, two and one-half inches long, of the deepest shade used; five pieces of ribbon of the same shade as first given, three inches long; five pieces of the next lightest shade, four inches long, and five pieces, the same length, of the lightest shade.

Gather one end of each piece of ribbon and plait the other end, as described for single roses. Begin at the outer edge of the stamped rose and sew down on each stamped petal a ribbon petal, using for this purpose the five four-inch pieces of the lightest shade of ribbon. Do not fold the ends of the ribbon petals together to fasten, but sew down the plaited end first, 
as described for single roses, then turn the petal over and fasten down the gathered end, a hair's breadth beyond where the first end was sewed down. After the five outer petals have been fastened into position, take a few running stitches on each side of each petal, at the base, and draw down toward the bottom of the petal and toward the center and fasten. Put in the next row of petals in the same manner, sewing down the plaited end so as to cover the gathered end of the preceding petal; use for this row the fourinch pieces that have been cut from the next deeper shade of ribbon. Inside this row fasten down, in the same manner, a row of petals of the deepest shade. Use the three-inch pieces for this row. Inside this, again, put in the petals that were cut two and one-half inches long, and which were also cut from the deepest shade of ribbon. This completes the rose to the center. Fill the center as follows: Cut a square piece of the ribbon, fold it diagonally across the center, making a half square; gather the two sides of the square into a point and fasten, giving the ribbon a little roll inward as the fastening is completed. Place this exactly in the center of the rose, and secure it firmly.

The different petals can be pinched into shape and fastened here and there with invisible stitches. The beauty of this work depends entirely upon the manipulation of the petals after they have been fastened into position. The more crumpled they are the better will be the effect.

\section{CALLA LILY.}

For banners or easel pieces there is no flower more effective than the calla lily, and better than almost any other flower does it lend itself to raised, effects. The background should be of plush or velvet, and the color should be rich and warm. A deep blue, crimson or green will throw the raised callas into relief.

In putting the design upon the material, stamp on only the leaves and stems; do not stamp the flowers. The material required for making one calla will be as follows: One square foot of screen wire-the finest mesh madethis can be procured at any hardware store; four dozen skeins cream white arrasene; one calla center, one skein light olive green chenille, one arrasene needle, one piece wash blond. The wire will be sufficient for two callas and one bud, but less cannot be purchased. 
ISS DAINTY WORK FOR PLEASIYRE AND PROFIT.

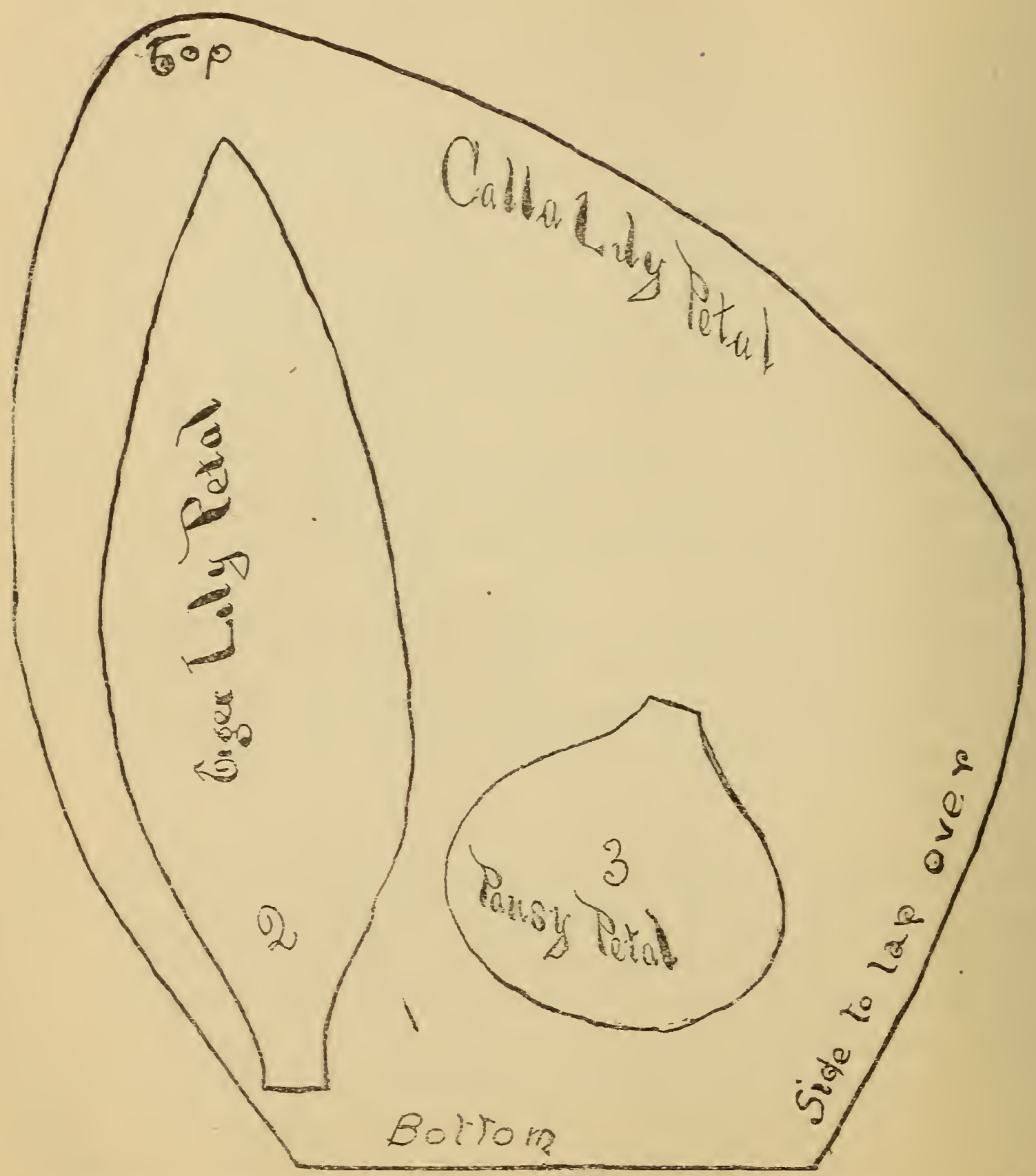

WOFHATG FATTERN TOB CRLLA, PANEY AHD TIGER LILY-SEE PAGE 137. 
Cut a piece of the wire into shape by the calla pattern on page 158 , cut with a pair of sharp shears, so as to get a good, clear edge. Cover this wire calla on both sides with the wash blond and secure with a few stitches. You are now ready to work the calla.

Thread the arrasene needle with a strand of the white arrasene. Begin at the top point of the flower and work over the edge, down one side; return to top of flower again and work down the opposite side, taking the stitch in to a depth of at least one or two inches. This operation will give a smooth, even edge to the flower, alike on both sides, and is worked as follows: Bring the needle up from the top of the wire about two inches from the top edge; throw the thread over the edge and bring the needle up again from the back. Repeat until the edge of flower has been worked. After this part of the work has been completed, begin to cover the wire on one side by filling in toward the center with several rows of short and long stitches, being careful to work both halves of the wire shape so evenly that there shall be no perceptible joining in the center, but the entire calla should present a smooth even surface. This completed, turn the wire shape over and work in the same manner on the reverse side.

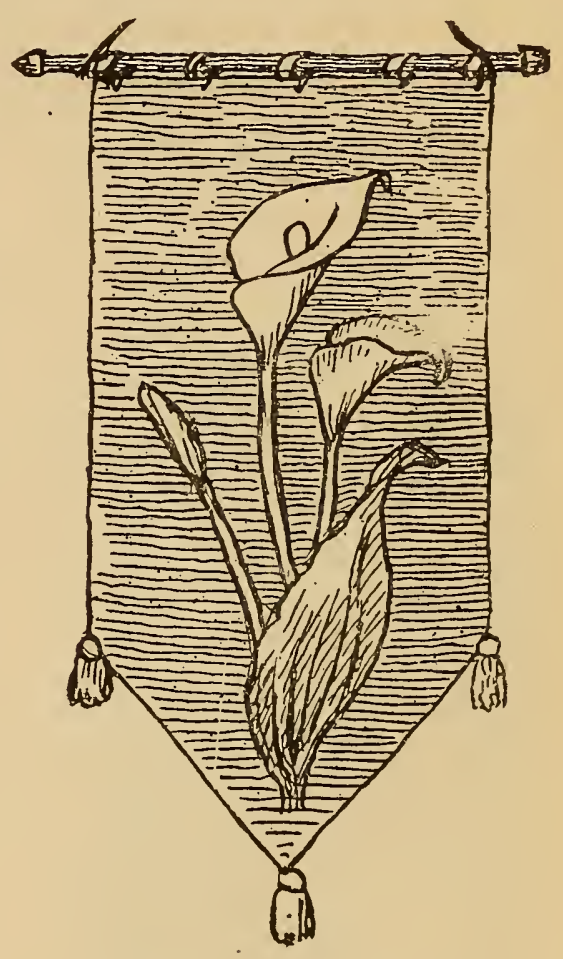

CALLA LILY. FIG. I55. In working, twist the arrasene in the needle. When the wire has been smoothly and solidly covered with the arrasene stitches on both sides, take the calla between the thumb and finger of one hand, near the top of the flower, and with the other fold it into shape, after the fashion of twisting a cornucopia; the wire is stiff and will retain the shape without trouble. Place the calla in position on the background and secure with a single stitch at the top and one at the base of the flower. This will be sufficient to hold it in place. Slip the calla center into position and catch with a stitch. With the green chenille, work the calyx of calla, carrying the stitches well up on the outside of the flower, working quite heavy at the base. This throws 
the flower into relief and gives it a rich effect. The stems of the calla should be worked with solid Kensington stitch.

Sometimes the calla is made of fine chenille instead of the arrasene, but it is never so satisfactory. Figure 155 shows a raised calla applied to a moss green plush banner, and will give an idea of grouping. These raised callas are very beautiful for Easter decorations.

When the callas are soiled they can be removed and cleaned by shaking in a pan of flour that has been previously well dried.

\section{TIGER AND JAPAN LILIES.}

The above varieties of lilies lend themselves to raised effects adnirably, and are easily made. First, stamp the design on the material to be embroidered - that is the leaves and foliage-be careful, however, not to

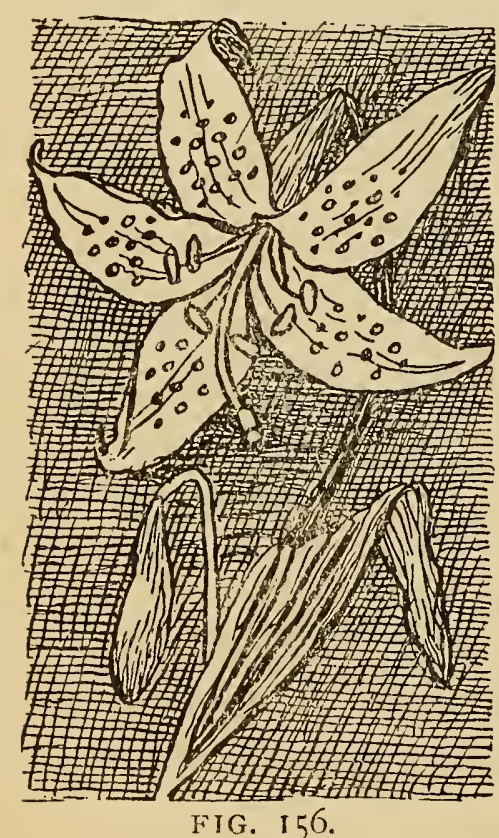
stamp the flower itself, as its position will be sufficiently indicated by the stems, and if the flower were stamped it would be difficult to cover the marks.

Figure 156 shows a correct shape by which to cut the wire petals for a tiger lily. It should be drawn from the figure and cut out of stiff cardboard so as to secure a pattern by which to cut the wire. For a Japan lily this pattern should be cut three times as large.

Materials required for a single Japan or tiger lily are as follows: Screen wire, finest mesh, blond net, arrasene and chenille. Both varieties of lilies are worked in the same manner, so that the instructions for one will answer for the other.

Three shades of orange yellow arrasene will be required for working the petals of the tiger lily, and one shade of pinkish white arrasene for the petals of the Japan lily. The brown spots marking the petals of both varieties should be imitated with reddish brown chenille, No. I-the finest made-with seed stitch.

The centers of the flowers can be purchased at any art store, though they may be very successfully made at home by grouping together pieces of fine hair wire for the stamens. Cut the wires about three inches long 
and bind them firmly togéther at the bottom; spread the wires apart at the top and fasten on the point of each a tiny bit of cotton wool; dip the whole in melted rosin, and then, while still wet, dip the very points covered with the wool in dry mustard, or sprinkle with gold powder.

For a tiger lily cut six petals out of the wire, by the pattern previously prepared, and cover them with the blond net. Work as follows: Begin at the tip of a wire petal with the lightest shade of the orange yellow, and work it downward in Kensington stitch to the base, using the three shades of orange yellow-the darkest at the base of petal. Work both sides of the wire petals alike. When finished, group them around the prepared center and secure with a few invisible stitches. After the stems and foliage have been worked with arrasene or chenille, punch a small hole at the point where the flower is to be placed and insert the stem of the lily, fastening it on the back of the material, and also catch a stitch or two lightly through the petals that lie next the material; curl the points of the petals outward, and work a few stitches on the lower part of flower with olive green chenille, to form the calyx. The Japan lily is made and placed in position in exactly the same manner.

Groups of either Japan or tiger lilies made in the above manner are suitable ornaments for the top of toilet cushions, banners or placques. To make one lily of either variety will require three skeins of arrasene, one skein of olive green chenille for the calyx, one thread brown chenille for the markings of petals, one yard hair wire and one-half foot of screen wire. Multiply the above quantity by the number of flowers on a pattern and it will give the necessary amount of material for that pattern. The background should be satin or velvet, either cream, blue or deep moss green.

\section{WATER LILY.}

Cut the shape of lily petal in fine screen wire, using the pattern of water lily on page 158 to get the shape of petal from. Cover the wire petal with white blond or rice net, smoothly, and beginning at the top of petal work downward in Kensington stitch with white arrasene; when one side of petal is worked, turn and work the reverse side in the same manner. For large lilies twenty-four petals will be necessary, using twelve for extreme outside row and eight for inside row, and the remaining four to 
surround the center, which can be purchased at any art store. The outside petals should have a few stitches of green arrasene near the base of each. The last or inner row of four petals should be much smaller than the outside row. In stamping the design do not stamp the lilies on the goods-just the leaves and stems-as in arranging the petals you would not be able to cover the marks made by stamping. Raised water lilies look best mounted either on white velvet or dark green satin. The leaves and stems should be worked with lily green arrasene or chenille. This flower, thus treated, is suitable for placques, banners, or for corners of plushcovered mirror frames. To arrange the petals on the foundation, commence on the outer edge of the space the flower is to occupy, and sew twelve of the large petals in a circle, fastening the petals to the fabric by taking a securing stitch in the very edge of base of petal. Curve the petal outward at the base and inward at the top, giving a cup-shape appearance to all the petals. Inside of this outer row of petals arrange eight petals, the same size, so that the center of each inner petal shall come exactly opposite the center of two outer petals, and last sew in the four small petals, arranging them so that each petal shall come opposite the joining of two petals in the preceding row; curve each row of petals as directed. After the petals are sewed in position, punch a hole with the stilletto in the exact center of the flower and insert the stem of the lily center and fasten it on the back.

\section{GOLDEN ROD.}

This most beautiful of all our autumn flowers is essentially national in its character, as it grows from east to west and from north to south; in good soil and in barren and waste places. It is loved by the rich and poor alike, and is a golden glory always.

For purposes of embroidery, where bright rich color is desired, there is no other flower that can quite fill its place. It allows of graceful arrangements of form, and is susceptible of the most subtle gradations of light and shade. It is especially beautiful when combined with other autumn flowers-as sumac, thistles or asters - the rich purples and bright gold forming a piece of gorgeous coloring that will most effectually brighten up any dull corner.

Golden rod may be worked in several ways, either raised or flat, and the materials may be arrasene, chenille, rope silk, filo floss or etching silk. 
To work with arrasene or chenille, commence at top edge of the highest branch of the golden rod and fill the surface of pattern with French knots, using the palest shade at the top and shading to deepest gold on the under branches; when it is desired to raise the flower, rope silk or filo floss must be used. First fill the surface of the pattern with large French knots of olive green rope silk; then, between each knot sew down thick strands of the silk, cut in lengths and crossed like the letter X; catch in the center of the $\mathrm{X}$ with a fastening stitch, pulling it down firmly, this will cause the ends of the strands of silk to spring up and cover the fastening stitches. Cover the surface of design thickly with the crossed strands of silk, which will be supported by the underfilling of French knots, which will also afford that undershading of green seen in the natural flower. To work a medium-sized branch of golden rod about four shades of filo floss will be required, viz: Nos. I26I, I262, I263 and I264. When it is wished to work this fiower on wash fabrics the work should be done with two or three shades of yellow etching silk in French knots; make the knots smooth and even, winding the thread around the needle twice only; place the knots close together, but do not crowd them. Made as above described, the knots will not be much raised from the surface, and work thus treated will laundry perfectly. The foliage and stems of the golden rod should be worked with silver or lily green silk in Kensington stitch.

\section{CATTAIL.}

This rather stiff and stately inhabitant of river courses and marshes is sometimes used for decorative purposes. It is suitable for frieze or dado for curtains, for banners or easel piece. When used on a curtain the leaves and foliage should be worked with solid Kensington stitch and the cattail with brown rope or et'ching silk in heavy French knots. When desired for banner or similar purpose, the cattail should be worked in plush stitch done with filo floss over a ground of French knots, made of brown zephyr. In fact, whenever plush stitch is used, the design should first be covered with French knots in order to give body and firmness when the work is finished, and as this stitch depends for its beauty upon the firmness and com pactness with which it is worked, this grounding is essential. The work, when the stitch is completed, must be evenly and carefully shaved and combed out. 
The foliage and spike or cattail may also be effectively worked with No. 2 chenille in browns for the cattail, and olive green, three shades, for foliage. Cover the design with French knots or with solid Kensington stitch.

\section{COXCOMB.}

This gorgeously-colored flower is peculiarly adapted for raised effects, but is most effective when combined with some other autumn flower. A favorite combination is coxcomb and thistles, or coxcomb and golden rod and sumac as shown in the illustration, figure 157.

Three shades of red filo floss will be required for working coxcomb, a deep red, a medium red and a bright scarlet. The scarlet is used to work

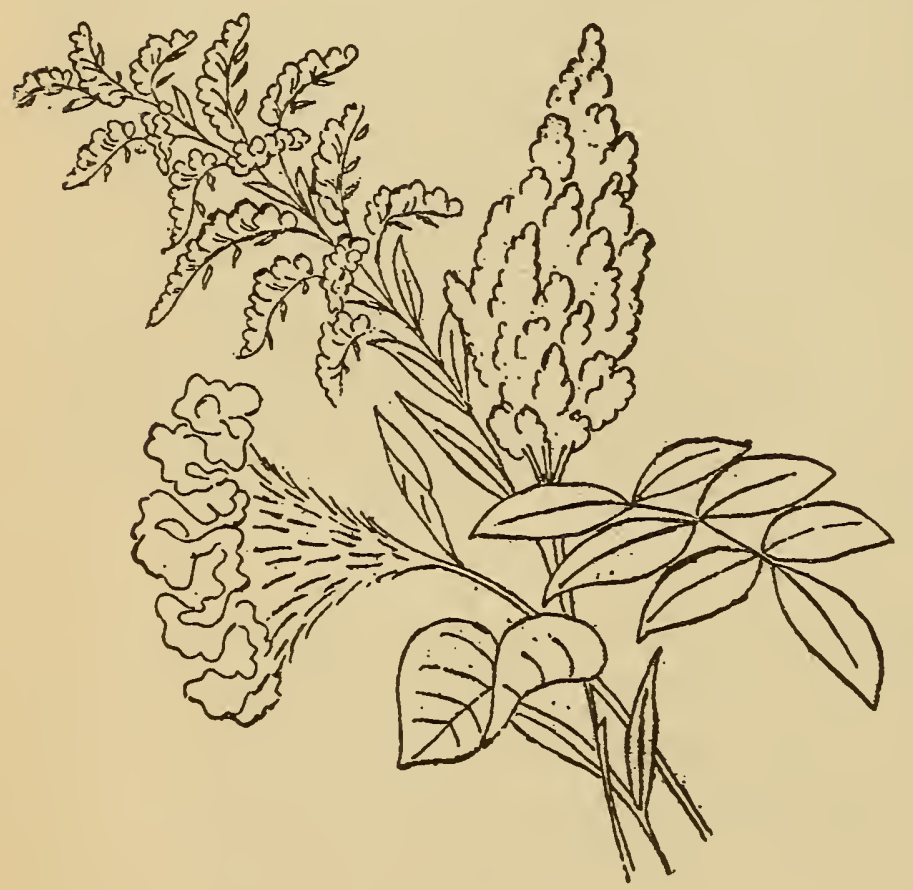

FIG. I 57 . that portion of the flower indicated by the heavy black line shown in figure 157 , at top of flower and following the outline. To work: Begin at the base of the flower where it joins the stem and work upward toward the top of flower with Kensington stitch, working lengthwise. Use deep olive green rope silk for this part of the work; when the base of flower has been solidly worked in Kensington stitch, work a few stitches, up and down, with a shade of red filo floss. This will simulate the markings of red in the natural flower. Above this base begin the flower by covering the design with heavy French knots done with red zephyr. Between the knots sew down heavy strands of filo floss or rope silk, crossed like the letter $\mathrm{X}$; cut each strand about one and one-quarter inches long. For one section of the $\mathrm{X}$ use the deepest shade of silk; for the other section the shade next lighter, and for the dark line shown in figure 157 use the scarlet silk, as before 
described. When the plush stitch is completed it should be clipped anc. shaved evenly with a pair of sharp shears, after which the silk shouic! be brushed with a comb or whisk broom. This will untwist the silk, which will lie fluffy and soft as the real flower itself.

\section{SUMAC.}

The foliage and bobs of the sumac are beautiful worked in autumn shades. The leaves and foliage should of course be worked with filo floss or Royal floss in solid Kensington stitches, using for the purpose dead yellows, deep crimsons, golden browns, orange, wood browns, and the bronze greens and browns; no two leaves need be shaded alike, and the effect of the entire foliage when worked should be a mass of royal coloring. The bobs are usually worked with raised effect in No. 2 chenille, using for the purpose the three deepest shades of yellow reds. To work the bobs: Begin at the top of the bob with the lightest shade, bring the needle to the surface of the material at the top and put it back to the wrong side again, close to where it came up, and pull it through until all the chenille has been drawn down, except a loop about one-half of an inch high; continue the operation to the base, working in the deeper shades, until the darkest has been used. Place the loops close together over the surface of the desionn, so that the bob. presents a mass of color from light to dark. No shaving or clipping will here be necessary. Another method of working this flower is to cover its surface with French knots of chenille, or rope silk, though the loops are by far the most effective. A beautiful combination would be a spray of goldenrod and sumacbobs, with foliage. This combination would make a beautiful banner or easel piece-or coxcomb, sumac, golden rod and thistles gracefully arranged and worked with raised effect, would be still more beautiful.

\section{THISTLES.}

Thistles are easily adapted for raised effects, and in combination with golden rod, or even used alone, they are always pleasing to the eye. The materials for making raised thistles are: one skein green zephyr, two skeins deep olive green etching silk, one skein silver green etching silk and three skeins of heliotrope filo floss. Do not make the mistake of using lavender shades the proper shade being a redelish purple, which is admirably 
imitated in that line of silks known as heliotrope. To work: Begin with the base of the thistle, cut the skein of green zephyr, and winc four or five strands over the thumb and first finger. Place the tip of the thumb on the

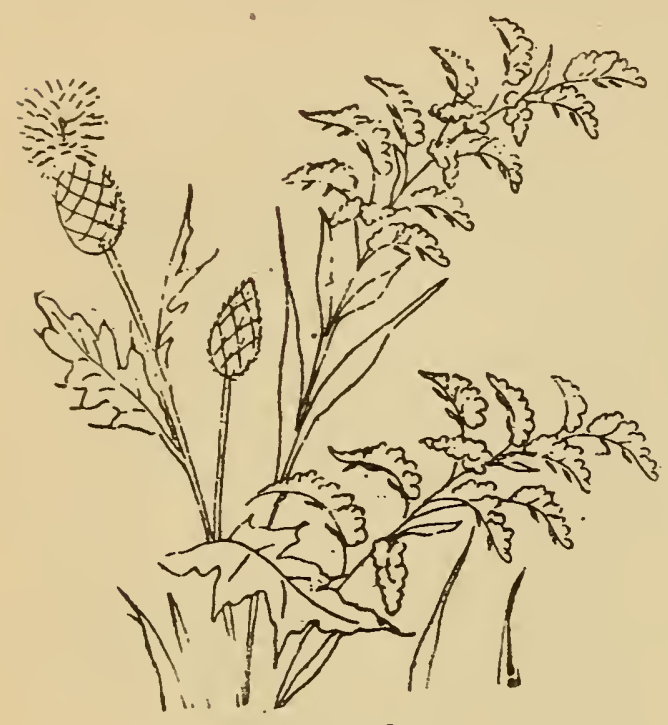

FIG. 158. bottom part of the base of thistle and the forefinger on the top of base, and slip the zephyr off, catching it into position by a stitch at top and bottom. This forms the padding that raises the base of thistle. With the darkest shade of green etching silk, work over this zephyr, taking a satin stitch from top to bottom of base, until the zephyr foundation is entirely covered with the silk; now repeat this silk covering by working crosswise of the base. Begin in the center of the base and work over and over to the top; place the needle in the center of base and work to the bottom of thistle base. After this has been done, so that the base presents the appearance of an oblong ball, of one solid even mass of stitches, mesh it off into small diamond-shaped spaces, with the skein of silver green silk. This completes the base of thistle, and if the work has been well done, a real thistle would find it hard to detect the fraud. To work the top of flower. Fill a semi-circle around

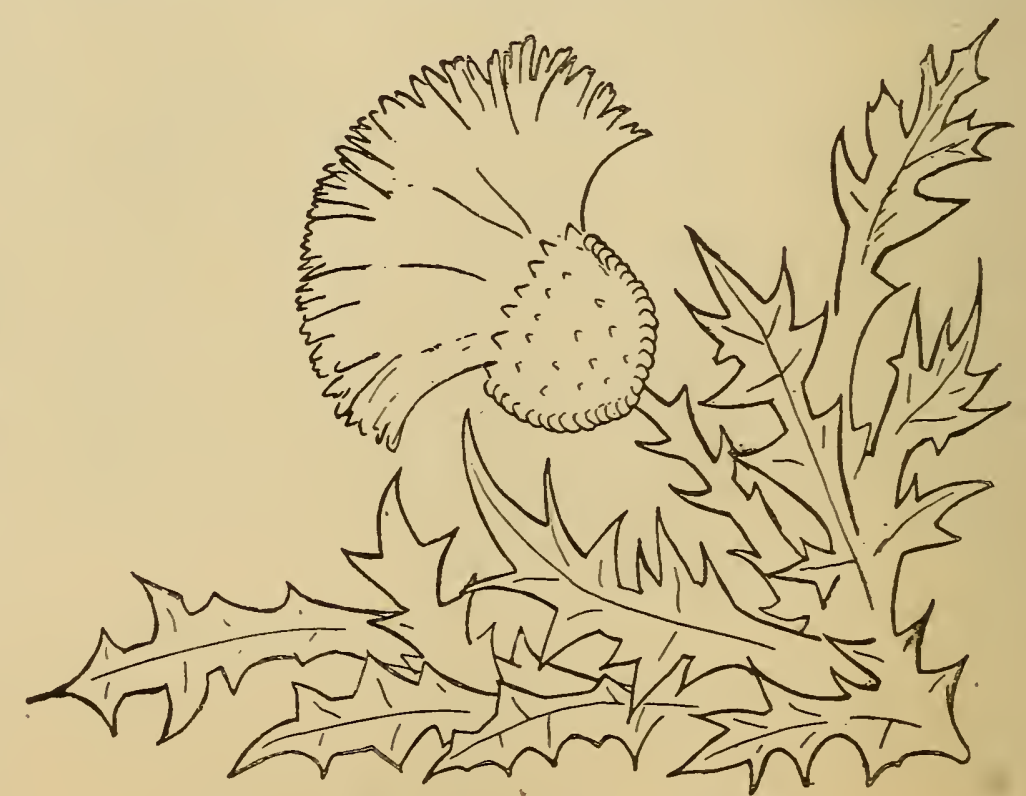

DESIGN FOR FLAT WORK. FIG. I59. the top of base with French knots, made out of green zephyr; fill in between these with heliotrope silks in plush stitch, in the same manner as described for coxcomb, using the very lightest shade at the top of flower. 
Carry out from the edge of the plush stitches a few simple straight stitches with one strand of silk. Shear off the uneven ends of silk and whip or comb out the flowers. This method gives the most natural effect of any employed, for making raised thistles.

\section{RAISED SNOWBALLS.}

Snowballs for ornamenting banners, corners of mirror frames, etc., are very effective and simple of construction. The background should be either plush or velvet of a rich dark color-as blue, red or green. For working the foliage five shades of olive green arrasene, from light to dark, will be needed; work in solid Kensington stitch. In stamping the design transfer only the foliage and stems, as advised for the lilies. To make the snowball will require a small piece of cheese cloth or scrim, six skeins of white arrasene, a skein of palest green arrasene, a knot of pale green zephyr and a piece of cotton

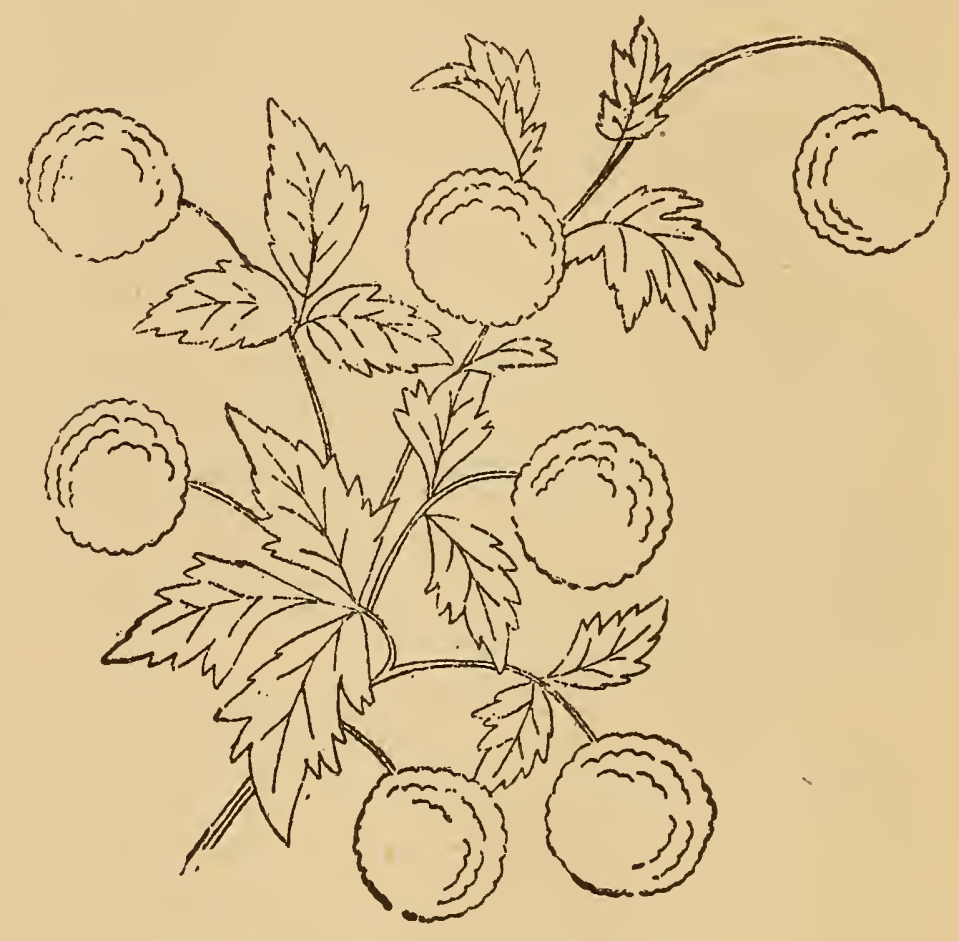
wool. To work: On the scrim or cheese cloth draw FIG. 160 .

a circle, using the top of a small-sized tumbler or water glass for a pattern; stretch the cloth firmly in a pair of small embroidery hoops and with the green zephyr work across the circle until the surface has been completely covered; over this prepared surface the white arrasene is to be put, and the method of doing this part of the work is identical with that described for making sumac, viz.: Commence at one side of circle; bring the needle, threaded with white arrasene, from the back of work to the surface and put down close to where it came up and pull through to the back, leaving a loop from one-half to three-quarters of an inch high on the right side; 
repeat this until the surface is thickly and solidly covered with these white loops; be careful not to arrange the loops so that they shall appear set in any particular direction, but so that they shall present a light, feathery surface. After this part of the work has been done, take the cloth from the frame and cut out the worked circle, leaving a margin of about one-sixteenth of an inch; turn this margin under and run in a gathering thread. Do not pull the gathering thread tight, but just enough to curl the edges inward; place the circle over the spot where the snowball is desired, and blind stitch it down, leaving a space of three inches at the point where it joins the stems. Into this space stuff the cotton wool, with the blunt end of the stilletto, until the snowball looks sufficiently raised, then sew down the space left and over it work in loops of the green arrasene. This will give the green cast observed at the base of snowballs.

The above method of working is very simple, and when the flower becomes soiled it is but the work of a few moments to remove it and clean in a dish of dry flour, when it can be replaced as good as new.

Snowballs are sometimes worked by putting heavy French knots of chenille over the surface, and sometimes plush stitch is employed, using filo floss to work with, but neither of these methods can compare in beauty with the one first given.

\section{RAISED PANSIES.}

For small banners, ornamental covers for toilet cushions and for the corners of small satin-covered photograph frames these flowers make beautiful ornaments. The leaves and stems should first be worked on the foundation with the finest chenille, No. I, in olive green, using Kensington stitch.

To work the pansy, select a perforated pansy pattern, and stamp a flower on stiff paper. From this cut out each petal separately and duplicate each one from fine screen wire by the pattern thus obtained. Work them with four or five shades of lavender chenille No. I, in Kensington stitch, putting in a few stitches of yellow chenille at the base of each petal. After all the petals are worked, arrange them on the foundation with invisible stitches, and curl the petals gracefully with the fingers; in the very center put in a French knot of the lightest shade of lavender. A cluster of pansies on a white satin banner, made in this way, is beautiful. For method of placing the petals, study one of the flowers in the perforated 
design. It is better not to attempt a too elaborate design-one or three pansies at most will be sufficient for any small article, and these flowers would not be suitable for large pieces. Both sides of the petals must be worked alike, as described for calla lily.

A toilet set of white satin, the mats of which are embroidered with pansies in filo floss, with Kensington stitch, and the cushion and bottles having a cluster of raised pansies, the whole finished with fine white lace, would make a wedding present that would delight the heart of any bride.

\section{RAISED FRUIT.}

Raised fruit may be used for panels for dining room or for over-mantel hangings, though, as a rule, it is not as effective as flowers.

\section{Cherries.}

A branch of cherries raised on a black velvet ground will make a very pretty placque ornament. To work: Stamp the design upon the velvet, cherries as well as foliage. Work the latter with silk, in Kensington stitch. Over each stamped cherry fasten a tiny ball of cotton wool rolled into the shape of a cherry, but a little smaller than the fruit should be when finished. Over this cotton work from the stem to the end of cherry with a strand of fine red chenille. Work these stitches until the cotton is completely covered, being careful to preserve the outlines perfectly. Some of the cherries may be made of a deeper red than others, thus giving a pleasing variety to the coloring.

\section{Raised Oranges.}

A panel of raised oranges, worked on a deep green velvet panel, will make a very beautiful over-mantel hanging for dining room. To work the oranges, two or three shades of golden yellow chenille No. 2, shades corresponding with 1263,1264 and 1265 of the Belding silks will be correct for this work.

Stamp the entire design upon the material, and work the stems and foliage with olive green arrasene, using about three shades of arrasene for this part of the work. Over the stamped oranges place a ball of cotton wool and fasten with securing stitches. The ball of cotton is made by rolling the cotton in the hand until it assumes the desired shape. Over this cotton ball work the yellow chenille, from the point where it joins the stem 


\section{I70 DAINTY WORK FOR PLEASURE AND PROFIT.}

downward, until it reaches the blossom end. Work a little knot of bronze green in the blossom end with filo floss. The stitch should be taken in a straight line, and the threads should lie evenly and not crowd each other. When it is desired to work oranges on table linen or other articles that must be laundered: (I) commence in the center and work around and outward in chain stitch, with yellow green etching silk; (2) work over the surface in three shades of filo floss, with Kensington stitch: (3) work over the surface with French knots, done with etching silk. For this style of work the foliage should be treated with outline or short and long stitch, in filo floss or Royal floss.

\section{Strawberries.}

Strawberries should be arranged with a suitable amount of foliage and a few blossoms, with one or two half-ripe berries and two or three fully ripe. Such a design thrown on white satin or black velvet is extremely effective.

Work the blossoms with cream white filo floss, in Kensington stitch; fill the center with a yellow French knot. The leaves and stems may be worked with filo floss, in Kensington stitch, using olive green, three shades. For the half-ripe berries use silk of a brighter shade of red than that used in the ripe fruit. Cover the stamped strawberry with a piece of cotton pressed into the shape of the berry, and secure with stitches. Cover this with filo floss of a true strawberry red, working over it, from stem to end, until the cotton is covered, after which put in seed stitches over the berry in irregular order, with yellow-green filo floss. Be careful not to rase the berry unnaturally, but follow the outlines of the stamped design. This fruit may also be worked with fine chenille, or it may be worked in solid Kensington, flat upon the surface of material, using either Royal floss or filo floss for the purpose.

\section{Grapes.}

Bunches of grapes, with foliage, make a beautiful design for panel. Stamp the entire design upon the fabric to be embroidered. Work the leaves and stems with bronze green and bronze brown arrasene. For the grapes, roll little balls of cotton, and catch down on each grape shown in the bunch, after which work over the cotton with chenille No. I, in the 
shade nearest like the variety of grape being copied. Deep purple grapes are the favorite for raised effects. Great care should be exercised to keep the form and outline of each grape perfect.

\section{COTTON PLANT.}

A cotton plant, with the ripe cotton bursting from the pod, is particularly beautiful, arranged as a panel, or as an ornament for a wall pocket, and figure I6I shows a particularly graceful arrangement.

The method of working is exceedingly simple and the materials quite inexpensive. The foliage and stems should be worked with solid Kensington stitch in arrasene, using bronze greens and bronze browns. The background should be of velvet or plush, of a deep rich shade, either blue, red or green.

The cotton ball for the design is the only difficult part of the work, and even this is simple enough, when one understands how it is done. To work: Cut out of pasteboard or stiff paper the pattern given in figure 162 . It is given the exact size, so no allowance is to be made. Having cut the pattern, place it on a piece of brown canton flannel, and mark it around the outlines, with a red pencil, or a piece of chalk; remove the pattern and stretch the canton flannel in a pair of small embroidery hoops, stretching as tight as possible. Work over the pattern marked on the flannel in solid Kensington stitch with arrasene, in dark and golden brown, and bronze green-a few stitches only of the latter

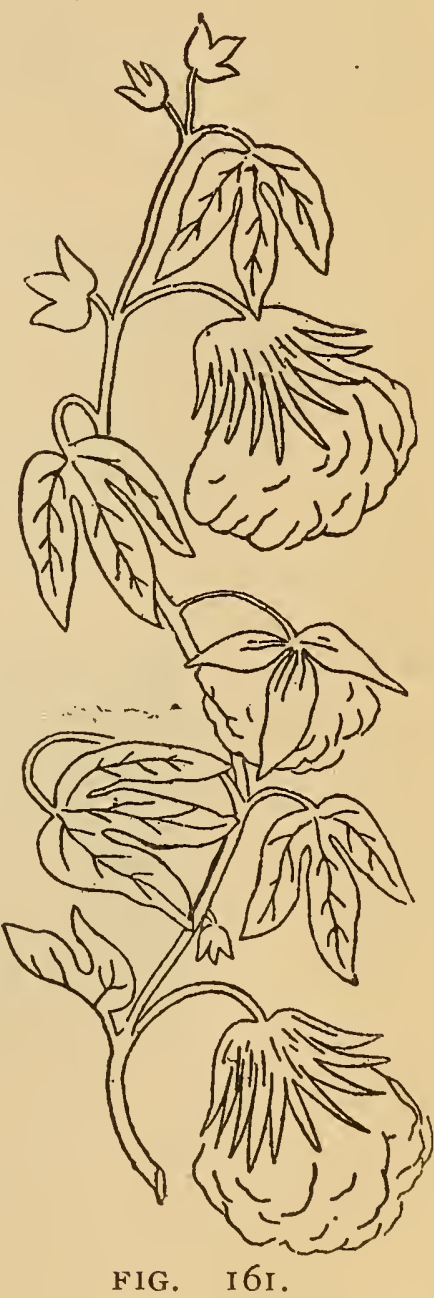
shade-work solidly, putting in plenty of stitches, and do not take them of too great length; carry one shade into another. When this has been done, remove the hoops and cut around the pattern, leaving a quarter-inch margin to turn down and hem with a running stitch, drawing the edges in slightly in the operation, so as to form a sort of bowl-shape receptacle for the cotton; now select a good sized piece of cotton wool that is free from dust or other specks, pull it apart until every fiber stands out distinct and 
light as a feather, place it in the pod or bowl just made, and catch it to the point marked $\mathrm{o}$, in figure 162 , with an invisible stitch. Half close the bowlshaped pod over the wool, pulling a portion of the wool beyond the points of the pod, and figure 162.

pends upon the manipuladesign shown at the beperfect guide. The balls be sewn to the foundation

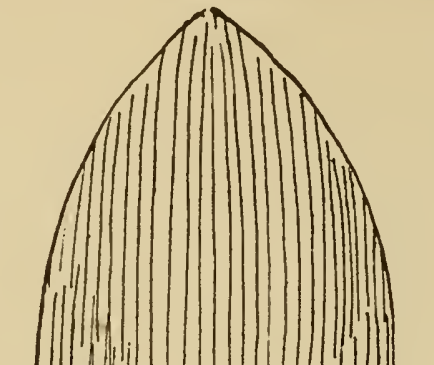

spreading it out as seen in

The entire effect detion of the wool, and the ginning of this article is a when completed should as indicated in the pat-

tern, though they it would be difficult to

The flowers of the white, shading to dull a little by working over crewel. This cotton and embroidered on plush or velvet, and framed with glass over it, makes a most effective and taking piece of needlework, for fairs and bazaars.

\section{THE DESIGNS AND INSTRUCTIONS}

For raised embroidery, given in this chapter, are not intended to exhaust the subject. Their province is rather to give practical help on specific articles of decorative work. It is needless to tell the woman of "ideas" that the instructions given for working raised pansies can be applied, with equal effect, to the working of single poppies, clematis, magnolias, and, in fact, any flower whose petals have simple outlines and unbroken surfaces. 
As before stated this form of work must be sparingly indulged in, and the proper place for its display should be well considered.

All raised embroidery should be worked in a square frame, both hands should be used-one under and one over the frame. When the work is completed it should receive a coat of thick mucilage on the back; this should be applied before removing from the frame and while the work is tightly stretched. This will secure ends of silk, etc., and give a smooth appearance to the article when finished. A most important point, and one that should never be forgotten, is to embroider all foliage and stems before the raised portion of design is commenced.

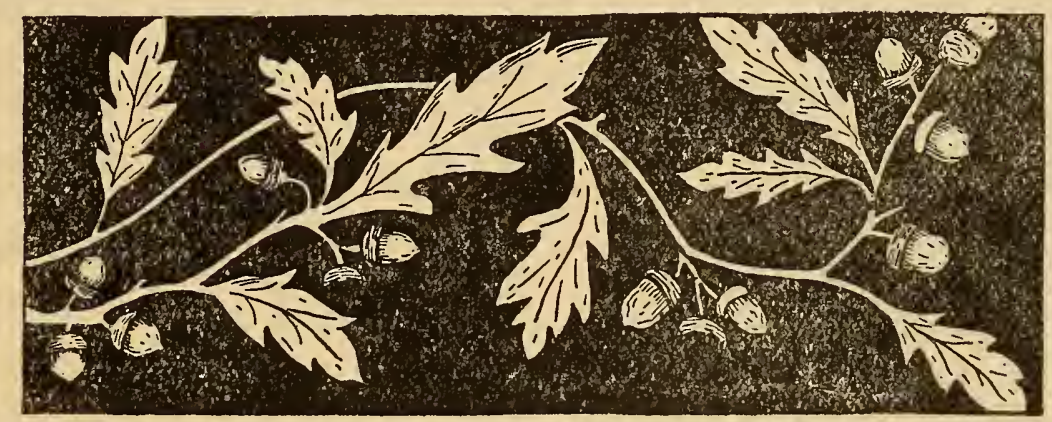




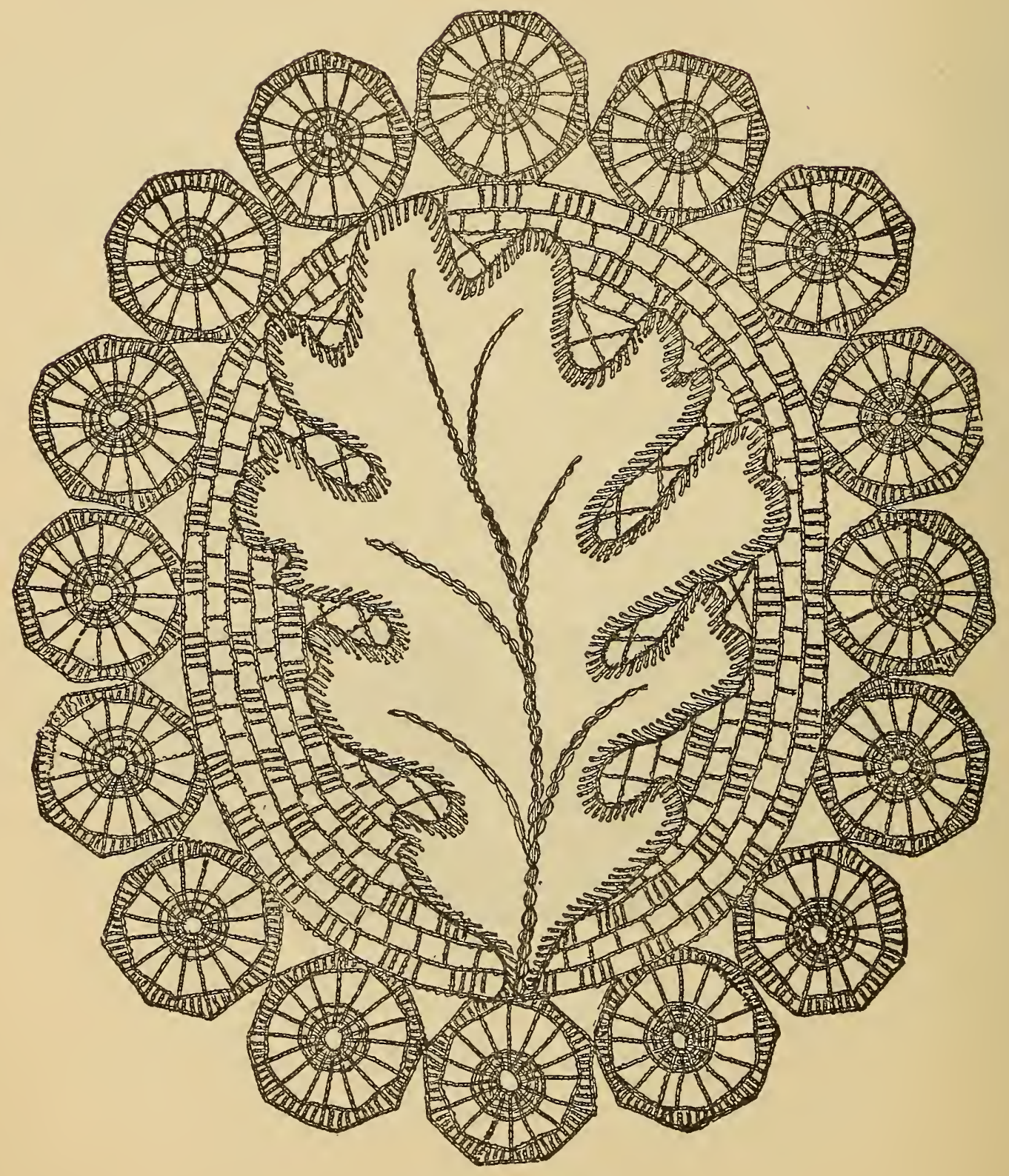

CARAFFE MAT. 


\section{CHAPTER XII.}

\section{DAINTY TRIFLES.}

HERE is always a demand from ladies for "ideas" on fancy work. They know how to embroider, but they do not know how to use the knowledge to advantage. Again, fairs, bazaars, Christrnas gifts, birthday and wedding remembrances are always in order, and "something new" is eagerly sought forHaving laid the foundation for a broad and systematic knowledge of decorative needlework in the preceding pages, we cannot do better service than to devote the present chapter to describing and illustrating a number of articles suitable for the purposes mentioned above. It is our experience that for fairs and bazaars, articles of useful ornament, made up inexpensively but showily, find quicker sale, and at comparatively better prices than articles of elaborate workmanship and expensive materials, while for birthday and wedding gifts the articles should be as daintily made as is possible for fingers to accomplish, and the best material of its kind should be employed. Thus, if the material be linen or lawn, a good quality should be purchased for the work; better far to make a smaller article, simpler in every way, than to cheapen the material. These gifts generally depend for their value upon the esteem in which the donor is held, therefore the best possible work should be done. Again, appropriateness to the occasion, to the age, condition and occupation of the recipient, should be considered.

We have not confined the articles illustrated in this chapter to those requiring embroidery for their decoration, but have included those that are to be painted as well, also some that require no decoration whatever, the aim being to give a number of inexpensive articles that can be made up with little expense; and also to utilize the contents of the scrap basket as far as possible. Very few novelties, so called, have been included 


\section{I76 DAINTY WORK FOR PLEASURE AND PROFIT.}

in the list; rather descriptions of articles that are always "in style," because always useful. The day has long gone by when a room was filled with a lot of useless, meaningless, dust-catching articles, called "fancy work." Nowadays, when an ornamental article is added to the furnishing of a room it must serve at least an ostensible purpose-in a word, it must have an excuse for its presence.

Whenever articles are illustrated requiring painting in either oil or water colors, specific instructions for this part of the work will be found in the chapter on "Brush and Palette."

\section{MEMORANDUM CARD CASE.}

These convenient racks make pretty remembrance gifts that have the

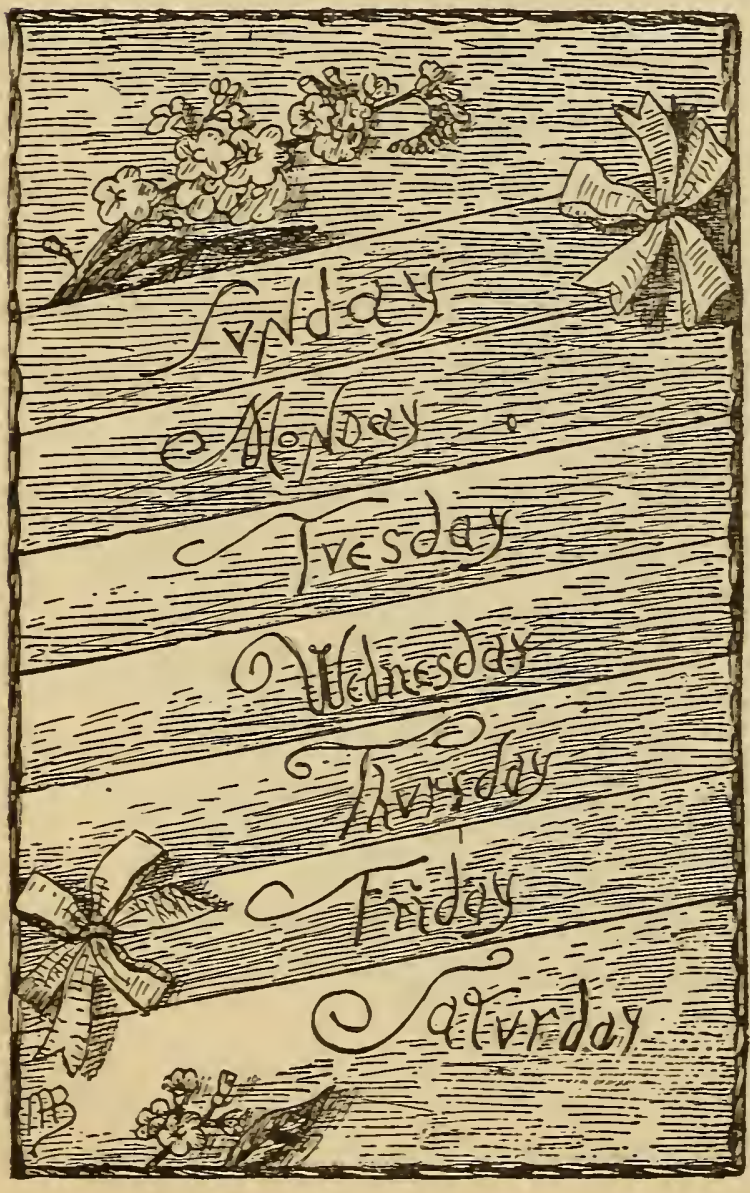

FIG. 163 . merit of being useful as well as ornamental. To make: Cut a foundation of stiff cardboard Io $\mathrm{x}$ 16 inches; line this cardboard on the back with bronze brown leatherette, which can be purchased at any art store for five cents per sheet. Line the front of the cardboard with gray linen; over this again, put folds of the linen diagonally from one edge to the other. These folds form receptacles for cards, one for each day in the week. The ornamentation may be done with either embroidery or painting. The model, figure I63, has a cluster of forget-menots on the top and bottom, worked with two shades of old blue filo floss, in satin stitch. The names of the days of the week are worked with outline stitch on the folds, with the same shade of silk, and the case is completed by bows of blue ribbon, disposed as shown in the illustration. The edges are bound with blue silk cord. 


\section{HANGING BOUQUET HOLDER.}

This novelty is designed to hold ferns, autumn leaves or pampas grass, and is made on a wire foundation, such as is used for making calla lilies. Cut the wire in shape and cover with soft cotton crepe, in pale blue or pink, or figured China silk may be used with good effect. Fold the whole in shape and secure with a few stitches. The wire gauze being stiff yet pliable, lends itself to any form of manipulation readily. Finish with a windmill bow, in harmonizing color, of soft ribbons. The wire is twenty cents a square foot. About two square feet will be needed, and one yard of the crepe or China silk. For bazaars or fairs this article would be attractive made up in a cheaper manner, as follows: Cut the shape out of pliable cardboard, cover with deep red crepe paper, fasten with a bow made of the same material. Fill with ferns and grasses. The crepe paper is beautiful, and exactly resembles expensive crepe.

The illustration is but one of many ideas that can be worked out for wall pockets. Very pretty catch-alls can be made from two palm leaf fans as follows: Cover one side of each fan with plain gray crash and gild the reverse side with liquid gold. On the gray' crash any pretty design may be embroidered. Join the two fans together at the handle by means of a ribbon bow, and add a few stitches on the side.

\section{WRITING BOARD FOR INVALIDS.}

This article, figure 165 , was designed for the use of invalids by a French lady, Madame Tignet. and will at once commend itself. The foundation is a light, plain board, $23 \% / 2 \times 151 / 2$ inches, covered with dark blue cloth-either denim or felt will answer. In the center is the blotting pad, held down at 


\section{8 \\ DAINTY WORK FOR PLEASURE AND PROFIT.}

each corner with triangular pieces of leather or cloth. The various pockets, intended to hold paper, envelopes, stamps, cards, etc., are fastened with buttons. A straight band of cloth, divided into compartments with small nails, holds the paper knife, pen holder, pencil and penknife. Next to thrs

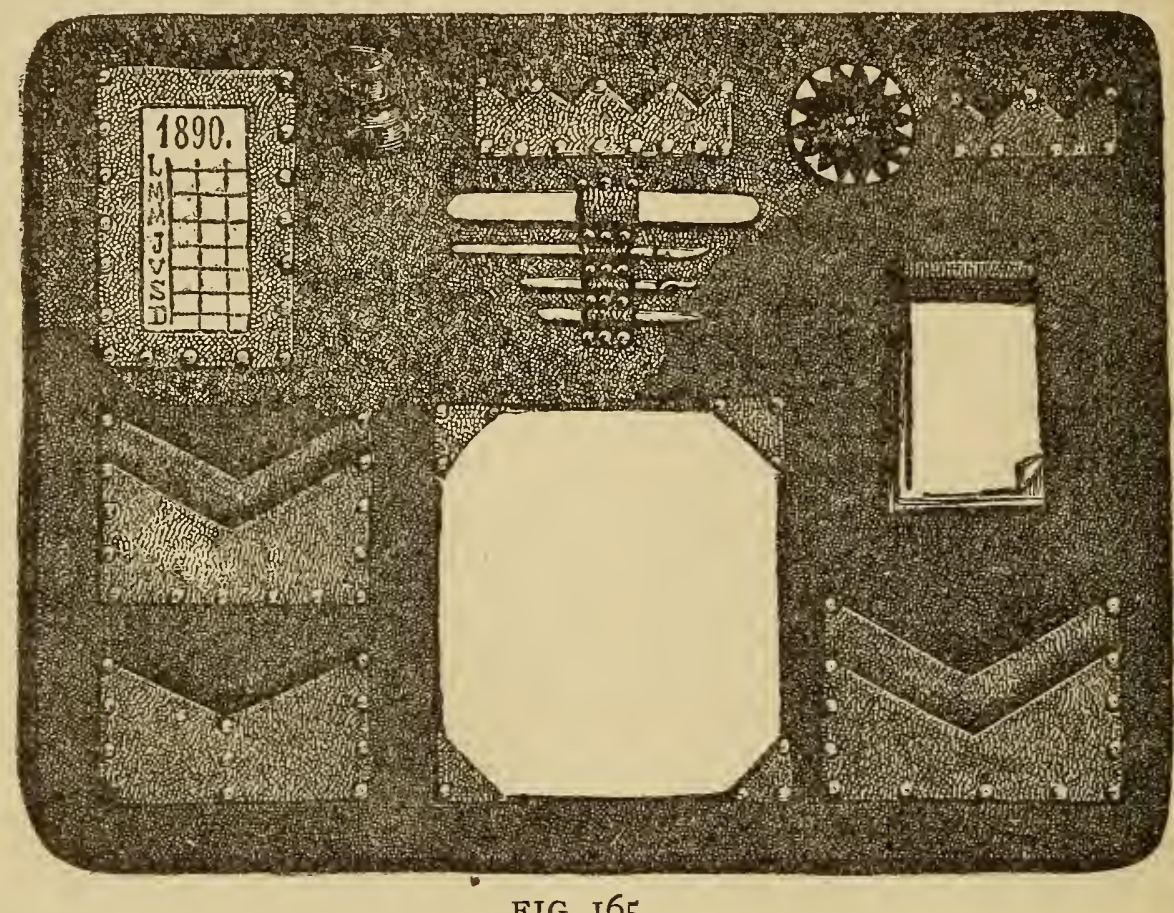

FIG. I65.

inkstand, held in place by a strap of leather or elastic, is a movable calendar, and on the opposite side is a penwiper and block note.

For an invalid friend no more acceptable gift could be devised. The expense is but trifling, the value of the article being in the loving care and thoughtfulness that prompts the offering.

FOOT STOOL.

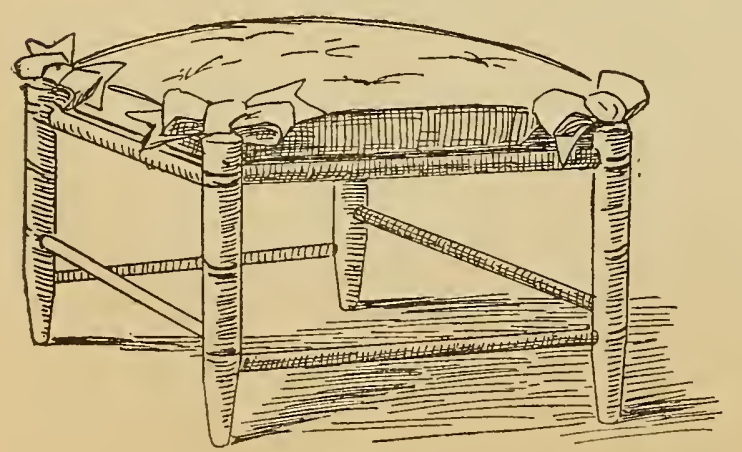

FIG: 166.

These little foot stools can be purchased in the rough for 50 cents each, and can be prettily decorated by means of a paint brush and a can of English enamel. One coat will be sufficient, and a pretty color will be old rose. The cushion is made of India silk, to just fit the top of stool; a pale gray blue or gold color will harmonize with the old rose. The 
cushion should be securely fastened to the corners of the stool to prevent slipping off. The filling of the cushion may be of sea moss or feathers, or, failing either of these, cotton wool will do nicely.

\section{PAPER STAND.}

This convenient paper stand is made of three bamboo rods, three box lids-either cheese or sugar-box covers are preferred-seven yards of twoinch ribbon and one bolt of very narrow ribbon. The bamboo rods are fastened to the box covers by means of fine wire threaded through holes bored into the covers and rods opposite each other. The places where fastened are concealed by the ribbon bows. The bamboo rods are wound from top to bottom with the narrow ribbon, a piece of the twoinch being fastened around top and bottom of rods. The inside of lids are covered with egg-shell paper, upon which has been painted a design in either oil or water colors; or, for those who do not paint, a very pretty substitute would be satin with floral design embroidered with the wash silks; the outside of rim can be painted with English enamel, or a band of embroidered ribbon can be fastened on with excellent effect. The colors to be used are a matter of taste, but should harmonize with the

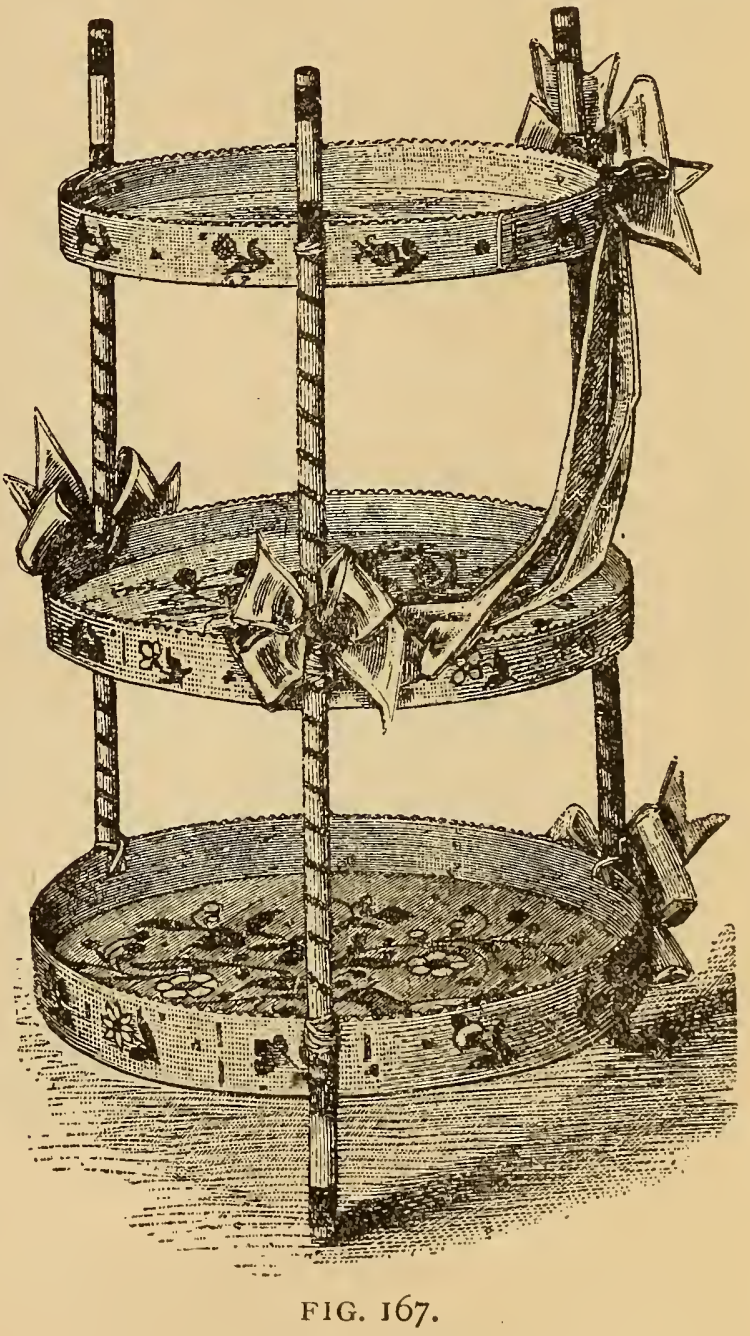
furnishings of the room. If bamboo rods are not obtainable, the handles of worn-out brooms will do nicely, sawed off to the desired length; they should be either ebonized or gilded. If the stand is to serve as a paper holder in a sitting room, a good substitute for the satin or eggshell board would be linen crash, embroidered with linen floss. The design should be tiny flowers, scattered rather than arranged in any set design. 


\section{SHOE BOX.}

We here give a design for a shoe box, which is ornamental as well as useful. An ordinary soap box will do for the foundation. It must have a cover fitted to it. Line the box inside with pale pink sateen, such as is

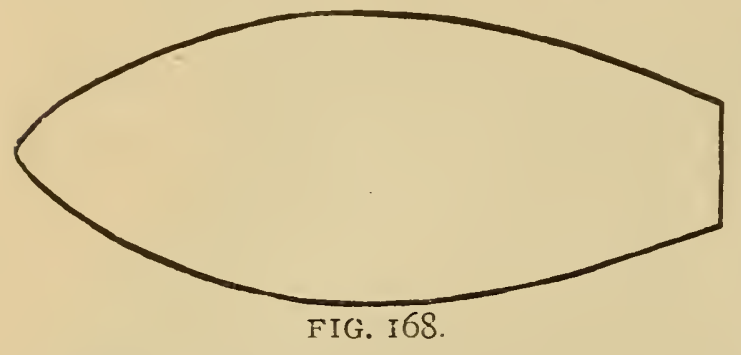
used for dress linings; cover the outside with pale blue sateen, and on this covering arrange flowers, as shown in figure 169; cut the flowers out of pale pink satin, using figure No. 168 as a guide. Cut eight pieces like the pattern and fasten them on to the background, slightly wadding each petal. The middle of each flower is made in knot stitch with tinsel thread, and the broken lines are made of the same material. A cording cutlines

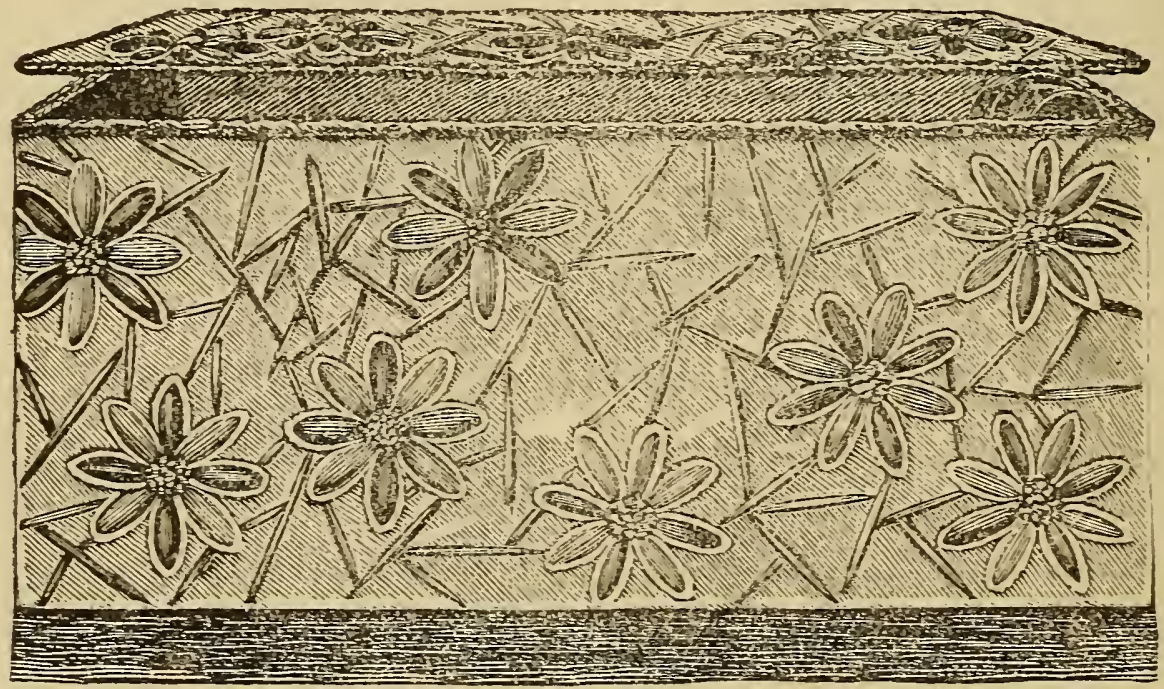

FIG. I60.

the box and conceals the sewing. Any other combination of colors or ornamental design may be substituted, keeping in mind always that the article is to be inexpensive.

\section{SUNFLOWER CUSHION.}

This little cushion for the dresser is very simple and can be easily made. The material is India silk, fine lace, silk bolting, one skein filo L:oss and one skein gold thread, and a small, round, soft cushion, not over 
four inches in diameter. First, stamp on the bolting the sunflower design, which, by the way, is not a sunflower at all, but is a conventional form, having pointed leaves like that flower. Around the edge of this design sew on the lace; this will give a circular cover; outline all the leaves or points with gold thread and fill them with darning stitch. Outline the center with gold thread and check it off into squares, couched down at the intersections. Any color of filo floss can be used for the embroidery-pink, yellow, brown, blue or green-but the silk lining must match in shade exactly. You are now ready to line the cover, which is done by cutting a circular piece of the silk the size of

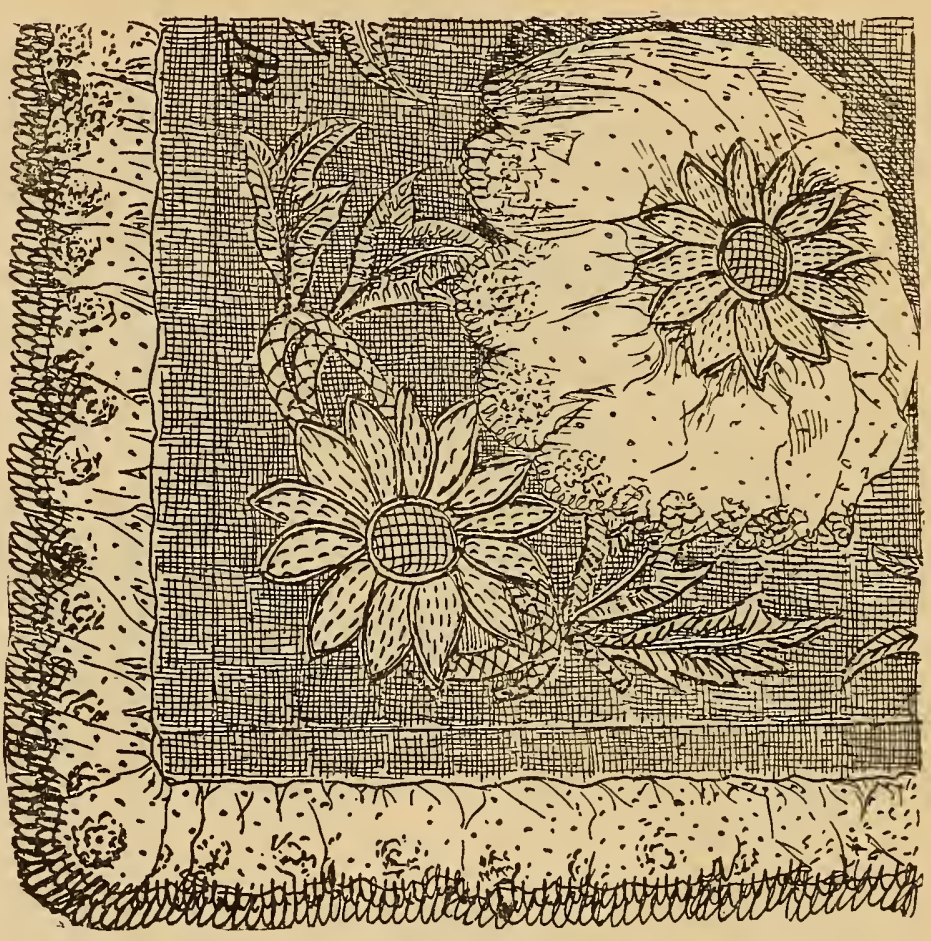

FIG. I70.

cover, lace edge included, and tacking lightly to the outside cover; pink the edge of silk all around. The cover, lining and all, is now fastened to the top of the cushion by tacking each point of leaf to the cushion proper; this gives the fluffy, full appearance of the flounced edge. Usually a dresser scarf is made to match these little cushions. Our design shows both scarf and cushion, the same design being used for both. The model was embroidered with gold filo floss, on white silk bolting, and lined with gold India silk,

\section{WORK BASKET.}

Figure I7I illustrates a collapsible work basket. This little basket, fitted with every convenience, will be especially appreciated by a lady who is visiting or traveling much. Four pieces, $9 \times 9$ inches at the top and $51 / 2 \mathrm{x}$ $5 \frac{1}{2}$ at the bottom, form the sides, and one piece $5 \frac{1}{2} \times 5 \frac{1}{2}$ the bottom. These pieces should be cut from pliable cardboard, not too stiff, and covered on the outside with gray crash or linen, or pongee silk in natural 
shade; these pieces can be embellished with a graceful design, worked out. with Real Scotch linen flourishing thread No. 8, in pink or red, with wood

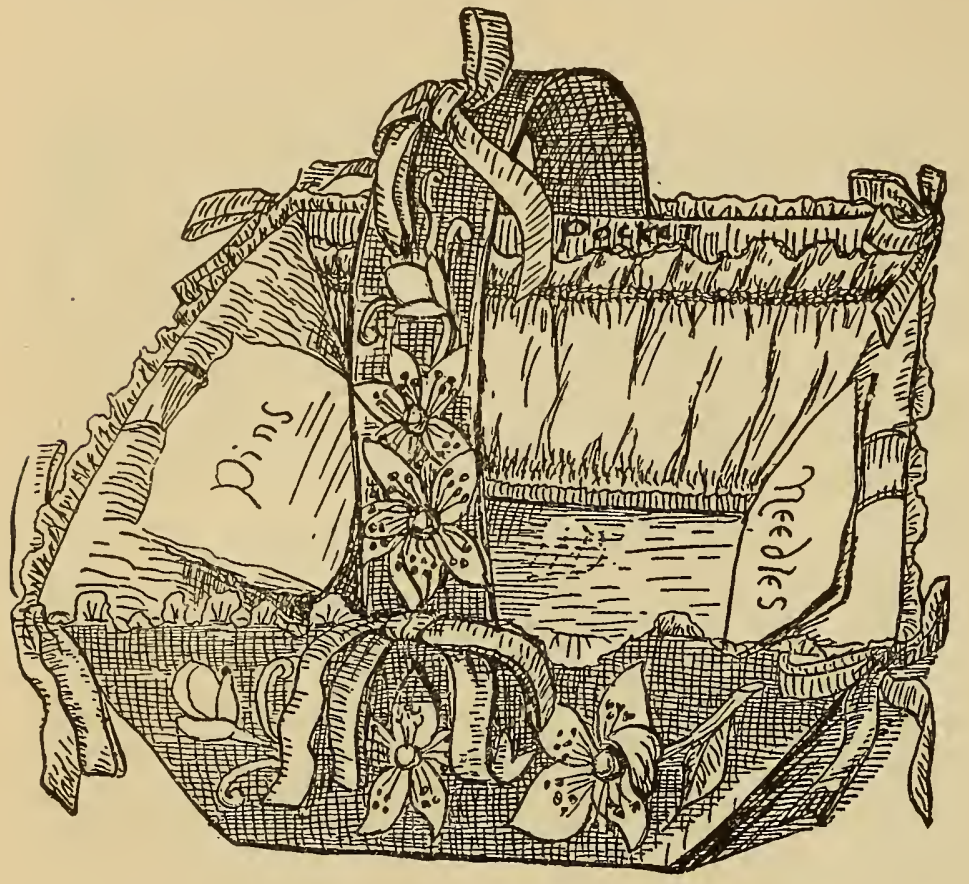

FIG. I7I. browns; the inside should be lined with pink silk, and the edges overhanded with silk to match; the bottom is covered in the same manner. The bottom edge of each side piece is then fastened to the bottom piece firmly, but the sides are not sewn together, only fastened at the top with bows of ribbon; a handle of cardboard, covered to match the basket, is fastened at the sides with bows, in a removable manner. When necessary to pack the basket, remove the handle, untie the bows fastening the side pieces at top, and fold the sides in, one over the other. This forms a neat, flat package that can be packed anywhere without injury. There should be two long, rather deep, pockets on opposite sides of the basket, on the inside, for spools, buttons, etc.; also on one end a neat needlebook, on the other a tiny square cushion, all of pink silk, and fastened securely. An acceptable gift for any occasion.

\section{HAIR PIN HOLDER.}

Figure $I 72$ is a small hair pin holder, the foundation of which is a tiny Japanese circular basket. Fastened on the inside of the opening are several pieces of narrow pink ribbon, on the ends of which are fastened small brass rings, crochetted over with pink etching silk. These ribbons are allowed to drop over the outside of

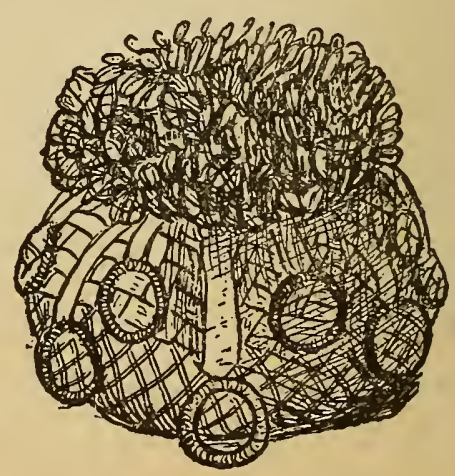

FIG. I72. basket, as shown in cut, and form a pretty finish; the top opening is then 
filled in with pink zephyr in tuft stitch; this is to receive the hair pins. The cost should not exceed fifteen cents.

\section{TWO SMALL CUSHIONS.}

These small toilet cushions can be made so daintily, ornamented with so little expense attached, that it is a real pleasure to write about them. Two or three of these on a dresser are much more convenient than one large magnificent affair that looks altogether too grand for mortal use. The model shown in figure 173 may be made of any color to suit the room or the fancy of the maker; but the puffed cover should be of soft silk, India or China, and the square in the center

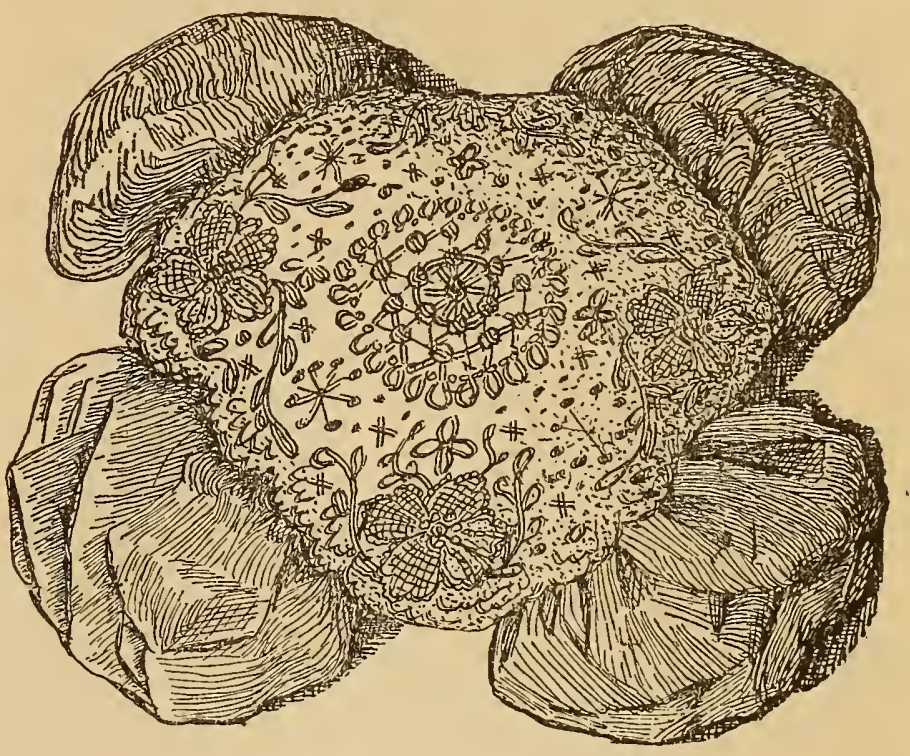

FIG. 173 . should be of wash material. The model was a cushion three inches square, covered with pale blue China silk; the center piece was a square of fine

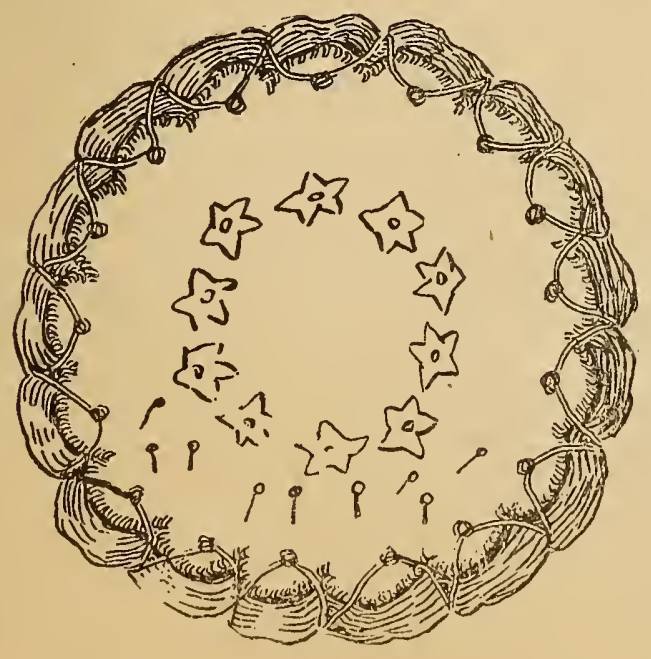

FIG. I74. linen lawn, embroidered with fancy filling stitches. As will be seen, the silk is cut larger than the cushion, and all the spare fullness is gathered into pompoms at the corners. The center square offers room for a variety of decorative work, and it is a good idea to have several of the centers for each cushion. Some may be worked with Roman embroidery, some with Dresden designs, and others may be covered with drawn work.

Figure 174 is a tiny round cushion, covered top and bottom with a plain white linen cover, embroidered with wreath of wood violets in natural shades. The covers are placed in posi- 
tion and laced together with a white silk cord over a puffing of lavender silk. The materials required for these dainty trifles are mere scraps from one's piece bag, and they will make most acceptable gifts for any occasion, at little expense.

\section{CHAIR BACK AND SEAT.}

Figures 175 and 176 show design for back and seat of chair. The material is a deep silver green Russian tapestry, and the design a spray of

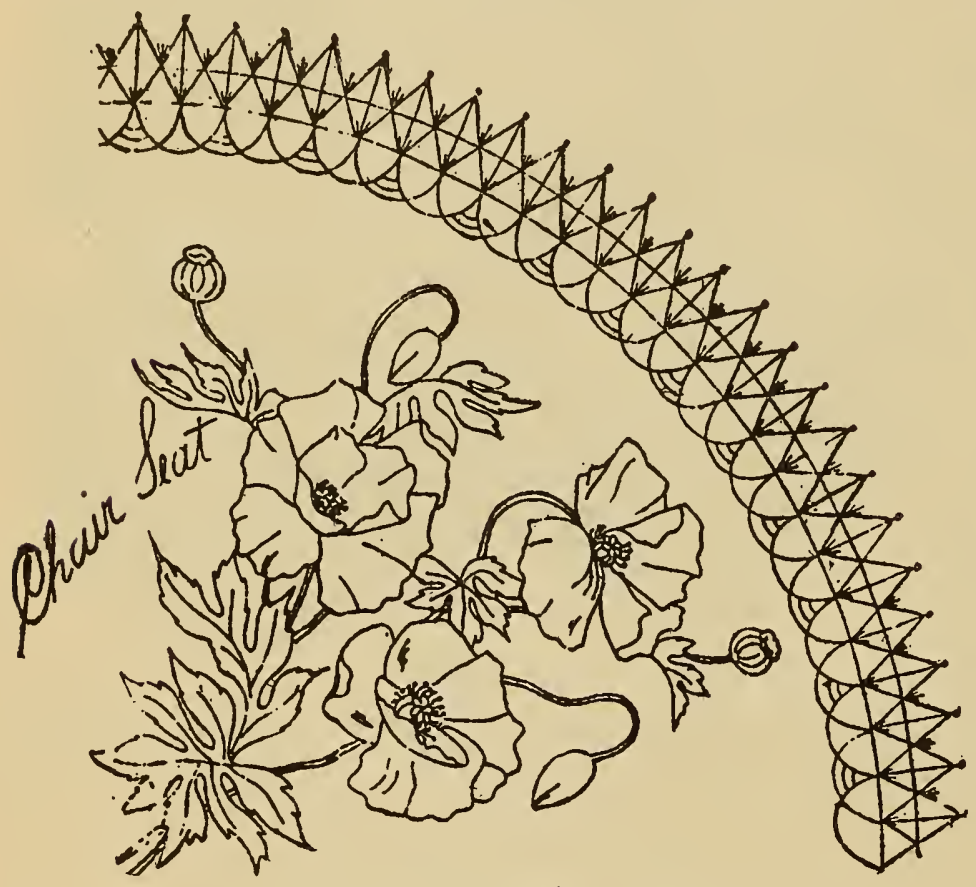

poppies. Treatment: Work the stems with No. I334, the leaves with Nos. I330, 133I and I332 silver gray green; the flower petals with Nos. I209, I210, I2II and I2I3; the centers of poppies with Nos. $\mathrm{I}_{2} 6_{3}$ and 1278 rope silk. Use solid Kensington stitch for the entire design. Work the fancy stitches around edge of chair with No. I263, with the exception of the three short stitches

FIG. 175 . at intersection of diamond-shaped spaces; for these use No. I209. The treatment is, of course, the same for seat and back, and filo floss should be used throughout. Of course, any other combination may be made to suit individual taste. The size of seat is I 5 XI9; of back, IOX I5.

The same pattern can be worked with other mate-

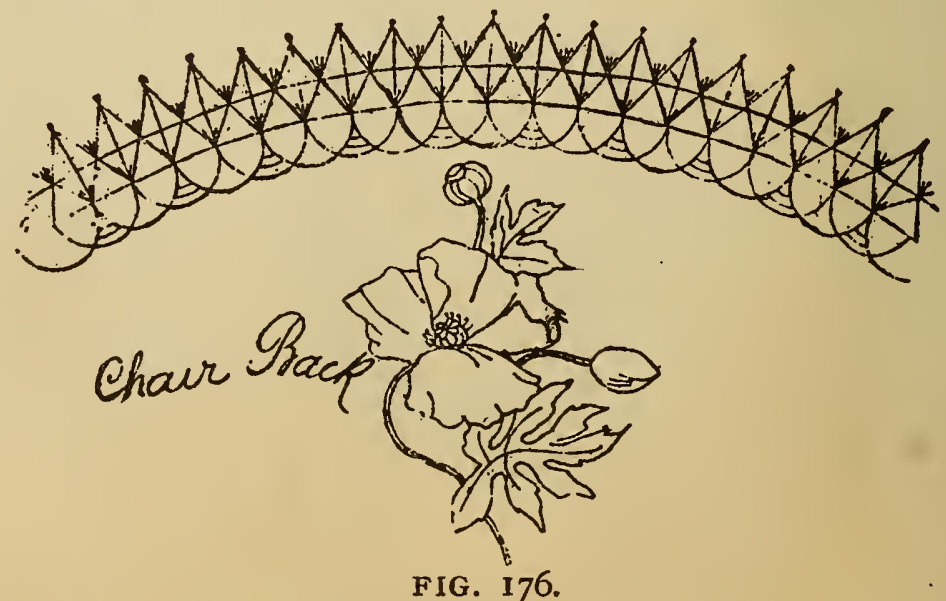
rials, as deep brown mail cloth embroidered in solid Kensington stitch with two or three shades of golden brown filo floss. 


\section{LAMP SHADE.}

The lamp shade shown in figure $\mathrm{I} 77$ is made of tissue paper in three shades of old rose. The paper is first creased by folding back and forth in small plaits and then drawing several times through the hands, or it can be purchased ready for use, but is more expensive that way. Place the three shades of paper, one over the other, the lightest shade first, the medium shade next, and for the outside the darkest shade. Gather the paper at the top to fit the neck of a wire foundation, which in turn is made to fit the top of a lamp chimney; fasten securely, and then drape up the outside layer as shown in illustration. The wire foundations

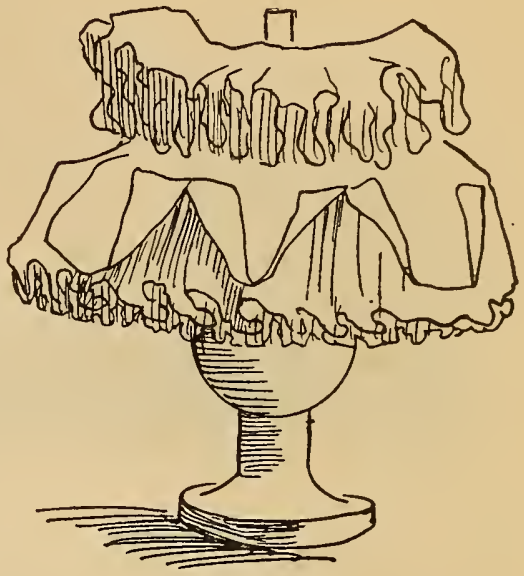

FIG. I77. can be purchased for twenty cents, and any color paper to suit furnishings of rooms can be used in place of the old rose.

\section{WALL POCKET.}

A convenient wall pocket for newspapers is shown in figure I78. Heavy

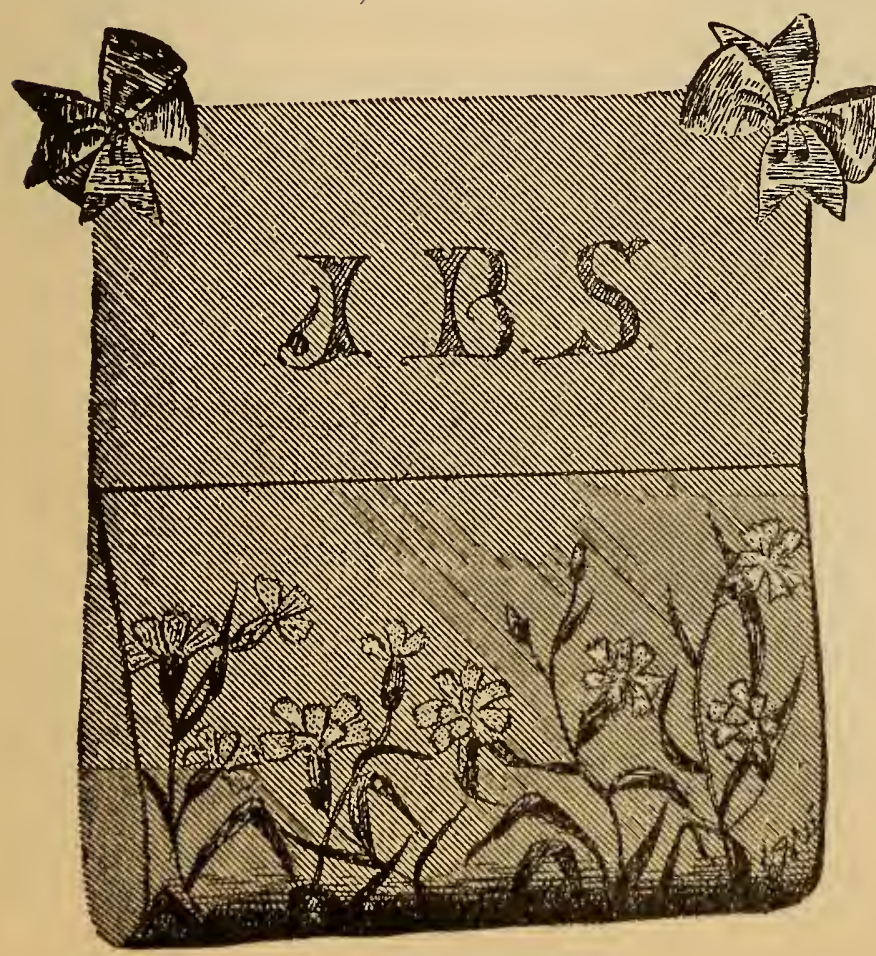

FIG. I78.

ing on the edges a row of Tyrol cord.

pasteboard is used for the foundation; the dimensions of the piece are 15 by 25 inches. The pasteboard should be covered on both sides with gray canvas or ecru linen; on that portion of the material that forms the pocket stamp a frieze of carnations; work the design with Real Scotch linen floss in the shades of red and olive greens. In covering the pasteboard with the linen the edges must be neatly turned in and overhanded together; the finish is made more complete by sewThe lower part is then turned 
over and sewed on the back as here indicated. Brass rings are sewed on the corners to hang it up by. Bows of ribbon, the darkest shade cf red used in the carnations, are placed on each corner.

\section{BOLSTER AND PILLOW.}

A bolster covered with white linen lawn is here shown. For a summer

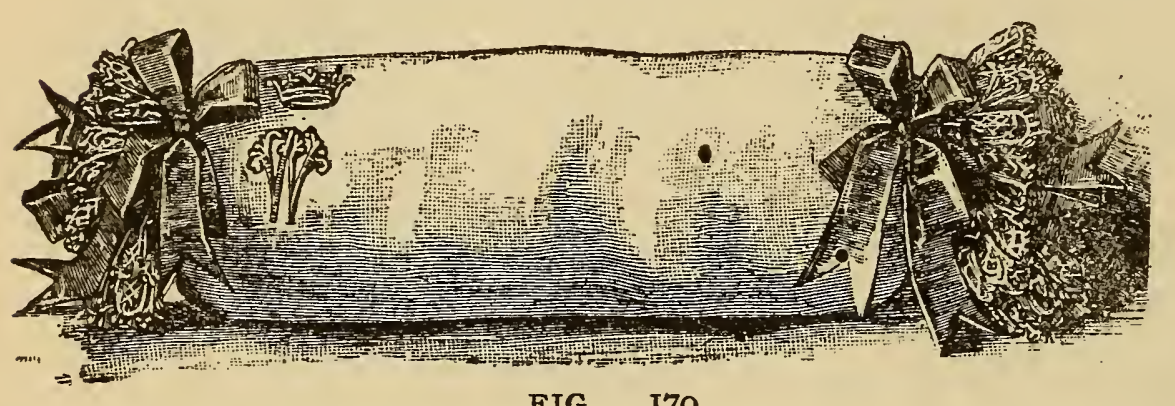

FIG. I79. bed nothing cooler or more inviting , could be imagined.

The ends are trimmed with wash lace and

bows of ribbon. Over the surface a design of very small daisies or carnations may be scattered and worked in short and long stitch with filo floss. The work must be light in effect and daintily executed.

The infant's pillow illustrated in figure 180 is a dainty affair, and consists of a square of fine shirt linen, around which is sewn a border of lace; the square

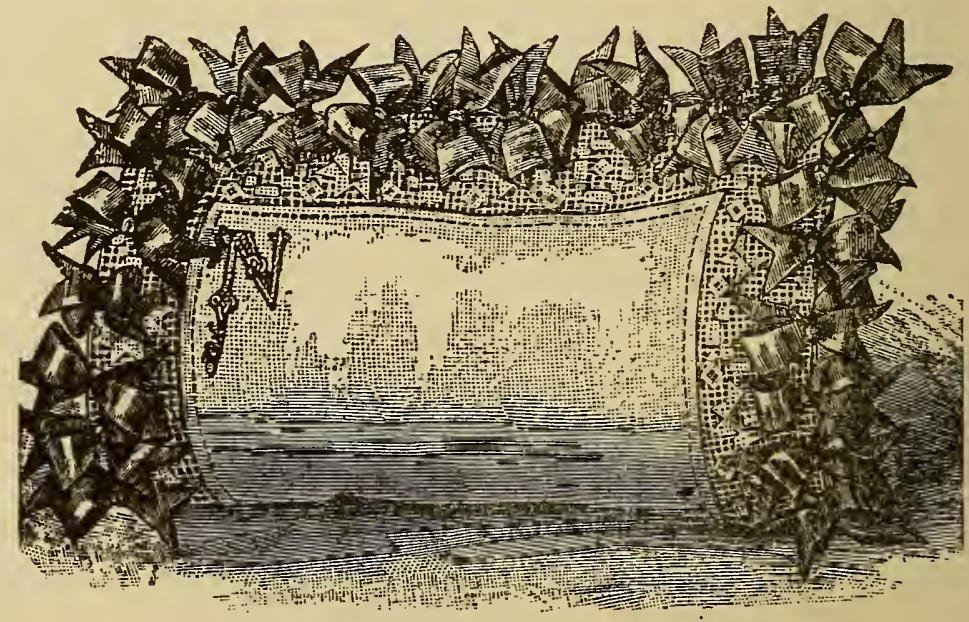
FIG. I80,

is then folded once in the middle and the edges sewn together and decorated with ribbon bows of any color desired. It can be removed when necessary to launder. A pretty design for ornamenting would be an arrangement of buttercups and daisies done with filo floss.

\section{HANDKERCHIEF HOLDER.}

Figures $18 \mathrm{I}$ and $\mathrm{I} 82$ show this little case in process of construction and completed, so that by studying them carefully the holder can very easily be duplicated.

Before commencing operations on the material to be used, it will be well to take a piece of white or light paper, twelve inches square, and pencil line it like figure I8I. First, fold the sides marked I, straightening out 
each before folding the next. Then turn the paper over and fold across cornerwise, like lines marked 2. Now turn the paper back again, first sidi up and fold in the sides according to creases, and fold the corners marked 3 back.

This will give a perfect guide for the folding of the case, without experimenting on the goods, which would show a misfold beyond repair. To make the case will require a piece of satin of pale cream twelve inches square, a lining piece, same size, of shrimp pink, and an intervening lining of stiffening-crinoline will do nicely. Place these together

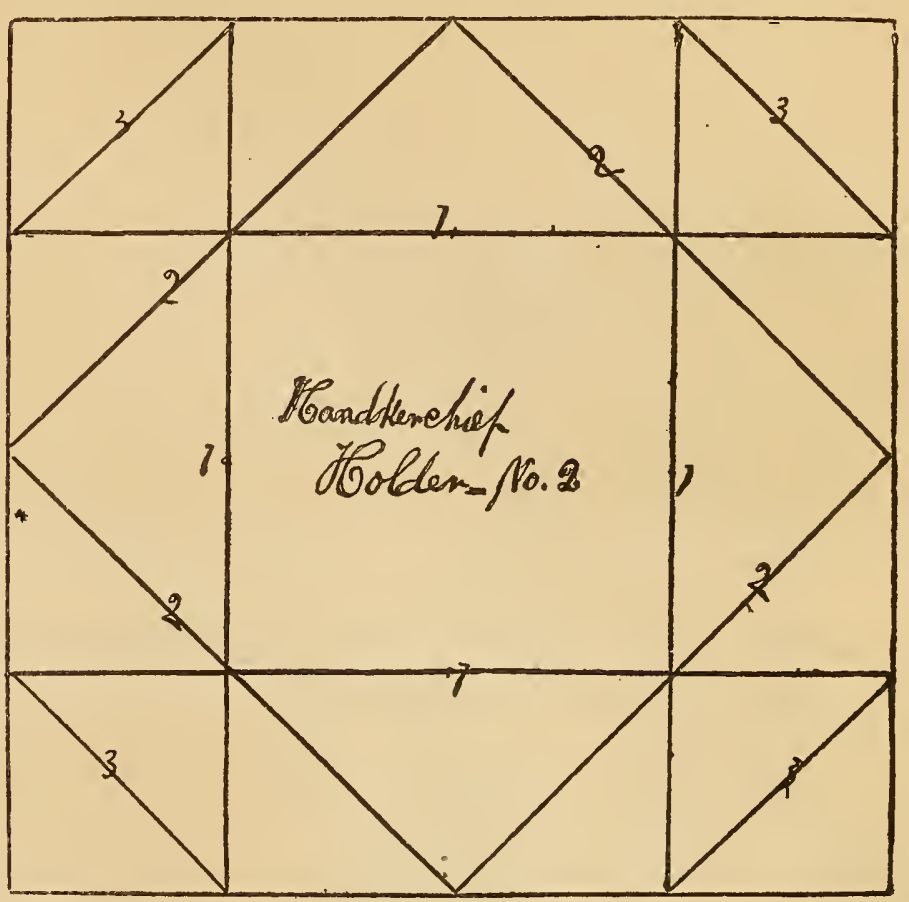

FIG. I8I. squarely and evenly; finish the edges with gold and pink silk cord and pro-

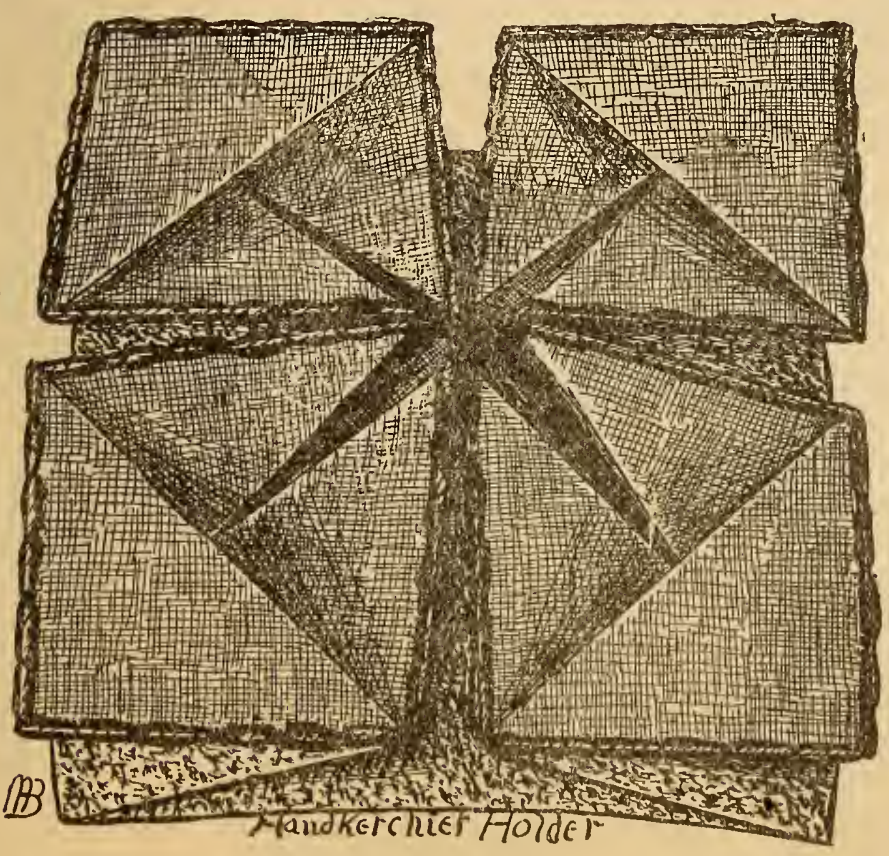

FIG. 182 . ceed to fold like the paper pattern previously prepared.

This will make a simple and inexpensive gift for friends, and but little time is required for the making, as no embroidery or painting is needed.

One-third of a yard of satin, twenty-four inches wide, and a like amount for the lining, will make two cases at an expense of sixtysix cents; two and two-thirds yards silk cord, at eight cents per yard, will bring the cost to about ninety cents for the two. 


\section{PHOTOGRAPH OR CALENDAR CASE.}

These cases are convenient for holding photographs and calendars, and are, beside, an ornament for mantel, table or bracket that will be appreciated by ladies who are interested in home decoration; also, they are always rapid sellers at fairs and bazaars, and make acceptable wedding,

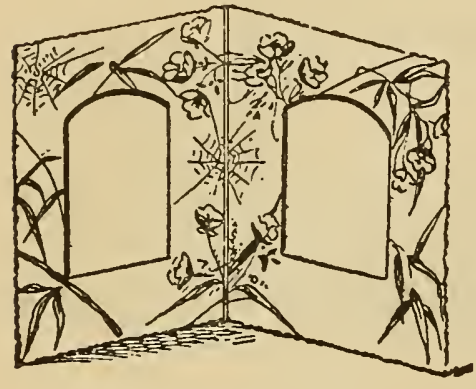

FIG. I83. birthday or Christmas gifts. The two-panel design shown in figure 183 is made of egg-shell board or linen canvas for the outside, and stiff cardboard lined with gray linen for the back. Egg-shell board is a stiff cardboard, having a crinkled enameled surface, and comes in cream, ecru, brown and old rose. A leaf from an old album will furnish a pattern to cut the panels by. Out of the egg-shell board or linen canvas cut two panels, each having an opening for the photograph, and out of the cardboard cut two pieces the same size, for a back to the panel. If linen canvas instead of egg-shell board is used for the front panel, then it must be first pasted smoothly over cardboard. Cover the two pieces that are to serve as backs for the panels with gray linen, on the outside turning the edges in on the inside of panel and gluing them down. When the front and back pieces are ready, place them one over the other and oversew the edges together with art cord. Join the two panels by oversewing down the back with art cord.

If the panels are covered with the linen canvas, they may first be beautifully decorated with needlework. The design should be worked before the covering is put on. Floral designs are best suited for this purpose, and may be worked solid or in outline, with silk or linen floss, in natural colors or in conventional shading. Brown silk floss on cream white duck is artistic to a degree, as is also pale gold, sage green, deepest shade, or old blue. If the egg-shell board is

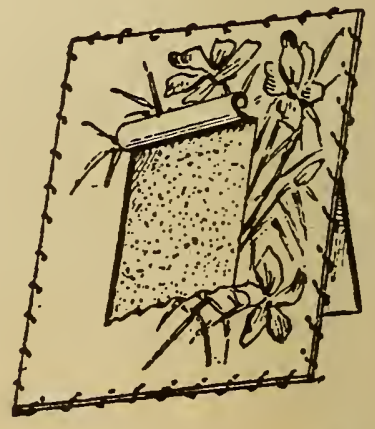

FIG. I84. used, it may be decorated with a design painted in oil or water colors, for which directions may be found in the chapter on "Brush and Palette."

Figure I84 shows a calendar case, made in same manner as described above, only in this case but one panel is used and it is covered with cellu- 
loid, instead of the opening being cut entirely away it is rolled up. This material may also be decorated with either oil or water colors. A printed calendar should fill the opening, being pasted in the opening on the back panel.

\section{NIGHT DRESS SACHET.}

This pretty sachet is made of white silk bolting lined with pale pink satin. The lining is quilted and covered on the reverse side with blue satin. The sachet is edged with a double frill of lace, and finished with bows of blue and pink ribbon. The silk bolting cover is decorated with a spray of Marguerites and rose buds, done with filo floss. Twenty-four inches of bolting and the same amount of blue and pink satin for lining, and one sheet of wadding, with five yards of ribbon for bows, will be required, together with one-half dozen skeins of pink, yellow and green filo floss - two skeins of each.

The shade may be used to make up other materials and colors by, and the outside cover may have a design painted instead of embroidered.

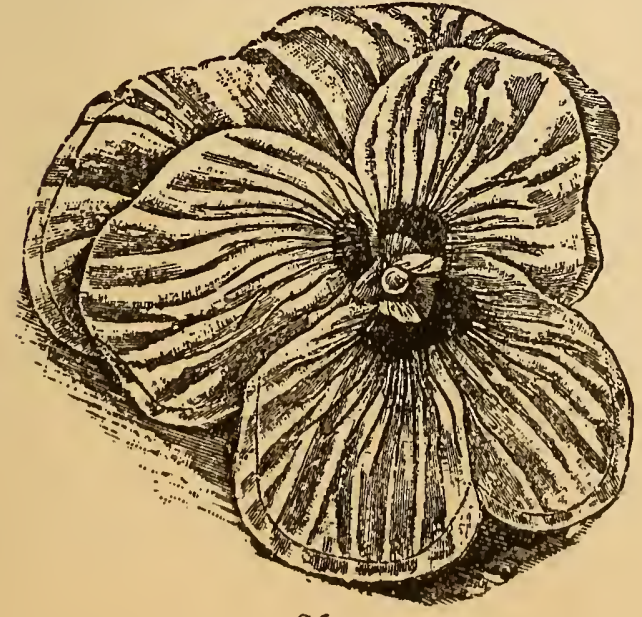

FIG. I86.

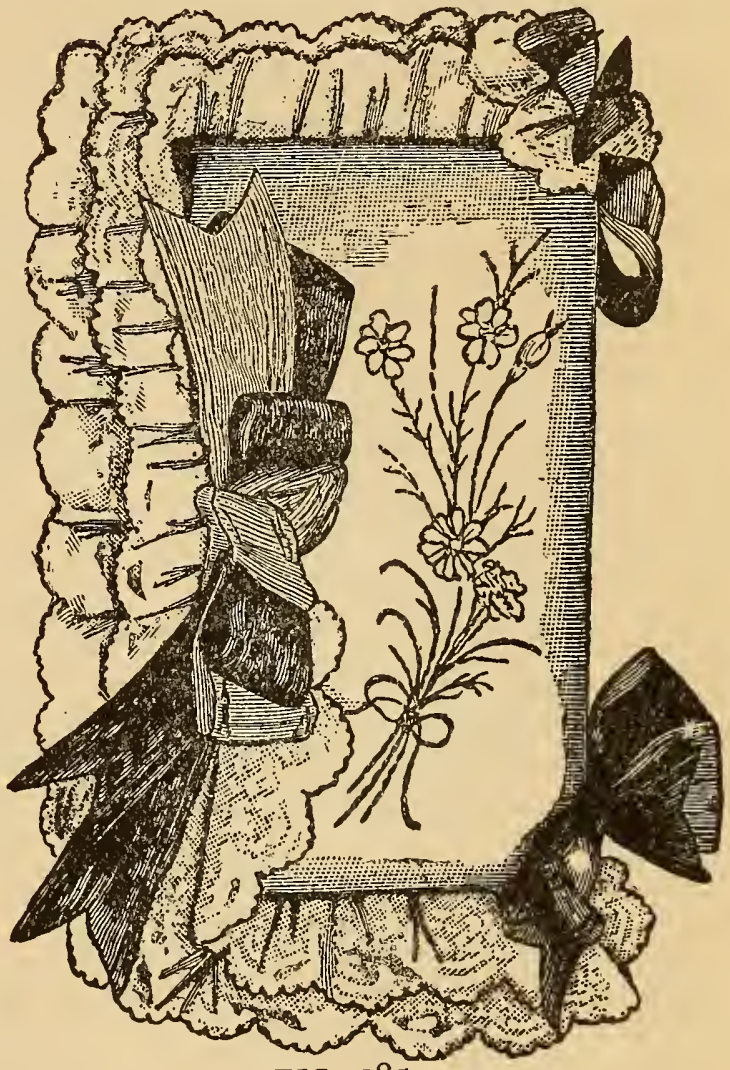

FIG. I85.

PANSY SACHET.

This pansy sachet is a charming litt's gift, and can be made of a bit of gold or rose-colored silk three inches square. Cut out the leaves separately, a little larger than the outlines shown in the illustration, using pattern of pansy petal given on page 138 , for this purpose; line each petal with pink satin, lightly wadding the space between the lining and outside with perfumed cotton. The lines on the petals may be made with a pen and 
violet ink. Arrange the petals so that they fall easily over each other, as in the illustration, and sew. a scrap of yellow silk bunched up in the center, and next to this irregular clippings of brown or purple velvet.

\section{PUFF CUSHIQN FOR HEAD REST.}

Soft, puffy cushions, circular in shape and made of India silk, make delightfully comfortable head rests. Figure 187 shows a very simple manner of making. Cut a circle, the size of a dinner plate, from cotton batting-not wadding -and cover with old rose India silk, as follows: Cut a square of the silk as large as the width of silk will allow, place the batting in the cen-

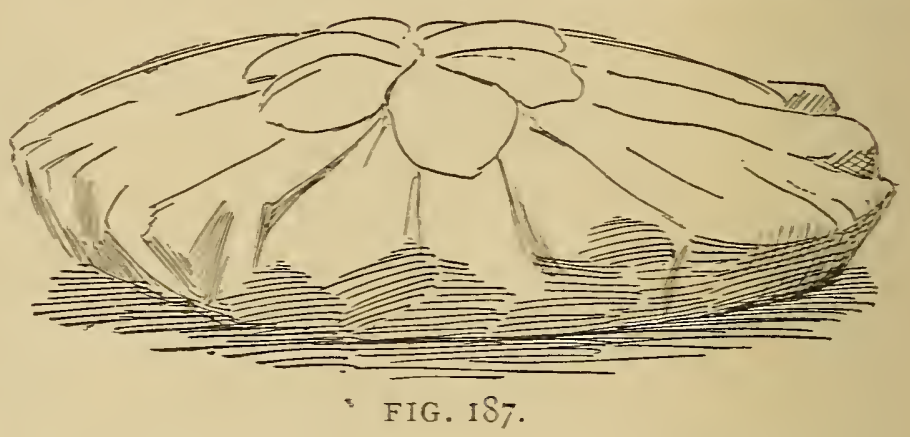
ter of the square and bring the four corners over to the center of top of wadding. Arrange the fullness into puffs, from center to circumference, by catching here and there with a stitch; over the top of this cushion fasten a large leaf or flower, cut from linen lawn or other wash fabric; work this with filo floss, in short and long buttonhole stitch, around the edges, and cut the material close to buttonhole edge. This piece can be easily removed for cleaning, and the entire arrangement is light and dainty.

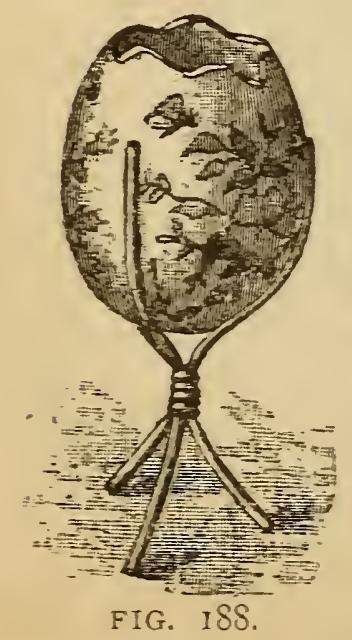

strewn grass seed.

\section{DECORATED EGG SHELL.}

The illustration clearly shows the idea. Care should be taken in breaking off the top of the shell not to make unsightly cracks and checks; a very sharp pair of scissors will obviate this difficulty. The wire standard can be made easily. Procure the wire at any hardware store, bend it into shape and twist around it a lighter wire to keep in shape. They can be painted in water colors or oils, and are dainty trifles for the mantel, and can be made to serve as unique vases by filling the shell with a piece of moistened sponge over which has been thickly 


\section{PANSY SACHET.}

A pretty trifle for toilet table is a pansy sachet, made as follows: Take a piece of cotton batting and roll it into a ball about the size of an egg, and sprinkle with sachet powder; cut from pale yellow tissue paper a square large enough to cover the ball and leave enough at top to form pansy petals $I / 2$ inches deep. After the square is cut, fold the paper back and forth into small plaits, like a paper fan, and then draw it through the hand several times; when unfolded it will present a soft, crepy surface; place the ball in the center of this and fold the paper to top of ball and tie closely with hair wire. Slash the paper that is left above the wire into four equal parts

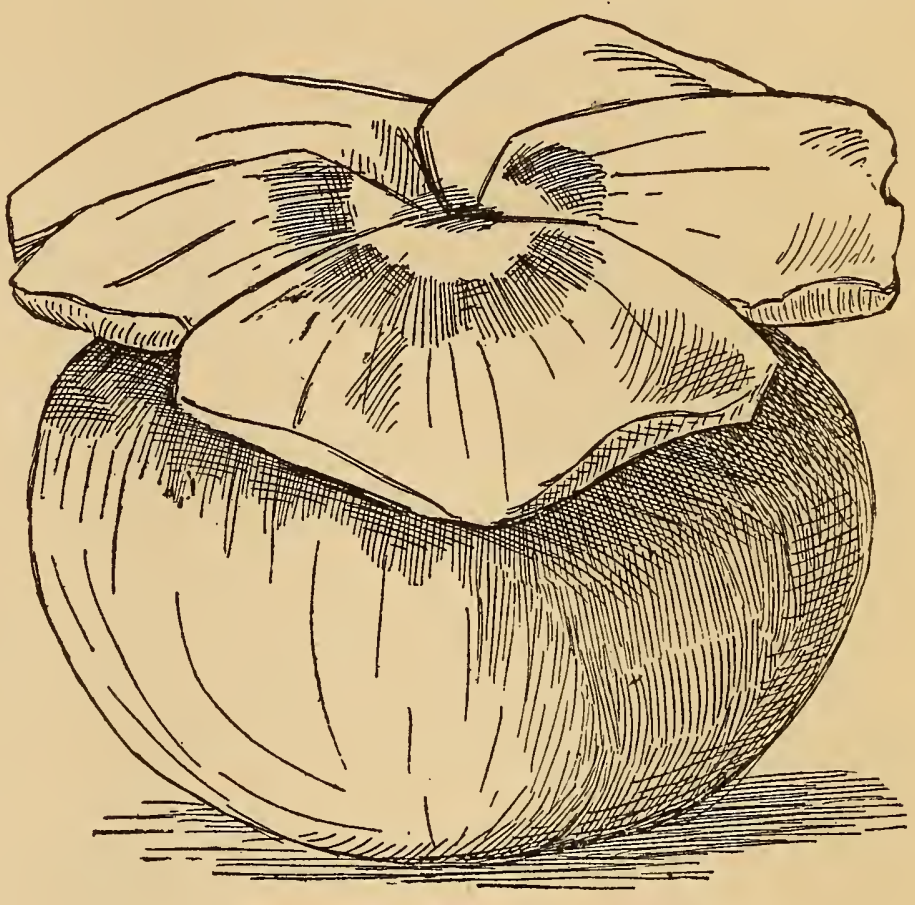

FIG. I89. and round the edges; cut one more petal from an extra piece of paper and mucilage it under and between two of the petals left on the bag. This forms a perfect pansy top to the ball, and the illusion is completed by painting each petal slightly with oil colors in pansy shades. This inexpensive trifle can be easily duplicated from the illustration.

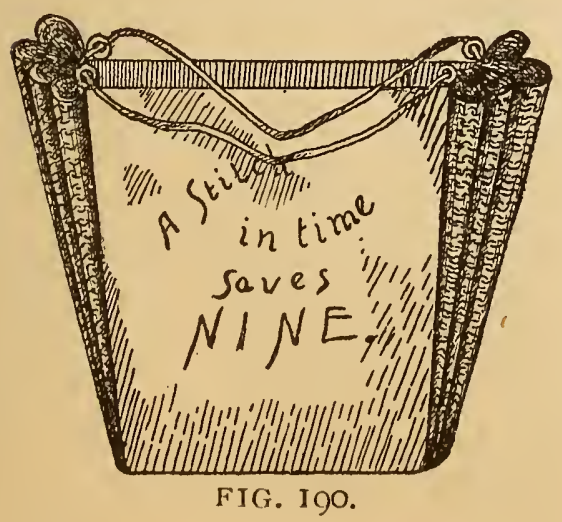

FIG. I9o.

\section{MENDING BAG.}

This simple and inexpensive mending bag would be a gift that would delight the heart of the busy housewife at any time. To make: Cut three pieces of cardboard, as follows: Two pieces for front and back, each I I 99 inches at the top and narrowed to $7 \mathrm{x} / 2$ inches at the bottom; one piece $3 \frac{1}{2} \times 7^{1} / 2$ for the bottom. Line these three pieces on both sides with plain sateen, pink, blue or brown, and join them together by overhand seams on 


\section{DAINTY WORK FOK PLEASURE AND PROFIT.}

the inside of bag. To make the accordion plaited ends, hem across the top a piece of cretonne I $3 \times 2 \mathrm{I}$ inches, and gather it at the bottom to fit the narrow end of the bottom piece of bag, to which it must be fastened. Sew the sides of the cretonne, which have been reduced to eleven inches by the hem across the top and the gathering at the bottom, to the sides of the front and back. This finishes one end of bag; the other must be treated in the same manner.

Across the inside of the back piece is placed a full pocket, eight inches deep, to hold balls, spools, etc. The top is finished with a shirring for an elastic band beneath a narrow standing ruffle. Above the pocket are tacked flannel needle leaves and scissors straps. Sometimes an interlining of wadding is placed beneath the inner cover of the back piece, and the whole used as needle-book or pin-cushion. Sometimes the pocket is omitted and a third piece, just like the front and back, is fitted in between the two to divide the bag into two equal or unequal compartments.

Small brass or silvered rings-or homemade ones of wound wire, covered with knitting silk or floss in closely worked double crochet-are sewed to each upper corner of the front and back, and three more are sewed crosswise to the hem of each side piece-one in the middle and one four inches from each corner ring.

A narrow stitched band of the plain sateen is run through the rings, and the ends are neatly joined. This forms the means of suspension, and also allows the bag to be opened easily and widely when in use. The lettering is worked with silk or floss in colors to match the figures in the side pieces.

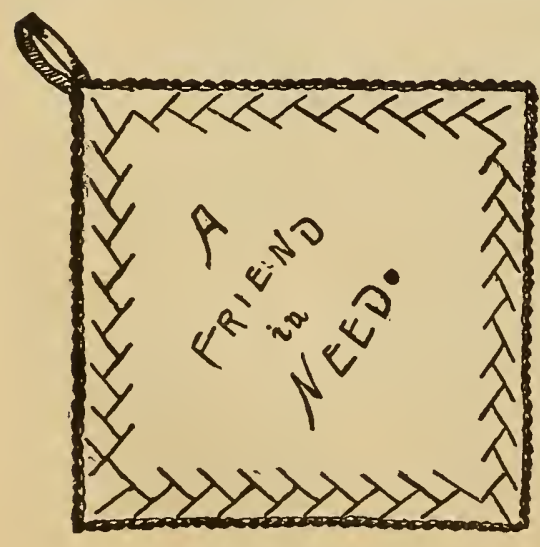

FIG. I9I.

HOLDERS.

These little articles do not find their field of usefulness bounded by the kitchen walls. They are quite frequently to be met with in the sitting room, in the library or living room, and sometimes in the parlor-but always in Sunday best, it must be confessed. Seriously, a good supply of holders, for various uses, are most convenient, and they may be made quite ornamental with little trouble. Our modei, figure I9I, is made of a square of dark brown felt, cut from an old felt hat that 
had been discarded. The edges are bound with a narrow red silk ribbon and ornamented with satin stitch in red etching silk. The letters forming the words are made in outline stitch with the etching silk. A loop of ribbon is added to hang it up by. These holders may be made of a variety of materials. Those used about the table should be made of two pieces of white canvas or linen, lined with sheet wadding on each side. Work one simple design in the corner; buttonhole the edge with linen floss. The merest scraps serve to make them.

Where it is necessary to make a large number of Christmas remembrances these inexpensive articles will serve for many of them. Of course, one would naturally make up ornamental holders for this purpose, rather than those for kitchen use, though even these would prove acceptable tc many a busy housewife. A good material is colored Hollywood drapery or the cream white if preferred.

\section{PAPER HOLDER.}

A piece of pasteboard, about $18 \times 22$ inches, is used for the back foundatıon. This is covered with garnet satin and decorated with brocaded ribbon of a lemon yellow; the front is made of garnet satin, lined with lemon yellow, and is slightly rolled back to show a portion of lining (see figure 192). Between the outside and lining put a stiff piece of buckram. On the front stamp a spray of yellow Marguer-

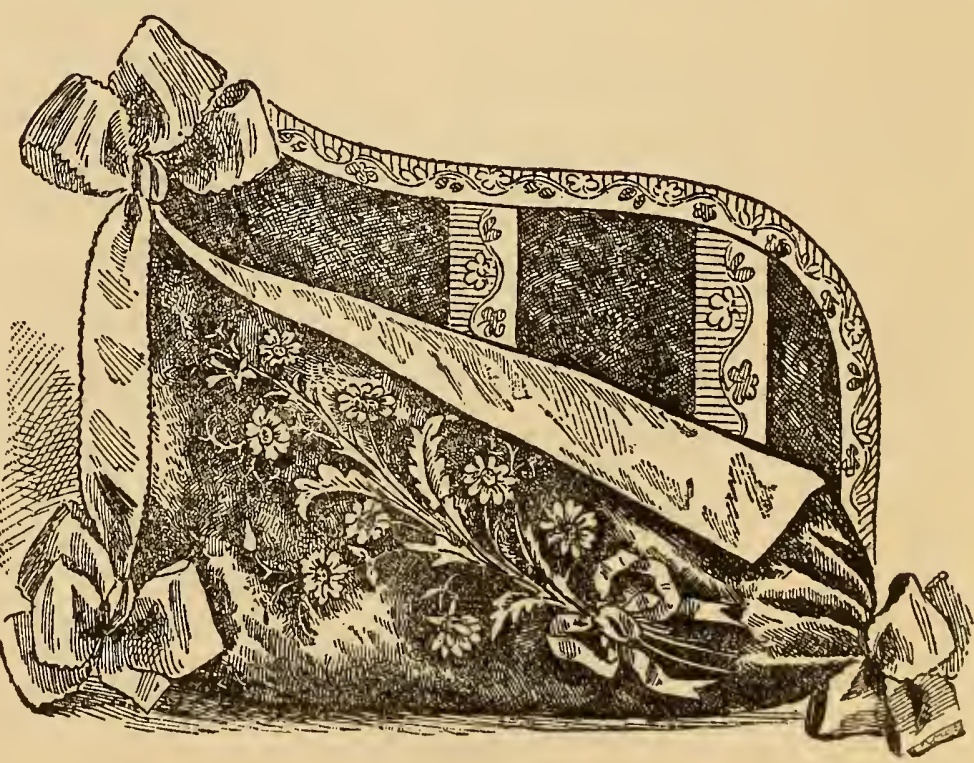

FIG. I92. ites; work with filo floss, Nos. I262, I 263 and 1 264, using brown for the centers. For the leaves use the olive green.

These holders are useful as well as ornamental, and may be made of a variety of materials. If satin and silk are too expensive, celluloid or eggshell board may be used, in which case the design must be painted. 


\section{HANDKERCHIEF SACHET.}

The illustration shows a handkerchief sachet made of cream white plush lined with quilted blush rose satin. The edges are bound with silver

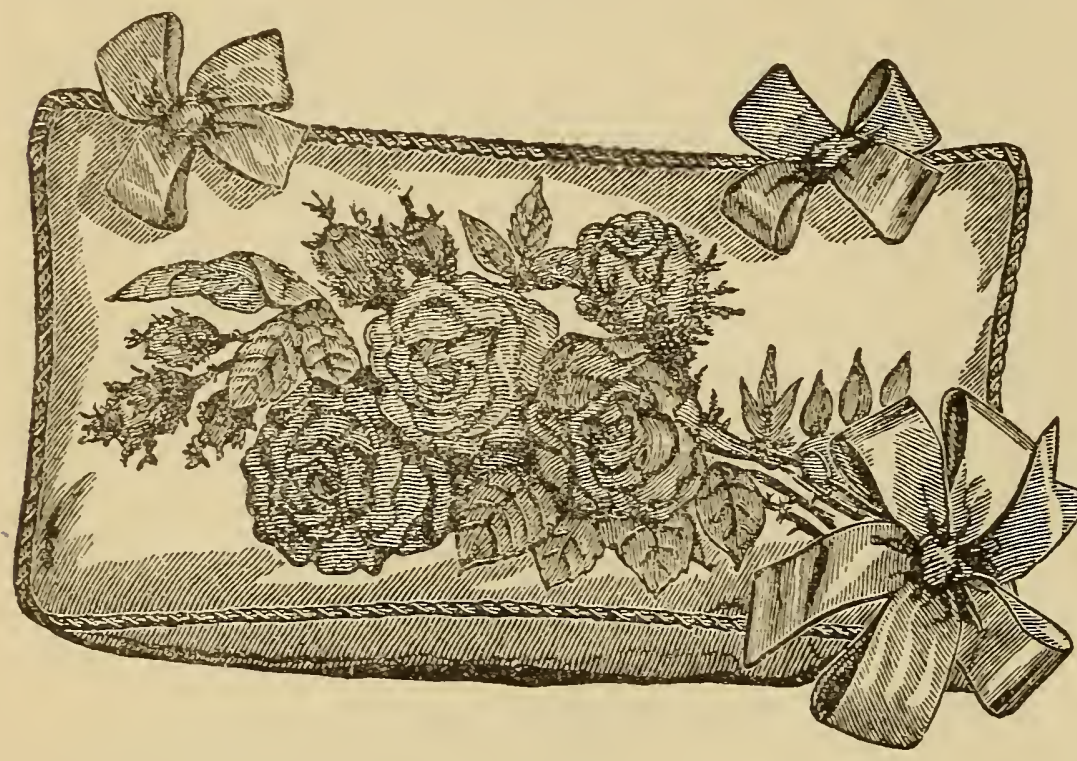

FIG. I93. cord, and bows of pale pink ribbon join the back edges together and ornament the front edge of sachet. The design is a cluster of double ribbon roses and buds with stems and foliage in chenille. The roses are made of pale pink faille ribbon. For directions for making see "Double Ribbon Roses," in Chapter XI. Dimensions of sachet are $12 \times 16$ inches. The design may be embroidered in solid Kensington stitch in Royal floss if preferred.

\section{FANCY TABLE.}

This dainty little table is at once useful and inexpensive, and best of all, easily made at home. The material need be nothing better than common pine, two pieces for top and shelf, $24 \times 24$ inches, one inch thick, and four pieces two-inch stuff for legs. The top, shelf and legs can be covered with art linen in yellow brown, old blue or shrimp pink, or the whole can be treated to a coat of English enamel, any color desired. The enamel is 40 cents a jar, and one jar will do considerable decorating. If the linen is used as a cover, a pretty decoration would be an all-over Roman embroidery design stamped on the cover and worked out with white linen floss. Instead

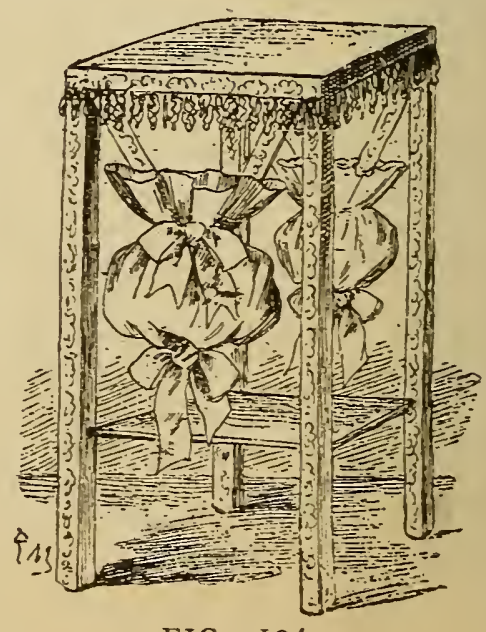

FIG. I94of cutting away the ground from back of the lace lines it would be better for this purpose to outline them directly on the material. 


\section{SUNFLOWER PENWIPE.}

Stamp on chamois skin any one of the many pretty sunflower patterns that can be had for five cents, and paint the petals with chrome yellow and burnt umber; cut the flower shape out, and finish center by putting in a circle of brown plush over a padding of wadding. This makes a useful as well as ornamental adjunct for the writing table.

The same design may be made from yellow flannel or felt for the petals and brown velvet for the center. To make from these materials it will be better to cut the

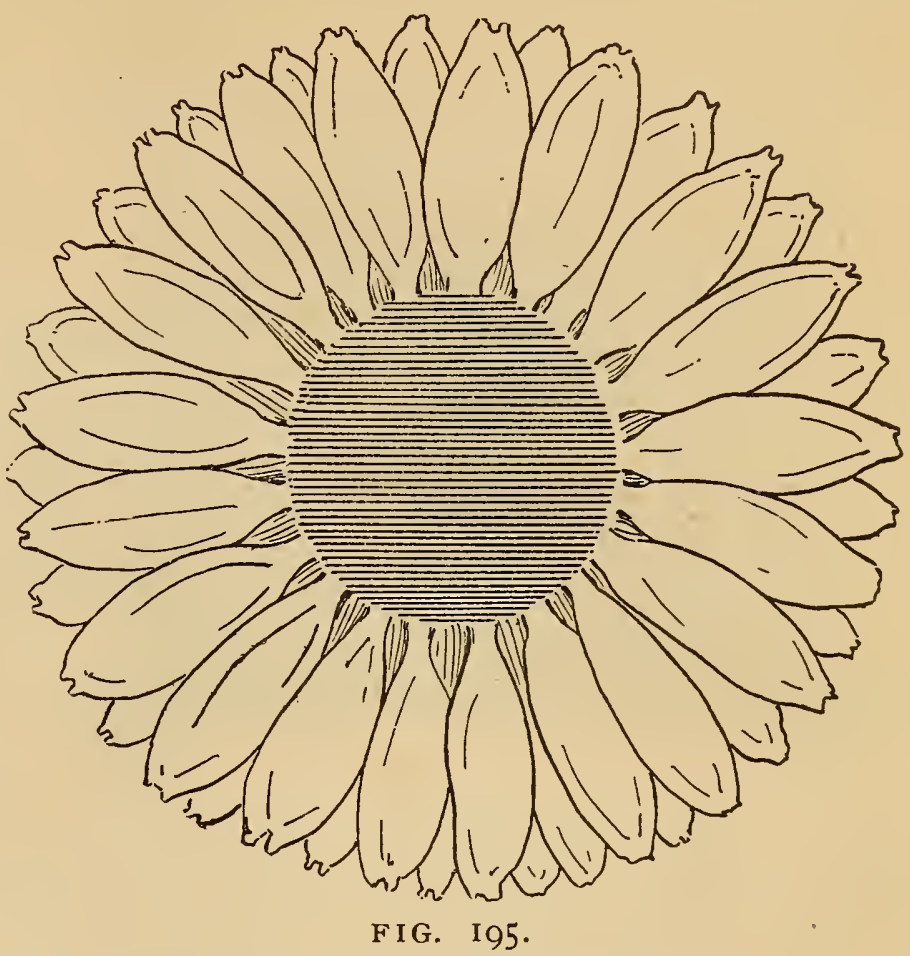
center from cardboard; cover it with the velvet, cut the petals out of the felt and fasten on to the back edge of center. This is a little more work than the first mentioned method.

\section{SOFA CUSHION.}

In figures I96, I97 and 198 we show three very comfortable as well as

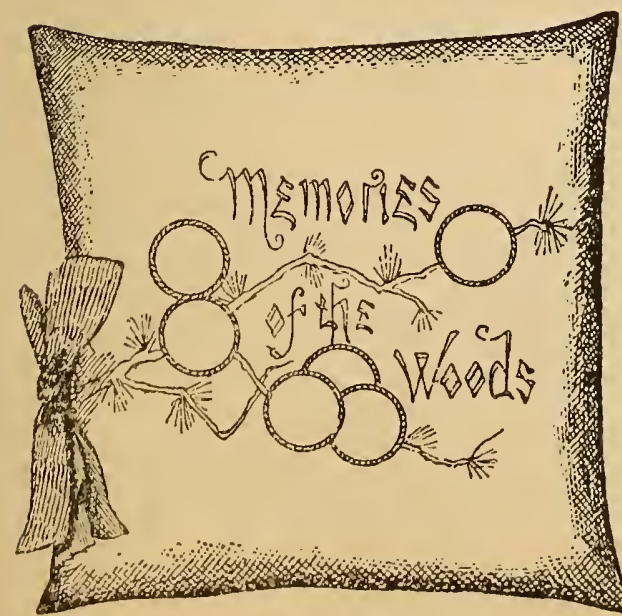

pretty sofa cushions. These particular cushions are not designed to repose in state, at a most uncomfortable angle on the best sofa in the best room. They are for everyday use; and, as comfort has been considered, the cushion proper has been made nearly square and lightly filled with feathers or hair. It is a great mistake to stuff a sofa cushion until it resembles a small mountain; it should be just high enough to fill the space between IFI G. I)6. the head and shoulders. As has before been stated the cover for cushions that are to be used should be made of 


\section{I96 DAINTI WORK FOR PLEASURE AND PROFIT.}

wash fabrics. Figure 196 is made on ecru twilled linen and the design shown is worked in outiine stitch with three shades of wood brown linen

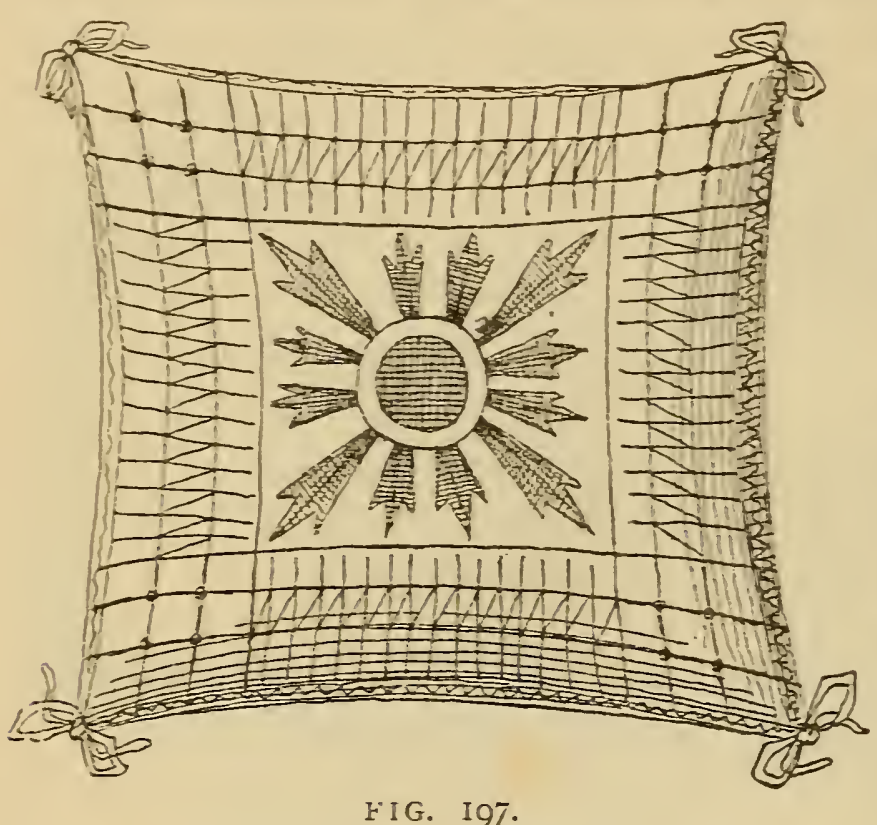

Hoss; a bow of brown ribbon finishes one edge. This is a very beautiful design, and amply repays all the work one chooses to put on it.

The cover for figure 197 is made of white linen having a border all around treated with drawn work; the center is embroidered with a set figure in white linen floss. The under cover is of the same material, without the decorative work, however; the "upper and under swers are laced together around the four sides and fastened at the corners.

Figure IgS shows a round cushion for sewing chair. The cushion is first made of unbleached cotton or cotton twill, and for comfort, filled with feathers; do not stuff it too full. Put on an outside cover of old blue tapestry linen, blind sewing the edges; fasten to the chair by means of straps on the under side caught to the seat; over the cushion fasten an embroidered cover of white linen edged with lace or drawn work. The design shown in illustration is powdered forget-me-nots worked

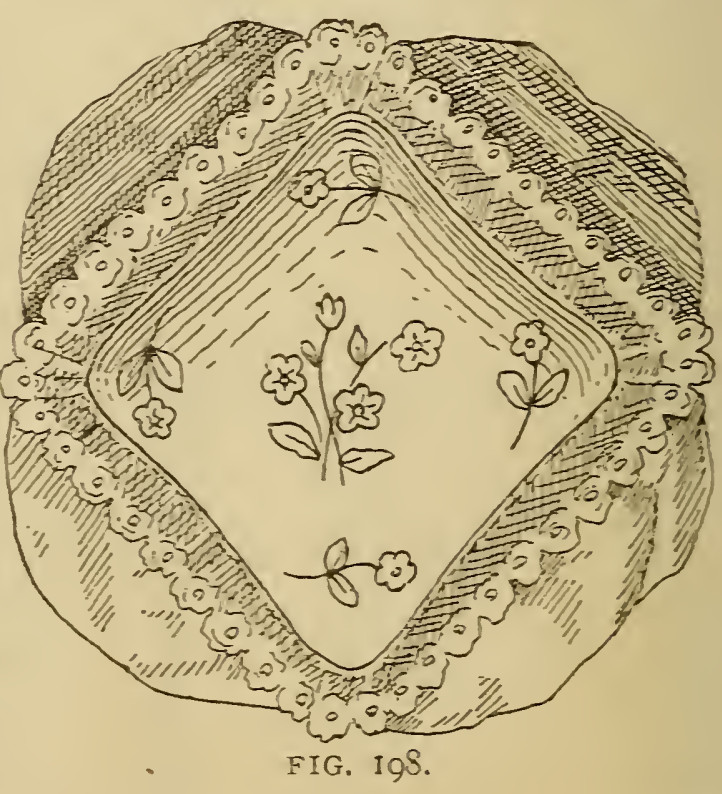
with old blue filo floss in satin stitch with a center of yellow French knots.

\section{FANCY WORK BASKET.}

The basket shown here is eggshape. The best way in which to prepare the foundation is to cut an oblong circle out of heary straw board, wet it 
thoroughly in cold water and roll up in the form of an egg; leave an opening to the side from end to end; secure in position with a string. Let it become quite dry, when it will retain its shape without trouble; prepare a lining of silk for the inside, which should be wadded lightly and quilted, fastcn this in the inside of the prepared shape. For the outside cut a circle out of cream satin the same shape as the foundation, stamp on it a cluster of pansies, work with wash filo floss in numbers I300, I30I, I302 with a dash of I $260, \mathrm{I} 264$; for the leaves and stems use

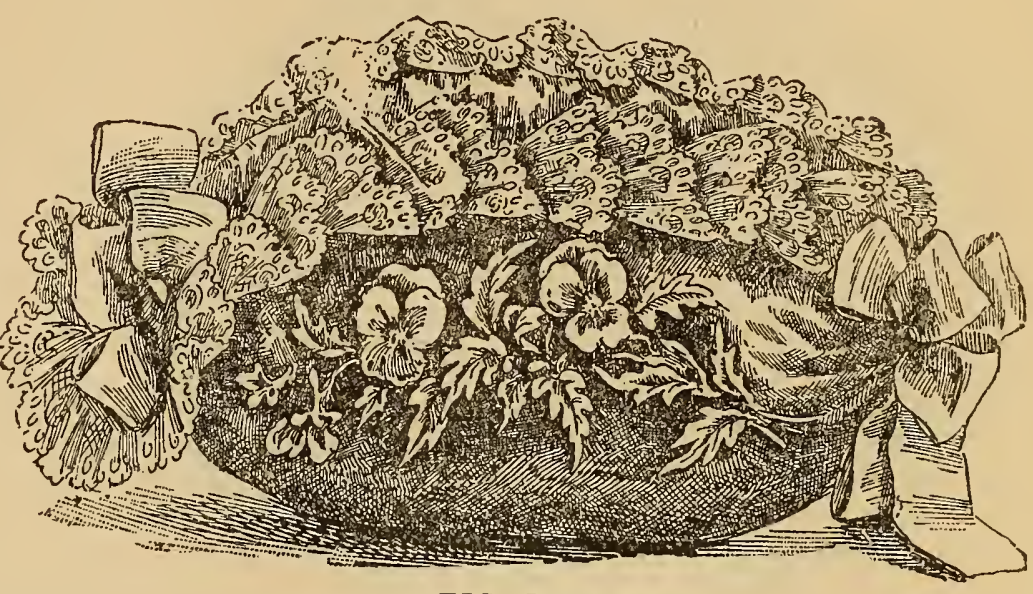

FIG. I99. numbers I248, I249, I25I. Stretch this cover over the shape, being careful not to leave any wrinkles; finish the opening with a frill of soft lace and ornament each end with bows of ribbon in two shades of numbers 1300 and I 30 I. The effect of this little trifle depends upon the neatness with which it is made. It should look light and dainty and not in the least useful, and, of course, is better adapted for fairs and bazaars, where it will be certain tu attract purchasers, than for sober everyday use. Made of more durable materials they are useful catchalls for the dressing table.

\section{FOOT CUSHION.}

Small cushions are much used in place of foot stools. The mode:

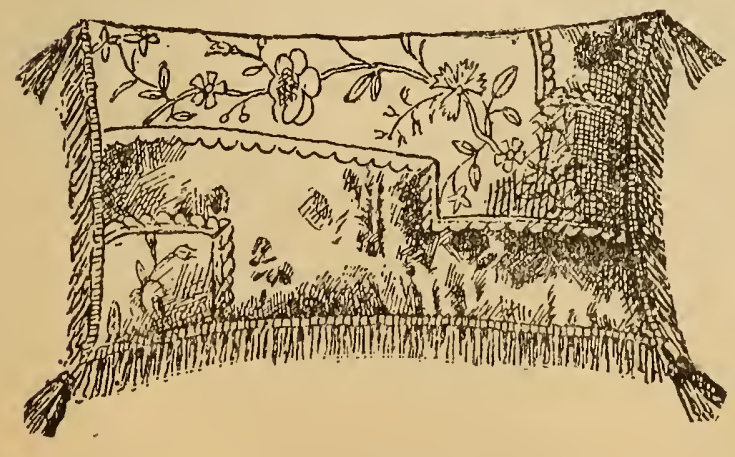

FIG, 200. shown in figure 200 is made of clianois skin. The cushion should be first made, and then covered on top only with the chamois cover. The designn should be worked out with golden anc bronze brown Royal floss and the scctions should be defined by sewing on flat gold braid. The ends and one edge of cushion should have a row of narrow gold fringe, and bullion tassels should finish the four corners, 


\section{OTTOMAN CUSHION.}

Round cushions for ottomans and foot-rests are always useful ornaments, and the two designs given in figures $20 \mathrm{I}$ and 202 are among the

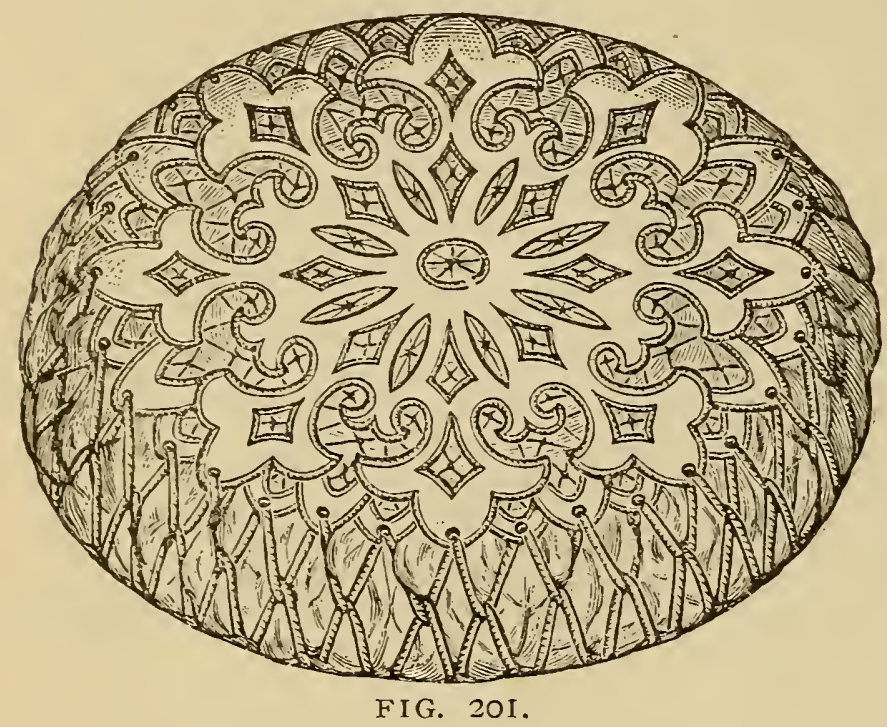

prettiest we have seen. Figure $20 \mathrm{I}$ is covered with Romar: embroidered cover, and the cover for figure 202 is treated with applique. The cushions can be purchased stuffed, ready for covering, for 75 cents each; however, if one prefers to do this part of the work at home, two circular pieces, the size the cushion is desired, should be cut from heavy twilled cotton and a bias strip, four to six inches wide, should be sewed around the circumference of one of the circles, the other circle should be sewed to the other edge of the strip, leaving an opening for stuffing. When the cushion is designed for a foot-rest, excelsior will be found a good stuffing; if for a seat to an ottoman, then feathers or hair will be more comfortable. After the cushion is made, the bias piece forming the sides of the cushion should be covered with a very full puff of China silk. The silk must be sewed to the cushion itself, as it is entirely distinct from the covers. For the covers cut two circles, the size of top of cushion from ecru linen; on one of these linen covers stamp a

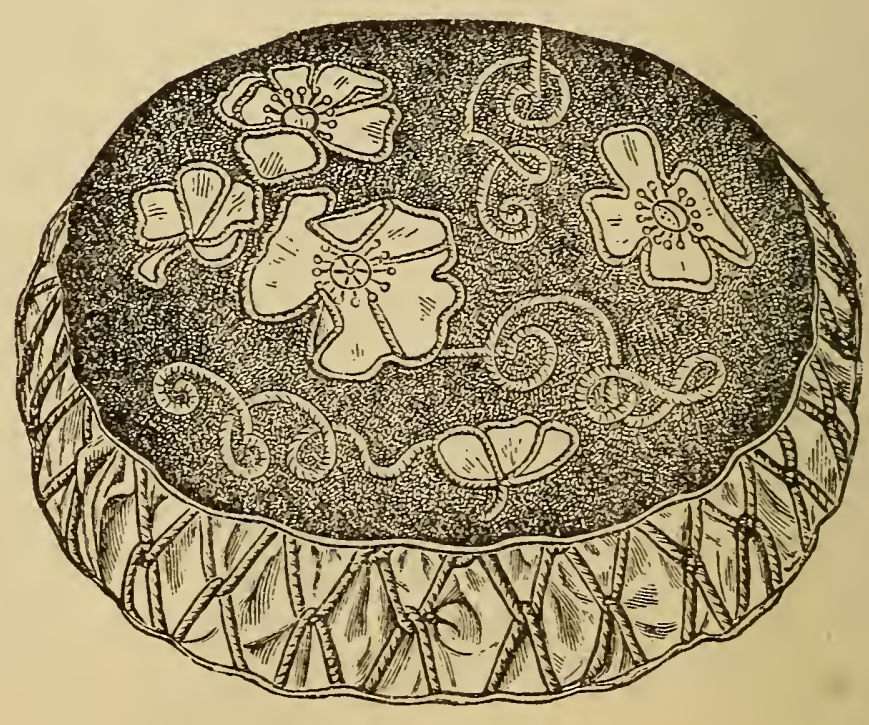

FIG. 202.

Roman embroidery design and work it with brown or white linen floss. Do not cut the material away from the background unless it is intended to line the cover with silk, but out line the lace stitches instead of carrying across 
from one outline to another. On the under cover work a row of buttonhole stitches, and at equal distances apart sew to the edges of both covers brass rings which have been crocheted over with the linen floss. Place the cushion in the center of the bottom cover, and over it put the top cover; lace them together over the cushion with heavy silk cord.

The cover for figure 202 is made of deep blue mail cloth on which is arranged a design of poppies, cut out of white ooze leather. Select any design having full blown poppies in one or two positions; stamp them on the leather, cut out with a pair of sharp scissors and glue to the foundation. When the design is in position, outline all the edges of the poppies with gold cord or bullion. Fill the center with French knots made of gold thread, and for the scrolls couch down white couching silk with gcld thread. The puffs should be white India silk and the covers should be laced together with gold cord.

These two cushions are very desirable for church fairs, etc. Of course the cushion may be made of other materials and combination of colors, and decorated to suit individual taste.

\section{SHAVING PAPER CASE.}

This little trifle is made of two pieces of cream tinted eggshell board, $7 \times 9$ inches in size. The design shown should be painted on the outside cover in oil paints; the edges of both front and back covers should be edged with gold paint; prepare a dozen sheets of pink and white tissue paper, the same size as cover, place evenly between the two eggshell board pieces and punch a hole in both ends, large enough to draw through a pink ribbon. Tie in small bows.

This makes a dainty gift for a gentleman friend at little expense.

Celluloid or leatherette may be used in place of the eggshell board; or, a cover can be made

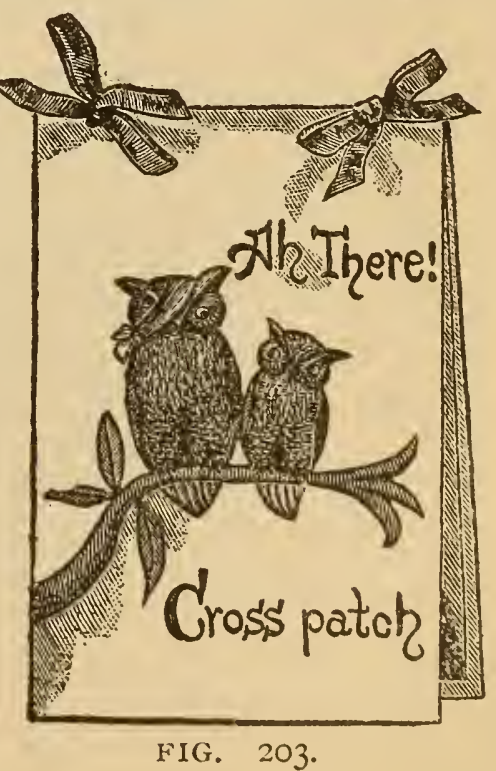
of white canvas, embroidered all over with a Dresden design; in this case, a pair of cardboard covers should be cut and the embroidered can vas fastened on the outside of each cover. 


\section{LAUNDRY BAG.}

Ornamental bags for small toilet articles, such as collars, cuffs and handkerchiefs are a necessary luxury, and the methods of making and

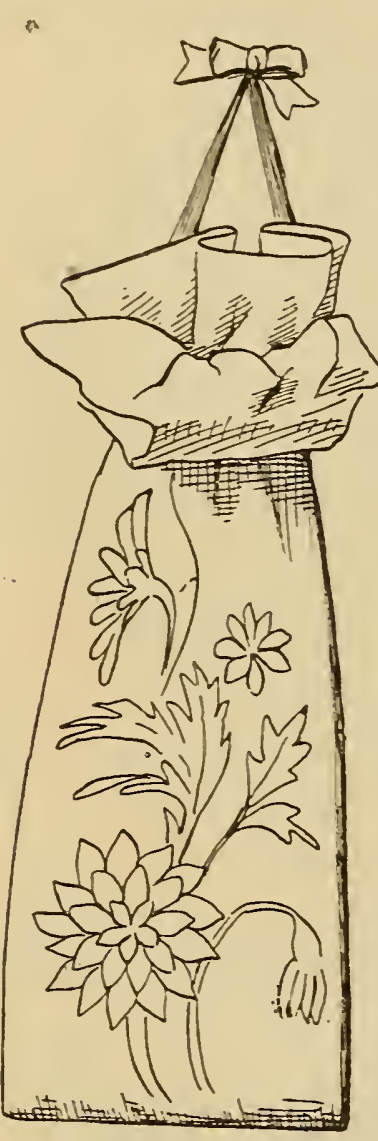

FIG. 204. decorating are almost numberless. It is, however, desirable to have them made in such a manner that they can be laundered when soiled. Figure 204 shows a bag made of a strip of cotton canvas, 36 inches long by 12 inches wide. The canvas is folded in the center of the greatest length; the two sides are sewed together, making a bag 18 inches long by 12 inches wide. Face the top of the bag to a depth of three inches with pale pink satin or silesia, and run in a shirring strip of pink ribbon at the bottom edge of hem, putting in two rows of stitches for that purpose. The ribbon should be long enough to tie in a bow and to form a loop to hang the bag up by. The decoration may be a simple floral arrangement outlined with silk or linen threads, or short and long stitch may be employed, if the article is desired to be very ornamental.

These bags may be made larger than the model when intended for larger articles than here mentioned, and Bargarren art cloth will prove a most serviceabit material for the purpose. The bottom of a bag made of this fabric coula be darned through each mesh, straight across the bottom, with Bargarren linen threads, to a depth of six inches. The top part that forms the hem could be treated in the same manner, and the center should have a design outlined with the same thread. Again, useful and durable laundry bags may be made of crazy patch work-silk and velvet pieces are not necessary, cashmere and other woolen material will work up with good effect. Put a chain stitch with etching silk over all the seams; face the hem with a piece of bright colored silk. A number of laundry bags of different sizes will always find sale at fairs and bazaars; beside they make a most acceptable Christmas gift to any woman. The expense of getting them up is but trifiing, provided one exercises common sense in selecting the materials 


\section{PUMPKIN CUSHION.}

Figure 205 shows a design for a pumpkin cushion. It is intended for use on the dressing table and is made as follows: Make a round cushion the desired size and stuff with cotton or hair; cut two semi-circles of yellow satin or surah silk and cover the cushion. Divide into sections as shown in the illustration by passing a long needle threaded with knitting silk or etching silk through the cushion as follows: Put the needle up through the center of cushion to the center of top; pull it up and carry the thread down the outside of cushion and secure it in place by put-

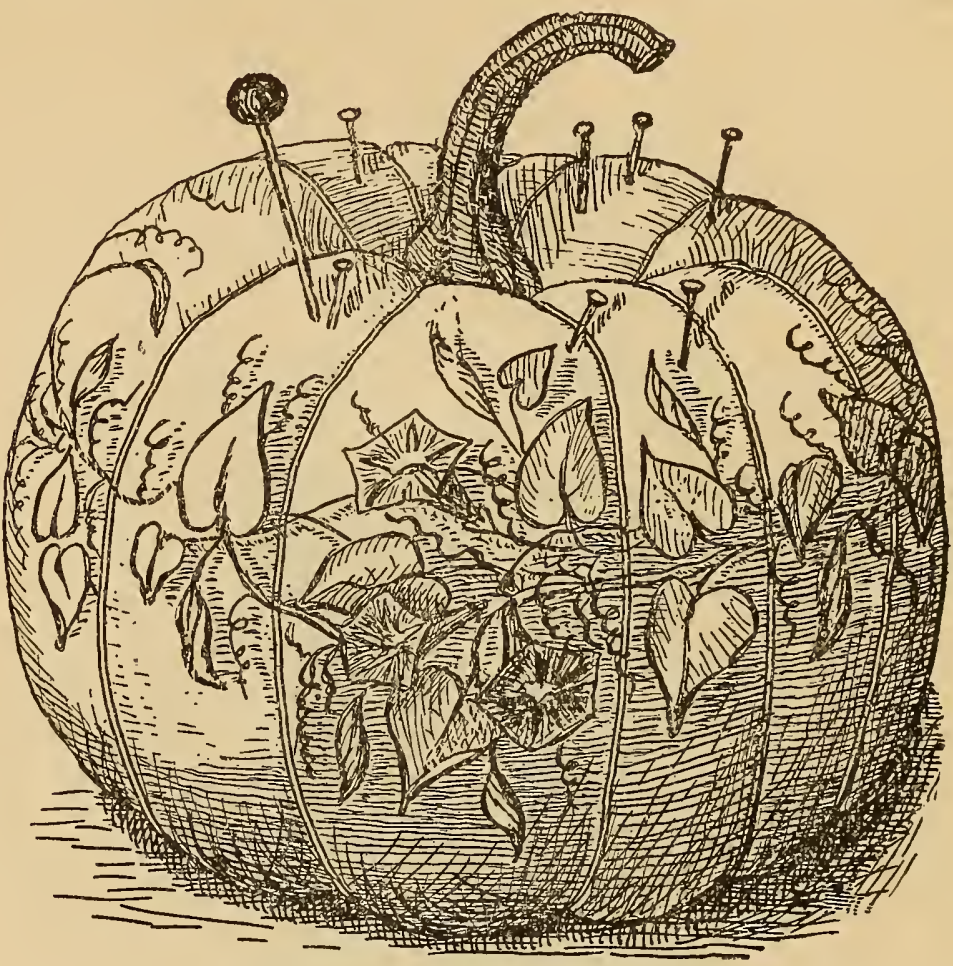

FIG. 205 . ting the needle up through the cushion to top again; this forms one section. Repeat this, carrying the thread down the sides of the cushion at equal distances apart, until you have marked off as many sections as are desired. Fasten the thread at the top of cushion where the handle is to be placed. Two curved pieces of cardboard should be covered with green silk and glued into position for a handle. The embroidery must be done before the cover is put on the cushion. The model shows a vine of morning glories worked with one strand of filo floss in short and long stitch; blue-palest shade-for the flowers, and two shades lily green for the stems and leaves.

Provided one can paint and would prefer that style of decoration, the cushion may be made of cream white satin, which should receive a ground. ing of deep yellow over which the design should be laid in.

A cushion made after this model would be sure to prove a great attrac. tion at any sale, or would be a very pretty gift for any occasion. They need not be so elaborately decorated unless desired. 


\section{PHOTOGRAPH FRAME.}

Figure 206 shows an easily made photograph frame, suitable for panel picture. To make: Cut a piece of cream white leatherette, $12 \times 15$ inches. Begin in the center and cut a slit toward the four corners, and bend the pieces of leatherette outward toward the side; this will form an opening, the size of which will depend upon the length each slit is cut. It must be

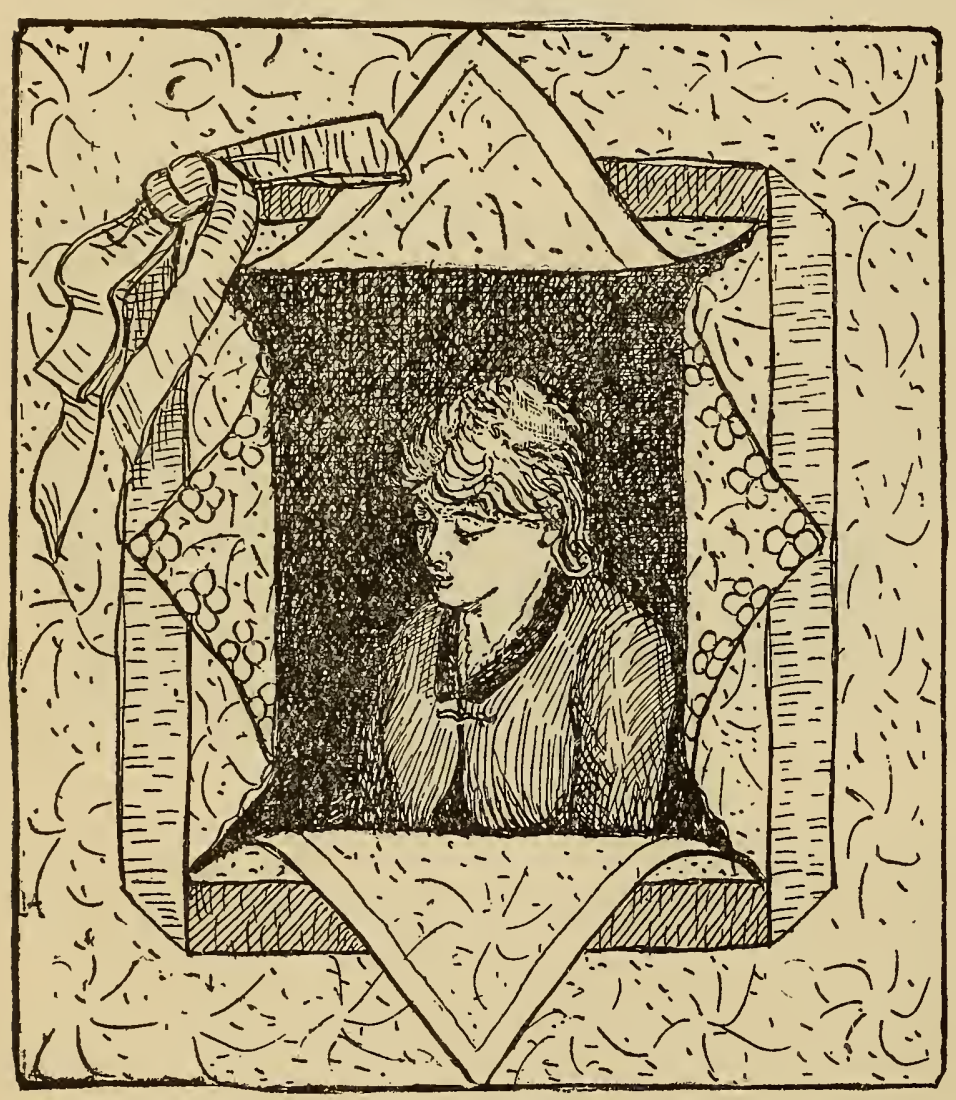

FIG. 206. cut to fit the picture the frame is designed for. The turned back pieces were decorated with gold paint -the upper and lower piece with a band of gold three-quarters of an inch deep and the sides a fioral arrangement of roses or pansies without foliagesee illustration-and painted with the gold paint. The piece of leatherette decorated as directed should be backed by a piece of stiff cardboard a size smaller, and the edges of the leatherctte should be folded back to the card. board and glued down; slip the photograph into the opening. Select two and one-half yards No. I2 satin ribbon of a rich gold color and place it squarely around the opening in the frame and tie a bow at the upper corner-complete by fastening a wire standard in the back to support the frame.

Any other material may be used for the frame, as eggshell board, celluloid, ivorine, plain cardboard covered with satin decorated with fat embroidery. The outer edges of frame, made as directed, should have a gold band one-quarter of an inch wide, painted all around; or the edges may be picked out in gold in vandykes. 


\section{PHOTOGRAPH ENVELOPE.}

An accumulation of photographs beyond the capacity of the family album is an embarrassment of riches to many people. A convenient receptacle for these "left-overs" will be found illustrated in figure 207. To make: Cut out of parchment paper a sheet 7 XI 3 inches; fold the two side flaps together down the center and cut into shape at the bottom edges; turn the bottom of the parchment up to one-half its depth and cut into shapesee illustration, figure 207. The flaps are to be left loose, as the envelope is to be placed upon a table, and this method allows the photos to be examined without fear of tearing their edges in removing from and returning to the envelope. The decoration may be made to suit the fancy; the model has an arrangement of

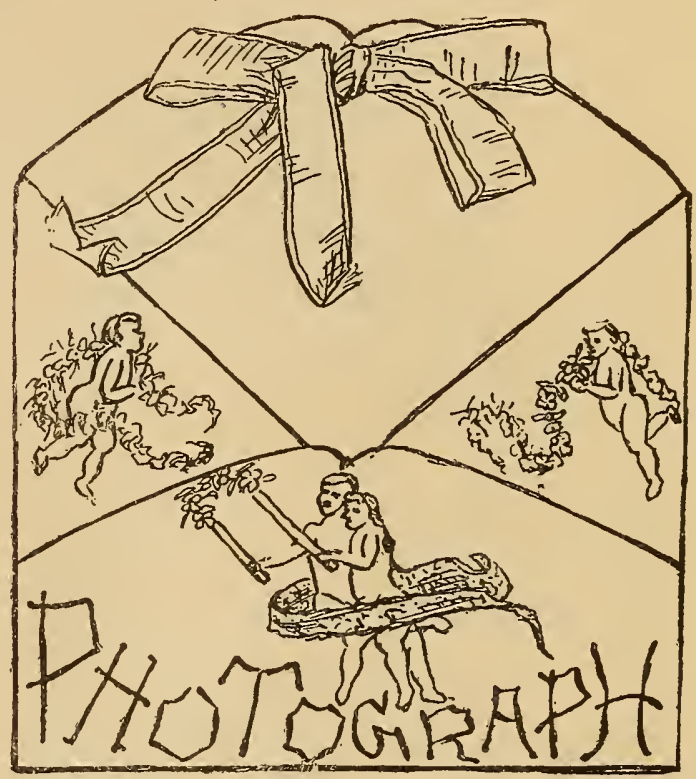

FIG. 207. cupids done in water colors; the lettering is done with gold paint, all of which can be easily accomplished by the merest novice with brush and pencil.

\section{PIN TRAYS.}

Small trays for holding pins and loose toilet articles are convenient for

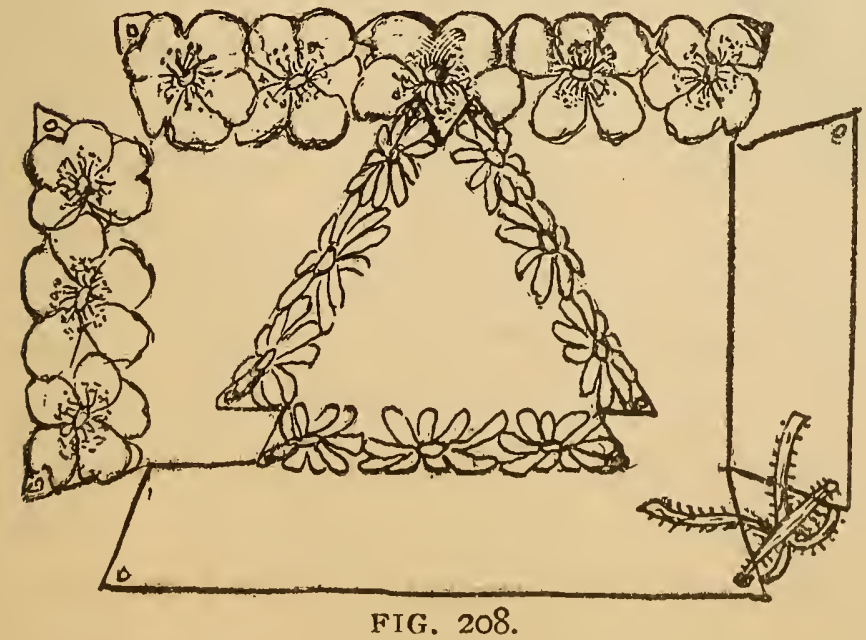
the dressing table. It is not always desirable to stick pins. big and little, into a handsome cushion, and these little trays make a most convenient receptacle. The model, figure 208 , shows two shapes, one inside the other, for making. The larger tray is made of a piece of ivorine six and one-half by nine inches. Slash the corners of the ivorine in to a depth of one and one-quarter inches, beginning one inch from the points; this will give a slant to the sides and ends when 
turned up; the edges should be turned up to one and one-quarter inches, forming a shallow pan. Use the polished surface of the ivorine for the outside of tray; on the inside edges paint a row of wild roses, without foliage, in oil colors-see figure 208. Cut the outer edges of the rose petals out to form an ornamental finish and fasten the corners together with tiny bows of ribbon. The tray in the center of illustration is cut in triangular shape, of the same material, and painted on the edges with a row of daisies. These trays can also be made of heavy linen twill; hem the edges and turn up to a depth of one and one-half inches; fasten the corners with a securing stitch and ornament the bottom with a Dresden design done with silk or linen floss. The twill should be heavily starched before turning the edges up; this keeps it in shape.

\section{GLASS HANDKERCHIEF BOX.}

The article illustrated in figure 209 is at once one of the simplest and most inexpensive gifts that could be devised. The boxes are made of

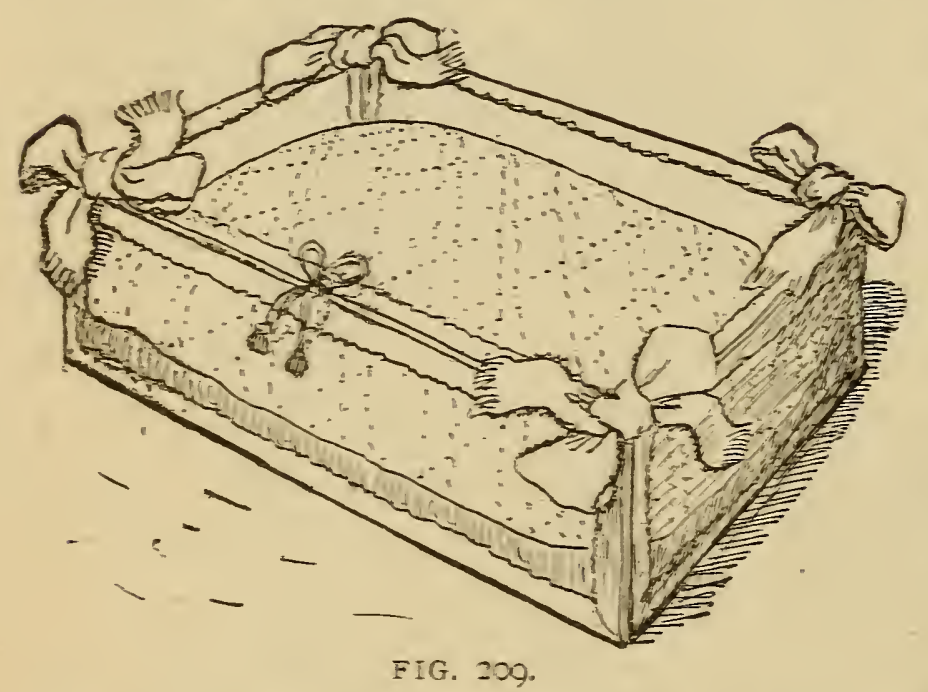

FIG. 200 . glass and may be any size or shape. The model show here was six and one-quarter inches wide, aine inches long and three and one-quarter inches high. To make this box will require six pieces of glass, as follows: Two pieces $61 / 4 \mathrm{x} 9$ inches for the top and bottom, two pieces $3 / 46 \%$ inches for the ends and two pieces $9 \times 3$ If inches for the side pieces. Bind each piece of glass with No. 9 ribbon, any color preferred; draw the ribbon tight while binding and over-cast it at the ends with invisible stitches. Fasten the pieces together by sewing at the corners through the ribbon, which has first been fastened to each individual piece of glass: this sewing must be done neatly and strongly. Finish the corner with ritan bows, also fasten two bows on the back edge of cover to serve as hinges; sew on a cord and tassel to lift lid by, and put in the bottom a suilted silk lining perfumed with sachet powder. 


\section{EIFFEL TOWER SCRAP BAG.}

The above illustrates a very pretty substitute for a waste, scrap or laundry basket, and can be easily made. To make: Cut from card or pasteboard three pieces, 7 $\mathrm{x} 2 \mathrm{I}$ inches in size, for the three sides; a triangular piece measuring seven inches on each of its three sides for the top or cover and a hectagon shaped piece for the bottom, measuring seven inches on each of its six sides. Cover the three sidepieces on one side with duck or canvas, and the other with silesia or anything else you choose, using the latter also tor the top and bottom pieces. Sew the three side-pieces to alternate sides of the hectagon with three half-yard breadths of Chinese or Japanese silk, properly lined, filled between the three boards, beginning seven inches from the top and fulled into the remaining sides of the hectagon. Gather the silk breadths closely i: at the top and fasten the upper portion of the three boards together. Put a ruffle of the silk around the triangular piece and fasten it to one side of the top for a cover.

The painting-it might be

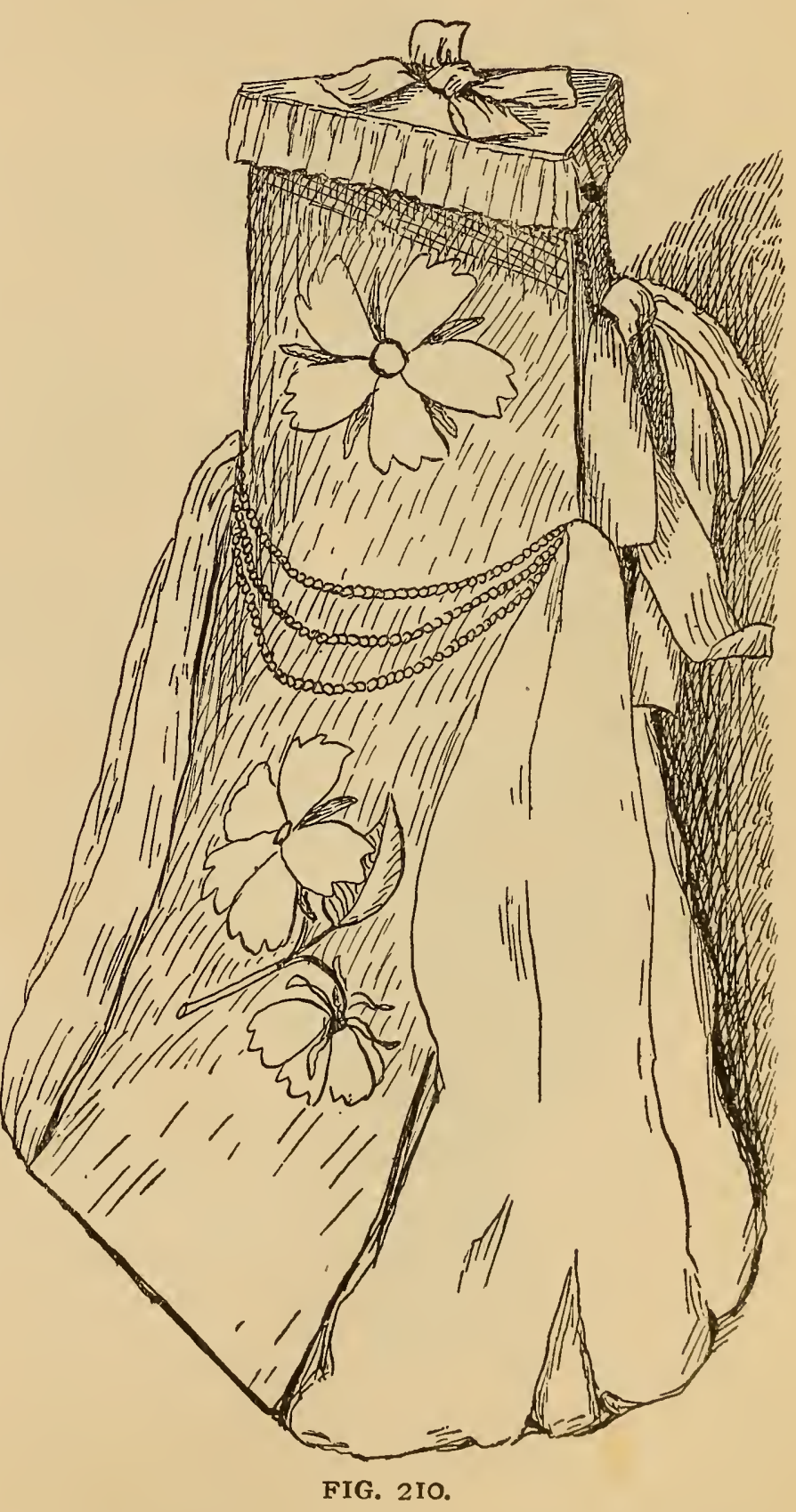
embroidery on appropriate material-had best be done before putting the parts together. The article illustrated has large, dull, yellow flowers, somewhat conventionalized and well shaded in different forms upon the three 
sides, the background being formed by painting the surface of the three pieces over with a delicate bluish gray. The silk is yellow, and yellow satin bows, one on the cover one hanging from the top by an ecru Tyrol cord, finishes the basket. The long lines of the sides are artistically broken by loopings of this cord, across from where the silk fullness begins. Other colors may be used, but all must harmonize or the basket will not be "a thing of beauty."

The most convenient way to paint the panels will be to first cover them entirely and evenly with the gray background; gray is simply red, blue and yellow combined, and is bluish, purplish or greenish, according to the predominating colors in the mixture. In mixing put your red and blue together with a very little ivory black and a good deal of white, and then tone with the yellow. When this background is dry, paint the flowers upon it. For the colors needed and the method of putting them on see chapter on "Brush and Palette."

Should embroidery be preferred, instead of painting, it must be done before the covering is fastened to the pasteboard. Any of the conventional forms preferred may be used. If yellow is the color chosen, Nos. 8I, IO, I $9,82,6$, if red is the color, then $87,58,70$ Real Scotch linen threads will be required. The work should be done in solid Kensington stitch. The leaves should be done in browns or olives. Of course, in case embroidery is used the canvas will not be painted.

\section{STAR QUILT.}

The subject of quilts is a most social topic of conversation. How many a romance has been stitched into patchwork squares will never be known, and the woman who has never solved the mystery of the "Irish chain," "fox and geese" and the "Greek cross" has missed half the sweetness of life. The old styles of patchwork quilts are still made, and probably always will be, but in these days of elegant fabrics and perfect embroidery materials at such fabulously low prices women have been tempted to stray beyond the limits of calico quilts, and indulge in something a little more ambitious. The popular quilt is now the star quilt, and for a raffle piece, for fairs or church festival, there could be no more attractive article. As the stars are worked separately and joined afterward, a number of people could 
work on the quilt at once, and then, too, there is such a variety of work necessary that even grandma's eyesight would enable her to add her mitc. The method of working is as follows: The materials used are first, four yards good quality domestic linen, quite heavy, with a nice smooth surface,

a quality worth about 35 cents per yard will do nicely, three bunches etching silk, or Real Scotch linen floss No. 8, two gross smallest size brass rings, one six-pointed perforated star pattern to stamp with.

Place the linen on a smooth surface, and stamp the star pattern over the surface, being careful to economize space, and yet leave room for working. After the stamping is done, cut out the stars on the square, that is, do not cut the points out, but cut a square with the star in the center, leaving about one inch margin fromthe end or point of star; this is for convenience in working. When the stars are all stamped and the squares cut out, you are ready to proceed with the fancy work. With the etching silk work a heavy buttonhole stitch (see illustration page 208) over a thread of Bargarren linen, or o size German cord around all the points of the star. Then, with the same silk fill each point of the star with one of the filling stitches, either fish net, brick cross, crows-feet, or any that is pre-

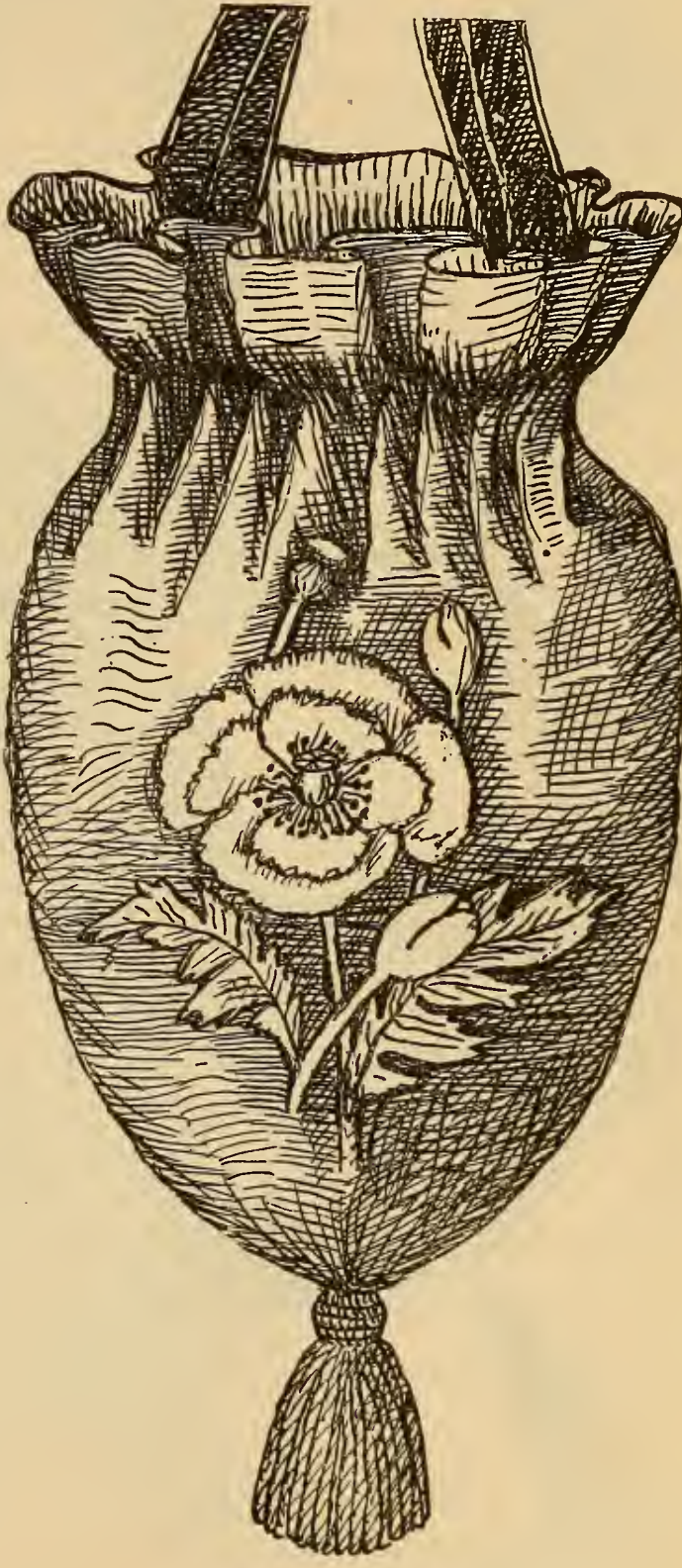

FIG. 2 II. ferred; each star however, should contain but one kind of filling stitch; but each star in the quilt may be of a different pattern, that is, use fish net in one star cross stitch in another, and so on. For the center part of star, crochet a sufficient number of the brass rings with the etching silk, and 
208 DAINTY WORK FOR PLEASURE AND PROFIT.

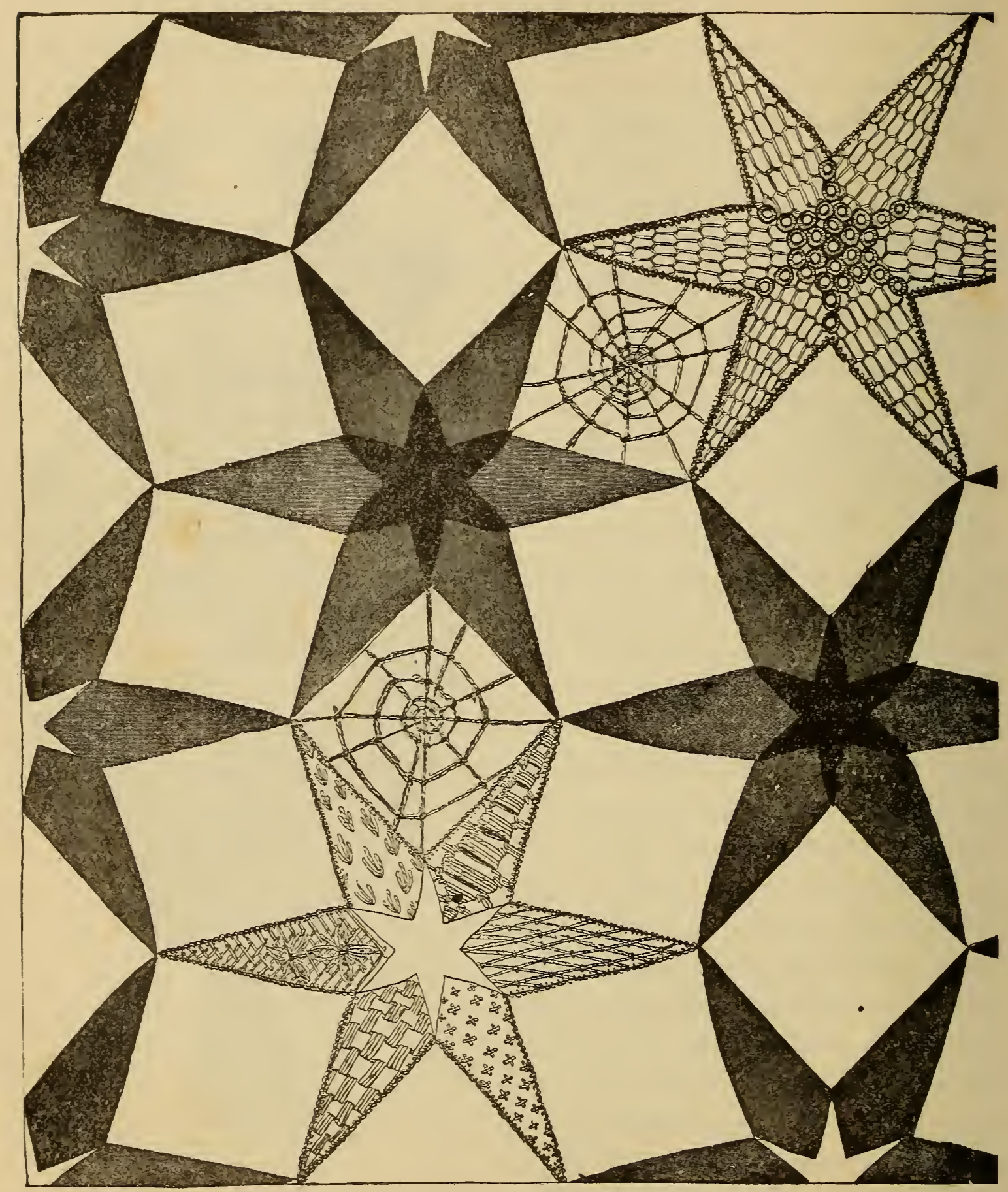

STAR QUILT-SEE PAGE 206. 
sew them in a circle to the linen. This makes a very effective finish. After the embroidery is done, cut out all the linen from the points of the star with a pair of sharp scissors; this will leave a six-pointed star only, w:th no surrounding linen. Thirty-six to forty-eight stars will be necessary for the spread and they must be set together by the points. The edges will, of course, be formed of the points, and from each should be suspended a tiny silken tassel. The colors of silk used is a matter of fancy, but the handsomest are gold on white, old rose and white, blue and white, green and white, and exquisitely beautiful is an all white quilt.

To bottonhole the edges of star will require about one skein etching silk, while the filling stitches in the points of star will require three to four skeins, and for the tassels, one skein etching silk will make three.

The illustration on page 208 shows two of the spaces formed in setting star together filled with cobweb stitch-this method is not arbitrary, as the quilts are quite as frequently finished without as with, but of course this method adds much to the beauty of the quilt when finished. As will be seen, threads are carried from different portions of the star points, across the open space, these are worked over with cobweb stitch, which is simply running a silk thread alternately over and under the crossing lines, loop over in each instance with buttonhole stitch-this will hold the silk in place. The cobweb can be made open or close.

The illustration on page 208 shows two of the stars worked, each after a different method. One has all the stitches alike, the other has six different stitches; the balance of the stars are not shown with stitches, they are merely added to show how to set a number of the stars together, and how to fill the spaces. We do not think there can be the least trouble in following these instructions.

\section{HANDY BAG.}

Fancy bags are very convenient receptacles for the odds and ends that accumulate, and which one occasionally has use for; suspended on the wall, in a corner or on a table in the sitting room they are useful and ornamental. These bags should be decorated with needlework and made of suitable material, art linen, silk, tapestry linen or cloth being best adapted for the purpose The mcdel, on page 207 , shows a bag having the bottom gath. ered in a point and finished with a tassel. The material is moss-green? 
satin, for the outside; the lining is of pink sateen, faced on the top to a depth of three inches with pale pink China silk. A shirring is formed at the bottom edge of the facing, into which an elastic rubber band is run. This draws the top of bag together. The handles are formed of No. I2 pale pink satin ribbon, fastened to the bag as shown in the illustration. The design ornamenting the front is a branch of pink daisies. The flower is worked out with four shades of pale pink filo floss, shading from the palest shade made down through four shades, in Kensington stitch. The center of flower is filled with French knots. The leaves and stems are done with the same stitch, in three shades of silver green. The buds are worked with the deepest shade of the green and tipped at the very point with a few stitches of the deepest shade of pink. The tassel at the end may be made of rope silk or crewels, as preferred.

Cheaper bags can be made by using art linen for the foundation and embroidering the design with linen threads.

\section{TOILET CUSHION.}

The cushion shown in the model, figure 212 , is made a little longer than wide, and is stuffed so that it is higher in the back and slopes at a gentle angle to the front. This effect is secured by sewing side pieces tr the top and bottom of cushion cover, having the back side pieces highe:

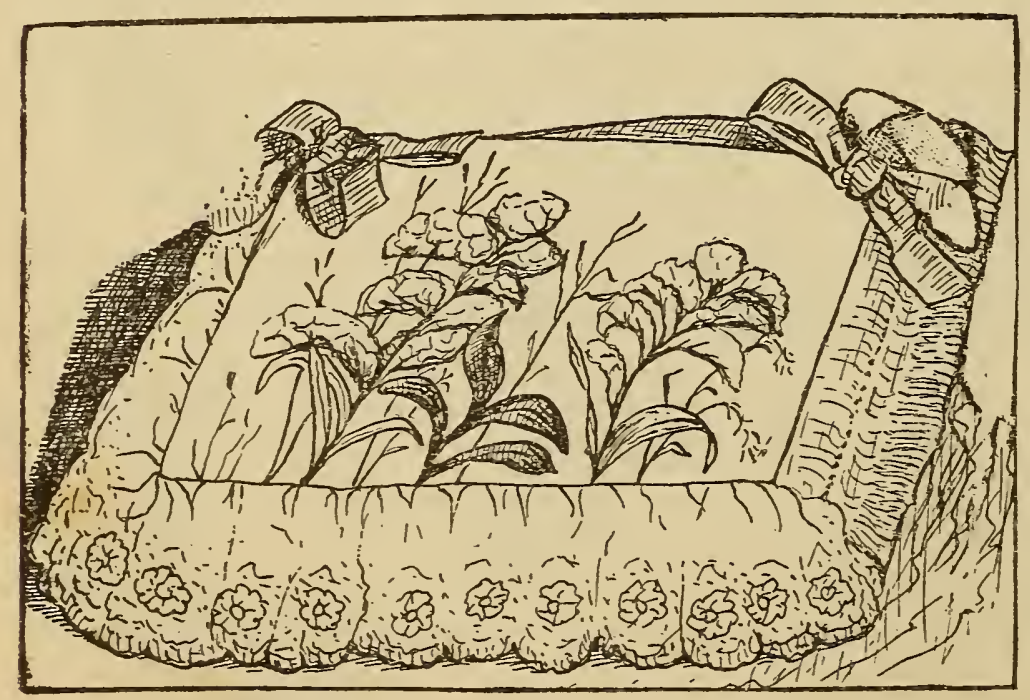

FIG. 2 I2. than the front. Cove: the cushion with pale yellow surah silk, and make for the top a removable cover of white silk bolting, bordered with fine white lace. The top of cover may have a design of golden rod and foliage painted in sil colors. Fasten to tri:e ushion with pale golden yellow bows. The design niay be embroidered, in which case the golden rod should be worked with French knots, and the foliage with short and long stitch. See chapter on "Raised Embroidery:" 


\section{EMORY CUSHION.}

A little novelty easily made is shown in figure 213 . It is an emory cushion, made in the form of a leaf. Make a cushion the same shape as here shown but twice the size, and fill with emory. Out of white cashmere cut two leaves a size larger than the cushion. Fill the surface of one leaf with French knots, done with etching silk-pale pink; vein the center with Royal floss, using a shade of pink a trifle deeper than for the French knots; place the cushion on the plain leaf and put over the cushion the embroidered leaf; buttonhole the edges of the two leaves together with pink Royal floss, and fasten a bow of narrow pink ribbon at the stem end. A number of these emory cushions embroidered in different colored silks will always prove a sell-

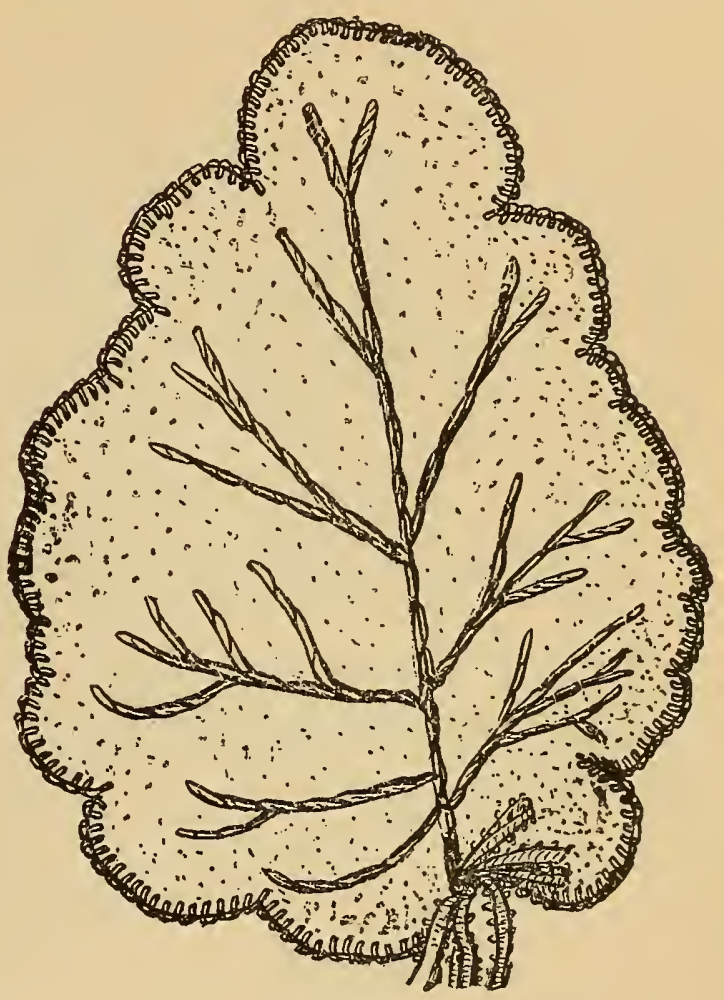

FIG. 213 . ing attraction for fairs and bazaars. Flannel could be used in place of the cashmere, or if it was desired to have something extra fine, wh te satin or silk could be used.

\section{BUTTERFLIES.}

Sprays of flowers, beetles, bugs and butterflies are used to decorate lampshades, to pin to the window curtains, to fasten on the wall over a picture frame; in fact, wherever a convenient spot affords a resting-place it is quite in order to fasten a bug of some kind-not the real thing, of course. but just as good an imitation as nimble fingers can fashion. We thow here, in figure 2I4, a butterfly which can be attached to a lamp shade, or any of the places mentioned, with good effect. Trace the shape of the wings by means of impression paper, but twice the given size. This can be accurately accomplished by means of a pantagraph. Cut the wings out of black veivet paper, or if preferred, black crepe paper; paint with liquid gold the markings shown in illustration, and fasten a fine hair wire around the wings 
on the wrong side. To make the body, roll a piece of cotton lengthwise until it is about twice the size of a lead pencil in thickness, wrap it around with the black paper and tie a piece of wire around the neck and at intervals down the length of the body; fasten in pieces of wire for feet, and two

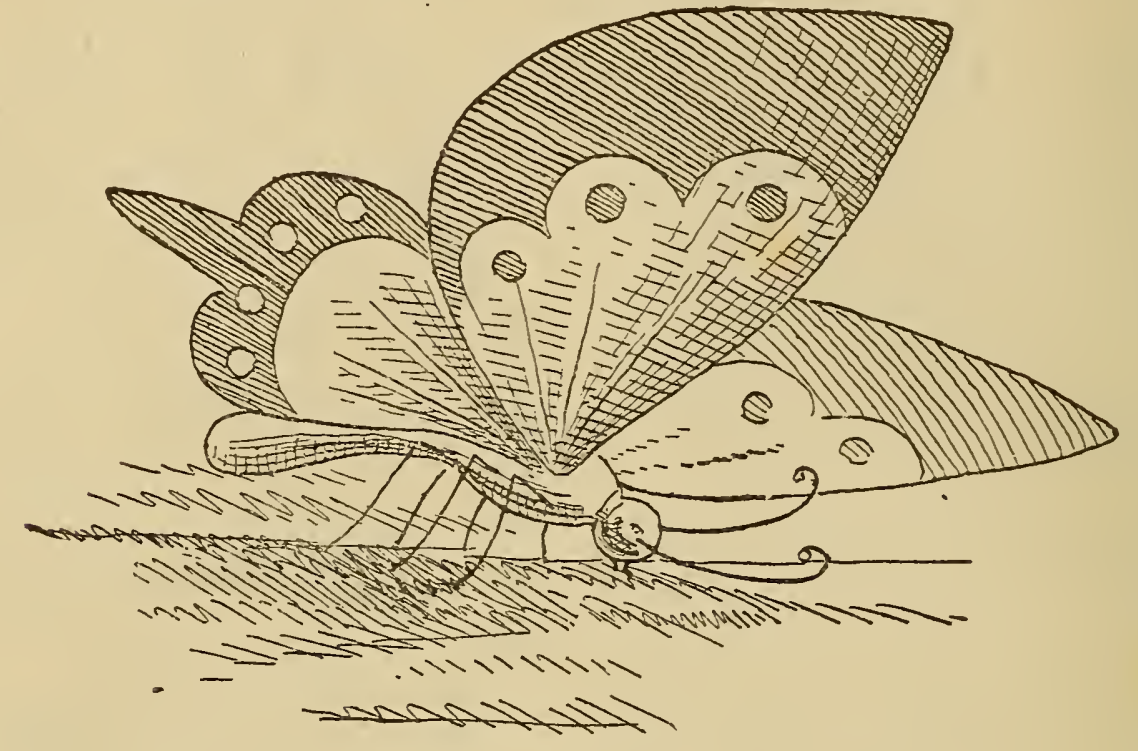

FIG. 2 I4.

wires in the head, as shown; these must be gilded. 'Fasten the wings to the body by means of mucilage. Several of these butterflies made up of different colored paper would be very useful for filling up bare-looking spaces.

\section{MUSIC STAND.}

To make the simple arrangement shown in figure 215 will require the service of one's husband, father or brother, as the frame work will be beyond the skill of most women,- note that we do not say all women. The four upright pieces that form the sides of the stand should be $31 / 4$ feet long and the side braces $2 \mathrm{r} / 4$ feet long. In the center of the side pieces bore two holes so that they come directly opposite each other; six inches from the bottom ends bore holes large enough to receive the ends of the side braces. Cross the two side pieces, like the letter $\mathrm{X}$ and fasten with a double-headed screw or with a riveted nail, and put the side pieces in. The material for the frame may be common pine or it may be old broom handles; in any event, when the frame is fastened together give it a coat of ecru-colored English enamel. For the pocket select a piece of tapestry 
linen, of a shade bordering on golden brown, four feet long by two feet wide. Line it with dark brown canton flannel, of a good quality, and finish the edges all around with a heavy silk cord. Fasten the four corners of the tapestry linen to the four side pieces of frame by means of ecru-colored ribbon bows. The sides of the pocket should have an elaborate design worked out with four shades of golden brown Royal floss, dashed with gold thread. This stand will be found very convenient, and inexpensive as well.

The colors given here are not arbitrary, nor are the materials; but it will be found to give better satisfaction if the embroidery is done with the same color

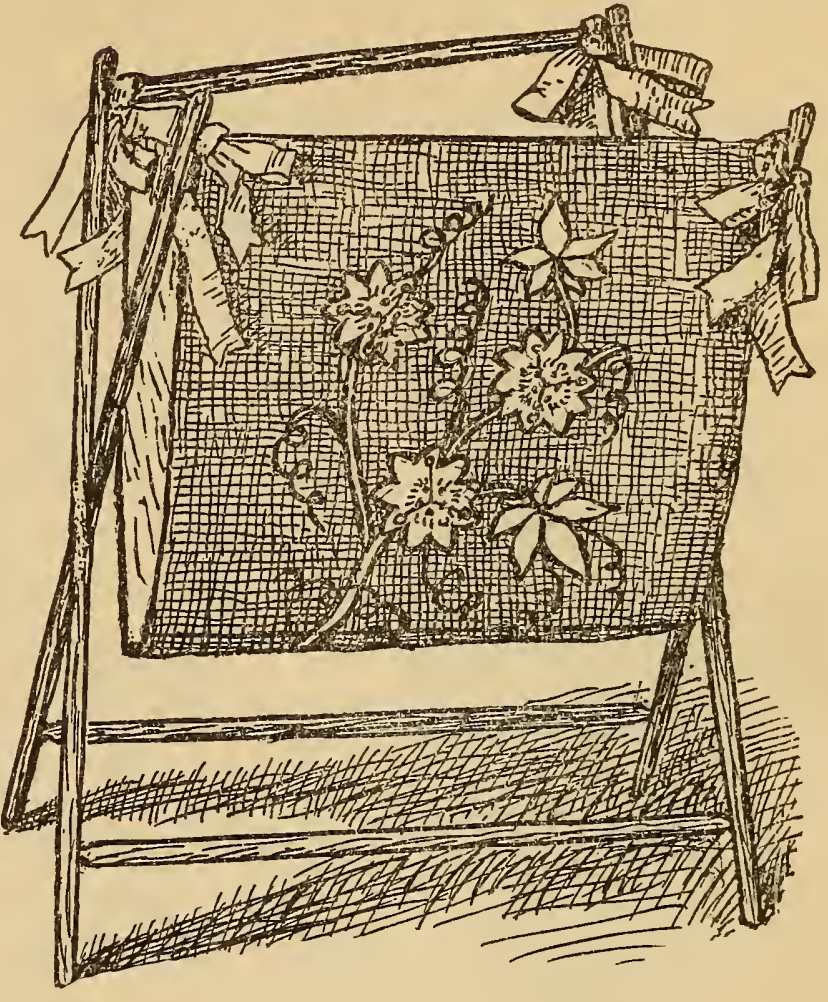

FIG. 2I5. as the foundation. Mail cloth, velvet, silk and ooze leather are all desirabie fabrics for the pocket of stand, but of course more expensive than the line:n. In case ooze leather is employed, two skins will be required. The decoration could be a conventional design stamped on the ooze leather and then gone over with gold paint. As this paint comes ready for use, a person need not be an artist to apply it. This leather can be had in ecru, white and bronze brown. The ecru treated as described would be effective. The inside of pocket could be lined with some pretty, bright color, either green, rose or blue china silk, and the edges finished with a heavy cord.

Artistic pockets for rack can be also made of Scotch homespun, which is a pure linen fabric, very heavy, but without any twill. The decoration could be a Dresden design of buttercups worked with yellow browns in silk or linen threads, or a design of music notes and violin could be worlied on one side. 


\section{SCISSORS CASE.}

A scissors case made as follows will be found a splendid article for farrs, Christmas or birthday gifts. The case, of course, must be cut to

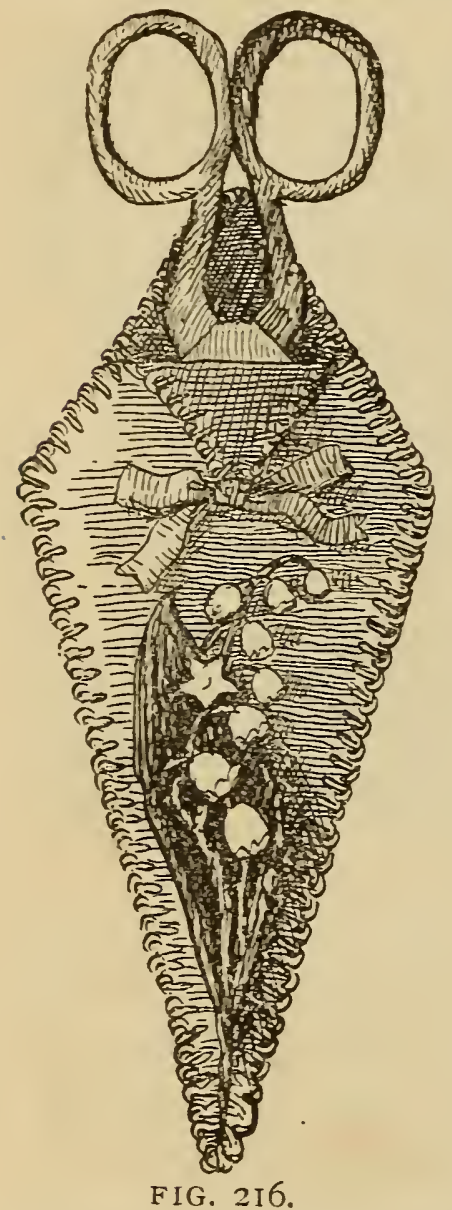
fit the scissors it is designed to hold, but the shape can easily be copied from figure 216 . Cut two pieces of cardboard, line them on the inside with a piece of chamois skin or velvet, cover the outside with any scrap of silk or cloth that may be at hand. join the two pieces together with a buttonhole stitch, done with rope silk or Royal floss. On the outside cover work a design in solid Kensington stitch with filo floss. The model shows lilies of the valley on moss green velvet. The lining is of pink satin, and the buttonhole stitching pale pink silk, though any culors one happens to have will do equally well. To embroider the design here shown will require onehalf skein white filo floss, one thread of green filo floss, and one-half skein rope silk.

The scissors case shown here, the laundry bag on page 200 , the mending bag on page I9I and the holder on page 192 would form a combination gift that would delight the heart of any woman with mind on housewifely cares intent-and the cost could certainly be compassed by a single dollar, and may be less-all would depend upon the condition of the scrap-bag. And here it may be well to state that a scrap-bag should be kept in every household. Pieces of ribbon-no matter how soiled or creased-should never be thrown away; carefully cleansed in gasoline, they can be utilized for various purposes; also velvet that has been used on bonnets, dresses, etc., can be steamed, when it will answer for making various trifling articles at once useful and ornamental; so cultivate the habit of saving all the scraps of old ribbon, velvet and cloth, and you will always have a resource in times of making up "iancy work." And be sure the scrap-bag will save many a penny in the course of a year. 


\section{CRAWLING RUGS.}

These rugs are the delight of every baby fortunate enough to possess one. The foundation may be made of an old dress skirt, a piece of felt or flannel, or even canton flannel will do nicely. It should be lined with old ticking and lightly wadded. Over the surface applique all sorts of figures-of toys, animals and familiar playthings. These figures can be cut out of any odd bright-colored scraps the rag-bag may afford. They should be buttonholestitched on to the foundation. Old pieces of silk, ribbon, velvet, flannel and cashmere can be used indiscriminately. Picture books and cards will furnish designs for cutting the scraps by, and the figures may be further embellished by adding a few embroidery stitches occasion-

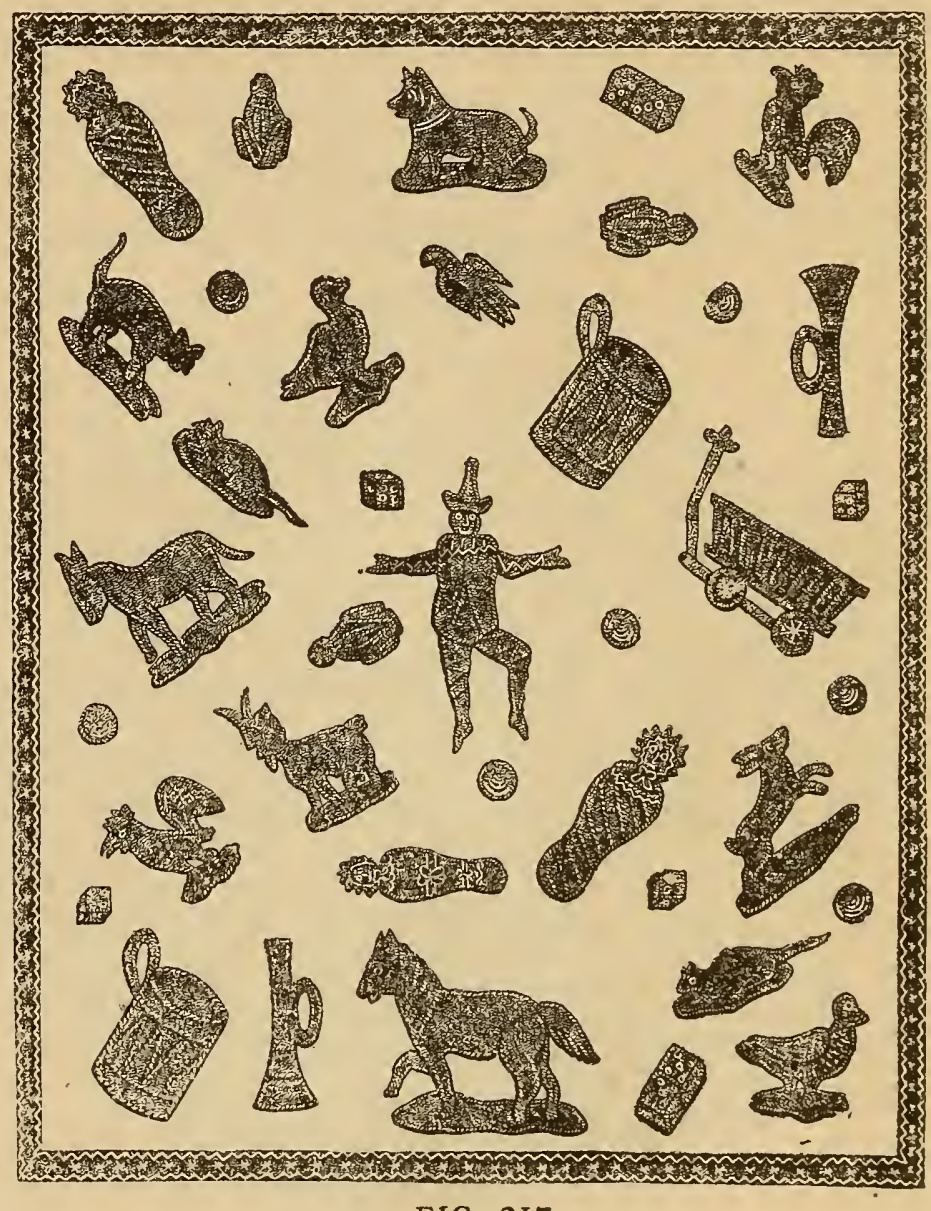
ally. But, made simple or elaborate, it is a most cunning article for the little tots who can just crawl about, investigating everything on their hands and knees. They will spend any amount of time patiently trying to pick a small boy, drum or wheel-barrow off the rug-sometimes developing a little latent temper when they don't succeed.

For fairs or bazaars they are a decided success. Where it is not desired to make up more than one rug for a bazaar a nice profit can be made by zutting sets of figures, ready for appliquing, out of bright felt or flannel. They could be sold very cheap, as the scraps and work would of course be sonated. 


\section{FOR THE DINING ROOM.}

Nowadays there are so many pretty novelties used on the dining tables and sideboards that one need never be at a loss what to make for Christmas, wedding or birthday gifts, for one's married, or about-to-be-married lady friends. In the chapter on "Flat or Art embroidery" will be found a list of the various linen articles that are commonly used in the dining room. In this chapter there are several ideas in the same direction that will be found simple and inexpensive in the working out.

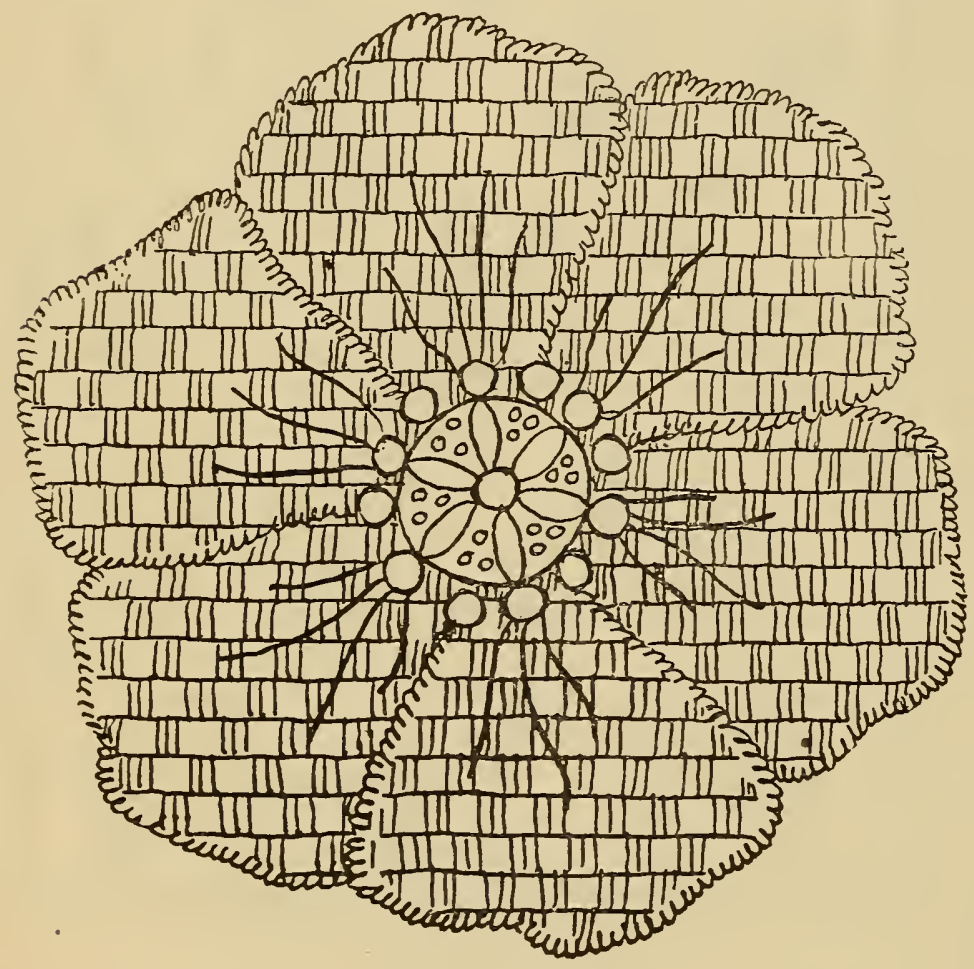

FIG. 2 I8.

Doyleys, mats of various sizes, lunch sets, tray and bread cloths, etc., offer a field for decorative embroidery whose variety scarcely knows a limit. The work required for these articles is not exacting and only simple coloring is required.

Roman embroidery is especially suitable for table linen and may be introduced in a center piece as a border, while the center of the article may be embroidered with Dresden

design. Some ladies prefer the appointments of their table to be all in white, while others prefer delicate shades of a color; but we would not advise going beyond this decided colors are not usually satisfactory used on table linen.

Doyleys are always in order; they may be of different sizes and used for a variety of purposes. It is a good idea to have several sets of different sizes on hand, but decorated with colors that will harmonize-so that in case it is necessary to use them all at one time, the colors will not swear at each other. If you are obliged to make a wedding gift, be sure that the bride will be delighted with any gift in this line. A set of six odd mars, a 
complete lunch set or a water server mat, will always find eager purchasers at fairs; and for one's self no more satisfactory fancy work can be done than making up "Dainty Trifles" for the dining room.

Figure $2 \mathrm{IS}$, on the foregoing page, shows a design suitable for center picce for luncheon. The working size is seventeen inches across, while plate or cup doyleys from four to six inches across may be embroidered to match. The material best adapted for this work is satin damask or Roman twill. To make a center piece and six plate doyleys will require two-sixths of a yard of the damask eighteen inches wide, three dozen skeins of etching silk, or one and onehalf dozen skeins Scotch linen floss, six skeins German cord, smallest size. First work the edges with buttonhole stitch over the German cord with the silk, after which fill in the surface with double basket stitch described in the chapter on "Stitches." The center should be filled in with spot stitch for the circles, out-

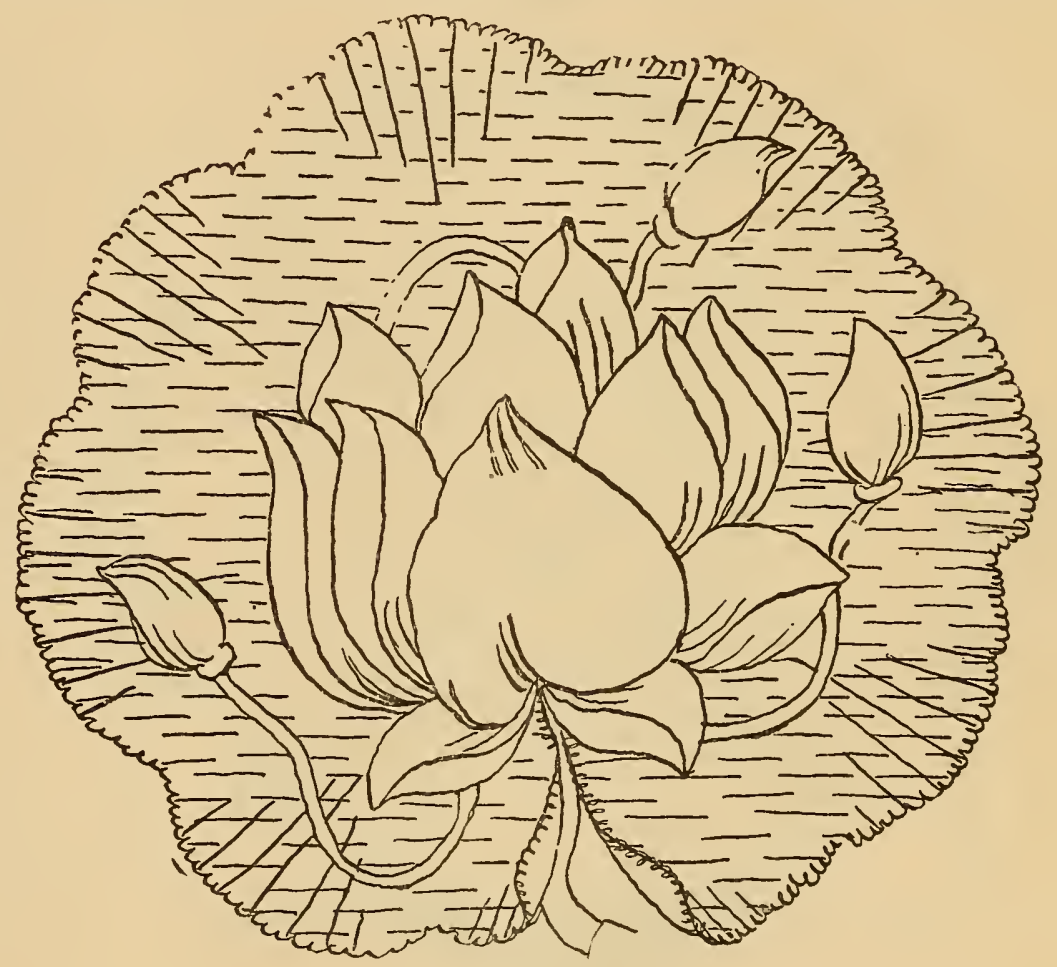

FIG. 2 Ig. line stitch for the straight lines, daisy stitches for the figure in the center, and French knots for the small circles in the center. The colors are of course a matter of taste, but pure white Royal floss for filling in the basket stitch, the same color etching silk for buttonholing the edges, and palest of green for filling in the center, would give a pleasing effect, or the pale yellows, pinks or blues would be effective.

Figure 219 is a very beautiful design which will require careful treatment to bring out its beauty. It is not desirable to use this design reduced in size for small doyleys. It is better to make the center pieces as shown 
and then have the cup doyleys in the shape of the leaf without the flower resting on it; silk bolting is a beautiful material for the foundation of this design. Treatment: First darn, in Queen Anne stitch, the entire surface of leaf with pale silver green filo floss, shading to nearly white; Work the edge with a light buttonhole stitch taken on the edge of material-nut over a cord-with the same silk; with a shade of silk a trifle deeper work in a few short and long stitches at regular distances around the edge of leaf, outline the petals of flowers with white Royal floss, working some of the petals with short and long stitch. For the shadows put in a few threads of

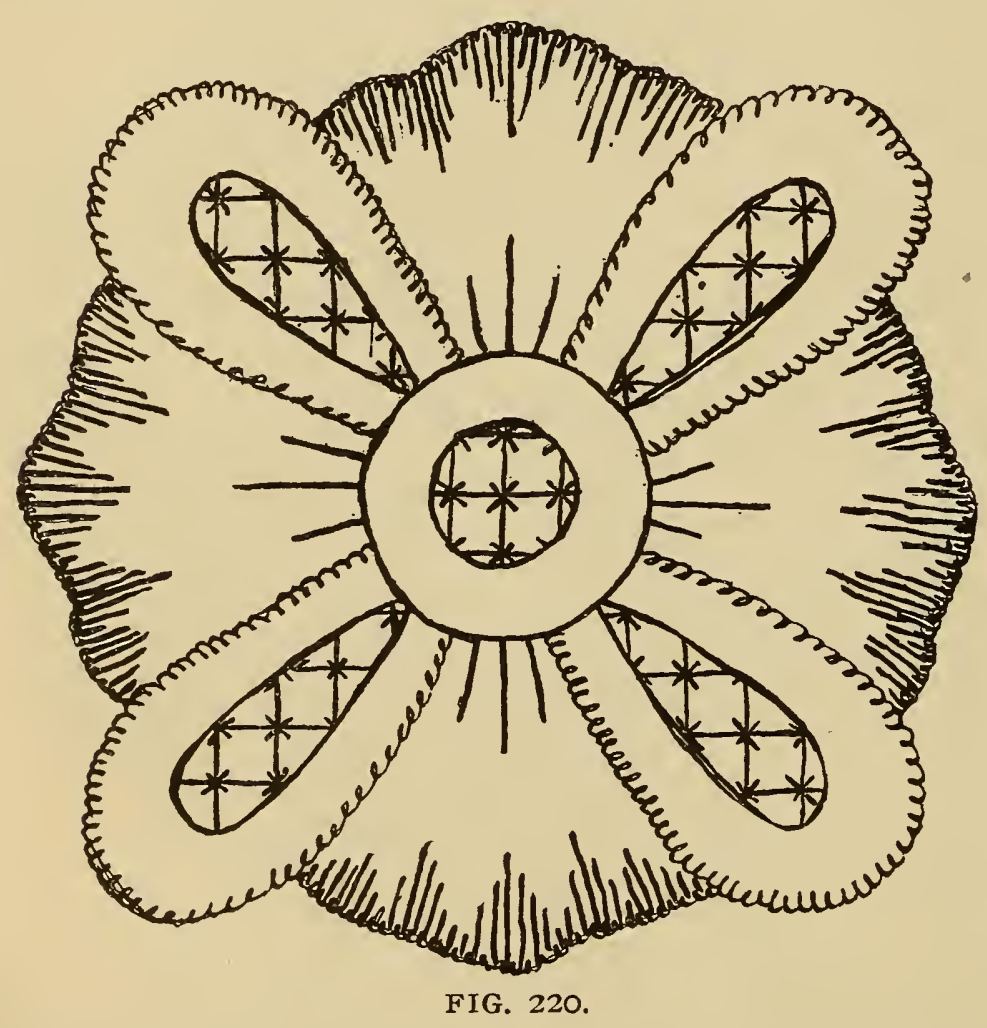
silver green filo floss; cut the edges out very carefully and line the center piece with pale pink silk or with a rich gold or a light green. The darning stitches should not be taken very close together, but about as indicated in the illustration. The silk lining may have the edges fringed or finished with a ruffle of fine snow-white lace-or both-arranging the lace so that it will fall over the fringe.

Figure 220 shows a

model that can be used as a center mat I7 inches across, or, reduced in size as a mat for bread plate, salad dish or fruit basket. The material for the foundation may be fine huck-a-buck linen, satin damask, or satin sheeting. To work the design as shown in the illustration will require, for a mat seventeen inches across, two skeins smallest size German cord, one dozen skeins etching silk or Royal floss. Work the edges of the center circle and the four loops with plain buttonhole stitch over a German cord, fill in the spaces in the loops and the circle with diamond couching, explained in the chapter on "Ecclesiastical Embroidery." Work the outside edges of the 
center pieces with pointed short and long , buttonhole stitch, previously described, using for this part of the work Royal floss. The rays spreading out from the circle between the loops will be effective in gold thread. This design is exquisitely beautiful worked with pure white for everything except the diamond couching and the rays, which should be of fine gold thread, although any other combination of colors may be used to suit individual fancy; as deep moss green and white for the couchings and rays, or, old blue and silver threads; pale rose and gold, or, yellow in two shades, one light and another shade at least two tones deeper. If linen floss is used in place of the silk about one-half dozen skeins No. 8 will be required.

Figure 22I shows a model for center piece that is very effective made up as a set, consisting of a center piece and six doyleys for cups, etc. Work the edges over the smallest size German cord with white etching silk: fill in the surface' of petals with honey-comb stitch in white etching silk; outline the center with outline

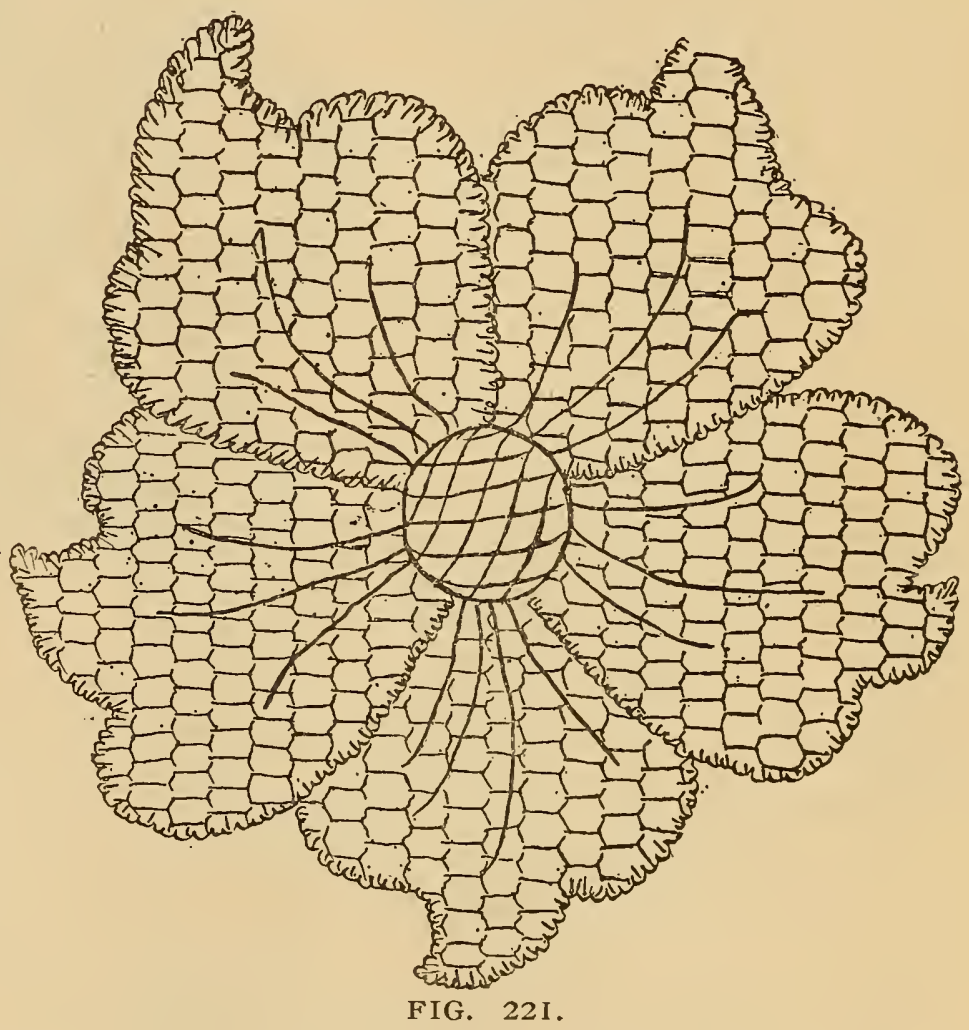
stitch and fill with plain diamond couching, done in gold thread; also work the veining lines that spring from the center down each petal in the gold thread. [ In using gold thread for table napery, or indeed wherever it will require washing, purchase the best, that which comes on spools giving better satisfaction than that put up in skeins.] White silk and silver thread would be a beautiful combination, worked out in this design. Of course the other mats, etc., used with this model should be decorated with the same or harmonizing colors or shades. For evening suppers or five o'clock teas the silver and gold thread light up wonderfully well, under the gas 
or candle light. Figure 222 shows a graceful design, suitable for center mat, in a size either I 7 or $2 \mathrm{I}$ inches in diameter; reduced to 12 to 14 inches a set of four mats could be most effectively used in combination with the center piece. The plate and cup doyleys could match or not as preferred. The edges of the petals should be worked all around with a row of buttonhole short and long stitches; into this should be blended two or three rows, so as to work into the petals at least I $\mathrm{I} / 2$ inches. Begin on the outer edge with the lightest shade of the color to be used, blend into this the next deeper shade, and into this row blend still a third and deeper shade. Fill the center with outline and spot stitches. Royal floss will be found

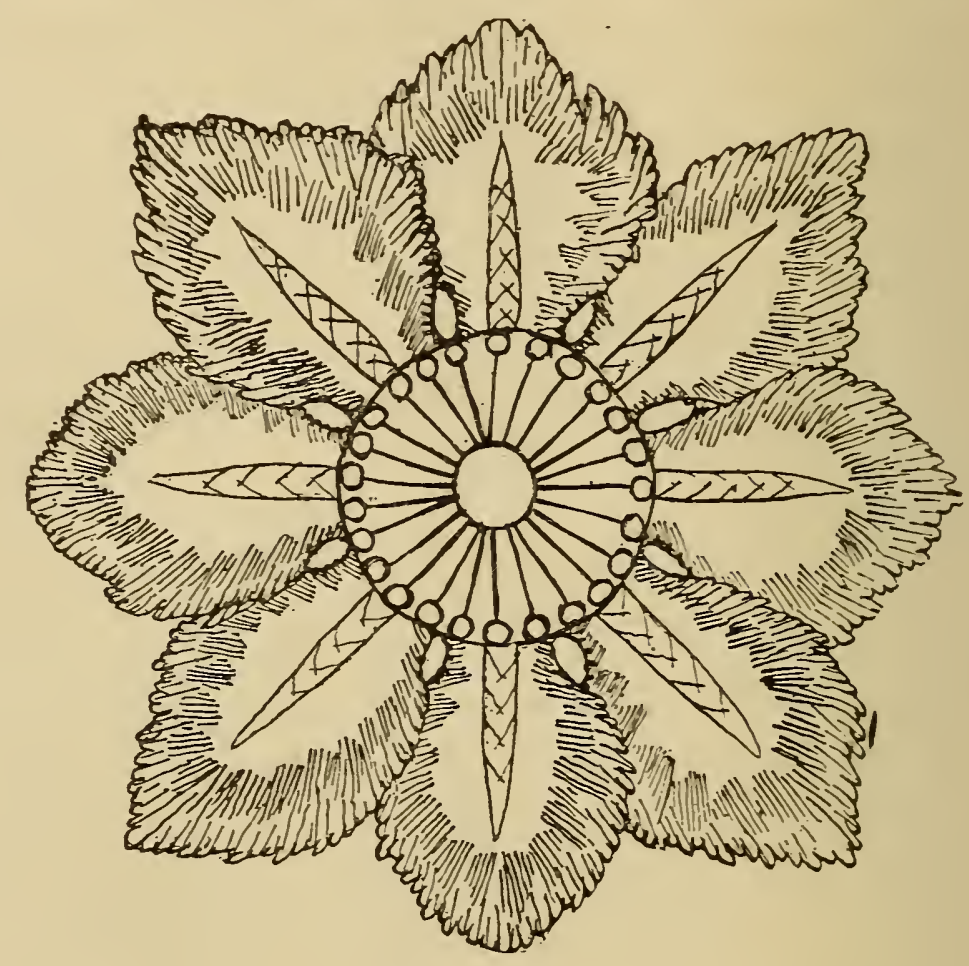

FIG. 222. the best adapted for working this design as indicated, and it will require a little more silk than the other, previously described.

A beautiful lunch set could be made by using this design in three sizes-for cups, plates and center pieces worked, as described, in three shades of lily green Royal floss on white satin damask. The combination of the delicate green and white would allow of any kind of floral decoration desired, and yet be a little change from the dead white so long used. If however the set is to be used over a white cloth, instead of on a polished table, a little color will lend a pleasing variety to the table, all the other appointments being in harmony; for this purpose pale dead rose, old blue, or certain shades of yellow are effective.

The set could appropriately be made of silk bolting, in which case the embroidery should be done with white silk and the doyleys and center pieces lined with delicate colored surah or China silk. 


\section{A SHOE BOX.}

A shoe box cannot properly be stylęd "a novelty" in itself, as these articles have been in use from time immemorial, but the one shown in figure 223 is both convenient and ornamental. A soap box I4xis inches will answer for the box, the inside of which should be lined with white oil cloth, tacked in neatly. Around the inside of the box, when lined, arrange a strip of the oil-cloth, as wide as a shoe is long, and tack it into position so as to form compartments or pockets, dividing each compartment by means of a strip of brass braid, such as is used to finish the edge of floor oil mats. A narroiv strip should also be fastened on the inside of the cover, for the purpose of holding brooms, brushes, etc.; for the bottom of the box, cut a strip of any dark colored cloth, canton flannel, Hollywood drapery or bolton sheeting as deep as the box is high, allowing for hem, and the strips should be as long as will go around the box twice. Finish the bottom edge with a buttonhole short and long stitch or a simple

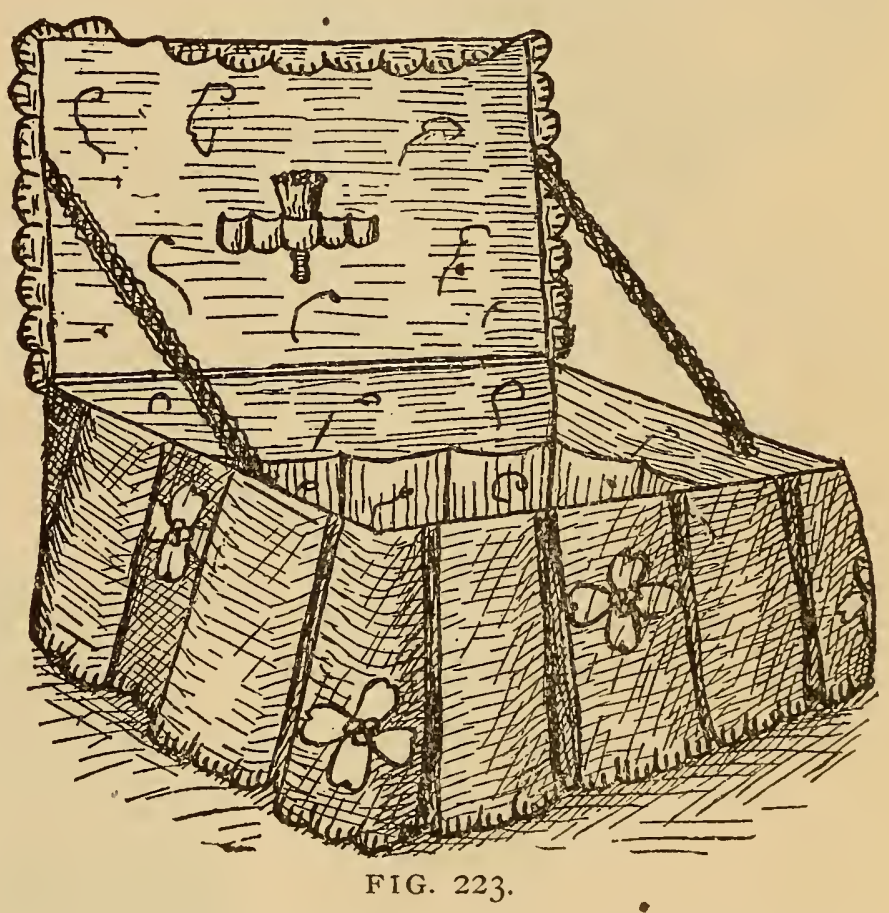
hem, and over the surface scatter dogwood blossoms and embroider them with white Bargarren art thread for the petals, and a shade of yellow for the center; arrange the strip around in box plaits, tacking on the top edge of box with brass headed tacks. The cover for the top should be cut three inches larger than the top of box, so as to allow it to fall over, scallop the edges and work with buttunhole stitch and embroider dogwood blossoms over the surface, fasten the cover to box by means of small hinges or strips of oil cloth, and fasten a stout braid from the middle of cover to the side of box, as shown in figure 223 . 


\section{BURNT MATCH HOLDER.}

Nothing presents a more untidy appearance than burnt matches scattered over dresser or commode. A pretty little affair to hold them is shown

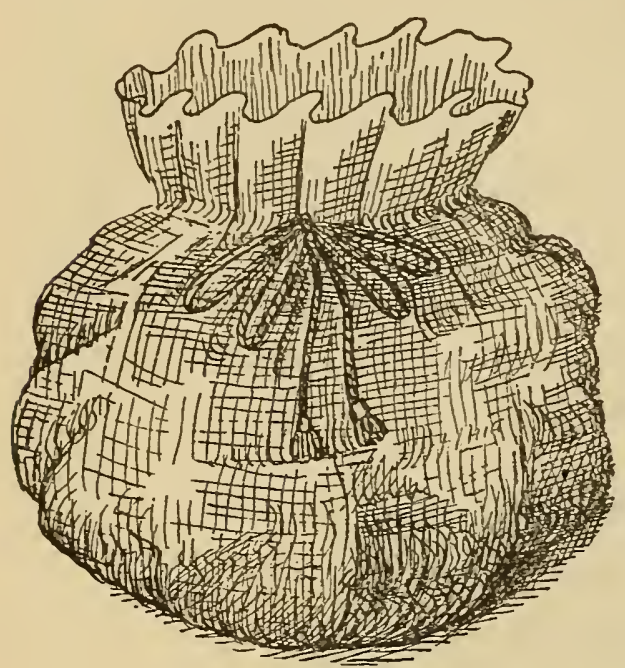

FIG. 224. in figure 224. It will be an acceptable gift to almost any lady, especially if she be of the busy practical sort who never "finds time to do fancy work."

The holder proper must be purchased and may be any pretty shaped glass, either white or colored; select a piece of colored china silk and cut a circle that shall be 12 inches across; hem the edges, and $\mathrm{I} / 2$ inches from the top run in a piece of ribbosene for a draw string; into this silk bag put the glass and draw the string and tie. What could be more simple and inexpensive? A most saleable article for fairs and bazaars.

\section{TOILET CUSHION.}

Figure 225 shows a very pretty round cushion which is about 4 inches high and 8 inches across. The cushion is first covered with some pretty-colored silk put on plain. Around the edges is fulled a flounce of white lace as deep as the cushion is high. The top of the cushion is then covered with a square of sheer linen finished around the edge with a narrow lace edge. In the corner crowsfeet stitches are worked with rope silk, or instead, a pretty idea would be to work a Dresden design

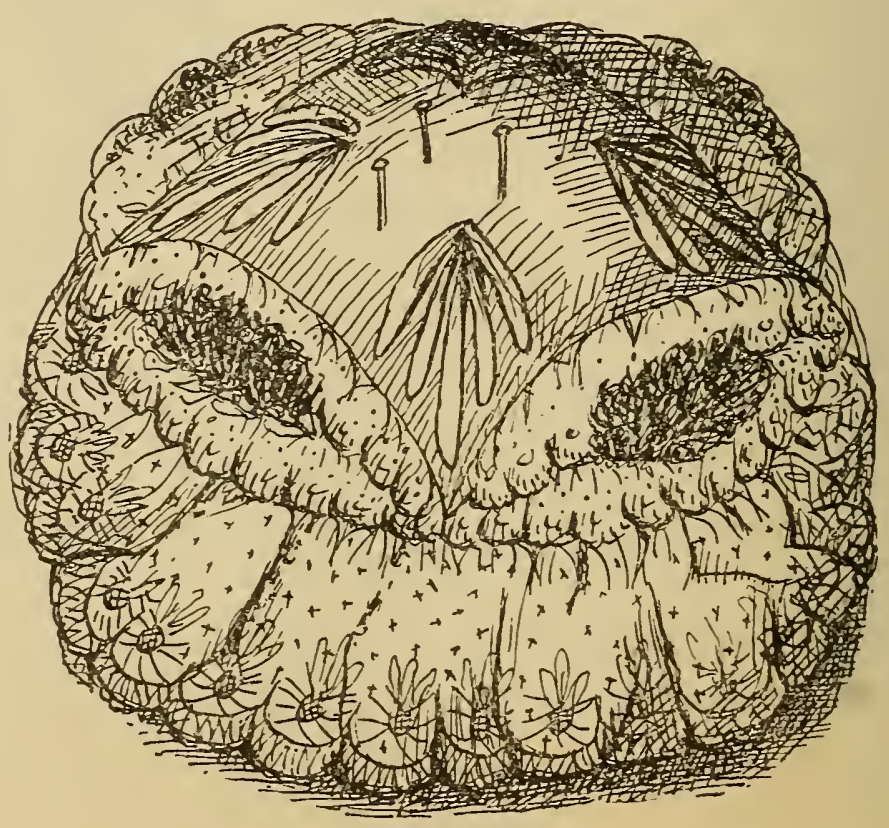

FIG. 225 . over the linen in solid Kensington stitch. In fact, it would be well to 
provide several of these small square covers for each cushion, each one worked differently, and as wash material will be used in their manufacture, the cushion can be kept ever fresh and clean with little trouble.

\section{FOOTSTOOL.}

These little footrests are made in divers shapes, and decorated according to individual fan'cy. The model has a frame painted. with white English enamel, a piece of canvas, tacked on to the frame, forms the bed for the cushion, which is made of ticking filled with sea moss, and covered with pale yellow silk, on one corner of which is embroidered a graceful arrangement of daisies, worked out with dull yellow filo floss, shading to a yellow brown. The cushion is kept in position by means of a scarf of pale yellow and golden brown silk, passed under the canvas and over the cushion and tied in a large, loose bow on top. The ends may be fringed or trimmed with lace. The frame of stool should be nine inches wide, twelve inches long and eight inches high.

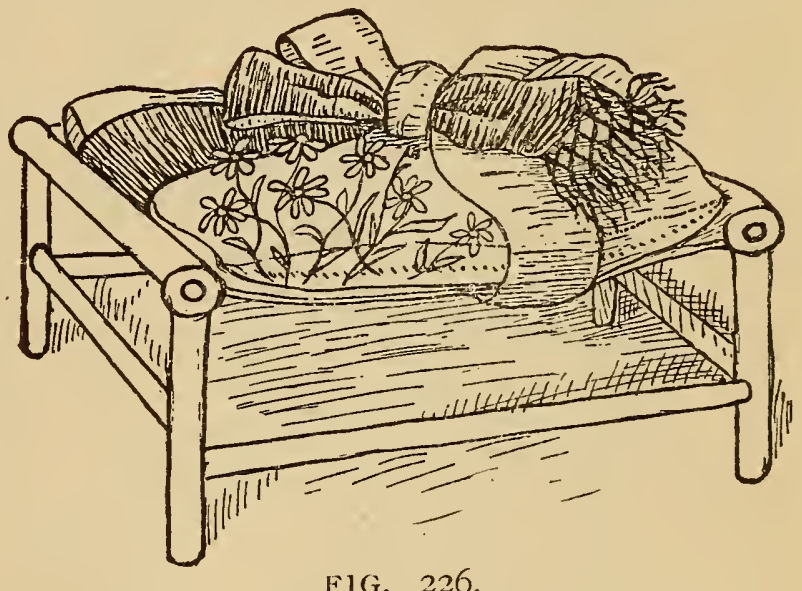

The material for cushion cover will be a piece of silk twelve by twenty-four inches, and the silk or ribbon to fasten cover on should be four inches wide and one and one-half yards long.

\section{CHILD'S CHAIR.}

At the season when the little folks are especially to be remembered the little chair here shown will serve as an idea which can be cularged upon to suit the fancy. The chair shown is one of the ordinary wooden affairs sold in stores for twenty cents. Taken home and treated to a coat of ecru-colored English enamel, and decorated as shown, it could not be purchased for $\$ 5.00$, and would make a gift that would delight the heart of the most exacting infant. The cushion for the seat is cut to fit, and filled with wool or feathers. The small cushion for the back should also be filled with the same mate- 
rials. The outside covers may be made of any material preferred; by far

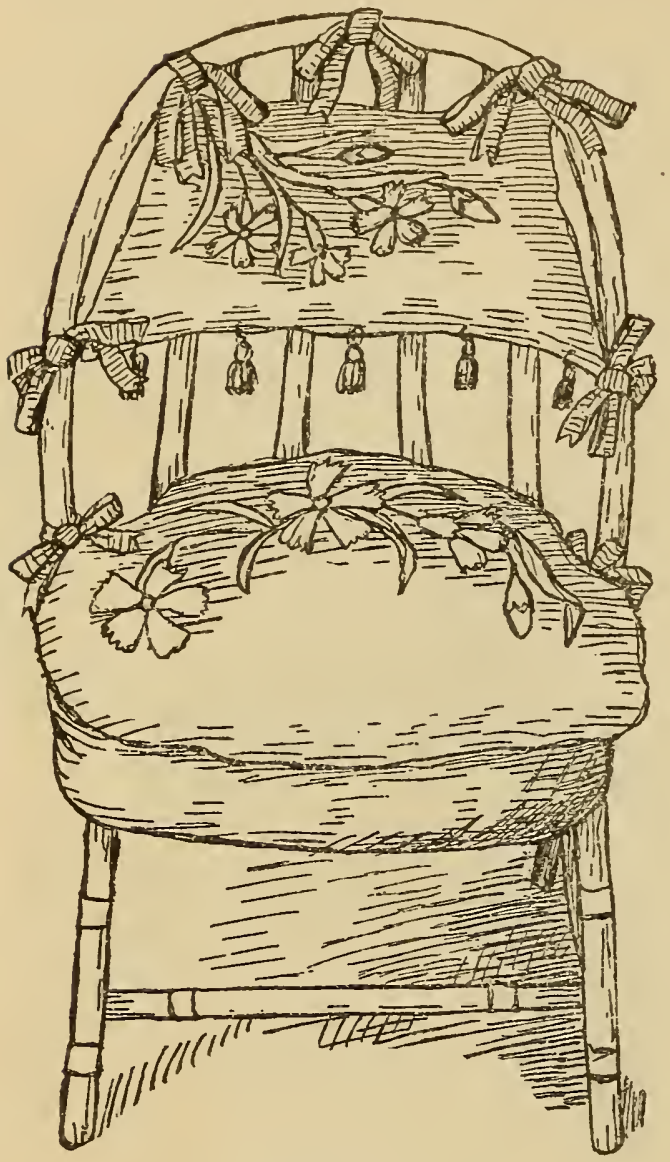

FIG. 227. the most sensible as well as most fashionable is fine white linen, embroidered with wash silks. The model shows an arrangement of carnations worked out with three shades of pale pink for the flowers, and two shades of brorin for the stems and leaves. The cushions are tied to the chair by means of brown and pink bows of ribbon. When soiled the covers can be easily removed and laundered.

\section{CATCH-ALL.}

A dainty little trifle for the table, which will serve as a catch-all for cards, odds and ends, etc. is shown in the accompanying illustration; it can be made of eggshell board, ivorine or cardboard covered.

To make one like the model will require five pieces of stiff cardboard, twelve by twelve inches each. Cover each piece on both sides with art linen of a dull rose or light tan shade; overhand the edges neatly. If the ornamentation is to be embroidery, this part of the work must be done before the cardboard is covered. The design shown is very effective, and should be wrought out with three shades of yellow filo floss. Sew the four pieces, forming the sides, together, about twothirds their depth, beginning at the bottom edge, and fasten a bow of ribbon at the termination of the fastening-see figure 228. Sew the bottom in and finish all the edges wi.h a row

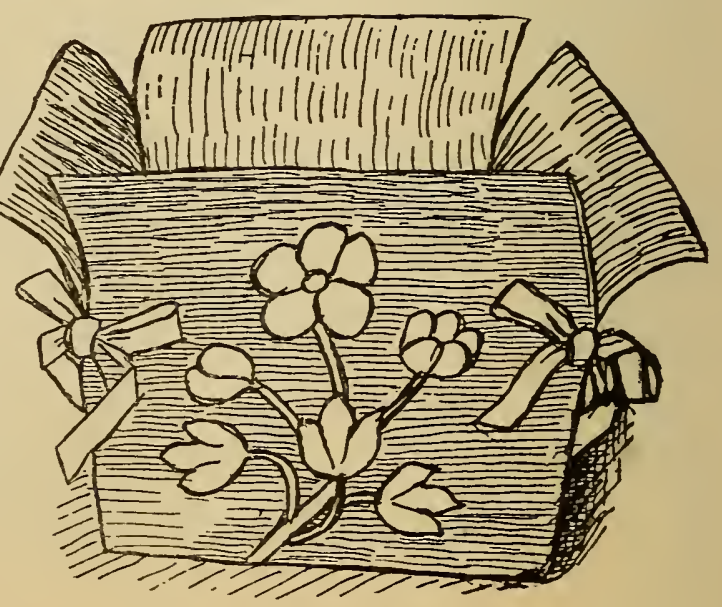

FIG. 228. of art cord, sewed on with invisible stitches. Curve the side preces out. 
ward slightly and the work is done. If celluloid or eggshell board is used the decoration must be painting, with oil or water color.

\section{HEAD REST.}

Head rests for backs of chairs are always in order, and those made long and somewhat narrow are preferable to the fancy shapes, cspecially when designed to be used on a sitting-room or library chair. The fancifullyshaped trifles are better suited for parlor, reception and drawing room chairs.

The rests are made from silk, satin, linen,-white or clored-velvet, chamois skin, bolting silk or tapestry cloth. The one shown in the model is made of a pale ecru-colored soft twilled silk, bordered with cream-colored lace and pale heliotrope ribbon bows. The design is an arrangement of conventional clematis, worked in solid Kensington stitch, with four shades of heliotrope filo floss. The decoration might be any other design preferred, or indced the entire scheme of color could be

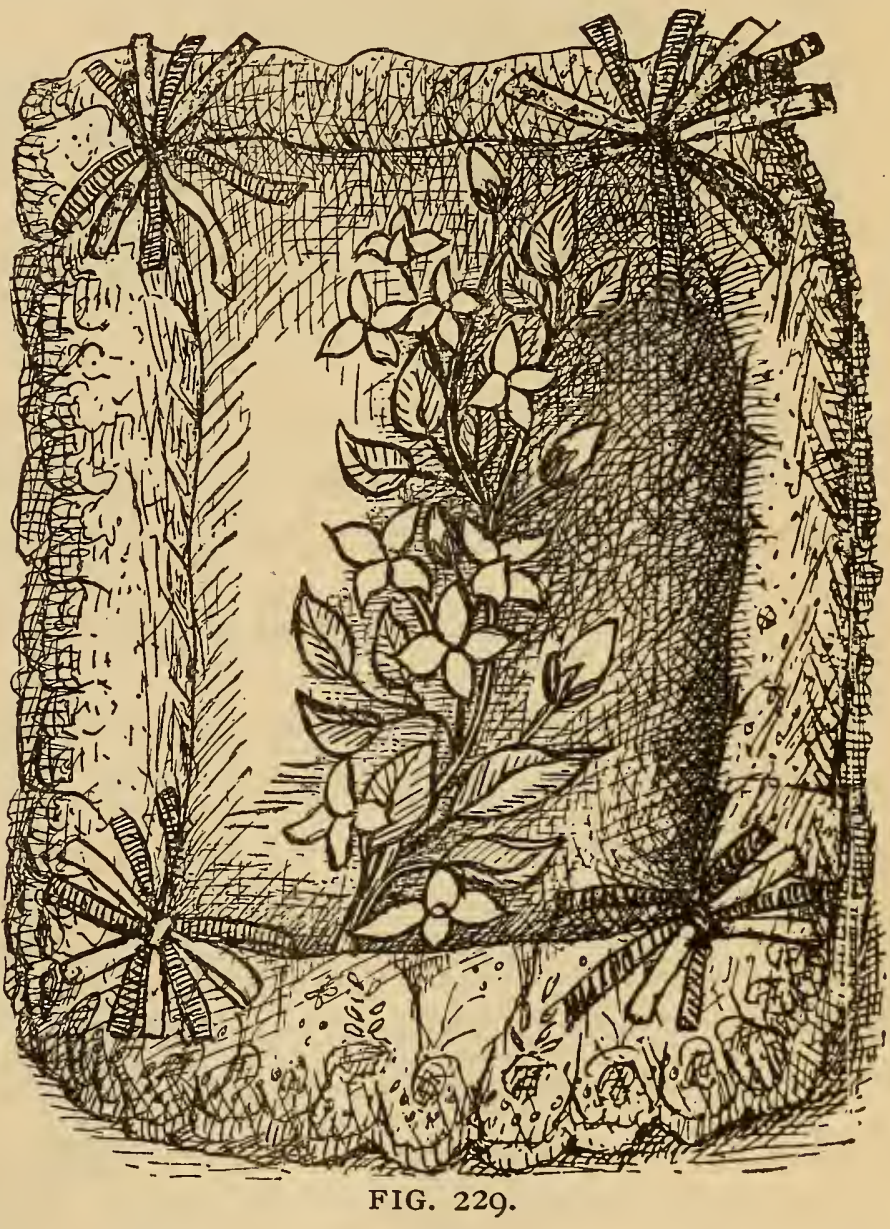
changed to suit individual fancy. The main idea is, of course, to have the thing rusemble its name, and be a head rest in fact as well as name, and to that end the cushion should be filled with eider down.

\section{HANGING PIN CUSHION.}

Figure 230 shows a convenient pin cushion, suitable for suspending from the wall or a corner of the dressing case.

To make: cut four pieces of cardboard $2 \frac{1}{2}$ inches wide and 7 inches long, pointed at each end. Cover each piece on both sides with soft silk, join 
the pieces as shown in figure 230, after which finish all the joinings with a cord terminating at the ends with loops and tassels. Suspend by means of a cord fastened at the ends. The model is covered with pale heliotrope

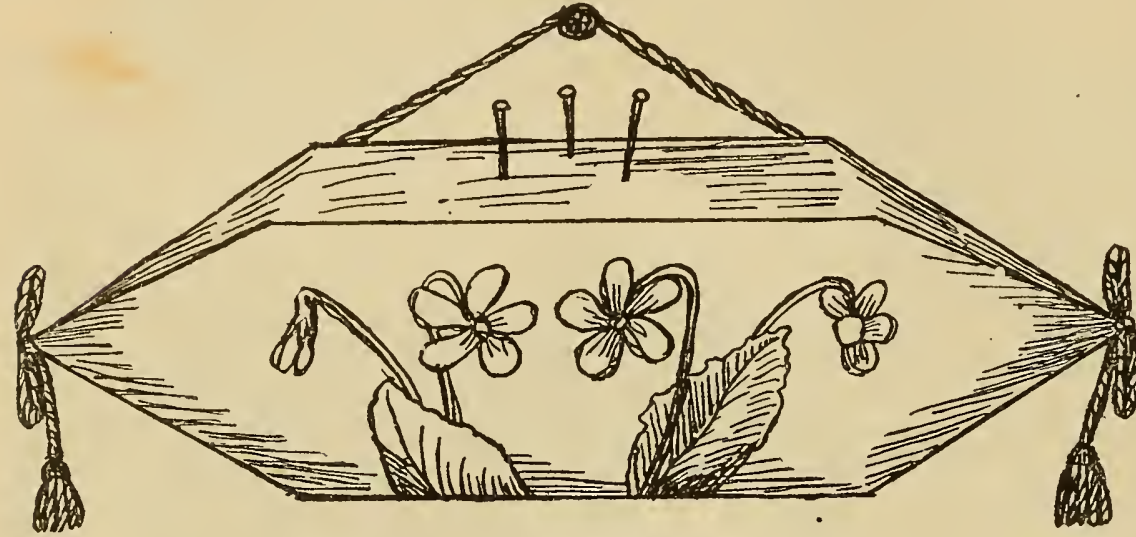

FIG. 230. satin on which is embroidered on the front edge pale wood violets. The pieces of cardboard should be exactly of a size, and to insure this it is better to cut one piece the required size and shape, and then cut the other pieces, using this as a pattern.

The entire effect of such articles as here shown depend upon the neatness of the work for their beauty almost entirely.

\section{FANCY PIN CUSHIONS.}

For fairs and bazaar sales the small inexpensive articles usually find readiest sale and yield greater profits than the larger and more expensive ones. The two designs, figures $23 \mathrm{I}$ and 232 show some pretty models for this purpose. Displayed in quantity, tastefully arranged in baskets they are an attractive addition to the fancy work booths. The illustrations show pin cushions made in the shape of apples and radishes of different sizes. They can be made of small scraps of

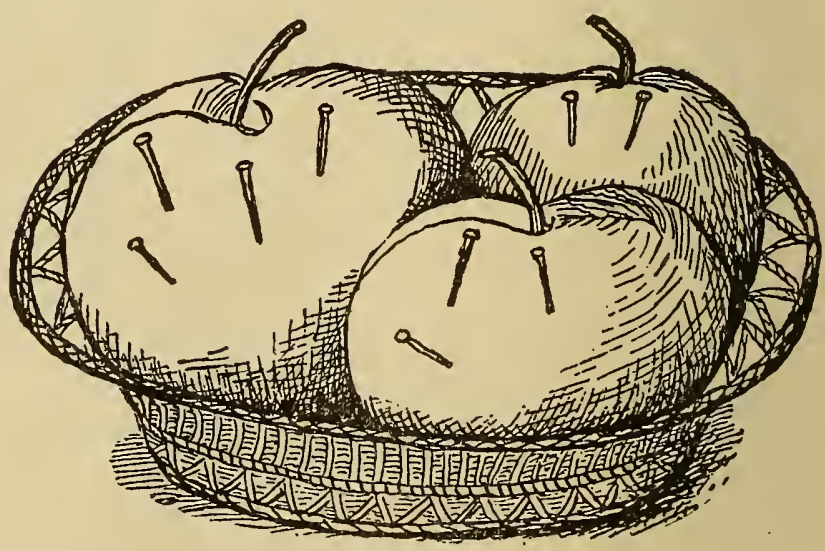

FIG. 33I. silk and a bit of cotton. To make: roll a piece of cotton in the hand until it.resembles the desired shape, then cover smoothly with soft china silk in the proper shape for the article represented, or in white silk tinted with diamond dyes to the required shade. Apply the dyes with a brush after the cushion is made. 
Other vegetables and fruits can be imitated in the same manner with equal success, for instance, tomatoles of the small, round varieties; strawberries, of exaggerated size; pears and peaches, can all be reproduced in velvet and silk for a trifling outlay of time and money. The strawberry and tomato shapes should be filled with emary for needles. The pumpkin

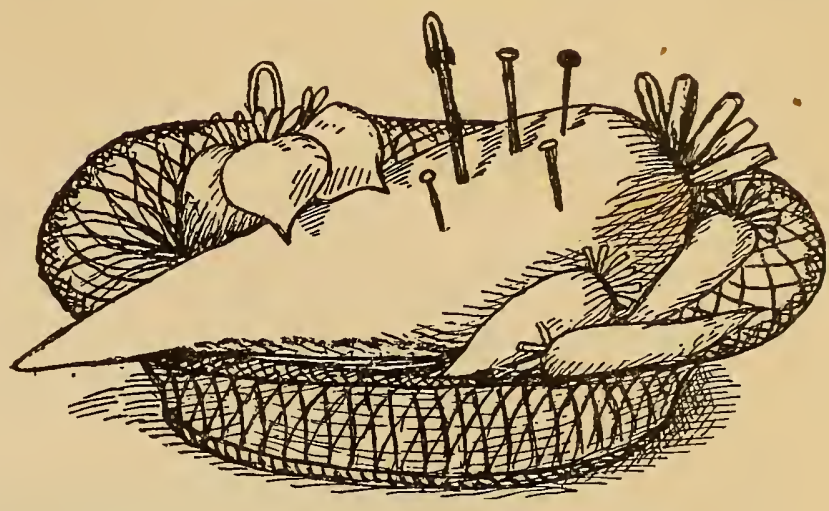

FIG. 232. pin cushion, shown on another page in this chapter, may also be made in a much reduced size, covered with yellow velvet and filled with emery.

\section{INFANTS' CRIB. COVERLET.}

Crib coverlets are appropriately made from flannel, eider down flannel, or lighter fabrics, as silk and merino, but in such cases they must be

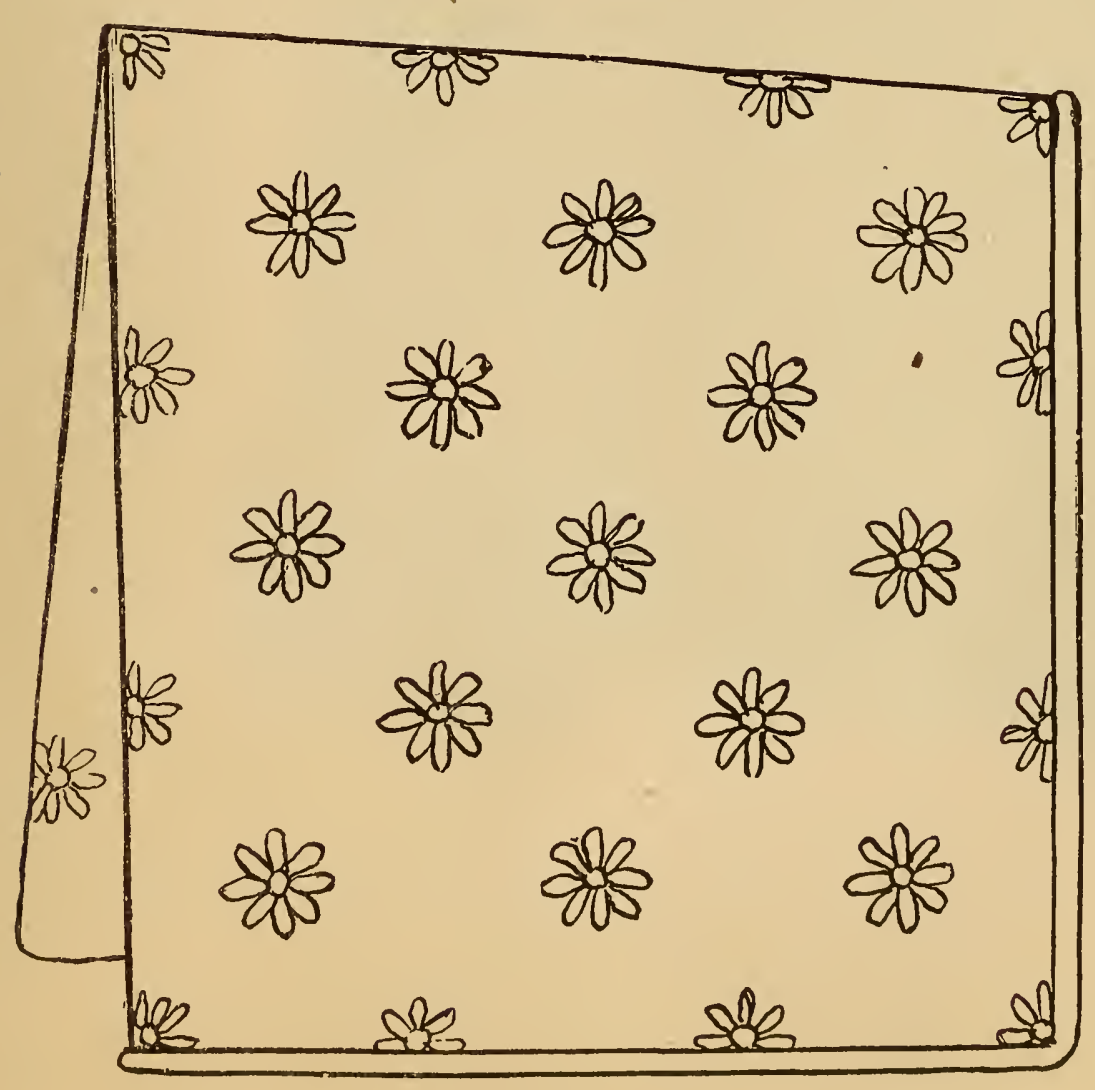

FIG. 233. lightly wadded and lined. There are various pretty methods of decorating these coverlets. The edges may be either bound with ribbon, or scalloped, and worked with a heavy buttonhole stitch with wàsh twist silk, and inside this border a scattered design of flowers over the entire surface is the prettiest and most simple method of making up. The illustration, figure 233 , shows a coverlet of heavy white flannel, having a design of daisies scat- 
tered over the surface, worked in heavy satin stitch with white wash twist silk. The edges are bound with white satin ribbon. Carnations, buttercups, furget-me-nots, and miniature morning glories are all suitable designs for this purpose.

\section{TABLE COVER.}

So much depends upon the size of the table, the place it is to occupy, the purpose it is to serve that it is hard to give. in general terms. directisns for making up table covers. Small tables having well finished legs or

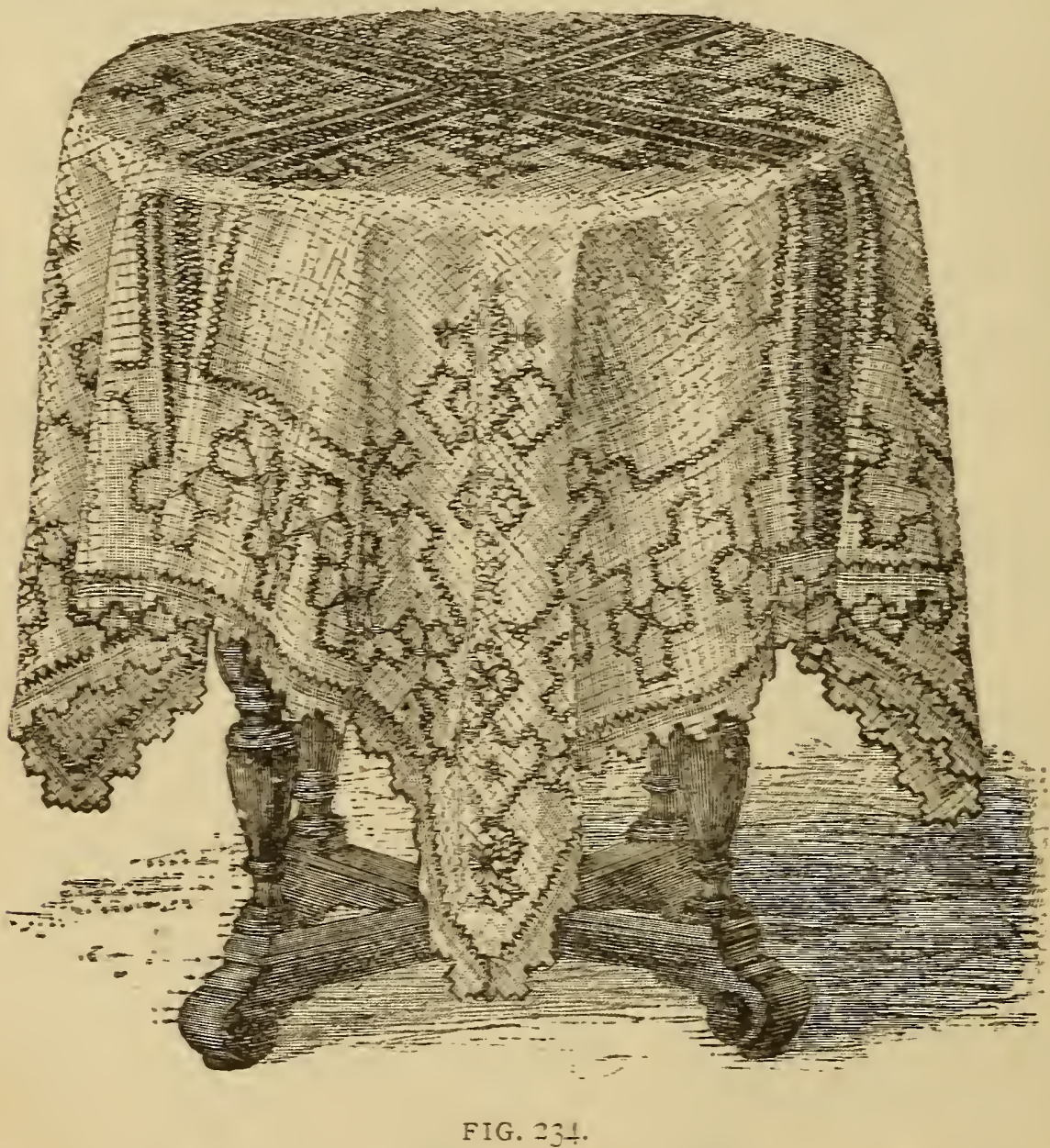

standards, are usually covered with a square of plush or silk, or other fabric, covered with embroidery, and edged with lace or fringe. Tables for dining and sitting rooms are more generally covered with a large cioth, reaching nearly to the Hoor, and made of either Bargarren art cloth. hollywooo Arapery, Devonshire art cloth, mail cloth or tapestry linen, and decorated with cross stitch borders, fringes and conventional designs. Figure 234 
shows a table cover made of No. 6. Bargarren art cloth, having a border worked out in cross stitch with three shades of brown Bargarren linen thread. Figure 235 shows detail of pattern, which can be easily copied. The design is carried across the top and worked in quarter sections. Any other colored thread could be used. The edge is finished with scallop: buttonhole stitched with the same thread as used for the cross stitch. This design is easy, and can be duplicated by a person having no previous knowl edge of embroidery, simply by following design and making a cross stitch in each mesh of the fabric.

Kensington embroidery is most effectively amployed on table rovers made of fabrics having a silken surface, as mail cloth, Russian tapestry or sateen, and the design selected should not be, for these large surfaces, broken by many details, but should be slightly conventionalized, both as to form and shading. See chapter on "Shading." Where it is not desired to put in as

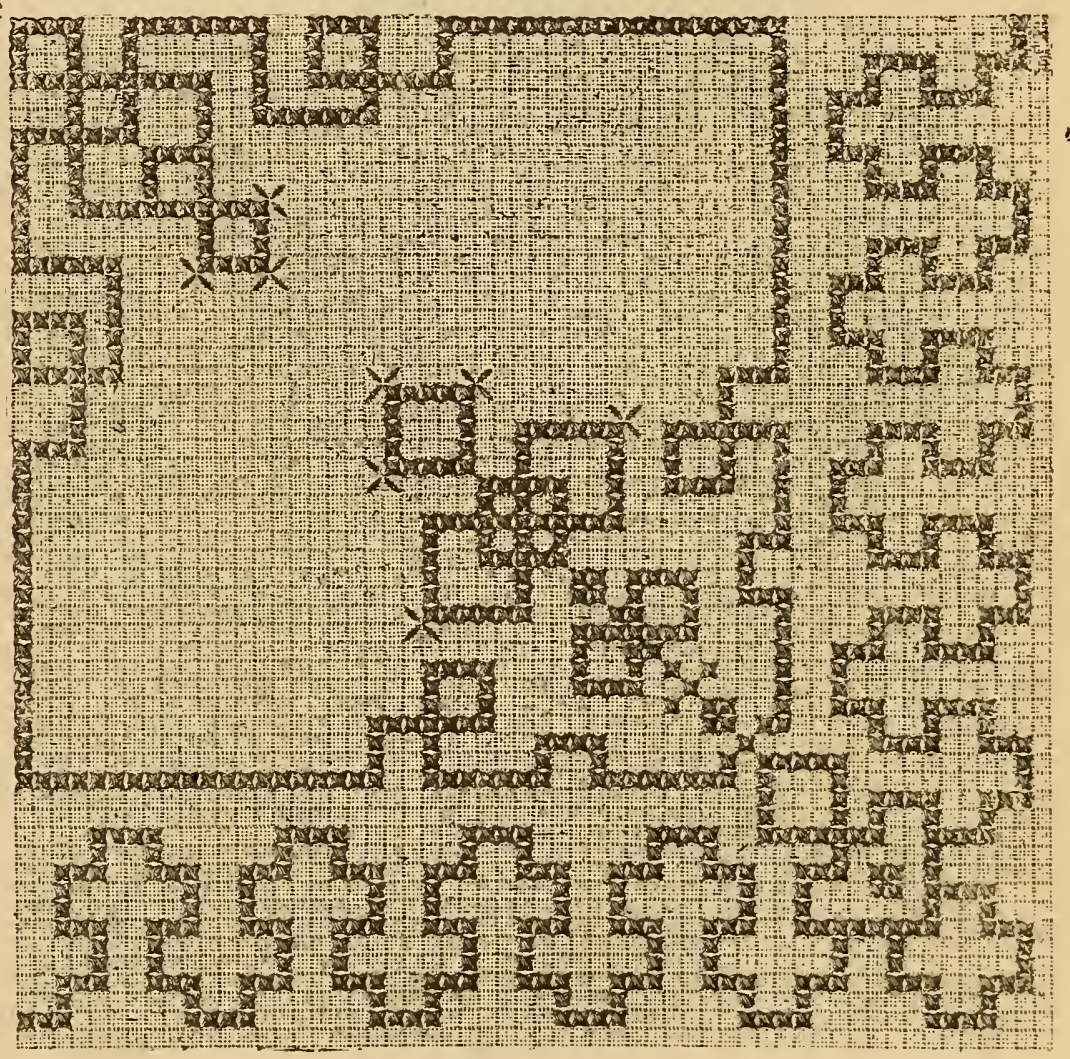

FIG. 235 .

many stitches as Kensington embroidery would require, short and long stitch will be found almost as effective, though, of course, a massed effect of color cannot by this method be obtained.

\section{CHICKEN PENWIPE.}

A unique little trifle is shown in figure 236. It consists of two or three leaves of chamois skin, on which is gracefully mounted a fluffy little chick. To make, cut from scraps of chamois skin three pieces about four inches square; paint around the edges a gold band, using a No. 3 brush and liquil 
gold paint. Fasten the leaves together by taking a few invisible stitches in the center. The next operation is the manufacture of the chicken. Cut a circle out of cardboard about the size of a small sauce dish. Out of the center of this circular piece of cardboard cut another circle. This will

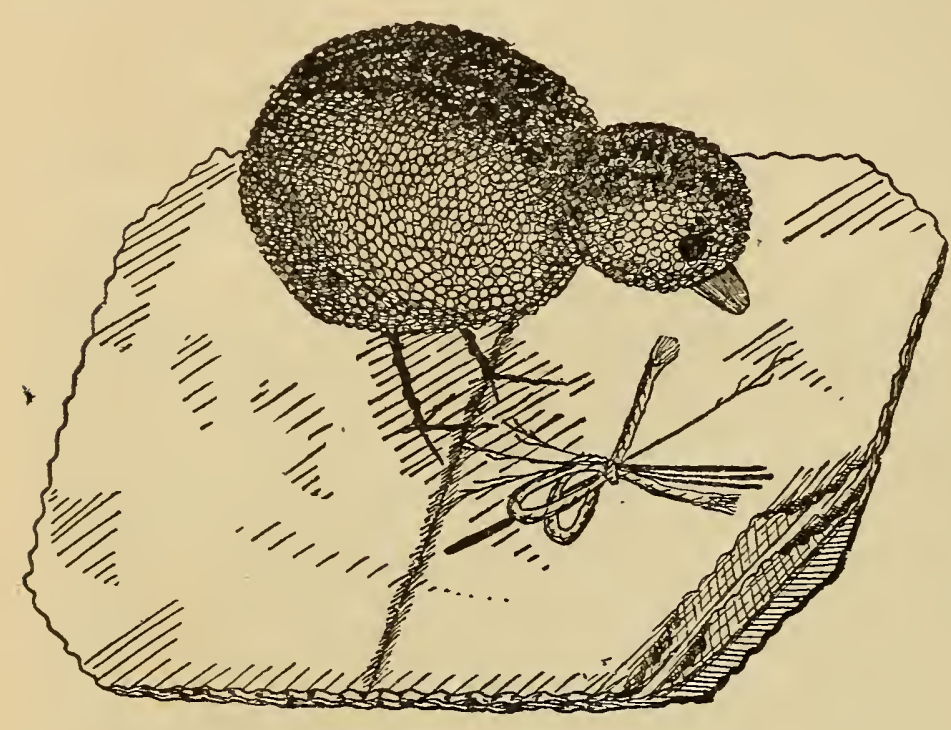

FIG. 236 .

leave a hole in the center. Around the edges of the circle wind pale yellow yarn until the center space will hold no more. Sew it on the inside edge and cut around the outside edge and pull out the cardboard in sections. The result will be a fluffy yellow ball, which will look like almost anything except a chicken, but at one end wind a piece of wire around to form the neck; put a black bead in both sides for eyes; crochet a beak out of brown wool or a bit of brown velvet, covered over cardboard and sewed on will answer as well. For the feet and legs select four strands of good, strong brown wire for each leg. Wind with brown silk thread, and at the bottom spread apart to form claws; the upper end of the wires must be fastened to the ball of wool. When the chicken is finished stand it in the center of the prepared leaves, and 10 ! you have the cutest possible penwipe.

\section{BRANCH OF FRUIT.}

There are many pretty little novelties that can be made from tissue paper-not the old-fashioned plain tissue, but the velvet and crepe papers, which are so beautiful and not at all expensive-that we think Dainty Trifles would not be complete without a few illustrations oi what can be done with this material. Figure 237 shows a branch of fruit which is effective, when nicely made, to pin to curtains, on a picture, or to cover a bare space on the wall. To make the spray shown here will require one-quarter yard green velvet paper, one-half yard orange-colored crepe paper, and a small piece of brown tissue paper and a piece of stout wire. Prepare the stem or 
branch by winding the wire with the brown paper, twisting it around smoothly and evenly. Cut the leaves from the velvet paper; they should be about three inches long. Cut the green paper into strips about four inches long by two inches wide, fold dow.. the center and taper the end to an oval point with the scissors, and the base of the leaf should also be sloped off. With a No. I brush vein the leaves with liquid gold paint, às indicated by márkings in the illustration. For the oranges roll a piece of cotton wool into a ball and cover with the orange-colored paper; fasten one orange to the end of the branch by inserting the wire directly in the center of the fruit; for the two remaining oranges insert a fine wire into each, and twist these wires around the main branch, where it is desired to have them; previously twist the small

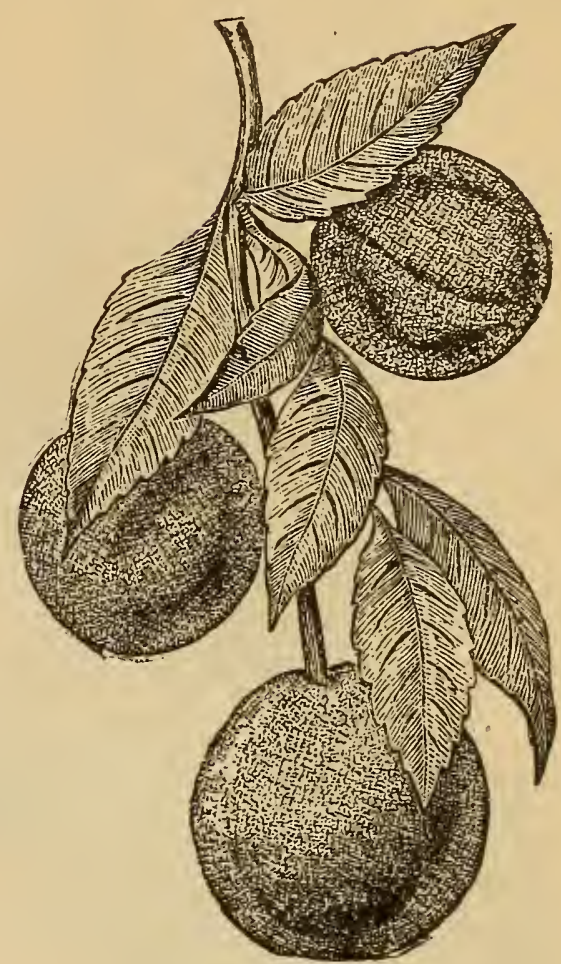

FIG. 237. wires with brown paper. Gum the leaves into position, and the branch is finished.

\section{DUST CAP.}

The little dust cap illustrated in figure 238 is not so very ornamental,

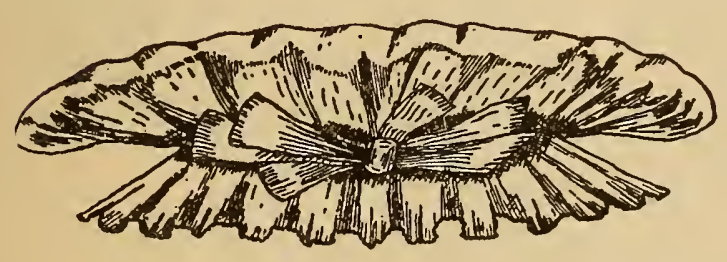

FIG. 238. but its usefulness entitles it to a place among Dainty Trifles, as no neat and dainty little lady who has dusting to do can dispense with one. To make, cut a good-sized circle-at least twenty-four inches across-from paper cambric.

Pink the edges, and two inches from.the edge run in a gathering ribbon, draw it up to fit the head of the wearer, and behold! the sauciest little mop cap imaginable.

\section{SACHET BAGS.}

The sachet bags shown in figure 239 are made of crepe tissúe paper and are pretty ornaments for parlor, mantel, or for the dressing case in a guest roon. To make the larger of the two shown, cut two squares of tissue 
vaper the same size; use a deep old-rose shade for the outer square and 3

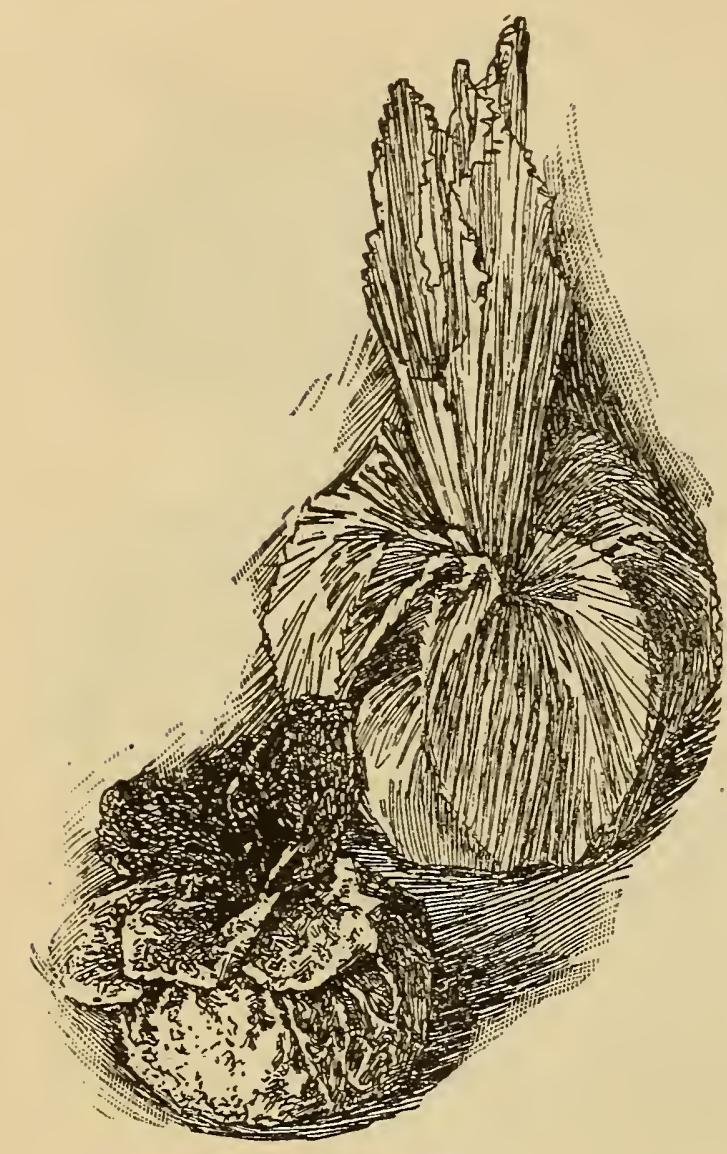

FI G. 239. light shade of the same color for the inner square. In the center of the squares laid flat upon a table place a ball of cotton wool, scented with some perfume. Gather the edges of the paper up and close to the ball of wool wind a wire tightly round and fasten. Pull the edges of the outer square downward to cover the fastening and to form a bottom trimming. Gather the edges of the inner square together, and dispose them so they will stand upright, as shown in the illustration.

The smaller sachet is made in the same manner as the one just described, only it is smaller and the edges of the paper are disposed a little differently. A pretty combination would be white crepe paper for the inner. square, and pale violet for the outer, and to carry out the illusion - or delusion-scent the wool with violet powder.

\section{TWO TOBACCO POUCHES.}

- Figures 239 and 240 illustrate two pretty little tobacco pouches, which can be utilized as Christmas or birthday gifts for the brother, big and little, one's own and the other body's brother, as the case may be. Figure 239 is made of olive plush, lined with oil silk, or sateen would do if more convenient. The design of clover, daisies and ferns should be worked out in solid Kensington stitch in wash silks in natural shades, and a drawstring completes the pouch.

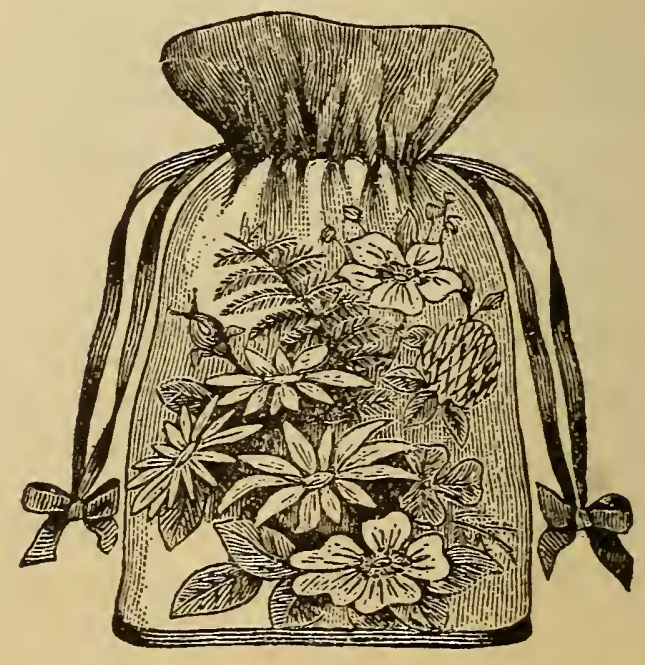

FIG. 240. 
Figure No. 24I is more simple. The pouch is made of chamois skin. without lining. The bottom edge is trimmed with a fringe of the chamois, made as follows: Select a strip of chamois as wide as the bag, and as deep as the fringe is to be when finished; paint over the entire surface of this piece, on both sides, with liquid gold paint. When dry, slash into narrow strips, to within a half-inch of the top edge, which is left for a heading; sew to the edge of pouch. The lettering on the pouch may be etched on, with wash twist, in deep brown, or it may be written

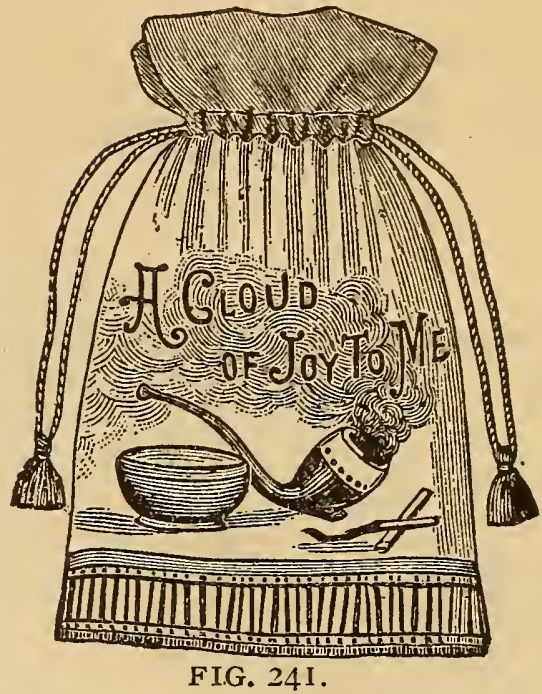
in with a brush dipped in the gold paint. The design shown in illustration can be transferred by means of a pantagraph direct to the chamois, and then painted in oil colors with one or two shades of brown and gray for bowl and clouds. See chapter on "Brush and Palette," for instructions for painting.

\section{LEAF PENWIPES.}

Figure 242 illustrates three very convenient and pretty penwipes. Cut
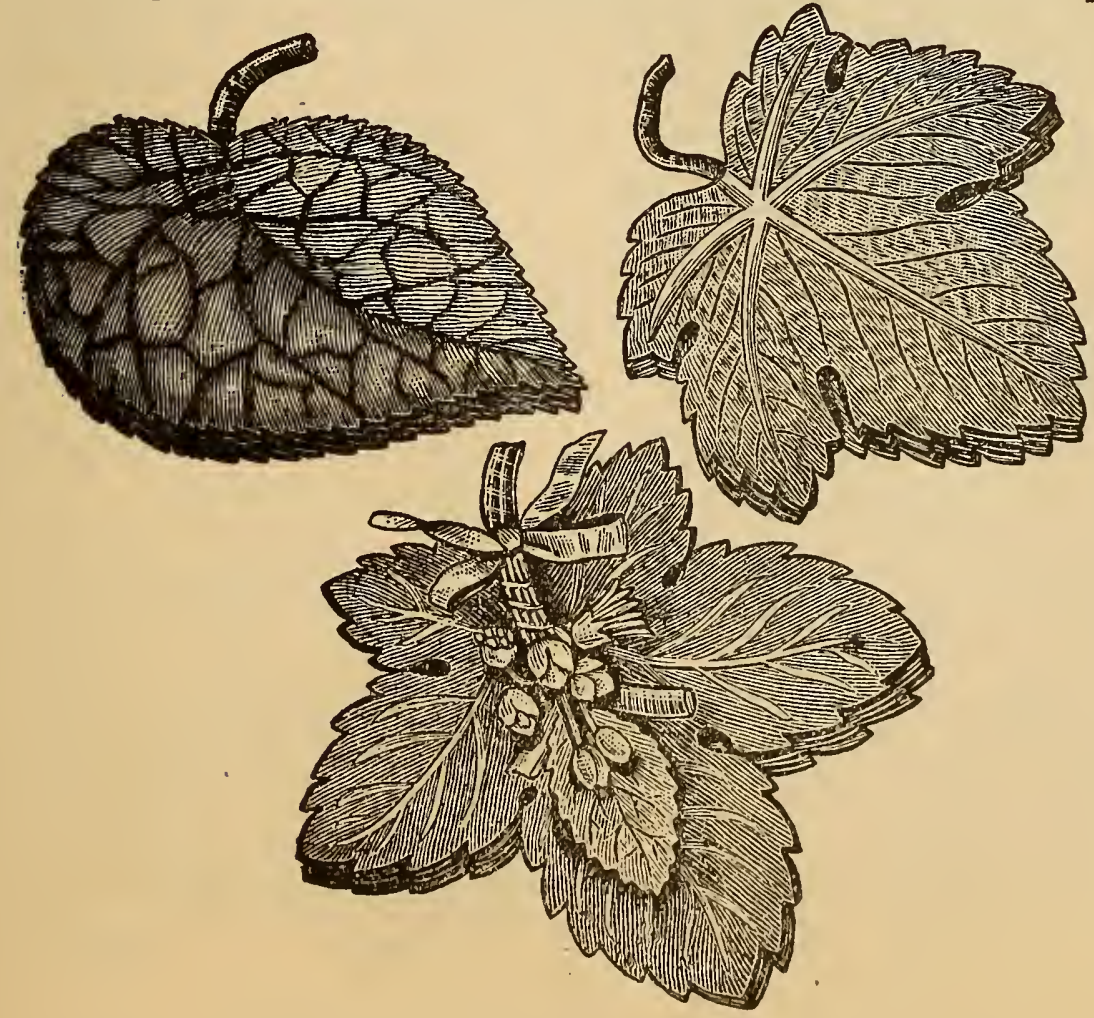

FIG, 242 . scraps of chamois skin into leaf shapes, as here shown. A natural leaf can be used as a pattern. For the covers, or outer leaves, use ooze leather, velvet or celluloid. Place the leaves together and fasten at the top, or stem part of leaf. Dip a No. I brush in liquid gold paint, and gild the edges of the leaves; also vein the outer or cover leaves with the paint, following the natural markings as nearly as possible. 


\section{TRAVELING CASE.}

For the convenient and serviceable traveling case here illustrated,

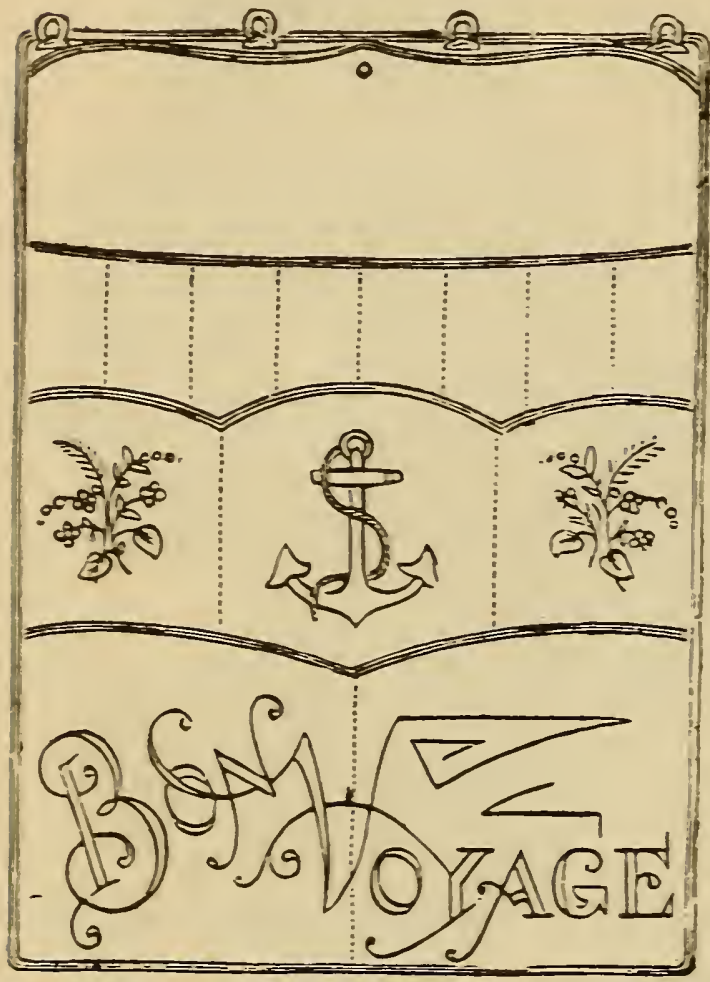

FIG. 243 .

select two pieces of good, strong, brown linen canvas, one for the outside, and the other piece for the pockets. The lower pocket is formed by facing up the inside of the case to a depth of fifteen inches with a piece of the canvas: round the top edge, and stitch duwn the center, forming two serviceable pockets. The middle of the case is divided into three pockets, for smaller articles, ard the space above is divided into eight sections. The case is bound with brown tape, and the pockets ornamented with lettering in satin stitch, done with Real Scotch linen floss, in a shade of brown lighter than the canvas. The middle pocket may be ornamented as shown in accompanying design.

\section{CHILD'S CHAIR.}

The chair shown in the illustration is such as can be purchased for twenty-five or thirty cents. Paint the frame with two coats of cream-white English enamel. For the seat and back make two cushions, filling them with feathers or cotton wool of the best quality. After the cushions are finished, cover them with white linen lawn cases, embroidered in solid Kensington stitch with filo floss. The model shown here had an exquisite design of forget-menots. worked out in natural shades. The advantage of these covers lies in the fact that they can be laundered as often as necessary, without fear of injury. Any other design than the one mentioned

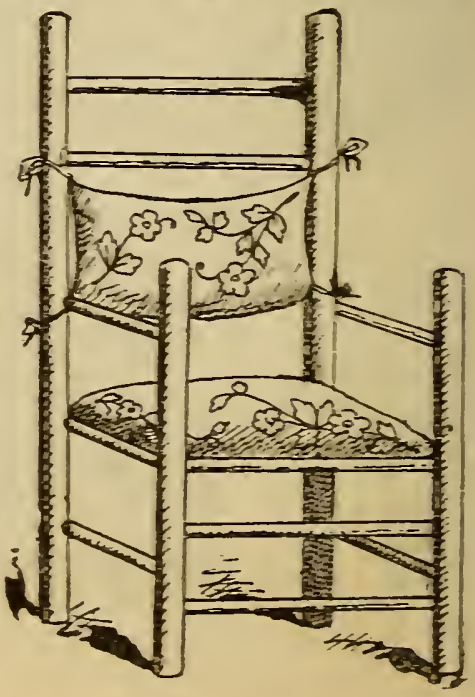

FIG. 24: can be used. It is ? matter of individual fancy-or taste, rather. 


\section{MAT FOR WATER PITCHER.}

The mat here illustrated may be made of heavy Roman twill linen. The outlines should be worked with buttonhole short and long stitch. The petals should also be marked into diamond-shaped spaces by means of outline stitch, and in the center of each diamond-shaped space, a tiny forget-me-not, embroidered in solid effect, adds much to the beauty. The bottom of the mat is worked with a spider web. The colors are a matter of taste, but white for the buttonhole stitch, blue and gold for the balance of the work, would be effective. If a round tray is used on which to rest the water bottle or pitcher, then

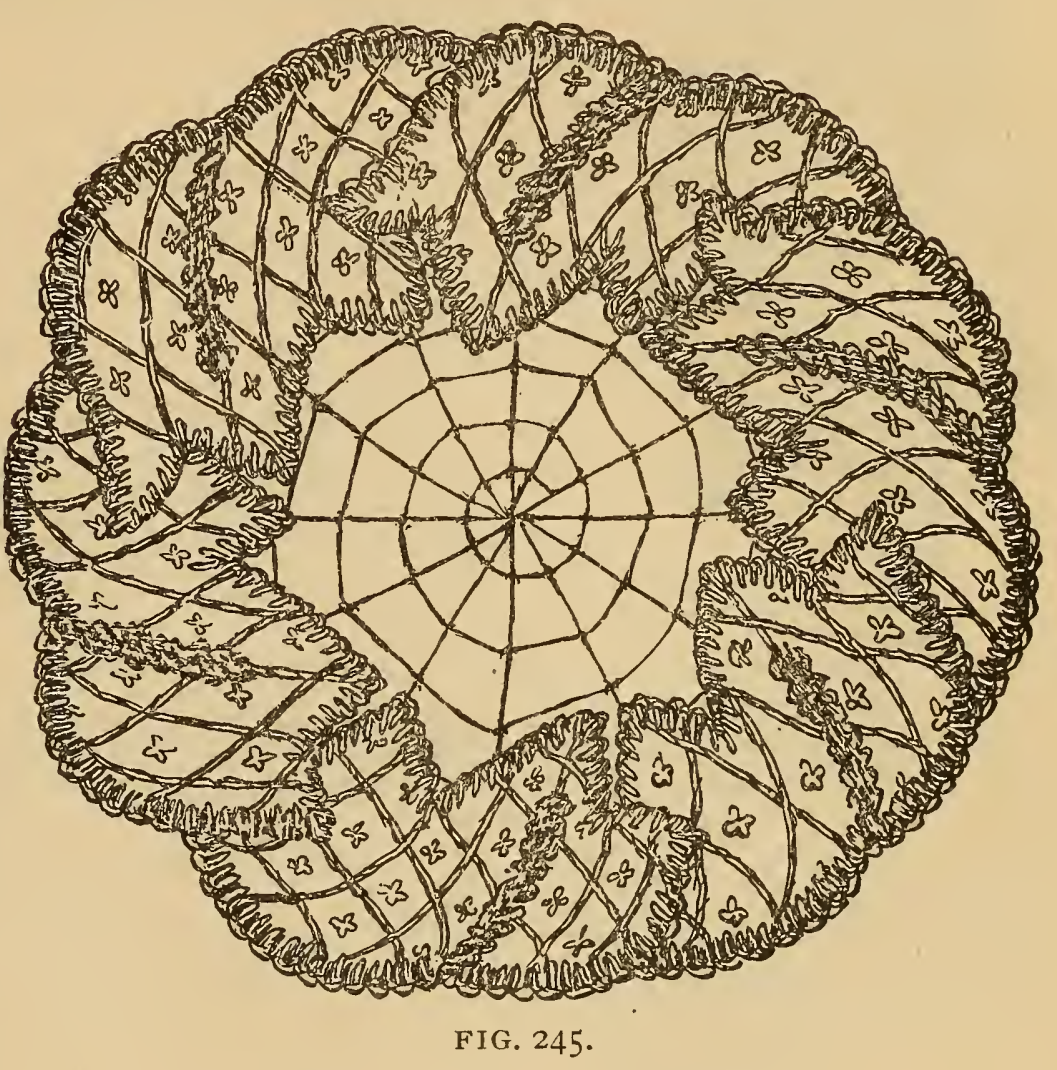
care should be observed to fit the mat to the bottom of the tray, and if "the bottom of the water receptacle can be made to fill the spider web portion of the mat, the effect will be even prettier.

NEEDLE CASES.

Figures 246 to 248 show two pretty needle cases. They are designed

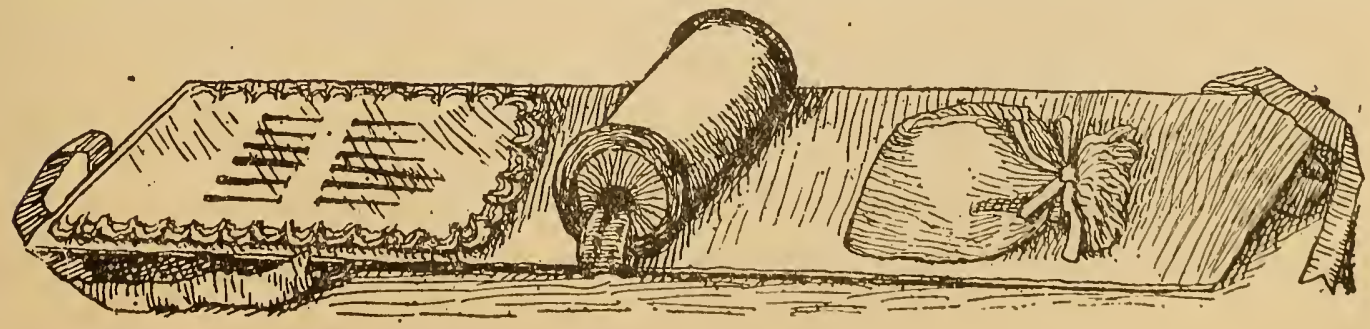

FIG. 246.

for utility, but may also be as ornamental as one chooses. Figures 246 and 247 show a needle case open and closed. To makc, cut a strip of velvet, 
celluloid or chamois skin eight inches long by three inches wide, lined with

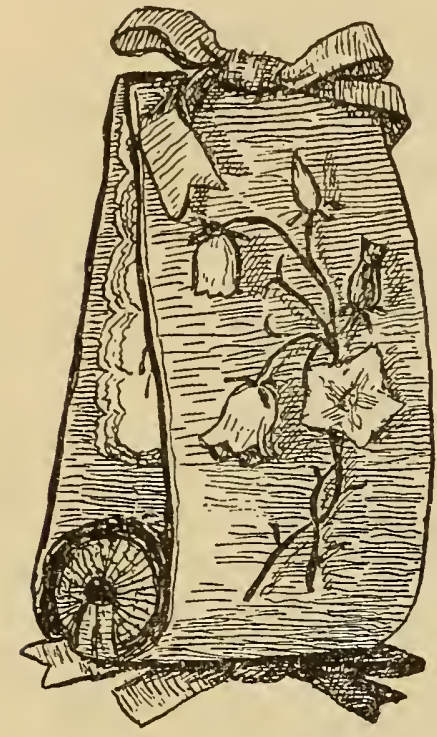

FIG. 247. a strip of red satin. In one end fasten a tiny silken bag for thimble, etc.; in the center fasten a spool of silk by running through it a ribbon, which is tied on the outside of the case in a bow; the other end of the case is furnished with a needle book, made of flannel leaves, having the edges scalloped and worked in buttonhole stitch with some pretty colored etching silk; ribbon is sewn on each end to fasten with. Figure 247 shows case closed. The ornamentations on outside of case may be any de. sign to suit the fancy, but for small articles of this nature, the pattern will look best worked out in natural shades in Kensington stitch.

Figure 248 shows a needle case somewhat similar to the one just described, but folding differently. Cut a piece of ooze leather or oil silk about three and one-half inches wide by six inches long. This will form the outside cover. For the inside lining stitch scraps of chamois skin or velvet in the form of pockets, as indicated in the illustration. Letter each pocket in outline stitch with etching silk. The leaves for the needles should be made of fine white flannel, buttonholed with blue or pink silk around the edges. The case should be neatly bound and supplied with two ribbons to fasten, with. This is a pretty little needle case and can easily be made, and is at the same time inexpensive.

The material indicated in the instructions for

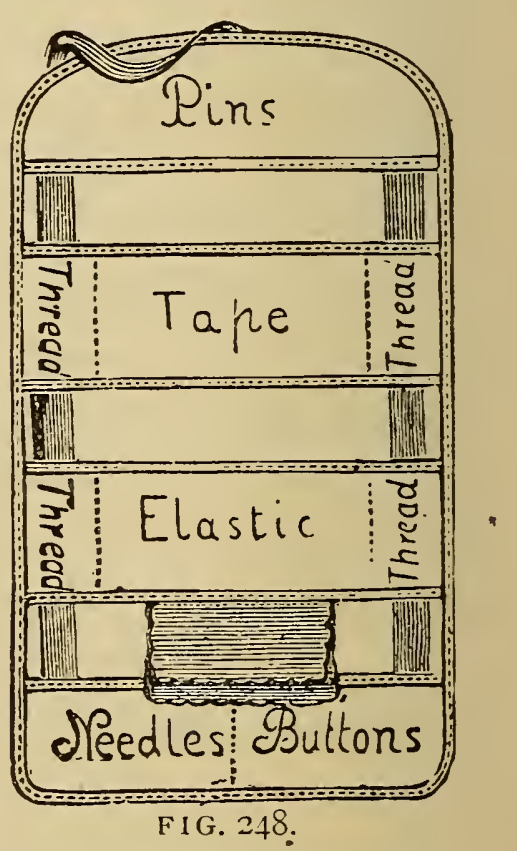
any article in this chapter may be varied to suit the convenience of the worker.

\section{KENSINGTON COVER FOR CUSHION.}

Figure 249 illustrates a beautiful design for sofa cushion cover, to be worked out in Kensington stitch with filo floss on mail cloth. The colors in the model were as follows: Foundation for cover, cream mail cloth; the roses were worked out with pinkish browns, which shades from a 
white brown to a pinkish brown; four shades were used in each rose; the forget-me-nots were done in old blue, with a tiny French knot in pale yellow; the star-shaped flowers were shaded with pale pink, and the ribbon

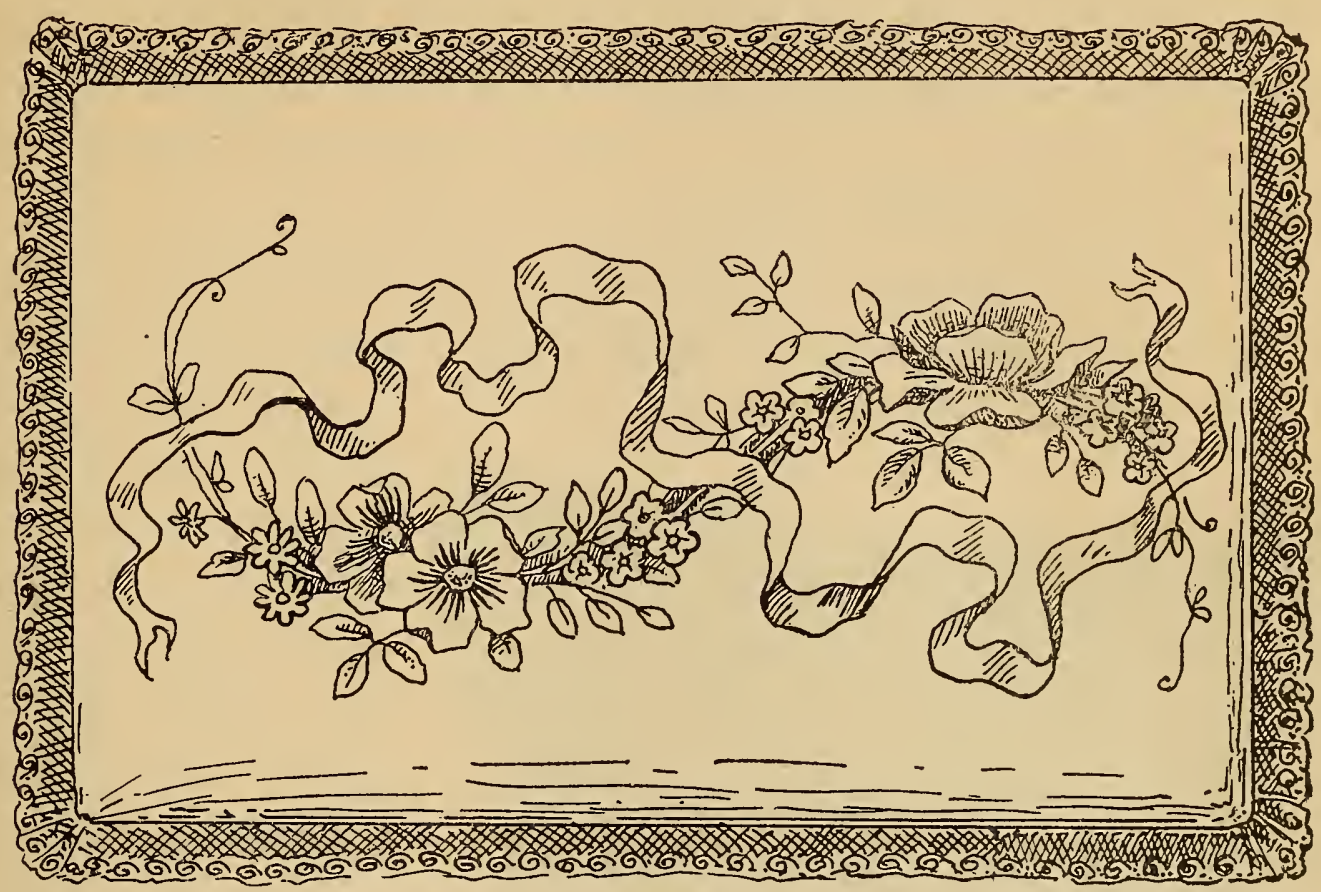

FIG. 249.

and foliage were treated with lily green in four shades. Anything more beautiful and artistic could hardly be devised, the effect of the semi-conventional shading being extremely rich. The edge was finished with a row of gold lace.

Furniture covers made of unbleached linen, of heavy quality, and with a decided twill. These covers are designed to protect handsomely upholstered furniture from the ravages of dust, light and moths, but they are sometimes also used to hide the ravages of time and hard usage. In either case they should be made to fit exactly, and the work should be done ncatly and thoroughly. There is no excuse for furniture swathed in an untidy mass of loose cloth. The covers may or may not be ornamented, according to the wishes of the owner, but where the covers are designed to be used throughout the greater part of the year, it will be more pleasing to the eye if there is a trifle of well-wrought needlework on each cover.

There are two methods of embroidering furniture covers, either of which will be recognized as artistic. The first is to decorate each piece with cross-stitch, done with linen floss in monotone. Pages I 40 and I4I show 
appropriate designs, and in the chapter on "Berlin Embroidery" will be found instructions for working. The second method is solid Kensington stitch, with wash flo floss silk. For this purpose natural forms of flowers may be selected, but the shading should be done in duil conventional shades, as described in the chapter on "Shading," as a constant sight of covers embroidered in glaring reds, yellows, pinks or blues will soon become an almost unbearable eyesore.

\section{NASTURTIUM CUSHION.}

The cushion shown in figure 250 is simple and elegant. The foundadation is a square cushion, rather flat, covered on both sides with dark blue

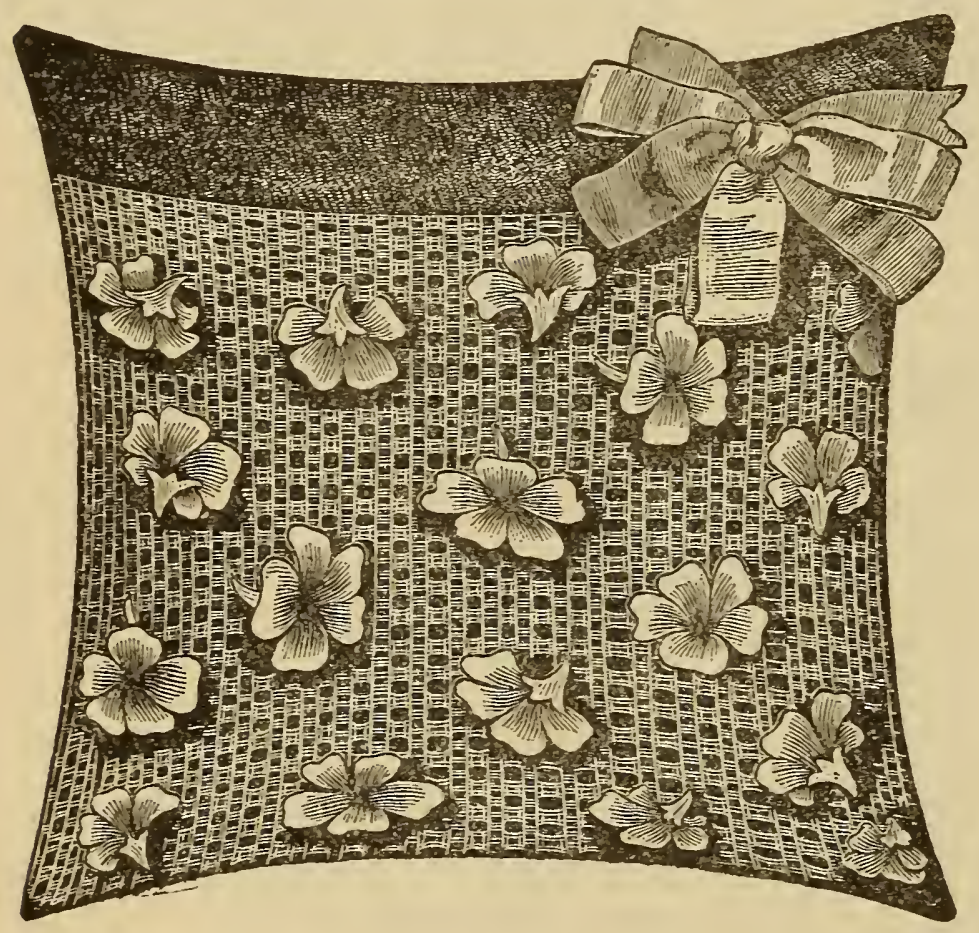

FIG. 250.

plush or velvet. The back cover is cut large enough to lap over on the front side, joining a facing or hem about four inches deep. The front of the cushion has a piece of pale blue fish net lace laid over the plush cover. Over this fish net are scattered nasturtiums, which have been just stamped on a piece of satin, the color of the lightest shade in a natural nasturtium, and then appliqued to the fish net by means of a buttonhole short and long stitch around the edges, with a shade of silk deeper than the satin flowers. The cushion is finished with a bow of ribbon, two shades, nasturtium yellow and pale blue.

In using, the fish-?et covering, any scheme of color may be carried out, and the effect of the net over satin is particularly pleasing. A beautiful cushion could be made in this manner by covering cream satin with cream white net, and appliqueing pansies in different shades of pansy purples, shading into pale heliotrope, with a dash of yellow; or, over the net, a 
powdered design of blush roses, worked in solid Kensington with rose pink filo floss. There is hardly a limit to the diversity of ornamenting cushions, but as has been frequently observed in these pages, the use to which each cushion is to be put must be carefully considered. Such. a cushion as described above would be suitable only in a reception or drawing room or guest chamber.

\section{INFANTS' TOILET CASE.}

This dainty little affair will at once commend itself to those mothers of little ones who visit or travel occasionally. Many small articles are needed for baby's comfort, and it is so necessary to have them all together. A piece of pale green moire silk, 27 inches square, lined with a stiffeningcrinoline will doand over this another lining of pale pink China silk, fitted on to the edges of the outside green silk, so that the pink lining will lay in little puffs

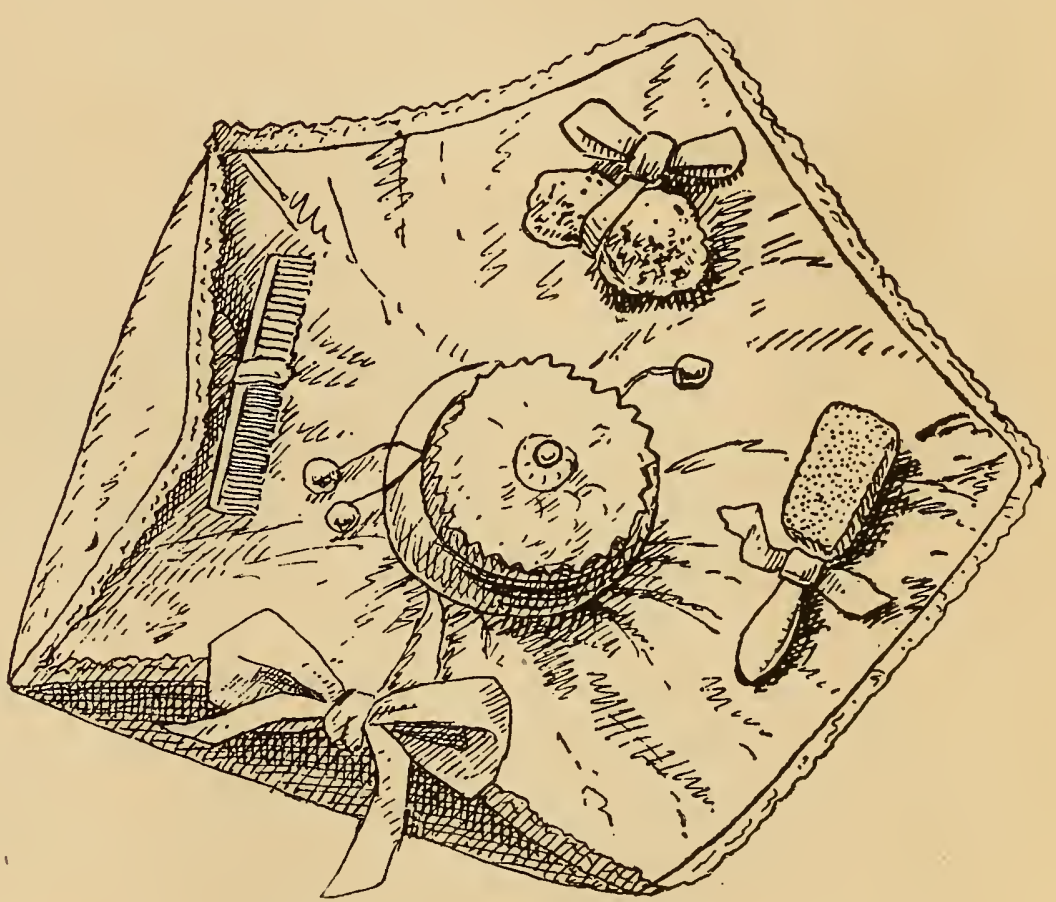

FIG. 25 I. of fullness. Straps of ribbons are fastened at convenient distances for holding sponge, brush, comb, powder box, etc. To close, fold the four corners together, and tie with a pretty bow. This will be found convenient for packing. A little case made after this model and fitted with brush, comb, sponge, powder and puff, would make a gift for an infant's trousseau that would delight the heart of the most exacting mother.

\section{KEEP A SCRAP BAG.}

In this chapter on "Dainty Work," many articles of easy manufacture have been illustrated and described, but, dear reader, do not for a moment imagine that the subject has been exhausted. What has been given should 
but serve as a stimulus to the inventive faculties of the worker, for each article illustrated can be made in as many ways as there are individual fancies. It is not expected that the woman with brains will confine herself slavishly to a given size, color or shape. She will but use the suggestions given as a foundation on which to work out her own taste. There are many novelties which the scope of this work will not permit us to enlarge upon, but we must be permitted one more word before we pass on to the next subject, viz., "Keep a Scrap Bag." The numbers of pretty little trifles that can be made from the accumulation of ribbon, velvet, silk, flannel, and the usual odds and ends that will accumulate, is indeed surprising. To women of limited means it is most discouraging to be compelled to purchase each item for making the smallest trifle of fancy work, and many homes go unadorned because of the neglect to care for the little scraps that an ingenious mind and nimble fingers could fashion into articles of use and beauty.

Again, despise not the simple and inexpensive means of decorating a mantel, shelf, table or dressing case. Tissue paper, scrim, muslin, crochet linen, etc., may all be used effectively, and it is not at all necessary to wait until you are able to buy silk, plush, velvet, etc., for the "work before attempting to make your home artistically and cosily pretty- "Cosily" is a good word applied in this connection, and the woman who fails to grasp its true spirit, is pretty sure to make a failure of her home beautifying.

The following chapters on "Drawn Work" and "Brush and Palette," should be carefully studied and thoroughly comprehended, as by means of the instructions there given, the question of expense is resolved to a minimum.

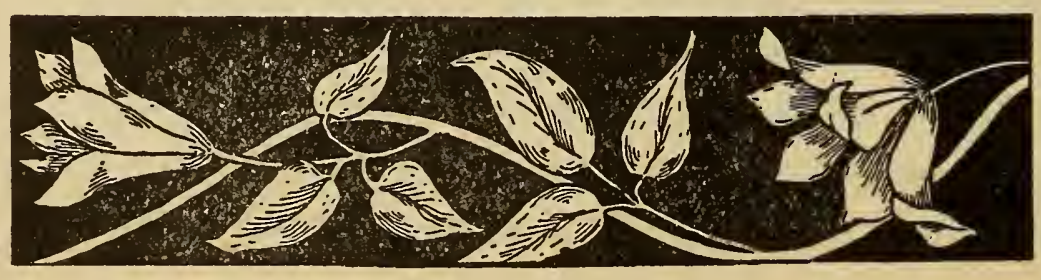




\section{CHAPTER XIII.}

DRAWN WORK.

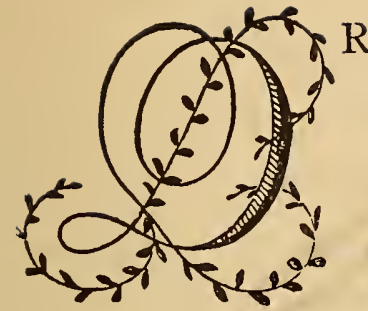

RAWN WORK offers a means of beautifying table linen, bed linen, towels, handkerchiefs, and many articles of a lady's wardrobe. It is the least expensive, as to actual outlay of money, of any form of decorative needlework, but to do elaborate pieces on fine materials will require time and patience, and prove a tax upon weak eyes.

The material for doing drawn work must be selected with care, but

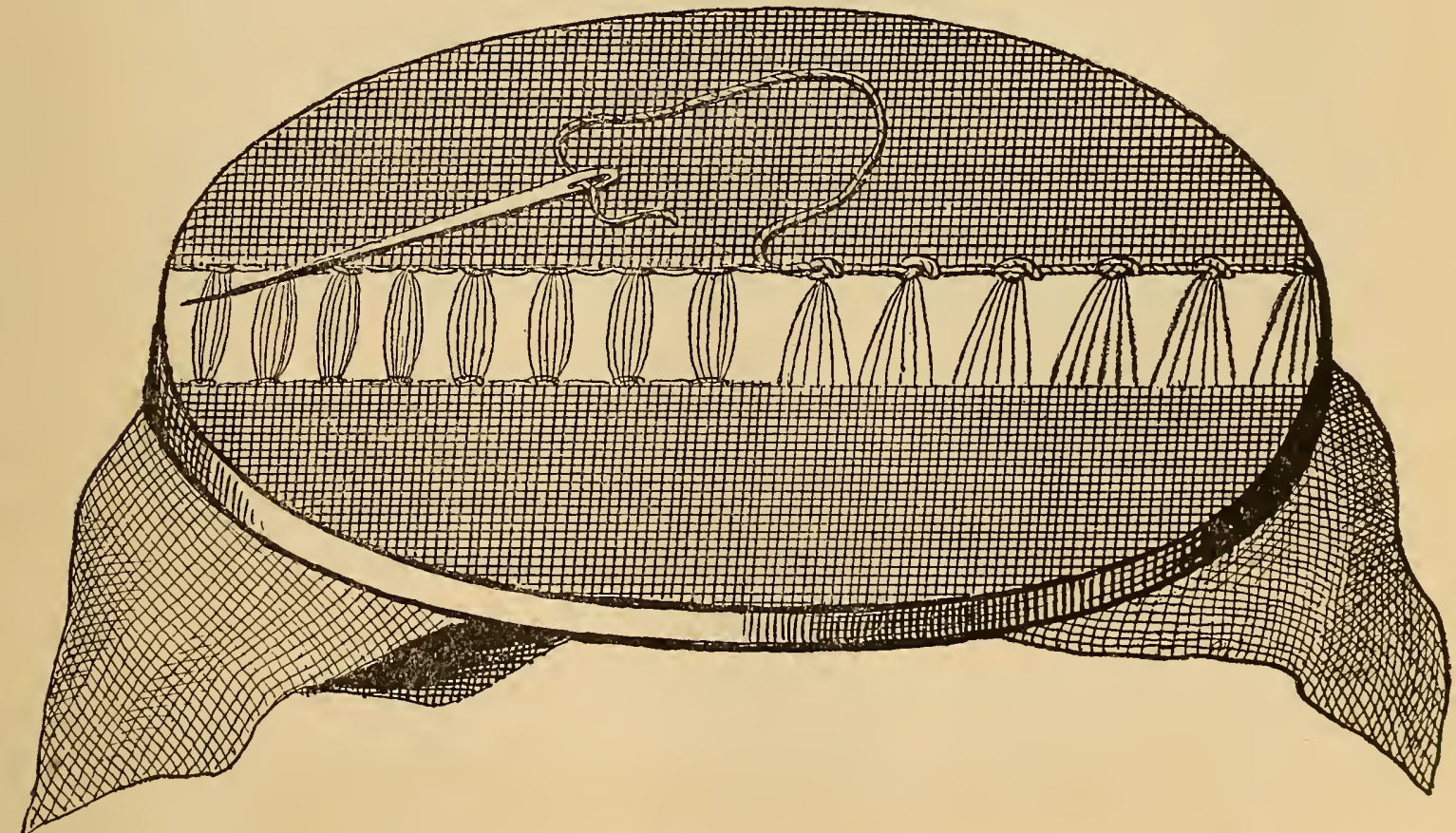

FIG. 252.

nearly all grades of linen may be used, provided the threads are even and the surface smooth. The quality of linen known to the trade as "round thread linen," is best adapted, as the threads are easily drawn, and the remaining threads are firm and smooth. Scrim, surah silk, lawn, mull-all may be decorated with drawn work. The work should be done in either a square 
frame, having the work stretched in it according to directions given in the chapter on "Implements," or in a pair of hoops, as shown in figure 252. To work with, linen crochet thread, No. 80, is most desirable to use, though the beautifully colored wash silks are frequently employed on white goods and always on silk and colored fabrics.

Madonna cotton is used where linen or silk thread is not desırable. It comes in small balls, in cream or pure white.

\section{Preparing the Work.}

Having decided upon the pattern, and the size of the article to be embellished with drawn work, the first step is to draw the threads. Figure 253

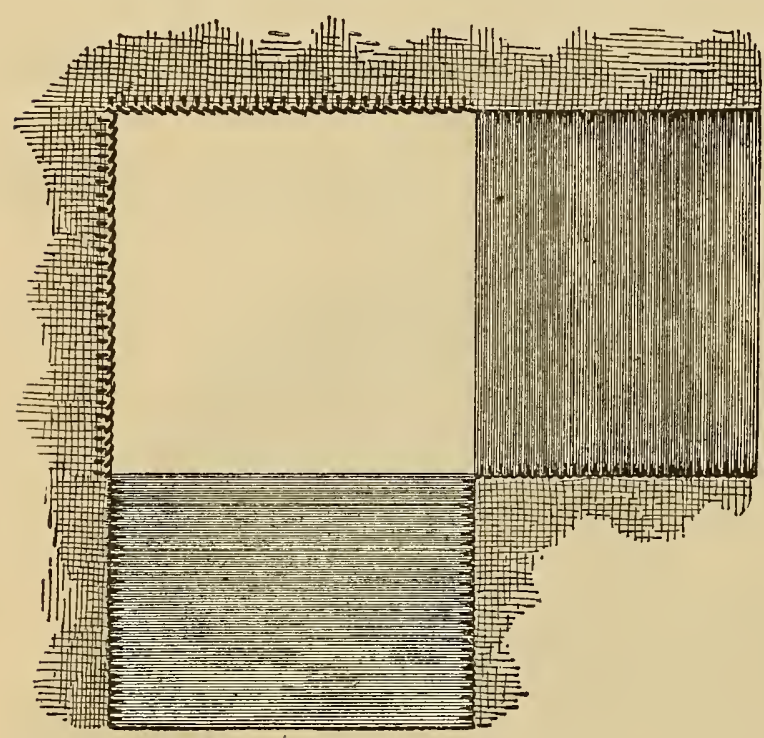

FIG. 253 . shows a border and one corner hav. ing the threads drawn ready for working. The space required for the corner is first cut out-see figure 254 . The threads are then drawn out from each side, until a space of drawn threads the required depth of the design is obtained.

This operation is always the same in all patterns, where a corner is desired; sometimes the corner is filled with alternate squares of linens and drawn threads, instead of being filled in with a solid design. Figure 255, illustrates method of doing this. First cut out a space the desired size for the first square, next leave an equal space of plain linen, repeat until the requisite number of squares is obtained, pull out the threads from each side; the pattern is then ready for working.

Where a border only is desired, the threads are drawn from edge to edge, see figure 256 .

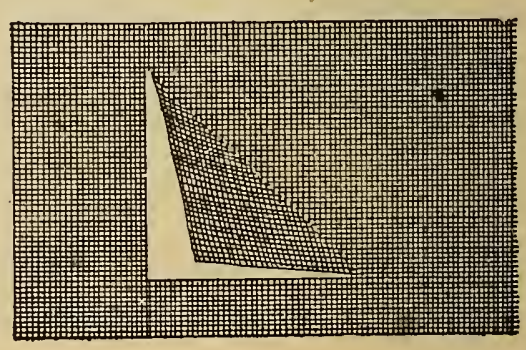

FIG. 254 .

The threads drawn, we are now ready to proceed to the work of making the patterns and this brings us to the question of stitches. 


\section{Foundation Stitches.}

The stitches used in complicated patterns of drawn work, are those given in the chapter on lace making. The number used in any given pattern is limited and when the work is done by use of the threads of the material, is confined to varieties of knot stitch and weaving stitch. For instance: the corner shown in No. 258 is made entirely from these two stitches-the varieties of these two stitches include hem stitching, ladder stitch, herringbone, duck's tail, spider web, raised wheel, flat wheel and woven wheels.

\section{Hem Stitching.}

The most useful of all drawn work stitches is the simple hemstitch shown in letter $c$, in figure 256 . This stitch enters into almost every pat-

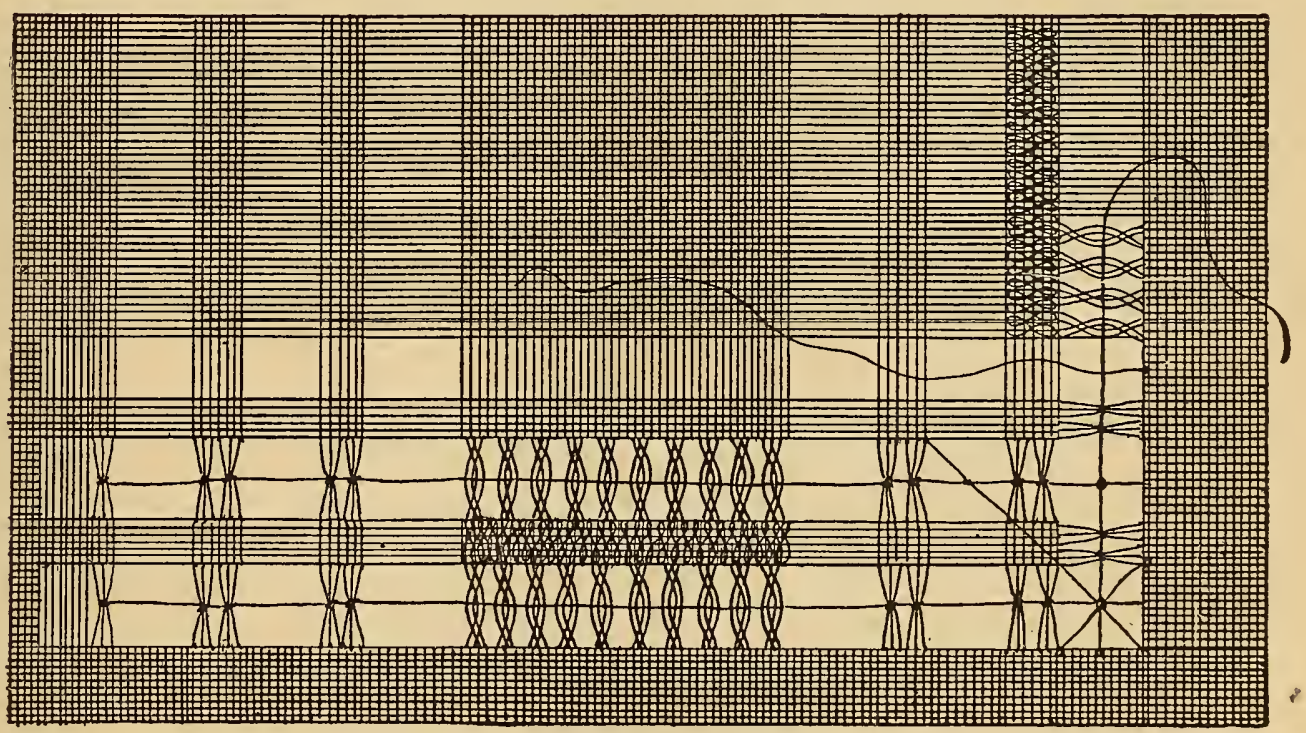

FIG. 255 .

tern for drawn work; for instance, when the threads are drawn for a design the first operation should be to hemstitch the edges of the material where the threads were drawn, to prevent the further loosening of threads, see letter $c$, figure 256 .

The method of making hemstitching is simple; commence at the left and work to the right is a rule to be remembered. Where hemstitching is to be applied as a finish to a hem of any article, a few threads only are drawn, the number in any case depending on the fine or coarse quality of the material. If very fine, more threads must be drawn, three to five, will in 
most cases be sufficient. The depth of the hem must be first determined, and the threads drawn twice the distance of the width of the hem from the edge. The hem should then be turned and basted into position close to

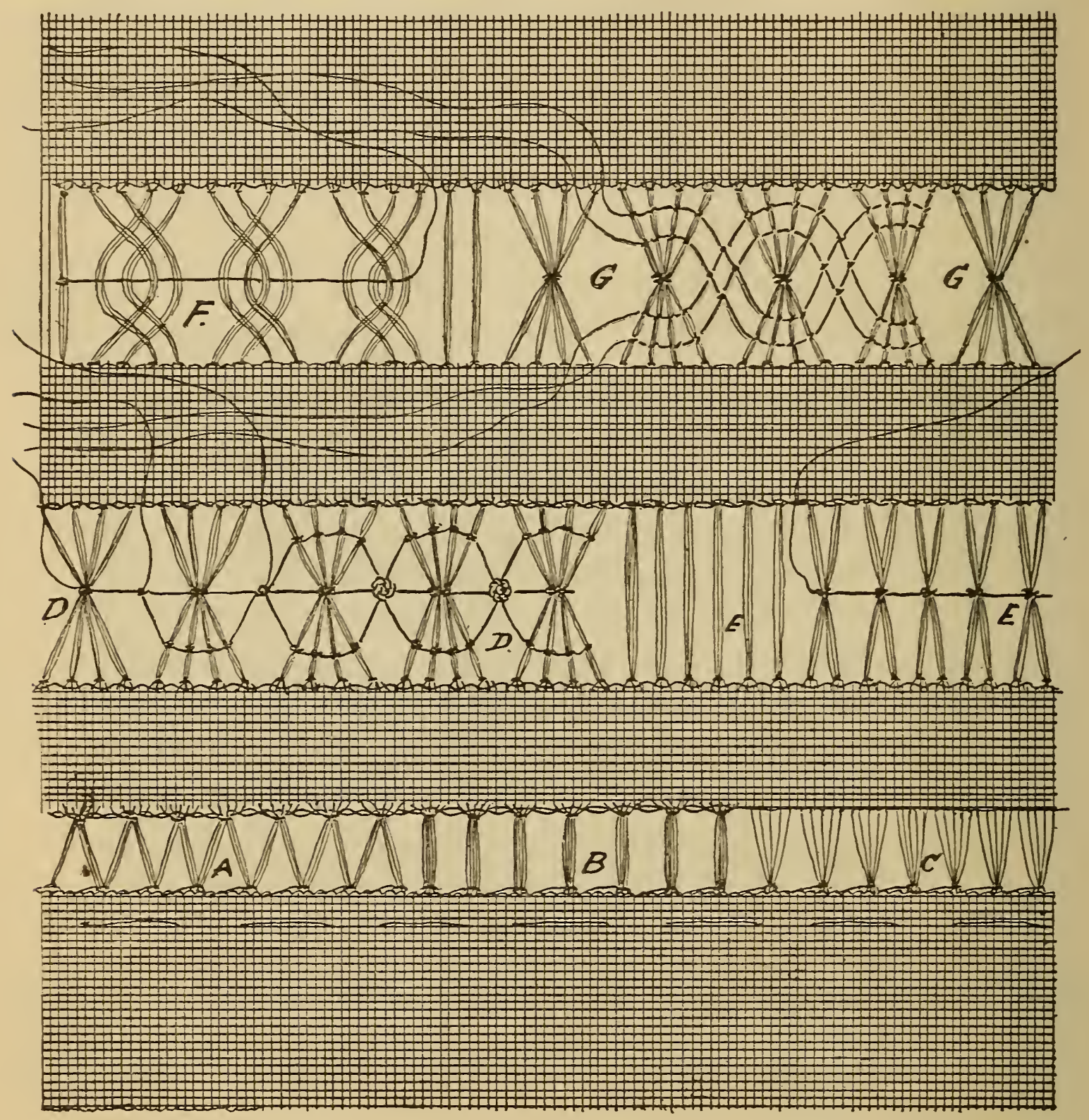

FIG. 256.

the first drawn thread, commence at the left and put the needle point at the back of the drawn threads, taking up three to four threads; be sure that the thread in the needle is to the right and under the needle, forming a halfloop. Pull the needle through towards you; repeat to the end. This 
forms a flat almost invisible stitcn, very useful for fine work. There is another variety of hemstitching which forms a row of raised knots on the edge of the hem. The method of working is described on page 36 , see figure 26.

\section{Ladder and Herringbone Stitch.}

Ladder stitch is simply a row of hemstitching on each edge of the drawn threads, see letter $b$, on figure 256 . This stitch is often used for making rows of hemstitching for trimmings.

Herringbone stitch is shown in letter $a$, on figure 256 . This stitch is made by first making a row of hemstitching on the lower edge of drawn threads. After this hemstitching is done, a row of hemstitching is made on the upper edge as follows:

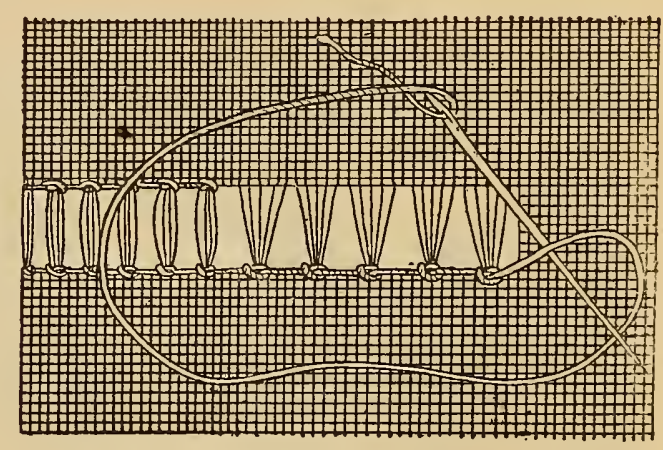

FIG. 257. one group of threads formed by the lower row of hemstitching is taken up on the point of the needle, together with one-half of the next group of

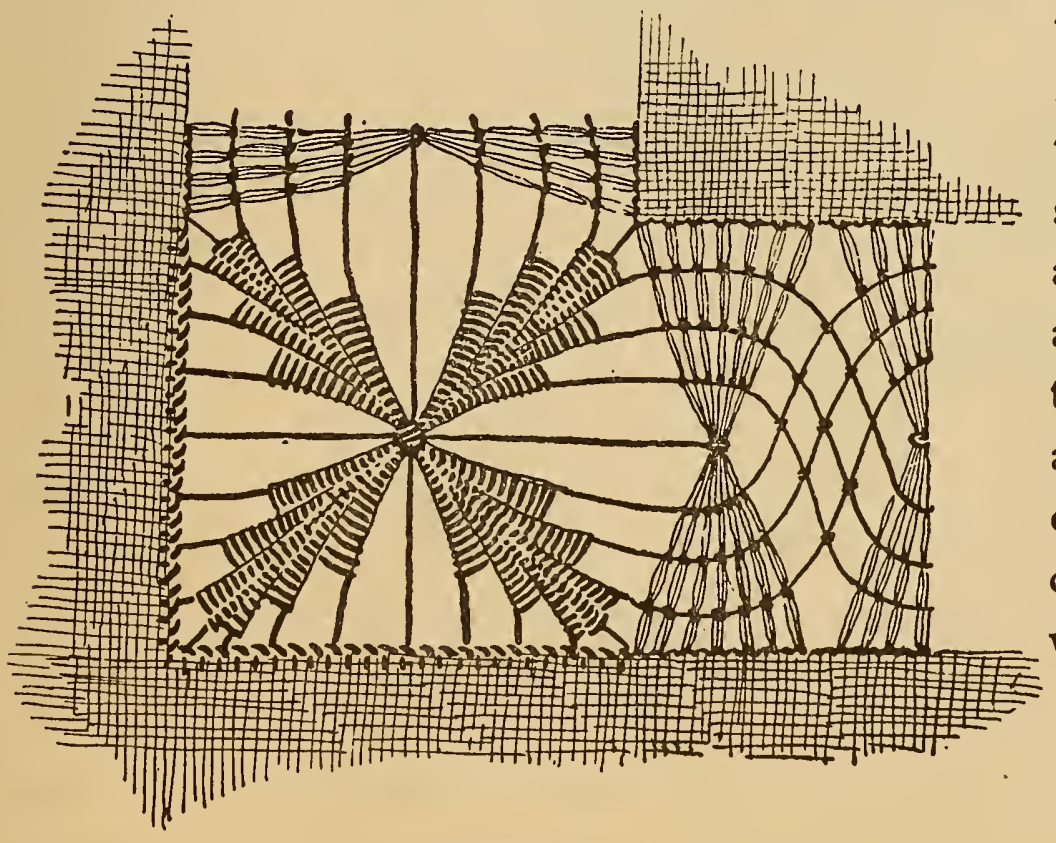

FIG. 258. threads, make a simple hemstitch and repeat to the end of row. This stitch, so applied, forms a very pretty insertion, and is especially effective where several rows are used, one above the other, leaving a space of plain linen, of equal width between.

\section{Knot Stitch.}

This is one of if not the most important stitch used in drawn work, and may be applied to the middle of drawn threads to form an insertion, when it is called duck's tail, or it may be used in succession to hemstitch threads, where no hem is to be formed, in this 
arrangement it has the appearance of knotted chain. Figure 257 shows the knot stitch so applied, as well as the method of placing thread to make. To make the knot stitch: carry the thread upward and to the left, forming a loop; insert the needle under the drawn threads to be tied and draw upward through the loop. The needle when inserted under the threads should point to the right and the thread should be drawn through carefully and firmly. Knot stitch will require some practice in order to make

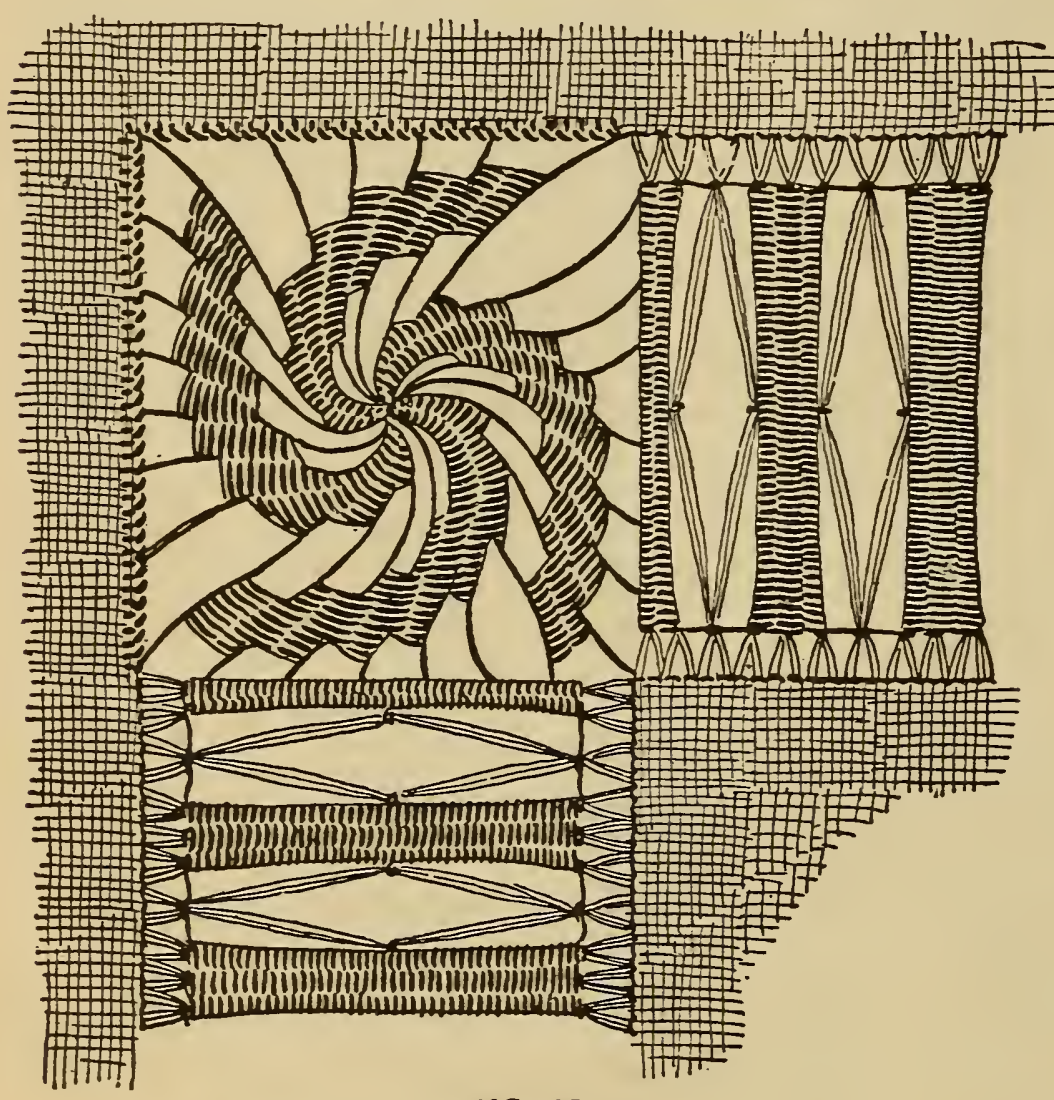

F1G. 259. it exact and smooth each time, as its beauty and usefulness depend entirely upon the nicety with which it is made. Letters $e$, figure 256 , show this stitch applied to knotting threads; letters $d d$, same figure, show the knots applied to forming patterns, and letters $g g$ show a more complicated use of the same knot.

In working out the design in letters $g g$, figure 256 , knot with knot stitch a group of four strands of threads, which have first been hemstitched on both upper and lower edges, knot in the center and twist the thread back to the edge of the material on one of the strands of threads, twist it down the first strand of the next group of four strands and knot again; continue this to the end of the drawn threads. The twisting back is done to avoid carrying the knotting thread across middle of the pattern forming. The difference will be noticed by observing letters $d d$, in figure 256. In this pattern the thread is carried from one group to the next, forming a strand through the center. The next step in forming this 
pattern is to commence at the first strand of the first knotted group and make a knot stitch about one-eighth of an inch from the upper edge, carry the thread across to the next strand and knot again; continue this knotting until the four strands have been knotted, when the thread must be carried down to the lower half (next the center knot) of the second group of fours, this thread is then knotted across each of the four strands, when it is carried upward to the upper half of the third group of fours. This operation must be continued until all of the groups have been knotted; commence again at the first strand of the first group of fours and repeat the knotting a short distance below the first row. The exact distance must of course be regulated by the depth of pattern. This will form two rows of knotting across the upper and lower portions of everyother group of fours. Begin at the first strand of the lower half of

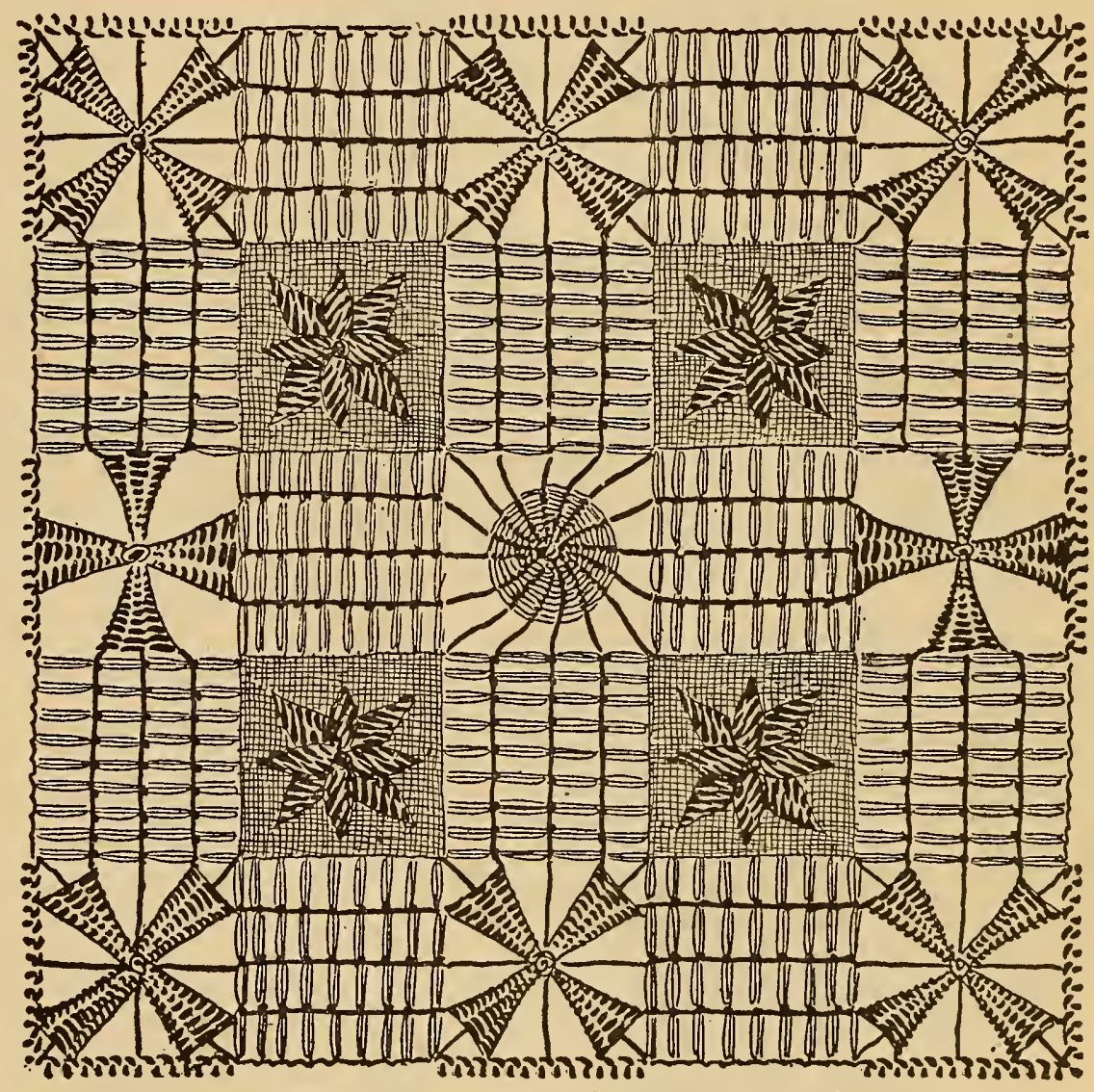

FIG. 260.

the first group and knot across, carry the thread upward to the upper portion of the second group-in crossing the threads that have been left between the two groups in the first two rows of knotting make a knot stitch-and remember it is a rule in drawn work to knot every time a thread is crossed. Continue the knotting across all the groups as before described and then return to the starting point and put in the last row. The number of rows must depend upon the depth of the design. In letters $g g$ the first and last group of strands have purposely been left unknotted. 


\section{Double Matrimony Stitch.}

Double Matrimony stitch is a simple and effective means of making rows of insertions, also to lend variety to designs that otherwise would appear too monotonous. Letter $f$, figure 256 , shows this stitch in detail, while it may be seen applied to a portion of insertion in figure 255 . To make: four strands of hemstitched threads will be required for each stitch -see letter $f$. Fasten the working thread in the edge of the material and carry point of needle under the third strand, twist point of needle back over the second and back under the fourth and over the first strand and draw the needle up through the center. The working thread running through the center of each stitch holds the twisted strands in place; work from the left to the right.

\section{Wheels and Spike Ornaments.}

Wheels and spike ornaments for corners and other open spaces are formed by means of weaving. Figure 258 shows a corner and section of

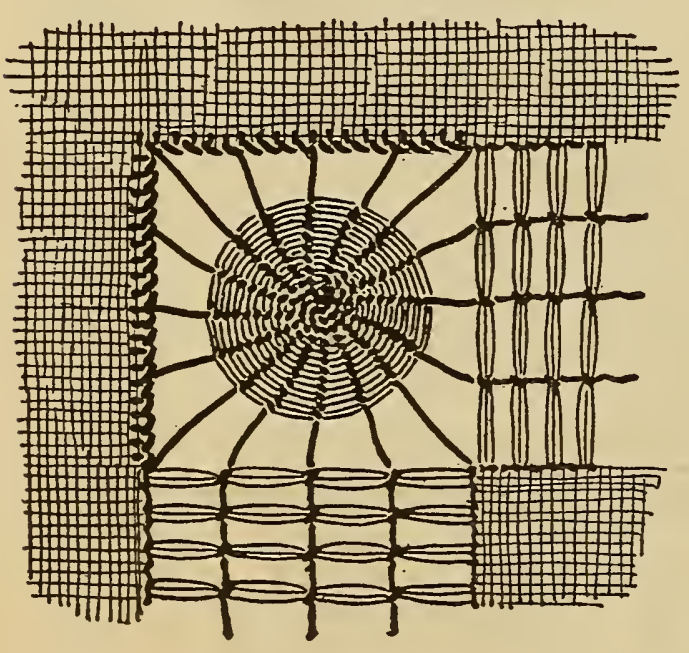

FIG. 26I. border. The space for the corner is first cut out-see figure 254. The cut edge is next buttonhole stitched to prevent raveling. The threads are then drawn from each way, as shown in figure 253. The border is first worked with knot stitch, as previously described, and the working thread, as each row of knotting is finished, is carried across the cut out corner and invisibly fastened into the edge of the material. To work the corner shown, commence in the center with the working thread and weave over and under all threads a few times; this will form a flat wheel. Then pick up seven threads and weave back and forth over and under them, until about one-third the distance from the center to the corner of design has been so treated. Now continue the weaving, but drop one thread at each side and weave over five instead of seven, proceed the desired distance-which is entirely a matter of personal choice-and then drop two more threads, one from each side, and finish to the corner, weaving over only three threads. To fasten, run the 
needle through the woven spike to the center and cut off at the back of the work. Leave one thread and take up the next seven and work as before, and so on until the four corners of the square are filled with woven spikes.

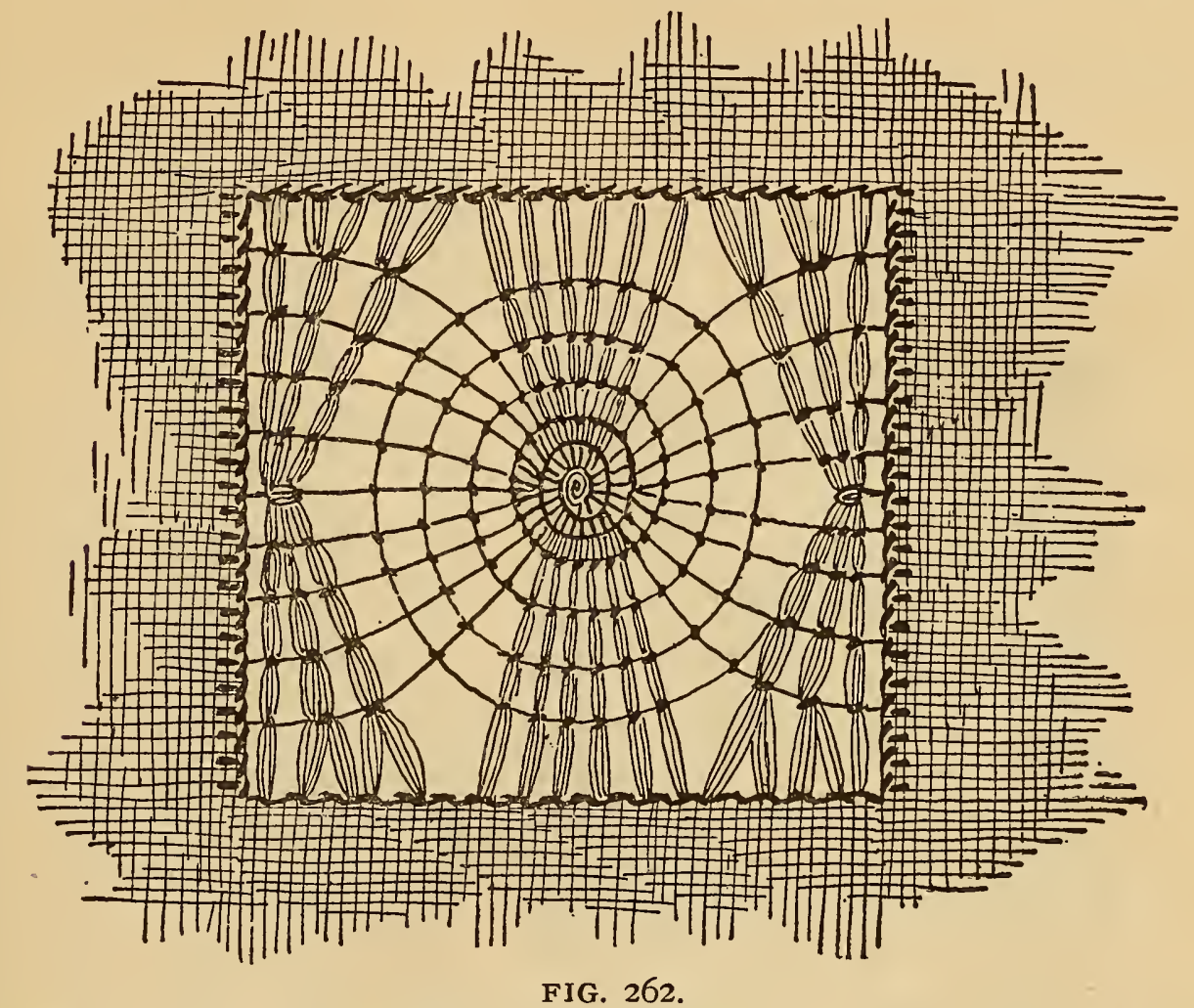

When the work is completed there will be left on each side one unworked thread-all similar ornaments are made in exactly the same way, being

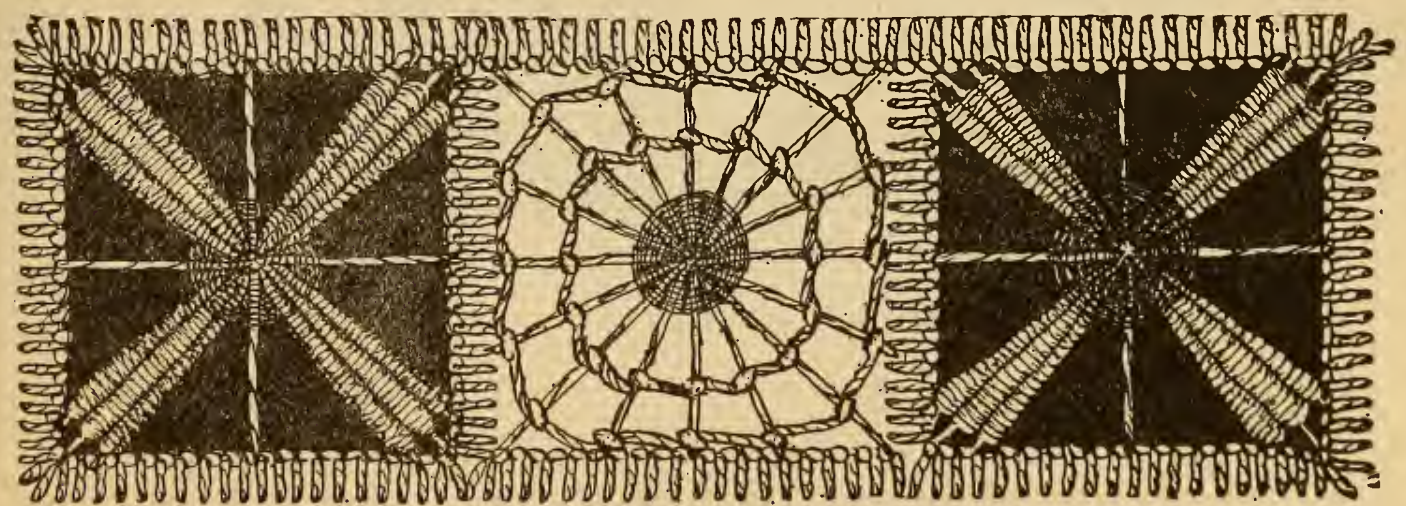

FIG. 263 .

varied only by the number of threads woven, and the different number of the rows of weaving. To weave, here means to put the point of needle under one thread, over the next, under the third, over the fourth, and so on, repeating back and forth. 
Figure 259 illustrates a corner and border different from any yet shown. In the construction are used four stitches, knot stitch, hemstitch, weaving stitch and buttonhole stitch.

The corner is prepared as described in the preceding. The two edges of the cut corner are first buttonholed, the threads for the insertion next
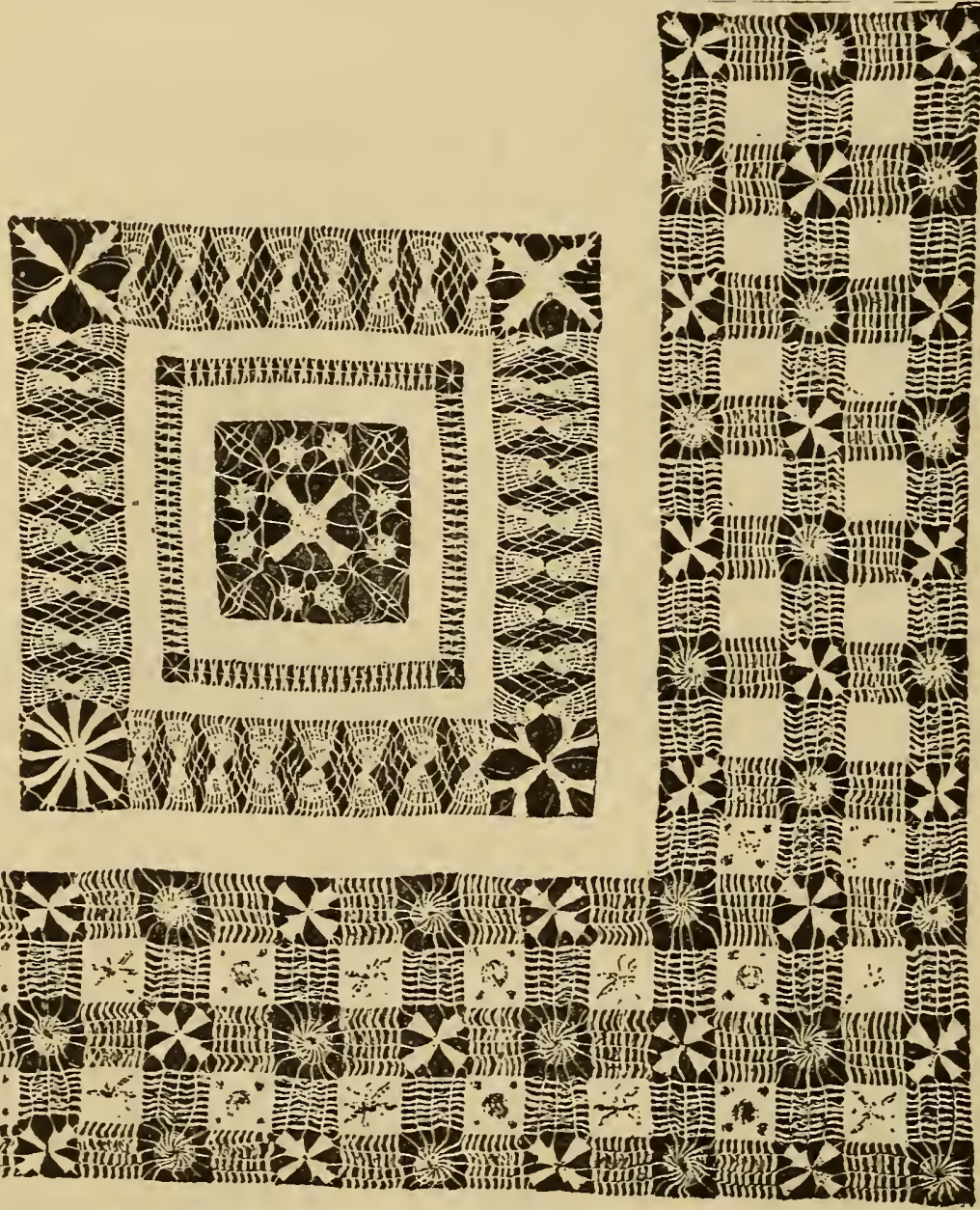

总

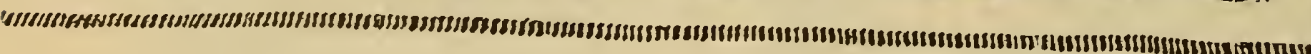

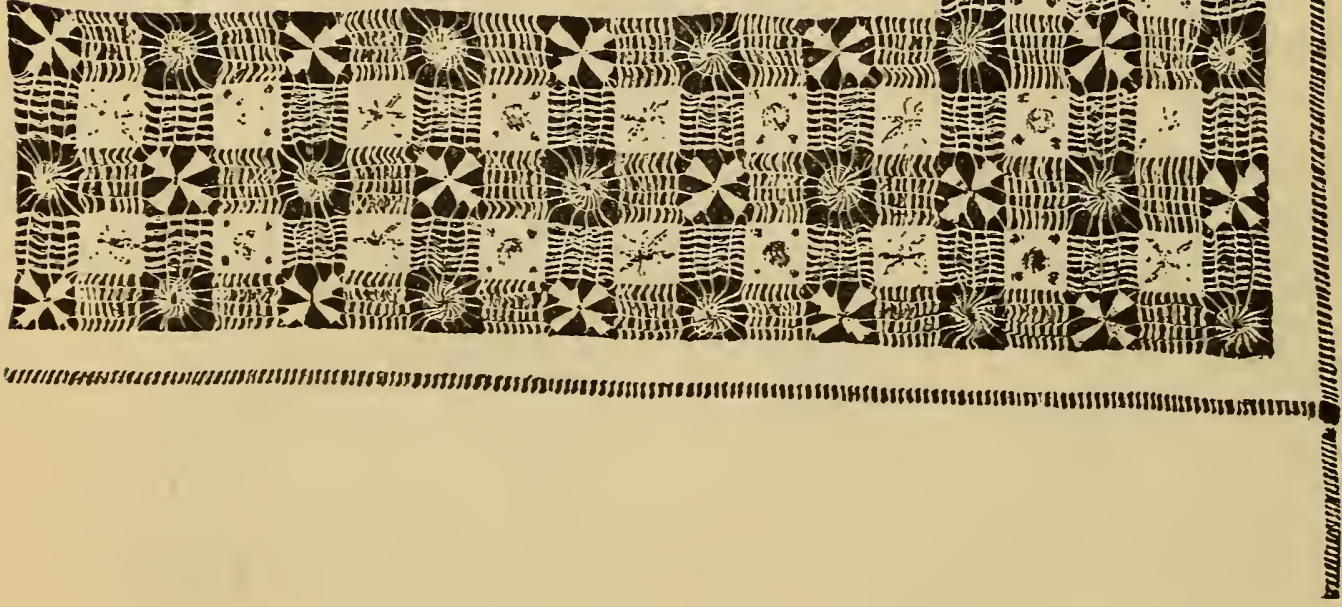

BORDER OF TEA CLOTH, AND CENTER OF DOYLEY. FIGURES 264-265.

drawn from each way. In the model the insertion is one and one-quarter inches deep. Both edges of the insertion are hemstitched and a row of knot stitch is worked top and bottom about one-eighth of an inch from the edge of the material, across the entire insertion. The border is made by 
weaving stitch; commence and weave over four strands of hemstitched threads, back and forth from top to bottom of insertion. When about hals way to the top edge of the insertion, weave in the fifth strand of threads with two rows of weaving, then drop it and proceed as before. Skip the sixth strand of hemstitched threads and begin at the bottom of the seventh
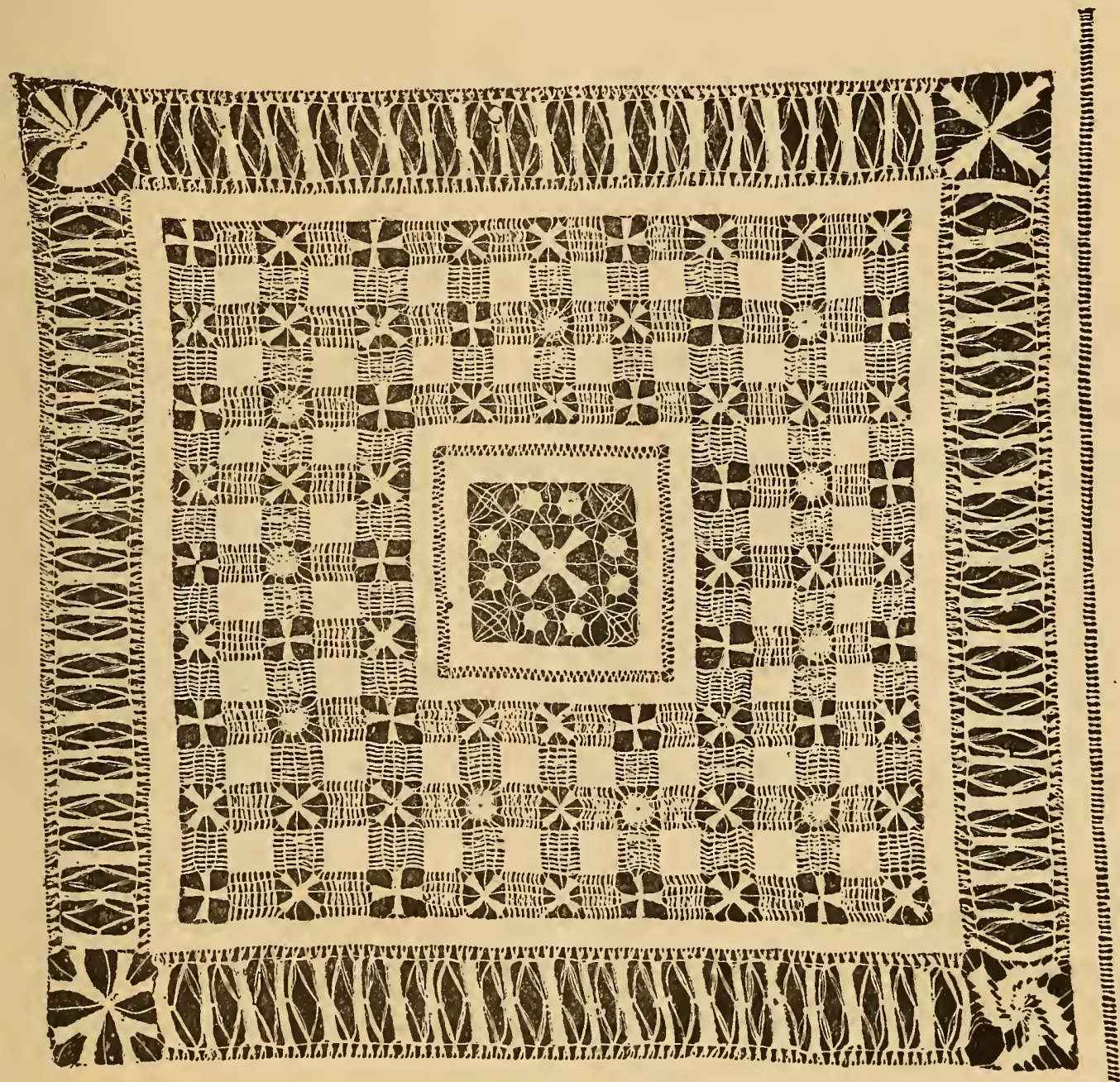

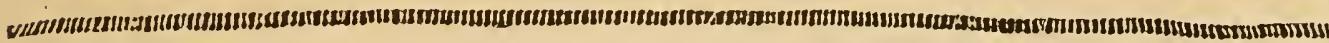

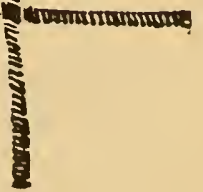

CORNER TEA CLOTH AND CENTER. FIGURES 266-267.

strand and weave back and forth over the four strands until the center is reached, when the sixth strand and the eleventh strand must be woven in with two rows of weaving; drop the sixth and the eleventh strands and proceed as before. 
To work the corners some extra threads must be put in; the corner requires 26 threads to form the pattern. Commence in the center and tie all the threads together with a knot stitch; then weave over eight threads as follows: starting from the center, weave over three threads for a distance, pick up the fourth and proceed as before; next take five threads and continue until the ten threads have been woven over. Go back to the center and begin the second spoke of the wheel, and so on until all the threads have been woven close to the edge of the material. A close following of the design will render this part of the work extremely simple.

\section{Good All-Over Design.}

Figure 260 shows a square suitable for a corner or for an all-over design, or a deep insertion. To prepare the threads for this design: the material must be cut for every alternate square and the edge of material buttonholed, the threads are then to be drawn each way; this will leave every alternate square of the material, and the other square of drawn threads crossways of the design, while there will be a square opening to be filled in, and a square of drawn threads, lengthwise of the pattern. The edges of the linen squares must be hemstitched; the drawn thread squares knotted with knot stitch, three rows being sufficient for a square fiveeighths of an inch each way. In knotting the drawn threads the working thread is carried straight across the cut-out square, and forms the foundation for the spikes and wheels, which are fitted in. The spikes are woven as described for the corner for figure 258; three threads being woven over for each corner. To weave the spikes and wheels commence in the center and weave outward. An unwoven thread is left on each of the four sides of the square.

\section{To make Raised Wheels.}

The woven raised wheel shown in the center of design 260 , is made by working over the threads carried across in knotting the drawn thread spaces. All the threads are tied together in the center with a knot stitch; the raised wheel is made by putting the needle under two of the threads, back again, under the last of the two threads, and one new one, and repeating, going around the center until the wheel is large enough. Figure $26 \mathrm{I}$ shows raised wheel in detail. 


\title{
CHAPTER XIV.
}

\author{
BRUSH AND PALETTE *
}

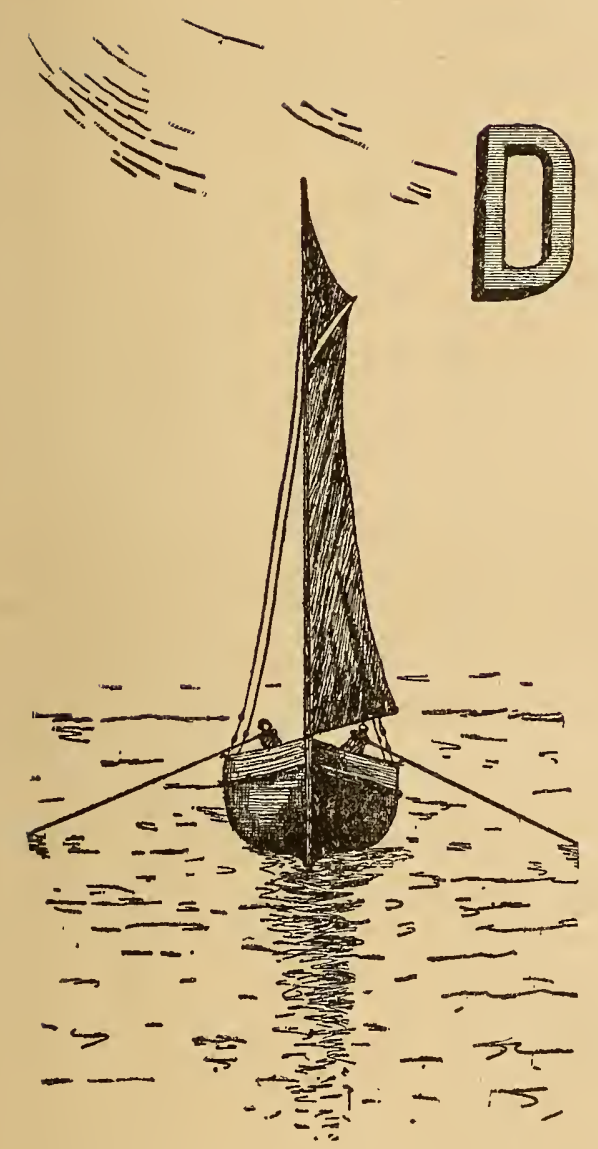

AINTY Work for "Pleasure and Profit" would not be complete without a chapter devoted to decorative work with the brush. It is not the intention of the writer to go into the subject exhaustively here, but to give simple, easily-followed instructions for doing decorative work for the home, without incurring the expense of a teacher. So much can be accomplished with a few colors and a set of brushes, in the way of decorative effects, and at such a small outlay of time and money, that every woman should make herself familiar with the method of doing the different kinds of decorative painting. Teachers have been and still are making money teaching painting; and painting on silk, satin, velvet, chamois skins, silk bolting, ivorine, celluloid, egg-shell paper, bristol board and canvas is used as means from which to derive a handsome revenue, by ladies who teach the art or do the painting for the trade. One lady in New York has, in the past few years, purchased a home and supported an aged mother and two younger sisters painting calendars, letter holders, telegram covers, etc., for a large wholesale firm in that city. There may not be much of what professional artists call "art," in this style of decoration, but it produces wonderfully pretty effects, at little cost, and so places the beautiful within the reach of the many, instead of confining it among the few wealthy fortunates.

* Note. - The illustrations given in Brush and Palette are described in detail in Chapter XVIII. 
The following instructions will be found practical, reliable and easily comprehencled.

\section{Designs for Painting.}

Many ladies imagine they cannot paint because they cannot draw. None of the paintings on fabrics displayed in the stores are drawn. The design is first stamped upon the fabrics by means of perforated stamping patterns, according to directions given in the chapter on "Designing." after which they are painted according to the instructions given in this chapter.

We will discuss first,

\section{Painting in Oils on Fabrics.}

Almost any fabric can be used as a background for painting, but of course some fabrics are better for this kind of decoration than others; but silk, satin, muslin, linen, silk bolting, wool and silk canvas, give the best results, while fairly good success may be obtained on plush (short nap) and felt. To paint upon plush, felt, silk or satin no preparation of the material is needed. Stamp the design lightly with a powder, dust it off lightly, and tack the fabric on a board, or stretch it in a pair of embroidery frames. When painting on silk or satin, to prevent the oil from the paints spreading beyond the design, put the paints first on blotting paper, which will absorb the superfluous oil. The materials necessary for painting on fabrics are few and simple, as follows:

\section{Materials for Painting.}

Brushes.-Red sable, No. I.

Red sable, No. 2.

Small flat sable brushes, Nos. 3, 4, 5, 6 .

One large bristle brush for backgrounds and large surfaces.

Palette.- This can be purchased at the art stores or a piece of smooth glass will answer the purpose equally well.

Medium.-For thinning paints for use on wash fabrics. Foster's medium is excellent for this purpose. One bottle poppy oil and some spirits of turpentine.

Colors.-A limited list of colors for decorative painting will be needed, as follows: zinc white, light red, vermilion, madder lake, cadmium, burnt 
sienna, terra verte, zinnober green, Vandyke brown, rose madder, Antwerp blue, raw umber, ivory black and mauve. Others may be added if desired.

STANDAGE, who is authority on mixing colors, gives the following table for mixing, which will be found very valuable for those who wish to do much work in this line.

\section{To Produce Blues.}

Cobalt Green, Cyanine Blue.-In series of blues of the turquoise tint, sparkling and tender, both beautiful and useful.

\section{To Produce Browns, Buffs and Yellows.}

Chocolate Brown.-Add lake or carmine to burnt umber, or take Indian red and black to form a brown; then add yellow to bring about the desired shade.

Olive Brown.-Mix one part of Saxony blue with three parts of burnt umber. Change proportions for different shades.

Snuff Brown.-Yellow, 4 parts; Vandyke-brown, 2 parts.

Ordinary Brown.-Red, 3 parts; black, 2 parts; Chinese yellow, 2 parts.

Light Buff.-Yellow ocher, lightened with white.

Deep Buff.-Yellow ocher, white and a little red.

Oak Color.-White, 8 parts; yellow ocher, I part.

Fawn Color.-White, 8 parts; red, I part; yellow, 2 parts; umber, I part.

Tan Color.-Burnt sienna, 5 parts; yellow, 2 parts; raw umber, I part.

Brick Color.-Yellow ocher, 2 parts: red, I part; white, I part.

Stone Color.-White, 5 parts; yellow, 2 parts; burnt umber, I part.

Portland Stone Color.-Raw umber, 3 parts; yellow ocher, 3 parts; white I part.

Yellow Lake.-Umber and white, equal parts; add Naples yellow and scarlet lake; glaze with yellow lake.

Fonquil Yellow.-Mix flake white and Chinese yellow, and add vermilion to carmine.

Golden Yellow.-White and yellow, tinted with red and blue.

Canary Yellow.-White and lemon yellow.

Lemon Color.-Lemon yellow, 5 parts; white, 2 parts.

Straze Yellow.-Yellow, 5 parts; white, 2 parts; red, I part.

Citron.-Red, 3 parts; yellow, 2 parts, blue I part. 
Clay Drab.--Raw sienna, raw umber and white lead, equal parts, and then tint with chrome green.

Ordinary Drab.-White, 9 parts; umber, I part.

Cream Color.-White, 5 parts; yellow, 2 parts; red, I part.

Orange.-Made with gamboge and burnt sienna. Useful for the autumnal tints, and to mix with the other greens, in order to vary their tone and depth, but not to be used in very warm sky tints.

\section{To Produce Grays.}

Indigo, lampblack, and madder lake.

Indig,o and Indian red.

Indigo and light red.

Indigo, lake and gamboge.

Indigo, lake and Roman ocher.

Indigo and Venetian red,

Indigo, raw sienna and lake.

Indigo, sepia and lake.

Indigo and purple madder.

Indigo, light red and lampblack.

Indigo, burnt umber and lake.

Indigo, madder purple and burnt umber.

Lampblack and madder lake,

Lampblack and burnt madder.

Lampblack and light red.

Lampblack and lake.

Cobalt green, cyanine blue, carmine madder, for silvery grays, through lilacs to purple.

The colors given in the first list answer for all kinds of painting for decorative purposes, no matter by what name it may be called, except for tapestry and china painting, a list or colors for which we give later. The designs that best lend themselves to decorative painting are all field and garden flowers, arranged in groups or used as single sprays, or formed into scrolls for border effects; also tiny landscapes.

Handkerchief cases and articles of a like nature, are made very 
beautiful by painting over the entire surface of the satin a background; it may be in some neutral shade, or it may be a sky effect, over which the floral arrangement is thrown.

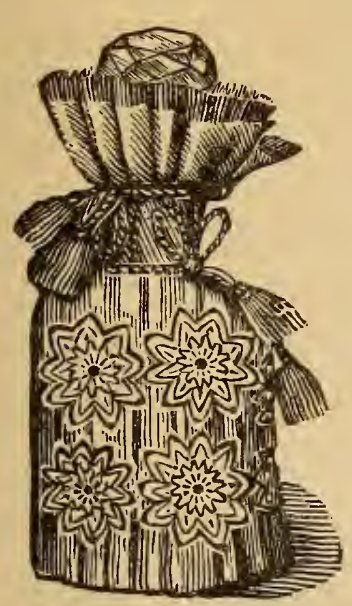

FIG. 27 I A.

Daisies, honeysuckle, apple or peach blossoms, delicate tea roses, feathery clematis, violets, white and purple lilacs, pansies, are all excellent subjects for silk or satin decoration.

A design of apple blossoms would be painted thus: Squeeze out upon the palette a good quantity of zinc white and a little rose madder, and for the green leaves, terra vert, cadmium, burnt sienna and Antwerp blue.

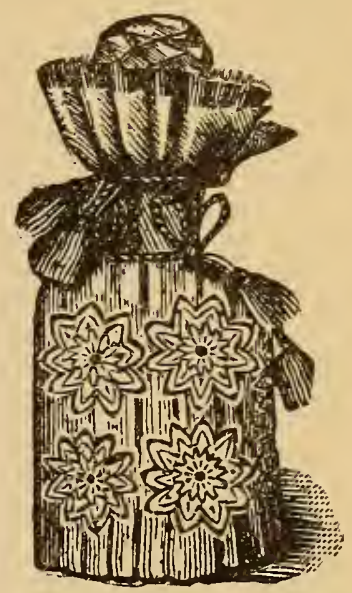

FIG. 27 I B.

Begin by putting in the general tone of the blossom with zinc white, rose madder and a trifle black. For the calyx use terra vert and cadmium, and in the center of flower burnt sienna and cadmium, with a touch of zinnober green. This for the yellow stamens. The high lights are painted with silver trifle madder leaves retransparenc $y$ For these use mium, burnt Antwerpblue. under side shows, use with the collittle ivory

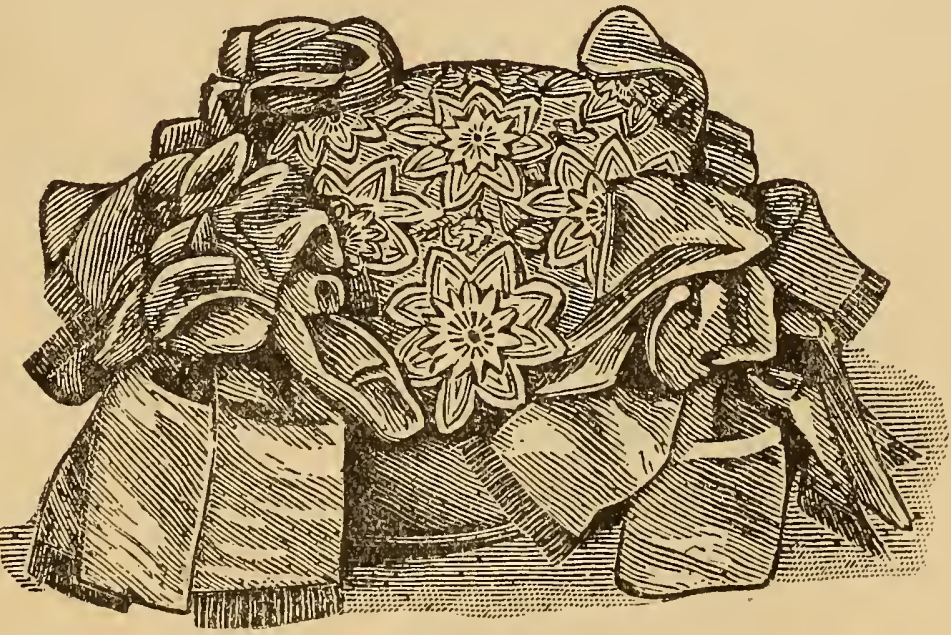

FIG. 27 I. white and a lake. The quire more of coloring. terravertcadsienna and Where the of the leaf silver white ors, and a black. The grayish branches are painted with Vandyke brown, silver white, and black, with raw umber and burnt sienna in the shadows.

To paint morning glories, which are a delicate pink, banded with white, use madder lake, silver white, yellow ochre, and a trifle ivory black for the local color. The shadows are painted with light red, raw umber, and white. The white stripes, or bands, with white, madder lake and a little ivory black. 
The lights are painted with the same colors. The centers are pale, almost white. For these use white, light cadmium and madder lake. The green leaves are painted with Antwerp blue, white, cadmium, light red, and ivory black, shaded with burnt sienna and black. The stems and tendrils, which are yellower in tone, with light zinnober green, white, light cadmium, and a little burnt sienna, and black in the shadows.

Apple blossoms and morning glories are both beautiful gracefully arranged for panels, photo frames, memorandum racks, cushions, drapes, etc., and the background effect spoken of previously enhances the effect a hundred fold. Any dealer in perforated designs will be certain to have several arrangements of these flowers, some one of which can be adapted for your use. Magazines, cards, etc., also frequently have pretty arrangements, which can be transferred by means of a pantagraph, as described in the chapter on "Designing."

\section{Sky Effect for Backgrounds.}

A good sky effect can be produced by going over the surface with cobalt and zinc white, with a little castle earth at the bottom. Over the blue scatter gray and white clouds; for gray see table for mixing colors. Mix the colors carefully, and do not work the strokes of the brush together very much, as the broken effect is much more desirable. This part of the work should be done with a large bristle brush, and must be allowed to dry perfectly before putting in the design, which may be stamped on through a perforated pattern with white powder. We will suppose a design of daisies to be painted on the background just described, the colors needed will be zinc white, pale cadmium, pink madder-the tiniest bit possible. Make a green for the leaves and stems by mixing on your palette a little Antwerp blue, cadmium, and ivory black, or terra verte and zinnober green may be used, modified with zinc white.

First, take out a small quantity of white on the palette, and we will say here, in taking paint from the tubes, always squeeze the paint from the bottom of the tubes; add to the white paint two or three drops of the poppy oil as a dryer. Mix thoroughly with the palette knife, and apply with a No. 2 brush. Begin at the top of the petals, laying on the color with smooth, even strokes, being careful not to go over the outlines. Take up 
the paint, on the point of the brush, which should be held almost perpendicularly. This method lays the color on heavily. When the petals are perfectly dry, retouch them with white, to which a very little pink madder has been added. The cadmium is for the center of the daisies. The leaves and stems are to be painted with the green mentioned.

Some artists use a great quantity of paint on textile fabrics, while others use very little. It is a question to be decided by individual preference. For white velvet the colors should be mixed with turpentine, and carefully painted over a coating of gum arabic, which has been previously applied to the velvet.

\section{Special Palettes.}

We have prepared instructions for painting a number of different

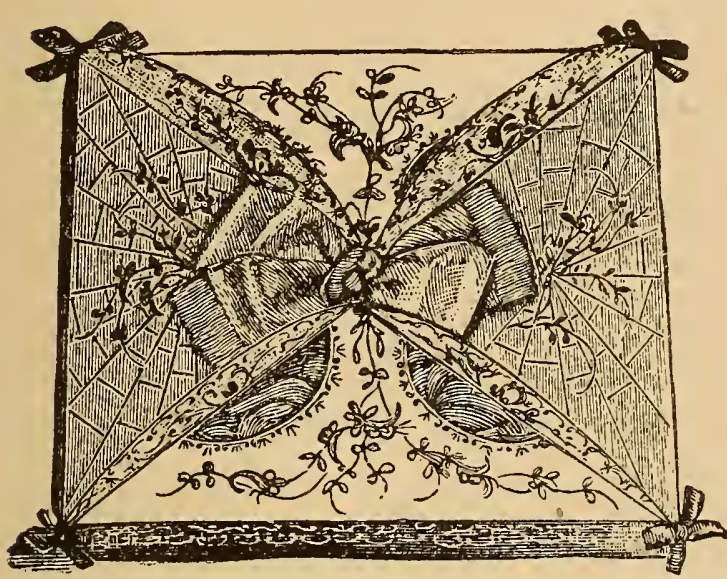

FIG. 272 . flowers and foliage, which can be applied to any purpose preferred, and the designs can be had in perforated patterns, very gracefully arranged. These instructions will frequently call for other colors than those mentioned in the list of colors. They can be added to the list if desired, or a substitute can frequently be made by using the table of mixing colors.

\section{Strawberry Spray.}

A pretty design for this spray, I7xi4, can be obtained in perforated stamping patterns. Bronze satin or plush would be a suitable background. The paints needed will be scarlet lake, emerald and chrome green, Chinese vermillion, lemon-yellow and white. If you wish to paint on satin, use soft brushes and drying oil; if on velvet or plush, use "clipped" brushes, and turpentine for mixing the paints.

Paint the leaves with emerald and chrome green, mixed, giving a lighter shade on one side by adding a very little lemon-yellow. The veins in the leaves must be made with the finest brush, using brown madder. When this is very dry, retouch them with pure chrome green. Use equal parts of Chinese vermilion and scarlet lake for the berries, lightening with a little white, where a tint is required. Where the lines cross, a fine point 
of yellow must be made, after the body of the berry is dry. This is put on with the finest brush, a very little paint being taken up directly on the point, and the brush held almost perpendicularly in the hand. The calyx of the berry is made in two shades of green. lightening with a little yellow, to suit the fabric used.

\section{Geranium Spray.}

Geraniums are exquisite painted on black, brown or white satin. In stamping patterns, there are small, medium and large sprays, so that one can adapt them to a variety of uses. We will suppose the pattern to be nicely stamped on the goods, and the goods neatly, firmly fastened to your painting board. As before stated, soft brushes are best suited to this class of work, and Nos. 2, 4 and 6 are good sizes.

There are a great variety of geraniums, but we will select one of the zonal species for this lesson. Its blossoms are pink and its leaves have zones of reddish-brown, separated from the edge by a very narrow border of green. The paints needed are zinc white, pink madder, lemon yellow, chrome green and brown madder.

Begin by laying out on your palette about a thimbleful of zinc white, Add to this five or six drops of the poppy drying oil. Now paint the entire design white, this being, of course, a mere body color. On satin of any shade, except white, it is best to give the design the body color. When the white is dry the natural shades are then laid on. For the leaves mix equal parts of chrome and emerald green, adding just a touch of lemon yellow. Paint the entire leaf with this color. When dry add the "zone" of pure brown madder. Do the veining with your finest brush, after all the rest is entirely dry.

A natural zonal geranium leaf aids one in attaining the best coloring.

For the blossoms use pink madder, lightened with white, laying on the color in smooth, even strokes, and being careful not to go beyond the outlines. The stamen in the center of each geranium may be represented by a small dot of yellow, made with the finest brush.

If you wish to paint scarlet geranium blossoms, use scarlet-lake, to which a small touch of carmine has been added.

An excellent siccative, made of equal parts of balsam of fur and turpentine, is used for varnishing work done on satin. It must only be applied 
when the paint is dry, and then very lightly. Work treated in this way is very durable.

\section{Butterflies.}

A small or medium-sized butterfly is a pretty decoration for a small cushion, perfume sachets, etc. A light yellow butterfly looks well on almost any shade of satin or velvet. Lemon yellow may be used for the body color, and when this is dry, spot with brown madder. The fine markings that are sometimes seen on butterflies, may also be made with madder, using a very fine brush. A golden brown butterfly is beautiful on pink, white or blue satin. For such a one add Vandyke brown to yellow, to make the desired color. Spot this butterfly with mauve lake or ivory black. Use only a few drops of poppy, oil in mixing the paints.

\section{Grapes on Black Velvet.}

For velvet painting the fine bristle brushes are used with good effect, but red sable brushes "clipped" are superior. To clip them, take a pair of small, sharp scissors, cut them off on each side and a little at the point. They must be rounded and firm to the touch, and at the same time have a good point. Three sizes of these brushes are necessary to do a variety of work on velvet. Cutting them lessens the size; therefore it is advisable to begin with medium sizes.

The colors for a design of grapes will be cobalt blue, mauve lake, chrome green, terra vert, brown madder" and silver white. Turpentine may be used for thinning the paints. Paint the stems and veins of the leaves with pure brown madder, then paint the grapes. To mauve lake add only a touch of white and two or three drops of turpentine.

Lay on the colors smoothly, and do not go beyond the outlines; shade a little (using another brush) with pure cobalt blue. For another shade mix equal parts of mauve lake and cobalt blue. Paint the leaves with chrome green, to which a little yellow has been added, and when this is dry retouch them with terra vert, to which a little chrome green has been added. Do not work your color in on the veining if you can help it. When the body of the leaves is dry go over the veins again with brown madder, to which a little yellow has been added. On velvet the grapes look well 
done in silver bronzing and the leaves in gold. Gold and silver paint may be purchased of all dealers in artists' supplies. A mixing liquid and directions for using accompanies it. Gold and silver bronzing are effective for painting borders to portieres, table scarfs, and piano covers.

\section{Pansies.}

Pansies are exquisitely dainty and lovely on bolting cloth, with satin under the bolting. They are also stylish painted in oil on the crown of an evening bonnet, with the ends of the strings painted to match.

The colors needed for this lesson in pansies are brown madder, pink madder, silver white, lemon yellow, mauve lake, blacklead, chrome green, and terra vert, the latter being a very dark shade of green. Some natural pansies or a good pansy study will be of great assistance in painting, as these blossoms admit of more variety of shades than any other flower.

To lemon yellow add a very little white and three drops of drying oil; with this shade paint one or two of the pansies, varying the color a little around the edges with brown madder; or only the lower half of the petals on each pansy may be painted with the light yellow. For some of the others mix mauve lake and pink madder, using one-third madder to twothirds of mauve lake. This makes a handsome purple; to brighten the shade add a little white, and to darken it add brown madder. The fine lines and markings are made with a very fine brush, using black lead. Put this on after the first color applied to the petals has dried. If there are any buds, paint them to correspond with the blossoms. Paint the leaves in two shades of green, making them a little lighter on one side. To vein them, add a little yellow to the chrome green; do your veining with your finest brush. If any sprigs of ferny appearances are visible, paint with light green. Paint the little oval in the center of each pansy with lemon yellow, to which just a touch of brown madder has been added. When this is dry draw a fine line of pink madder around it. The same rule would apply it the same work was to be done on white or cream-colored satin.

\section{Purple Thistles.}

To paint the purple thistle in oil colors use permanent blue, madder lake, white, raw umber, and a little ivory black for the purple blossoms. In the shadows add to these colors burnt sienna, and use less white. Put 
in the high lights last, using permanent blue, madder lake, white, and a very little ivory black. Do not blend these lights, but paint them while the surrounding tone is fresh. The leaves, stems and calyx, which are rather a light, silvery gray green, are painted with permanent blue, white, cadmium, madder lake, and ivory black. In the sbadows add burnt sienna and raw umber; use less cadmium and white.

\section{Pond Lilies.}

To paint pond lilies in oil colors, first paint in the white blossoms in one general tone of light, warm gray. Afterwards put in the deep accent of shadow, and add the high lights with crisp touches; do not blend. Paint the general tone with white, yellow ochre, a little ivory black, cobalt and light red. In the shadows substitute burnt sienna for light red, and use less white and yellow ochre. The high lights are painted with white, a little yellow ochre and a little ivory black. The green leaves, which are gray and rather dark in quality, are painted with permanent blue, with a little cadmium, madder lake and ivory black. Use the same colors in the shadows, adding a little raw umber and burnt sienna. Paint under side of leaves with yellow ochre, white, cobalt, a little ivory black, and light red.

\section{Purple Clematis.}

Colors: Permanent blue, white, madder lake, and ivory black for the general tone. Shadows, add raw umber and burnt sienna.

Paint the high lights in white, permanent blue, madder lake, a little yellow ochre and ivory black. In the centers use a little light cadmium with raw umber; for the shadows add white, ivory black and a little burnt sienna. For the green leaves use white, cadmium, Antwerp blue, vermilion and ivory black. Shadows of leaves, substitute burnt sienna for the vermilion, add raw umber, and use less less cadmium and white.

\section{Oxhart Cherries.}

To Paint White Oxhart Cherries: Lay in a general tone. Mass the lights and shades very simply. Put in the high lights with crisp touches, and add the deeper accents of dark in the shadows. For the local tone use white, yellow ochre, a little vermilion, and a very small amount of ivory black. For shadows use white, raw umber, yellow ochre, a little ivory 
black, and madder lake. Paint the high lights with white, yellow ochre, a very little madder lake, and the smallest possible quantity of ivory black, There will probably be soft gray half parts. Use for these white, yellow ochre, ivory black, cobalt and light red. In the deeper accents of the dark use burnt sienna with the other colors. If there is a faint tinge of red on one side of the cherry, add some madder lake to the other colors. To paint the black Oxhart cherries, use for the local tone madder lake, ivory black, a little cobalt, burnt sienna, and a very little white. The high light is made in white, yellow ochre, a little madder lake, and a very little ivory. black. There is a gray surface light, which must be carefully observed, add more white and cobalt to the other colors in painting this.

\section{Leaves and Berries of Virginia Creeper.}

To paint the leaves of the Virginia Creeper, when they are turning red and yellow in autumn, use for the local green parts Antwerp blue, white, cadmium, vermilion, and ivory black. The shadows are painted with the same colors, but burnt sienna is substituted for vermilion, and raw umbet is added. Less white and cadmium is needed. The red tones are painted with vermilion, madder lake, light red, white, a little ivory black, and raw umber. In the deeper tones add burnt sienna, and in the cool gray half tints use white, ivory black, a little cobalt, light red, and yellow ochre. The yellow tones are painted with light cadmium, yellow ochre, white, and a little ivory black for the lightest tints. In the deeper yellows add a little madder lake or light red, and substitute a darker cadmium for the light shade. In the shadows add raw umber and burnt sienna to the same colors, omitting yellow ochre. To paint the dark purple berries use madder lake, permanent blue, ivory black, white, and yellow ochre for the local tone. In the shadows use the same color, but add burnt sienna and raw umber. The reddish-brown stems are painted with burnt sienna, permanent blue, white, and ivory black.

\section{Peacocks.}

The oil colors used in painting a peacock are as follows: The deep, rich, irridescent blue tones in the center of the eye are painted with Antwerp blue, a little madder lake, and ivory black. The bronze tones are painted with raw umber, yellow ochre, burnt sienna, a little ivory black, and as 
much white as may be needed. For the brilliant emerald greens use Antwerp blue, white, light cadmium, a little vermilion, and a little black. The delicate purple tints are painted with permanent blue, white, madder lake, a little yellow ochre, and a very little ivory black. In the shadows add raw umber and burnt sienna. The beautiful irridescent color on the breast, which is partly blue and green, is painted with Antwerp blue, white, a little light cadmium, a little madder lake, ivory black, and burnt sienna. For the beady, black eye use ivory black

\section{Golden Rod and Asters.}

Set the paletce with white, chrome or king's yellow, cadmium, cobalt, Prussian blue, Indian red, burnt sienna, mauve, castle earth, and black.

Use a background, I $5 \times 24$-larger or smaller, only keeping that proportion. If you do not draw, stamp the design with powder. There are many pretty perforated patterns to be had for 15 to 25 cents. With a large bristle brush paint the background, using cobalt and white for a bit of sky effect near the top of the right side of the canvas, and surround it with white and light gray clouds, making some darker towards the left upper corner. For gray use white, black, a little cobalt, Indian red, and yellow ochre.

Mix the colors carefully, and do not work the strokes of the brush together very much, as the broken effect is much more desirable. Paint the background entirely first, using castle earth toward the lower edge of the canvas to darken it; also, draw in some grasses before it is dry, in pale, yellowish green or gray, using brighter green for the nearer bits of grass or leaves.

Paint stems of light green, shaded with castle earth and burnt sienna. Use chrome yellow and black for most of the leaves, using white or a little cobalt where they are lighter. Use a small, flat bristle, No. 3, for painting leaves, drawing the stroke out from the stem.

Paint in the golden rod flower with an undertone of cadmium, using a small bristle brush, well loaded with the color, or else use a corner of the flat brush, and touch the color, not put it on with a stroke. Finish with chrome yellow for highlights, and where the flowers are full bloom, add a few touches of burnt sienna for shadows, taking care to mix the colors as little as possible. 
For asters, use mauve and white, putting each petal on with one stroke of the brush. A red sable, about No. 2, is most convenient for this part of the work. The center of the flower has the lower part, or shadows, in burnt sienna, and chrome yellow above. Leave the background to dry before attempting the flowers, if you feel that you can succeed better by doing so.

\section{Panel of Hollyhocks for Screen.}

A very good background for a panel of hollyhocks can be made from cream white hollywood drapery, which should have a background painted over its entire surface, thinning the paints with Foster medium, scrubbing them into the material with a stiff brush, after which the hollyhocks can be stamped on the panel.

Set your palette with white, chrome yellow, yellow ochre, permanent and Prussian blue, burnt umber, vermilion, light or Venetian red, crimson lake, rose madder, raw umber, castle earth, and black.

If you wish a sky background with clouds, paint in the blue where you prefer it, using permanent blue, white, and a little black; cloud of blue, Venetian red, yellow ochre, and most of all white. Soften edges of clouds - and blue carefully together; or, the background may be painted with a pale shade of green.

Below center of panel darken gradually towards left-hand corner, if your study is painted with the light striking from the right side.

After background is finished for the first painting, begin at highest buds and paint down buds and stems, carefully putting in the bright colors in buds before laying in green leaves. For pink flowers and buds use rose madder with white, using sugar of lead as a drier-a very little with each brushful of color. Use flat, soft brush on flower leaves, pressing the color firmly down at outer edge and drawing brush towards the center for large petals. For dark red flowers use crimson lake; shadows, mauve or purple lake; under side of leaves, crimson lake and white. For center of stamens use burnt sienna, yellow ochre and chrome yellow.

Paint aves in with Prussian blue and chrome yellow, or chrome yellow and black, using blue in shadows. Paint stems a little lighter, using white with other colors.

After it is dry use castle earth to scrumble the background on lower half of panel. 
The foregoing instructions can be applied not only to painting on fabrics, but are equally suitable for painting on celluloid, ivorine and glass, eggshell board, wood, curtain holland, and also for

\section{Washable Painting}

on linen, bolting silk, scrim and muslin; very beautiful effects are also obtained by painting in oil on wash fabrics. Curtains, pillow shams, toilet sets, and stand covers can be decorated in this manner at very little expense. The painting is done as directed for oil painting, and the same colors are used, with the addition of the Foster medium, which renders the colors almost liquid and allows them to be absorbed into the fibers of the cloth. Only flat floral effects, without perspective, should be attempted for this purpose.

\section{To use the Medium.}

Pour a thimbleful of medium to use from, as it must be used but at the one sitting; dip brush in medium, then in color on palette, working a little. Repeat same if necessary until color is thin; then tap brush on blotting paper until the paint will not spread, or until you can make a hair line. This will come with little practice. Hold brush perpendicularly, firm, but with a light hand, and, as a general rule, make quick, short strokes. Care should be taken to dip brush in medium often, and judgment used not to get too thin-i. e., without body of color, as colors shade lighter on fabric. Always put the dark shade lines in first. The colors, by use of the medium, act as a dye, and fill the fibres of fabric; therefore go over work but once. What is put on in excess of first coat-or more color than cloth will absorb --will wash off. If color does not seem to penetrate cloth so as to show well on wrong side of work, dip clean brush in medium, tap on blotting paper, and carefully go over work on wrong side just as if you were painting; or if you wish to blend colors, follow same directions as on right side. Let painting dry thoroughly - thirty to forty days - before washing: then if the work fades it is because enough medium was not used while working. In painting from nature or a study, in most cases, any defect can be remedied by painting over. With this process this is impossible. Therefore, as an easy, quick way of doing, we suggest the sketching or tracing outlines of study or object on thin paper, placing paper between 
fabric and sheet of glass, placed on easel, or held in any manner, the work may be done without fear of mistake.

WASHING-Make a warm suds, wash the article, rinse and hang uf; when nearly dry iron. Do not nub soap on painting direct. In short, take the same care you would with a delicate handkerchief.

\section{Water Color Painting.}

This method of painting is easily learned, and can be applied to many ornamental objects. Squares, scarfs, etc., of bolting silk, linen lawn, and China silk, tinted delicately with a wash of water colors, make desirable backgrounds for embroidery in outline, while drawing paper, leather, ivorine, and eggshell board, made into calendars, letter covers, telegrarn holders, photo frames, etc., and decorated with designs in water colors, are within the reach of all who are willing to bring to the work patience and application.

The materials necessary are brushes, from No. I to No. 6, china palette, having cups in which to mix the colors, a list of colors as follows:

"The pigments, which you will find most useful, are comprised in the following list: Vermilion, orange and scarlet, extract of vermilion, crimson lake, rose madder, or extract of madder, carmine, light red, Indian red, gamboge, yellow ochre, Indian yellow, raw sienna, lemon yellow, cadmium, burnt sienna, purple madder, cobalt and indigo, brown madder, biste, Van. dyke brown, sepia, burnt umber, neutral tint, ivory black, Prussian blue, French ultramarine, Chinese white."

There are other colors which may be added, as desired, but the above will answer all the requirements of an amateur.

In stamping the design for water color painting, great care must be observed. Stamp with white powder, and then with a brush charged with a pale tint of the local color of the flowers, go over the outlines lightly. If the painting is being done on cardboard or drawing board, the card must be wet in all the parts intended to be colored. This is done by passing a wide, flat brush, moderately full of water, gently over it; boiled water is best for this purpose. When the card is thoroughly dry, each flower and its foliage should be washed over with a tint, matching as closely as possible the lighter tones of its own local color. This wash should be begun at the upper left-hand side, proceeding quickly to the right and downward. 
Beginners generally cxperience a little difficulty in this operation at first, but if attention is paid to keeping the brush equally full of color until it is completed, the difficulty will be easily overcome.

Beginners frequently exhaust the color in the brush before filling it afresh, the consequence of which is that the new supply of color flows back into the former, leaving, when dry, a distinct mark, which it is impossible to obliterate without sponging the whole entire ${ }^{1} y$ out with clean water.

Fresh color should, therefore, be taken so frequently that no difference can be perceived between the tint of that which flow's from the brush and that which is already laid on.

The large pool left when the wash is completed can be best disposed

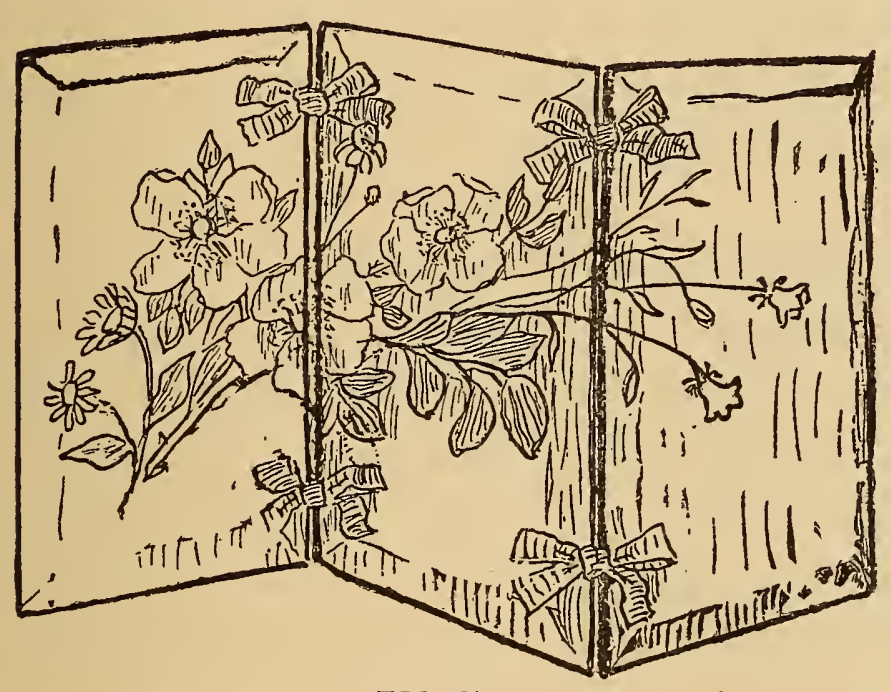

FIG. 273. of by drawing the brush gradually to a fine point against the extreme outline.

When the color just laid on is perfectly dry, the shadows must be carefully painted in and washed in the manner already described, pains being taken to match the colors with those of nature. The shadows generally appear in parts to be insensibly lost and blended with the pure color of the flower. This effect may be produced by passing over the edges while still wet a clean brush rather dryer than that with which they have been painted. The local color may then be deepened where necessary, and the darker shadows may be worked upon until they are of the proper depth, and finished by small touches wherever great nicety is required. Some artists endeavor to finish their work entirely with soft washes, whilst others execute them with large or small touches, called stippling, but a middle course between these extremes will give a result more akin to nature.

The leaves must be executed in the same manner as the flower. When of a glossy surface and affording brilliant lights, as well as shadows, they must be washed over with a very pale shade of cobalt and Indian red mixed. 
the veins only being left (if they appear of a very bright green), and those lights which appear absolutely white. When this is dry the local color, composed of chrome yellow, No. I, and indigo, must be laid on carefully, the lights only being left. The shadows must then be painted in with the same color, or a little gamboge and indigo, with a very small addition of Indian red; then the veins, if dark, with the same color, and the whole finished with small touches. Some leaves are of a silvery grayish tone; for these the local color must be composed of cobalt and chrome yellow, with an almost equal amount of Indian red.

With respect to the use of body color, there is a great diversity of opinion. The earlier artists disdained its use altogether, while some later artists used it on every possible occasion, loading the paper with it, both foreground and background. This method undoubtedly gives more force, and certainly is more expeditious. When white is used it should be the permanent Chinese white.

We give a few studies with palettes, suited to beginners.

\section{Roses.}

In pink roses, the local color is best imitated with pink madder, a pale tint of which must be washed over the flower, leaving only the perfectly white lights. When quite dry, the darker petals must be again covered with a deeper hue, and again with a still deeper hue the dark ones near the center. Sometimes the color of these is extremely red; if so, a little scarlet must be washed over them first. Next lay on the shadows, but so great is the transparency of the petals, that very little gray will be perceived in them. A very small proportion of cobait and Indian yellow must, therefore, be mixed with the madder with which they are painted, and for the dark ones carmine alone may be used. For dark roses, crimson lake will be found the best color, instead of pink madder or carmine.

Yellow roses should be painted in the same way, with either lenion yellow or chrome No. I, for the local color, the darker shades of yellow being finished with gamboge. The shadows can be painted with the same gray recommended for other flowers.

\section{White Flowers.}

In painting white flowers it is totally impossible to produce the brilliant whiteness of nature. The paper on which the drawing is to be executed and 
the purest white pigments, are only of the same degree of whiteness as the flower in its half shade. If, therefore, either of these is used for the prevailing tone, which it most resembles, there will be no means left to represent or even to attempt to imitate the high lights. The whole flower, therefore, to be in keeping, must be painted a degree lower than natural, and washed over with a pale gray, just as the local color is laid on in other flowers, leaving only the high and most brilliant lights. For this gray use pink madder, cobalt, and a very little Indian yellow, mixing the two former toyether first, and then adding the yellow cautiously. Then lay on the shadows with a much warmer gray-that is, with a less proportion of blue.

In painting a white lily, chrome yellow, No. 3, may be used for the anthers; but in the white azalia, or other white flowers where they appear pale, a little Chinese white, mixed with chrome No. I, will be better.

\section{The Primrose.}

Outline the flower, beginning in the center; wet all the parts intended for coloring with water; wash over with a thin tint of lemon yellow, such as will match the depth of the larger portion of the flower, leaving only those parts, if any, which appear to be white, or nearly so. The color must be softened into these with a clean brush, as already'explained. The color for the shadows must be composed of cobalt, pink madder, and a very little gamboge, the two former being mixed together first, and then the yellow added, always matching the tints used with those of the flower itself. When quite dry, the yellow must be deepened where necessary, the green in the center painted in with a little gamboge and indigo, and the deep yellow marks, which surround it, with gamboge alone. The local color for leaves may be composed of chrome yellow, No. I, and indigo, with a very slight admixture of Indian red. For the darker shadows a little gamboge will be required, instead of the chrome yellow.

\section{Blue Flowers.}

For the blue nemophila, cobalt alone will be the nearest approach to nature in the local color. For some campanulas, cobalt and a little lake; for others, French blue, and as much lake may be used as appears best to match the color of the species to be represented. For the salvia, smalt or ultramarine must be used, no other colors being sufficiently brilliant in the particular tone required. 


\section{The Convolvulus.}

Cobalt or French blue, with a slight mixture of crimson lake, may be used for the local color, but, of course, the more blue this is, the fresher and nore newly expanded the flower will appear. The shadows should be painted with the same colors, substituting in the darker tones indigo for the cobalt or French blue.

\section{To Paint Figures in Water Colors.}

For designs having figures, as cherubs, cupids, Diana, etc., flesh colors will be required, and the following directions from A. H. WALL's treatment will be found to give desired results, although the minute details given need not be sofaithfully carried out. Use the broad, general effect, and put in only such details as are needed to accentuate the work.

The flesh colors are rose madder and raw sienna, Indian yellow or burnt sienna; vermilion and raw sienna; vermilion; extract of vermilion, alone or with rose madder and raw sienna; Venetian red, crimson lake and yellow ochre; rose madder and Naples yellow.

The required mixture of colors prepared carry a clean, flat wash of it over the flesh, using the large brush charged with just enough color tocover the space at once.

"The first wash being dry, take a little rose madder and extract of vermilion, and with a few tenderly applied touches, or washes, put the color in the cheeks and lips; then, but with extreme delicacy and faintness, increase the depth very gradually and softly-touching upon the chin, the nostril, the upper eyelid, just over the eyebrows, the tips of the ears and fingers, knuckles, etc., with the same tint, rendering the effect more or less perceptible, according to the complexion desired.

"Mix a little raw sienna and cobalt (substitute lemon yellow or yellow ochre for raw sienna, if the study is a dark one) and with the same delicate touches go over the outer portions of the shadows and retiring surfaces of the face, over the entire sockets of the eyes, the lower jaw, and the lines where the hair and flesh combine, making the blue a little more perceptible when touching the temples, the lower lid of the eye, between the eye and nose, etc., where the skin is thin and the veins perceptible.

" Upon the darker shades a little vermilion and raw sienna may now be touched, and upon the darkest a little pure vermilion used thin and faint. 
"With a little rose madder and cobalt the retiring portion of the cheek and chin, the lower portion of the socket and upper lid of the eye, may be carefully and tenderly touched.

"A little indigo and raw sienna may be next used, with the same delicate care and faint, fairy-like scarcely perceptible touches upon the edges of the deeper shadows, and where the hair and flesh meet, blending and softening with patient skill one into the other.

"Here and there the color in the cheeks and lips requires strengthening. For this use a little crimson lake, or crimson lake and vermilion, or the extract of madder carmine, as your thoughtful consultation of the model may decide.

"Put in the pupils of the eye with indigo; outline the iris with either the same color or sepia, according to its color, stronger or fainter, as your subject may indicate. If the eye is blue, use cobalt, or cobalt and indigo, as may be required; if gray, use a little indigo and burnt sienna, or neutral tint; if brown, umber, burnt or raw, as may be most desirable, or burnt sienna, or Vandyke brown, or brown madder, being guided by the tone of the part to be covered, and the color to be attained. Sometimes a little Chinese white will be required, mixed with either of the above.

"Proceed to strengthen all the lines, such as those of the eye and eyelids, the line dividing the lips, that of the nostril, the under or shadow line of the eyebrow, the soft line of shadow seen where the hair is thin and the skin seen through it, using a little Indian red or brown madder. A little madder brown may also be used to give strength to the cast shadows at this stage of your progress, and a little sepia to strengthen the outer lines of the upper eyelids.

Very artistic figures can be had in perforated designs. "Aurora, cupid, cherubs-arranged in designs for wall panels, fans, easel pieces, etc.

\section{Fabric Tinting.}

Many ladies find profitable employment in tinting white goods with water colors, Paris tints, or even diamond dyes, which latter certainly have the advantage in point of cheapness.

The tinted pieces are usually small and either square or round in shape. They are used as head-rest covers, toilet cushion covers, toilet mat, chair 
mats, etc. The fabric selected should be very fine linen lawn, mull or bolting silk.

The article to be tinted is first tacked to the drawing board, being careful to stretch it smoothly and firmly. Sponge the surface of the fabric with water until it is evenly wet all over; then, commencing at the upper portion, apply the tint with a large, flat brush, which has been heavily charged with color. This part of the work must be done rapidly, and the brush must be refilled before the first charge is exhausted, in order to secure an even, uniform tint over all the surface. Always begin at the top and work downward.

A variety of effects are obtained by deepening the tint on the edges or in the center. This is done by going over the work several times with a stronger wash of the color desired. After the pieces are tinted they are allowed to partially dry, and are then pressed between two pieces of thick white flannel.

They are now ready for receiving the design, which should be stamped on the tinted background with the black liquid stamping fluid, as this makes the finest and, at the same time, most legible line; see chapter on "Designing." The designs for this purpose should be light and of a most fairy-like daintıness-a blackberry bramble or a wild rose and bud, a flight of butterflies, cte., being suitable.

The tints for the background may be a primrose yellow, a wild rose pink, a pale green or a delicate blue, but be careful to have only a tintnever a decided color. The water colors that come in the little pans are more easily managed than the colors sold in cake form.

If a rose tint is desired, place a little pink madder in a butter chip or on the china palette, if one is at hand. Dilute with water until the lightest possible pink tint is obtained; wash this over the fabric as described; then to deepen the edges add to the pink madder a little cobalt and the tiniest bit of Indian yellow. This wash must also be diluted with water and washed over the edges, carrying the stroke toward the center in irregular and broken lines. The general directions given under the head of "Water Color Painting" will be of assistance for this kind of work and should be carefully studied. In tinting it is convenient to have at hand a piece of cloth on which to try the paints before applying them to the article, as if it happens to be too deep it will not be possible to remedy the defect. These 
tinted novelties find ready sale, and when the work is nicely done, and stamped with artistic designs, and done up in assorted lots of one dozen each, the wholesale houses become eager purchasers at fair prices.

One lady known to the writer has added materially to her income by this means. She knew nothing about painting, but one day being engaged in dyeing some white cotton cloth for carpet rags, she observed that different tints were obtained with the same color by adding water. Naturally of an inventive and ingenious disposition, she began to experiment, and from the crudest possible first results, she has succeeded in producing some of the most beautiful tinted articles ever put on the market, and her customers may be found in almost every state.

Aside from the tinted backgrounds, beautiful decorative effects are easily obtained by the use of the Paris tints, described below, or the water color paints may be substituted with as good effect if the artist has them at hand. The water colors are in the end the cheapest, as they can be used for a greater range of work than the Paris tints, which are useful only on fabrics, while the water colors can be used on fabrics, celluloid, wood, etc., and offer a greater range of tints, shades and colors. Many teachers insist upon pupils using the Paris tints for fabric and water colors for other backgrounds. The reason is obvious, as the use of the two entails an extra lesson or lessons and an extra outfit, which the teacher usually supplies the pupils for a "discount."

\section{Paris Tinting.}

This is a style of painting applied particularly to bolting silk, sheer lawn, and China silk, and by means of the tints, the daintiest possible effects can be obtained, and if washed carefully the color will not run or fade.

The designs most effective in this work are flowers that do not require minute shading to bring out their beauty. Pansies, fleur de lis, wild roses, poppies, morning glories, and trumpet vines are particularly effective.

The paints necessary for the work are as follows:

PARIS Tints-Put up in outfits of twelve bottles each. There are two sets, viz., $A$ and $B$.

Set " A" contains the following shades:

Orange, dark red, bronze, blue, black, violet, lemon, scarlet, peacock, Bismarck, dark green, and mordant. 
Use the mordant to lighten the color of any shade.

Set "B" contains the following shades:

Yellow, old red, olive, navy, drab, seal, flesh, pink, yellow green, purple, magenta, and mordant.

Use the mordant to lighten the shade of any color.

The above sets, with two brushes and two saucers, are sold in the art stores for \$I.00 each.

To use these tints, first stamp the design, moisten a fine sable brush with the color desired, and go over the space a number of times. Do not charge the brush too full of color, as it will then run over the outline and spoil the material.

The material to be painted should be placed on the drawing board or table over a piece of blotting paper. Plenty of soft, clean rags should be at hand to wipe brushes on. Be careful not to dip the brush from one bottle of paint into another without first cleaning, as this will ruin the tints.

The same effects can be obtained with the common Diamond dyes, sold in the drug stores at Io cents a package. To paint light tints of a color, dilute with water, and apply in the same manner as the Paris tints.

\section{Painting on Celluloid or Ivorine.}

Celluloid or ivorine offers a beautiful background for painting in either oil or water colors. No preparation is needed for oil painting, while for water color painting it is well to go over the surface with a piece of fine emery paper. This will give what artists call a "tooth" to the material. The celluloid should be cut the desired shape and size, and tacked firmly" to the drawing board, as otherwise the edges would curl. The design can be stamped upon the material, and the outlines gone over with a finepointed sable brush with white paint. This will be necessary, as the powder will not adhere to the smooth surface of the celluloid.

To paint with water colors on any highly polished surface, as celluloid, ivorine or glassine, first wash it over with a strong solution of borax water.

Celluloid and ivorine are both inexpensive, and many beautiful articles can be manufactured from them. See chapter on "Dainty Trifles."

\section{Lilacs in Oil Colors on Celluloid.}

This flower in large, rich masses offers a beautiful and effective design for painting either on fabrics, cardboard or canvas. If the artist is com- 
pelled to depend upon a stamping pattern for the design, a special pattern should be made to order, as those usually sold are intended for needlework only, and show each portion of flower in detail. While for brush work masked effects are necessary, and if a little detail and perspective be in the pattern, all the better, as a fence, through which a distant bit of foliage can be seen.

Begin the first painting by laying in the general masses, and do not attempt to paint the blossoms in detail at this time; that should be reserved for the finishing. For the second painting begin with the blossoms, and finish each part, if possible, while the paint is wet. For the purest white of the white lilacs use zinc white, softened with a little yellow ochre, light cadmium, madder lake and cobalt blue. For the shadows the same colors with ivory black and raw sienna. For the clearest purple mix a tone of zinc white, cobalt blue and madder lake, which should be modified with yellow ochre and ivory black. A little vermilion will be found useful in the darker blossoms. For the pale green leaves use zinc white, yellow ochre, light cadmium, and raw sienna. For the darker greens add some burnt sienna, deep cadmium, Antwerp blue and ivory black. The distant greens seen through and beyond the fence, are painted with the same colors, the grayer tones being made by using more of the yellow ochre, cobalt blue and ivory black. If a sky background is to be desired, the principal color needed will be zinc white, light cadmium, yellow ochre, cobalt blue and vermilion, some madder lake and ivory black will be found useful for the dark warm purple. For the fence use Vandyke brown, yellow ochre, raw sienna, ivory black and zinc white. Any parts which cannot be finished at the second painting should be allowed to dry for several days and then have a little linseed oil rubbed over them before proceeding again with the work It will be best to avoid turpentine for these final touches.

\section{Lilacs in Water Color on Celluloid.}

A deep cream or pale ecru will be most suitable for the small and delicate forms of the lilacs. Moisten the celluloid with borax water and then start with the fence, using Vandyke brown, lamp-black, yellow ochre and cobalt blue. Next paint the leaves which come in contact with it, using cobalt blue, light cadimum, raw sienna, Indian yellow, burnt sienna, indigo and yellow ochre. All the greens used can be made 
with this palette by varying the mixture. To paint the leaves outline cach one with the green paint and then fill in quickly before the outline dries and leaves a hard line. A full brush should be used. In painting the lilacs pass a delicate wash of cobalt blue and rose madder, modified with yellow ochre and lampblack. Tint the wash, paint the shadows, using the same colors intensified, and adding vermilion and brown madder. Keep the portion of the study you are working at wet, or the outlines will look hard, and be careful to observe the roundness of the bunch. If the bunch shows white and purple flowers, the background should be toned with lampblack and yellow ochre, for the white bunches, of which a very delicate wash should be made. For the shadows use the same colors, adding cobalt blue and light red.

\section{Lustra Painting.}

This method of decoration can be applied to wood, pottery and velvet, felt or satin, though velvet offers by far the best background.

The materials necessary are the colors which come in the form of a dry powder. They can be had, put up in bottles, at the art stores, or in ounce packages, which latter is somewhat less expensive than the former method. The colors or powder are of two kinds-brocades and flitters. The brocades are a fine, impalpable powder of irridescent hues: the flitters are brilliant flakes of the same colors as the brocades. The colors are as follows:

Brocade Powders-Antique green, brown, copper, crimson, gold brocade, green gold, lemon, light green, orange, rich gold, scarlet, silver, sparkling silver, steel.

The above colors are each 15 cents per bottle.

Dark purple, pale purple, emerald green, pale blue, ultramarine.

BRoCADES-Gold, silver, copper, orange, fire, carmine, crimson, cambric (assorted), light green, light blue, dark blue, blue violet, red violet, new green, dark green, straw yellow.

Flitters-Gold, silver, copper, orange, reds, blues, greens.

Dry Colors-Black green, dark green, dull green, dull blue, dull red, light green.

General medium, special medium.

A china palette for mixing colors will be necessary. Where a palette 
is not at hand small butter dishes will do nicely. Two flat bristle or sable brushes, Nos. 5 and II, will be found most suitable for this work.

The colors are liquified by mixing them with the general medium. Use the china palette or saucer for this purpose (for pale pink, madder pink and carmine, the special medium must be used for mixing). The paints must be applied with the flat of the brush as much as possible. Keep the edges clear cut by taking fresh color in the brush frequently. Take long, firm strokes, laying the color on the surface only.

In painting on plush the colors should be mixed with the medium until about the consistency of syrup. Select a plush with short, close pile, and paint with the pile. In painting silk, satin and linen, the colors must be mixed thinner and but little color taken in the brush at a time. Care must be taken that the color is not too thin with medium, or it will spread beyond the drawing.

These colors are very brilliant and will last as long as the foundation to which they are applied.

In the list of colors are given all that are in the market, but it is very seldom that one will require more than a dozen different colors for a large and varied amount of work. Good taste demands that the brocades should be used sparingly on textiles. They are more suitable for decorating pottery, wooden articles, etc., though for white flowers and brilliant foliage they can be made very effective and beautiful. The best artists, however, incline to the duller, more sombre tones.

White flowers should be painted all over first with silver, using more paint where a high light will be than in the shadows; afterward glaze the high light with a mixture of one-third silver and two-thirds sparkling silver, and glaze the deepest shadows with steel.

For yellow flowers use rich or pale gold, for the high lights, fire for the deepest shadows, blend with a middle tone of lemon or orange, to suit taste.

In attempting any new kind of work, without personal instruction, the student must be prepared to do a little experimenting, to exercise considerable patience, and bring to the task all the common sense she can muster. There are ever pitfalls for the feet of the unwary, and many a puzzling point will come up for settlement in the work of each individual student. which cannot be anticipated in a work of this character, but with the pa19 
tience to follow the instructions that have been given, point by point, and item by item, there is no excuse for not obtaining satisfactory results.

\section{Kensington Painting.}

This style of painting, as its name indicates, is a close imitation of Kensington embroidery, and for some purposes quite as effective as that work and much less expensive.

The painting can be done on plush, velvet, satin, felt and any smooth surface cloth, with oil paint, the same list of colors given for painting on "fabrics," answering for this style of work also.

Brushes Nos. 6 and 7, a palette knife, a porcelain palette, spirits of turpentine, a bank pen and some soft rags for wiping brushes, will complete the materials necessary for "Kensington Painting."

Stamp the design on the material, using floral arrangements, and tack the material smoothly to the drawing-board. If the piece to be painted is small it can be stretched in a pair of embroidery frames instead.

The paints should be applied with a stiff bank pen for the detail of the work, though where a broad surface is to be gone over, the paint may be applied with a brush, and afterward worked up with the pen.

Squeeze out on the palette, or a common plate, a little of each of the colors to be used; if it is necessary to mix any of the colors to get any desired shade, it should be done with the palette knife. To do the painting hold the pen bottom up; take the paint up in the hollow of the pen, shovel fashion; wipe the paint from the back of the pen with a piece of old cotton cloth, kept for the purpose. Then draw the point of the pen, with the back toward the material, over the outline, turning the pen slightly as you draw it, in order to make the outline clear and sharp. Next fil the pen and apply the paint to cover the inside of the petal or leaf, drawing the pen toward the center, as the stitches are taken in embroidery. Apply the several shades used in this manner, blending them together with the pen. After this scratch the leaf over with the point of the pen to give it the appearance of stitches, remembering to make the lines run towards the center, as in embroidery. If you wish to make the work finer, a needle can be used to do this part of the work.

In painting petals or leaves, begin from the outer edge and paint to- 
ward the center. Lay on the principal colors first, afterwards the darker shades and high lights, blending with the pen.

For such flowers as golden rod, coxcomb, sumac, and for the pollen or anthers of other flowers, the paint is best applied by dabbing it on with the end of the brush. The knack will be caught with very little practice, and then no further trouble will be met with.

The "feather stroke" is made by taking the paint up in the hollow of the pen, and from the outer edge of outline of design make a clear stroke to the center, twisting the pen in the fingers in order to make the paint stand out roundly from the material. Place another stroke beside the first in the same manner, but do not attempt any other blending of colors.

Use turpentine or poppy oil to thin the paint with when necessary, also to act as a dryer.

\section{Parrots in Kensington Painting.}

A large parrot is a good design for fire screens or wall banner, and small ones form an effective decoration for the blocks of a fancy quilt, cushion or wall pocket. A moderately coarse pen and the following paints will be needed: Silver white, Vandyke brown, chrome yellow, Chinese vermilion, chrome and emerald green, Prussian and cobalt blue.

Paint the branch with Vandyke brown, to which a touch of chrome green and white have been added. This and the leaves can be done with a brush. Make the leaves of chrome green, and shade them by adding a little white and a touch of yellow. Paint the bill pure white, using the brush. For the front and back of the head use Chinese vermilion, applying the paint with the pen, doing the "feather stroke." Take the paint up on the under side of the pen, place the pen down firmly on the goods and draw it toward you. Use chrome yellow for the eye, and paint the neck with chrome yellow, lightened with a little white. For the breast, mix equal parts of emerald green and chrome yellow. Use a little brown and yellow for the feet. Edge the wing with a stroke of Chinese vermilion, and then one of yellow. Paint the inner part of the wing with Prussian blue and green, mixed. Paint the inner tail feathers with the chrome green. The back of the wing should 
be greenish-blue, made by mixing chrome green and cobalt blue. Paint the tail itself with a first stroke of Vandyke brown, then one of white, and another of Chinese vermilion. Around the eye draw a circle of light brown, made of white, mixed with Vandyke brown. A fine brush may be used for this. The same directions would apply for large or small parrots.

\section{Crayon Painting-Portraits.}

Portrait painting is a branch of "Brush and Palette" entirely distinct from the decorative methods we have been considering. Portrait painting should not be attempted without either a natural talent or instructions by a competent teacher, yet crayon portraits are frequently made by persons who have had no training whatever. The work is much simplified by the use of solar prints.

Having secured a good photograph of the subject you desire to paint, send it to any first-class photographer with orders for a solar print, being careful to state the size you wish the picture enlarged to. A solar print is a shadow picture without details.

The solar print secured, the following instructions for treatment will be found to yield satisfactory results. It is from the methods of one of the most successful portrait painters, and we submit it to our readers, feeling certain that it will be of far greater value than a half-dozen lessons from an inferior teacher. Practice, patience, perseverance, observation, are watchwords that should ever be borne in mind. Without them it were better not to attempt portrait painting.

"First, as to materials required. For finishing in monochrome or black and white, finely powdered pumice powder will be required to give a tooth to the paper. Some paper stomps of various sizes, with at least six stippling stomps, a stick of crayon sauce, and Conte crayons, Nos. I, 2 and 3.

"Have the print mounted upon good stout cardboard and well rolled -not burnished. Pin it to a smooth drawing-board, and sprinkle lightly with the pumice powder. With a circular motion rub the surface of the print all over, till there is an even tooth over the surface of the print. Take a little crayon sauce and mix some of the pumice powder with it, in proportion of one of pumice powder to two of crayon sauce. Put this upon a piece of cardboard, to form a palette, and rub the tips of two fingers into it, 
and rub on the print, upon the background, over the shoulders. Never mind its going into the drapery or face; it can be removed. With vignette portraits a light, cloudy effect over each shoulder is best, making it a trifle darker where it approaches the lighted side of face, and lighter on the shaded side, to give contrast. Make it darker than you intend it to remain to allow for the after treatment with the pumice powder.

"When you have got in the effect of light and shade sprinkle a little of the pumice powder all over the picture, and lightly rub all over. This loosens the crayon and gives softness and distance. Take the Conte crayon No. 3 and go over the deeper shades of the drapery, marking them in very

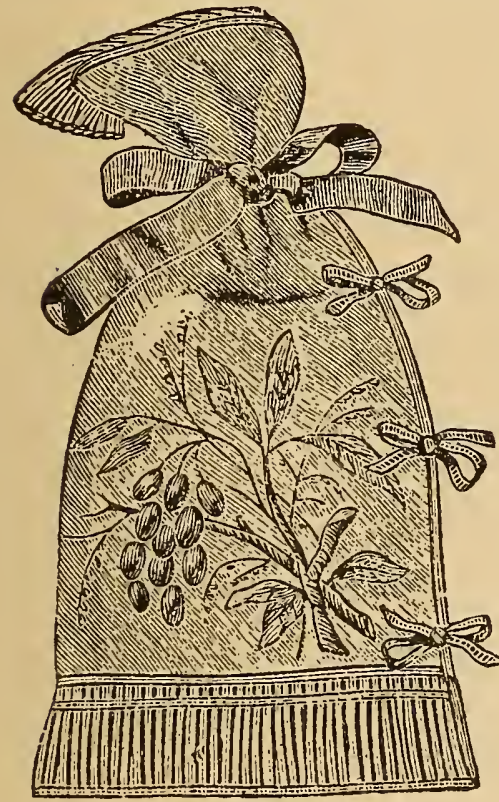

FIG. 274. strongly; then soften them with the fingers, graduating them where it may be wanted.

"Next take the Conte crayon No. I, cut it to a rough point with a knife, and finish upon a piece of No. I emery cloth. With this deepen the line of the eyelash, the marking of the nostrils, the division between the lips, the eyebrows and marking of the hair, softening where you think it necessary with one of the small stomps or the fingers.

"Take Conte crayon No. 2, make a point in the manner already described and despen the pupils of the eyes and the lines around the eyes. Mark in the shadows in the ear and under it, around the chin and upon the neck, under the chin and under the eyebrows, and down the shadow side of the nose and forehead, and any other shadow which needs strengthening, softening and graduating where necessary.

"Now pause and go back over the whole work. You will see that, starting with a strongly printed solar print, the whole work has been nothing more than deepening the photographic shadows and graduating them into the lights. If this has been done carefully there will be no loss in resemblance, and the picture has gained vastly in strength.

It is now time to add finish, and that is done by filling up with the point of an $\mathrm{H} \mathrm{H}$ lead pencil any breaks in the graduation of the shadows, and in taking out the highlights. This part is done by making a fine point 
with the emery cloth, and working out any spots with a light circular rubbing touch. As you go on with the finish you will find, perhaps, that your shadows want deepening. This can be done best with the stomp and crayon sauce for broad effects, and the No. 2 Conte crayon for decisive touches.

"For taking out highlights a piece of hard ink-eraser will be wanted, mounted in a porte crayon, and cut to a flat point. The lights to be taken out are upon the forehead, down the nose, upon the cheek bone, and upon the chin. Clean off with the ink-eraser white drapery or white collar, and with a fine point take out the highlight on the eyes. Sometimes on light hair a few judicious touches with the ink-eraser upon the highest lights will effect a great improvement.

For the finishing touches go carefully over the face, filling up any breaches in the graduations, deepening, if necessary, with a stippling stomp and crayon sauce, the upper lip. Shape the lower one with the point of the stomp, and deepen the shadow under it. Round the lower part of the chin, and put a little delicate shadow on the light side of the forehead and the shadow which is upon the cheek from the ear to the curve of the nostril. Keep the ear back by keeping it in soft, delicate shadow. Go over the halfshades of the hair with the same stomp and crayon sauce, and when all these delicate half-tints have been attended to, finish by a few vigorous touches with Conte crayon No. 3 upon the darkest shade of the drapery, the pupils of the eyes, the deepest shadows of the hair, the shadows under the eyebrows and down the nose, the nostrils and under the chin.

"This treatment is for vignettes of a half or three-fourths length.

"The background will want somewhat different treatment. 'The crayon sauce and pumice powder may be utilized all over the background, keeping the lightest side of the figure darkest in the background, making the lower part of the background graduate darker as it gets lower, and finishing by broad hatching with the stomp and crayon sauce. Keep the lower part of your picture lower in tone altogether than the upper part, so that the concentration will be upon the face as the powerful part of the picture and let all your touches be more and more undefined as they recede from it. All the sharp, crisp touches should be upon or near the face."

There is no form of art work that yields better returns for time and 
skill required than crayon portrait painting. An expert artist easily paints three to four full-length portraits in a week, and the average price ranges from $\$ 10$ to $\$ 25$ per picture. The solar prints cost $\$ 1.50$ to $\$ 2.00$ each, so it can be seen that it will repay the time spent in learning how to work with the crayon. Again orders can be secured for portraits when all other paintings are a drug on the market. We do not pretend to have exhausted the subject, but merely to have pointed out the road to follow in order to reach success.

\section{Painting on Birch Bark.}

The inner layers of birch bark offer a beautiful background for sepia painting. Sepia is a beautiful dusky brown, very powerful and harmonizes perfectly with the tawny color of the bark. It is diluted with water and used like any of the water colors.

The bark should be selected and prepared with great care. Three thicknesses will be sufficient to leave togeiher, while if it be more finely subdivided it will not have sufficient body for making into ornamental articles. This dividing and splitting must be done when the bark is first pulled from the tree, and it should be immediately pressed flat under weights in order to prevent the edges from curling. As the bark from young trees is the most desirable, it follows that the pieces secured are in small sections, therefore the designs selected must be reduced to miniature, which can be done by means of the pantagraph.

To paint: Place the bark on the drawing board, carefully secured at the corners. Place the board in a slanting direction, and sponge the bark over with clear water. This is done in order to make the bark take the color.

Small landscapes are best suited for this background and should be painted in regardless of small details. Cover the pincipal outline with the sepia, carried in a No. 2 brush. A larger brush may be used for $c^{\prime}$ sud effects, which should be put in with Chinese white for the light oncs and warm sepia for the dark ones; the natural bark wiil serve for the general sky tint. If the shadows are too deep absorb the superfluous color by pressing over it a bit of blotting paper. Aside from landscapes, floral designsvery simple in arrangement-are exquisite painted on the bark. Follow the directions given for water color painting. The decorated pieces of bark 
can be made up into numberless pretty trifles-as a cover for tablets, an inlaid panel on a blotting pad cover, a telegram holder, or a pretty easel picture can be made by placing the bark in the center of a square of ecru

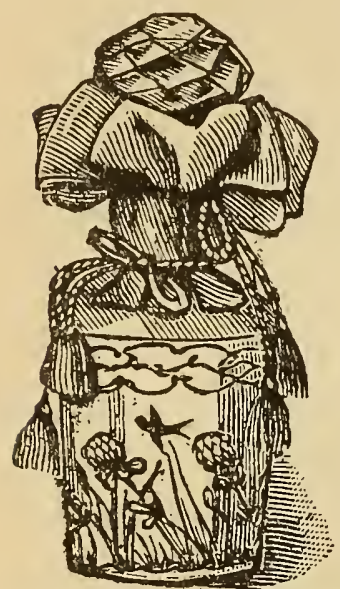
rice board or leatherette. To mount the bark slit the leatherette or board in two diagonal lines near each corner, and slip the edge of the bark through.

\section{Scorch Painting.}

This form of painting is enjoying a revival of popularity and bids fair to become a popular craze. It is a revival of the ancient art of pyrography, and, as DETAIL A, FIG. 275 . its name indicates, is done by burning

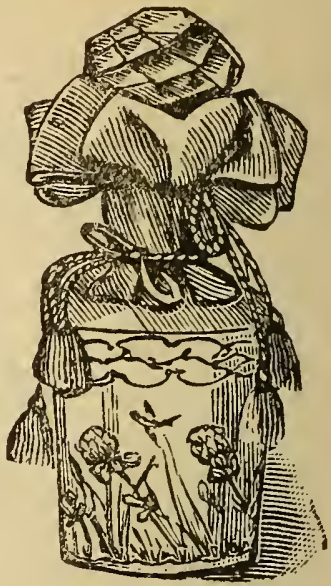
the design on wood with a hot poker or platinum point.

Scorch painting offers teachers an almost unexcelled opportunity for forming classes at profitable prices, as the work is fascinating in the doing, beautiful and artistic when done, and easily learned. Aside from the profit of teaching, well-executed designs find ready sale.

Very little is known of the method of doing scorch painting, and the field is practically in the hands of the energetic artist who chooses to give the time to paintand forming ples are all essary to enmost conser-

The outfit for scorch consists of c a 11 e d the which supnecessary for

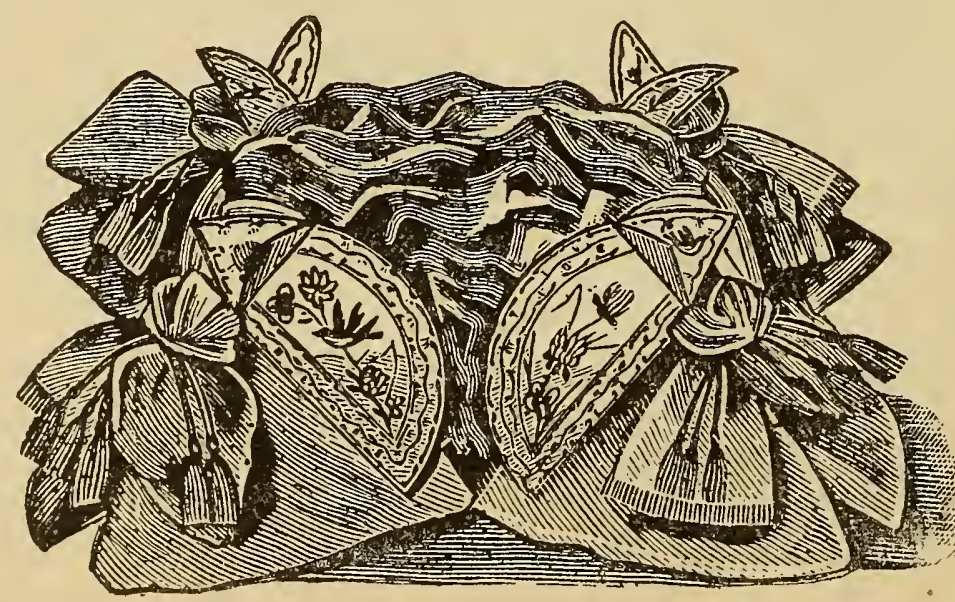

FIG. 275 . ing samples classes; samthat is necthuse the vative. n e cessary p a inting a machine "Vulc a n," plies the heat heating. the platinum points or pencils, and regulates the intensity of heat desired. Its manipulation is very simple and easily learned. In addition to the "vulcan," points of platinum, which come in a box of assorted sizes, and patterns, will also be necessary. The entire outfit, consisting of the vulcan, assorted 
platinum points and benzoline, can be procured for $\$ 5.00$, and there the expense for materials ends. Of course, the article to be decorated is not included.

The woods best suited for scorch painting are holly, sycamore, lime and even bass wood. This latter wood is cheaper than the others, and is best adapted for practice work. Other wood may be used with good results, as oak, ash, elm, chestnut, cedar and teak. Sycamore is by far the best for fine, delicate work.

The platinum points should be used with extreme care, as they are the most costly part of the outfit.

The designs for scorch painting may be floral, landscape, animal or figures, either natural or conventional; the detail should not be minute, but tather the design should be clear-cut and expressed by sweeping strokes. The design may be stamped on the wood by means of perforated patterns, where the worker does not draw-and indeed for purposes of class work it is by far the better plan to stamp the design at once.

Full instructions for using the vulcan and points are supplied with the outfit. When starting the scorching, the point must be heated in the spirit lamp, but afterward it will not be necessary to repeat the operation, as the bellows maintain and regulate the heat.

In teaching oneself or others the mode of scorch painting, it is excellent practice to make strokes, oblique, straight, circular, etc., with the different points, as they will show gradations of tone and will give ideas for treating rounded surfaces and backgrounds. The strokes must be bold and firm. The background may be either dark or light, but if light, then the design must be dark, and vice versa.

Effective results are obtained by burning away the background deeply, leaving the design in low relief.

Scorch painting will occupy a high place in decorative work, as it can be used for so many architectural details, as paneling fur dining room or library, frieze for hall or library, while tops of tables, pianos, etc., can be rendered beautiful by its employment.

The teacher who intends to undertake scorch painting for profit, must be thoroughly prepared at all points. First, she must have becume proficient in the different strokes; the use of the different points, and the 
manipulation of the vulcan, all of which can be learned without other aid than here given, if perseverance and common sense be brought to bear upon the subject. The learner's best aid first, last and all the time, is patient practice, coupled with observation.

As will be seen, beautiful effects can be obtained by light and shade, in the scorching, so that attention should be paid to this part of the work. High lights and shadows should be sharply accented, otherwise the work will present the appearance of flat lines, scorched on a flat surface.

Simple outline designs should be tried first, after the various strokes have been practiced, and the burning in of shadows should be approached gradually. The same rule for perspective, roundness and body given for oil painting obtains in scorch work, and should be applied, or else the work will not have been brought to its best possible state of perfection, and half its artistic beauty will be lost. In preparing for a class, the teacher should see to the selecting of the practice wood; in fact, the better plan is to supply each pupil with a piece of clean wood for the purpose of practicing the strokes, and another piece on which has been stamped a simple landscape design-a road, a tree, a bit of fence answering admirably. Such patterns are kept in stock by the best pattern houses. Do not supply pupils in the same class with the same design, as the work will become too monotonous. In addition to the two pieces of the wood the teacher should also supply the outfit of tools for the work, where at all possible, as by this means all the pupils will be uniformly accoutred for work, and there will be no hitch or delay because some pupil has forgotten to supply herself with some part of the outfit.

The usual price for a course of five lessons in scorch painting is $\$ 6.00$, and a teacher can do full justice to a class of six pupils at one sitting.

\section{Tapestry Painting.}

Tapestry painting is one of the most practical of the decorative arts, as larger schemes of decorative work can be carried out by its use than by any other form of painting within the reach of the masses. In this chapter no exhaustive details can be gone into, but in the few pages devoted to the subject sufficient instruction will be clearly given to enable one who has had some experience with the brush in either oil or water colors to prosecute 
the work successfully without other aid; while for the woman who has never used the brush the hints here given will save many dollars and much time and, it may be, some discouraging failures in experimenting, either alone or with the aid of the teacher. We advise personal instruction for the novice to the extent of a few hours at least, yet the pupil should go as far as possible alone, using the instructions here set down. In cases of rare art talent further aid might not be needed.

Tapestry painting, as its name implies, is painting with specially prepared dyes on wool or silk ribbed canvas, to imitate the old woven tapestries. It is used for furniture covers, wall hangings and screen panels.

The designs are usually landscape, figure, or large floral, fine details and miniature designs not being suitable. Historical scenes are by far the most satisfactory designs for this work-as Queen Mary of Scot's meeting with Elizabeth in the garden after the signing of the death warrant, scenes from Richelieu, Romeo and Juliet, the Chase, showing hunters and dogs, etc., are all effective. As very few, if any, perforated patterns are made for this form of work, the old engravings and wood prints to be found in French journals and books will serve as excellent studies. A simple subject should be selected first, and the engraving or cut transferred to the canvas by means of the pantagraph, and this should be done with the utmost care so as to secure an exact tracing. For the beginner it will be safer to transfer the picture the

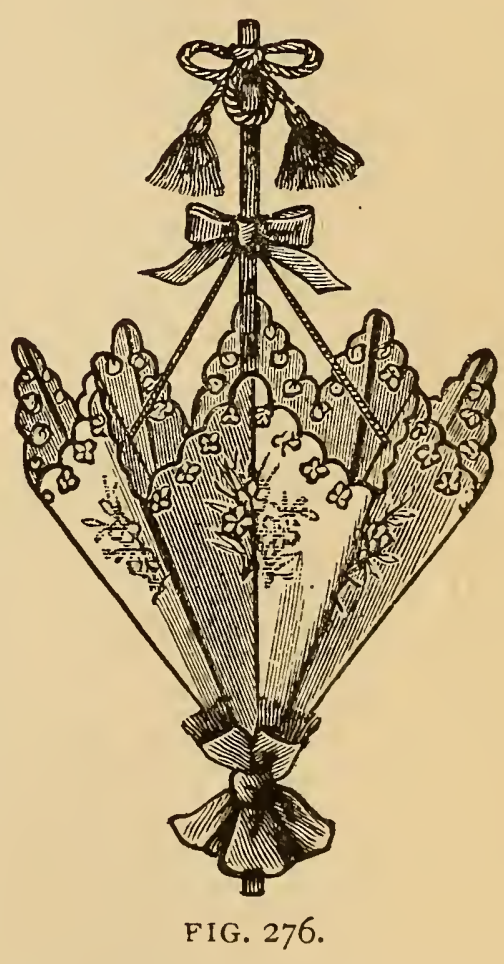
desired size to good bond paper first, after which the lines can be pricked through with a fine pointed needle, which will give a perforated pattern, by means of which the design can be stamped on the canvas, using the powder for this purpose.

\section{Canvas for Tapestry Painting.}

There are various kinds of canvas sold for tapestry painting, but the best will be found to be Binant wool canvas. This canvas can be had in 
294 DAINTY WORK FOR PLEASURE AND PROFIT.

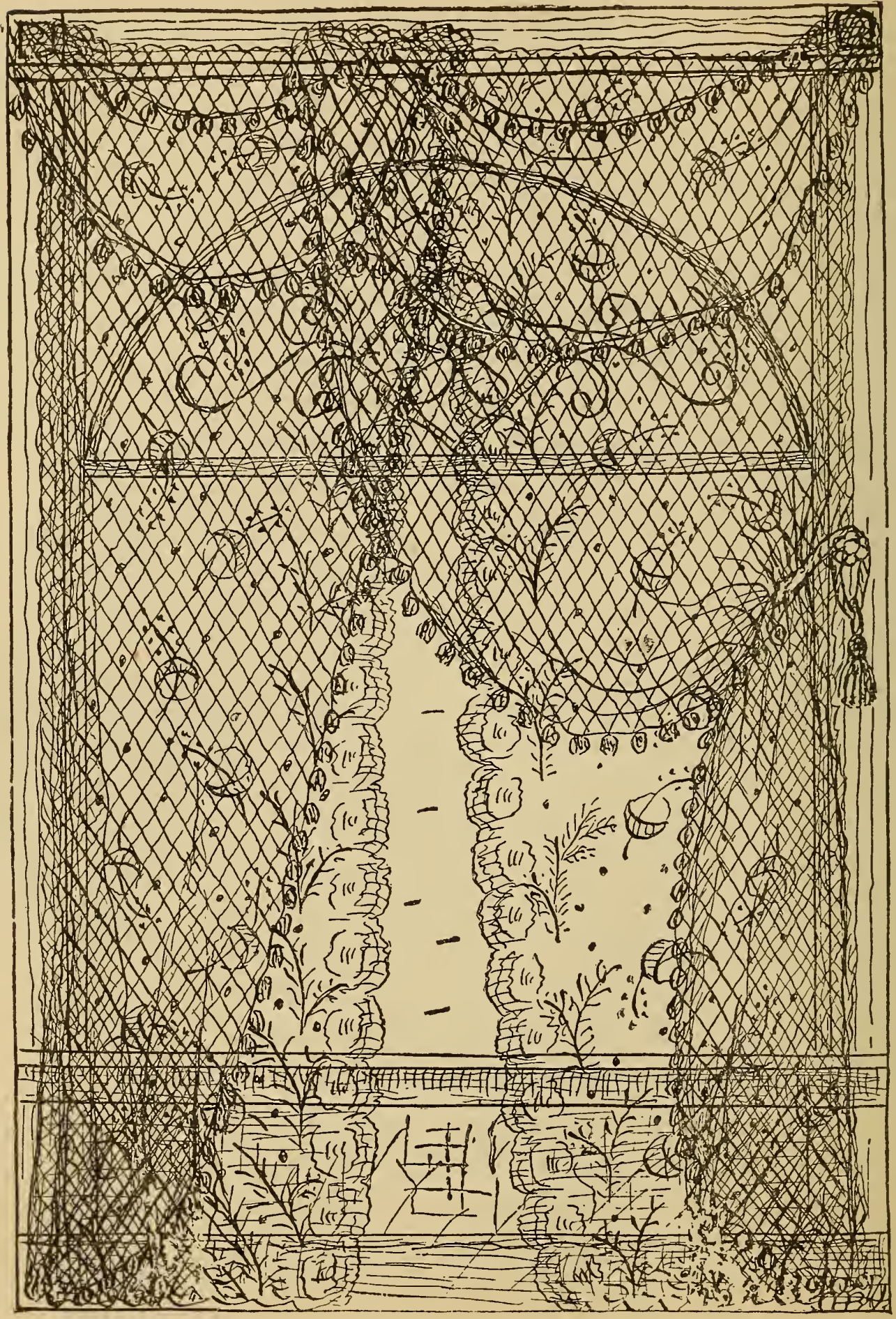

WINDOW DRAPERY OF WABAN NETTING OVER LACE. 
various widths and for various prices. The following table, from an art supply catalogue, will be of assistance in selecting canvas.

No. I, 84 inches wide, coarse thread, best quality, per yard, \$6.15; No. I, I 22 inches wide, coarse thread, best quality, per yard, $\$ 8.00 ;$ No. 2,84 inches wide, fine thread, best quality, per yard, $\$ 6.15$; No. 5,56 inches wide, coarse, dark, best quality, per yard, $\$ 3.75$; No. 7,36 inches wide, fine, best quality, per yard, $\$ 2.00$; No. 8,84 inches wide, fine, best quality, per yard, $\$ 4.00$; No. 9,54 inches wide, medium, best quality, per yard, $\$ 3.00 ;$ No. 14 , 50 inches wide, silk, ecru color, per yard $\$ 8.00$.

There are other widths and qualities made which any dealer can procure.

Nearly all art stores have small pieces of these tapestry canvases ready stretched in stretchers, which will be found cheaper to commence on than to purchase by the yard, as in this case the whole width must be paid for whether one has use for it or not.

For large subjects the coarse vanvas will give best results.

To prepare the canvas for painting is a most particular part of the work. For small pieces the canvas can be siretched in an embroidery frame exactly as for a piece of embroidery; see chapter on "Implements." If the subject is large a pair of light quilting frames may be utilized. The frames must of course be clamped to the desired size. Tack the canvas to the ends and lace it to the sides. Secure the corners firmly in place before lacing.

\section{Dyes.}

The dyes used for tapestry painting are French dyes, but there are different methods of preparing them, and this constitutes the difference in their artistic values. M. T. Wynne advises the use of the Grenie dyes, and having used them in our own work with uniform success we endorse the opinion of our esteemed confrere.

The Grenie dyes are advertised as indelible, and sc far as our expericnce goes we have found them to possess this quality. There are thirteen colors, indicated by numbers, from one to thirteen inclusive, as follows: indigo, ultramarine, rose, vermilion, cochineal, Indian yellow, burnt sienna, brown, gray, violet, green, gray green, black. 
The Grenie dyes are a little more expensive than others on the market, but knowing that the best is ever the cheapest, in painting materials at least, we do not mention others. The cochineal and indigo are sold for 30 cents a bottle, while the other colors are only 20 cents. There is a medium for mixing the colors that is indispensable; it is sold in pint bottles, and costs 40 cents per bottle.

\section{Brushes.}

Next to the colors in importance is the question of brushes. It means absolute failure to attempt tapestry painting with the ordinary brushes. At least one dozen brushes, assorted sizes, from No. I to No. I2, made especially for this purpose, should be procured. They are made of stiff bristles, and cut in the shape of a chisel. For large surfaces a double varnish brush, cut down a little to stiffen it, is recommended by artists for skies. Glass palettes should be procured, and also several glass jars for mixing the colors; two or three of a larger size, for mixing large washes in.

\section{Mixing the Dyes.}

The dye colors are exceedingly crude in the pure state, and therefore should be toned down with complementary shades. The colors that are made by mixing are: brown, made by mixing indigo, burnt sienna and vel. low; gray, made by mixing indigo, cochineal, yellow and a little burnt sienna; gray green, made by mixing indigo, yellow and cochineal. The shade required of each of the foregoing colors must, of course, be determined by the proportion of the mixture.

All colors in which a sediment settles in the bottom of the bottle must be shaken before being used.

No color should be used without first mixing it with some of the medium, and a certain amount of water. A pen knife and steel eraser, not too sharp, and rounded at the points, are necessary and important articles of the tapestry painting outfit.

When the painting is finished, Wynne strongly insists upon the necessity of steaming the work, either at home, over a specially constructed steamer, or failing in this method, advises sending the work to a regular dying establishment. Doubtless this procedure would add to the indelibility of the work, but in our experience we have found the following much more simple and quite satisfactory: 
Place the painting on the table, face up; wring out a thin woolen cloth in cold water and place it smoothly over the work; over this again place a dry cotton cloth, then with a hot iron go over the whole until the woolen cloth is thoroughly dry. This method serves to subdue, soften and mellow the dyes, taking away the crude and glaring effect that is sure to be felt by a person of artistic susceptibilities, unless this subduing process is resorted to. Flesh Colors.

As figures are largely used as subjects for tapestry painting, a few remarks on flesh painting will be acceptable to the amateur, at least; and as our own knowledge of the art has been gained by a close following of the method employed by Wynne, we cannot do better than introduce the reader to his method for flesh, skies, foliage, etc.; adding from time to time points that have been learned from that best of all masters, "experience." To proceed, then:

\section{Flesh Painting.}

The brushes for painting flesh colors should never be used for any other purpose, as the entire work depends upon their being free from even a suspicion of other colors, and they should occasionally be cleaned with soap and water. The colors used for painting flesh are, burnt sienna, rose, yellow and indigo; the palette is extremely simple, but other color or colors must never be substituted for those given.

Place on the palette two shades of burnt sienna. For fine work use the finest brush, and put in clearly and with a firm hand the deepest markings of the eyes, nostrils, lips, ears and fingers. Then with a large brush wash in the broad shadows, using the paler shade of the burnt sienna. Be careful not to touch the broad highlights. If the eyes are brown they may be put in with burnt sienna, also. This part of the work is called modeling, and after it is quite dry the local tone is washed over all the flesh, including shadows, using again the burnt sienna, but so much diluted as to merely tint the medium, which should be added to it without any water. For a dark, sallow complexion, befitting an Othello or a Spanish knight, add a touch of yellow to the sienna. In applying this local wash it should be scrubbed into th: canvas until every thread is thoroughly colored. The shadows must be counteracted with green made of indigo and yellow. 
${ }^{2} 98$ DAINTY WORK FOR PLEASURE AND PROFIT.

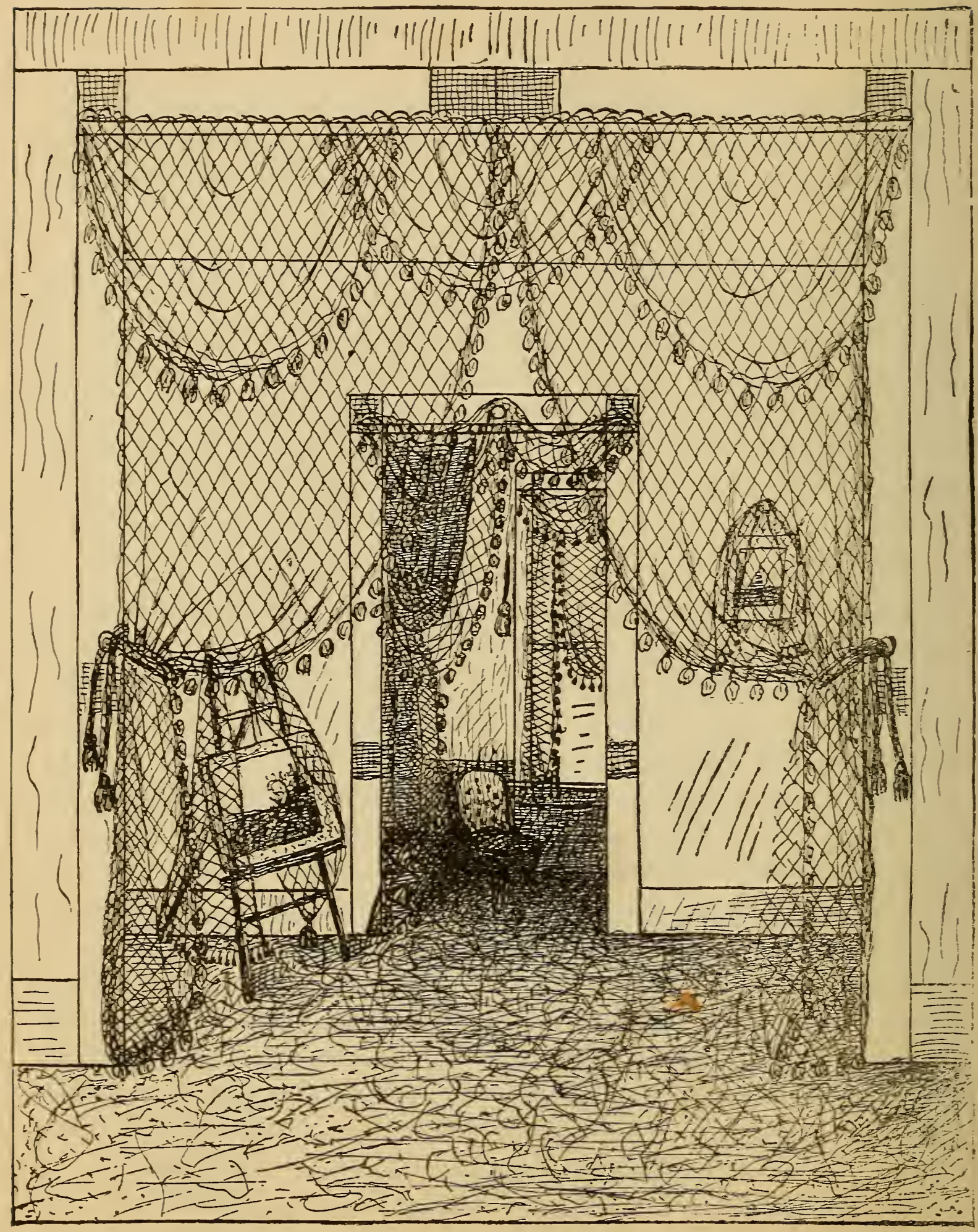

LIGHT DRAPERY FOR PORTIERES. 
Make two shades of the green of about the same relative strengtis as the two shades of burnt sienna used for putting in the shadows. Before applying the green, tint the cheeks with rose. Do not allow the greens to run beyond the shadow color.

If the red in drying works up too much, add more green; but if the green is too strong, work in more burnt sienna.

The sharp markings must not be painted in until the previous washes are nearly dry, or the color will spread.

When the first flesh painting is perfectly dry, and you must be sure about this, add a touch of rose for the lower lip, model the highlights by scraping very gently with the rounded point of the knife or eraser. This gives the picture artistic finish, and is effective, as the color still remains in the ribs of the canvas, while the knife removes only that color lying on the surface, giving a stippled effect. You are now ready for the second flesh wash. Put on the glass palettes the four colors previously given for flesh painting, and apply. Make another tint by mixing green and red together. This mixture will be needed only for touching up and delicate modeling. The markings of eyes, ears, lips, nostrils and fingers must be strengthened. The use of the knife between the shadows and highlights will produce the pearly tints necessary for softening the flesh tones and blending massed light and shar'e.

\section{Hair and Drapery.}

In painting hair, lay in the color in broad masses of light and shade. For golden hair, make a palette of a pale yellow and a very small amount of rose. The shadow color is made by mixing yellow and brown and a little rose with these colors. Any shade of hair, from light flaxen to golden brown, can be made by simply varying the proportions of the same color. While Hayward advises the use of brown, yellow and rose for the shadow color, yet in our own work we frequently omit the rose, finding the yellow and brown sufficient; but in case there is difficulty in getting just the satisfactory proportions of each color, the addition of a very little rose will often solve the difficulty, and give the exact shadow required. It will modify the greenish effect caused by too great preponderance of yellow in the 
first mixture. For dark hair, mix the burnt sienna with the medium, and much or little water, according to the shade required. If nearly black hair, add a little indigo to the brown, and for the local wash add gray to the brown.

For painting in draperies, we have followed the method laid down by Wynne for beginners. It was the one first practised in our work, and having always given satisfaction we see no reason to change it in our later work: Model all the shadows before putting in the local tint. Put in the darkest shadows first, then the next tone, leaving the delicate half-tones to be added when the local wash is nearly dry. Lay the local tint over both light and shade. This will complete the first painting. There remains only to accentuate the shadows and scrape the highlights wherever needed, to give brilliancy. There are no definite rules laid down to guide the artist in regard to the coloring of draperies; the harmony of colors must, of course, be considered, but we will say in this connection that all kinds of fabrics may be reproduced in tapestry painting by simply giving attention to the falling of lights, as all the difference lies in the fact of their being soft or sharp. All the tapestry dyes can be mixed with each other, and the most satisfactory method is to mix and mix, strengthen and weaken-trying the colors on a bit of canvas-until the desired colors and tones are secured.

We will append here the result of a few experimental mixings.

\section{Colors for Draperies, Greens, Earth, Marble, Stone, Sky, Fore- grounds, Trees, etc.}

Blue.-Local wash, indigo diluted until very pale. For shadows, indigo blue, gray and a little orange. Make the orange with burnt sienna and yellow.

Salmon Pink.-(This is a color very effective for draperies, slashings, etc.) For the locai tone use rose diluted with medium and water until very pale, to which a very little green has been added. For the shadows use brown, to which has been added a little rose.

White.-For shadows, gray; the canvas will do duty for highlights.

Violet.-Local wash, rose and ultramarine; for shades and shadows, indigo and cochineal, with a touch of burnt sienna in the shadows. 
Yellow.-Use a mixture of brown and yellow for shadows; use pure yellow for local wash.

Pale Lavender.-Light wash ultramarine and rose; shadows, ultramarine, rose and burnt sienna.

Heliotrope.-Ultramarine and rose, and burnt sienna for shades; for the local wash omit the burnt sienna.

Terra Cotta.-Brown, rose and yellow. Use less brown for the local wash.

Sapphire Blue.-Indigo, rose and ultramarine.

Olive Green.-Burnt sienna, yellow and indigo.

Turquoise Blue.-Emeraude green and ultramarine much diluted for the local wash; for the shadows add ultramarine and orange.

Ashes of Roses.-Local wash, rose diluted with water to a mere tint; for the shadows mix brown, rose and gray.

Greenish Gray.-Indigo, yellow and cochineal.

Mahogany.-Mix brown, yellow, burnt sienna and rose. For the highlights omit the brown; add a little indigo for the darkest shadows. The effect of polished mahogany can be obtained only by paying attention to the reflected lights.

Crimson.--Cochineal for local tone, adding a little rose for highlights and brown in the deepest shadows.

Golden. Brozen.- Mix brown, yellow and rose for the shadows, and yellow and rose for the local tint.

Gray Green.-Mix indigo, cochineal and yellow.

Deep Crimson.-Use cochineal, adding a very little rose, for the lights, and brown in the deepest shadows; if too warm soften with indigo.

\section{To Paint a Sky.}

In painting in a sky the methods of different teachers vary. That recommended by Hiller will perhaps be more readily comprehended by the beginner. "Select a large brush and commence at the right-hand cornes of the canvas, and with indigo mixed with the medium and much diluted with water, soak the canvas thoroughly. Be very careful about putting in the first wash; scrub it into the canvas thoroughly and evenly, 


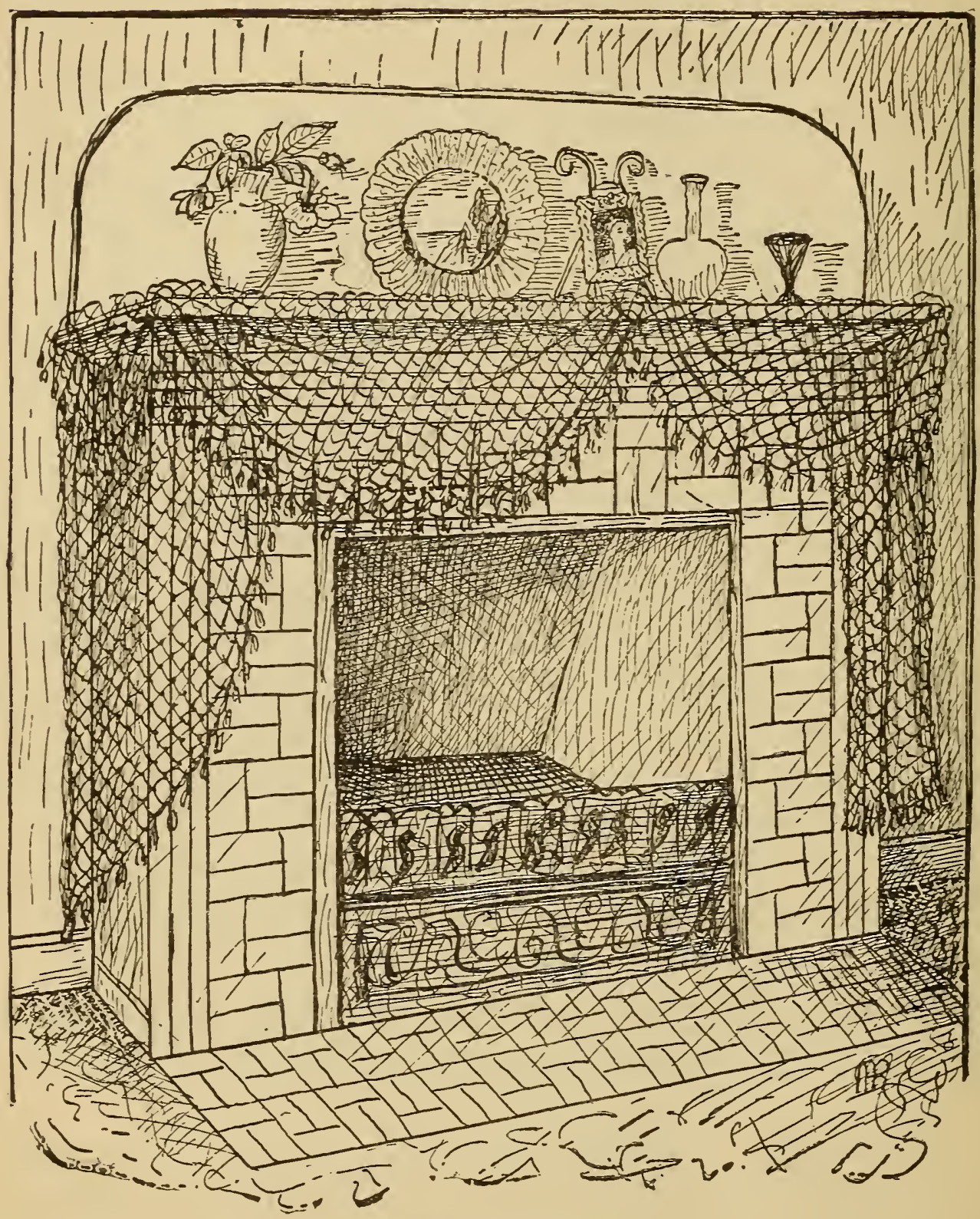

DRAPED MANTEL. 
otherwise it will dry with a spotted appearance. Carry this deepest shade as far down as necessary, then dilute it a little and carry it a few inches further down. With a clean brush wet the canvas a little below the horizon line with medium and water. Some workers omit the medium for purposes of economy. using the water only; but this is a policy that will not answer; satisfactory results cannot be obtained without the use of the medium. Soak the canvas thoroughly. Dilute yellow to a pale shade, also a little rose; place each in a glass by itself. Begin a little below the horizon line and paint in the yellow. Do not carry this tint as far as the biue. Next paint the rose color into the yellow, carrying it above the yellow, and blend it with the blue. The above will give the rosy, purple shade seen in sunset skies.

\section{DECORATIVE PAINTING FOR PROFIT.}

In the chapter on "Dainty Work for Profit" the subject of decorative painting is not touched upon for the reason that it more properly belongs under the head of "Brush and Palette," and is of sufficient importance for a more exhaustive consideration than could have been given elsewhere.

The ideal art bazaar or "fancy goods" store is not complete without a good assortment of artists' supplies, painted decorative articles, etc., and yet, strange to say, we rarely find the two combined. The stock is more easily handled, the profit greater, and the loss from out-of-date supplies less than in a needlework stock, yet women who enter the decorative business fight shy of it.

This state of affairs probably arises from the fact that, as a rule, women are better posted on needlework than on painting. The latter art is enshrouded with a good deal of mystery, from the fact that artists treat the subject technically, and the poor layman or laywoman is terrified by the sound of unfamiliar terms, and at once conceives the idea that painting is an art to be attempted only by fortunates especially endowed by the gods with a "peculiar genius."

This may be all very well from an ideal art standard, but from a decorative point of view is sheer nonsense. Any woman with brains enough to run an art bazaar or store, has talent sufficient for doing a line of profitable 
decorative painting. A good eye for the harmony of colors and a steady hand, trained to follow outlines, is all that is necessary for this branch of work.

Lack of knowledge in the art of drawing has always been the great stumbling block to endeavor in this direction, but the beautiful artistic designs gotten up in perforated patterns does away with this obstacle and opens the wide field of decorative painting to the dealer in art supplies.

The dealer who can use the brush has at her command an infinite variety of ways in which left-cver scraps of silk, linen, ribion, etc., can be worked up into saleable novelties with the least possible outlay of time and money. The decorative painter need never be at a loss for fresh and attractive window display, and her show case will be filled with small novelties that find ready sale at good profit in their respective seasons. Classes in decorative painting are always a powerful factor in drawing trade, and stimulating interest in fancy work generally. Many ladies who would never think of embroidery are easily enthused on the subject of painting.

A word of warning may not come amiss at this point: Do not make the mistake of undertaking too ambitious flights in the realm of art butconfine your efforts to simple decorative painting, which will leave no room for criticism.

The instructions given in this chapter are sufficient to give the dealer or teacher full command of the best and easiest methods of doing the different styles of painting. In giving lessons the different methods of decorative work with the brush should be kept carefully distinct from eachother. Thus pupils in a class of water color painting on fabrics should be kept faithfully to that subject, and not allowed to talk about Paris tinting or oil painting on celluloid.

In the present chapter will be found illustrations of several beautiful novelties that will repay the dealer who will make them up and put them on exhibition in show case or window.

The amount of painting supplies to be carried in stock will, of course, depend entirely upon the extent and kind of trade each dealer has, but the following are staples and will be needed by all. Other novelties can be added from time to time, as the demand for this kind of work increases: 
Oil Paints in Tubes-A good assortment of Winsor \& Newton's oil colors should be carried in stock. Those colors given in the preceding lessons will be found practicable. It will not be found sound business policy to induce customers to purchase a large line of made colors, when three or four will answer as well, provided a little instruction in mixing them is given, and this should be given, for the less expense attached to any kind of decorative work, the more of that work will be done, and small profits and quick sales is the surest and best policy always, especially is this true in a trade that depends upon women for its best patronage.

Water Colors - The moist colors that come in little pans will give better satisfaction than those put up in cakes. The necessary colors for a varied line of decorative work are as follows; Antwerp blue, burnt umber, burnt sienna, Chinese white, chrome yellow, chrome orange, emerald green, gamboge, ivory black, yellow ochre, terra vert, Vandyke brown, venetian red, vermilion, brown madder, carmine lake, crimson lake, scarlet lake, purple lake, lemon yellow, pink madder and sepia.

FOR PARIS TINTS-Outfits consisting of the tints in bottles, with the diluting medium.

Lustra Paints-Brocades, powders and flitters, as given under the head of "Lustra Painting."

English enamels, gold paint in leaf and powder, gold size for mixing the gold powder, poppy oil, turpentine, should always be carried in stock, put up in convenient sized bottles. A good assortment of brushes, flat and round sables, from No. I to No. I2; a few badgers, palette knives, easels, palettes and brush cleaners should be included also.

If any interest is manifested in tapestry or fabric painting, then supplies for that work should be added, the colors and materials for which will be found under the head of "Tapestry Painting."

A small supply of ivorine, glassine, celluloid, parchment, bristol board, leatherette, eggshell board, chamois, ooze leather, glass and wood, should be kept constantly on hand, as nothing so much conduces to a falling off in interest on the worker's part as being obliged to wait while the dealer sends to some distant city for supplies with which to prosecute the work. A small assortment of china and porcelain placques, easel stands for small 


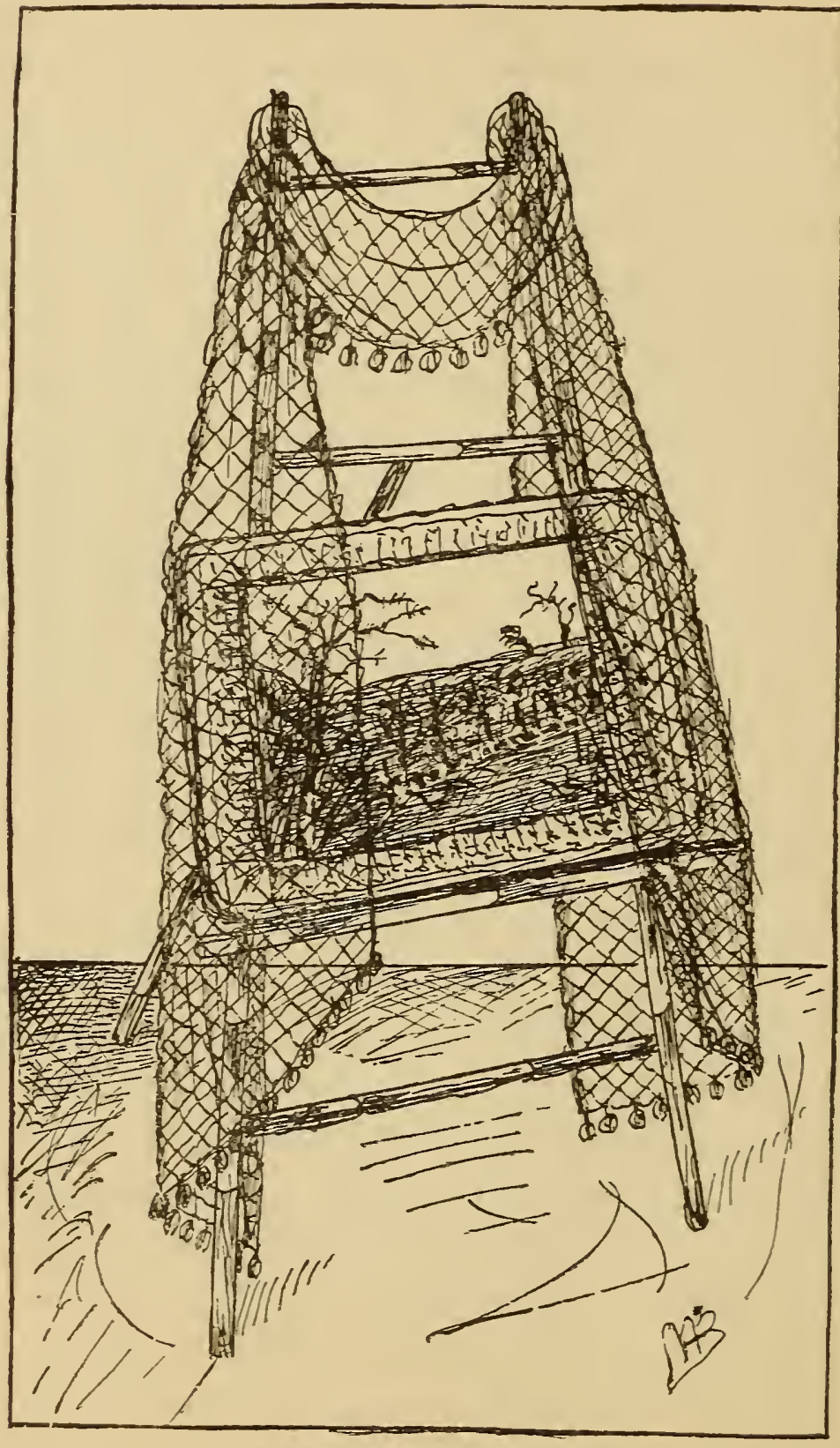

DRAPED EASEL. 
pictures, a few plush frames, etc., decorated, will make a most attractive display for little outlay of money. A very fair artist's supply or stock can be put in for from $\$ 75$ to $\$ 100$, and can be made to yield a profit of nearly, if not quite, 50 per cent, and this aside from money obtained from teaching, and profits accruing from the ability to work off out-of-date fabrics for needlework.

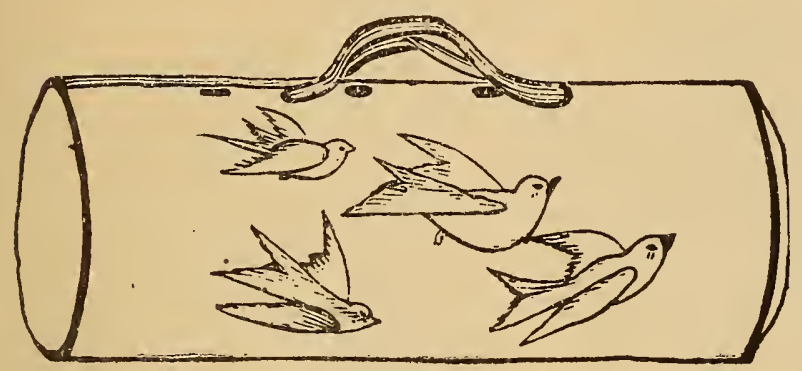

FIGURE 277.

In addition to the above enumerated articles it will be a profitable investment to add a choice selection of designs, perforated, especially suited for decorative painting. Designs for painting are somewhat more expensive than those for needlework, but the dealer receives more for stamping from them than from the ordinary designs. If the business is exclusively devoted to artist's supplies a more complete line of artists' materials must be carried in stock than where decorative needlework supplies are combined with it. Chapter XVII will supply all necessary instructions pertaining to that subject.

Samples of decorative painting are a great stimulus to trade, and should be such as to appeal to the average woman, because there are more average women than there are exceptional ones, and it is from the former class that the

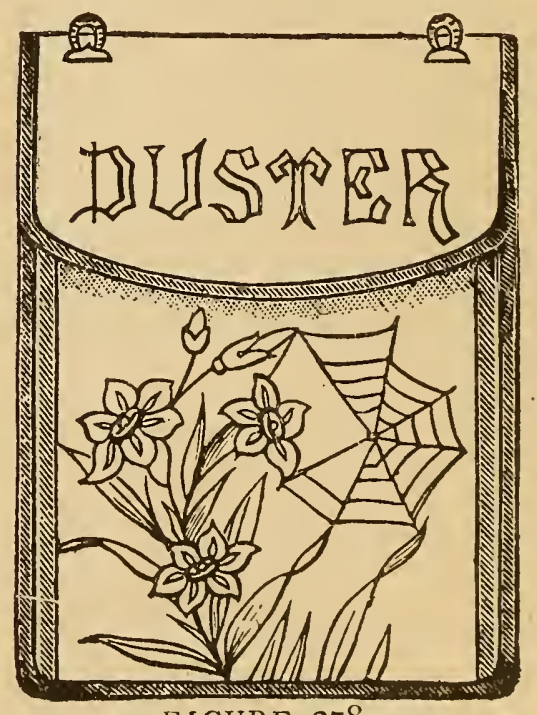
FIGURE 278. lealer receives the larger share of patronage. A panted plush-bordered table cover, a pair of painted pillow shams, a parasol cover delicately painted, a paper holder of cellulaid also painted will appeal to ten women where a beautifully painted landscape would not appeal to more than ten women out of a hundred, and this is not because of a vitiated taste in art matters so much as a feeling that the lower form is within the compass of thcir talents, while the greater work seems so far away and beyond their grasp.

A varicty in the painted samples and a frequent change is desirable. 
Tapestry painting is most attractive when well done, as also tinted articles which have been described on previous pages.

Flower and fruit painting the artistic grasp of most pretty bright bits of dechave a few studies of fruit

In leaving this subject ers that the subject of dec. by no means exhaustively introduced to your notice, that the industrious, intelherself proficient in those of and at the same time road leading to the higher words written shall but issue with the beautiful, repaid for her labor in this

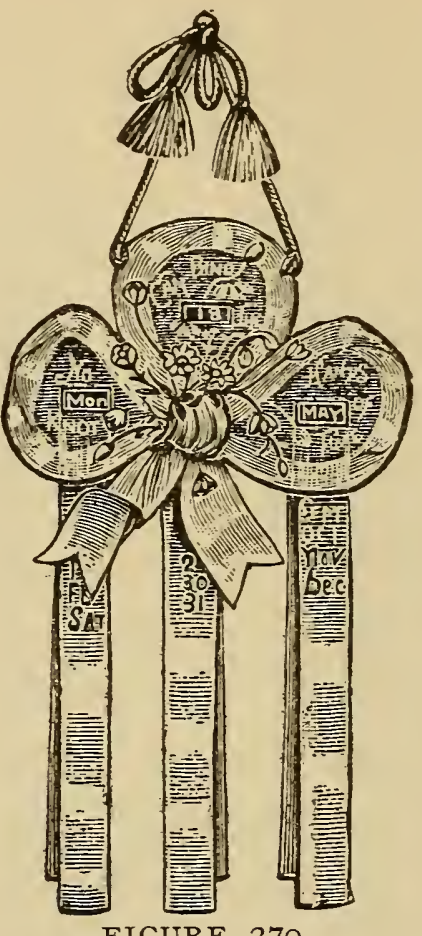

seem to be quite within women, and as they make orative work, it is well to and flowers.

we must remind our readorative painting has been treated, rather merely but in so practical a form ligent woman may make forms of painting treated make progress on the forms of art work. If the inspire one woman to try the writer will feel amply direction, not alone because of the added profit to the dealer, but because of a wider knowledye of Art, which will be disseminated through her advocacy of it.

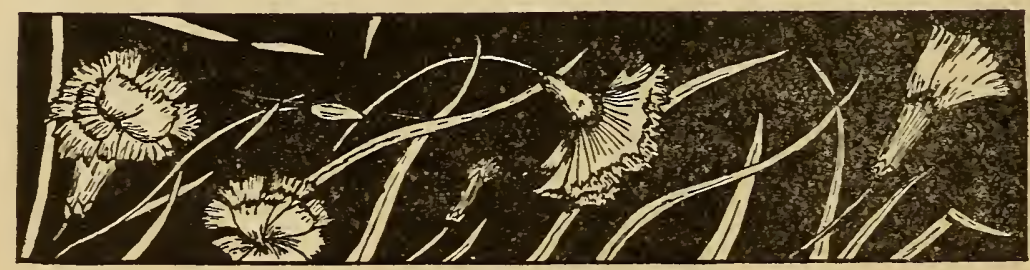




\section{CHAPTER XV.}

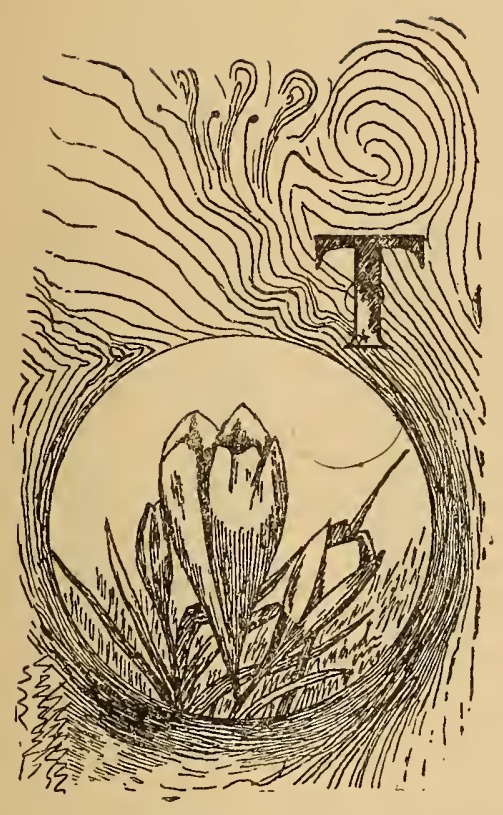

\section{LACE STITCHES AND DESIGNS.}

HE FASCINATING work of lace-making has become quite the rage during the past few years, and as knowledge of the work becomes more general its beauty and great value will induce many ladies to undertake its manufacture who could never be induced to investigate the mysteries of other forms of decorative work. In the following pages we have attempted to give detailed instructions, profusely illustrated, for making the stitches most commonly employed in this work. The designs of patterns given for handkerchiefs, centers, borders, ends, etc., may be transferred to the tracing cloth, enlarged to any size desired, by means of the pantagraph, and form a very valuable feature of this chapter, as the perforated designs are quite expensive. The enlargement of the designs in this chapter should be four times the size here given, each way, for the best effect. There has been but little printed on the subject of lace making in this country. Mme. Guyon, a French lady, brought out a few years ago, a small pamphlet on the subject, and from its pages nearly all the magazine articles on "Lace Making" have bccn taken, in a form so incomplete and mutilated as to be of little practicable value.

The instructions necessary for a beginner are simple, and must be exactly followed. Deftness of manipulation can be acquired only by paticnt practice, and this practice must be gone through with, as exactness of detail is absolutely necessary for the securing of beautiful results. 

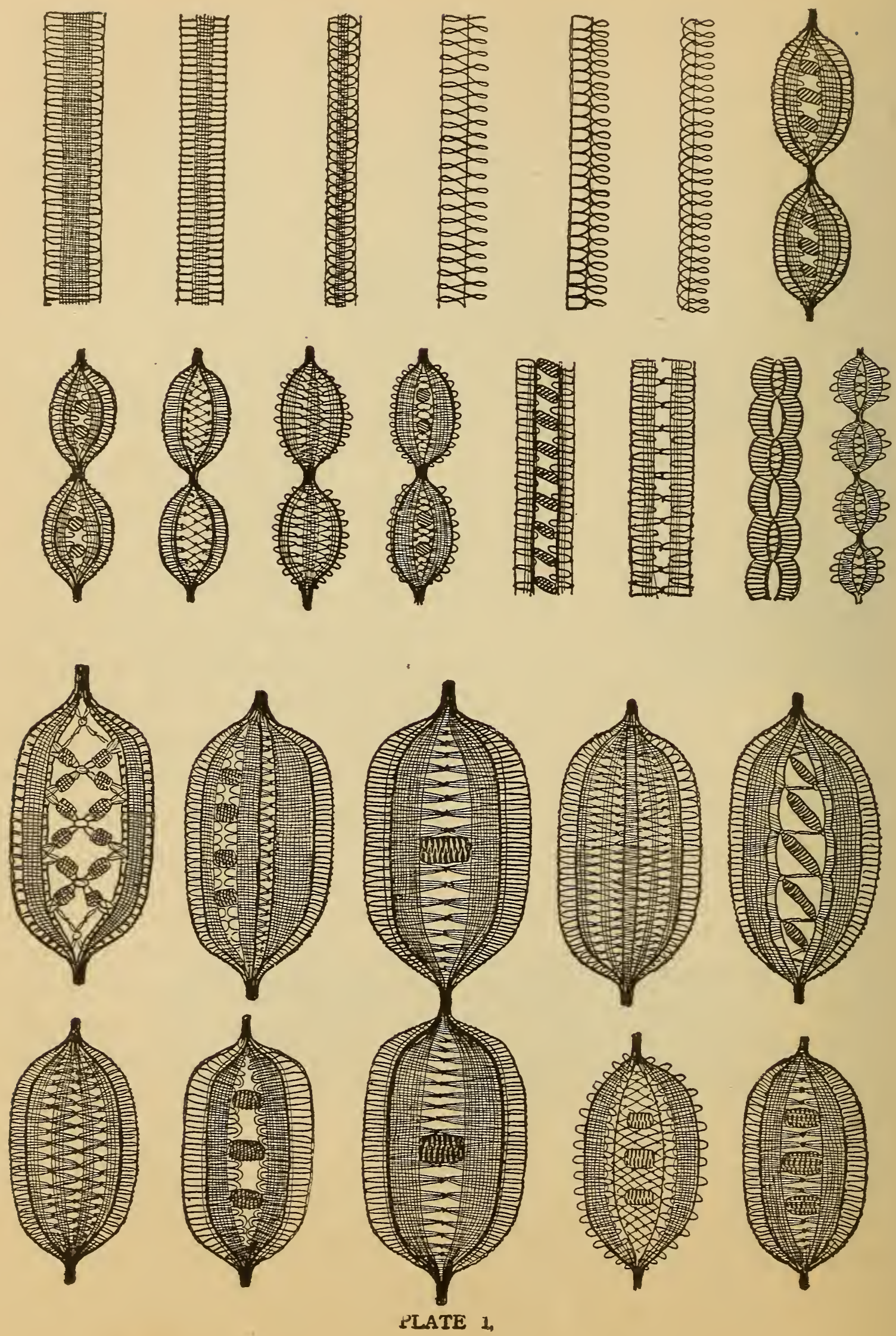
Point lace and Honiton lace are made on a foundation design of braid which is sewed to the outlines of patterns, and the spaces are then filled in with the various lace stitches. In brief this is all of mystery of lace making there is about the work, yet how much is covered by these brief words.

Materials for lace making are simple, and consist of tracing cloth, a foundation of soft oil cloth, such as is used for table and shelf covers, or leather will answer, but is more expensive, braids of various styles and sizes, lace threads and designs.

Figures I, 2, 3, shows several of the braids used in this work. As will be seen they are of varying sizes and styles and are designated by numbers. Each manufacturer using a different number. The numbers given below can be readily placed from the accompanying illustrations.

No. $4722,420,402,347,652,145,697,4886,52,505,503,388,287$, III, I $28,564,155,319,510,416,344,384,367,368,414,365$. The prices for which the best English braid can be secured ranges from 7 cents to 50 cents per piece of six yards.

The thread-for working the lace stitches should be of the best linen, and is sold in balls. The threads are of different sizes, indicated by numbers, 300 to I 500 . No. 300 indicating the coarsest thread used and No. I 500 the finest. These threads should not cost more than 5 cents per ball, as that is the price usually charged by the large establishments carrying a line of lace-making materials.

Tracing cloth is made 18 inches wide and may be purchased by the yard, the usual price being 25 cents.

Designs: special designs for point and honiton lace may be had from the stamping pattern houses. They are made on good strong tracing cloth, which is all ready for tacking to the oil cloth foundation previously mentioned. The designs are the most expensive part of the outfit. A handkerchief pattern costing 50 cents, a design for infants cap 35 cents, a collar 35 cents, a fischu 75 cents, a deep border $\$ 1.00$, and so on. Therefore it will be seen that where good lace designs can be copied from books or magazines a considerable saving will be effected.

\section{Laying the Braid.}

Outlining the design with the lace braid is the most particular part of the work as upon the neatness with which it is done depends the entire 


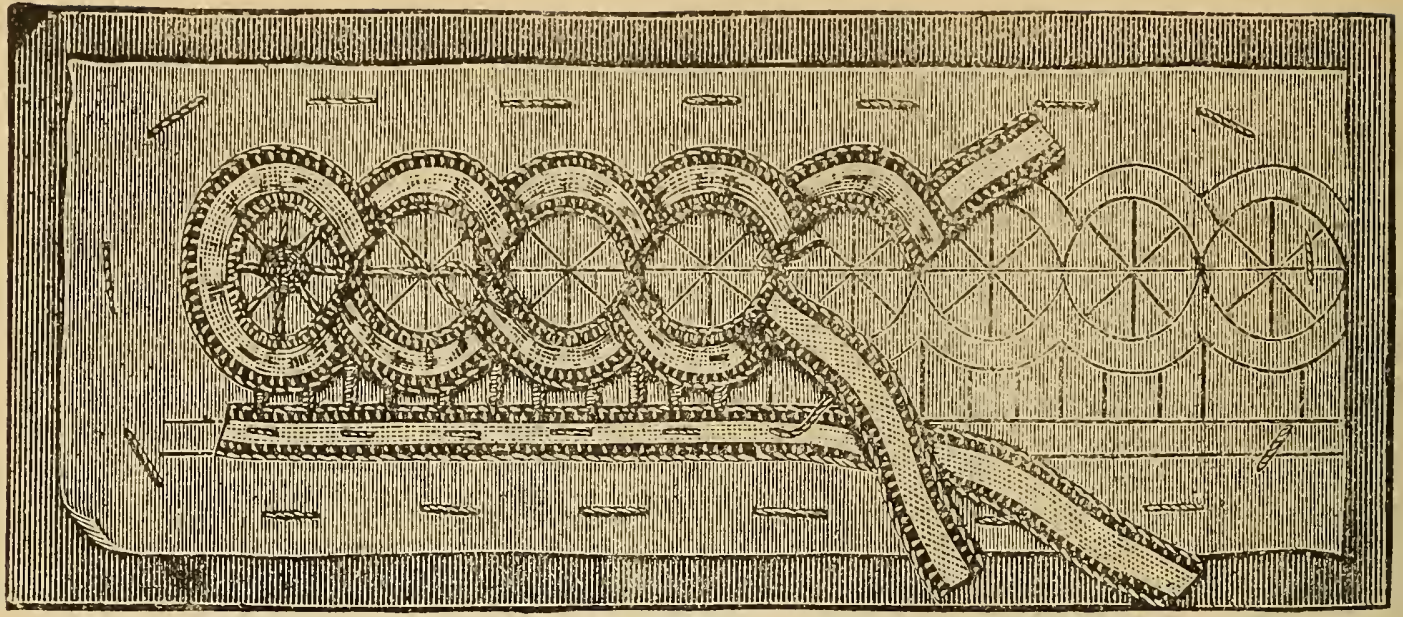

1

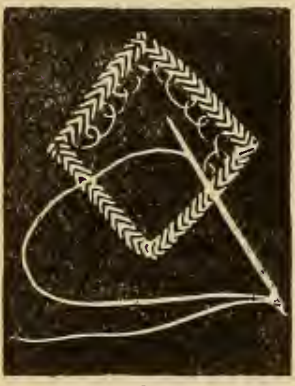

2

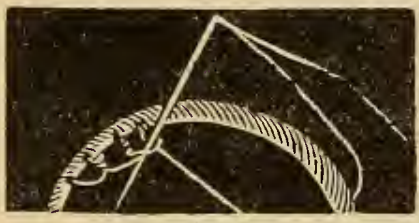

5

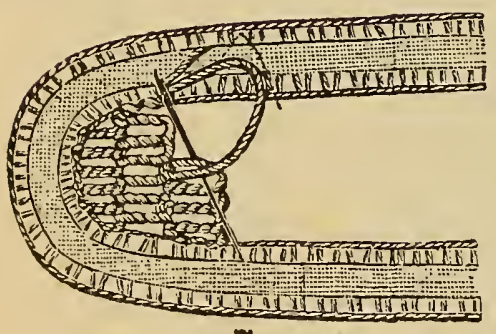

7

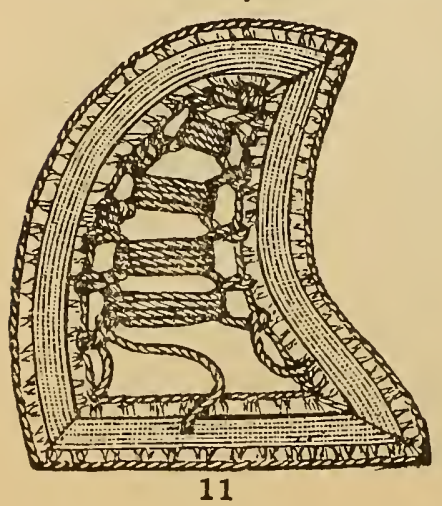

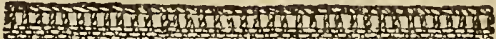

112 (1) (1) 3

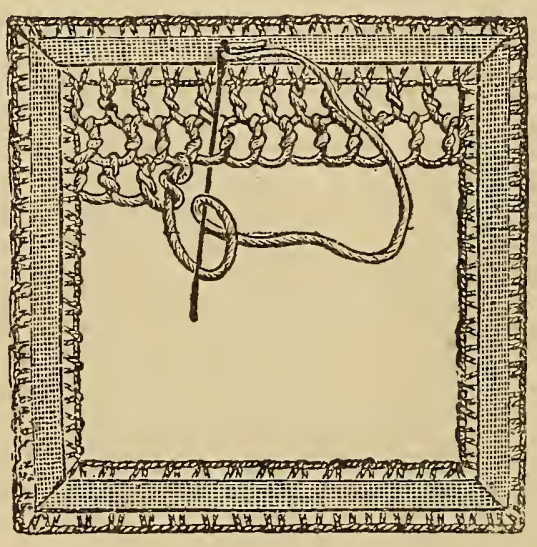

6

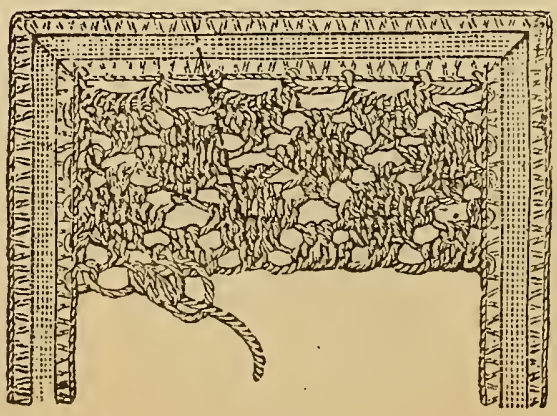

10

PLATE 2 .

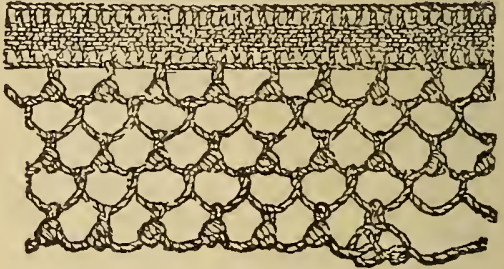

4

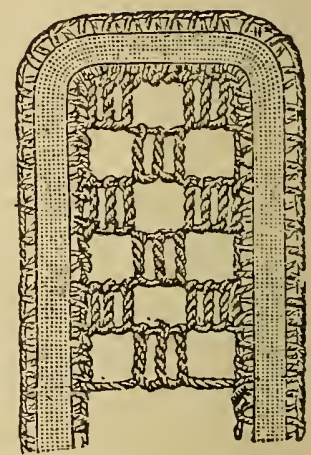

8

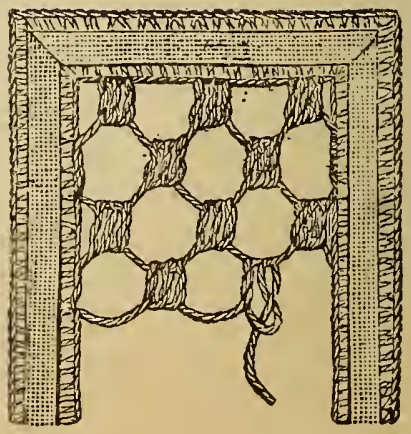

9 
effect of the finished piece. When possible, piecing the braid should be avoided; sometimes it is absolutely necessary, and great care must be exercised in this operation. To join: stitch the braid together firmly, open

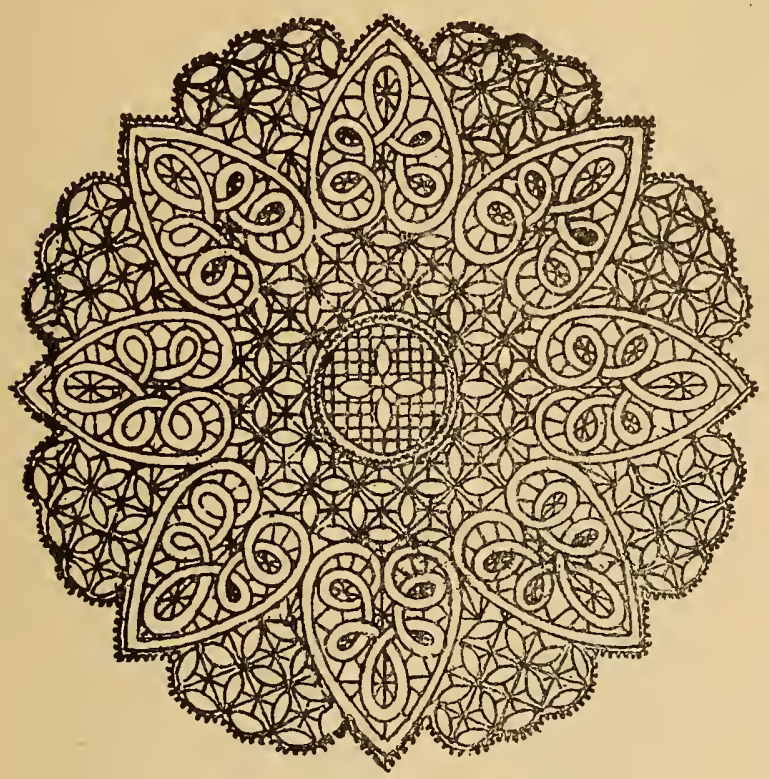

HANDKERCHIEF CENTER. the seam and turn back the edge, stitch the edge down separately. In passing the needle from one part of the braid to another run along the center of the braid, keeping the stitches as nearly invisible as possible. In commencing the laying of braid make a few stitches, leaving the end of the thread on the wrong side and cutting it off afterward. In fastening the finishing make a tight buttonhole stitch, run on two or threestitches, take the needle to the back of the work and cut off.

Time, care and patience must be given to this work.

Fig. I, plate 2, illustrates the method of outlining design with braid. The illustration shows the pattern stamped on tracing cloth, which, in turn, is tacked to a foundation of oil cloth. Commence the design by running on a straight line of braid across the bottom of the pattern, (some teachers instruct pupils to run the braid on in the center, and this method is shown in the figure, but the safer rule is to fasten each edge with the tiniest imaginable over-stitches.) Commence to lay the braid from left to right. For the circles in the design take another piece of braid and lay it in position by running stitches through the center being careful to keep the braid as smooth and

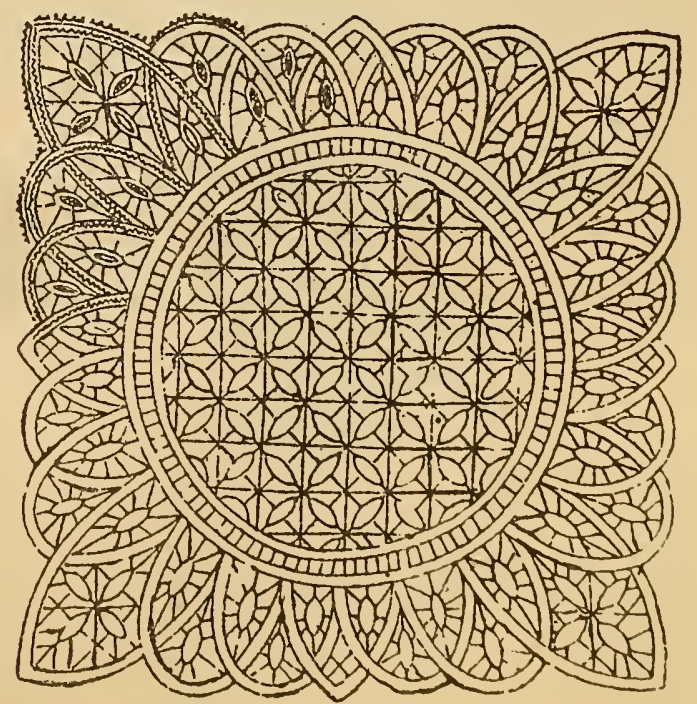

HANDKERCHIEF SQUARE. even as possible. There will be no trouble with the outer edge, but the inner edge will present some difficulty to a beginner. 


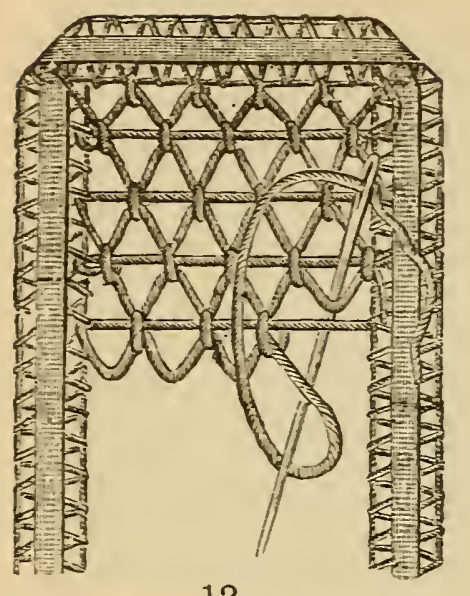

12

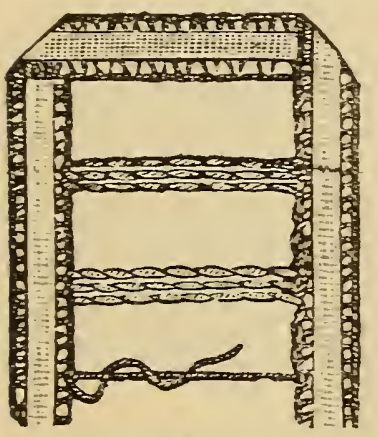

15
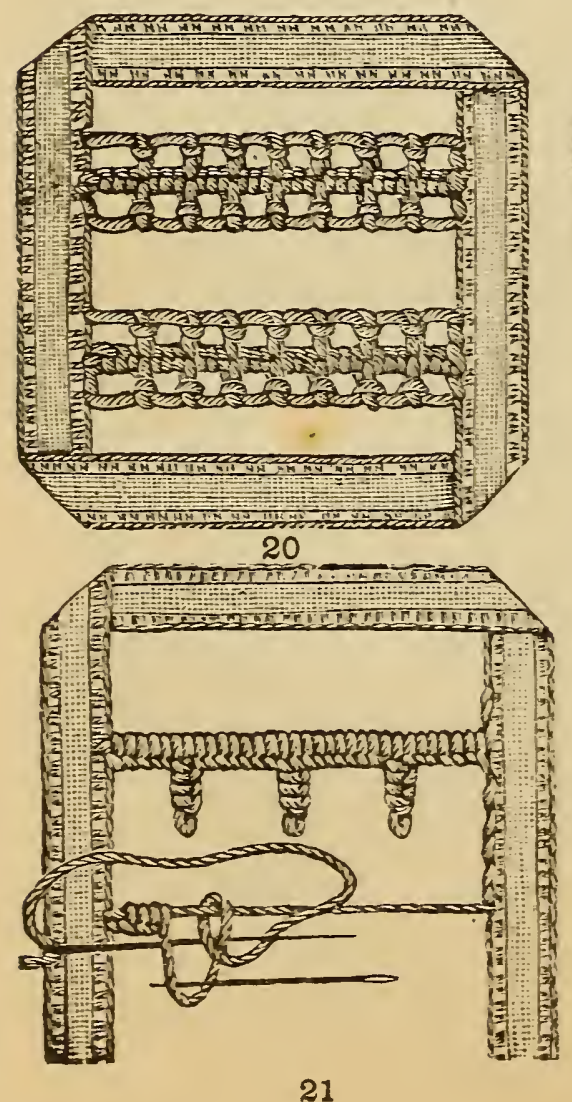
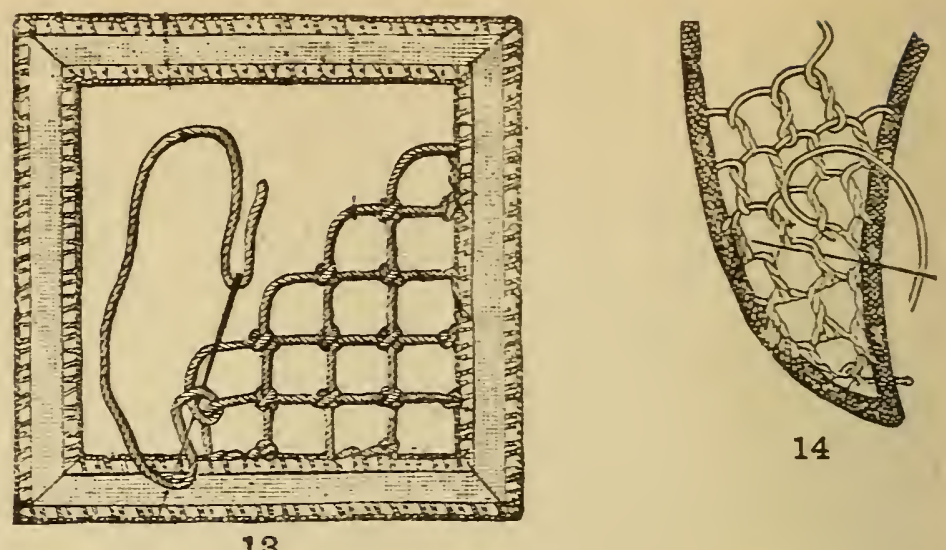

14

13

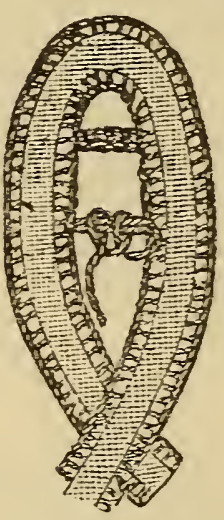

17

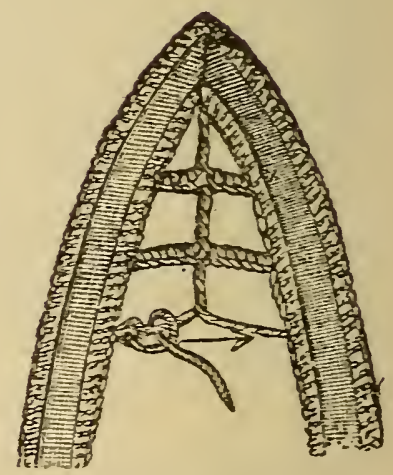

18
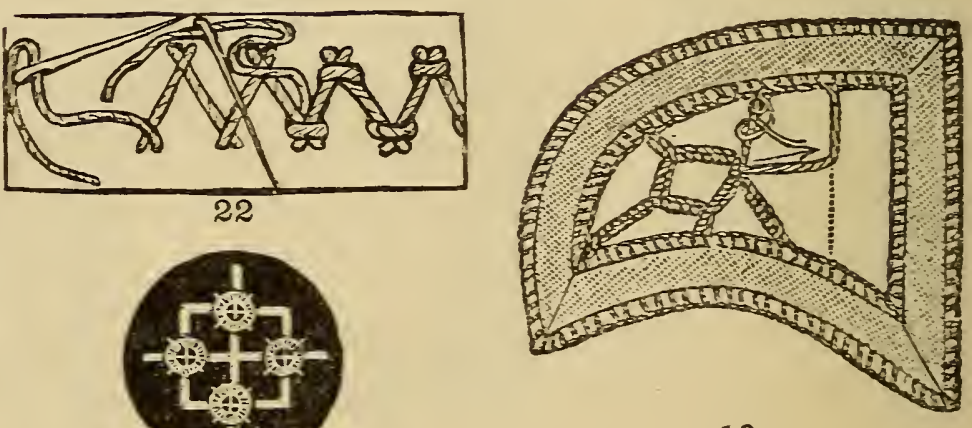

19

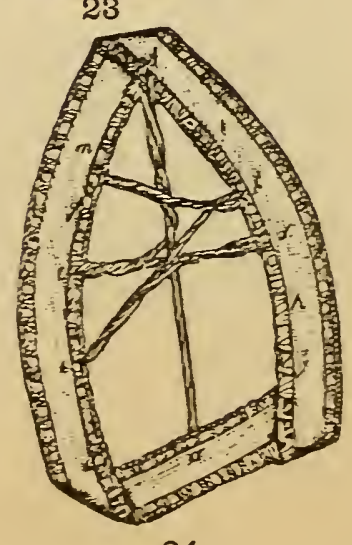

24

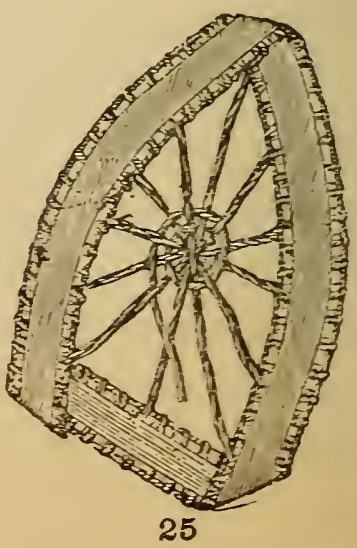

PLATE 3. 
In order to make the inner edge lie even and smooth thread a No. 9 needle with No. 500 lace thread, fasten the needle at one point and take a few running stitches along the edge of the braid, as a fine gathering, and draw the thread up slightly, this will force the braid into position. The same method must be followed by laying braid on a curve or half circle and two or three fastening off stitches should be taken. When each curve or circle is finished the gathering thread mìst remain on the work.

\section{LACE STITCHES.}

Having illustrated the method for laying the foundation of a design, the next step in the work is naturally the stitches to be used for filling-in. No large or important piece of lace work should be attempted until the stitches have been thoroughly mastered by persistent practice on small samples.

The stitches used in point lace are divided into four sections, as follows: stitches proper, or points; finishing edges, connecting bars, wheels, rosettes. As there are over Ioo stitches used in making old point lace it will be seen that much study and many copies of old point must be gone through with to master all the beautiful varieties of stitches, and in a work of this character all that can be successfully attempted is to instruct the novice in the simpler methods, illustrating the principal stitches, and the proper application of same. Sufficient knowledge may be obtained from this chapter to enable any lady to manufacture beautiful specimens of old point and houiton lace.

The finer the braid used in the work the more value will the finished piece possess. And here comes in a wice point for discrimination, viz.: where to use the coarser braids? It will be readily seen that lace for personal use needs to be of finer texture than that employed for purely decorative purposes, hence for handkerchiefs, fichus, collars, lace edgings, etc., the finer braids and most delicate and lacy stitches should be employed. For bed spreads, shams, dressing case sets, etc., the heavier and coarser braids will be found more effective as well as more appropriate. The finest threads should be used with the finer braids always, while for ornamental articles thread as coarse as No. 300 may be employed.

The first stitch to be practiced in lace making is the Brussels point. This stitch is the foundation of nearly all the many lace stitches, and is 


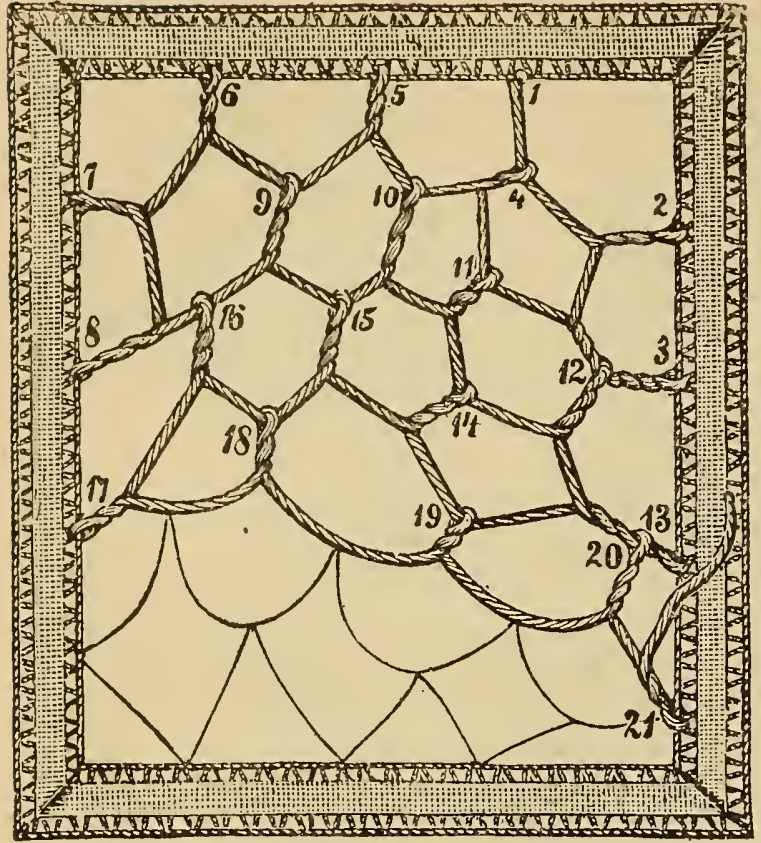

26-Raleigh Bars

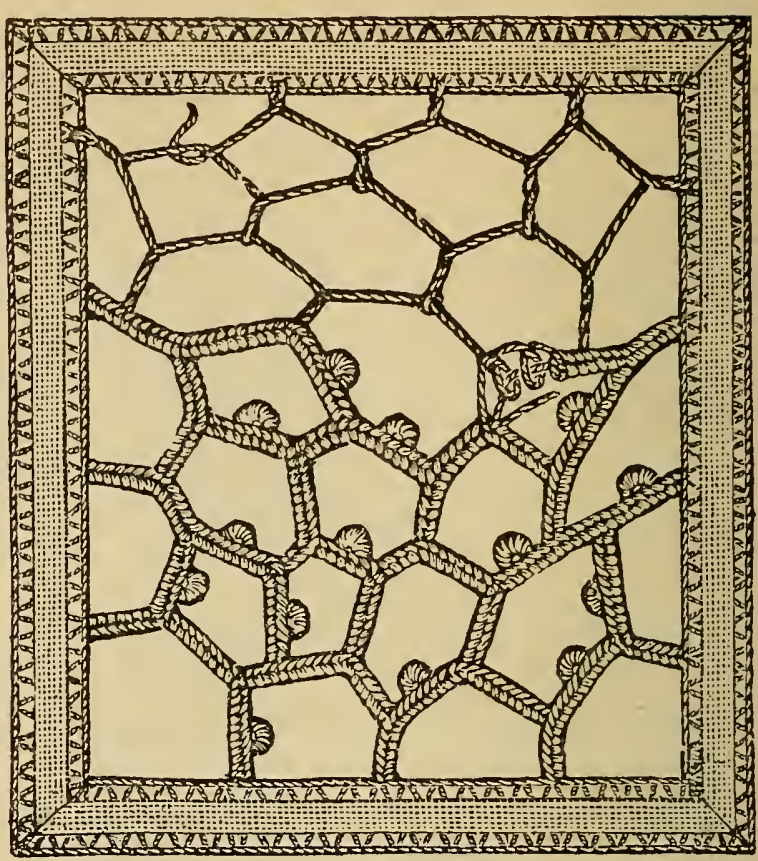

27-Raleigh Bars.
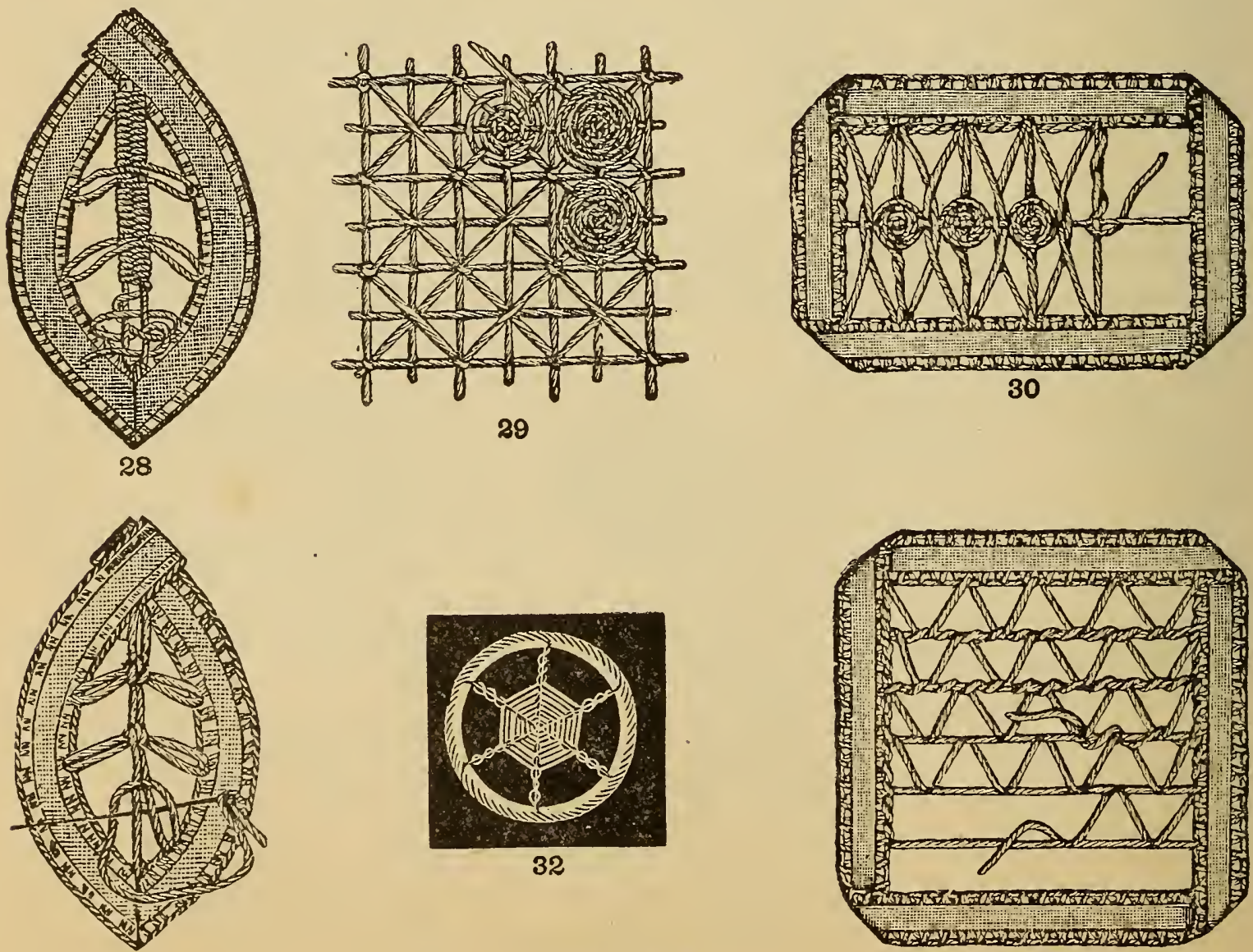

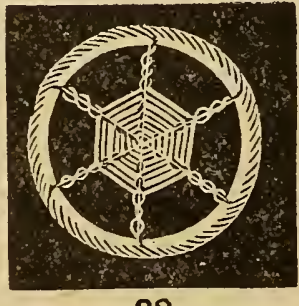

32

PLATE 4

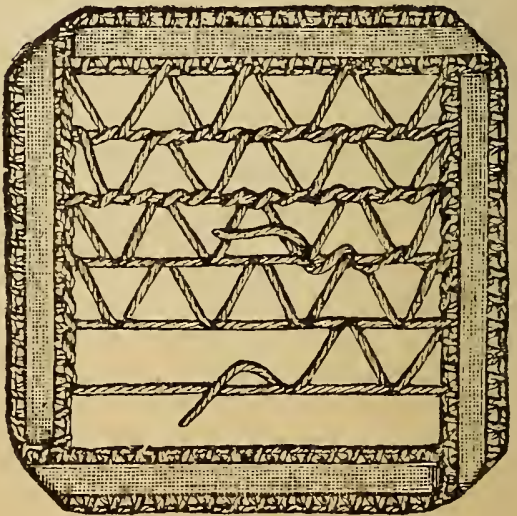

33 
worked as an edging or in rows backwards and forwards to fill spaces, or as a ground work. Brussels point consists of a simple buttonhole stitch worked loosely and evenly. Fig. 2, plate 2, shows method of working clearly. Fig. 3, plate 2, shows the stitch worked in rows; Fig. 4, plate 2, the Venice point. This stitch is worked from left to right as is also the Brussels point. Work one loose buttonhole stitch, and in this again work four buttonhole stitches tightly drawn up: next work another loose buttonhole stitch, and four more buttonhole stitches in it as described for the first stitch. This operation must be repeated to the end of the row and the thread fastened off. This will be found to make a most useful filling stitch.

Figure 5, plate 2, illustrates Little Venice point, which is adapted for filling small spaces. It is worked in the same manner as the Venice point, the only difference bain? that one tight buttonhole stitch only is worked into each loose one.

Spanish point is worked from left to right as follows: insert the needle in the edge of the braid, keeping the thread to the right, bring out the necdle inside the loop, (see figure 6, plate 2.) The needle must pass from the back of the loop through it. Pass the needle under the stitch and bring it out in front, by this means twisting the thread twice. This operation gives the stitch the desired cord-like appearance. The thread must be fastened to the edge of the braid at the end of each row. Return to the opposite edge without breaking the thread by sewing back, inserting the needle once in every open stitch.

Figure 7, plate 2, shows Close Spanish point which is worked in exactly the same manner as the regular Spanish point, but so close are the stitches worked that the needle can but just pass through in the next row. Fasten this stitch also at the end of each row and sew back to opposite row. Begin the work at the left and proceed to the right.

Figure 8, plate 2, illustrates still another variety of the Spanish point stitches. It is called Treble Spanish point and is worked in the same manner as the two preceding stitches. The arrangement forming the distinctive features; begin at the left, work three close stitches, one open, three close to the end of the row; fasten off and run back to the left edge. In the second row begin with one open, follow with three close, then one open to the end of the row, fasten off and sew back. Repeat as niany rows as 
are necessary to fill the desired space being careful to alternate the beginning of each row as indicated above. Great care must be observed to see that the close and open stitches follow each other in regular order. Various patterns may be formed with this stitch, as stars and diamonds.

Grecian Point is illustrated in figure 9, plate 2. This stitch is worked from left to right, working backwards and forwards. To begin, make one loose Brussels point and follow with three close Spanish point stitches, then one Brussels point stitch and repeat, alternating the stitches to the end of the row. Work back in the same manner, only commence the second row with the three close Spanish points instead of the loose Brussel point. This is the first occasion, so far given, where two stitches are combined to form a pattern, but these combinations are largely used in all patterns, and lend variety to the work. We shall have more illustrations of combinations further on.

Valenciennes stitch, figure IO, plate 2 , is simple when understood though at first it may appear a little difficult. To work: ist row, commence at the left hand and make six Brussels point stitches at equal distances, every alternate stitch must be larger. Second row: upon the first large stitch work nine close buttonhole stitches, follow with one short Brussels point stitch under the one above; repeat nine close stitches and so on to the end of the row, (right to left.) Third row, work five close buttonhole stitches in the nine of the previous row, follow with one short Brussels point, two close buttonhole stitches in the Brussels point, one short Brussels point, five close buttonhole stitches, one short Brussels point, two close buttonhole stitches, one short Brussels point, five close, one short, two close, one short, repeat. Continue the rows in the order given until the desired space is filled.

Point d'Alencon is a lace stitch used to fill up narrow spaces where lightness is necessary to a good effect. This stitch is worked over and under in alternate stitches, as in hemstitching.

Figure 12, plate 3, shows the Turkish stitch. This is an easy stitch and equally effective for large or small spaces. The thread should be varied in size according to the size of the space to be filled, even in the same portions of a design. To work first row: work a loop bringing the thread from right to left, pass the needle through the twist, and through the loop, draw 
up tight and repeat. Second row: one straight thread from right to left. Third row: work in same manner as described for first row, using the straight thread in place of the braid, Pass the needle through the loop of previous row.

Point de Reprise is a stitch much used and will be found illustrated

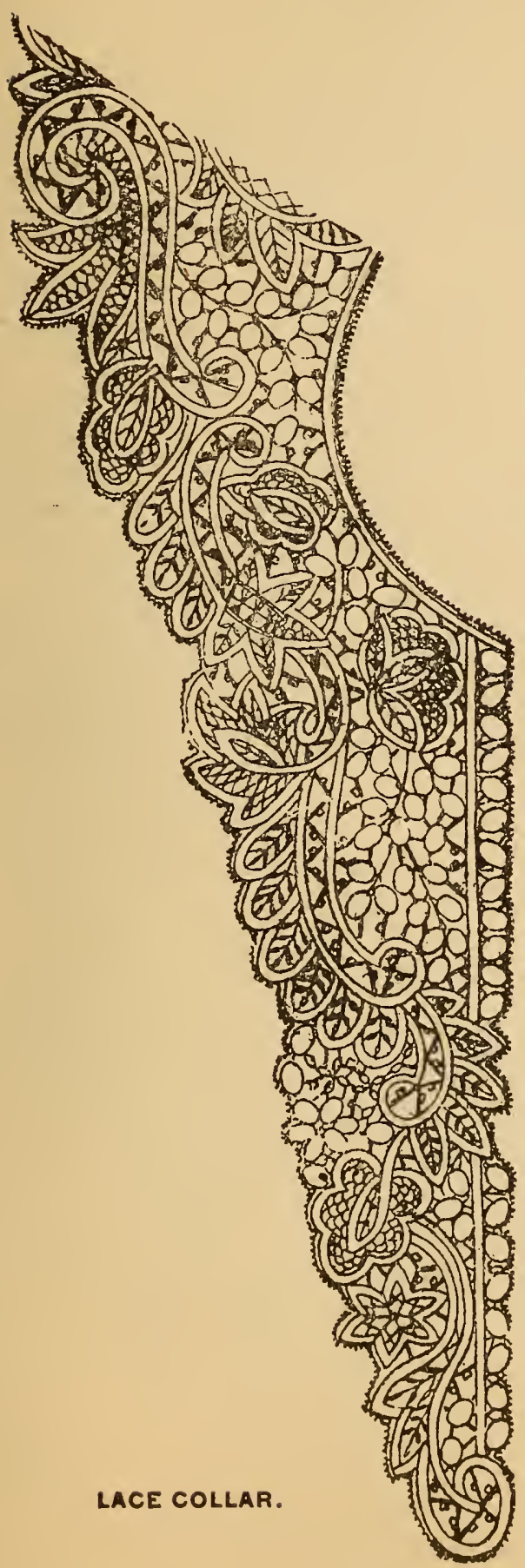
and described in the chapter on "Applications of stitches."

Net Groundwork Stitch is effective for ground work where Brussels net is not imitated. To work: commence in the corner or crosswise of the space to be filled, work a loose Brussels point, fasten it to the braid, passing twice through the braid as shown in figure 2, plate 3. Work in rows backwards and forwards as follows: one Brussels point before making the next stitch, pass the needle under the knot, over the thread and again under it, see illustration. This stitch fills up space rapidly, figure 13 , plate 3 , thus shows the stitch applied as a filling or ground work over which is worked a few point de reprise stitches.

Point de Tulle. This pretty stitch is used as a ground work for very fine work. It is made by working in rows backwards and forwards, using open Spanish point stitch. See figure 14, plate 3. When this part of the work is completed it is worked over a second time, as follows: insert the needle under one twisted bar, bring it out and insert again at * and bring out again at the dot. This makes a close double twist which when done with fine thread is very effective.

Mechlin lace stitch, see figure 23, plate 3 . This is one of the prettiest stitches used in lace work and is also one of the 
most difficult. Work a number of diagonal bars in buttonhole stitch, on a single thread, in one direction. Then in the opposite side begin in the same manner and work five or six stitches past the spot where the two lines cross each other. Pass the thread around the cross twice, under and over the thread, to form a circle, work in buttonhole stitch half of one quarter, make a dot by putting a fine pin in the loop instead of drawing the thread tight, and in the loop held open by the pin, work three buttonhole stitches; take the pin out and continue as before. The dot is the difficult point in the work and it will be better for a beginner to omit this until the plain wheel can be worked smoothly and evenly: Mechlin wheels are effective worked in rows upon horizontal and parallel lines of thread.

WHEELS, ROSETTES, "BARS, ETC.

We come now to the introduction of combinations in our work, and will commence by introducing to the worker the Sorrento wheel. The method of working this stitch is clearly shown in figure 24 , plate 4 . The thread is put in the space to be filled at the points indicated by letters. Commence at $a$ and fasten the thread, carry it to $b$, twist back upon the thread to the center of it and fasten at $c$; carry to center again by winding around or twisting

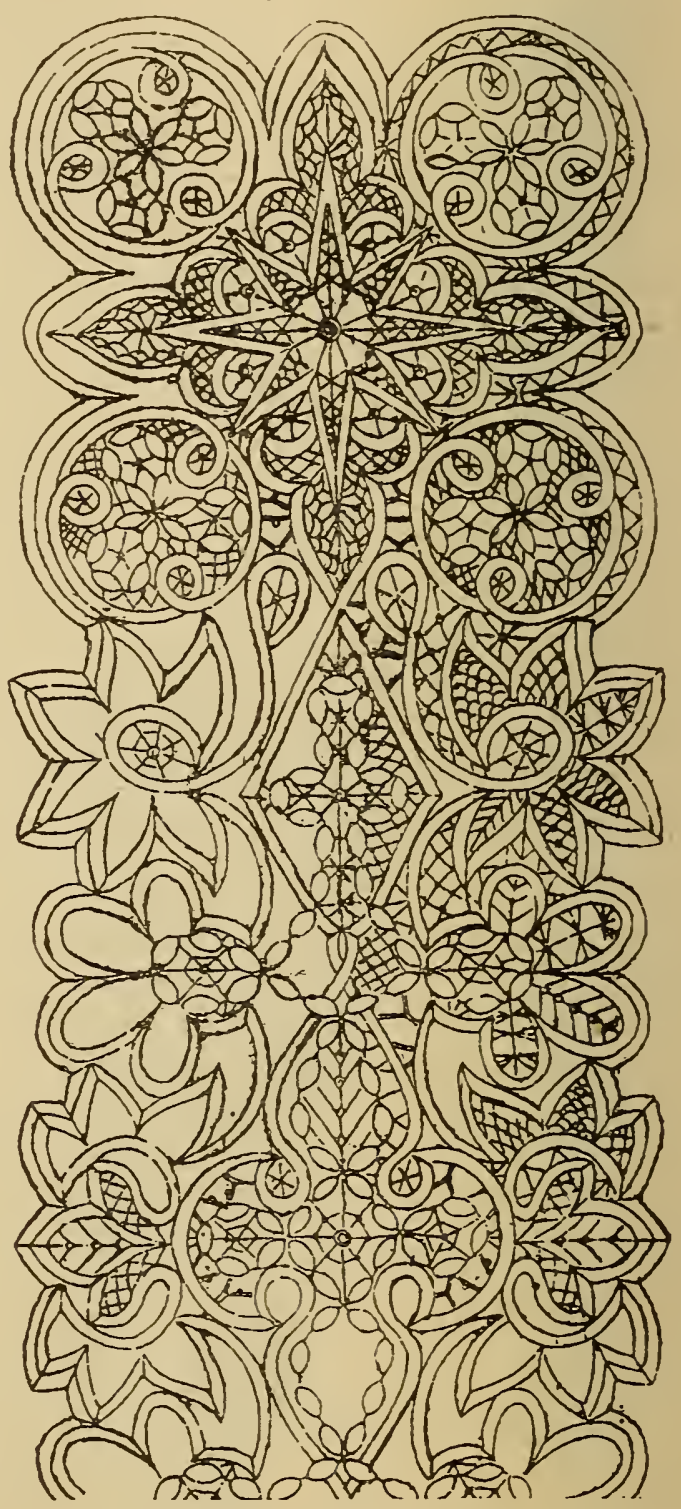

LACE INSERTION. around itself, and so on until all the lines are in, as marked in figure 24 . Figure 25 shows method of forming wheel, which is very simple; the thread is worked over and under the crossed threads, repeating the operation until a wheel the desired size has been obtained; see plate 4 for figures 24 and 25 . 
A very pretty wheel called spider web is shown in the chapter on "Application of Stitches."

The various stitches used to connect the different parts of point lace work are called bars, and upon the suitability of the bar to the special place it is made to fill depends the beauty of the work.

The Sorrento bar is one of the simplest used, and consists of a single thread carried from right to left and fastened in the edge of the braid; the return row is made by simply twisting back to the starting point on tive thread just carried across. These bars are usually made in groups of three placed close together, with an equal distance between each group. Figures I 5 and 16, plate 3, show two applications of the Sorrento bar.

Point d'Alencon bars are useful, but as they have been referred to before, we will pass on to the Venetian bar, which, while so simple as to hardly need'description yet has an important place in lace making. Figure I7 shows this bar applied to a small space. It consists of two straight threads worked over in reverse directions with buttonhole stitch. Figure I 8 shows the bar applied to the veining of a leaf which is worked upon sorrento bars. See plate 3 .

- Figure 19 shows Venetian bars worked to form squares, triangles, etc., in buttonhole stitch upon a straight thread. The arrow in the illustratiori points to the direction for working the next.

Venetian bars edged. Figure, 20, plate 3, illustrates this method of working. Begin at the right and carry a thread straight across to the opposite side; fasten off in the edge of the braid with a tight buttonhole stitch. Upon this thread work a succession of tight buttonhole stitches. In every third stitch work a Venice point stitch.

We come now to the dotted lace bars, and a very important feature of bar is this dot.

Dotted Venice Point: Stretch the thread across the space to be filled from right to left. Upon this thread work five tight Brussels point stitches. In the fifth stitch insert a pin to hold it, open and loose; pass the needle under the loose stitch and over the thread as shown in figure 2I. In this loop work three tight Brussels point stitches; work five more tight Brussels points on the thread and make another dot; repeat to end of row. 
322 DAINTY WORK FOR PLEASURE AND PROFIT.

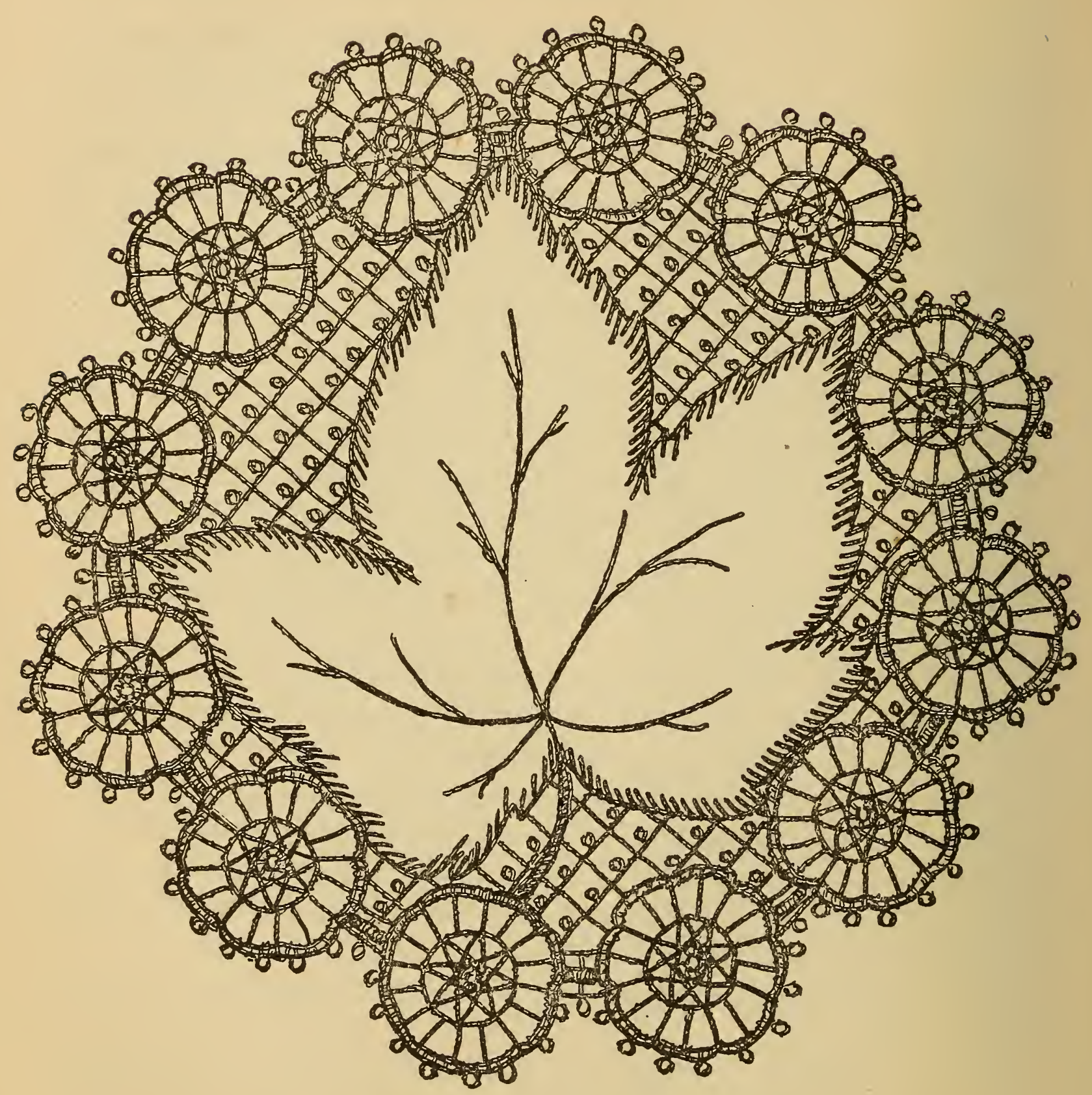

MAPLE LEAF PLATE DOYLEY. 
Raleigh Bars are worked over a foundation of net work, made of coarse thread, twisted in places so as to more easily fall into the desired form. Figure 25 shows a figured diagram for placing threads; and by following the numbering from $I$ to $2 \mathrm{I}$ in the diagram, a square space may be easily filled. Portions of the arrangement can also be applied to any shaped space. Upon the groundwork make tight Brussels point stitches, and upon these the dot must be worked. To work the dot either of three methods may be followed. First method: work five tight Brussels points, one louse Brussels point; pass the needle under the loop and over the thread, as shown in the dotted Venice bars; draw up, leaving a small open loop as in tatting. Work five tight Brussel points and repeat.

Second method of making dots: Proceed as described for first method, but instead of continuing the tight Brussels point work two or three tight buttonhole stitches in the loop thus formed.

Third method of making dots or picots: work four tight Brussels points and one loose; through the loose stitch pass the needle point, wind the thread three times around the point, press the thumb tightly on this, and draw the needle and thread through the twists. This method very closely imitates the real Spanish lace.

Figures 27 and 30 represent two very beautiful fancy filling stitches, which are so simple that they can readily be copied from the illustrations. Figure 27 is called Point d'Anvers, and figure 30 Greek point.

Many of the lace filling stitches are sisceptible of other uses than making lace, for instance the most beautiful drawn work is that wherein occur many lace stitches, also in conventional embroidery they are especially useful for filling large surfaces.

The lace insertion on page 320 will require 3 yards of Honiton, and 39 yards of point lace braid. This material will make a piece one yard long.

For the collar shown on page 319 there will be required braid as follows: Honiton, $23 / 4$ yards: point, $173 / 8$ yards; purling, 41/2 yards. 
324 DAINTY WORK FOR PLEASURE AND PROFIT.

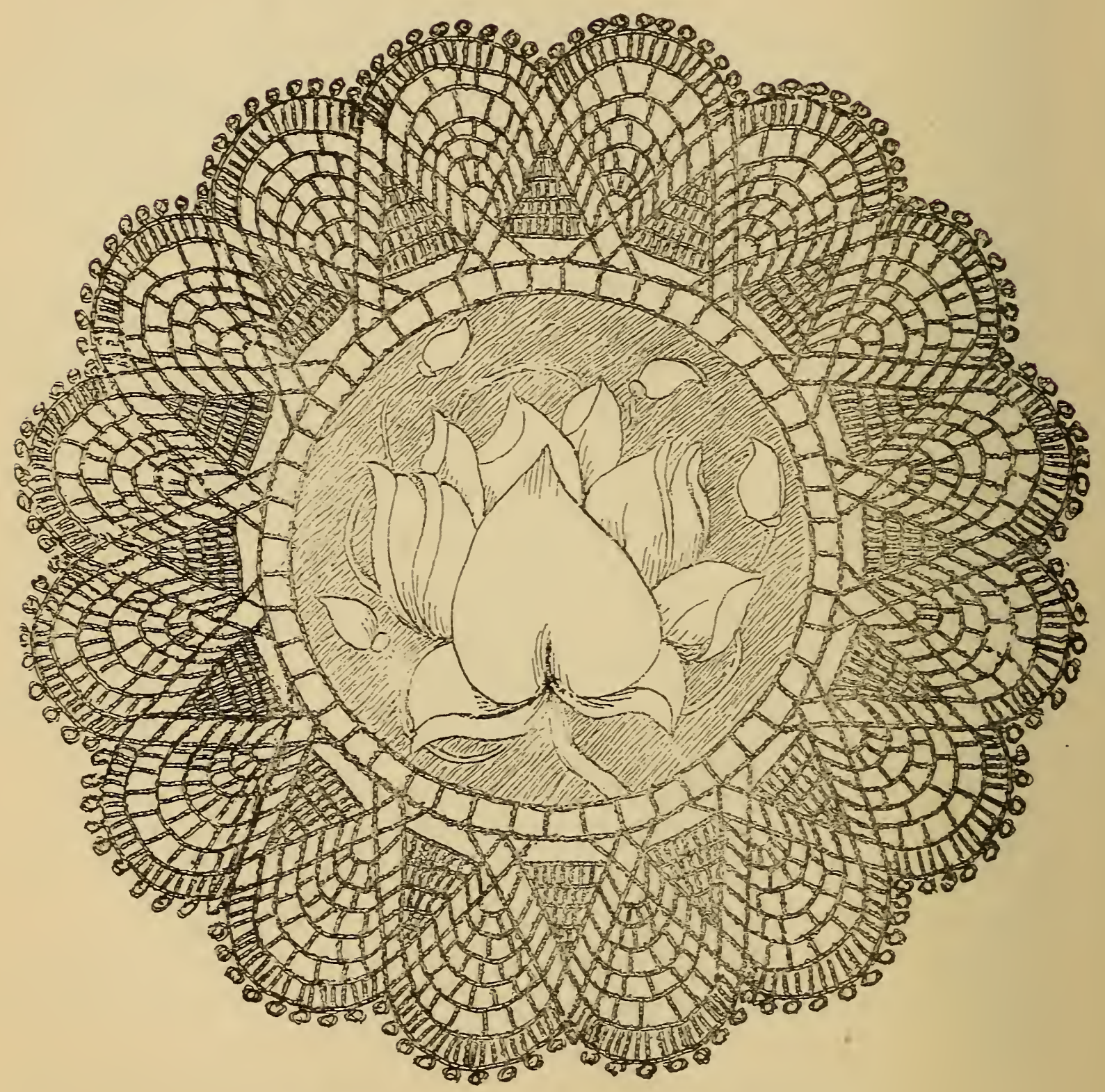

MÁGNOLIA PLATE DOYLEY. 


\section{CHAPTER XVI.}

KNITTING AND CROCHETING.

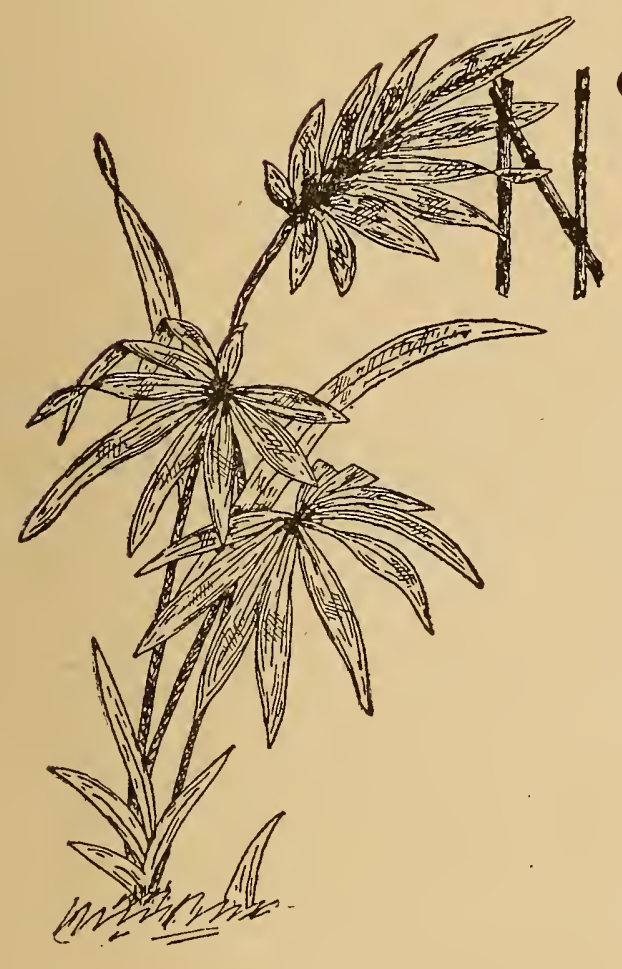

OTHER form of "Dainty Work" is so conducive to sociability and a general feeling of bonhomme as a piece of knitting or crochet work. It occupies the fingers, yet leaves the mind free for "idle converse the while." Aside from every other consideration this form of fancy work offers a means for decorative efforts in different directions that would otherwise be beyond the ability of limited resources to compass. One has first to try what can be accomplished with a ball of linen or silk crochet thread and a crochet needle to have a proper respect for the day of "little things." Women, even of the most limited financial means, have leisure hours, or it may be minutes, which can be utilized in making trimmings for the garments of the wee mites who demand so much of a loving mother's thought and care, or árticles of more absolute necessity, as petticoats, sacques, stockings, dresses, etc., can be fashioned from a few cents' worth of wool. In this day of cheap Hamburg embroideries it is a relief to see underwear decorated with fine linen thread lace, either knitted or crocheted, and the possession of a variety of such hand-trimmed garments betokens a refined taste in the possessor. Aside from articles of actual use, there are many others of mere ornament that add much to the home-like comfort of one's rooms, such as toilet sets, scrap bags, bracket covers, table covers, etc., that can be made by means of knitting or crocheting, and these can be man- 
ufactured so cheaply, and in the odd minutes that would not otherwise be employed, that the cost is not appreciated.

In the chapter devoted to this subject, no attempt at originality has been made, but much time and labor have been expended in securing the prettiest and most useful, as well as most ornamental articles that could be found in two continents. The many friends of the writer in the United States-from Maine to California, and from British. Columbia to the Gulf Coast-have been laid under requisition, and most generously have they yielded their treasures to her insatiable demands for "more," and she wishes nere to thank the ladies who so kindly came to her assistance, sending samples for illustrating, and instructions for making so many of the articles here given. To friends in England, Scotland and France she is also indebted for like favors.

The idea in preparing this chapter has been to give such instruction as could be most generally adapted to the greatest number of purposes. No effort at classification has been made; the different articles have been erited and grouped just as they were received, and form a very good index of what women are doing in this line all over the country.

For the benefit of tinose among the readers who do not understand knitting and crochet work, we append here the abbreviations used in giving the instruction for making the different articles, together with their meaning.

Some ladies have a deep-seated conviction that they cannot learn anything from printed instructions. This is nonsense. If the instructions are given clearly and concisely, there will be no difficulty in following them. Staring at the cold type with an I-never-can-do-it expression will not do it, certainly; the only way to reduce printed instructions to knowable quantitie:s is to prove them. Take a crochet needle and a piece of coarse thread and. proceed to follow the instructions as given below, beginning at the first one and proceeding to the last. Never give up trying until the correct result has been obtained. You will then be prepared to use the terms whenever you meet with them in the succeeding articles, illustrated in this chapter.

Having disposed of the terms for crochet work, proceed to solve the difficulties of those given inr knitting. Once actually at work, practically trying to follow instructions, you will be surprised to see how easily difficulties that seemed insurmountable melt into nothingness. 


\section{TERMS USED IN CROCHETING.}

Ch.-Chain: a straight series of loops, each drawn with the hook through the preceding one.

Sl.-Slip stitch: put hook through the work, thread over the hook, draw it through the stitch on the hook.

Sc.-Single crochet: having a stitch on the hook, put the hook through the work, thread over the hook, draw it through the work, thread over, draw it through the two stitches on the hook.

Dc.-Double crochet: having a stitch on the hook, put thread over the hook, then put hook through the work, thread over and draw it through two stitches, thread over, draw it through the two remaining stitches.

Tr.-Treble crochet: same as double crochet, except that the thread is thrown twice round the hook before inserting the hook through the work. The stitches are worked off two at a time, as in double.

L. t. c.--Long treble crochet: twine the thread three times round the hook, work as in treble, bringing the thread through two loops four times.

Picot: made by working three chains, and one single crochet in first stitch of the chain.

Cl.ch. st.-Close chain stitch: insert the hook in the loop of preceding row, and draw thread through it and the loop on the needle at the same time.

Half dc.- - Half douhle crochet: like double crochet, only pass the cotton through the three loops on the needle, instead of passing it through two loops twice.

\section{EXPLANATION OF ABBREVIATIONS AND TERMS USED IN KNIT'TING.}

K.-Knit plain.

N.--Narrow, knit two stitches together.

P.-Purl or seam.

PN.-Purl or seam two stitches together.

S. and B.--Slip and bind, slip one stitch, knit the next, and pass the slipped stitch over.

O.- Thread thrown over as if about to purl.

S.- Slip the stitch off without knitting.

Round.-When the work is done with four needles in a tubular web, this expression describes one circuit of the web from the first stitch of the first needle to the last stitch of the third needle inclusive.

Row.-This term is made use of only when the work is done on two needles in a flat web.

Repeat.-This word, following a description of round or row, means that the same work is to be done again, not only once, but throughout the round or row. In other places the word implies a repetition of all rows or rounds preceding it in that rule.

Cast off.-This is done by knitting two stitches, passing the first one over the second, and repeating as required. 


\section{CROCHETED DRESS FOR CHILD.}

The top and skirt are crocheted separately, yet each is made of stripes of crochet, afterwards joined together. For the skirt I 5 such stripes sloped off in the width are required. Figure 282 shows the widest part of each

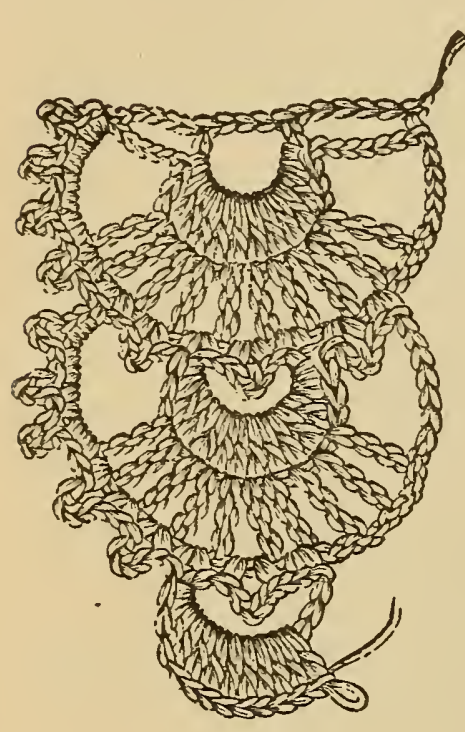

FIG. 280 . stripe at the lower edge of the skirt, and figure 280 the upper narrowest part where begun. The cro chet is made in rows, backwards and forwards, the firm center on the right side being made directly with the scollop points, while the left side can only be crocheted in one with the inner chain scallops (see figure 280); the other scallops follow later on, as shown in figure 282 , on page 329 , but also here each picot scallop is finished separately. Only a few directions for the stitches will be required, as the designs very clearly show the way the work is made. $9 \mathrm{ch}$. are always to be worked for the rings, into each of which is crocheted I sc., I half high dc., Io dc., I half high dc. and I sc. Then follow io long stitches each separated by I ch. Each scallop on figure 282 is crocheted over, later on, with sc. and picots requires $8 \mathrm{ch}$. The smallest part of the scallop consists of 3 rows of scallops ( 3 rows of scallops); then follow scallops of 4 rows and lastly I scallop of 5 hole rows. The stripes are joined by carching together the picots in the hollows of the scallops; this is done by adding a ring crocheted separately, as seen in figure 28I, at the upper edge of the top. The ring consists of $18 \mathrm{ch}$, round which are worked 24 sc., interrupted after $3 \mathrm{sc}$. by a picot

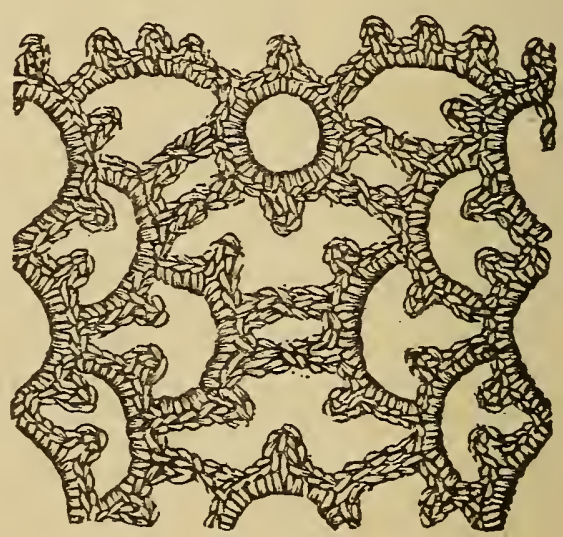

FIG. 28 I. of $4 \mathrm{ch}$. The upper edge of the skirt is secured by I row of $\mathrm{ch}$. and dc., this drawing the width into about $253 / 4$ inches, and I open dc. row (I dc., I ch.) For the back on the left side a stripe 6 scallops long is required, and each scallop here, as everywhere else, on the top worked of 3 hole rows. These six scallops give a length of about $9 \frac{1}{2}$ inches. On the second stripe I scallop more is worked for half the shoulder strap (the stripes are joined 
as on the skirt), to this follows a stripe of only 5 scallops to form the armnole, on which the scallops are narrower above, so that the top becomes somewhat tighter. A stripe of 8 points is now made to complete the shoulder; the two shoulder stripes are then sewn together. The middle of the

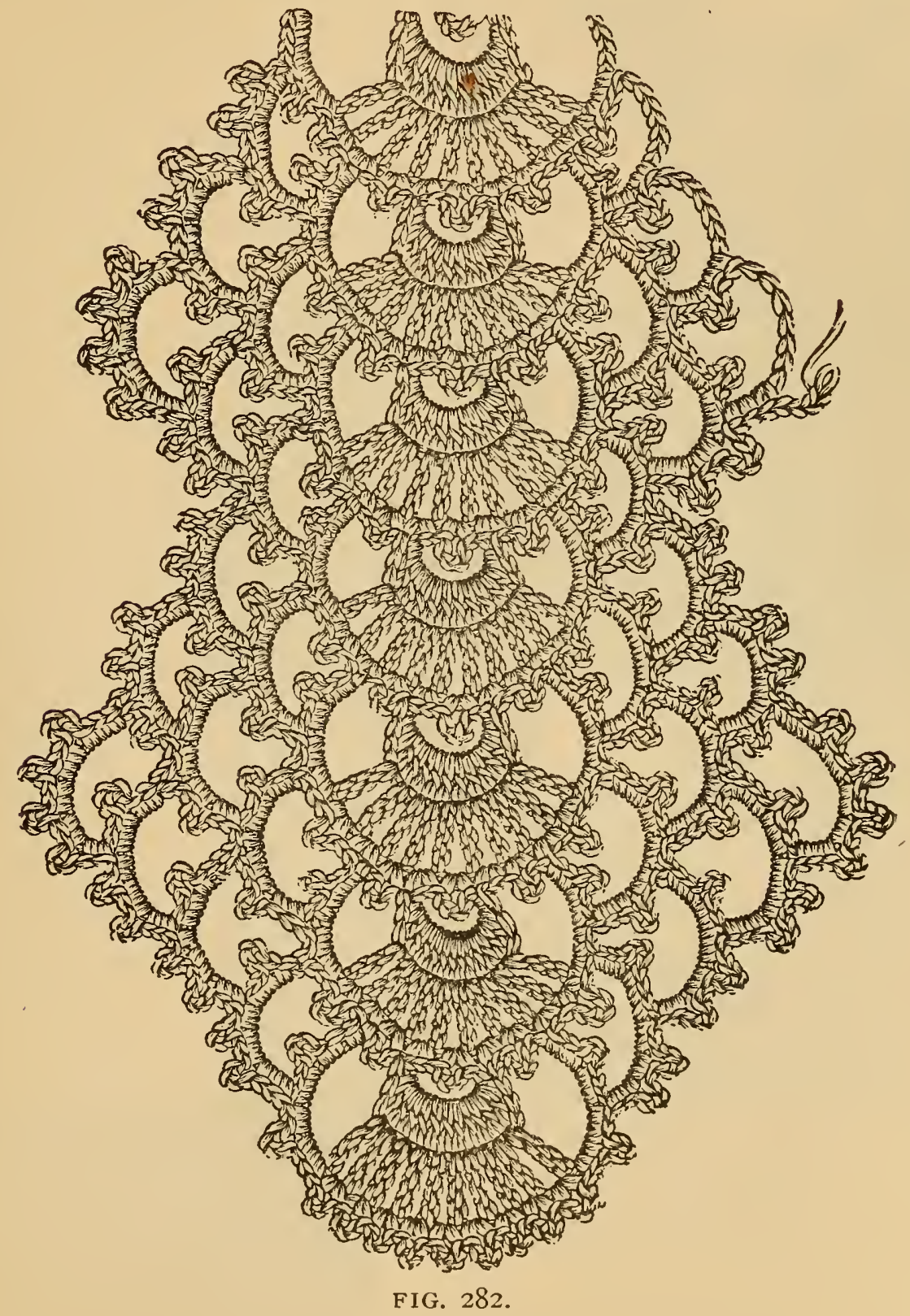

top, which is now finished in an opposite direction, is reached after a stripe again of 6 scallops. The two middle stripes are only to be joined to the shoulder straps at the tops of the points; the rings are left out here, as ribbon is afterwards drawn through the openings, An extra stripe is made 
on the right back for the button fastening, but this is finished at the back edge of the top only with the inner row of scallops, as seen in figure 280 .

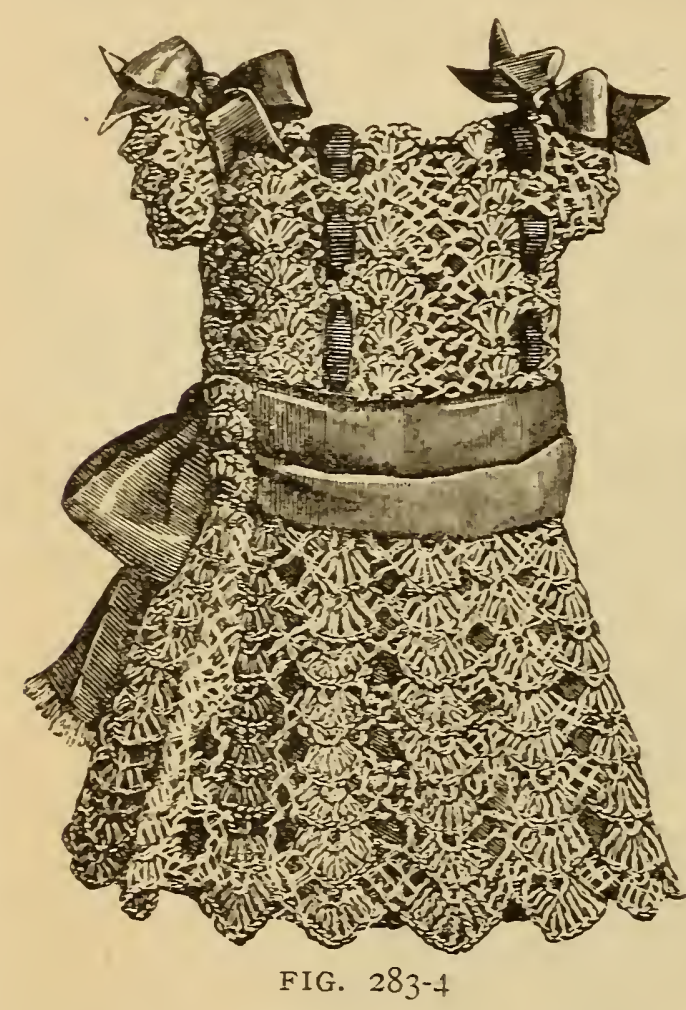

The above mentioned stripe is put below a little over the first one on the left back. before sewing the top and shirt together. The sleeves require each 7 to 9 pointed scallops, the picot edges of these being made on one side only. The upper edge of the top is finished with small picot rings and scallops like those seen in figure 281. For the tabs holding the sash at the sides, a stripe 4 or $43 / 4$ inchies long is to be crocheted with only one scallop on each side, as seen in figure 280 . Figure 284 shows the dress completed.

\section{PURSE.}

Materials: $x / 2$ ounce lnitting silk and a fine steel hook. Make $36 \mathrm{ch}$., and I row of dc. $2 \mathrm{~d}$ row; I ch., $2 \mathrm{dc}$. in back edge of the first and last dc. of the foundation. a dc. in each stitch between.

$3 \mathrm{~d}$ row, I ch. and a dc., to end of row; 4 th row to $32 \mathrm{~d}$ rows, like second and third alternately; $33 \mathrm{~d}$ row, like third; 34 th row, dc. across row, missing the first and last stitches; 35 th to 65 th rows, like thirty-third and thirtyfourth rows. This completes the center part. For each of the side pieces make $28 \mathrm{ch}$.

Ist row, dc. turn the work, I ch., dc. in back edge of foundation stitches; 4 dc. in last stitch of foundation, $27 \mathrm{dc}$.

3rd row, turn the work, I ch., then dc. to end of row, widening in the center of the work as required.

4th to 12 th rows, like the preceding. join the pieces together on the wrong side with dc.

These purses can be made of any color, garnet and brown being the most popular. They are also extremel! pretty when covered with glittering steel beads, one being placed on every stitch. 
A novel idea is to carry one of these little purses worked in silk exactly matching the dress, and as so many delicate shades are produced in silk; this can very easily be done. A monogram in initial letters worked on one side in beads, is also a very pretty device, adding much to the beauty of the purse.

\section{BABY'S SACQUE.}

Materials: 4 ounces knitting silk, and three yards satin ribbon No. 4 . The yoke of the sacque is in reversible spider stitch.

Ist row, take 5
ch., to turn, make
loops on the first 5
ch., draw the silk
through 6 loops, then
through $2 *$ Take up
3 loops on side of spider and 2 loops through ch., draw the sik through 6 loops, then through 2. * Repeat.

To Widen.-Take up five loops with the one on needle, then repeat, making two spiders over one, being careful to finish

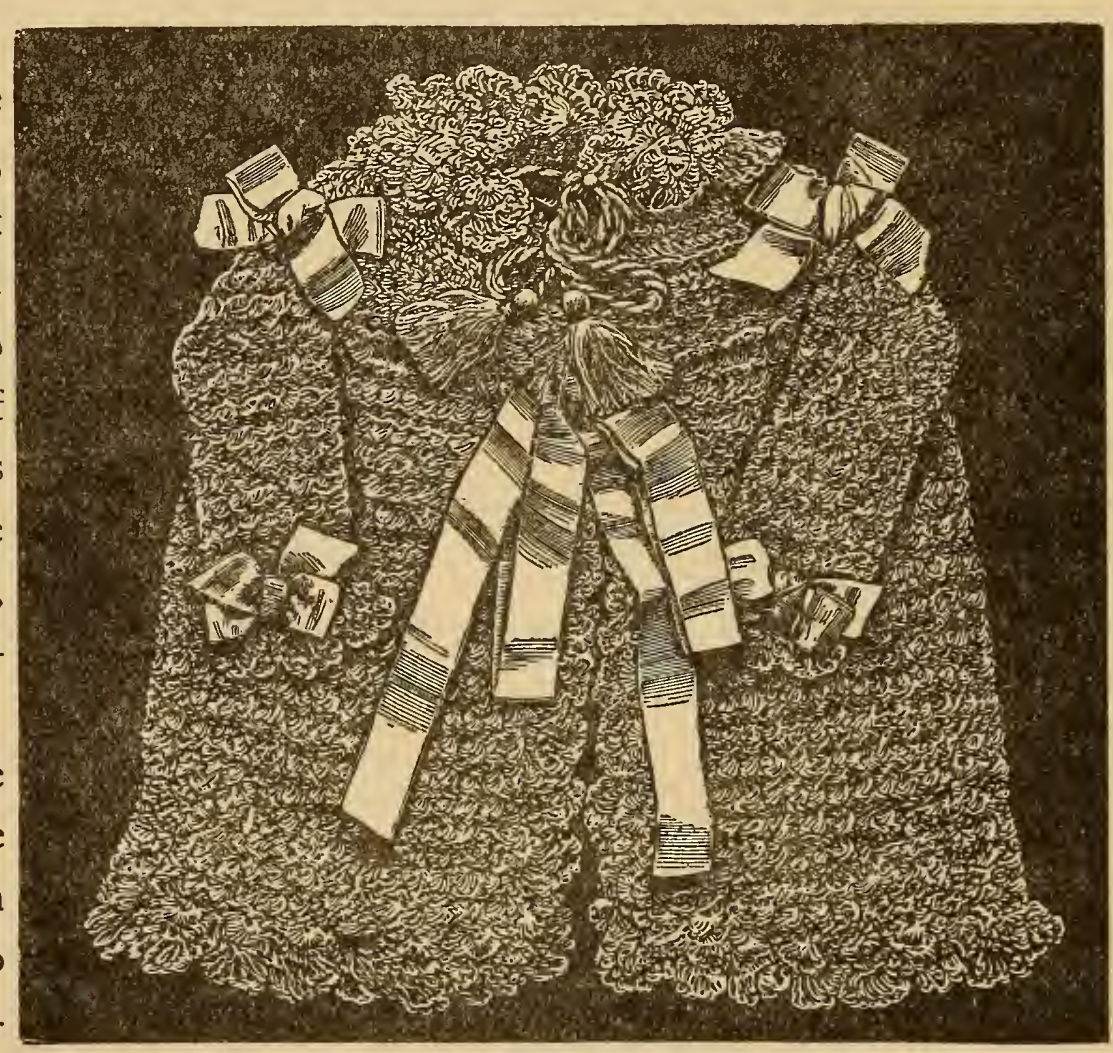

FIG. 286. the two spiders at the end of the one spider of last row.

$2 \mathrm{~d}$ row, I 5 plain, widen twice, I plain between; 3d row, I 5 plain, widen twice, 2 plain between; 4 th row, 5 plain, widen twice, 3 plain between.

$5_{\text {th }}$ row, 15 plain, widen twice, 4 plain between; 6th row, 15 plain, widen twice, 5 plain between; 7 th row, I 5 plain, widen twice, 6 plain between.

Work the front and back separately, leave sixteen spiders for front: seven spiders for arm-holes, the rest for back. Make six rows on each. All the yoke is worked back and forth, making a reversible spider stitch. 
Skirt of Sacque.- This is worked crazy stitch; on the first row join the back and fronts together at the side. Ist row, repeat first of baby's hood, on page 338 , making a crazy stitch pattern over every spider, which produces the fullness required for the skirt. Make 38 rows as above.

Sleeve.-Make I7 crazy-stitch patterns round arm-hole; being at the joining of back and front, turn the work at the end of each row and workbackward, which will produce the raised appearance of the stitch. Narrow the ninth row in the center of the side of sleeve by taking the top of two shells together and working as if they were one. Make twenty-four rows alike, except the last one, where you put 2 tr. instead of 3 for spider. 25th row.- 2 dc. into every spider. * Repeat for 4 rows. Make 5 shells round sleeve.

For Shells._.* I dc., miss 2 into 3, Io tr. into same, miss 2, I dc.; * repeat.

Last Row.-Turn over needle twice. Repeat last row of Baby's Hood. on page 338 .

For Shells around Cape.-Repeat instructions for shells, also same for collar. Make I row of spiders belween yoke and skirt for an edge. Make two rows of holes with I ch. between.

For Neck.-Make shells for collar between these rows. Place a tied bow of ribbon on each shoulder, a bow witi long loops where yoke and skirt join in front, and a small bow on sleeves.

\section{BABY'S SHIRT.}

Materials: 2 ounces "pure thread" knitting silk, and steel needle, Nos. I7 or I8. Cast on 164 stitches.

2nd row, p.; 3rd row, knit plain; 4th row; sl. I, n., k: 2, * thread over, k. I, thread over, k. 2, n. 2, k. 2; repeat from* across the needle.

5th row, p.; 6th row, like the 4 th; 7 th row, p.; 8th row, like the 4th; gth row, knit plain; Ioth row, p.; I Ith row, k.

This makes one row of shells. Repeat until you have four rows, then p. 2 and k. 2, 30 times across. Now divide the stitches, putting 82 on a third needle (as you must knit up the two halves separately), and k. 2, p. 2, 26 times across. Then narrow on each end of needle for shoulders, knitting once plain between each narrowing. Knit the shoulder any length you desire. Knit up the other side like this; then taking the stitches on 
one needle, begin on the wrong side, knit across, then purl across, then knit across, k. 2, thread over, narrow; ${ }^{*}$ k. 2, thread over, narrow; repeat from * to the end of needle; knit back, purl across, and cast off.

The row of holes around the neck are to put a ribbon in to draw it up. Sleeves.-Cast on 64 stitches. For short sleeves k. 3 rows of shells like the body. For long sleeves k. 2, p. 2, 26 times across; then put in fine needles and k. 2, p. 2, about Io times across. Cast off.

\section{LADY'S VEST.}

Materials: $6 \frac{1}{2}$ ounces "pure thread" knitting silk, $2 \frac{1}{2}$ yards of pink satin ribbon about one inch in width, and $2 \frac{1}{2}$ of very narrow pink satin ribbon to run in the neck. Two steel needles, No. I7, twelve inches in length, one fine steel crochet hook and one small skein of pink filo floss, for sewing the seams. The knitting silk is too hard a twist to sew with, and if used will make an uncomfortable seam to come next the skin.

The directions for making this veșt are very simple. It is made in two pieces, front and back, which are knit perfectly straight up and down upon two needles, until the neck is reached, where shaping begins, as directions will show. When the front and back are completed they are joined by a seam under each arm. The narrow strips which pass over the shoulder and form the sleeve are joined by crocheting. The hip or lower part of vest is widened by gussets, which are knit separately, one being placed in each side seam. The neck, sleeves and hem of vest are finished with crochet edges.

Formula.-Cast on with two needles, loosely, 200 stitches. This is the lower edge of vest. In second row across arrange in ribs of threes and twos. Every sixth row is purled all the way across. Continue with this pattern until you have a straight piece twenty-four inches long; here arrangre the work so that in beginning to knit the wrong side of the fabric will be next you. Now knit off in the usual way for 50 stitches, using an extra knitting needle. Leave the remaining I5O stitches on another needle; secure them by placing a small cork on each end of the needle. For the present this needle of 150 stitches is left, and you continue the pattern with the 50 stitches, thus shaping the neck and the short sleeves of the vest. Each time the edge is reached (the one which comes next the neck, not the outer sleeve edge), narrow once by knitting 2 stitches together Repeat 
till you have the stitches reduced to 20. Upon these knit the pattern for six inches, then cast off.

This makes a long slip, which is to form the sleeve or shoulder band Next cast off loosely ioo stitches from the extra needle of I50. Upon the remaining 50 stitches knit a strip to correspond to the strip already described. Narrow only on the side next the neck.

One half of the vest is now completed. For the other half of the vest, knit exactly as for the half already described. The lower part of the neck between the shoulder-bands is filled with a pattern crocheted as follows beginning in the lower right-hand conner: Raise Itr.; make $5 \mathrm{ch}$., I tr. repeat to left-hand corner. In making the tr., arrange so as to bring I tr. each side of every rib of 3 stitches. Break the silk at the ind of every row already described.

Now with a fine crochet hook fill in the space between sa two long strips with a square open-work pattern, described below and shown in the accompanying illustration. Do likewise with the other half of the vest.

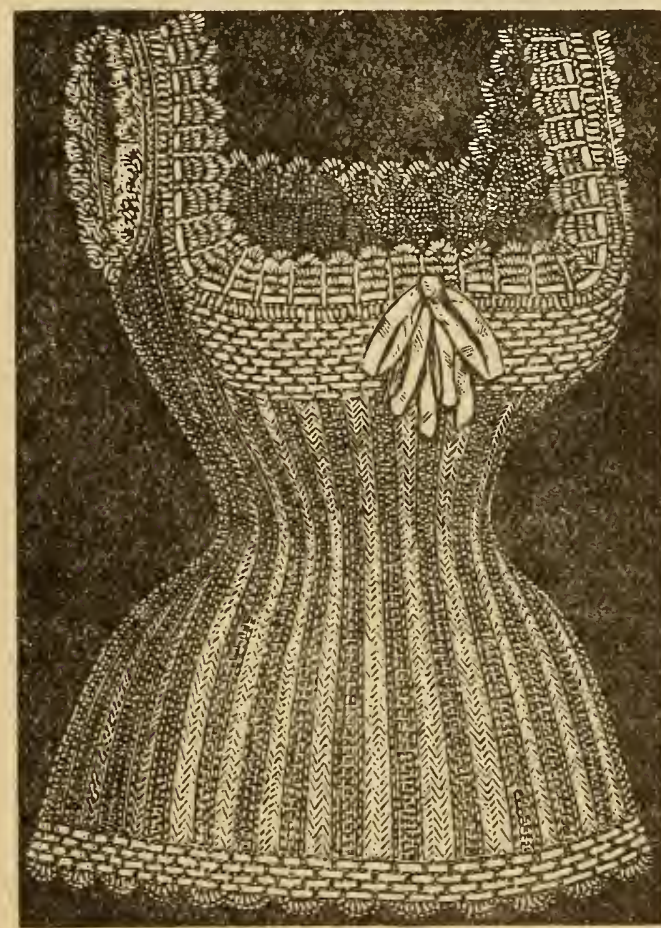

FIG. 287.

To make the open-work with which this space is filled, upon the row already made, work another row same as first, except that eacn tr. is worked midway of each ch. of 5 of preceding row. So alternate for 9 rows; but in beginning and ending each row you increase a ch. of 5 and I tr. This will be readily seen in working. If you do not increase, the space will not be entirely filled. Now join the strip over the shoulder in this way: Make a ch. of the required length to reach over the shoulder from front strif to back strip and secure. Then upon this work a ch. of 5 and I tr. clear across. Now to this add another row same as first except bringing each tr. midway of ch. of 5 of previous row. So alternate for 5 rows and you will have a pattern the same as the space is filled in with for the front and back described; also will have formed the sleeve of 
band. The other strips are joined just the same. Now work around the edge next the neck I row of tr., setting them close together. To this row add another, which work as follows, viz.: Make I tr. into each of 2 stitches or tr. of preceding row; 5 ch.; pass over 5 tr.; repeat.

To this row add another by working thus: I tr. into each of the 2 tr. of last row; I ch.; 4 tr.; each separated by I ch. into the third of $5 \mathrm{ch}$.; I ch.; repeat.

Next add another row. I tr. into each of the 2 tr. of last row; I ch.; 4 tr.; each separated by $1 \mathrm{ch}$. into the center; I ch. between the tr. of last rows; I ch.; repeat. Repeat this last row once more.

Now to this add a row of scallops, making about 15 tr. into I stitch for each scallop. Fasten down by I sc. to tr. of previous row. So work all around the neck. Add a similar row of scallops to outer edge of strip-band or sleeve. This completes the working of the band or sleeves. Run ribbons through holes and tie in pretty bows in front. Next sew up the seams under the arms, leaving a space on the lower part of each seam for insertion of gusset, say about five inches.

Gussets.-Cast on loosely 50 stitches on 2 needles; knit in ribs of threes and twos as you have for the other part of vest. Make this pattern for about one inch. Then narrow once at the beginning of every row; that is each time you turn the work. This narrows the work off to a point, or one stitch. When the gusset is finished, make one more for the other side. Sew into seam at lower or hip part of vest. These gussets are only necessary for persons of large size. Now add to the edge of vest open-work and scallops, as made above. This completes the work.

These garments are often knitted in cream white, pale pink or pale blue silk, and will be found to be durable and elastic, fitting very neatly to the figure.

\section{LADY'S BASKET PATTERN VEST.}

Use the same materials as given above. Begin by casting on III stitches for bottom of front of vest, and work for the border: always slip the first stitch of every row: Ist row, plain; $2 \mathrm{~d}$ row, p.; $3 \mathrm{~d}$ row, plain; 4th row, sl. I. * make one, k. 3, sl. I, k. I, pass the slipped stitch over; k. 2 togethe!, k. 3, make I, k. I; repeat from * to the end. 
5 th row, p; 6th row, same as 4 th row; 7 th row, plain; 8th row, p.; 9th row, plain; repeat trom the 4 th to the 9 th row.

I6th row, k. 2 together, k. I, k. 2 together, * k. 2 together, k. 9, and repeat from * till within 7 stitches of the end of the row, when $\mathrm{k}$. together, k. $2, \mathrm{k} .2$ together, k. I. Now the border is finished, and there are 98 stitches on the needle.

Work for the Basket Pattern as follows: Ist row, k, 5., p. 3, * k. 7, p. 3, and repeat from * to the end; 2nd row, k. 3, p. 7, and repeat, and end with p. 5 only.

3d row, same as the first row; 4th row, plain; 5 th row, p. 3, k. 7, and repeat, and end with $\mathrm{p}, 5$ only.

6 th row, p. 5, k. $3, *$ p. $7, \mathrm{k} .3$, and repeat from * to the end. 7 th row, same as the 5 th row.

8 th row, plain. Repeat these 8 rows till you can count 18 blocks of the basket pattern, I 44 rows; or 2 I blocks of the basket pattern, I 68 rows; according to the length required.

Then for the Shoulders: Ist row, k. 5, p. 3, k. 7. p. 3, k. 7, p. 3, k. I, cast the last stitch but one over the last stitch, and continue casting off till 26 stitches remain on the left-hand needle, then $\mathrm{k}, 3, \mathrm{p} .3, \mathrm{k} .7, \mathrm{p} .3, \mathrm{k} .7, \mathrm{p} .3$ 2nd row, k, 3, p. 7, k. 3, p. 7, k. 3, p. 2, p. 2 together.

3 d row, k. 3, p. 3, k. 7, p. 3, k. 7, p. 3; 4th row, k. 24, k. 2 together.

5 th row, k. 7 , p. 3 , k. 7 , p. 3 , k. 5 ; 6th row, p. 5 , k. 3, p. 7, k. 3, p. 5, p. 2 together.

7 th row, k. 6, p. 3, k. 7. p. 3, k. 5; 8th row, k. $22, \mathrm{k} .2$ together.

9th row, p. $3, \mathrm{k}, 7$, p. 3, k. 7, p. 3; Ioth row, k. 3, p. 7 k. 3 , p. 7 , k. r, k. 2 together.

I Ith row, p. $2, \mathrm{k} .7$, p. $3, \mathrm{k} .7$, p. 3 ; I 2 th row, k. $20, \mathrm{k} .2$ together.

I 3 th row, k. 3. p. 3 , k. 7 , p. 3, k. 5; I4th row, p. 5, k. 3, p. 7, k. 3, p. I, p. 2 together.

I 5 th row, k. 2, p. 3, k. 7 . p. 3, k. 5 ; I6th row, k. 20 ; 17 th row, k. 7 , p. j. k. 7, p. 3; I8th row, k. 3, p. 7, k. 3, p. 7; I9th row, k. 7, p. 3, k. 7, p. 3; 20th row, plain; cast off.

Now work upon the other shoulder, beginning inside the neck (the first row being already knitted).

2nd row, k. 2 together, p. 7, k. 3, p. 7, k. 3, p. 5 ; 3 d row, k. 5, p. 3, k. ;. p. $3, \mathrm{k} .7$, p. $\mathrm{r}$. 
4th row, k. 2 together, k. 24 ; 5 th row, p. 3, k. 7, p. 3, k., 7, p. 3, k. 2.

6th row, p. 2 together, k. 3, p. 7, k. 3, p. 7, k. 3; 7th row, p. 3, k. 7, p. 3 . k. 7 , p. 4 .

8 th row, k. 2 together, k. 22 ; 9 th row, k. 5, p. 3 , k. 7 , p. 3, k. 5 .

Ioth row, k. 2 together, p. 3, k. 3, p. 7, k. 3, p. 5 ; i ith row, k. 5, p. 3, k. 7. p. $3, \mathrm{k}, 4$.

I2th row, $\mathrm{k} 2$ together, $\mathrm{k}$. 20 ; I3th row, p. $3, \mathrm{k}, 7, \mathrm{p} .3, \mathrm{k}, 8$.

I 4 th row, p. 2 together, p. $6, \mathrm{k} .3$. p. $7, \mathrm{k} .3$; I 5 th row, p. $3, \mathrm{k} .7, \mathrm{p} .3, \mathrm{k}$. 7; I6th row, k. 20; I7th row, k. 5. p. 3. k. 7, p. 3, k. 2 .

I 8 th ruw, p. $2, \mathrm{k} .3$, p. $7, \mathrm{k} .3$, p. 5 ; Igth row, k. 5, p. $3, \mathrm{k} .7$, p. 3, k. 2 ; 20th row, plain. Cast off.

Knit another similar piece for the back of the vest. Sew the shoulder pieces together, and sew up the sides of the vest, from the bottom, leaving space for the armholes.

For the Sleeves.-Cast on 69 stitches, and work 15 rows of the border. the same as at the bottom of the vest, but doing I additional plain stitch at the beginning and at the end of each row.

I6 h row, plain knitting, and take 2 stitches together once in the course of the row, to bring 68 stitches on the needle. Now knit 8 rows of the basket pattern, and cast off loosely. Make a little gusset by casting on I6 stitches, and knitting 26 plain rows, and cast off. Knit another sleeve and another gusset in the same manner. Sew up the sleeves, taking care that the gussets are placed properly. Crochet a dainty edge around the neck, having first a row of holes to run in ribbon.

\section{BABY'S HOOD.}

Materials: I $1 / 2$ ounces knitting silk and a steel crochet hook of moderate size. Make $25 \mathrm{ch}$.

Isí row, * I dc. into Ist ch., 2 ch.; 3 tr. into same ch., miss 2,2 ch., I dc. into $3 \mathrm{~d}$; ; $*$ repeat.

2nd row, ${ }^{*} 3$ ch., 3 tr. into first dc. of last row, I dc. into 2 ch. of last row. * Repeat, making 25 rows which form crown of cap. Break off and tasten on at the ch. stitches, which must form back of cap, and make a mesh as follows:

To begin, make $5 \mathrm{ch}$, take up one loop as for a tr., putting the needle in third.ch,, then, still holding the loop on needle, take another loop, put- 


\section{DAINT WORK FOR PLEASURE AND PROFIT.}

sing the needle in first stitch, draw the thread through 2 loops at once until only one is icft on the needle. 2nd mesh, make I tr. into every $3 \mathrm{ch} ., 2 \mathrm{ch}$. between each mesh, making it wide enough to take satin ribbon No. 4; work

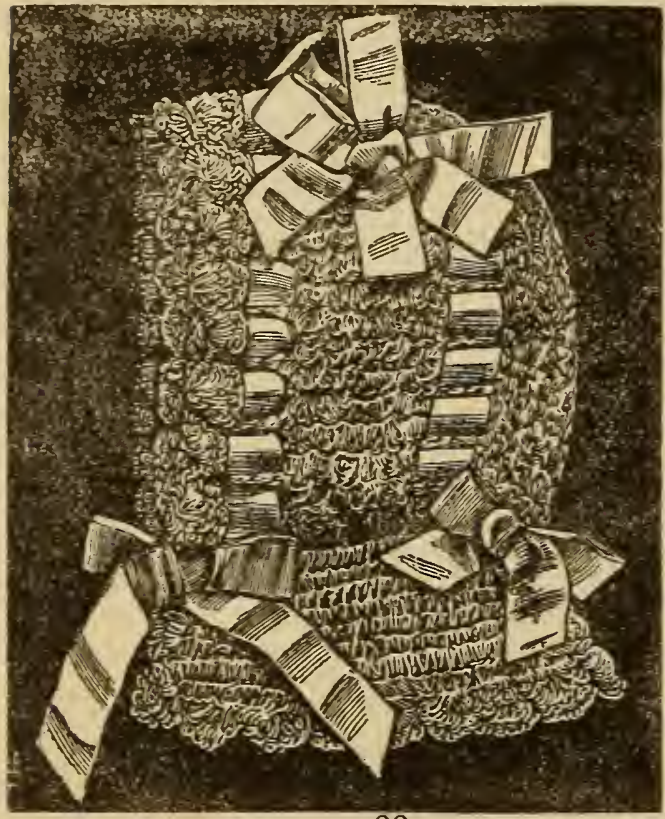

FIG. 288 . mesh around the crown.

Top of Head.--Repeat second row. working every mesh until twelve rows are done. Repeat row for mesh.

Repeat 2nd row three times. Make I row of dc. round neck of cap.

Cape.--Repeat $3 \mathrm{~d}$ row of Baby's Sacque, on page 33I, making 7 rows.

8th row, repeat 2nd row of Baby's Sacque, on page 33I, taking up three loops on each side instead of two. 9th row, repeat 7 th row. Round the front of cap make shells to meet those already made, making a double front.

Edgt.-.* Make Io tr. for shell, turning over the needle twice into third ch., I dc. into next third ch. * Repeat round the cap and round cape, miss two cross-stitches into third.

Last Row' -3 ch. and a dc. into every tr., missing the dc. between the shells. Under Cap.- 25 ch., 25 tr., for five rows, then making tr. round the crown all but the neck for head. Make 7 rows. Sew in with silk. The woolen lining makes the cap warmer, but if preferred, a wadded silk lining can be used, made separate from the cap, instead of the woolen under-cap. Split zephyr or Shetland is used double.

\section{FANCY BAG.}

Materials, I I/2 ounces knitting silk, I yard silk, satin or plush, and $4 \mathrm{I} / 2$ yards of ribbon $\mathrm{I} / 2$ inches wide. To make the large rosettes, make $\mathrm{I} 3 \mathrm{ch}$; join.

Ist round, $3 \mathrm{ch}$., I tr. in first stitch, $4 \mathrm{ch} ., 2 \mathrm{tr}$. till there are seven points to the star; join.

2nd round, $2 \mathrm{ch}$. and a row of str. around the star by making 4 str. in each loop, and 2 str. on the upper edge of the points of the star; join.

3d round, 3 ch., I tr. in first stitch, 2 tr., 3 ch. and 2 tr. in every fourth stitch to end of round; join. 


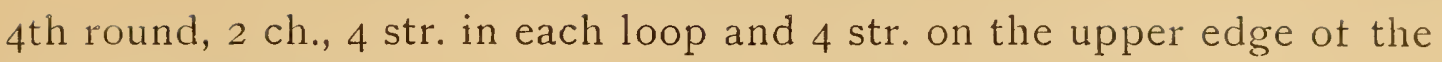
tr. to end of row; join.

5 th round, 3 ch., 2 tr. in first stitch, 4 ch., 2 tr. in the third and fourth stitches to the end of the round; join.

6th round, 2 ch., 4 str. in loop, and 2 str. in upper edge of tr. to end of round; join. 7 th round, $3 \mathrm{ch}$., I tr. in first stitch, $6 \mathrm{ch}, 2 \mathrm{tr}$. in fifth and sixth stitches to end of round; join.

8 th round, I str. between the tr. in the foundation, $8 \mathrm{tr}$. in each loop to end of round.

To make the small rosettes, make $13 \mathrm{ch}$; join. Ist round, $2 \mathrm{ch}$., I tr; in every first stitch, $4 \mathrm{ch}$. and $2 \mathrm{tr}$. till there are seven points in the star; join.

2nd rcund, 3 ch., I str. in every first stitch, 4 str. in loop, 2 str. on the upper edge of tr. to end of round; join.

$3 \mathrm{~d}$ round, $6 \mathrm{ch}$. in every third stitch to end of round.

These rosettes are arranged upon the bag as illustrated, and after making a row of ch.stitches, and a row of tr. with $2 \mathrm{ch}$. between them, on the lower edge of the second row of rosettes, a less number of rosettes is placed below on the gathered end of the bag, as shown in the cut. The rosettes should not be crocheted too loosely. The width of the satin or plush forms the length of the bag, and the hem above the gathers on the upper end may be lined with satin of a contrasting color, matching the rosettes. Fawn color or light brown on blue, or cardinal and old gold on brown velvet, are very clegant combinations.

\section{FRINGE.}

This fringe is designed for the ends of pillow scarls, diessing-case scarfs and towels, made from silk-finish embroidery linen, which come in widths from 18 to 80 inches. The fringe can be made from cotton thread, but is much handsomer made from

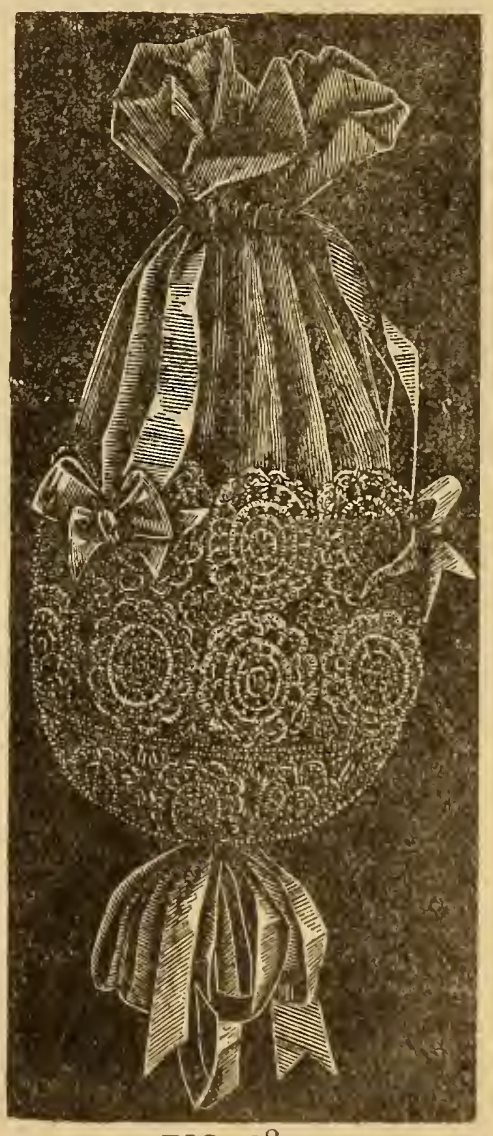

FIG. 289. the Bousfield Scotch linen thread, while some ladies are so extravagant as to usc knitting silk. 
Make a chain of 35 stitches.

Ist row, 3 dc. in fourth stitch, 3 ch., 3 dc. in same stitch; fasten to next third stitch of chain, 6 ch., miss 6 ch., 3 dc. in seventh, 3 ch., 3 dc. in same; fasten to next third chain, $6 \mathrm{ch}$., miss 6 , put $3 \mathrm{dc}$. in seventh, $3 \mathrm{ch}$., $3 \mathrm{dc}$. in same; fasten to next third stitch; $6 \mathrm{ch}$., miss $6 \mathrm{ch} ., 3 \mathrm{dc}$. in seventh stitch of chain, 3 ch., $3 \mathrm{dc}$, in next chain stitch, $5 \mathrm{ch}$. stitches and turn.

2nd row. $3 \mathrm{dc}$. around next chain of three, worked between the last 3 dc. in preceding row, 3 ch., 3 dc. around same; fasten around first stitch of next chain of six, 6 ch., 3 dc. around next chain of three, 3 ch., 3 dc. around same chain, fasten, 6 ch., 3 dc. around next chain of three, $3 \mathrm{ch}$., $3 \mathrm{dc}$. around same chain, fasten, $6 \mathrm{ch} ., 3 \mathrm{dc}$. around next chain of three, $3 \mathrm{ch} ., 3$. dc. around same, work $6 \mathrm{ch}$; t turn.

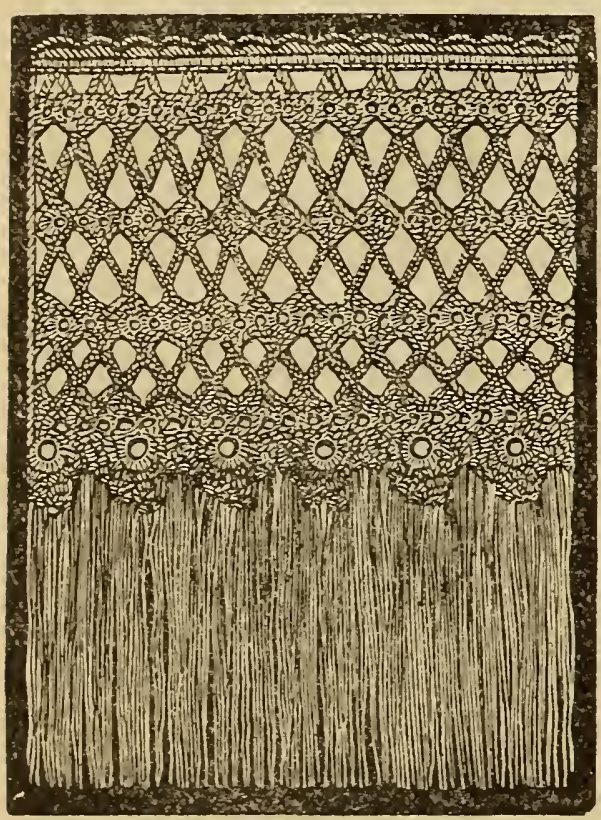

FIG. 290.

3d row, 3 dc. around next chain of three of second row, 3 ch. 3 dc., around same chain, fasten, 6 ch., $3 \mathrm{dc}$. around next chain of three, 3 ch., $3 \mathrm{dc}$. around same chain, fasten, $6 \mathrm{ch}$., $3 \mathrm{dc}$. around next chain of threc, $3 \mathrm{ch}$., $3 \mathrm{dc}$. around same, fasten, $6 \mathrm{ch} ., 3 \mathrm{dc}$. around next chain of three, $3 \mathrm{ch}$., $3 \mathrm{dc}$. around same, fasten in first stitch of chain of five of last row.

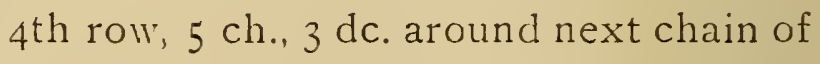
three of third row, 3 ch., 3 dc. around same, fasten, 6 ch., 3 dc. around next chain of three, 3 ch., 3 dc. around same, fasten, 6 ch., 3 dc. around next chain of three, 3 ch., $3 \mathrm{dc}$. around same, fasten, 6 ch., 3 dc. around next chain of three, 3 ch., 3 dc. around same; work around next chain of six, * 2 dc., 3 ch. *. Repeat five times, stopping before $3 \mathrm{ch}$. Fasten by I sc. in last third dc. of first row.

$5^{\text {th }}$ row, turn, * I sc., 3 dc., I sc. around chain of three, *. Repeat four times; 3 ch., 3 dc., around next chain of three of last row, *3 ch., $3 \mathrm{dc}$. around same, fasten, $6 \mathrm{ch}$., $3 \mathrm{dc}$. around next chain of three, repeat to the end of the row.

Finally, make 5 ch. instead of 3 . 
Begin again from $2 \mathrm{~d}$ row.

For the heading, fasten the silk to the end of the work, then crochet I sc. in every stitch of the edge.

The second row of heading is worked as follows:

Turn, * 3 dc. in next sc. of last row, miss next sc., fasten in following sc., miss I, * repeat to the end of the row.

\section{PINE APPLE EDGE.}

Make a ch. of 24 stitches; turn.

Ist row, I dc. into the sixteenth, I sc. into each of 2 next stitches; turn. 2nd row, 9 tr. under loop of ch., $2 \mathrm{ch} .9$ tr. under same loop of ch.; turn.

3d row, $3 \mathrm{ch}$. I tr. into each of $9 \mathrm{tr}, 3$ tr. under $2 \mathrm{ch} .2 \mathrm{ch}$., 3 tr. under same 2 ch., I tr. into each of 9 tr., pass over 2 ch., I sc. into each of 3 next stitches; turn.

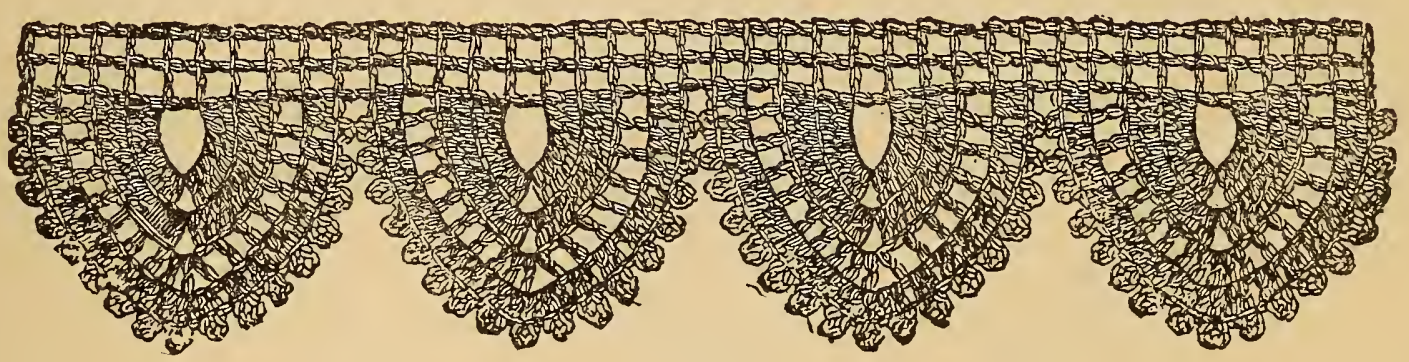

FIG. 29 I.

$4^{\text {th }}$ row, 2 ch. I tr. into first stitch, 2 ch., I tr. into next stitch $*, 2 \mathrm{ch}$., pass over I stitch $I$ tr. into the next, repeat from * 4 times more, 3 tr. each separated by $2 \mathrm{ch}$. under $2 \mathrm{ch} ., 2 \mathrm{ch}$. I tr. into the next tr. *, 2 ch., pass over I stitch I tr. into the next, repeat from ${ }^{*} 5$ times more, $2 \mathrm{ch}$. I tr. into end stitch; turn.

5 th row, 3 ch., 2 tr. under each 2 ch., repeat all round, work 3 instead of 2 tr. under the $2 \mathrm{ch}$. on each side the center tr., I sc. into each of the 3 next ch.; turn.

6 th row, 4 ch., pass over I stitch, I sc. into each of the 2 next stitchcs; repeat all round.

For the heading:

Ist row, I tr. into the end of first row of scallops $*, 2$ ch. I tr. into cncl of next row, repeat from * to the end of the row.

2nd row, I tr. into tr. of last row, $2 \mathrm{ch}$, repeat to the end of the row 


\section{INSERTION CROCHET.}

Nake a ch. of 13 stitches, turn back and work I dc. into the seventh: 3 ch., pass over 2 stitches, I dc. into the next, $4 \mathrm{ch}$., one dc. into end stitch; turn.
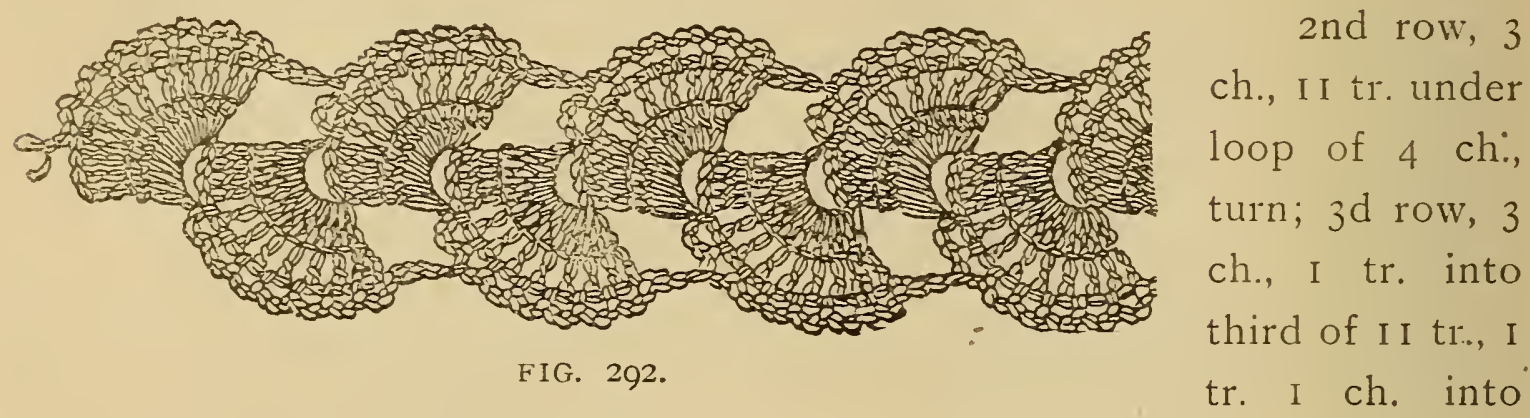

cach of 8 tr. of last row; turn.

4 th row, 4 ch. I dc. between 2 tr. (see figure 292), 3 ch., I dc. between 2 next tr., repeat from ${ }^{*} 5$ times more, $4 \mathrm{ch}$. I dc. into end stitch, repeat from second row for the length required.

When commencing the last row of next and following patterns, join to preceding, pattern by working $3 \mathrm{ch}$. I sc. into second loop of $3 \mathrm{ch}$. (see figure 292), 3 ch., I sc. into first of previous $3 \mathrm{ch}$.

\section{BORDER CROCHET.}

Commence with I of the oblongs. Make a ch. of 16 stitches, work 14 rows backwards and forwards with I dc. into the back horizontal loop of each stitch; for the oblong forming the point, work I7 dc. at one side of the first oblong (see figure 293), work I 3 rows; for the third oblong, work I6 dc. into the side of the last worked oblong, 3 ch., work backwards and forwards on the ch, and the dc. for I 4 rows; do not break off the cotton, but commence the ncxt pattern with $16 \mathrm{ch}$. as you did the first.

For the heading:

Ist row, I dc. into a point (see figure 293), 2I ch., I dc. into fifteenth 3 ch., I dc. into the sixth, 2 ch., I dc. into side of oblong (see figure 293), $8 \mathrm{ch}$., I dc. into the third, one sc. into each of $3 \mathrm{ch}$. before second picot, 9 ch., I dc. into the third, 2 ch., I sc. into side of next oblong (see figure 293), $8 \mathrm{ch}$., I dc. into the third, I sc. into each of 3 next ch., 6 ch., I dc. into the first, I sc. into each of 3 next stitches, $\mathrm{I}^{-}$ch. repeat from the beginning of the roil, 
2nd row, I tr. into a stitch, I ch., pass over I stitch and repeat.

3rd row, I tr. into each stitch; 4th row, I tr. into a stitch, I ch., pass over I stitch and repeat.

For the edge:

Ist row, * I doubie tr. into the fifth stitch at side of oblong, $2 \mathrm{ch}$., pass over 2 stitches, repeat from * I5 times morc, at the point work 7 instead of $2 \mathrm{ch}$., and do not pass over a stitch (sec figure 293).

2nd row, I dc. into each of 9 stitches, 4 ch., I sc. into the first, I dc. into each of 3 stitches, 9 ch., work back with I sc. into the third stitch before the picot, under the loop of $9 \mathrm{ch}$. work 2 dc., * 4 ch., I sc. into the first, 3 dc. under 9 ch., repeat from *

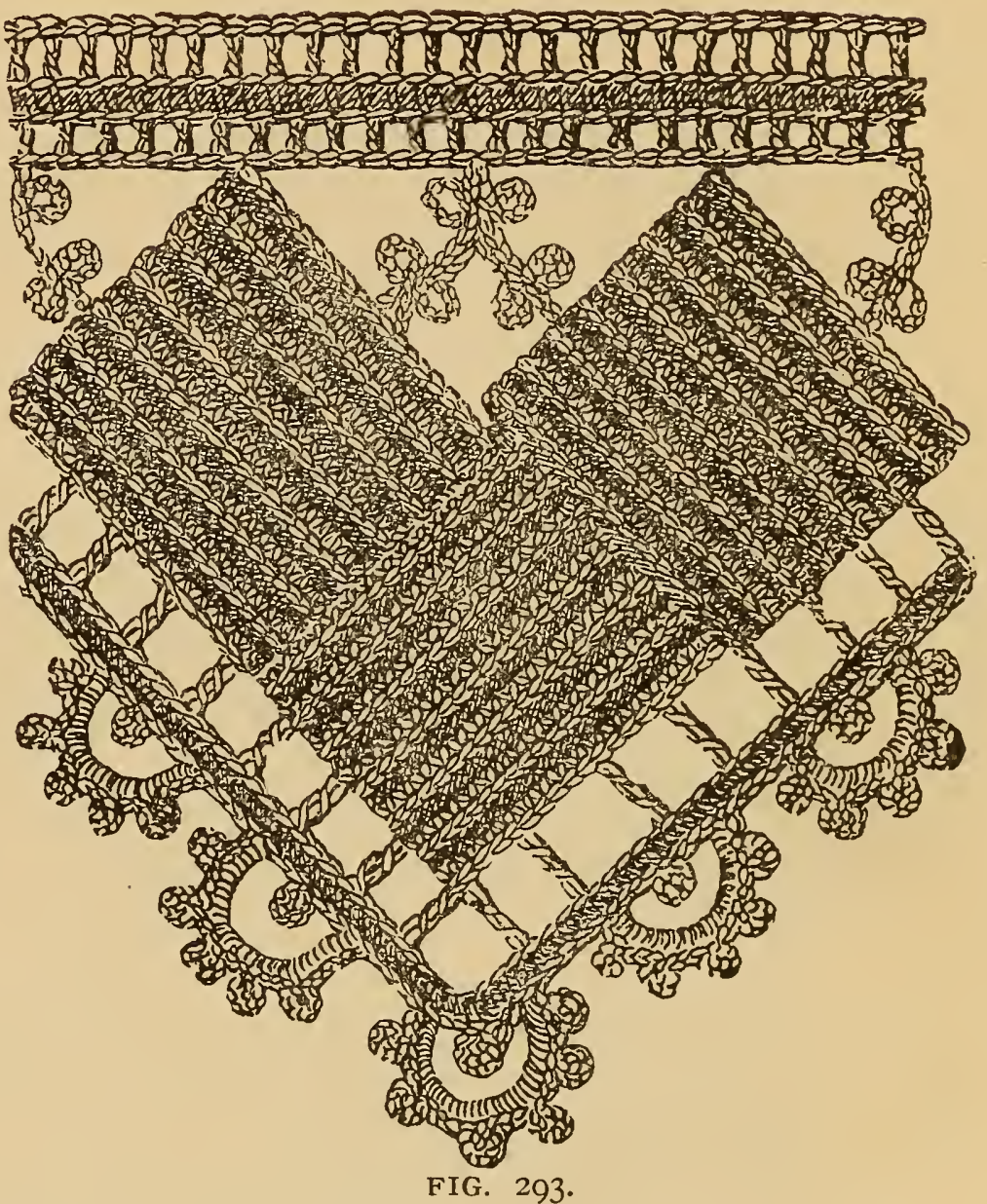
4 times more, I dc. into each of 7 next stitches of last row; work 4 more of these scallops or the vandyke.

\section{TRIMMING CROCHET.}

Make a chain the length required.

Ist row, I dc. into each of I9 stitches, 9 ch., pass over 8 stitches, and repeat from the beginning of the row. 2nd row, I dc. into the second and I 6 following dc. of last row, 3 ch. 2 tr. separated by 4 ch., into center of 9 ch. $3 \mathrm{ch}$., repeat from the beginning of the row.

3 rd row, I dc. into the second and i 4 following dc. of last row, 2 ch. 6 tr. each separated by $1 \mathrm{ch}$. over the $2 \mathrm{tr}$. and $4 \mathrm{ch}$. of last row (see figure 294), 3 ch.; repeat from the beginning of the row. 
4th row, I dc. into second, and I into each of the 12 following stitches of last row, $2 \mathrm{ch}$., 9 tr. each separated by one ch. over the tr. of last re: 2 ch.; repeat from the beginning of the row.

5 th row, I dc. into the second and I into each of the Io following dc. of last row, $2 \mathrm{ch}$. Io tr. each separated by I ch. over the tr. of last row, 2 ch.; repeat.

6th row, I dc. into the second and I into each of the 8 following dc. of last row, 2 ch., I 3 tr. each separated by i ch. over the tr. of last row (see figure 294), 2 ch.; repeat from the beginning of the row.

7 th row, I dc. into the second and I into each of the 6 following dc. of last row, 2 ch. I 4 tr., each separated by i ch. over the tr. of last row (sec figure 294), 2 ch.; repeat from the beginning of the row.

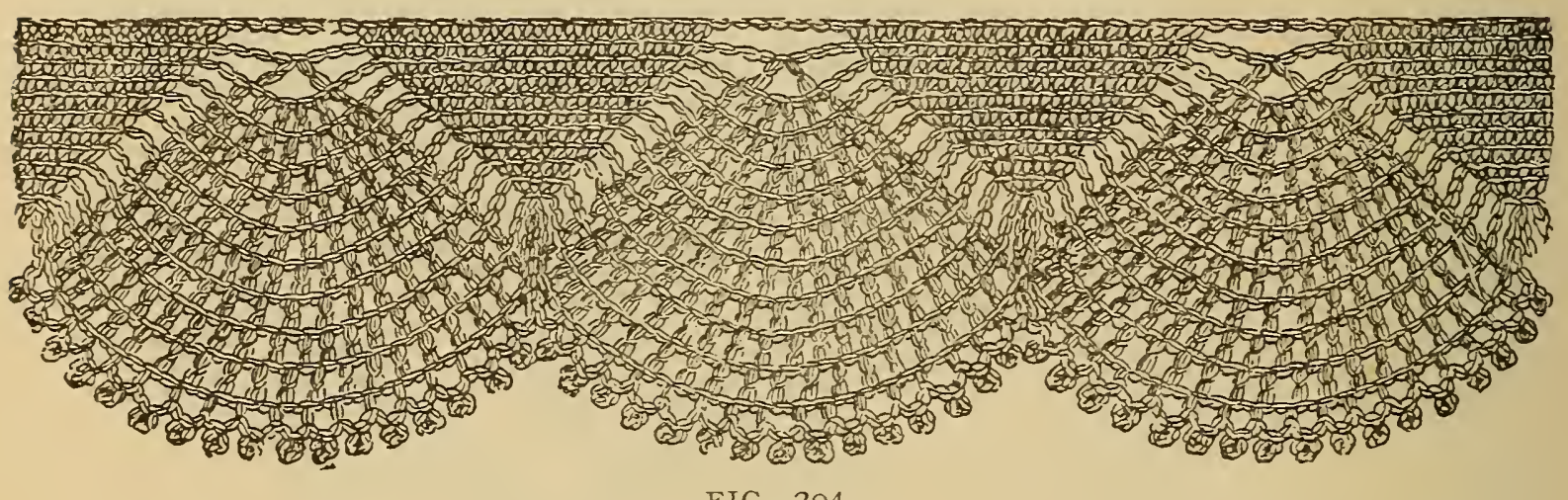

FIG. 294.

8th row, one dc., into the second and I into each of the 4 following dc. of last row, $2 \mathrm{ch}$., I7 tr. each separated by I ch. over the tr. of last row, $2 \mathrm{ch}$; repeat from the beginning of the row.

yth row, I dc. into the second and I into each of the 2 following dc. of last row, 2 ch. I 8 tr. each separated by I ch. over the tr. of last row, 2 ch.; repeat.

Ioth row, I dc into each of the $3 \mathrm{dc}$. of last row, keep the top loops on the hook, draw through all together, $3 \mathrm{ch} .20 \mathrm{tr}$. each separated by I ch. over the tr. of last row, 2 ch.; repeat.

IIth row, I dc. between 2 dc., 4 ch. 20 tr. each separated by i ch. over the tr. of last row, $4 \mathrm{ch}$; repeat.

I2th row, I dc. into first ch. between 2 first tr., 3 ch. I dc. between the third and fourth tr. ${ }^{*}, 4 \mathrm{ch}$, I dc. into the second, I ch. I dc. into the ch. between 2 next tr., repeat from * I4 times more, $3 \mathrm{ch}$. I dc. between 2 next tr.; repeat from the beginning of the row. 


\section{KNITTED SQUARE FOR COUNTERPANE.}

D. M. C. knitting cotton No. IO, and pins No. I3 are a nice size to use for a counterpane. The squares are sewn together when all are worked, and the counterpane is finished with a knitted border, or with fringe.

Commence with the raised patterns, cast on 3 stitches.

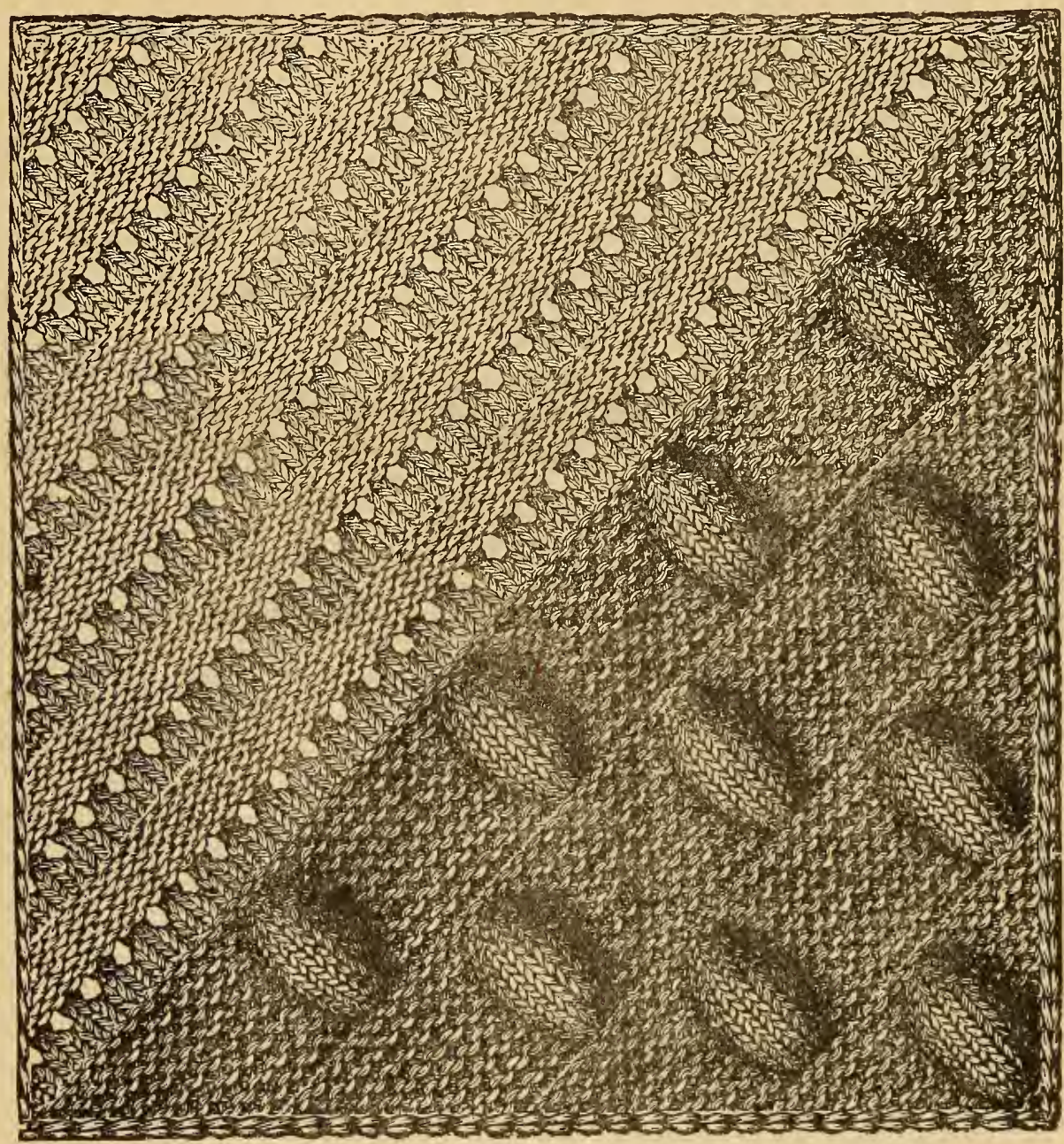

FIG. 295 .

Ist row, k.; 2nd row, sl. I, make I by knitting the horizontal thread iying under next loop (all the increases at the beginning and end of each aiternate row are made in this way), knit the next stich, make I, k. I.

3d row, sl. $\mathrm{r}, \mathrm{k}$. to end of row, the first stitch is slipped in every row:

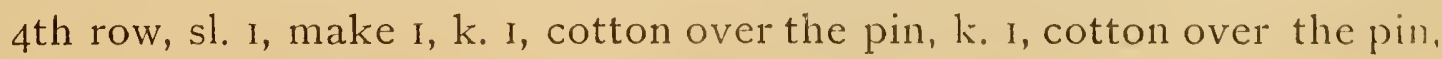
this commences the first raised pattern, k. I, make I, k. I.

"5th row, sl. I, k. 2, p. 3, k. 3; 6th row, sl. I, make I, k. 2, cotton over the pin, k. 3 , cotton over the pin, k. 2, make 1, k. I. 
7 th row, sl. I, k. 3, p. 5, k. 4; 8th row, sl. I, make I, k. 3, cotton over the pin, k. 5 , cotton over the pin, k. 3 , make $\mathrm{I}, \mathrm{k}$. I.

9th row, sl. I, k. 4, p. 7, k. 5 ; Ioth row, sl. I, make I, k. I5, make $1, \mathrm{k}$. I. IIth row, sl. I, k. 5, p. 7, k. 6; i2th row, sl. I, make I, k. 5, k. 2 together at the back, k. 3, k. 2 together, k. 5, make I, k. I.

I3th row, sl. I, k. 6, p. 5, k. 7; I4th row, sl. I, make I, k. u, k. 2 together at the back, k. I, k. 2 together, k. 6, make I, k. I.

I 5 th row, sl. I, k. 7 , p. 3 , k. 8 ; I6th row, sl. I, make I, k. 7, k. 3 together, k. 7 , make I, k. I; I th row, sl. I, k. to end of row.

I8th row, sl. I, make I. k. 3 , cotton over the pin, k. I, cotton over the pin, k. 9, cotton over the pin, k. I, cotton over the pin, k. 3, make I, k. I.

Igth row, sl. I, k. 4, p. 3, k. 9, p. 3, k. 5; 20 th row, sl. I, make I, k. 4 , cotton over the pin, k. 3 , cotton over the pin, k. 9, cotton over the pin, k. 3 , cotton over the pin, k. 4, make I, k. I.

2Ist row, sl. I, k. 5, p. 5, k. 9, p. 5, k. 6; 22nd row, sl. I, make I. k. 5, cotton over the pin, k. 5, cotton over the pin, k. 9, cotton over the pin, k. 5 , cotton over the pin, k. 5, make I, k. I.

23 rd row, sl. I, k. 6, p. 7, k. 9, p. 7, k. 7 ; 24 th row, sl. I, k. 35 , make I, k. I.

25 th row, sl. I, k. 7, p. 7, k. 9, p. 7, k. 8; 26th row, sl. I, make I, k. $7, \mathrm{k}$. 2 together at the back, k. 3, k. 2 together, k. 9, k. 2 together at the back, k. 3 , k. 2 together, k. 7 , make I, k. I.

27 th row, sl. I. k. 8, p. 5, k. 9, p. 5, k. 9; 28 th row, sl. I, make I. k. 8, k. 2 together at the back, k. I, k. 2 together, k. 9, k. 2 together at the back, k. I, k. 2 together, k. 8 , make I, k. I.

29th row, sl. I, k. 9, p. 3, k. 9, p. 3, k. I0; 30th row, sl. I, make I. k. 9, k. 3 together, k. 9, k. 3 together, k. 9, make I, k. I.

3 Ist row, sl. I, k. to end of row; 32 nd row, in this row 3 raised patterns are commenced. Sl. I, make I, k. 5 , cotton over pin, k. I, cotton over pin. k. 9 , cotton over pin, k. I, cotton over pin, k. 9, cotton over pin, k. I, cotton over pin, k. 5, make $1, k$. I.

Continue to work the 3 raised patterns as described for the preceding. still makins the increase at the beginning and end of each forward row In the $44^{\text {th }}$ row you complete the 3 raised patterns. 
45th row, sl. I, k. 46; 46th row, sl. I, make I, k. 7 *, cotton over the pin, k. I, cotton over the pin, k. 9, repeat from * 3 times more, except that in the last repeat, you k. 7 instead of 9, make I, k. I.

Continue to work the 4 raised patterns as described for the previous raised patterns.

For the second half of the square, in the first forward row after the raised patterns are finished, knit 2 together at the beginning and end of the row.

2nd row, purl without decrease; 3d row, knit, decreasing at the beginning of the row.

4th row, purl without decrease; 5th row, k. 2 together, * cotton forward, k. 2 together, repeat from * till within 2 stitches of end, k. 2 last stitches together.

6th row, knit without decrease; 7 th row, purl, decreasing at the beginning and end of row.

8th row, knit; $9^{\text {th }}$ row, purl, decreasing at the beginning and end of row; Ioth row, knit, without decrease.

Then repeat from the first row of second half until 3 stitches only remain, then cast off.

\section{WHEELS FOR TOILET SET:}

Make a chain of IO, join with s. s.; Ist row, ch. 4,24 dc. into ring, join with s. s.

2nd row, ch. 5, I dc. into joining stitch of preceding row, * ch. I, I dc. into second stitch from preceding, ch. I, I dc. in same stitch as before *, repeat from * to * until there are twelve couplets, which should bring one back to starting point; ch. I, join with s. s.

3d row, * ch. 2, I dc. into center of first couplet, ch. 4 , join with s. s. into stitch from which ch. 4 starts, ch. 4 , join in same way in same place, ch. 4 ,

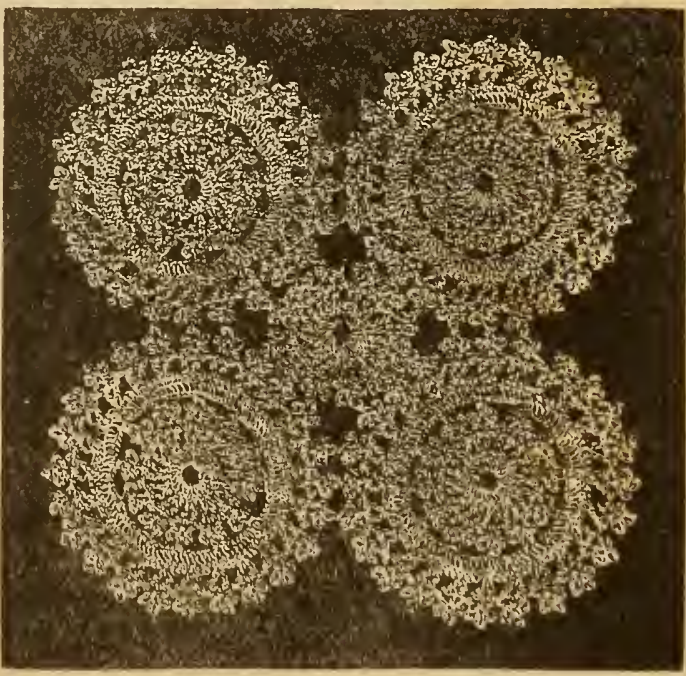

FIG. 296. join in same way in same place, ch. 2 , join with s. s. into ch. I between first and second couplet of preceding row $*$. This forms a picot. 
Repeat from * to * until 12 picots are made. When this is done a small wheel is completed.

To make the large wheel, go on from this point by making a ch. 4 and fastening it in center loop of first picot from the back with a s. s.

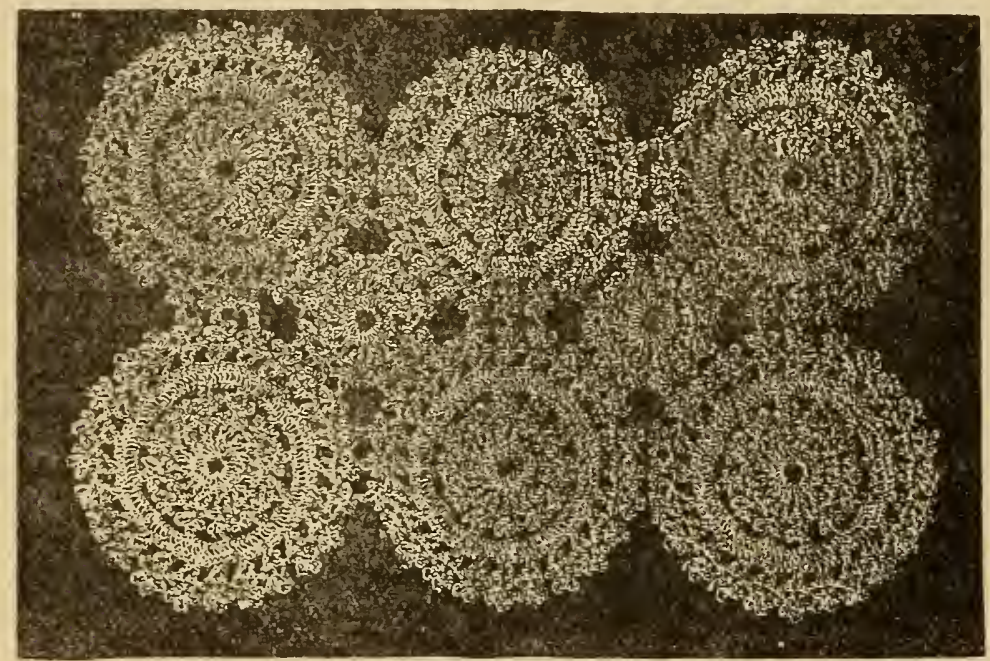

FIG. 297.

4th row, ch. 7 , join in center loop of second picot with s. s. ch. 7 , join in center loop of third picot with s. s., and so on until starting point is reached, when there should be 96 stitches; join with s.s.

$5^{\text {th }}$ row, ch. 4 , I dc. into each of 96 stitches: join with s. s.

6 th row, ch. 5, I dc. into joining stitch of preceding row, * ch. 2 , I dc. into fourth stitch from preceding, ch. I, I dc. into sime stitch as before *, repeat from * to * until there are 24 couplets, which should bring one back to starting point, ch. 2; join with s. s.

7 th row, same as 3 d row, except there will be 24 picots instead of 12 .

The wheels may be joined while making, or afterwards, as one chooses.

\section{EDGING.}

Make a chain of 40 stitches.

Ist row, dc. into 7 th stitch of ch., 3 dc. into next 3 stitches, * ch. 2 , dc into third stitch $*$, repeat from $*$ to $* 4$ times, 6 dc. into next 6 stitches, repeat from * to $* 2$ times, 3 dc. into next 3 stitches; turn.

2nd row, ch. 3,3 dc. into last 3 dc. of previous row, (the ch. counts as I dc. whenever used for turning, so that there will always be 4 dc. upon 4 dc. at this point, ) * ch. 2 , dc. into third stitch *, repeat from * to $*$ once, 3 dc. into next 3 stitches, repeat from $*$ to $* 7$ times, I 4 dc. into loop; turn (Throughout the further rows "from * to *" will mean the same as in these two rows.)

$3 \mathrm{~d}$ row, ch. $3,7 \mathrm{dc}$ into next 7 stitches, ch. 5, 4 dc. into 4 stitches imme. diately following the 7 stitches; repeat from * to * 3 times, 6 dc. into next 
5 stitches, repeat from $*$ to $* 2$ times, $3 \mathrm{dc}$. into next 3 stitches, repeat from * to * 3 times, 3 dc. into next 3 stitches; turn.

$4^{\text {th }}$ row, ch. 3,3 dc. into next 3 stitches, repeat from $*$ to $* 5$ times, I2 dc. into next 12 stitches, repeat from * to * 3 times, I4 dc. into loop; turn.

5 th row, ch. 3, 7 dc. into next 7 stitches, ch. 5, 4 dc. into next 4 stitches, repeat from * to $* 4$ times, I 2 dc. into next 12 stitches, repeat from $* t_{0} * 5$ times, 3 dc. into next 3 stitches; turn.

6th row, ch. 3,3 dc. into next 3 stitches, repeat from * to * 3 times, $6 \mathrm{dc}$. into next 6 stitches, repeat from * to * I time, 6 dc. into next 6 stitches repeat from $*$ to $* \mathrm{I}$ time, $6 \mathrm{dc}$. into next 6 stitches, repeat from * to * 3 times, 14 dc. into loop; turn. $7^{\text {th }}$ row, ch. 3,7 dc. into next 7 stitches, ch. 5,4 dc. into next 4 stitches, repeat from * to * 3 times, I $2 \mathrm{dc}$. into next 12 stitches, repeat from * to * 2

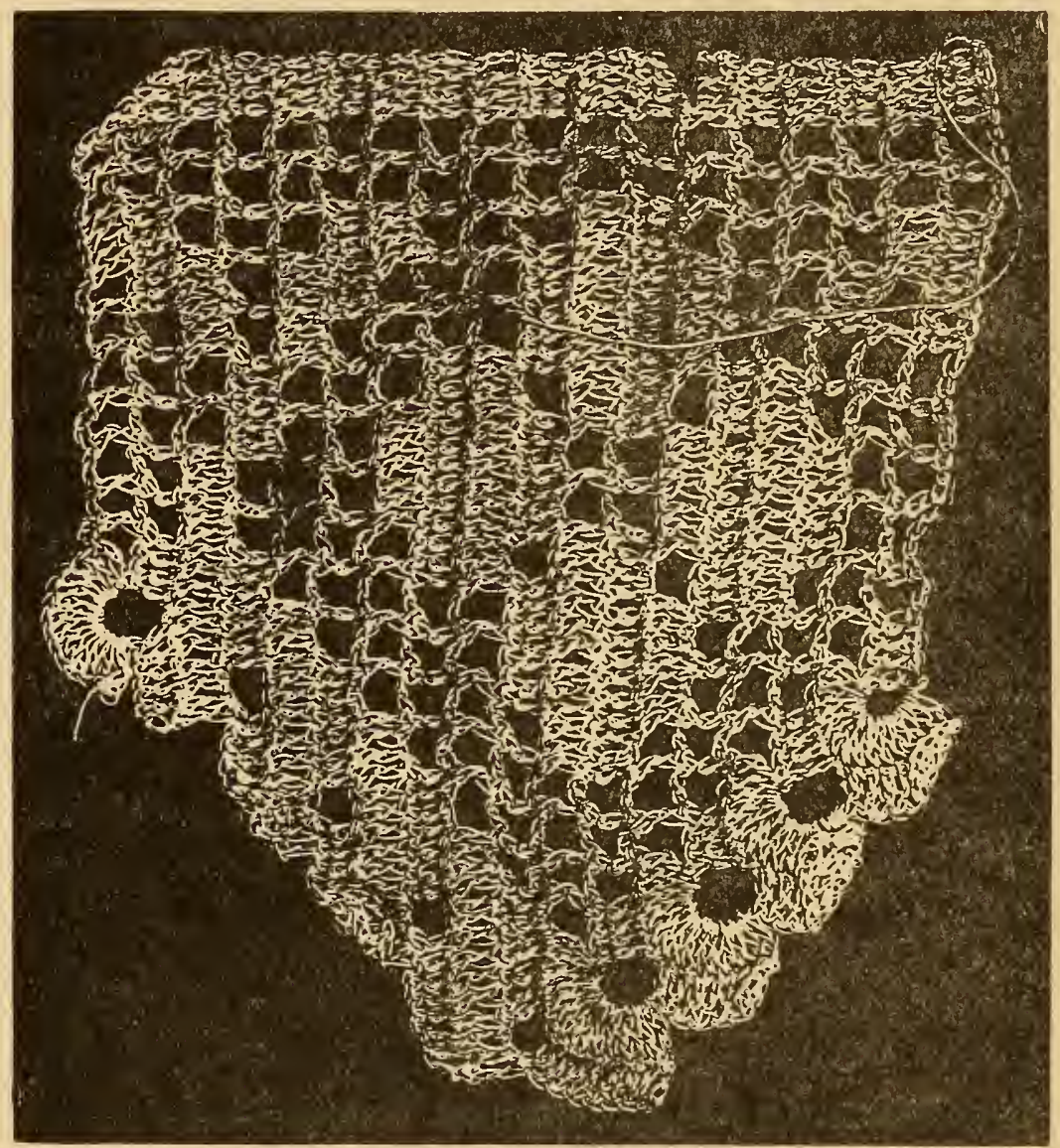

FIG. 298. times, 12 dc. into next 12 stitches, repeat from * to $* 2$ times, 3 dc. into next 3 stitches; turn.

8 th row, ch. 3,3 dc. into next 3 stitches, repeat from * to * 2 times, 12 dc. into next 12 stitches, repeat from $*$ to $* 2$ times, I 2 dc. into next 12 stitches, repeat from $*$ to $* 4$ times, I4 dc. into loop; turn.

9 th row, ch. 3,7 dc. into next 7 stitches, repeat from * to * I time, 7 dc. into next 7 stitches, repeat from $*$ to $* 4$ times, 6 dc. into next 6 stitches, repeat from * to * I time, $6 \mathrm{dc}$. into next 6 stitches, repeat from * to * I 
time, 6 dc. into next 6 stitches, repeat from * to 3 times, 3 dc. into next 3 stitches; turn.

Ioth row, ch. $3,3 \mathrm{dc}$. into next three stitches, repeat from * to $* 5$ times, I 2 dc. into next 12 stitches, repeat from * to * 5 times, I 3 dc. into next I 3 stitches; turn.

IIth row, ch. 3,7 dc. into next 7 stitches, repeat from * to * I time, 6 dc. into next 6 stitches, repeat from * to 4 times, I2 dc. into next I2 stitches, repeat from $*$ to $* 5$ times, 3 dc. into next 3 stitches; turn.

I2th row, ch. $3,3 \mathrm{dc}$. into next 3 stitches, repeat from * to * 2 times, 3 dc. into next 3 stitches, repeat from * to *3 times, 6 dc. into next 6 stitches, repeat from * to * 4 times, I3 dc. into next I 3 stitches; turn.

I 3 th row, ch. $3,7 \mathrm{dc}$. into next 7 stitches, repeat from * to I time, 6 dc. into 11ext 6 stitches repeat from * to $* 7$ times, 6 dc. into next 6 stitches, repeat from * to *2 times, 3 dc. into next 3 stitches; turn.

I4th row, ch. 3,3 dc. into next 3 stitches, repeat from * to *3 times, 6 dc. into next 6 stitches, repeat from * to $* 5$ times, I3 dc. into next I3 stitches; turn.

I 5 th row, ch. 3,7 dc. into next 7 stitches, repeat from * to * I time, 6 dc. into next 6 stitches, repeat from * to * 2 times, 6 dc. into next 6 stitches, repeat from * to $* 5$ times, 3 dc. into next 3 stitches; turn.

I6th row, ch. $3,3 \mathrm{dc}$. into next 3 stitches, repeat from * to 3 times, 6 dc. into next 6 stitches, repeat from * to *2 times, 15 dc. into next 15 stitches; turn.

I7th row, ch. 3, 5 dc. into next 5 stitches, ch. 5, 4 dc. into next 4 stitches, repeat from * to $* 5$ times, $6 \mathrm{dc}$. into next 6 stitches, repeat from * to ${ }_{2}$ times, $3 \mathrm{dc}$. into next 3 stitches; turn.

I8th row, same as 2 nd row, etc.

\section{DIAMOND EDGING.}

Make a chain of 50 stitches; Ist row, 5 dc. into sixth stitch, * ch. 2, 5 dc. into third stitch from preceding *, repeat from * to * 4 times, ch. 2,2 dc. into third and fourth stitches from preceding, repeat from * to *6 times, ch. $2,2 \mathrm{dc}$. into third and fourth stitches from preceding, ch. 2, I dc. into last stitch of ch. 50 ; turn.

2nd row, ch. 5, 4 dc. into the 4 stitches immediately preceding shell *, ch. 2,5 dc. into next ch. $2 *$, repeat from * to * 4 times, ch. 2,6 dc. into the 
6 stitches following the shell, repeat from $*$ to $* 5$ times, join with s. s. to second dc. of last shell; turn.

3 d row, ch. $3, * 5$ dc. into ch. 2, ch. $2 *$, repeat from * to * 3 times, Io dc. into the Io stitches following the shell, ch. 2 , repeat from * to * 4 times, $6 \mathrm{dc}$. into the first six stitches following shell, ch. 2 , I dc. into third stitch of loop; turn.

4 th row, ch. 5,8 dc. into the 8 stitches immediately preceding shell, * ch. 2,5 dc. into ch. $2 *$, repeat from * to * 2 times, ch. 2,14 dc. into the 14 stitches following the shell, repeat from * to * 3 times, join with s. s. to 2 dc. of last shell; turn.

5 th row, ch. $3, * 5$ dc. into ch. 2 , ch. $2 *$, repeat from * to * once, $18 \mathrm{dc}$. into 18 stitches following shell, ch. 2, repeat from * to * 2 times, Io dc. into first 10 stitches following shell, ch. 2, I dc. into third stitch of loop; turn.

6 th row, ch. $5,12 \mathrm{dc}$. into the 2 stitches immediately preceding shell, ch. 2,5 dc. into ch. 2, ch. 2, 22 dc. into 22 stitches following the shell, ch. 2, $5 \mathrm{dc}$. into ch. 2 , join with s. s. into second dc. of last shell; turn.

7th row, ch. 4,4 dc. into last dc. of last shell in preced-

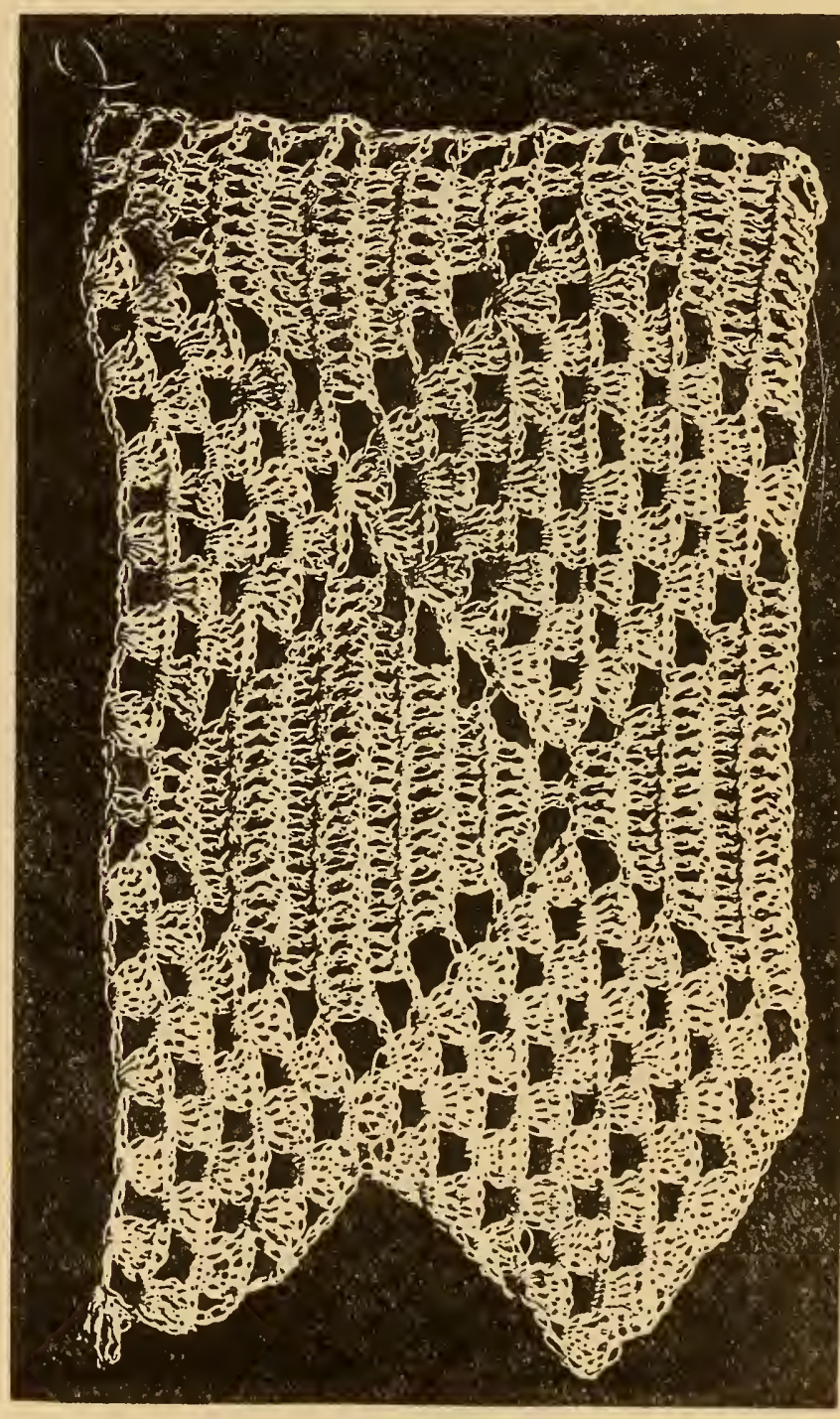

FIG. 299. ing row, (the 4 dc. with ch. 4 make the shell of five, as usual,) ch. 2,5 dc. into first dc. of last shell, ch. 2 , pass over 4 stitches and make i $8 \mathrm{dc}$. into next is stitches, ch. 2, 5 dc. into last dc. of shell, ch. 2, 5 dc. into first dc. of shell, ch. 2, pass over 4 stitches and make ro dc. into next Io stitches, ch. 2, I dc. into third stitch of loop; turn. 
Sth row, ch. 5,8 dc. into the first 8 dc. of preceding row, " ch. $2,5 \mathrm{dc}$. into first dc. of first shell, ch. $2,5 \mathrm{dc}$. into ch. 2, ch. 2, $5 \mathrm{dc}$. into last dc. of last shell, * ch. 2 , pass over 4 stitches and make I4 dc. into next I4 stitches, repeat from * to * once; turn.

9th row, ch 4, 4 dc. into last dc. of last shell, * ch. 2, 5 dc. into ch. 2, ch. 2,5 dc. into ch. 2 , ch. 2,5 dc. into first dc. of third shell, ch. $2 *$, pass over 4 stitches and make $10 \mathrm{dc}$. into next 10 stitches, ch. $2,5 \mathrm{dc}$. into last dc. of first shell, repeat from * to * once, pass over 4 stitches and make $6 \mathrm{dc}$. into next 6 stitches, ch. 2, I dc. into third stitch of loop; turn.

Ioth row, ch. 5,4 dc. into first 4 dc. of preceding row, ${ }^{*}$ ch 2.5 dc. into first dc. of first shell, ch. 2,5 dc. into ch. 2, ch. 2, 5 dc. into ch. 2 , ch. 2,5 dc. into ch. 2 , ch. 2,5 dc. into last dc. of fourth shell, * ch. 2 , pass over 4 stitches and make $6 \mathrm{dc}$. into next 6 stitches, repeat fiom * to * once; turn.

IIth row, ch. 4,4 dc. into last dc. of last shell, *ch. 2,5 dc. into ch. $2 *$, repeat from * to * 3 times, ch. 2 , 5 dc. into first dc. of fifth shell ch. 2 , qas:; over 4 stitches and make 2 dc. into next 2 stitches, ch. $2,5 \mathrm{dc}$. into last dic of first shell, repeat from * to * 4 times, ch. 2,5 dc. into first dc. of fifth shcll, ch. 2, pass over 4 stitches and make 2 dc. into next 2 stitches, ch. 2, I dc. into third stitch of loop: turn.

I2th row, same as 2nd row, etc.

\section{FERN TRIMMING.}

The heading of this trimming is worked shortwise, and the scallop edge lengthwise.

Make a chain of 7 stitches, join round. Ist row, $5 \mathrm{ch} ., 8 \mathrm{tr}$. under the ch.; turn.

2nd row, $4 \mathrm{ch}$. *, pass over I tr., I tr. into the next, I ch., repeat from * 3 times more, $4 \mathrm{ch}$., $8 \mathrm{tr}$. under $5 \mathrm{ch}$; t turn and repeat from second row for the length required.

For the edge, which is worked lengthwise:

Ist row, I dc. into a point of heading, $6 \mathrm{ch}$., I dc. into next point; repeat to the end of the row.

2nd row, I tr. into a stitch, I ch., pass over one stitch and repeat.

$3 \mathrm{~d}$ row, I dc. into each of 13 stitches, $5 \mathrm{ch}$., pass over 5 stitches, I tr. into each of 3 stitches, $5 \mathrm{ch}$., pass over 5 stitches repeat from the begin. ning of the row. 
$4^{\text {th }}$ row, I dc. into each dc. of last row, $4 \mathrm{ch}$., I tr. into last of $5 \mathrm{ch}$. and each of 2 next stitches, $3 \mathrm{ch}$., I tr. into same stitch last tr. was worked into and I into each of the 2 next stitches, 4 ch.; repeat from the beginning of the row.

$5^{\text {th }}$ row, I dc. into the second of $13 \mathrm{dc}$. and I into each of Io following stitches, 4 ch., I tr. into each of 3 next tr., I ch., 3 tr. into center of $3 \mathrm{ch}$., I ch., I tr. into each of 3 tr., 4 ch.; repeat from the beginning of the row.

6 th row, I dc. into the second and each of 8 following dc., 3 ch. *, r tr. into each of $3 \mathrm{tr}$., I ch., repeat from * once more, $3 \mathrm{ch}$., I tr. into same stitch the last tr. was worked into and I into each of 2 next stitches, I ch., I.tr. into each of 3 next tr., 3 ch.; repeat from the beginning of the row.

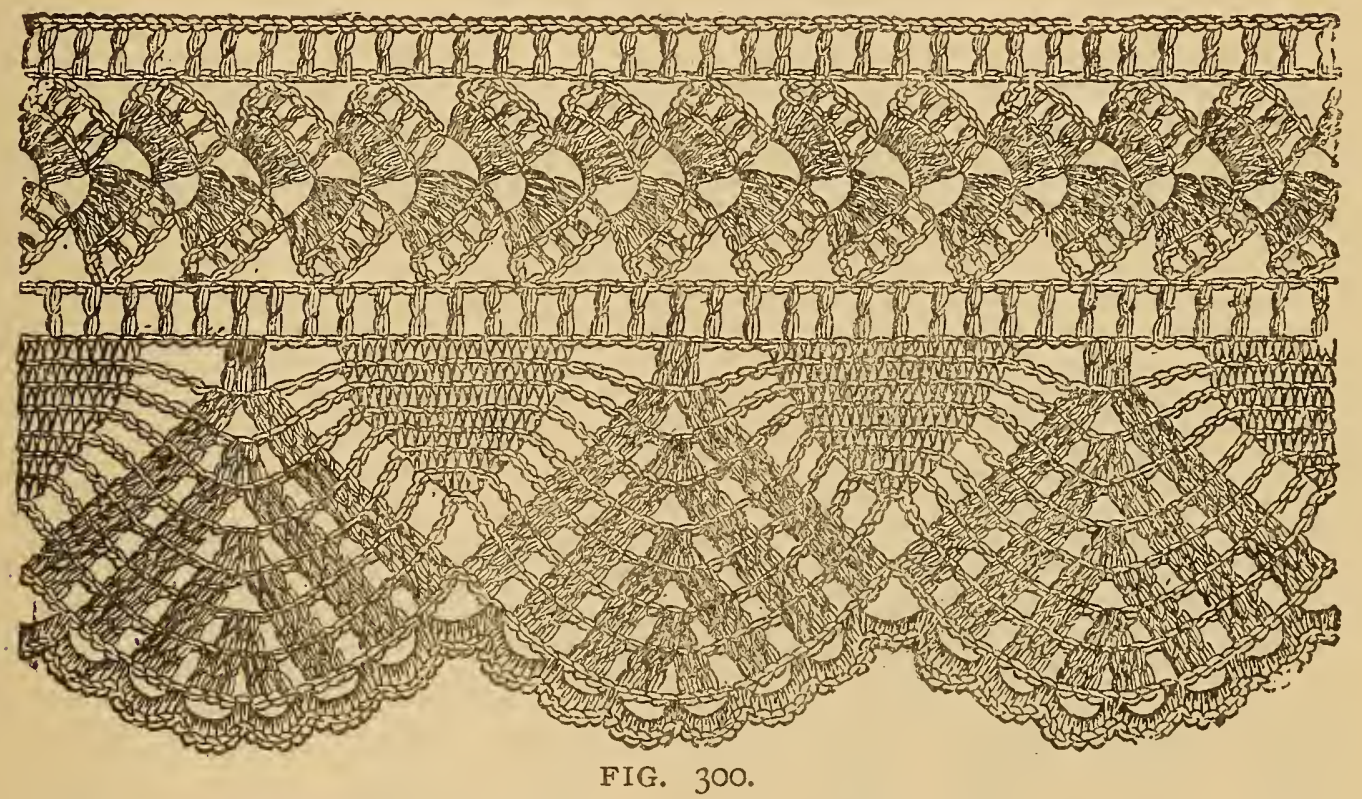

7 th row, I dc. into the second and each of 6 following dc., $3 \mathrm{ch}$. *, I tr. into each of 3 next tr., I ch., repeat from * once more, I ch. 3 tr. into center of 3 ch., I ch.. I tr. into each of 3 next tr., I ch., I tr. into each of 3 next tr., $3 \mathrm{ch}$; repeat from the beginning of the row.

8 th row, I dc. into the second and each of 4 following stitches, 3 ch. *, I tr. into each of 3 next tr., $2 \mathrm{ch}$., repeat from * once more, 2 tr. into next stitch, I into the next, 3 ch., I tr. into same stitch last was worked into, 2 into the next $*, 2$ ch., I tr. into each of 3 tr., repeat from last $*$ once more, 3 ch.; repeat from the beginning of the row.

9th row, I dc. into each of 3 center stitches of dc., 2 ch. *, I tr. intc each of 3 tr., repeat from * 6 times more, working the center 3 tr. into cen- 
ter of $3 \mathrm{ch} ., 3 \mathrm{ch}$.; repeat from the beginning of the row.

Ioth row, * I tr. into each of 3 tr., 2 ch., repeat from * twice more, 2 ch., 2 tr. into next tr., I into the next $2 \mathrm{ch}$., I tr. into same stitch last worked into, 2 into the next $*, 2$ ch., 2 tr. into each of 3 next tr.; repeat from last * twice more, then repeat from the beginning of the row.

I I th row, I dc. under first I ch. of scallop *, $4 \mathrm{ch}$., I dc. under the next $2 \mathrm{ch}$; repeat from the beginning of the row.

I 2 th row, $6 \mathrm{dc}$. under each $4 \mathrm{ch}$. of last row.

On the other side of the heading stripe work 2 rows like the 2 first rows of edge.

\section{CROCHET TRIMMING.}

This trimming is worked lengthwise. Make a chain the length required.

Ist row, I tr. into a stitch, 2 ch., pass over 2 stitches and repeat.

2nd row, I dc. into each stitch of previous row.

$3 \mathrm{~d}$ row, I sc. into each of 6 stitches, 16 ch., work down the chain with I sc. into first stitch, I dc. into next, I half tr. into each of Io next stitches, I tr. into each of the next stitches, pass over I stitch of last row, then repeat from the beginning of the row.

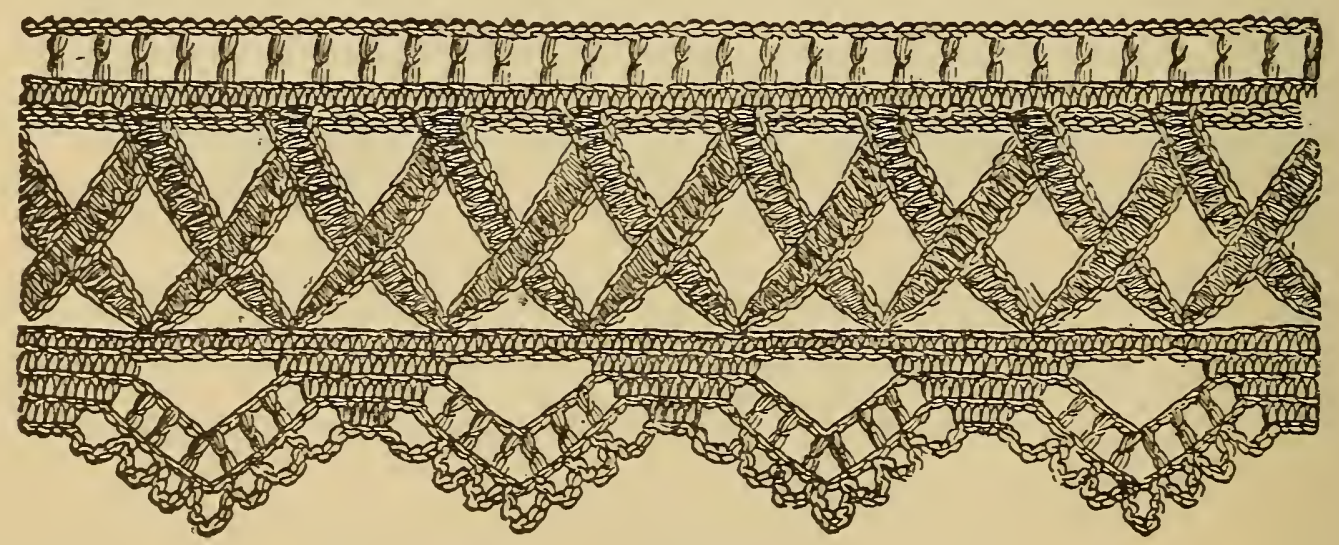

FIG. 30I.

Turn the work, and work a row of points at the back of the last row in exactly the same way as already described.

4th row, turn the two points which lie.together, one to the right, the other to the left, and interlace them, as shown in the illustration, work I sc. into 2 points together, $7 \mathrm{ch}$; repeat from the beginning of the row. 
$5^{\text {th }}$ row, I dc. into each stitch of last row.

6th row, I dc. into each of 9 stitches of last row, I I ch., pass over 9 stitches and repeat from the beginning of the row.

7 th row, I dc. into each of the 7 center stitches of 9 dc., * 2 ch., pass over I stitch, I tr. into the next, repeat from * twice more, $3 \mathrm{ch}$., I tr. into same stitch last tr. was worked into, *2 ch., pass over I stitch, I tr. into the next, repeat from last* once more, 2 ch., then repeat from the beginning of the row.

8th row, I dc. into each of 3 center stitches of 7 dc., 4 ch., pass over 4 stitches, I dc. into the next, * 4 ch., pass over 2 stitches, I dc. into the next, repeat from * 5 times more, $4 \mathrm{ch}$.; repeat from the beginning of the row.

\section{NARROW EDGING.}

The edging is worked backwards and forwards as follows: I4 ch., turn; I sc. in the ninth, ch. (the last stitch is reckoned here and afterwards as the first stitch), 7 ch., I sc. in the I $4^{\prime} h \mathrm{ch}$.; turn; * $7 \mathrm{ch}$., I dc. in the sixth of these $7 \mathrm{ch}$., I ch., I sc., in the fourth of the next $7 \mathrm{ch} ., 4 \mathrm{ch}$., I dc. in the last but one of these $7 \mathrm{ch}$., I ch., I sc. in the fourth of the $8 \mathrm{ch}$.; turn.

$8 \mathrm{ch}$., I sc. in the first of the $4 \mathrm{ch} ., 7$ ch., I sc. in the first ch. of the following ch. curve beside the top of the dc., I 3 ch.; turn.

Loop on to the last ch. beside the

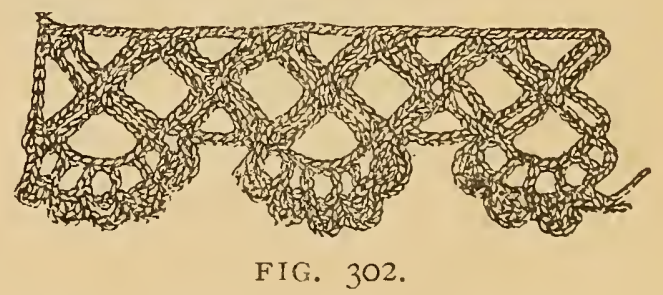
foot of the dc., I ch., I sl., I sc. and I dc. in the second-fourth ch., 2 ch., in the course of the work loop here on to the last picot of the foregoing curve, 2 ch., I sc. in the last dc., I ch., I dc. (this like the following dc. are worked into each of the next ch.), I picot (here and later on of $4 \mathrm{ch}$., and I sc. in the last dc.), I ch., I dc., I p., I ch., I dc., I p., i ch., I dc., I p., I ch., I dc., I sc. and I sl. in the next 2 ch., 4 ch., I dc. in the last ch. but one, I ch., I sc. in the fourth of the $7 \mathrm{ch}$., $4 \mathrm{ch}$., I dc. in the sixth of the same 7 ch., I ch., I sc. in the fourth of the $8 \mathrm{ch}$; turn; $8 \mathrm{ch}$., I sc. in the first of the $4 \mathrm{ch} ., 7 \mathrm{ch}$., I sc., in the first of the next 4 ch., turn, and repeat from star.

\section{BROAD EDGING.}

The scallops of the wide cotton braid are to be laid over each other as seen, and in the straight edge thus made are crocheted alternately I dc. and I ch. 
2nd row, repeat constantly I dc. in every second dc., $3 \mathrm{ch}$. and $4 \mathrm{dc}$. round the single dc.

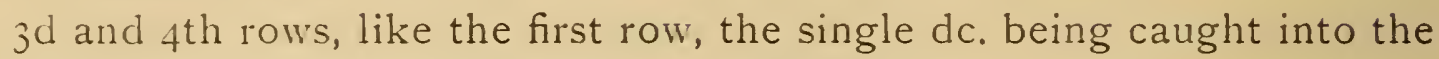
first of the $4 \mathrm{dc}$.

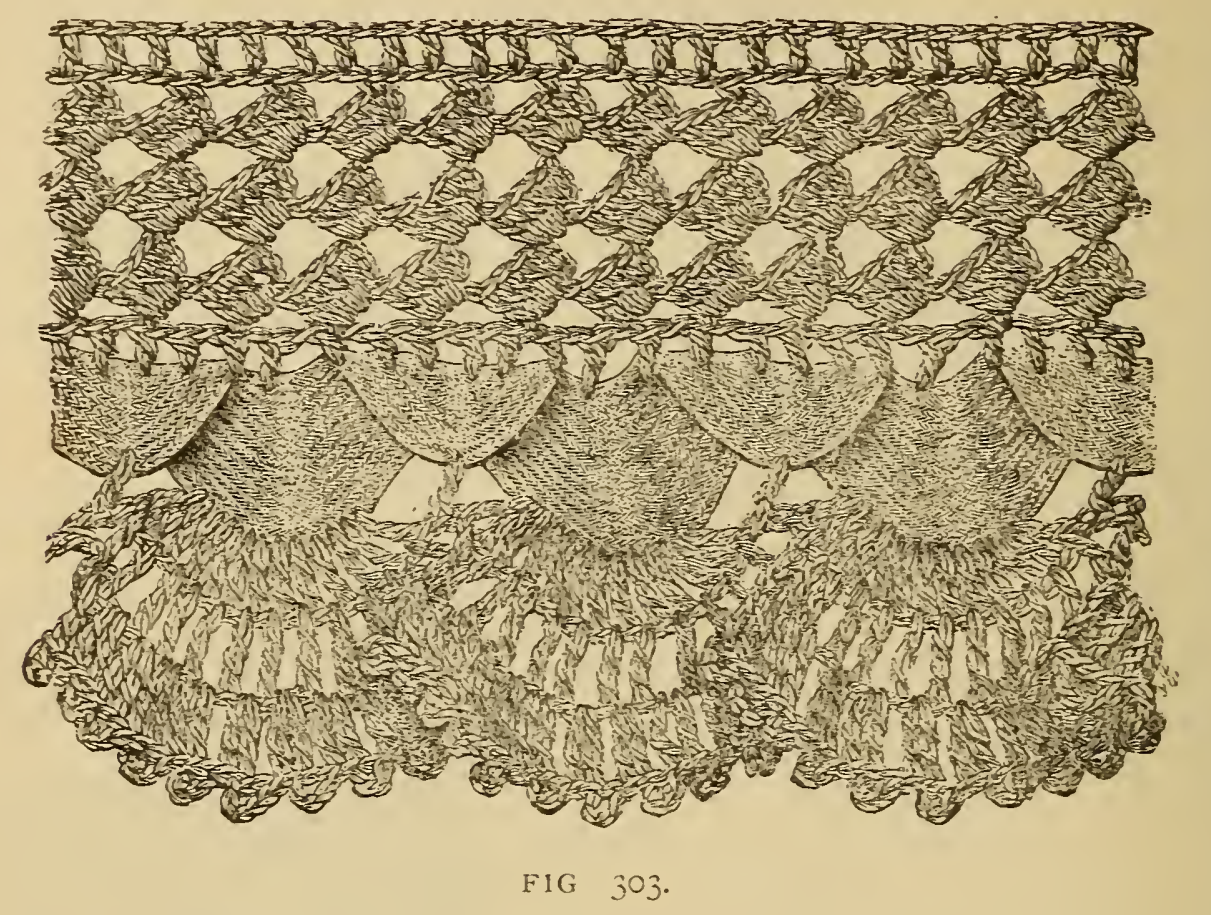

5th row, alternately I sc., in the first of the $4 \mathrm{dc}$. and I ch; 6th row, alternately I dc. and I ch.

The lower, curved edge fulled somewhat, requires: Ist row, alternately I tr. in the braid curve turned over, and 13 tr., separated by I ch. in the next curve not turned over.

2nd row, round each single ch. I tr., and between these $2 \mathrm{ch}$; $3 \mathrm{~d}$ row, round each of the $2 \mathrm{ch}$. $2 \mathrm{tr}$, and between these groups of dc. a picot of 4 ch. and I sc, back into the last dc.

\section{NARROW CORAL EDGING.}

This edging is worked of Io ch., I sc., back into the ninth ch., II ch., I sc. in the third last but one of the Io ch., I ch., I dc., in the last of the ro ch., *广 5 ch. loop on to the sixth of the II ch. (the stitch to be looped on had better be slipped off first, the hook inserted into the link required, then the stitch taken up again and both stitches now on the hook meshed 
off, putting the cotton over once), 5 ch., I double long tr. in the dc., 5 ch., loop on as before, II ch. again loop on as before, $5 \mathrm{ch}$. $t$, loop next on to

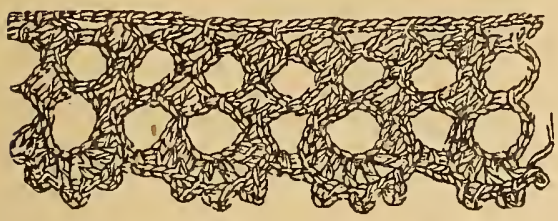

FIG. 304 . the first sc., later on always at the place where the $6 \mathrm{ch}$. were slung on, $9 \mathrm{ch}$., loop on to the sixth of the II ch. left hanging; turn. $5 \mathrm{ch}$., I dc., work three times $2 \mathrm{ch}$. and I dc. (these $4 \mathrm{dc}$. are worked into each of the second of the $9 \mathrm{ch}$.), 2 .ch., I sc. worked first into the first sc., later on again after the 6 ch., turn.

5 picots of $4 \mathrm{ch}$. and I dc. back into the last.sc., between the picots 1 sc. always in to the dc. after the last picot, I sc. in the sixth of the II ch. already caught up, II ch., I sc. in the ch., before the double long tr., I ch., I dc. in this double long tr.; repeat from cross to cross, then loop on to the sc. after the last picot, $6 \mathrm{ch}$., loop on to the sixth of the II ch., II ch., I sc. in the last ch. before the double long tr., 1 dc. in the double long tr., and repeat from star.

\section{RETICELLA CROCHET.}

The pattern here given is at once beautiful and of practical service as it can be varied in width and used for many different purposes. Figure 306 shows the design as a border, and figure 305 shows it used as an insertion for trimming dinner cloth in place of drawn work. This crocheted lace has this great advantage over the same amount of drawn work, that it requires less than one-quarter the time to make, is comparatively no tax on the eyes, and looks equally well; made of fine Scotch crochet linen thread it will last a life-time. If any one wishes a narrow insertion one row of the square and stars bordered by an edge will be very pretty.

This trimming is not difficult, yet requires some practice in crochet. One row of the three pattern shapes of which the trimming is composed give alone a pretty insertion, and are crocheted separately, beginning in the middle, yet must be joined in the course of the work, see the illustration. Each square is begun with I $2 \mathrm{ch}$. closed to a ring; then follow: $5 \mathrm{ch}$., I tr. in the last ch., which finishes the ring, ${ }^{*} 5$ ch., 2 tr., meshed off looping the cotton over once, in the first ch., $7 \mathrm{ch}$., r sc. in the next ring-ch., y ch., 2 tr. meshed together looping cotton over once, in the next ring-ch. and repeat three times from star. . 
358 DAINTY WORK FOR PLEASURE AND PROFIT.

10. 4.

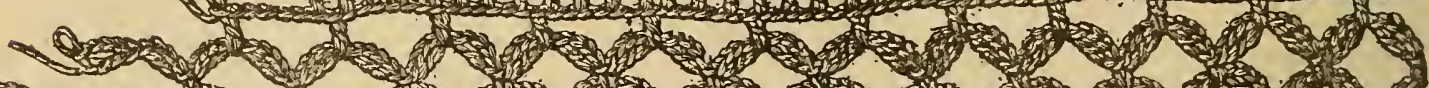

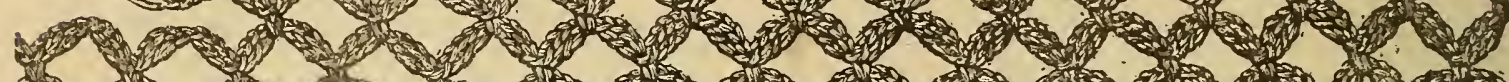
1.

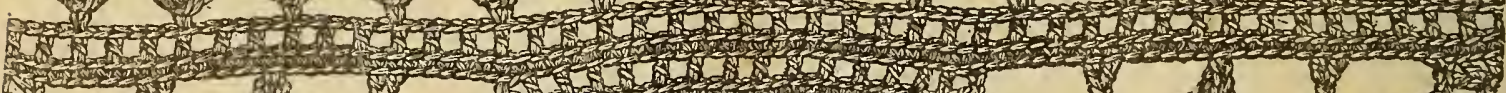

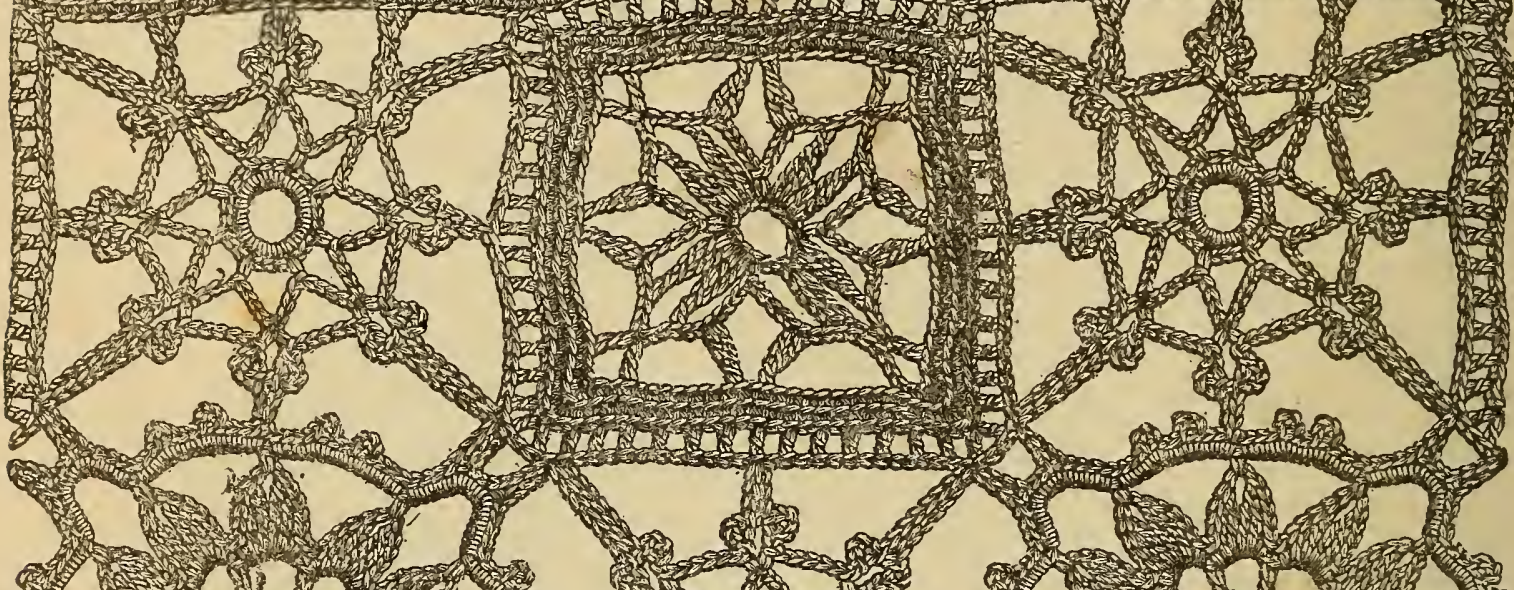
4. (4)

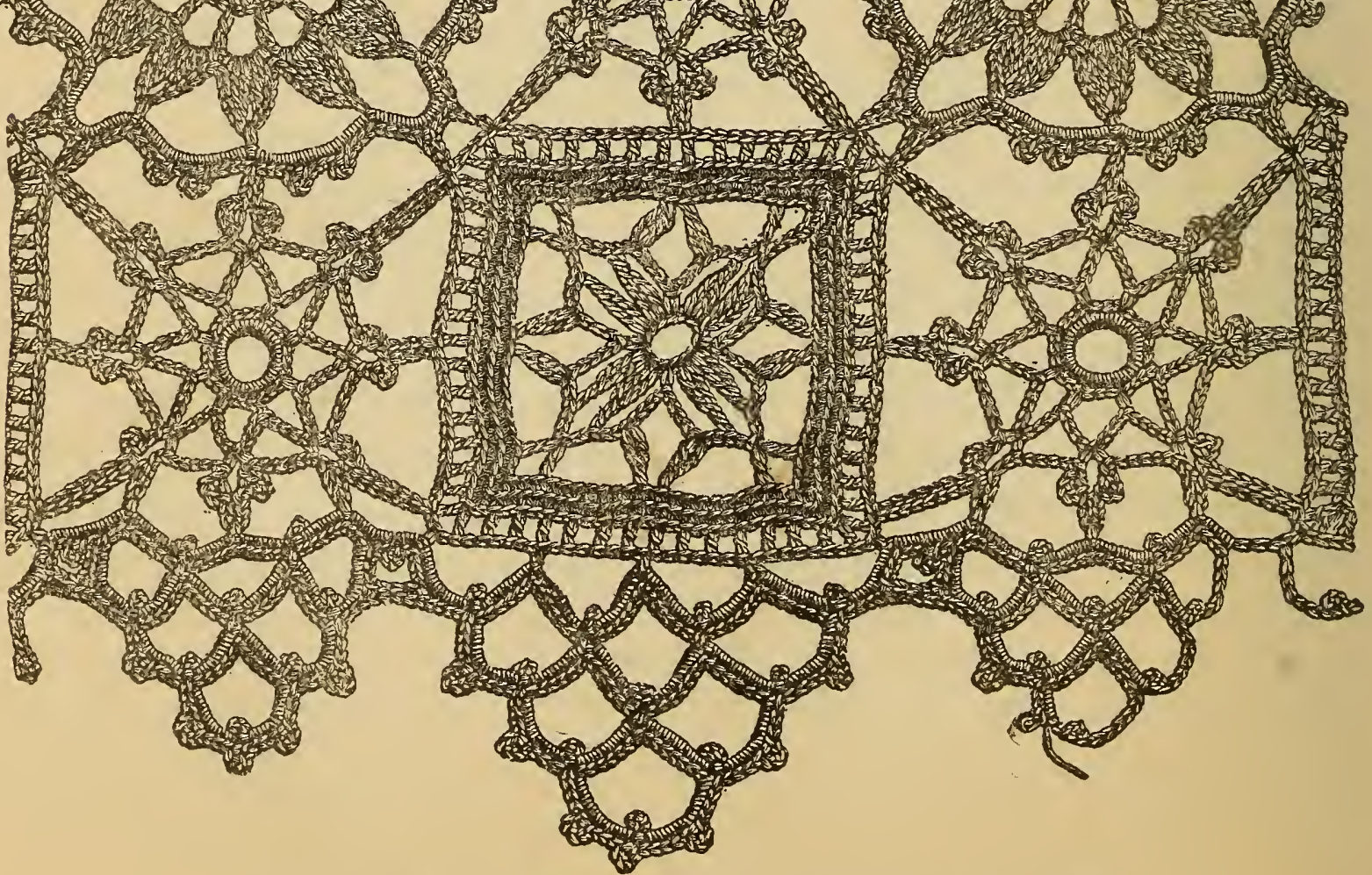

CROCHETED RETICELLA LACE. FIG. 306. 
In repeating for the third time after the second $7 \mathrm{ch}$. pass over with 3-4 sl. the first group of bars and the next $2 \mathrm{ch}$., then, as a foundation for the thick edge, work $8 \mathrm{ch}$., I dc. in the third of the $5 \mathrm{ch}$., therefore beside the last sl., $7 \mathrm{ch}$., work always twice 2 tr., meshed together, looping cotton over twice; the first group of bars is worked into the third of the first $7 \mathrm{ch}$., the second into the fifth of the next $7 \mathrm{ch} ., 7 \mathrm{ch}$., I dc. in the third of the $5 \mathrm{ch}$., $5 \mathrm{ch}$., I dc. again in the third ch. and repeat from star. After the last $7 \mathrm{ch}$. I sl. in the third of the $8 \mathrm{ch}$. with which the row was begun. 2 rows of sc. are now crocheted stitch for stitch, and for the corners $3 \mathrm{sc}$. always worked in the third of the $5 \mathrm{ch}$. A row of I dc., I ch. completes the square, 2 dc. being always worked in each corner mesh.

The rosette-like shapes are begun with a ring of 8 -10 ch. into which are worked after $3 \mathrm{ch}$. (in place of first dc.) $23 \mathrm{dc}$. Then I sl. in the third ch., $3 \mathrm{ch}$. (in place of first dc.) and I I times $3 \mathrm{ch}$. and I dc. in every second dc., 3 ch., I sl. in the fourth ch., * I sc. round the 3 ch., 4 ch., 2 tr. each meshed together looping the cotton over once, 3 ch., again 2 such tr., 4 ch., and I sc., these and the group of bars round the same $3 \mathrm{ch}$., as also the I sc., repeat I I times from star. Work up to the top of the first scallop with sl. here work I sc. round the $3 \mathrm{ch}$., then 12 times $7-9 \mathrm{ch}$. and I sc. round the $3 \mathrm{ch}$., I sl. in the I sc., round each scallop 4 sc., I p., ( $4 \mathrm{ch}$. and I sc. back into the last sc.) 3 sc., I p., 4 sc., then loop on to the first sc., fasten the thread and cut off carefully.

The third star-like shape begins with a ring of 8-10 ch., in which are worked 24 sc. Now follow I sl. in the first sc., * 9 ch., I sc. in the third following sc. but one; repeat from star; the last sc. is caught into the sl., then work $5 \mathrm{sl}$. in the next $5 \mathrm{ch}$., * I p., I 3 ch., I sc., in the foot of the picot I p., I sc. in the fifth ch., which catches up the last sl., $5 \mathrm{ch}$., I sc. in the fifth ch., I p., 5 ch., I sc. in the foot of the picot, I p., I sc. in the fifth ch. already caught up, $5 \mathrm{ch}$., I sc. in the fifth ch., and repeat from star. The first row at the lower pointed edge is worked backwards the whole length. To each square are joined 4 scallops of 6 ch., I p. turned downwards, $6 \mathrm{ch}$. and I sc. (see figure 306), while above each star shape the following stitches are crocheted: 5 ch., I sc. in I p., 4 ch., I p.turned downwards (see figure 306), 4 ch., I sc. in I p., again 8 ch. scparated by I p., again I sc., 5 ch., and the first sc. in the corner of the square. Beginning with the small point, this is now completed separately, and in rows backwards and forwards, as 


\section{DAINTY WORK FOR PLEASURE AND PROFIT.}

already mentioned above. Round the chain curves are worked 6 as also I 2 sc., where they are not interrupted by a picot, yet where this takes place after figure 306, 4 sc. are worked before the picots and between, and after these always $3 \mathrm{sc}$.

The curves on the large pointed scallop are each of 6 ch., I p. turned downwards, and 6 ch. They are crocheted where the picots are wanting, with 7 and also I4 sc., while $4 \mathrm{sc}$. always go before, separate, and follow the picots.

Figure 306 explains the upper edge, therefore it is only $\mathrm{n}$-cessary to add that the small leaf-curves, each separated by I sc., are of $4 \mathrm{ch}$. and $\mathrm{I} d \mathrm{~d}$. back into the first ch. worked twice.

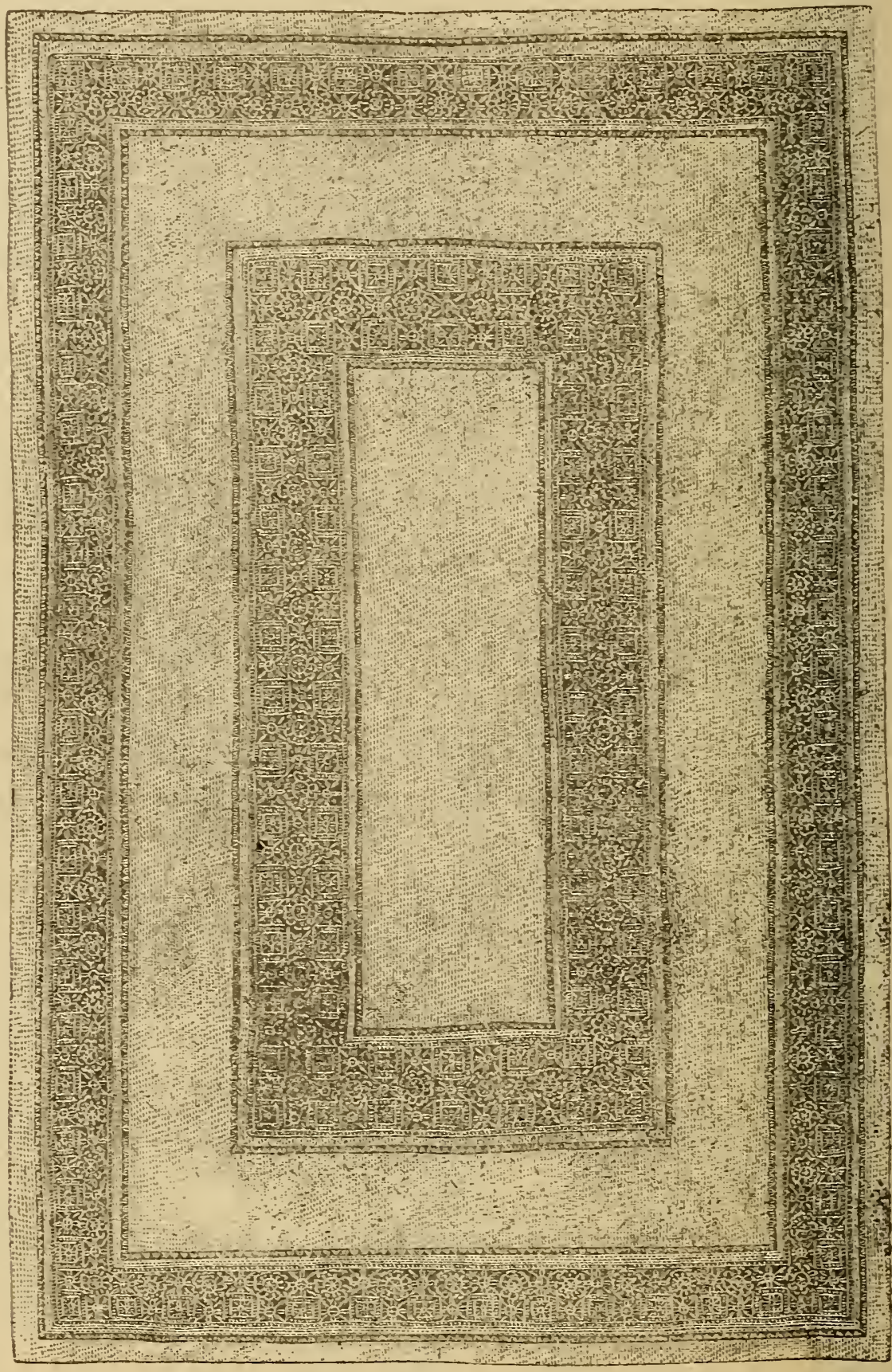

TABLE COVER. FIG. 305.

The above description has been very carefully verified and there will be no trouble in following. When used as an insertion for table cloth, it should be set in with a double hem on the outside with a row of hemstitching on both edges. 
CROCHET TOP PETTICOAT FOR CHILD. FIGURES 307-309.

Crochet pattern of bodice, figure 307. Materials: I $1 / 4$ pound of red wool, and a few skeins of white Berlin wool.

This petticoat is to be worn under the dress, and is not at all difficult to crochet. The work is begun at the neck-opening with a foundation of I29 ch., then working forwards, after 2 ch., more, alternately 4 dc., separated in the middle by 2 ch., in the iast foundation mesh and each fourth following mesh but one, so that 33 groups are made on the whole. The whole bodice is crocheted, going backwards and forwards, in this simple pattern, but from here the $4 \mathrm{dc}$. are worked round the $2 \mathrm{ch}$. in the former row. Each row is begun with $2 \mathrm{ch}$.

In the and row the worker has to increase the meshes for the width of the shoulder at the fifth group of dc., and to crochet instead 3 times 2 dc. always separated by $2 \mathrm{ch}$. round the $2 \mathrm{ch}$., this being repeated at the Ioth, 24 th and 29th groups, the row is finished with 4 further groups.

With the $3 \mathrm{~d}$ row which has 37 groups of dc. on account of the increase, the shoulder strap is completed.

In the $4^{\text {th }}$ row II ch. are cast on for

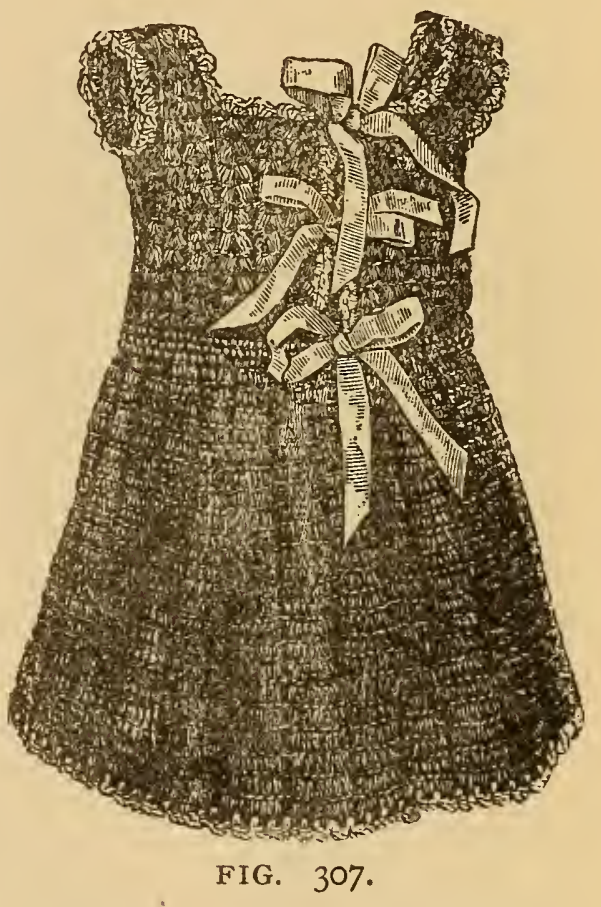
the armhole after 5 groups of dc., and then 7 groups of the former row

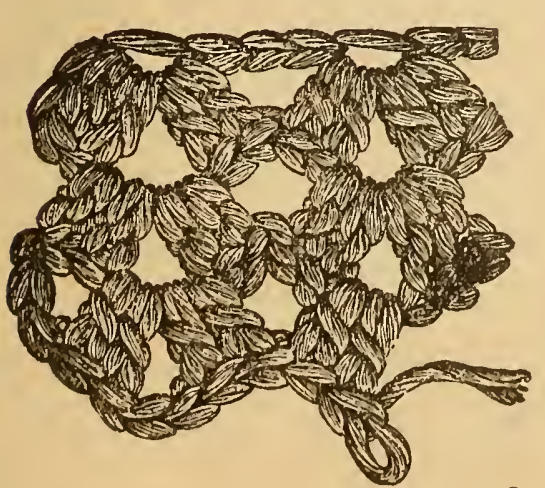

DETAIL OF WAIST. FIG. 308. passed over; when 13 groups of dc. have been crocheted II stitches are again cast on for the second armhole and 7 groups passed over.

$5^{\text {th }}$ row, a fresh group of dc. is always worked in the fourth and eighth foundation mesh after 5 groups of the same and this is repeated after I3 groups; this row is of 27 groups including the next following 5 groups.

The 6th to 3 th rows have the same number of groups and complete the bodice. For the waistband follow r row of sc., and over each group of dc. come 5, over the first and last always 4 sc. 
these being caught into the upper link of each mesh and I row of dc. into the whole link; each of these rows has 133 meshes. The skirt is now begun and requires larger groups of dc., increased gradually in size going lownwards, as seen in the full size on figure 307 .

Ist row, I dc. in the first mesh, $3 \mathrm{dc}$. in the following one, again I dc. in the next mesh; this group is repeated 26 times passing over 2 meshes every time.

and to 4 th row, like the Ist; the three dc. are each worked into the second of the $3 \mathrm{dc}$. of the former row. As the skirt slit is now long

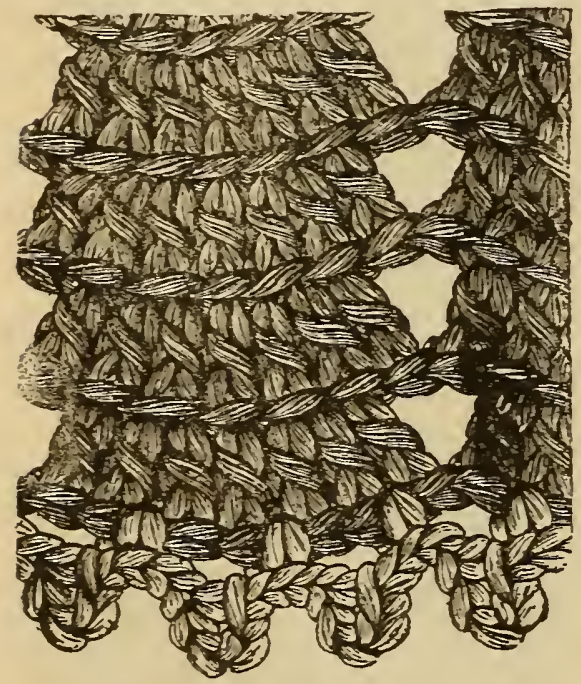

DETAIL OF SKIRT. FIG. 309. enough, the crochet is worked round from this time.

$5^{\text {th }}$ to 7 th row, like the former one.

8th row, alternately I group of $j \mathrm{dc}$. and I group of $6 \mathrm{dc}, 2$ dc. being always worked at the last one of these into the three middle dc. of the former row.

gth row, only of groups of $6 \mathrm{dc}$, these being caught alternately into the 3 middle meshes of the 5-part group, and into the 2nd-5th dc. of the 6-part group.

Ioth to $5^{\text {th }}$ row, groups of $6 \mathrm{dc}$., like the last described; I6th row, groups of $7 \mathrm{dc}$., which catch up the second $\mathrm{dc}$. singly, the 3 rd- 5 th dc. always in twos.

I 7 th to 22 nd row, like the I6th row of groups of $7 \mathrm{dc}$., the first, fourth and seventh dc. being caught into the second, fourth and sixth dc. of the former row, the second and third dc. into the third dc. of the same, and the fifth and sixth dc. into the fifth dc. The skirt is now finished and picots each of I sc., 5 ch., I sc. back into the second ch. and I ch., are crocheted on at the edge with white wool. Curves each of 2 sc., I half dc., I dc., I half dc. and 2 sc., the latter being each caught round the ch, are also worked with white wool round the neck-opening and back edges of the bodice. An edging of 2 rows is worked round the sleeve.

Ist row, groups of dc. like those on the bodice; 2nd row, 4 to $5 \mathrm{sc}$. each made round the $2 \mathrm{ch}$. between the dc. The petticoat is fastened at the back with strings. 


\section{CROCHET TASSEL.}

This illustration shows in the proper size, and without tassels, a trimming for bordering small tables. It is crocheted with German wool in three shades of brown. The left side of the work is turned to the outside when the trimming is used, and enriched with gold tinsel. The upper scallops are made first with the middle shade and in constant repetition, as follows: $5 \mathrm{ch}$. and in these going backwards and leaving the last unworked,

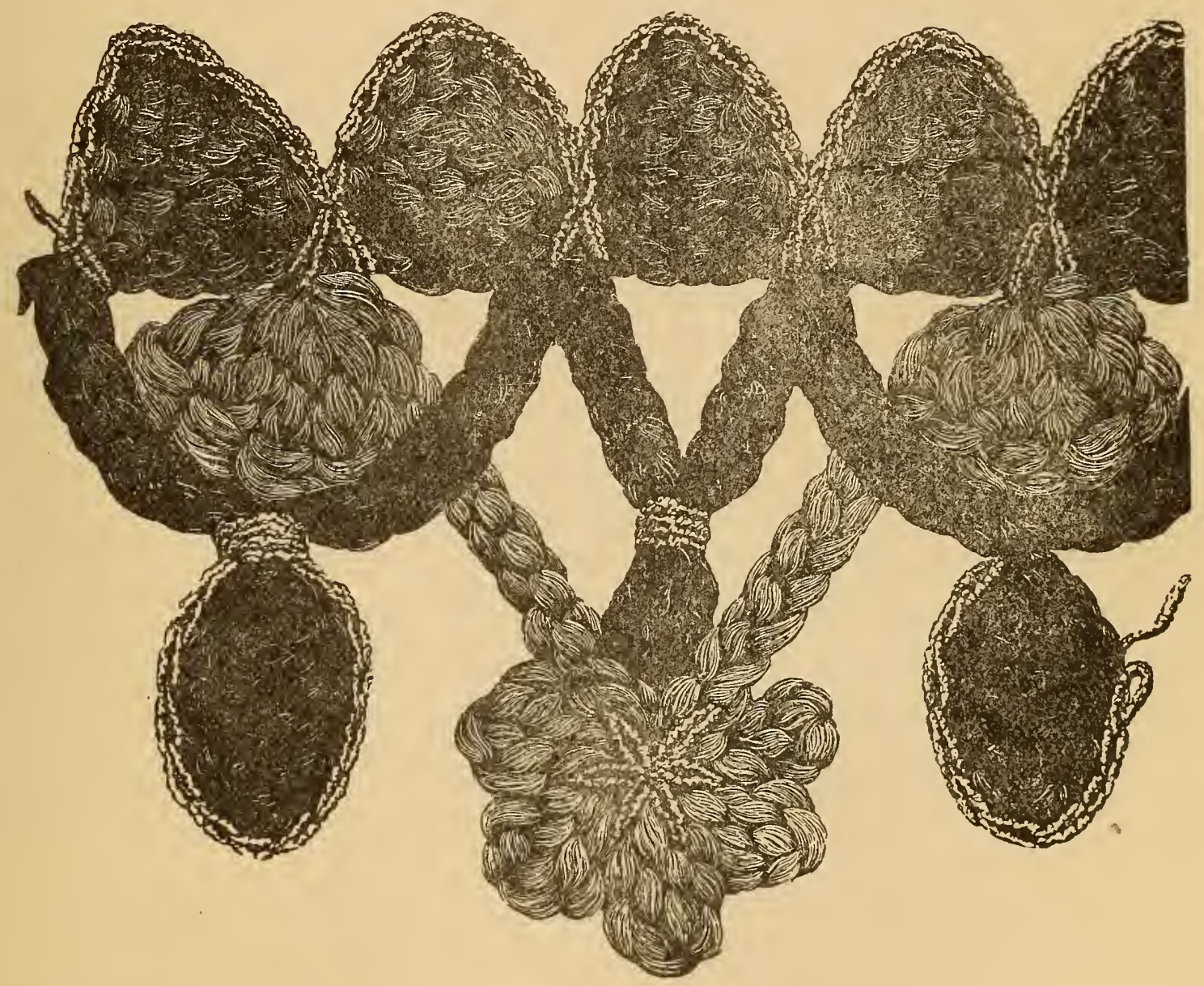

FIG. 3 IO.

I sc., I half tr., I dc., and I tr. At the foot of the points the next row is worked with the darkest wool: I sl. in the hollow of a scallop, * i 2 ch., passing over the last, back into the Irth-7th ch., I sc., $3 \mathrm{dc}$. and I sc., $6 \mathrm{ch}$. I sl. in the second next hollow of a scallop but one, 13 ch., take the hook out of the thread loop and put this from below to above through the sixth ch., 5 ch., I sl. in the next hollow of a scallop and repeat from star. 
The 3 d row now follows with the lightest wool, leaving the second row at the back untouched: * In the hollow of the scallop, which is not caught up by the darkest wool, $5 \mathrm{dc}$, $6 \mathrm{ch}$. in the fourth of the $7 \mathrm{ch}$. which form a ring of the darkest wool, 6 sl. each separated by $5 \mathrm{ch}$., $6 \mathrm{ch}$. and repeat from star. Chain stitches of tinsel thread border the upper scallops and the ovals in the second (darkest) row; the rows of chain above the same being wound over several times with tinsel thread, after which, starting from the left side, the middle mesh in the group of bars in the lightest row is united invisibly with the darkest by a few stitches, fastening at the same time both ends of the tinsel thread. The half rosettes of the light wool are then ornamented with long star-like stitches of the latter, and the chains of the two dark shades united by twisting them several times with the same. To make tassels to susqend from each of the points, take about 40 threads, each from $43 / 4$ to $5 \frac{\mathrm{T}}{2}$ inches long of the middle shade; twist over very tight in the middle with black thread, and then about $3 / 8$ inches from below with tinsel thread. Fasten each tassel on the left of the light half rosette by drawing a loop of wool through the black thread.

\section{DAISY CROCHET LACE.}

Commence with the circles which form the heading, * 4 ch., I sc., into the second, repeat from * 7 times more, draw through the first $\mathrm{ch} ., 3 \mathrm{ch}$. to take the place of a tr., * 5 ch., I tr. between 2 next picots, repeat from last * 6 times more, $5 \mathrm{ch}$., draw through third of first $3 \mathrm{ch}$.

In working the next and following circles, draw through the center of one of the $5 \mathrm{ch}$. Continue to work the circles until you have the length required.

For the Ist straight row of heading, work I double tr. into center of 5 ch. at left hand of a circle (see figure $3 \mathrm{II}$ ), I double tr. in center of $5 \mathrm{ch}$. of next circle, $6 \mathrm{ch}$., I tr. into center of next $5 \mathrm{ch}$., $6 \mathrm{ch}$., repeat from the beginning of the row.

2nd row, I tr. into a stitch, I ch., pass over I stitch and repeat. Work 2 rows like these on the other side of circles.

$3 \mathrm{~d}$ row, I tr. into a stitch, $2 \mathrm{ch}$., pass over 2 stitches, and repeat.

4 th row, I dc. into each of 16 stitches, $9 \mathrm{ch}$. pass over 6 stitches, and repeat from the beginning of the row. 
$5_{\text {th }}$ row, I dc. into the second and each of 13 following dc., 2 ch., pass over the first of $9 \mathrm{ch}$., I tr. into each of 3 next stitches, 2 tr. separated by 2 ch. into the next, I tr. into each of 3 next stitches, 2 ch.; repeat from the beginning of the row.

6 th row, I dc. into the second and each of II following dc., 2 ch., I tr., I ch. into the second and each of 2 next tr., I ch., 2 tr., $3 \mathrm{ch}$. under $2 \mathrm{ch}$., I ch., I tr. into each of 3 tr., 2 ch.; repeat from the beginning of the row.

7 th row, I dc. into the second and each of 9 following dc., 3 ch., I tr., I ch. into each of 4 tr., I ch., I tr. into next stitch, I ch., 2 tr. separated by $2 \mathrm{ch}$. into the next stitch, I ch. I tr. into next stitch, I ch. I tr. into each of 4 tr., 3 ch.; repeat from the beginning of the row.

8th row, I dc. into the second and each of 7 following dc., 4 ch., 22 tr. over the tr. of last row the 2 center tr. are worked into, I stitch, and are separated by 2 ch., 4 ch.; repeat from the beginning of the row.

gth row, I dc. worked into each of the 3 center dc. of last row, 5 ch., 24 tr. each separated by I ch. over the tr. of last row, the 2 center tr. are separ-

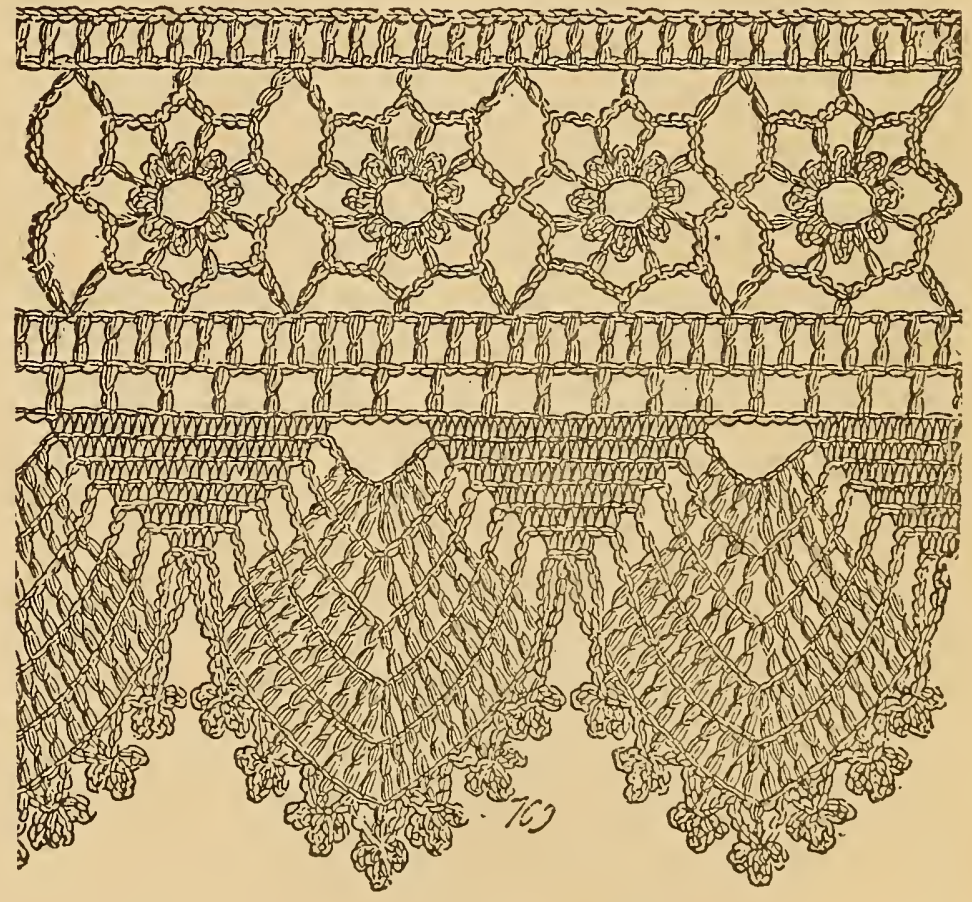

FIG. 3II. ated by $2 \mathrm{ch}$. are worked into I stitch, $5 \mathrm{ch}$; repeat from the beginning of the row.

Ioth row, I sc. into center of 3 dc., 6 ch., I sc. into first tr., 3 ch., pass over 3 stitches, I dc., into next stitch, 3 ch., I sc. into the first, 4 ch., I sc. into last sc., 3 ch., I sc. into last sc., I dc. into next stitch of last row, 3 ch., pass over 2 stitches, I dc. into the next, * 7 ch., I sc. into the fourth, 4 ch., I sc. into last sc., pass over 2 stitches of last row, I tr. into the next, 3 ch., I sc. into the first, $3 \mathrm{ch}$., pass over 2 stitches and repeat from * 4 times more, 
then work anoiher cluster like the first cluster of scallop, $6 \mathrm{ch}$; ; repeat from the beginning of the row.

\section{CROCHETED GUIPURE LACE.}

This design is suitable for border for table cover or bedspread. To work, use Scotch linen crochet thread, No. 70 . It is also effective crocheted with coarse cotton and gummed, after which pin it out on a board to dry;

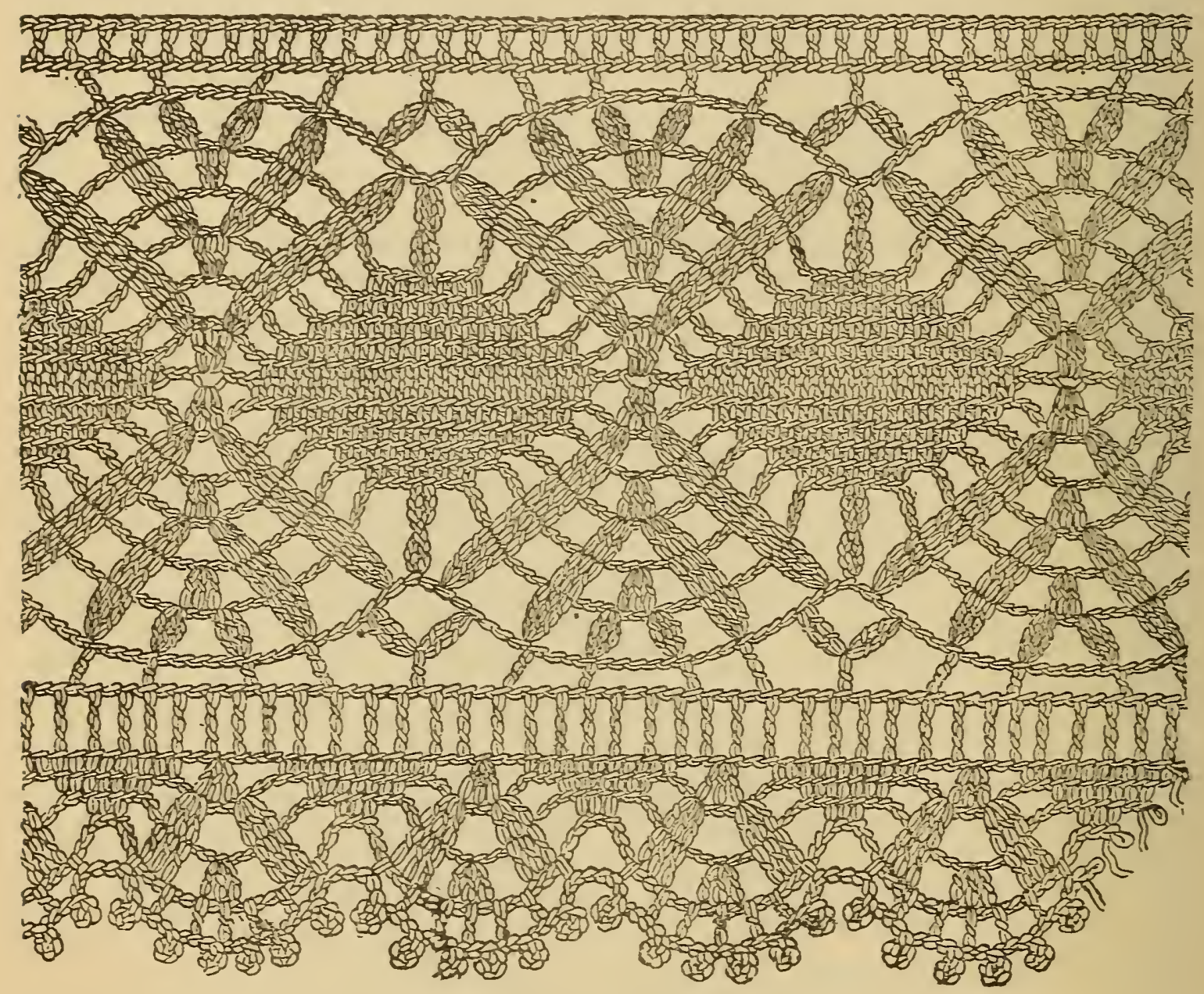

CROCHETED GUIPURE LACE. FIG. 3I2.

when dry and stiff, coat with gold paint. This will make a most novel and effective drapery for brackets, wall baskets, etc.

Commence in the center of the pattern with a chain the length that you wish the finished work to be.

Ist row, work I dc. into each of 22 stitches, 4 ch., pass over 3 stitches, $3 \mathrm{tr}$. into the next, $4 \mathrm{ch}$., pass over 2 stitches and repeat from the beginning of the row. 
2nd row, I dc. into the third and each of the 17 following dc., 3 ch., 3 tr. into the last of $4 \mathrm{ch} ., 4 \mathrm{ch}$., 3 tr. into the first of next $4 \mathrm{ch}$., $3 \mathrm{ch}$.; repeat from the beginning of the row.

$3 \mathrm{~d}$ row, I dc. into the third and each of $\mathrm{I} 2$ following dc., $3 \mathrm{ch}$., I tr. into each of 3 tr., 3 ch., 3 tr. into center of next 4 ch., 3 ch., I tr. into each of 3 next tr., 3 ch.; repeat from the beginning of the row:

$4^{\text {th }}$ row, I dc. into the third and each of 7 following dc., $3 \mathrm{ch}$., I tr. into each of 3 next tr., 3 ch., 3 tr. into next tr., 3 ch., pass over I tr., 3 tr. into next, $3 \mathrm{ch}$., I tr. into each of 3 next tr., $3 \mathrm{ch}$; repeat from the beginning of the row.

$5_{\text {th }}$ row, $I$ tr. into the second and into each of the 5 following dc., 3 ch., I tr. into each of the 3 next tr., 4 ch., I tr. into each of 3 next tr., 3 ch., 3 tr. into 3 ch., 3 ch., I tr. into each of 3 next tr., 4 ch., I tr. into each of 3 next tr., 3 ch.; repeat from the beginning of the row.

6th row, 9 ch., I sc. into center of dc., 3 ch., I sc. into fourth of $9 \mathrm{ch}$., $3 \mathrm{ch}$., I sc. into second of $9 \mathrm{ch}$., I double tr. into each of 3 next tr., keep the top loop of each on the hook, and draw through altogether, 6 ch., 3 double tr. worked like the last into next tr., 3 ch., 3 double tr. worked as before into next tr., 4 ch., pass over I tr., 3 double tr. worked as before into next tr., 3 ch., 3 double tr. worked as before into next tr., 6 ch., 3 double tr. worked as before into next tr.; repeat from the beginning of the row.

7 th row, I double tr. into second cluster of double tr. (see design), 5 ch., I tr. into next cluster, $7 \mathrm{ch}$., I tr. into next cluster, $5 \mathrm{ch}$., I double tr. into next cluster, $6 \mathrm{ch}$., work one quadruple tr. into fifth of $6 \mathrm{ch}$., work off two loops, work a double tr. into same stitch, work 2 double tr. into corresponding stitch of next scallop, work off the rest of the loops of the quadruple tr., 6 ch.; repeat from the beginning of the row.

8th row, I tr. into a stitch, I ch., pass over I stitch and repeat.

Commence the other half on the other side of the foundation chain; it is worked exactly like the first half.

For the edge:

Ist row, I double tr. into a stitch, I ch., pass over one stitch and repeat. 2nd row, 3 tr. into a stitch, $4 \mathrm{ch}$., pass over 4 stitches, I dc. into each of 11 stitches, 4 ch., pass over 3 stitches; repeat from the beginning of the row. 
3d row, 3 tr. into first of 4 ch., 5 ch., 3 tr. into next ch., 3 ch., 7 dc. over dic. of last row, $3 \mathrm{ch}$; repeat from the beginning of the row.

$4^{\text {th }}$ row, I tr. into each of 3 tr., 3 ch., 3 tr. into center of ch., 3 ch., 3 tr. into tr. of last row, 3 ch., 3 dc. into center of dc. of last row, $3 \mathrm{ch}$.; repeat from the beginning of the row.

5 th row, I dc. into the first tr., 5 ch., I dc. into the second, I ch., I tr. into third tr., * 7 ch., I dc. into the third ch., I tr. into next stitch, repeat from * four times more, 2 ch., I dc. into the last tr., 2 ch.; repeat from the beginning of the row.

\section{CROCHETED TABLE COVER.}

This cover is about twenty-four inches square and crocheted with cream linen thread No. 70. The center and edging are worked of the same transparent star design, and are separated by a thick band, one and one-half inches wide. The work is begun with the single stars in the center, afterwards united by a guipure ground.

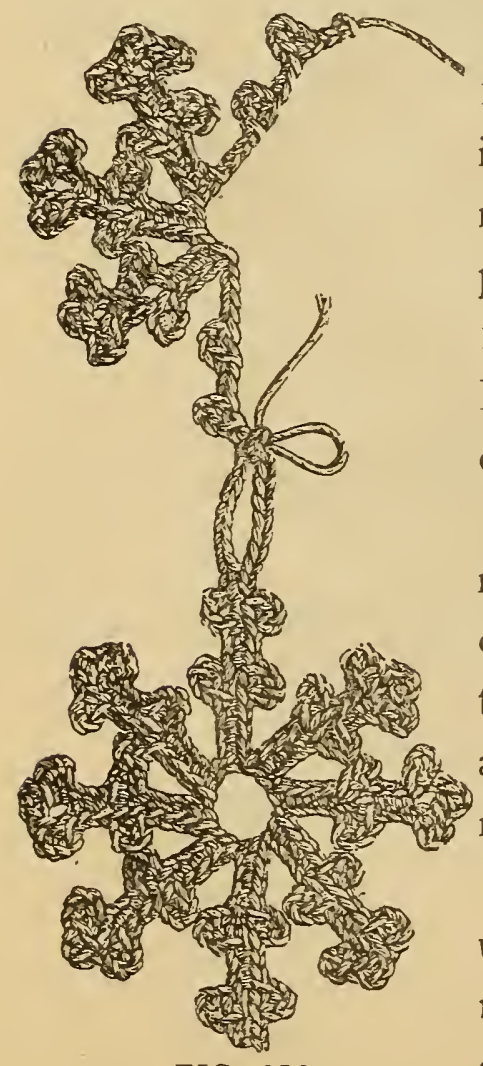

FIG. 3 I3.

Each row of stars requires: $2 \mathrm{ch}$., I picot, $2 \mathrm{ch}$., I picot, 8 ch., I picot, 2 ch., I picot, 2 ch., I sc. in the top of the last sc., I picot, 2 sc. in the 2 next ch., I picot, $4 \mathrm{sc}$. in the next $4 \mathrm{ch} .,{ }^{*} 4 \mathrm{ch} ., \mathrm{I}$ picot, 2 ch., I picot, 2 ch., I sc. in top of the last sc., I picot, $2 \mathrm{sc}$. in the $2 \mathrm{ch}$., I picot, $4 \mathrm{sc}$. in the $4 \mathrm{ch}$. Repeat once more from star, so that then three rays of a star are finished.

Then follow: 4 ch., I picot, 2 ch., I picot (a half ray), I I ch., I picot, 2 ch., I picot, $8 \mathrm{ch}$., I picot, 2 ch., I picot, 2 ch., I sc. in the last sc., I picot, 2 sc. in the $2 \mathrm{ch}$., I picot, $4 \mathrm{sc}$. in the next $4 \mathrm{ch}$., by which again one ray of the second star is finished; two more following are made exactly like these.

The worker must then repeat from double dot until the number of stars required for one row are made-on the pattern cover there were eight-yet in crocheting the last star seven rays instead of three are made, one after another, and the half-star then completed, going forwards as follows: * 4 sc. in the next 4 ch., I picot, 2 sc., I picot, 2 sc., 7 ch., 2 sc., I picot, 2 sc., I picot, 4 sc. 
Three rays now follow these meshes, which are repeated from star, until the row is finished.

The rows of stars are crocheted together, beginning with I sc. worked into the point of the last finished ray, and require: I I ch., I sc. in the following point of the ray, * work twice I I ch. and I sc. each in the next ray,

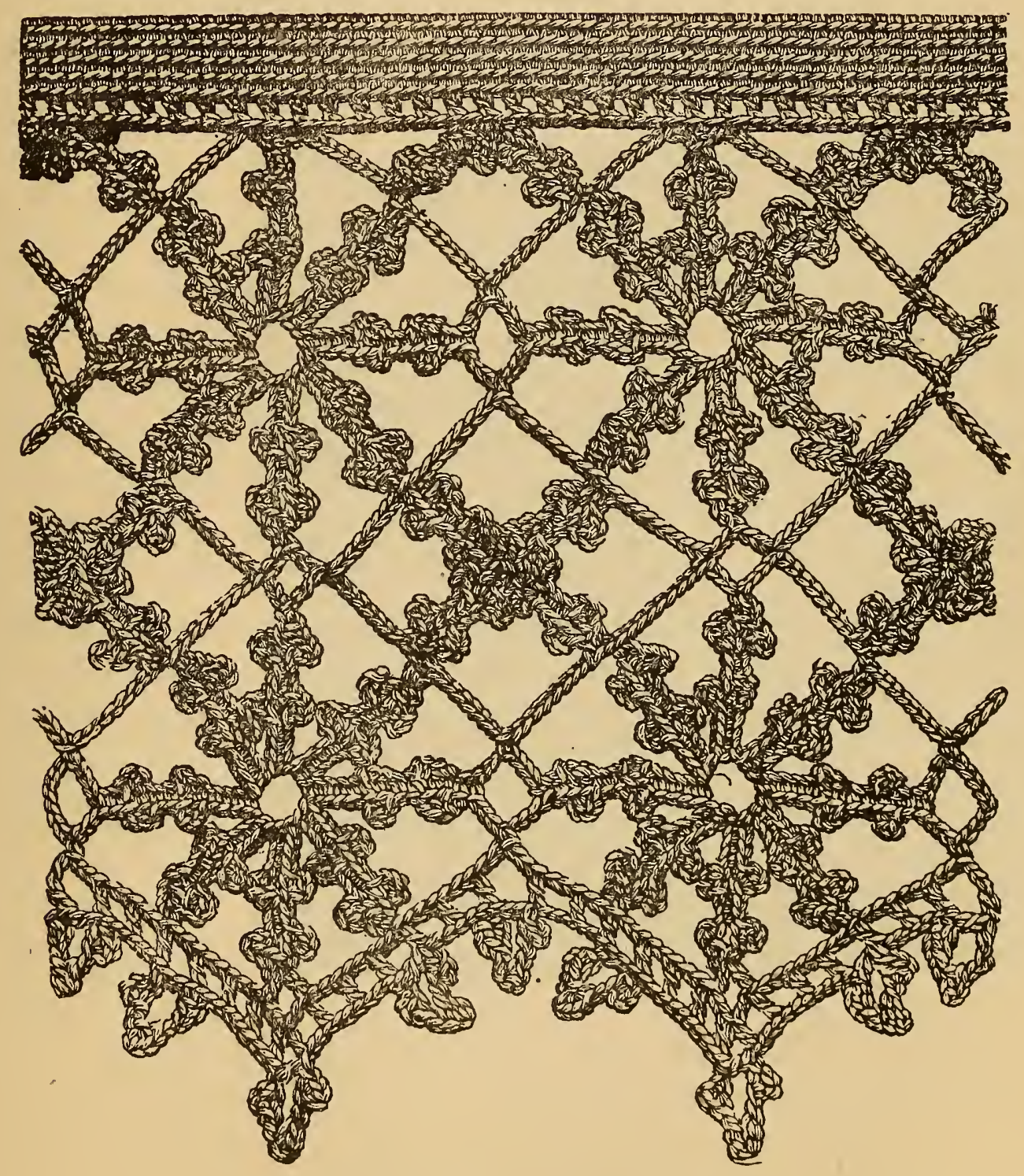

EDGE FOR TABLE COVER. FIG. 314.

$8 \mathrm{ch}$., I sc. in the fourth of the $7 \mathrm{ch}$. between the stars, $8 \mathrm{ch}$. I sc. in the next ray of the second star.

Work four times $2 \mathrm{ch}$., ana I picot, then loop I ch. on to the sc. which are caught into the last ray of the foregoing star before the first $8 \mathrm{ch}$., going 


\section{DAINTY WORK FOR PLEASURE AND PROFIT.}

forwards I sc. into the I ch., then work four times I picot and $2 \mathrm{ch}$.; now repeat from star until the whole row is bordered round.

At the end of the row, as at the beginning, three times II ch. and I sc. in the point of the ray.

For the second row crossing the just-described one, the thread is again put on at the point of the first ray for the second row of stars and the crochet continued thus: I I ch., I sc. in the next point of the ray, work four times $2 \mathrm{ch}$. and I picot. The illustrations will make this quite clear.

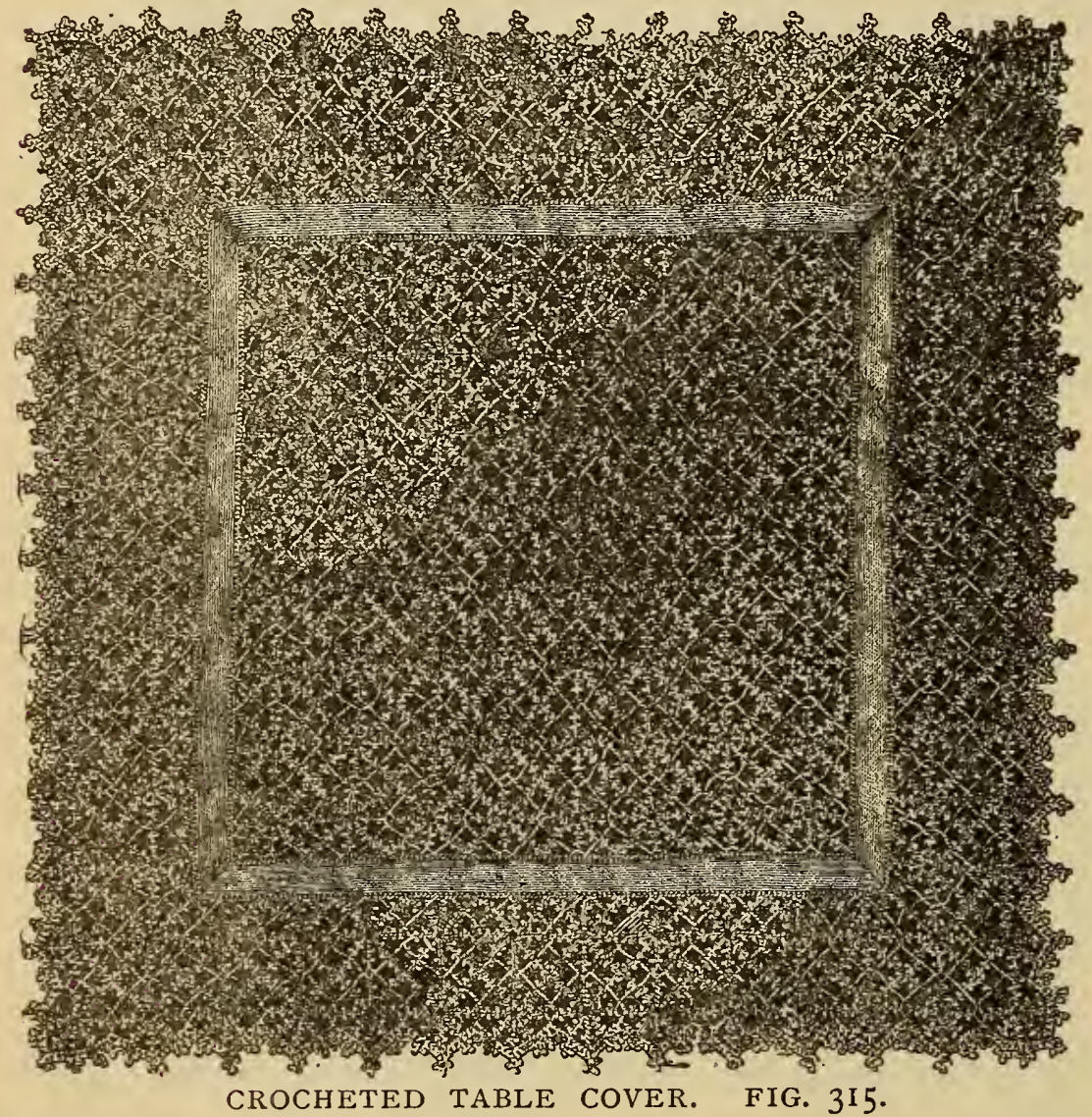

Then I ch., loop on to the sc., which catch up the last ray but one of the first row of stars, going forwards I sc. in the I ch., four times I picot and $2 \mathrm{sc}$. in the $2 \mathrm{ch}$. (the picot bar thus made is caught up later on between the second and third picot by one sc. of the first row of the band), * $8 \mathrm{ch}$; loop on to the $3 \mathrm{ch}$. of the opposite chain length of the former row, 3 ch., I sc. in the point of the next ray, $3 \mathrm{ch}$., loop on to the $3 \mathrm{ch}$. of the opposite chain of the former row, $8 \mathrm{ch}$., I sc. in the next ray point, twice 2 ch. and I picot, then I ch., loop on between the second and third picot of the whole picot bar of the former row, going forwards I sc. in the I ch., twice I picot and $2 \mathrm{sc}$. in the $2 \mathrm{ch}$., $8 \mathrm{ch}$., I sc. in the fourth of the seventh ch., $8 \mathrm{ch}$., I sc. in the next ray point of the following star, twice $2 \mathrm{ch}$. and I picot, then I ch., again loop on between the second and third picot of the above-mentioned picot bar, going forwards I sc. in the I ch., twice I picot 
and $2 \mathrm{sc}$. in the $2 \mathrm{ch}$; repeat from star until the row is finished; at the end of the same a whole picot bar must be crocheted, as at the beginning, which is again to be looped tight to the first star row.

It must be remarked that at the outer edge of the first and last row of stars, the first uniting row through a half-picot bar is required at each corner of the cover (see figure 315).

When the center is finished the band is crocheted, beginning at one of these half-picot bars at the corner: I sc., in this $*$ I I ch., I sc. in the third last $\mathrm{ch}$. but one of the next II ch. of the center, $5 \mathrm{ch}$., I sc. in the third $\mathrm{ch}$. of the following I I of the center, I I ch., I sc. between the second and third picot of a whole picot bar.

Repeat from star.

In the second row I dc. comes alternately with I ch., in the corner meshes 3 dc. are always to be crocheted.

Now follow Io rows of sc., worked into each hind mesh link, and in each corner mesh again $3 \mathrm{sc}$.

The last open bar row (double) is shown, figure 3I4, with the edging. For this two rows of stars are to be set together (see figure 315 ).

Figure 3I4 shows clearly how the row margining the edging inside is joined at once to the band.

Figure 31 3 gives the corner of the same always made of three half-picot bars; also the execution of the outer part of the edging offers no difficulty after figure 315 .

\section{PETTICOAT: KNITTING AND CROCHET.}

Materials required: 12 ounces of peacock German wool, one pair wooden pins, No. 12, and one pair No. 10, steel.

This petticoat is very convenient to work, being knitted in stripes, eight of which are needed. The stripes are sewn together, and the border is worked separately, and is sewn on to the edge of stripes.

Commence at the bottom of the stripe; cast on 40 stitches, with pins No. IO.

Ist row, k. 5 , p. $4, *$ k. 2 , p. 3 ; repeat from ${ }^{*} 5$ times more.

2nd row, the stitches that were purled in the previous row are to be knitted, and those that were knitted purled.

$3 \mathrm{~d}$ row, like Ist row; 4 th row, like 2 nd row. 
5 th row, k. 5, p. $5, *$ k. 7 , p. 3 ; repeat from * to end of row.

6th row, knit the purl, and purl the knitted stitches of previous row. 7 th row, like 5 th row: 8th row, like 6th row.

Repeat from the Ist row until you have worked 16 inches, then begin the decrease by knitting 2 together in the basket patterns, leaving the broad knitted and purled stripe the same width throughout; the decrease

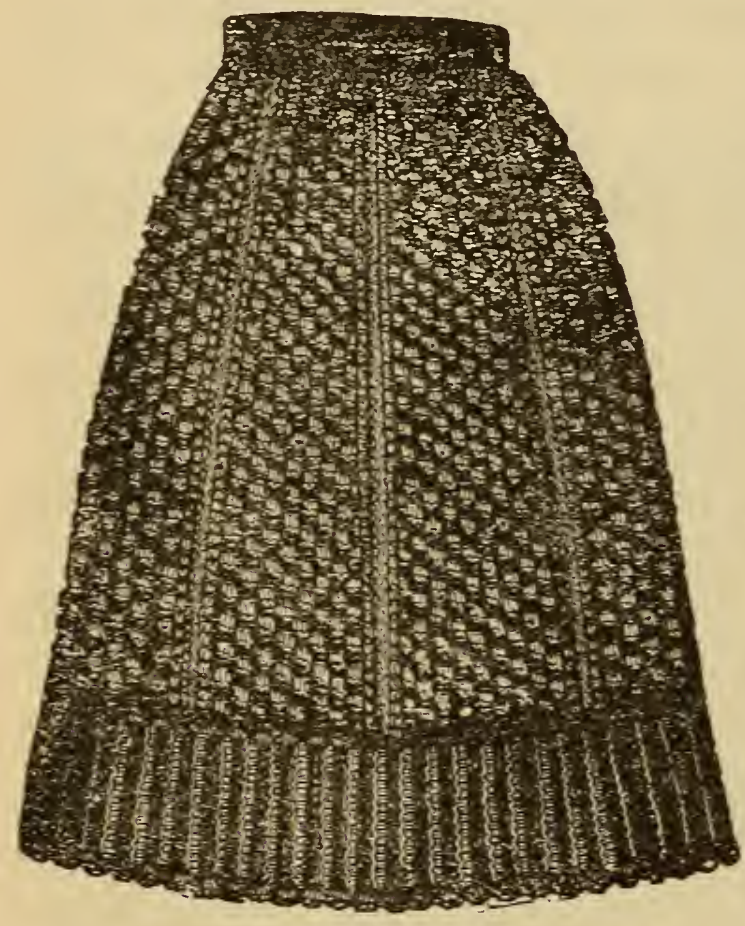

FIG. 3 I6. is made in every 4 th row, until you have only 3 of the purl squares in a row; work without decrease until the petticoat is long enough, after reckoning for the border, then cast off.

Sew all the stripes together, stitch by stitch, with a needle and wool.

For the border, cast on 30 stitches with pins No. I2.

Ist row, knit; 2nd row, purl; these 2 rows are repeated twice more.

7 th row, purl.

8th row, knit, repeat from 7 throw twice more, then repeat from the Ist row until you have worked a length sufficient to go around the lower edge of petticoat. Sew the border to the petticoat and work an edge of crochet scallops: 5 trebles into center row of one stripe, I double into first row of next stripe; repeat from the beginning of row.

Leave the two back stripes of petticoat unjoined for about 12 inches, bind the edge of 'opening with a piece of satin ribbon, then sew the top of stripes into a silk band, and fasten with a button and buttonhole.

\section{TRIMMING: CROCHET AND BRAID.}

Materials required: Linen thread, No. 80, a narrow point lace braid, and a fine crochet hook.

Commence in the center of a rosette with $3 \mathrm{ch}$; join round.

Ist round, I2 dc. under the ch.

2nd round, $2 \mathrm{dc}$. into each stitch of last round. 
$3 \mathrm{~d}$ round, I dc. into a stitch, $3 \mathrm{ch}$., I dc. into next stitch; repeat from beginning of round.

$4_{\text {th }}$ round, I dc. into center of $3 \mathrm{ch}$., $5 \mathrm{ch}$; repeat all round.

5 th round, I dc. into center of 5 ch., 9 ch., I dc. into the fifth $*, 5$ ch., I dc. into the second, repeat from * twice more, I sc. into fourth of $9 \mathrm{ch} ., 3$ ch.; repeat from the beginning of the round.

6th round, I dc. between second and third picots of a loop of last round, * 5 ch., I dc. into the second, repeat from * 4 times more, then repeat from the beginning of the round, pin to first stitch of round, break off the

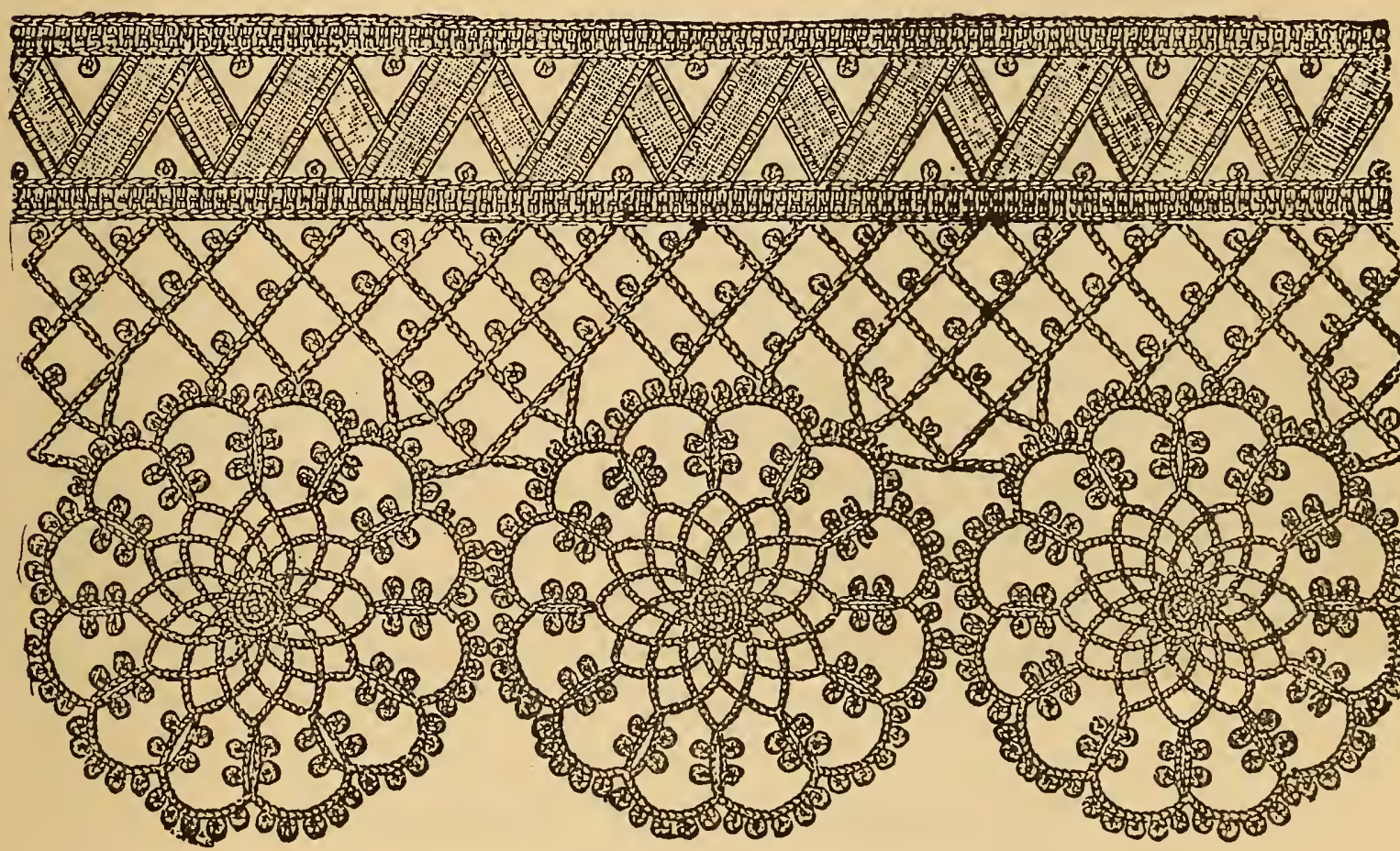

FIG. 317.

thread and fasten securely at the back of the work; when working the last round of the next and following rosettes join to previous rosette by drawing through a picot on each of two scallops-see figure 3I7.

For the heading:

1st row, hold the rosettes in the left hand, work I dc. into the center picot of first scallop after the joining of second rosette $*$, 12 ch., I dc. into the eighth, 5 ch., I dc. into next picot, I 2 ch., I dc. into the eighth, 2 ch., I dc. into the center of $5 \mathrm{ch}$., I I ch., I dc. into the seventh, $12 \mathrm{ch}$., I dc. into second picot of next scallop before joining of the first rosette, $3 \mathrm{ch}$., I dc. into 
fourth of $12 \mathrm{ch} ., 4 \mathrm{ch}$., I dc. into fifth of $\mathrm{I} 2 \mathrm{ch}$., $4 \mathrm{ch}$., pass over I picot of same scallop, I dc. into next; break off the cotton and fasten neatly at the back of the work.

- 2nd row, I dc. into center picot of the first of top scallops of first rosette (see design), II ch., I dc. into the seventh, 2 ch., I dc. into second picot of next scallop, I3 ch., I dc. into the ninth, $2 \mathrm{ch}$., I dc. into center of loop of $9 \mathrm{ch}$. (see design), * I2 ch., I dc. into the eighth, $2 \mathrm{ch}$., I dc. into third stitch past next picot, repeat from * once more, I 2 ch., I dc. into the eighth, 2 ch., I dc. into the center picot of next scallop of following rosette; repeat from first * to the end of row.

$3 \mathrm{~d}$ row, I dc. into fifth of $12 \mathrm{ch}$. of last row, I2 ch., I dc. into the eighth, $2 \mathrm{ch}$; repeat from the beginning of the row.

$4^{\text {th }}$ row, like $3 \mathrm{~d}$ row.

$5^{\text {th }}$ row, I dc. into fifth of $12 \mathrm{ch} ., 7 \mathrm{ch}$; repeat from the beginning of the row.

6th row, I tr. into each of II stitches, $4 \mathrm{ch}$., I dc. into the first; repeat from the beginning of the row.

For the vandyke take a length of point braid, fold it as shown in the illustration, sew with a fine needle and cotton by the folded part to the stitches between two picots.

7 th row, work $5 \mathrm{sc}$, into the folded braid at the other side of vandyke, $7 \mathrm{ch}$., I dc. into the fourth, $3 \mathrm{ch}$.; repeat from the beginning of the row.

8 th row, I tr. into each stitch of last row.

\section{INFANT'S KNITTED JACKET.}

Materials required: $2 \frac{1}{2}$ ounces knitting silk, 2 knitting needles, double pointed ( $\operatorname{size} 8$ ), 2 yards satin ribbon.

This jacket is a most useful little garment for infants to wear under cloaks, or even under robes in very cold weather.

Commence at the bottom; cast on 94 stitches on one needle.

Ist row, k.; 2nd row, k. 2, p. 2 throughout the row. These 2 rows are each repeated 9 times more.

2 Ist row, k. I, * silk forward, k. 2 together, repeat from * to end of row, knitting the last stitch. 
22nd row, k.; 23d row, k. I4, k. I and p. I, in the next stitch, k. 24, $k . I$ and $p . I$ in the next stitch, repeat from the beginning of the row once more, end with $\mathrm{k}$. I4.

$24^{\text {th }}$ row, k.; $25^{\text {th }}$ row, like 23 d row, with the exception that you k. 15 instead of 14 , mentioned in 23 d row.

26th row, k., continue to increase with a plain row between, until you have increased 9 times in all; then increase only in the back, until you have increased at the back in all 13 times; now work 4 rows on all the stitches. Then for the right front, work on 35 stitches.

Ist to Ioth row, k.; inth row, leáve 8 stitches on another pin, and for the shoulder on the remainder of the stitches, k. 2 together, k. to end of row in each alternate row for 5 times; k. Io rows without decrease, then cast off.

Work the other shoulder in the same way as described for this. Take 13 stitches from each side of back stitches, work on the remaining 42 stitches, Io rows, then decrease at the beginning and end of each alternate row for 18 rows more, sew

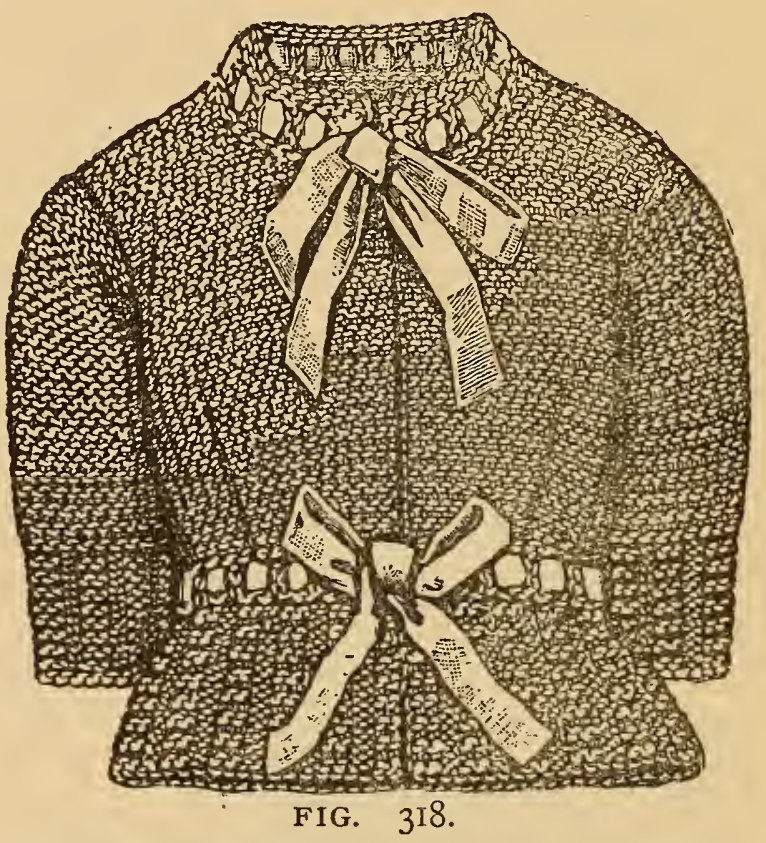
up the shoulders, pick up I stitch at the end of each ridge of both fronts, and knit across in a row with the 24 stitches of the back to form the neck.

Ist row, k.; 2nd row, silk forward, k. 2 together throughout; repeat the Ist row 3 times more, then cast off. For the sleeve commence at the wrist; cast on 27 stitches.

Ist row, k.; 2nd to 2oth row, k. 2, p. 2 throughout.

2Ist row, k. I and P. I, in the first stitch, k. 25, k. I, and p. I in the last stitch.

22nd to 26th row, k., repeat from the 2 Ist row 5 times more, work 8 more rows without increase, then cast off; sew up the sleeves, and sew into the arm-holes. Run ribbon into the holes at the neck and waist. 


\section{MOSS EDGING: CROCHET AND BRAID.}

For the edge, work as follows, using No. 80 linen thread:

Ist row, work I dc. into 2 picots together, 5 ch.; repeat; 2nd row, 2

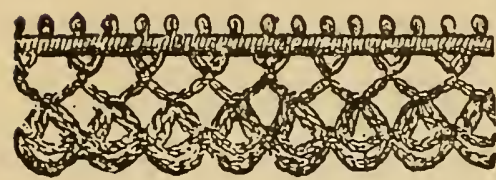

FIG. 3I9.

tr. into center of 5 ch., 4 ch., 2 tr. into same stitch last tr. was worked into; repeat from the beginning of the row.

3d row, I dc. between the little scallops, 6 chain; repeat. See figure 319.

\section{FERN EDGING: CROCHET AND BRAID.}

For the edge, work as follows use linen thread No 70 :

Ist row, I dc. into 2 picots together, 2 ch., 2 tr. separated by 4 ch.; into next picot, $2 \mathrm{ch}$; repeat from the beginning of the row.

and row, 2 dc. under 2 ch., * I dc. under 4 ch., 4 ch., I dc. into first, I dc. under same $4 \mathrm{ch}$. the last was worked under, repeat from * 3 times

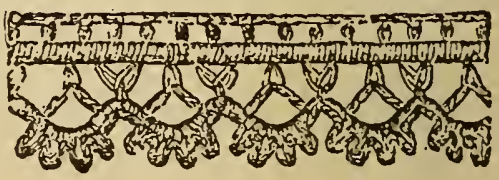

F IG. 320 . more, 2 dc. under next 2 ch.; repeat from the beginning of the row.

For the heading: I dc. into a picot, 2 ch.; repeat.

\section{FRENCH EDGING: CROCHET AND BRAID.}

For the edge, work as follows with linen thread No. 80:

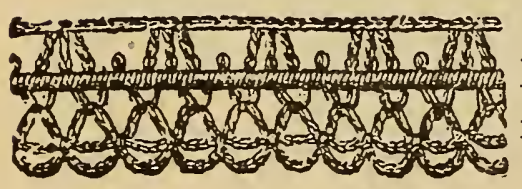

FIG. 32 I.

Ist row, work 2 tr. separated by $3 \mathrm{ch}$. into 2 picots of braid together; repeat from the beginning of the row.

2nd row, I dc. between 2 tr., 5 ch.; repeat. For the heading:

I tr. into each of 3 picots of braid, 5 chain, pass over I picot and repeat.

\section{EDGING: CROCHET AND SERPENTINE BRAID.}

Into a length of serpentine braid work a row of cross tr., as follows: Work as for a double tr. into I point of braid, draw through the first loop, work a tr. into next point of braid, work off all the loops, one at a time; 2 ch., I tr. into center of cross tr., 2 ch., repeat

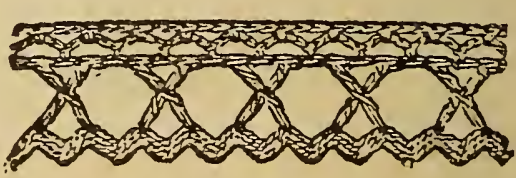

FIG. 322 . from the beginning of the row; 2nd row, I dc. under a ch., $3 \mathrm{ch}$., I dc., under next ch.; repeat; $3 \mathrm{~d}$ row, I dc. under $3 \mathrm{ch} ., 2 \mathrm{ch}$.; repeat. 


\section{EDGING: CROCHET AND FANCY BRAID. No. 1.}

This fancy braid has a long loop, with three small picots at the top. Work I dc. into the last picot of one cluster and first of next cluster together, under the center picot work $3 \mathrm{dc}$., $3 \mathrm{ch}$., I dc. into the first, $3 \mathrm{dc}$. under same $3 \mathrm{ch}$. the last dc. were worked under, repeat from the beginning of the row.

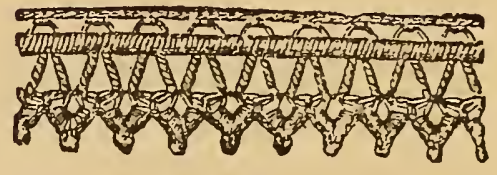

FIG. 323 .

For the lheading: work $2 \mathrm{dc}$. into picot on the other side of braid $2 \mathrm{ch}$; repeat. See figure 323 .

EDGING: CROCHET AND BRAID. NO. 2.

Ist row, 3 tr. into I picot of fancy braid, I ch., pass over I picot and repeat.

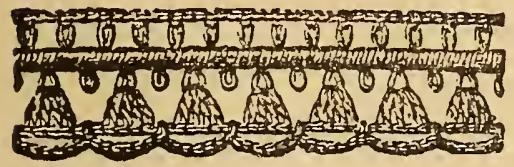

F I G. 324. 2nd row, I dc. under I ch., 5 ch.; repeat.

For the heading:

I dc. into a picot, 2 chain; repeat. See fig. 324

\section{SHELL TRIMMING: CROCHET.}

Make a chain of 32 stitches.

Ist row, I tr. into 26 th, 2 ch., 2 tr. separated by $3 \mathrm{ch}$. into the 2 Ist, *2 ch., pass over 4 stitches, 2 tr. separated by $3 \mathrm{ch}$., into the next repeat from * 3 times more; turn.

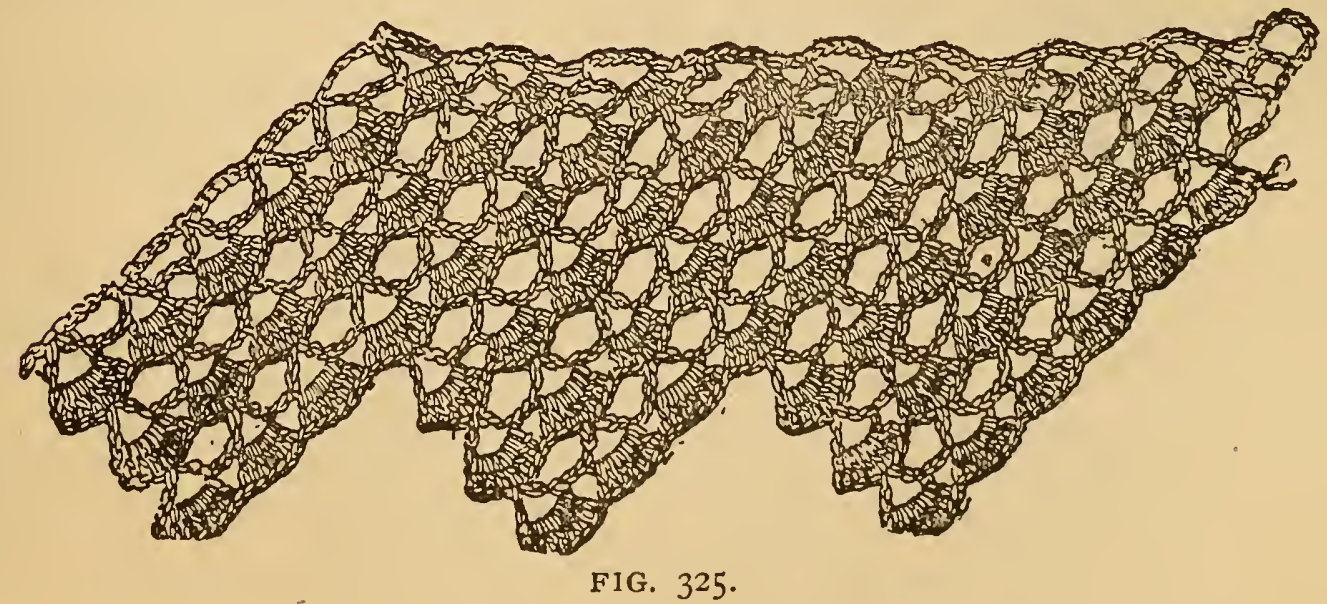

2nc row, 3 ch., 7 tr. under each $3 \mathrm{ch}$. of last row, 5 tr. under ch. at the end of the row, $3 \mathrm{ch}$., $1 \mathrm{tr}$. under same ch.; turn. 
3 d row, 5 ch., I tr. under 2 ch., I ch., 2 tr. separated by 3 ch. into center of $5 \mathrm{tr}$., * 2 ch., 2 tr. separated by $5 \mathrm{ch}$. into center of $7 \mathrm{ch}$., repeat from $* 4$ times more; turn.

$4^{\text {th }}$ row, like 2 nd row, except that there will be 1 more cluster of $7 \mathrm{tr}$.

5 th row, like $3 \mathrm{~d}$ row, repeating from ${ }^{*} 5$ instead of 4 times; 6 th row, like 2 nd row, with 2 more clusters of $7 \mathrm{tr}$.

8 th row, like $3 \mathrm{~d}$ row, repeating from * only 3 times; repeat from the 2nd row for the length required. See figure 325 .

\section{PALM TRIMMING: CROCHÉT.}

Commence with the oval in the center of pattern, make a ch. of 14 stitches.

Ist row, work down the chain with I half tr. into the last stitch but one, I tr. into each of II next stitches, I half tr. into the nexxt.

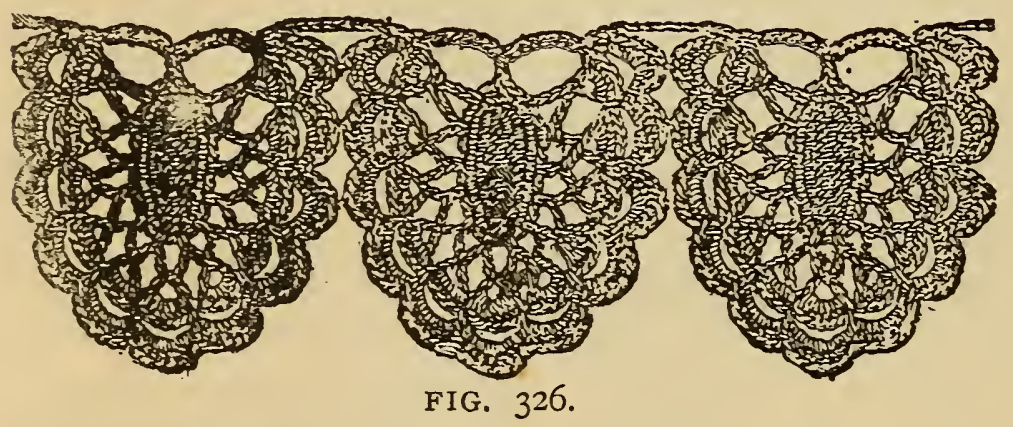

2nd row, I dc. into stitch last half tr. was worked into, I dc. into the next and each of I2 following stitches, 2 dc. into each stitch at end of oval, I into each

stitch at the other side, I sc. into the end.

$3 \mathrm{~d}$ row, I dc. into first stitch at side, * Io ch., I sc. into the 5 th, $4 \mathrm{ch}$., pass over 2 stitches, repeat from * Io times more; work I sc. into the first stitch of row.

4th row, $7 \mathrm{ch}$, 6 half tr. under each loop of ch. of last row, $5 \mathrm{ch}$., I sc. into the second and each of 5 next stitches of $7 \mathrm{ch}$., * I dc. into first half tr., $4 \mathrm{ch}$., I dc. into the last of 6 half tr., repeat from * Io times more.

Work as many patterns as are needed for the length of trimming. Then to work the edging row and to join patterns, work I dc., 6 half tr., and I dc. under each loop of $4 \mathrm{ch}$., I $2 \mathrm{ch}$.; repeat from beginning of the row. When working the next and following patterns, draw through the center of 3 first scallops of previous patterns, when working corresponding scallcps See figure 326. 


\section{KNITTED HOOD FOR CHILD FROM ONE TO TWO YEARS OLD.}

This little hood is knitted with white Saxony wool, and trimmed with inch-wide white satin ribbons.

Figure 328 half of crown and 329 half of front.

Prepare a stiff paper pattern, according to figures 328 , same size as shown, and $329-63 / 4 \times 7$ inches in size-cutting each piece on the double. Begin at the lower edge of figure 329 , using coarse needles. Cast on the required number of stitches ( 44 in the model), and knit to and fro as follows:

Ist row, * k. I ; out of the next stitch work 3 , namely, I k., I p. and I k.; repeat from $*$.

2nd row, knit plain throughout.

3d row, right side of the work; alternately knit I, and knit 3 together crossed (for crossed, insert the needle at the back and downward instead of from the front and upward).

4th row, purl throughout.

$5_{\text {th }}$ to 7 th rows, knit so that all stitches appear purled on the right side.

8th row, purl throughout.

Continue to repeat the Ist to 8 th rows, widening or narrowing as the pattern requires.

Begin the crown at the lower edge of figure 328 with the requisite number of stitches ( 32 in the model), and knit in the same pattern as the front.

For the cape begin at the top, casting on 50 stitches, and knit to and fro, first 9 rows of plain

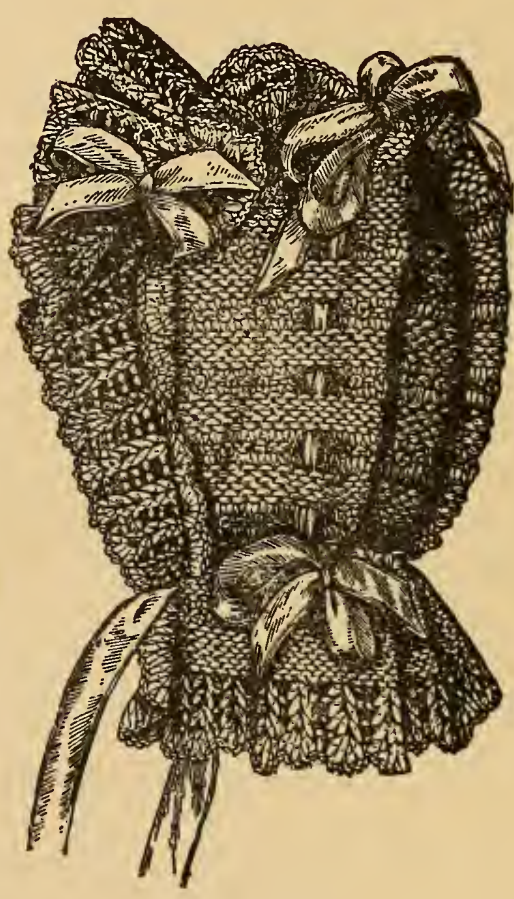

FIG. 327. knitting, then 3 rows in which all stitches appear purled on the right side, then 14 rows of plain knitting, after which cast off. Turn down the last 13 rows on the wrong side, and catch the cast-off stitches to the foundation stitches.

Edge the front and bottom of the cape with a crochet edging of 5 rows as follows:

Ist row, by turns, $2 \mathrm{dc}$. separated by I ch. on the following edge stitch, I ch., pass 2; widen at the corners as required. 
2nd row, 2 dc. separated by $\mathrm{I}$ ch. around every chain separating a pair of dc. in the last row, and I ch. between. -

$3 \mathrm{~d}$ row, $3 \mathrm{dc}$. around the ch. separating a pair of dc; I ch. between.

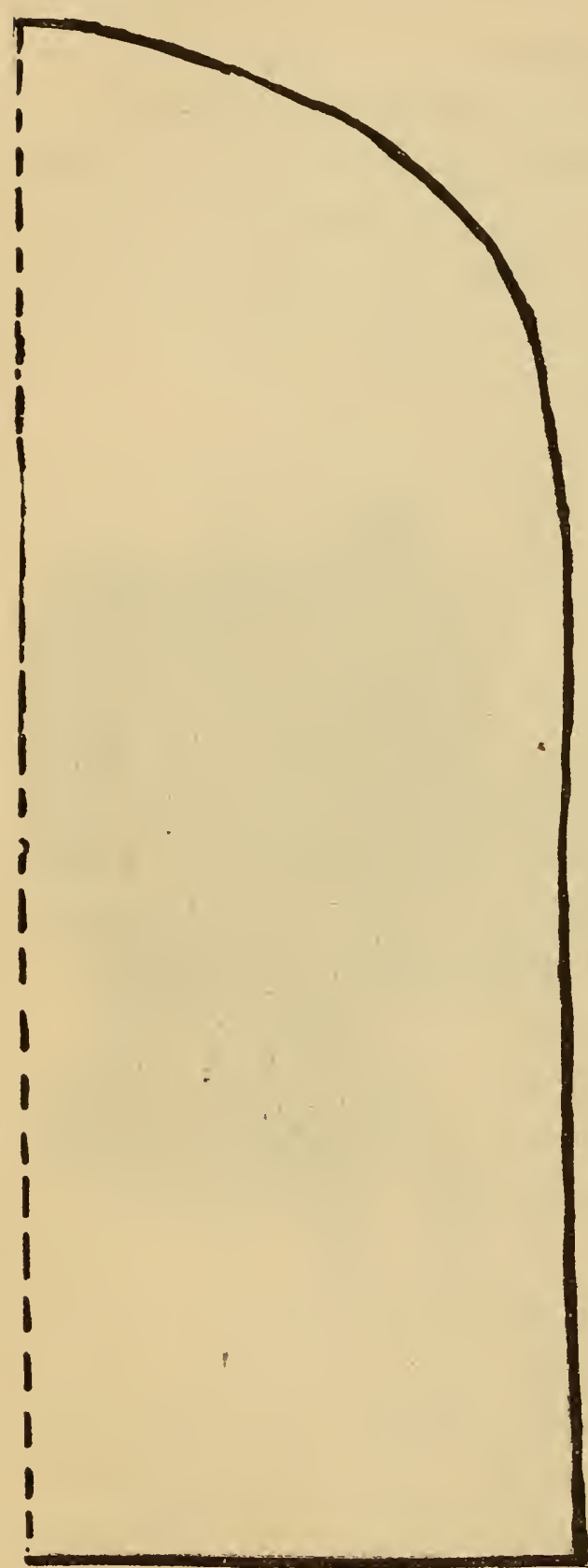

FIG. 328 .

$4^{\text {th }}$ row, $3 \mathrm{dc}$. on the middle one of the next $3 \mathrm{dc}$. in the last row, I ch., I sc. around the following ch., I ch.; repeat.

$5^{\text {th }}$ row, I sc. on every stitch.

For the frill at the front edge make a chain 32 inches long, turn, and for the ist row pass 5 , I dc. on the next, then by turns I ch. and I $\mathrm{dc}$. on the following second stitch. All the other rows are begun at the same end and fastened off at the other.

2nd row, on the middle 26 inches of the 32 , work like the first row of the edging.

$3 \mathrm{~d}$ row, like the second row of the edging, but make it 3 patterns longer at each end than the last.

The other rows are worked full length; the 4 th to 6 th are like the preceding row, and the 7 th to 9 th like the $3 \mathrm{~d}$ to 5 th of the edging.

On the other side of the foundation chain on the middle 16 inches work two rows like the Ist and 2nd of the edging, then work at full length two rows like the last two of the edging.

Turn down the front on the wrong side along the dotted line, join it to the crown, then join the cape to both.

Knit a lining in plain knitting according to figures 328 and 329 , but work the front only from the front edge to the plain line. Set in the lining, then crochet two rows of dc. around it, and on these at the front edge set the frill, as illustrated, box-pleated at the top. Trim the hood with narrow ribbons as illustrated. 


\section{CROCHETED HOOD FOR CHILD FROM 2 TO 3 YEARS OLD.}

This hood is crocheted with white zephyr wool. It is worked in three pieces-the side of the crown, the back, and the frill which surrounds the edge.

For the side prepare a stiff paper pattern from figure 33I, dimension $91 / 4 \times 53 / 4$ inches at the widest point and $91 / 4 \times 4 \frac{1}{2}$ inches at the narrowest point. The illustration shows shape. Cut it on the double. Begin at the lower edge with a chain of the length required, and crochet back and forth as follows:

Ist row, 3 ch., take a loop each through the second and first of these 3 ch., take a loop each through the next $2 \mathrm{ch}$. stitches of the foundation,

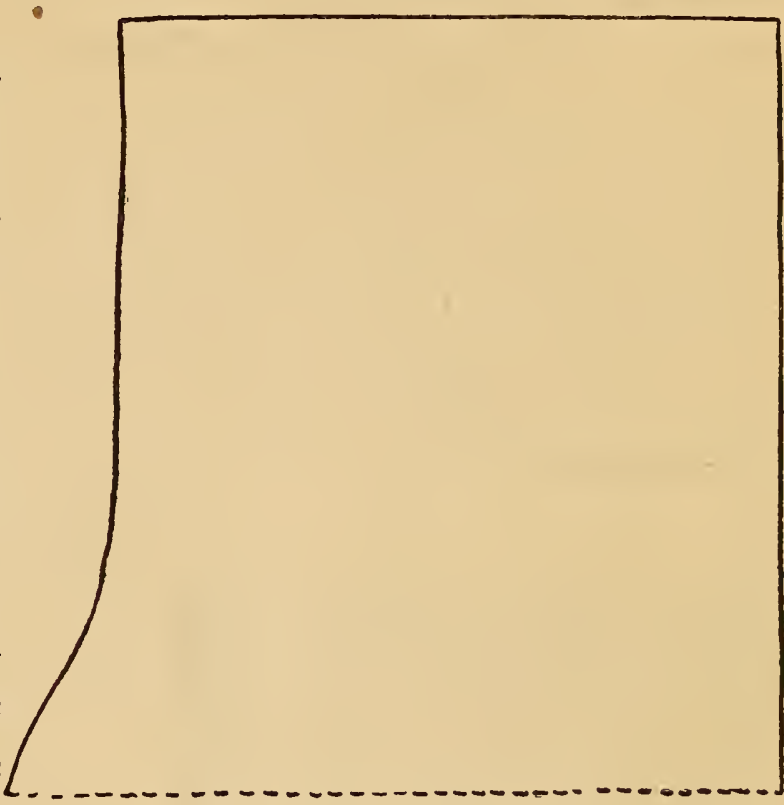
pull a loop through all the loops now on the needle, work off this loop by

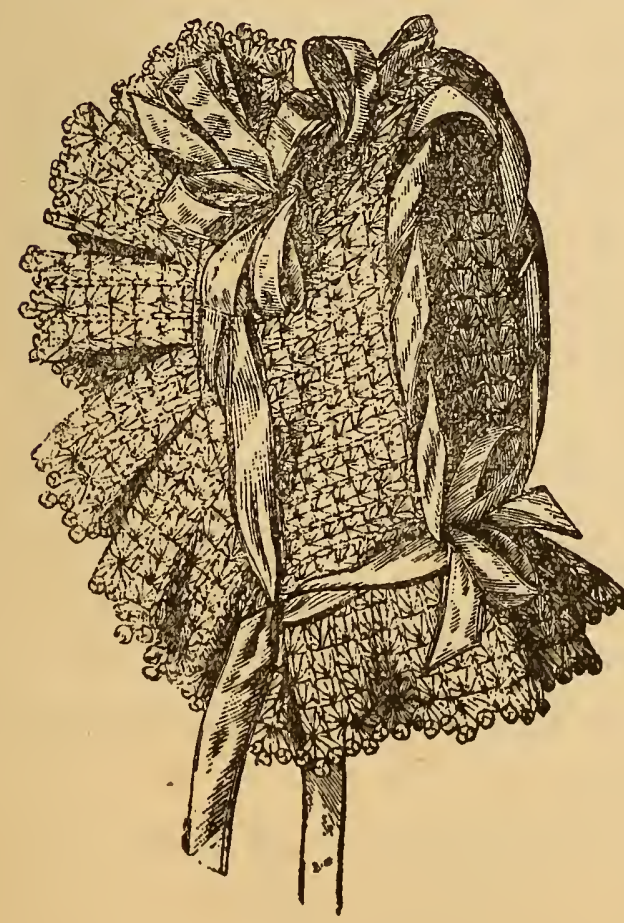

FIG. 330 . pulling another through it, * with a loop on the needle, take up 4 loops, the first through the mesh above the group of loops previously worked off, the second through the back mesh of the last of the 4 preceding loops taken up, and the third and fourth through the next $2 \mathrm{ch}$. of the foundation, pull a loop through all the loops now on the needle, work off this loop; repeat from *.

2nd row, I ch. to begin, then by turns I sc. on the next worked-off loop, and I sl. st. into the back mesh of the following stitch.

Continue to repeat these two rows in turn, widening or narrowing as the pattern requires.

For the back of the crown make a chain of 28 stitches, which forms one side edge, and work back and forth in the same stitch as the side, 4 patterns deep. 
For the frill around the edge make a foundation of $14 \mathrm{ch}$., and work in the same stitch as the rest, making a strip three yards long, which edge along the outer side with a row of scallops, as follows: * I sc. on the next siitch on the edge, I ch., on the next second stitch work 4 dc. with a picot between every two of them (for a picot $3 \mathrm{ch}$. and I sc. around the upper perpendicular meshes of the preceding dc.); repeat from *. Underlay the back of the crown with foundation muslin, and join the side and back; fulling the side from the middle to *.

Work a lining for the hood, which consists of a band and a loose crown piece; for the band make a chain of 70 stitches, and work 5 rows in tricot

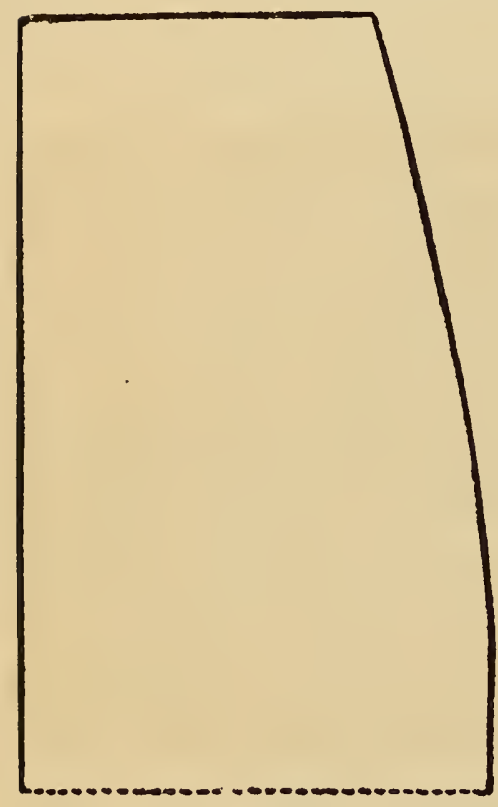

FIG. 33I. or afghan stitch, which is the well-known stitch in which loops are taken up going forward and worked off coming back, the two forming one row of the stitch. After the last of the 5 rows work a row of sl. st., one into every perpendicular mesh of the last row, and on the foundation chain work chain scallops, 2 sc. on the next 2 stitches, $6 \mathrm{ch}$.

For the crown piece of the lining make a chain of 36 stitches, and work 18 rows in tricot stitch, but with a slight variation in the stitch; when taking up the loops going forward take them through the horizontal meshes at the back of the chain stitches of the preceding row instead of the perpendicular meshes on the surface.

Join the crown piece to the band of the lining, gathering the crown at the middle as needful, then set in the lining. Set the box-pleated frill around the outer edge, as illustrated. Cover a piece of ribbon wire with white silk flannel binding, and set it around the inner edge of the hood; at the front run it into the chain scallops at the front edge of the lining, and at the back let it cover the edge of the lining.

Trim the hood as illustrated, with twisted bands and bows of white watered ribbon an inch wide. The two hoods described on pages 379 to $38 \mathrm{I}$ may be made of knitting silk instead of wool in which case the linings should be knitted in a light weight Saxony yarn. 


\section{SQUARE FOR PILLOW SHAM OR BEDSPREAD.}

This bedspread is made in squares, joined to each other, having four leaves and four rosettes. See figure 332.

The squares are joined to the picots by means of a slip stitch.

For each one of the leaves make a chain of 20. Going back on them, work as follows:

Miss I ch. st., I $8 \mathrm{sc}$. in the next $18 \mathrm{ch}$. on one side of the chain, $3 \mathrm{sc}$. in the first ch., then $16 \mathrm{sc}$. in the 16 remaining chain loops on the other edge of the chain, * I ch.; turn the work. Going back on the preceding stitches, make I7 sc. in the back loops of the next I 7 stitches. All sc. will be made in passing the hook in the back loops of the stitch. Make $3 \mathrm{sc}$. in the middle one of the last $3 \mathrm{sc}$. coming together; $16 \mathrm{sc}$. in the next 16 stitches; repeat 6 times from *, and then repeat once more until the $3 \mathrm{sc}$., worked in one loop, have been reached.

Fasten the thread and break it.

The remaining three leaves are made in the same manner. At the end of the fourth leaf, in order to fasten the leaves together, make I ch., I cl. ch. in the stitch of the next leaf; repeat this four times.

Each rosette is worked as follows: make I chain of 4 ; join with I $\mathrm{cl}$. $\mathrm{ch}$.

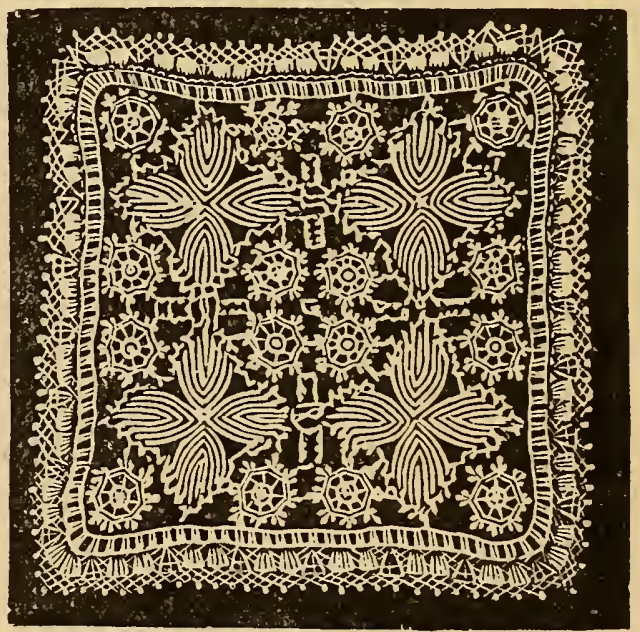

FIG. 332 .

Ist round, 2 sc. in every ch.; 2 nd round, 2 sc. around every sc. of the last round.

$3 \mathrm{~d}$ round, $8 \mathrm{ch}$., the first 3 will serve as the first dc.; alternate 7 times, I dc. around the second stitch of the last round, $5 \mathrm{ch}$.; finally I cl. $\mathrm{ch}$. in the third of the first $3 \mathrm{ch}$. of this round.

4th round, * $3 \mathrm{ch}$., I dc. in the middle one of the next $5 \mathrm{ch}$. of the last round, I cluster of 3 picots. (The picot is made in $5 \mathrm{ch}$., I sc. in the last worked dc.)

Make $3 \mathrm{ch}$., I sc. around the next dc.; repeat 7 times from *. Fasten the thread and break it.

Then work around the edge of the four leaves: * I sc. in the thirteenth sc., counting from the middle end of the leaf; † I ch., I picot (making $5 \mathrm{ch}$., 
and I sc. in the first of them; I ch., I sc. in the next point of the leaf; repeat four times from $\uparrow$, but when coming to the middle stitch of the third picot, join to the middle stitch of the next picot of the rosette.

Work next I ch., I picot, I ch., I sc. in the same point in which the preceding sc. has been worked.

$\dagger$ I ch., I picot, I ch., I sc. in the next point; repeat 3 times from $\dagger$, joining the ninth picot to the next picot of the next rosette.

Make I ch., I picot, I ch., I sc. in the following fourth stitch; $2 \mathrm{ch}$., I picot, joining it to the following picot of the same rosette; repeat 3 times from*, joining at every repetition the third picot to the next picot of the same rosette which has been joined last; and at the last repetition, join the ninth picot to the last picot of the rosette which has been joined first.

In this way one square is already made. Each next square is made in the same mannir, joining the picot of the square to the corresponding picot of the rosette, as is shown in figure 332 .

Then a picot pattern is also worked in the center of the four rosettes, coming together, as follows:

Starting from the middle stitch of the picot of a rosette, make $\dagger 2 \mathrm{ch}$, I picot, 2 ch.; join to the picot of the next rosette (see figure 332); repeat three times from $\uparrow$. At the last repetition, make $\mathrm{I} \mathrm{cl}$. ch. in the stitch from where one started first, instead of making a slip stitch.

The picot on the point of the leaves has to be joined by another picot to the corresponding picot on the point of the leaf belonging to the other square. To do this, start from the middle stitch of the first picot mentioned, make $2 \mathrm{ch}$., then join by I sl. st., $2 \mathrm{ch}$. and I cl. ch. in the stitch from where one started. Then work from the next point of the leaf, I ch., 3 picot, I ch.; join to the cluster picot of the rosette; 2 ch., I picot, 2 ch.; join to the opposite cluster picot of a rosette; I ch., 3 picot, I ch.; join to the corresponding picot of the leaf belonging to the opposite square; $2 \mathrm{ch}$, I picot, $2 \mathrm{ch}$., I cl. ch. in the stitch from where one started.

This pattern is very effective carried out in dainty colored Scotch linen crochet thread No. 70, or in knitting silk, while if cheaper materials are required, fine macrame cord will give very pretty effects.

If a bedspread and sham of this pattern be considered too much work it can be utilized for making toilet sets. 


\section{TABLE MATS.}

The set consists of six mats-three sizes of two each-and look best when crocheted with No. 9 macrame twine. The directions given are for the smallest size.

For the next size commence with 20 , and the largest with 24 stitches.

Ist row, make a chain of I6 stitches; miss the I6th ch. stitch; work I sc. in next I 4 ch. stitch; 2 sc. in next I 5 th; I sc. in following 14 stitches on the other side of the ch.; fasten in first sc. of this "row.

2nd row, turn; I ch. stitch, I sc. in last sc. of last row, passing the hook in the back loop of the stitch; 2 sc. in next; I sc. in following $12 \mathrm{sc}$; $2 \mathrm{sc}$. in I 3 th.; I sc. in next sc.; 2 sc. in the following one; I sc. in next sc.; 2 sc. in following sc.; I sc. in next 12 sc.; 2 sc. in 13 th; I sc. in I 4 th; fasten in first sc. of this row.

3d row, turn; I ch. stitch; I sc. in next 2 sc.; 2 sc. in third; I sc. in following 13 sc.; 2 sc. in I4th; I sc. in next 2 sc.; 2 sc. in third; 1 sc. in

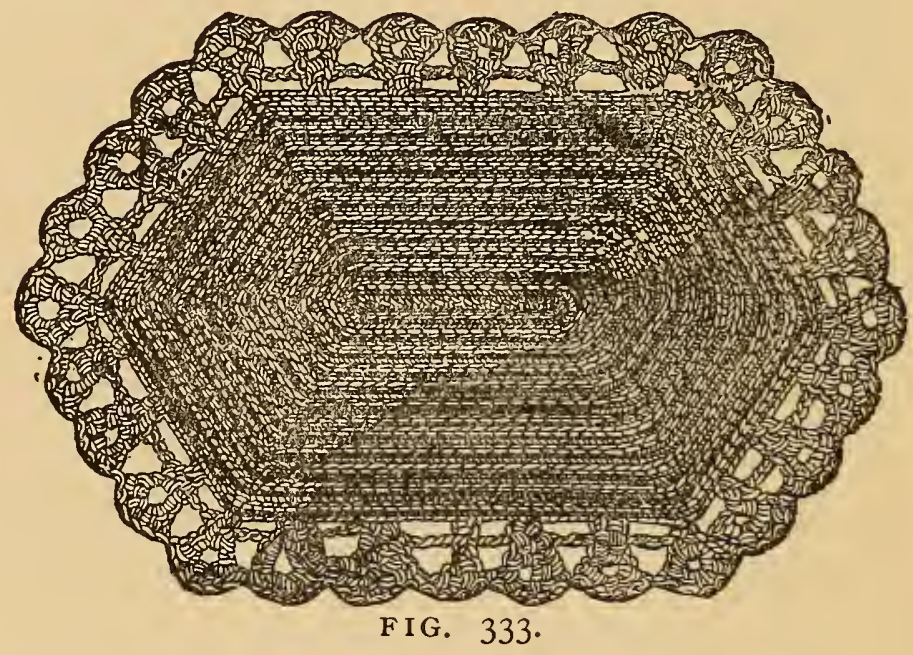
next $2 ; 2$ sc. in third; I Sc. in next I3; 2 Sc. in I 4 th; I sc. in next 2; fasten in first sc. of this row.

$4^{\text {th }}$ row, turn; I ch. stitch; I sc. in next 3 sc.; 2 sc. in fourth; I sc. in next 14; 2 sc, in I 5 th; I sc. in next 3;2 sc. in fourth; I sc. in next $3 ; 2$ sc. in fourth; I sc. in next I4; 2 sc. in I 5 th; I sc. in next 3 sc. Fasten as before.

Continue working the following rows in the same manner, always widening with $2 \mathrm{sc}$. in one of the sc. belonging to the last widening.

\section{BORDER TO MAT.}

Ist round, make $2 \mathrm{ch}$. stitch, which will serve as I dc.; I dc. in next stitch. Alternate to the end of round; 2 ch. stitch; $2 \mathrm{dc}$. in third and fourth stitch of the edge of the mat.

2nd round, work $6 \mathrm{dc}$. around the chain of 2 of last round, fastening every time in the middle of the $2 \mathrm{dc}$. of last round. 


\section{CROCHETED DOLL.}

Materials: double zephyr; bone hook, as small as can be conveniently used.

Commence at the waist with thirty-six chain stitches; dc. the Ist row; turn and work three more rows.

5 th row, dc. Io stitches, increase in the IIth stitch, dc. 16, increase, dc. 10; 6th row, work without increasing.

7th row, similar to 5 th, except that you knit I I instead oi ro. Continue thus until you have 46 stitches, 5 widenings under each arm. [You will see after knitting that two rows form a sort of stripe. See that these are uniform. A row across and back make this stripe, as the stitches are taken through the two loops of the chain, not the single one, as in ordinary crochet.]

Divide in three parts for the back and two fronts, 12 stitches on each front and 16 across the back; 3 are left under each arm. For the back, work four rows without increase.

5 th row, add one at each end of the row; 6th row, without increase.

7 th row, as the 5 th; 8th row, without increase.

9 th row, increase as the 5 th, making 22 stitches; roth row, without increase.

For the front work four rows on the twelve stitches.

5th row, add one stitch at the arm, keeping the front edge straight.

6th row, without increase.

7 th row, add I, making it 14 ; 8 th row, without increase.

gth row, add I; Ioth row, without increase.

Commence at the front and work across both fronts and the back, joining them at the shoulder. Crochet back; narrow at each shoulder at the I4th stitch (counting from the front edge) every other row until you have 27 stitches.

For the collar crochet a row across and back, leaving two stitches each side of the front.

For the skirt commence at the waist, work a row across the whole waist then back to the center of the back; work back again 12 stitches; next row knit II; each row one less until you have 6 (observe that this narrowing is at one side-the center is kept straight). Work the other side of the skirt similarly. 
For the sleeve commence at the wrist with 12 stitches-four rows without increase. Add I stitch at each end of every other row until you have 24 stitches and 16 rows, not stripes. [A stripe is two rows.] Work 6 rows, leaving off 2 stitches at each end; this adds 3 stripes to the 16 for the longest part of the sleeve. Crochet the sides together on the wrong side and sew in the arm-hole. Crochet a row around the wrist with black, with pale pink or flesh-colored single zephyr. Work the hand in the form of a small mitten: work two or three rows; divide in half and narrow it off in two places every row. Close at the end, join together as in a mitten.

The jacket is worked in red. Now, with black, work a row all around the collar fronts and skirt. It is best to cut a doll down to fit; slip the jacket on, crochet together down the front; sew three small gilt buttons on each side, two at the back and one on each sleeve.

For the pantaloons make 36 stitches for the waist; work back and forth as in the jacket.

Ist row, widen one stitch at each hip and in the center of the back; 2nd row, without increase.

3 d row, similar to Ist; 4 th row, like 2 nd.

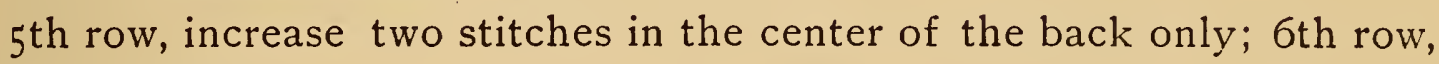
without increase.

7 th row, like the 5 th. Continue thus until you have 50 stitches. Make I4 rows. Break the wool and tie it at the hip; work 20 stitches; work back I8, leaving one off at each end. Next row, 16 and back I4. Tie the wool in front, work a row across all around, taking in the slope formed by the added rows; work back.

For the leg, divide in the center of the seat, 25 stitches on each leg. Work three rows; narrow at each end of the 4 th row; work 3 , narrow; thus until you have 20 stitches. The leg is 24 rows or 12 stripes long. Crochet together on the wrong side, turn them on the right side; $;$ with black double zephyr crochet a row around. Thus with red single zephyr you make the stockings; you can stripe them, if preferable. Make Io rows; reduce from 20 to 18.

For the boot take black double zephyr. Work two rows without increase; in the $3 \mathrm{~d}$ row you widen two stitches each side of one center stitch. You proceed thus until you make the foot as long as you require, say eight 
rows; then work one row without increase. Join the two sides together; work a row of black above the red stocking.

It is better to use a doll's body instead of stuffing the crocheted form, as it prevents stretching and is much more durable. A rubber or china head can be used, as preferred.

\section{KNITTED TORCHON LACE.}

Cast on 18 stitches. Knit back plain.

Ist row, sl. I, k. 2, over twice, p. 2 together, k. 5, narrow, over, k. 3 , over, k. 3 .

The 2nd and alternate rows are alike: i.e. knit plain until there are only 5 stitches on the left needle; then over twice, p. 2 togethcr, k. 3 .

3 d row, sl. I, k. 2 , over twice, p. 2 together, k. 4, narrow, over, k. 5 , over, k. 3 .

5th row, sl. I, k. 2, over twice, p. 2 together, k. 3, narrow, over, k. I, narrow, over, k. I, over, narrow, k. I, over, k. 3 .

7 th row, sl. I, k. 2, over twice, p. 2 together, k. 2, narrow, over, k. 1, narrow, over, k. 3 , over, narrow, k. I, over, k. 3 .

gth row, sl. I, k. 2, over twice, p. 2 together, k. I, narrow, over, narrow, k. I, over, k. 5, over, narrow, k. I, cver, k. 3 .

IIth row, sl. I, k. 2, over twice, p. 2 together, k. 2, over, narrow, k. I, over, narrow twice, over, k. I, narrow, over, k. I, narrow, over, k. 3 .

I 3 th row, sl. I, k. 2 , over twice, p. 2 together, k. 3, over, narrow, k. i over, narrow, k. I, narrow, over, k. I, narrow, over, narrow, k. 2.

I th row, s!. I, k. 2, over twice, p. 2 together, k. 4, over, narrow, k. I, over, sl. I, narrow, slip the slipped stitch over the one last knitted; then over, k. I, narrow, over, narrow, k. 2.

I 7 th row, sl. I, k. 2 , over twice, p. 2 together, k. 5, over, narrow, k. 3 , narrow, over, narrow, k. 2.

Igth row, sl. I, k. 2, over twice, p. 2 together, k. 6, over, narrow, k. I, narrow, over, narrow, k. 2.

2 Ist row, sl. I, k. 2, over twice, p. 2 together, k. 7, over, sl. I, narrow, draw the slipped stitch over the one last knitted; then, over, narrow. k. 2.

Repeat from first row. 


\section{SMALL COMFORT FOR LOUNGE.}

Take knitting needles No. 5 and double zephyr wool, in any pretty contrasting colors. The comfort is knitted in stripes which are crocheted or sewn together.

Cast on eleven stitches for each stripe.

Ist row, k. 3, make one, p. 2 together, k. I, p. 2 together, wool forward, k. 3. The 2nd and other intermediate rows are all purled.

3 d row, k. 4, make I, p. 3 together, wool forward, k. 4.

5 th row, k. 3, p. 2 together, wool forward, k. I, make I, P. 2 together, k. 3. The 7 th, 9 th and $\mathrm{I}$ th rows are like the $5^{\text {th }}$.

I $3^{\text {th }}$ row, k. 3, make I, p. 2 together, k. I, p. 2 together, wool forward, k. 3 .

I $5^{\text {th }}$ row, like the I $3^{\text {th. }}$ Repeat from the Ist row.

\section{PORCUPINE STITCH.}

Cast on in twelves. Ist row, plain.

2nd row, * over, k. 2 together, repeat from *.

3 d row, purl; 4th row, plain; 5 th row, purl.

6th row, sl. I, k. 2 together, pass the slipped stitch over, k. 4, over, k. I, uver, k. 4 ; repeat.

7 th row, p. 3 together, p. 4, over, p. I, over, p. 4 ; repeat.

8th row, like 7 th; $9^{\text {th }}$ row, like 6 th; Ioth row, like 7 th.

Repeat from second row. Very pretty.

\section{DIRECTIONS FOR MAKIVG WHEEL.}

Make a ch. of 12 stitches, join with sl. stitch, 3 ch., 47 dc. into ring, join in top of three ch. with sl. stitch and break off thread.

For the squares, make $9 \mathrm{ch}$., I sc. into any stitch of the circle, holding the wrong side of the wheel towards you, * turn and work back and forth upon the 9 ch., making 8 rows of sc., always taking up the back thread of stitch, to make the ridge, I $\mathrm{ch}$. at the end of every row. When the 8 rows are finished, make $9 \mathrm{ch}$., I sc., into the eighth stitch of the circle from that last worked into. Repeat from * until there are six squares, join the corner of the last square to the corner of the first with sl. stitch, and break off the thread. 
Ist round, (I sc. into the point of a square, 9 ch., I tr. between the squares, 9 ch.) 6 times; sl. stitch into first sc. of round. There should be I 2 loops of $9 \mathrm{ch}$., making Io8 $\mathrm{ch}$. in all.

and round, I sc. into every stitch of last round, join with sl. stitch; turn.

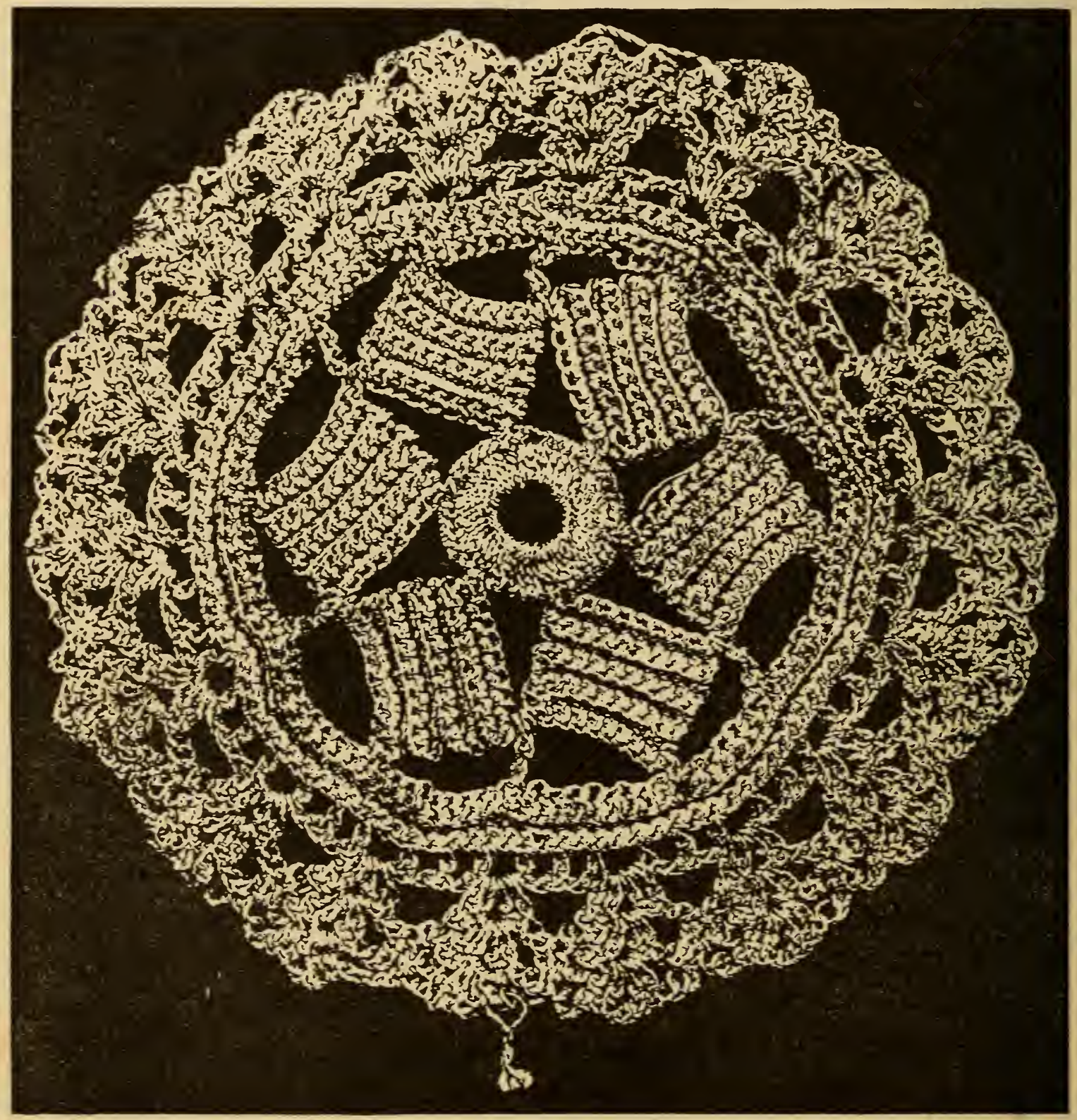

FIG. 334.

3d round, I sc, into every stitch of last round, taking up back part of stitch, join with sl. stitch; turn;

$4^{\text {th }}$ round, like $3 \mathrm{~d}$, but do not turn the work when the round is finished. 


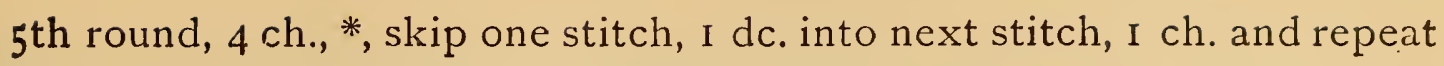
* all around last row, join with sl. stitch in third stitch of $4 \mathrm{ch}$. at the beginning, making 54 spaces around.

6 th round, 3 ch., I dc., 2 ch., 2 dc., into first space, ${ }^{*}$ ch. of 5 , skip 2 spaces, 2 dc., 2 ch., 2 dc. in third space and repeat from * all around, join with sl. stitch in top of $3 \mathrm{ch}$. at the beginning.

7 th round, 3 ch., 1 dc., 2 ch., 2 dc. in middle of shell made in row previous, * 3 ch., catch with sc. in middle of 5 ch., 2 dc., 2 ch., 2 dc. in middle of next shell and repeat from *; when finished there should be 8 shells around.

8 th round, like 7 th row. When done fasten and break off the thread.

\section{CROCHET: PETTICOAT FOR CHILD FROM 1 TO 2 YEARS OLD.}

Materials: $\quad 1 / 2$ pound German yarn, a medium size bone crochet hook and one yard of ribbon.

Commence at the waist, make a chain 25 inches in length, work in shell pattern.

Shell pattern is worked as follows: Draw up a loop through each of 5 successive stitches, draw through all the loops on the hook, close the cluster with one chain, * draw up a loop through last chain, another through back perpendicular loop of last stitch, and one through each of the two next stitches of chain, draw through all the loops on the hook together, close with one chain, repeat from * to the end of row.

The wool is broken off at end of each row, and every row is commenced from the same side. Work 5 inches without increase or decrease, that is until you reach the under part of arm-hole.

For the left half of back, work on one-quarter of the stitches for 3 inches, then decrease for the shoulder, by working all but the last shell at the end of each row for $1 / 2$ inch more. Work across the front for 3 inches, then decrease at the beginning and end of each row for $\mathrm{I} / 2$ inch more. The right front is worked like the left, but the decrease is made at the beginning of the row instead of the end.

For the sleeves, make a chain 8 inches in length, work in shell pattern for 8 inches, shape the top of the arm by leaving one shell pattern on each side unworked for three rows. Sew the sleeve together on the wrong side. sew up the shoulders, and sew in the sleeves. 
For the skirt: Ist row, into the edge of bodice work 2 tr., separated by 2 ch., into every other stitch.

2nd row, 2 tr. separated by $2 \mathrm{ch}$. under $2 \mathrm{ch}$; $3 \mathrm{~d}$ and following rows,

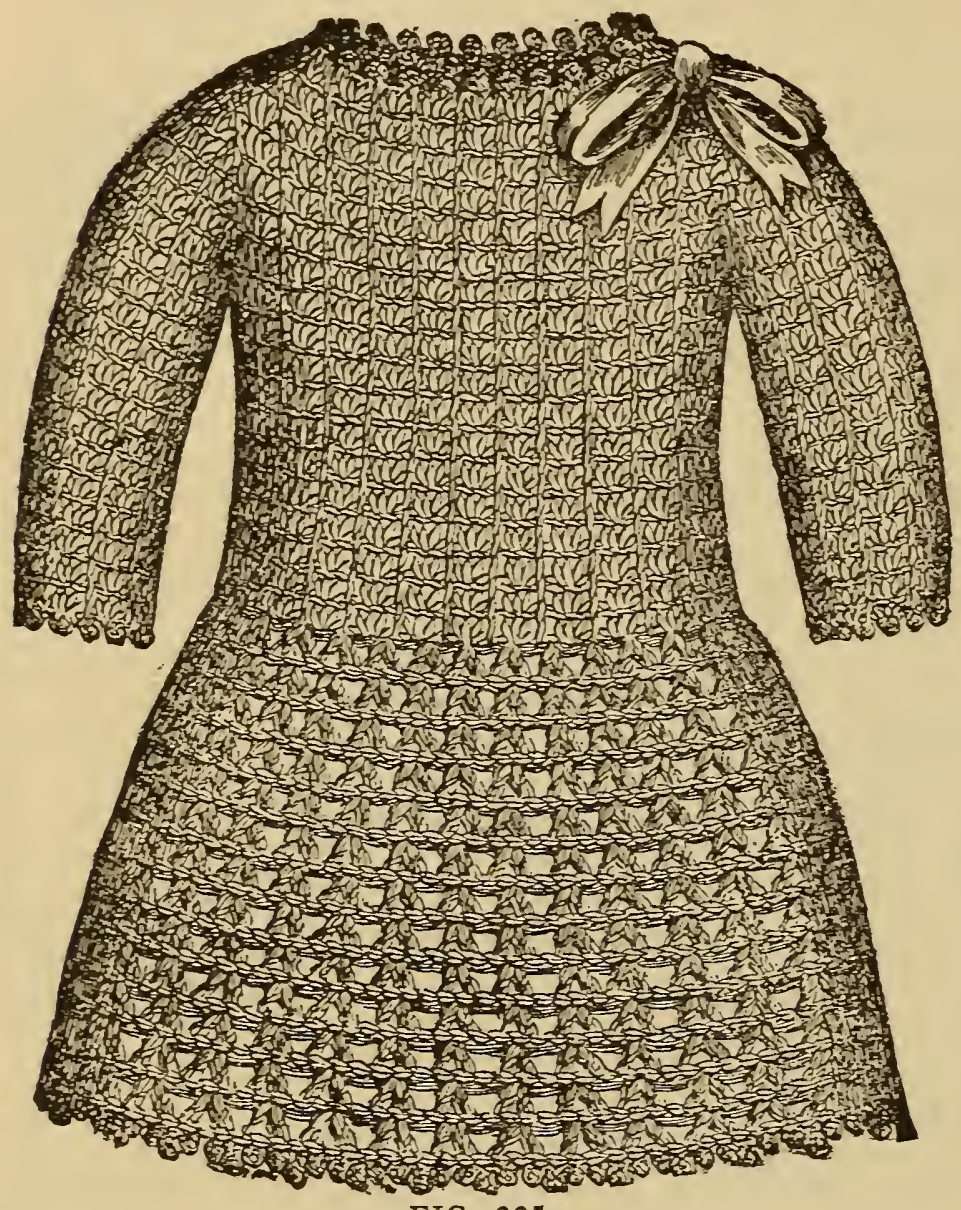
2 tr., 2 ch., and 2 tr. under 2 ch., I ch.; repeat from the beginning of the row.

When the skirt is the length required, for the edge, into the last row, work I dc. into a stitch *, 3 ch., I dc. into next stitch, 4 ch., I dc. into next stitch, 3 ch., I dc. into next stitch, repeat from * to end of row. A row of scallops is worked round the neck and wrist. Sarsnet ribbon, about I inch wide, is run through the work at the neck and waist. Sew two buttons to the left side of back, and in the same place on the right side, press the stitches apart and work two buttonholes.

\section{CROCHET: BORDER AND CORNERS FOR TABLE COVERS, \&C.}

Materials: Scotch crochet linen No. 70 and medium-sized crochet hook.

This border is suitable to edge table covers, toilet cloths, etc. The rosettes are joined when working the last row of each.

Commence in the center of a rosette with $4 \mathrm{ch}$., join round.

Ist round, $6 \mathrm{ch}$., $7 \mathrm{tr}$., each separated by $2 \mathrm{ch}$. under $4 \mathrm{ch}$., $2 \mathrm{ch}$., draw through the fourth of $6 \mathrm{ch}$.

2nd round, $2 \mathrm{dc}$. under $2 \mathrm{ch}$., $4 \mathrm{ch}$., draw through the top of last dc., 2 dc. under same $2 \mathrm{ch}$. the last were worked under, I dc. into top of tr.; repeat from the beginning of the round 7 times more. 
3d round, $9 \mathrm{ch} . *$, I tr. between 2 picots, $5 \mathrm{ch}$., repeat from * 6 times more, join to fourth of $9 \mathrm{ch}$. with I sc.

$4_{\text {th }}$ round, Io dc. under each $5 \mathrm{ch}$. of last round.

In working the next and following rosettes work I half tr. into 2 scallops of previous rosette when working last round (see figure 336). The

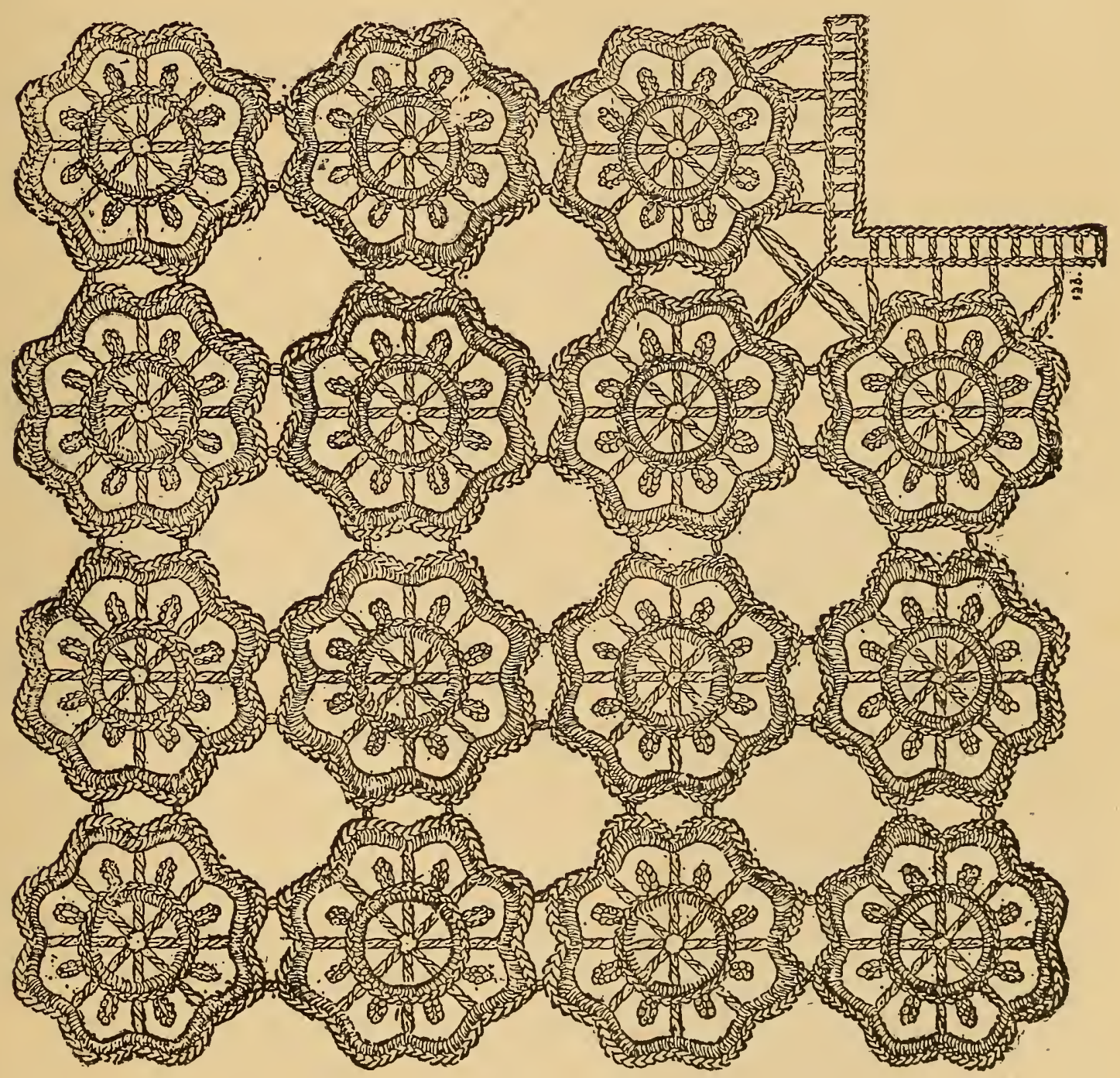

FIG. 336 .

method of joining the rosettes to form the corners will be clearly seen from the illustration.

For the heading, work I tr. into center of the first scallop at the top of rosette, $4 \mathrm{ch}$., I tr. between 2 scallops, $6 \mathrm{ch}$., I double tr. between 2 next scallops, 12 ch., I sc. into each of 2 next stitches of scallop, 5 ch., I sc. into seventh of $12 \mathrm{ch}$., 5 ch., I sc. into each of 2 stitches between 2 scallops of 
next rosette in the corner, 4 ch., I sc., into first of $5 \mathrm{ch}$., $5 \mathrm{ch}$., I sc. into each of 2 stitches in depth between 2 scallops of next rosette (see figure $336), 5 \mathrm{ch}$., I sc. into seventh of $\mathrm{I} 2 \mathrm{ch}$. and each of 2 following stitches, 4 ch. *, 3 tr. separated by 5 ch. into next rosette (see design), 5 ch., I quadruple tr. into depth between 2 scallops, work off 2 loops, I double tr. into next stitch but I, work off 3 loops, work 2 double tr. into corresponding place of next rosette, work off all the loops, $6 \mathrm{ch}$., repeat from last* until you reach the next corner, which must be worked as directed for first corner.

2nd row, I tr. into a stitch, I ch., repeat.

\section{CROCHETED SKIRT.}

The design shown here is one of the prettiest, as well as the best fitting we have seen. As it is fitted to the figure there is no extra fullness about the hips and waist, and it is light and warm.

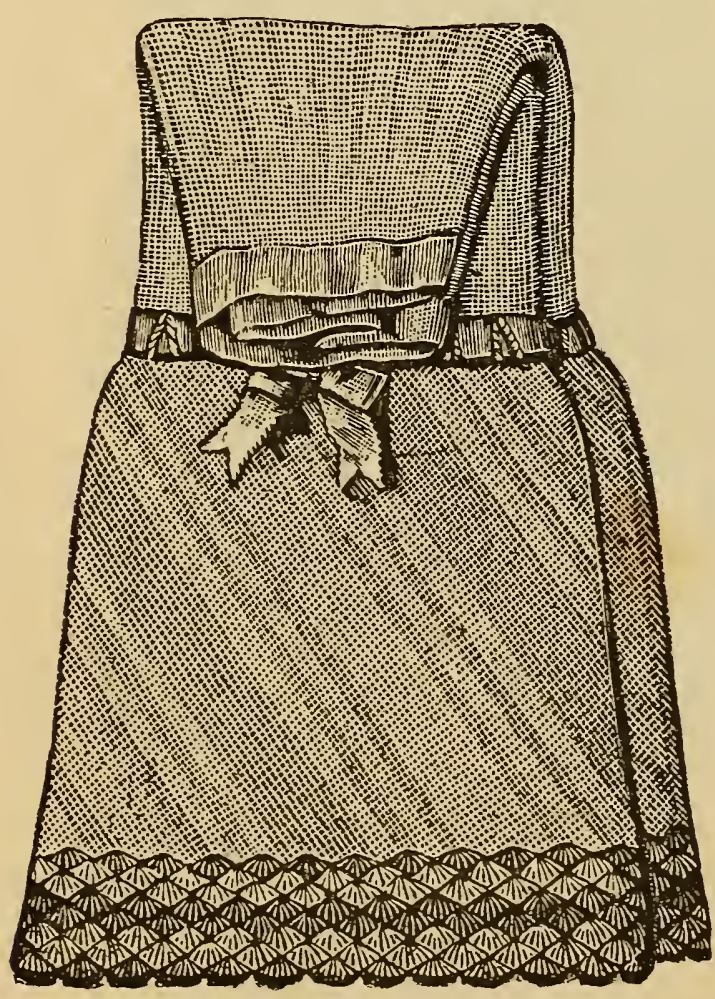

FIG. 337 .

Materials: Germantown wool-4 skeins of blue and 4 of drab; coarse tricot needle.

Yoke-Make a chain of 55 stitches; * with the blue and work 3 rows in afghan-stitch. Next work one row with drab to the eighth stitch from the end of the row, which forms the top of skirt, then one row to the Igth stitch from the top in drab. Repeat from * until there are 33 stripes of drab. Finish with 3 rows of blue.

Flounce - Make a chain of 75 stitches; * with drab wool; work 8 rows; then work 2 with blue, 2 drab, 2 blue and 2 drab. Next work 8 rows with blue, 2 drab, 2 blue, 2 drab and 2 blue. Repeat from * until you have 6 broad stripes of drab and 6 of the blue, and then join together. In making the flounce, the following directions must be exactly followed: (See page 382 for afghan stitch.) 
Ist row, plain tricot; after that make I chain at beginning of each row to form an extra stitch, and leave off a stitch at end of each row, keeping the same number of stitches all the time, but giving the flounce a bias appearance. Fasten yoke to flounce with chain of 5 stitches, caught first in the flounce and then to the yoke.

Through the openings made by these chains of 5 , a blue ribbon No. 7 , may be run and tied in a bow.

At the waist finish off the skirt with one row in dc., putting I ch. between. Through this a cord and tassel may be run. The opening at back of yoke may be finished with a scallop.

Border of Flounce-I row in drab shells of 5 stitches, 2 rows of blue, 2 of brown and 3 of blue; the last blue row having 6 stitches in each shell.

\section{INFANT'S BOOT: KNITTING.}

Materials: Three balls knitting silk and three skeins wash twist embroidery silk, four steel knitting needles No 18 , and a yard of narrow ribbon.

Commence at the top of leg, cast on 40 stitches on 3 needles, that is I 4 on each of 2 needles, and 12 on the third.

Ist and 2nd rounds, k.; $3 \mathrm{~d}$ round, bring the wool forward, $\mathrm{k}$. I in the front, I at the back, and again I in the front of next stitch, making 3 stitches in I, put the 3 stitches back on the left-hand pin, knit them, put them a second time back, and knit them, bring the wool forward, k. 3; repeat from the beginning of the round.

$4^{\text {th }}$ round, k.; 5 th round, cast off $4, \mathrm{k} .3$; you will have 4 stitches on right-hand needle; repeat from the beginning of the round.

6 th round, k.; 7 th round, k. 2 before beginning the raised pattern as described in $3 \mathrm{~d}$ round, this makes the patterns lie between those of $3 \mathrm{~d}$ round; end the round with wool forward, k. I. Continue to work as described, until you have 9 raised patterns in a diagonal line.

For the next round, k. 5, make I by bringing the wool forward, repeat, this increases the number of stitches to 46 in the round.

For the ankle, k. 22 rounds plain. For the heel, Ist row, k. 28; turn. 2nd row, k. I, p. $26, \mathrm{k}$. I. Repeat these 2 rows until you have worked 18 rows. 
I9th to 22nd rows $k$; ; this forms 2 purl ridges; $23 \mathrm{~d}$ row, k. I9; turn. $24^{\text {th }}$ row, $k$. IO, this leaves 9 stitches each side of heel; $25^{\text {th }}$ row, k. 9 ,

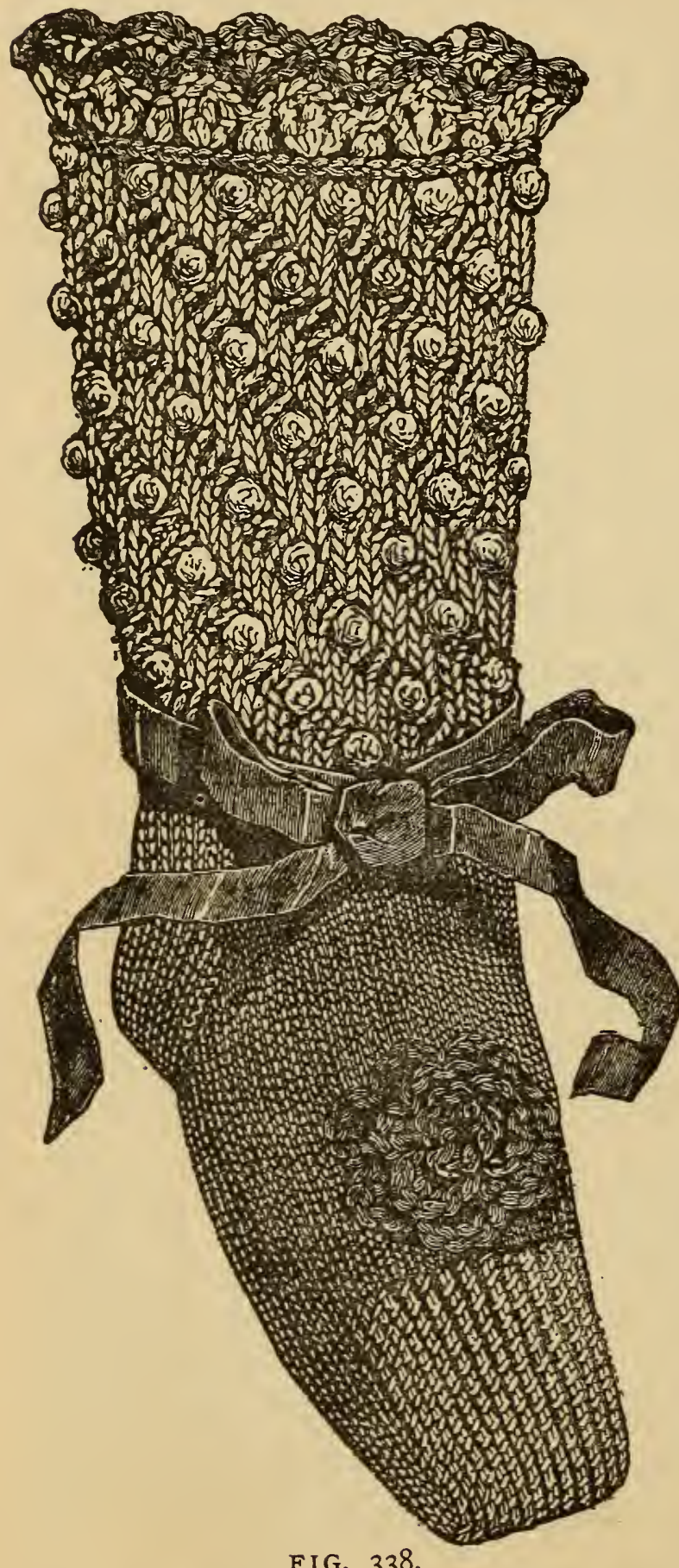
$k$. the next stitch, and I of the side stitches together, repeat this last row until all the side stitches are worked in, leaving Io stitches only on the heel needle.

Now pick up 13 stitches down each side of heel, $\mathrm{k}$. for 24 rounds. $25^{\text {th }}$ round, $\mathrm{k}$. the stitches for top of foot, k. 5 of the sole-stitches, k. 2 together, k. 22, k. 2 together, k. 5 , k. 3 rounds without decrease.

In the next round decrease again at the same places, but there will be only 20 stitches between the two decreases this time; continue to decrease 4 times more with 3 plain rows between each decreasing row; then at the top k. I, p. I across (for the remainder of the boot) reversing the stitches in each alternate round, but continuing the decreasings at sole for another 4 times, making in all ro decreasings on each side.

Turn the boot inside out, put 2 stitches from each side of sole stitches to the top stitches, k. together, I stitch from the top, and I stitch from the bottom, then 2 from the top, and I from the sole (casting off each time) across the whole stitches, until there are left but I top and I bottom, k. these 2 together, and cast them off.

For the crochet trimming round the top work with wool:- 
Ist round, I double into a stitch, I ch., pass over I stitch, 3 tr. each separated by I ch. into next stitch, I ch., pass over I stitch and repeat all round.

2nd round, with silk, I double into each stitch. For the line below the scallops, with silk, work I double into each stitch.

For the rosette, with silk, make a chain of 14 stitches, turn, work I double, 3 tr., and I double into each of 3 stitches, I double, 4 tr., and I double into each of 4 stitches, I double, 5 tr., and I double into each of 5 stitches. Roll the work round, with the smallest scallops in the center, sew securely at the back of work, and sew to the toe of boot. The ribbon round the ankle is sewn to the boot at the back, and is tied in front.

\section{CROCHET MOP FOR DUSTING PICTURES.}

The above mop may also be used for washing pictures, and is com. posed of a wooden or cane stick one and a half inches thick, and any length desired, covered with a strip of crochet. One hole is made at lower end of this stick, through which is run a red ribbon and tied in a bow; and two more holes, one and five-eighths inches apart, for fastening on the mop itself, at the upper end. The crochet is made of knitting cotton, No. I, in red and white. The crochet for the stick is begun with $4 \mathrm{ch}$. closed to a ring, into which are worked 2 sl. st., 3 sc. and 5 dc., the crochet is then continued in this manner the desired length (three rows of white come alternately with one of red); the work is then drawn over the stick and again closed with I sc. The single strips, for the mop part, which are four and three-quarters inches long, are each worked in two rows, on each side of a foundation of I 8 or $20 \mathrm{ch}$. In the second row, however, each sc. is worked over the foundation chain and the front link of the sc. 1ying underneath; in this way the strip becomes a little rounded and firmer. Twenty red and the same number of white strips must be made and then strung

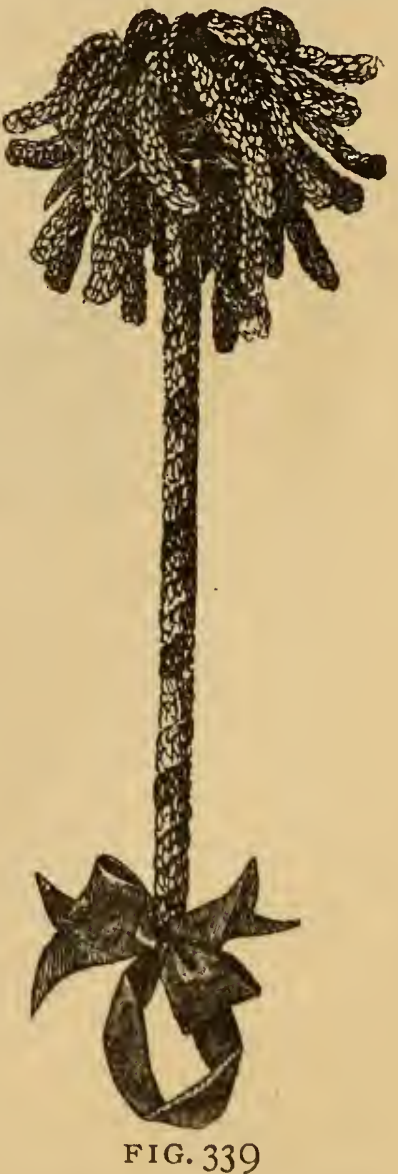
in pairs of each color on a piece of thick cord, put through the two holes 
already mentioned, and twisted around the stick, the ends being fastened at these holes.

\section{CROCHETED NOVELTIES.}

Many pretty little articles are made by crocheting forms, and then stiffening them with glue or gum arabic. Bottle covers, slippers for watch cases, scarf bags, and boxes are made in this manner. Very pretty covers for square bottles may be made from almost any design given in this chapter. A piece, large enough to go around the bottle, is made from fine linen crochet thread. Stretch it on a board, and paste with gum arabic. When dry, paint with gold powder, wet in a medium. The edges should now be joined together, and a piece of silk gathered into the bottom, when the cover is ready for the bottle. Small baskets are made in the same manner. Very pretty effects are obtained by crocheting a pattern of an oven design, and lining with bright-colored silks.

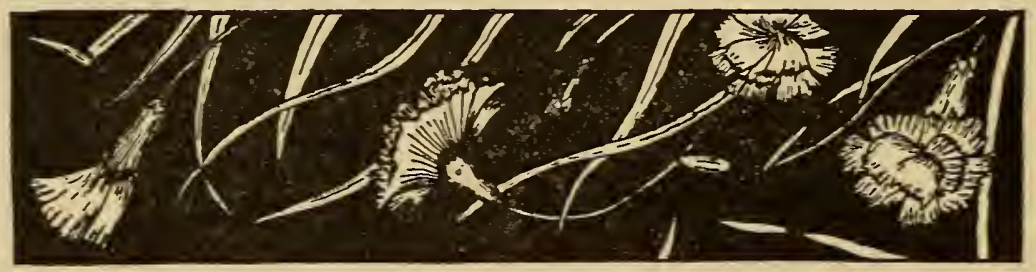




\section{CHAPTER XVII.}

\section{DAINTY WORK FOR PROFIT.}

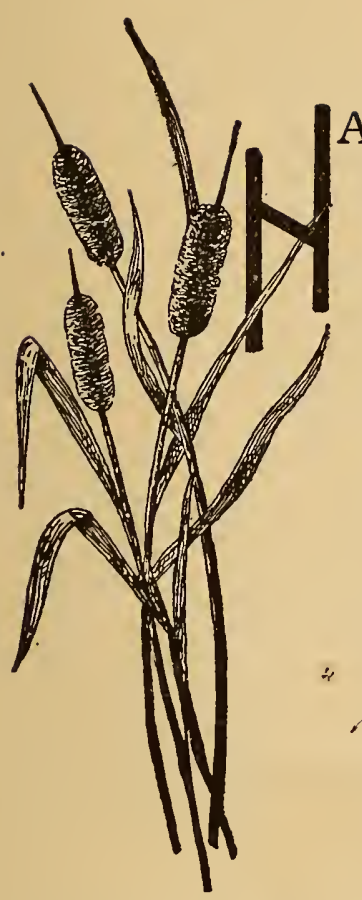

AVING discussed "Dainty Work for Pleasure" or amateur home adornment, we will now deal with it from a practical standpoint, or as a means of producing dollars and cents. "Dainty Work for Profit" is a subject that will appeal to the great army of women in our country who feel the need of adding their mite to the family exchequer, either as wife, mother, daughter, or sister. These women may be divided into two classes. First: those who have only a limited portion of time to devote to any work outside the regular home duties, and who could not be absent from home for any regularly stated time. Second: those women who have to assume the support of families or portion of families, and whose time must be given to that work, leaving home cares and duties to others. For both these classes there is a note of hope and good cheer in the words "Dainty Work for Profit." By this it must not be assumed that all women are fitted to become dealers or teachers of art work, either in painting or embroidery. There are some women totally unfitted by previous occupation, as well as inclination and natural taste or aptitude for this kind of work, and it is manifest that such would not make a success; but these cases mark the exception to the rule, and need hardly be considered, as they would be far from likely to adopt decorative art work as a means of livelihood.

We will discuss first "Dainty Work for Profit" for the

\section{Stay-at-Homes.}

There are hundreds of homes of wealth in the country that have not within them the means of artistic decoration. It may be the mistress has 
passed the period of life when "fancy work" had a charm, she may be a woman of "ability" who scorns the "little trifles" of life, or it may be the mother and daughters have, by years of pinching economies, helped to upbuild that elegant home of brick and mortar, and so have not had time to give it the home-look of habitations where womanly love and care presides over all departments. It would be unjust to say that the inmates of these bare-looking homes do not desire beautiful pictures, graceful draperies, pretty nick-nacks and all the little touches that help to make the real home. They do desire these things, as the orders daily received by the leading upholstery houses in the large cities attest, and here is the opportunity for the woman who has a love for making beautiful things, and the time to give to it. She can exchange her brain work, her artistic abilities for the dollars and cents of her wealthier neighbors by taking orders for making their homes beautiful, "and filling them within the shelter of her own home."

\section{The First Start.}

To make a beginning is the hardest part of the battle, and as so much depends upon the right kind of a start we will here give a few general hints, which, while they may not fit each individual case, can be modified to meet almost any requirement for doing work at home, generally called "order work," sometimes including "teaching."

In the first place the woman who intends to do "order work" to. make it pay, must know what she can do, and must have absolute confidence in her ability to do it and do it well. People do not care to pay for art work or decorative work unless it is what it claims to be, therefore the very first step to take on the road to success is thorough preparation. It is absolutely neccessary that the worker should understand hem-stitching, Roman embroidery, Kensington embroidery, satin stitch and novelty stitches; she should be capable of making up the different articles after the work of embroidery has been completed.

Understanding all the above requirements she should carefully consider her territory, go over every inch of the ground mentally and decide what articles will be most likely to find sale. The next thing to consider is the question of 


\section{Commenced Samples.}

This is a very important point and one upon which depends much, if not the entire future success of the worker. The item of expense of these articles is one of serious moment, and in many cases offers almost unsurmountable obstacles, but very little can be accomplished without them, and the difficulty might as well be met first as last. However, there are relative degrees of cost and some very attractive samples can be gotten up with little outlay of money, and it is here the writer wishes to give some very practical words of advice. Stamp and commence only such samples as you think there is a reasonable chance of selling. For instance: in your mental survey of the field of possible customers, you have decided that Mrs. A., who has a "craze" for decorated table linen, and cares little or nothing for articles of mere ornament, will most likely give you an order for some doyleys or a center piece. Your wisest course will be to stamp on fine, firm linen a design for center piece. The design may be Dresden or Roman embroidery or a conventional design filled with fancy filling stitches, in either case a section should be neatly worked, then on good brown manila paper stamp twelve different doyleys to go with the center-this saves spending the money for the linen for the doyleys and will answer as well.

These articles will make a practical beginning for your samples; but you have provided for the taste of only one class of customers; there is another class who have an especial pride in bed-room furnishings. To meet this possible demand stamp two pillow shams-not mates, but one of two different sets-and treat each one differently in the sampling. Again, some ladies have a penchant for hem-stitched hems, therefore one of the shams at least should be so finished, it may be the means of obtaining orders for many yards of hem-stitching. Then, as towels with handsomely worked initials are dear to the heart of every woman worthy the name of home-maker, have at least one sample worked, and let the stitches be set "on honor" every time, as in no other form of decorative work does the effect depend so much upon the neatness and exactness with which the design is wrought.

If these instructions have so far been followed the worker will have as samples: one center piece, $27 \times 27$ inches, one set of twelve doyleys stamped on paper, two pillow shams and one embroidered towel. These sam. 
pies, if stamped with attractive designs and having a portion of each neatly sampled or commenced, to show how the work will look when finished, will afford sufficient working capital for a start, and should not cost to exceed $\$ 2.00$. This estimate does not include sufficient embroidery threads to finish. In addition to the above it is a most satisfactory plan to have at hand a neatly arranged book of samples of different fabrics, as several qualities of linen, satin damask, scrim, crash, plush, sateen, and any new material that may be suitable for decorative needle-work; have each sample neatly labeled, with name, width, and price per yard. In addition there should be a line of samples of all the various embroidery threads, which will be found named and described in the chapter on "Materials." Have these threads arranged so that they can be displayed without much handling, as that will detract from their lustre and finish. These samples can be procured for an additional expenditure of $\$ I$.

\section{Designs for Order Work.}

There is yet another question, and a most important one, viz.: Designs. The successful order worker should have a selection of stamping patterns of exclusive designs, that is she should be careful to supply herself with such patterns as are not to be had elsewhere in her territory. These designs should include three center cloth patterns, twelve doyleys, (different designs for each) butter, egg, fish, hot-roll and cake doyleys, one lunch set, three designs for shams, two Dresden designs that can be used for any purpose, three cushion cover designs-one floral, one conventional and one Roman-and half a dozen all-over patterns that can be used anywhere, on large or small articles.

Do not allow these designs to become common. If you use one design for a center piece for one customer, do not repeat that design for another customer in the same neighborhood, or even in the same social circle; no lady likes to spend money for an article she fondly hopes is the latest thing in art work, only to find it duplicated on half a dozen tables. The moment one of your designs is to be had in the shops, discard it. Your only hope for order work and good pay for same, lies in your ability to give the ladies something they think cannot be had elsewhere. To keep your designs exclusive and fresh will require time, care and eternal vigilance, but be sure it will pay. 


\section{Soliciting Orders for Embroidery.}

Having a knowledge of art embroidery, and being perfectly posted as to the prevailing "fads" in the decorative line, and in possession of the commenced articles, and a line of samples, you are prepared to enter the list with a fair chance of winning a brilliant victory; but there is one quality we have, as yet, not mentioned, and one without which success is impossible, viz.: confidence; confidence, not in your customers, but in yourself. And here is where failure is likely to stare you in the face at the outset; but do not give up in despair, if you have not "confidence," then you must cultivate that necessary quality. It is always better in this work to make a personal canvas of your customers, taking your samples with you and soliciting the order in person. In working your samples, you have had in mind certain persons whom you thought it more than probable would be interested to the extent of ordering the sample finished. Call upon such first; state clearly, without embarrassment or false pride that you intend to establish a parlor for private order work, that you will furnish only exclusive designs, and that you wish to secure the lady as a patron; show your samples, which are bound to interest any woman, no matter how much she may be determined not to give you her orders, or how much she may think there is nothing in the world she needs in your line. The ice once broken, the matter is easy. Interest grows with every word you utter on the subject. Be sure to lead the conversation, holding it well in hand, and close to the line of home beautifying; be enthusiastic, talk of what is being used for this and that purpose, discuss bed-room furnishings, suggest novel and pretty ideas for wall and mantel ornaments, a cushion cover, an ottoman, any and everything in the line of art work. Be decided in expressing an opinion as to the relative merits of shading, stitches to be used, materials-in a word, leave upon the mind of your listener the firm impression that what you don't know about interior decoration isn't worth considering, and all the while this should be the truth, for you have no business in the field unless you are posted, unless you do know that your ideas are bright, original and practical. An hour spent in such conversation with the right person is "capital well invested."

The first day's work in soliciting may not result in obtaining any definite orders (this is hardly probable) but a beginning will have been 
made. The ladies called upon will be sure to talk about the work, the ideas you have advanced, and the samples you have shown, and it will not be long before the seed sown will bear fruit in more orders for decorative needlework than one pair of hands can well accomplish.

\section{Teaching Embroidery.}

If you have the time, it will pay to interest the ladies to do some of their own embroidery, and to this end classes should be formed, of two or three ladies for a class, not more, unless you have been accustomed to teaching. Induce them to commence some article, rather than to work on a sampler; stamp the design clearly, according to instructions given in chapter on "Designing," and lay out the proper shades and select a suitable embroidery hoop; all this should be done previous to the hour appointed for the lessons, so that no time may be lost. Aside from the profit accruing from the lessons, it will be a certain means of extending your fame as an art needleworker abroad, and will not in the least diminish your chances for obtaining order work.

The most embarrassing question connected with this method of doing business is the one of

\section{What to Charge and how to Collect}

and especially is this the case where parties are acquaintances, and may be friends. The social relation should not be considered in this connection, and the only successful method is to adopt a rule of procedure to be applied to all cases, and apply it impartially. Never accept an indeffinite order, as "my dear Mrs. A. I believe I will have you get me up something for my dressing case, something pretty!"

"What would you like?"

"Oh, I'm not particular, just anything that would suit you!"

Never be betrayed into filling such an order. Insist upon the name of the article being specified, the nature of the work, the scheme of color, the amount of work, cost and nature of materials, and last the price of the whole when completed. Make a memoranda of the items in the form of an order, read it to the lady and pass it to her for her signature. This will preclude the possibility of any mistakes being made, and leave no loopliole for misunderstandings to occur when too late to rectify them. Be 
sure to fill all orders according to agreement, make out a bill for the work and present when the work is delivered, and insist gently upon payment at that time; if this is not possible have a deffinite time set when you are to call for the amount, and be punctually on hand. Women, as a rule, are apt to be careless in regard to the payment of such bills, and allow them to run into indeffinite time, but they will none the less be your customers because you insist upon your rights in a dignified manner.

Where the parties are not known to you insist upon an advance payment of at least one half the price when the order is taken, and the balance upon delivery.

Do not work too cheap, neither be exhorbitant in your charges. Makc the estimate of cost upon the time occupied in filling the order; at the rate of $\$ 1.50$ per day is a fair consideration. When possible furnish the embro:dery silks, linen or other threads used in your work; by this means you will always be certain of having correct shading and then, too, the profit on the same will be an item worth considering at the end of a year, as you will bc able to make arrangements to secure these materials in small quantities of - the wholesale houses.

The chapters on "Shading," "Designing," "Stitches," "Materials," "Art Embroidery," including "Roman Embroidery," "Kensington and Satin Embroidery," "Application of Stitches," and "Drawn Work," contain all the instructions necessary to thoroughly equip one for "Ordcr Work," without the aid of a teacher, if even the learner has had no previous instructions in embroidery, provided she has any natural aptitude for the work.

\section{Art Bazaars or Fancy Goods Stores.}

We come now to the second class of women, who need to earn money, and have an inclination to turn their energies to interior decoration and art needlework. For such the" "Art Emporium," "Fancy Goods Bazaar," or "Decorative Art Store" offer a field that well repays careful cultivation.

The first consideration for such a departure is that of Capital, usually, a word of discouraging import to women of ambition, as so few women ever have anything except brain, energy, pluck, faith and hope, to work wi h; but even the want of capital can, in a measure, be overcome by good bus- 
iness ability. Of course there must be some ready money, but the amount actually necessary can be brought down to quite modest proportions, provided, always, one knows how to go to work, and has a good reputation at home for integrity and honesty of purpose in all the relations of life.

In opening a store for the sale of any line of merchandise there are many things to be considered, but most important of all is that of location; and in no other business is it more important-we had almost said as important-as in that of decorative art supplies. Women are creatures of impressions as well as of impulses, and in the matter of patronizing art stores it is certainly true. A good field for a lucrative trade may be utterly ruined simply by having the store on the wrong side of a street, or because it is located next to some objectionable place, that has been put under the ban of the fair ones of the town. Then, in selecting a store, give the matter earnest consideration; stand upon a corner near the location you prefer on a fine day and watch the pedestrians; note especially the lady shoppers and the side of the street they affect; note also the particular locality on the chosen side they longest linger, and most congregate, and there pitch your tent, if possible, even if the obtainable location there does not offer half the conveniences to be had elsewhere. Better stay out of business than locate it on the wrong side of the street, or on the wrong street, for that matter. The location secured, the next step to consider is the proper

\section{Store Fittings.}

These may be very expensive,or simple home-made affairs, as the purse of the proprietor can afford. It is a matter of fact in these days of enterprise and business push that the richer the furnishings and the better the impression of business prosperity that can be sent abroad the better the business is sure to be in point of fact as well as reputation; "nothing succeeds like success," says Emerson, and a better business motto could not be adopted. If plate glass and brass-trimmed show cases and handsome wall cases are out of the question at first, then the general air of well-to-do prosperity must be imparted to the store by some other, less expensive means.

A good wall case for the display of large articles is an absolute necessity, as it serves to protect from dust such articles, while it, at the same time, allows of their examination without handling. Such a case can be 
made by any carpenter, and common pine will do for the frame work. The case should reach almost to the ceiling, and should be lined inside with tar paper, for the purpose of keeping out moths; brass hooks should be placed at intervals around the inside of case, and at different heights, for the purpose of suspending articles. There should be suspended from the top of the case a few "swings," through which to throw handsome fabrics, embroidered or not. The swings allow of most graceful arrangements, but they should not be so placed as to interfere with the view of articles hung on the back wall of the case. The doors should be paneled with glass, and the frame stained or ebonized. Very pretty show cases, nickel trimmed, can be purchased for almost any price from $\$$ I 2 to $\$ 25$, and of these there should be at least three, as they help to give an air of "fullness" to the establishment. For the show windows there should be several side brackets, to fasten to the window frame, one or two small stands that can be placed in the window for the purpose of displaying scarfs, covers, etc., two or three swings for each window, and two small easels, which can be purchased for 50 cents each.

The store room should be divided in the rear so as to enclose a space for a work room. This is quite necessary, and the partition should be strong and have a door that can be fastened, when necessary. Do not be deluded into using screens or a curtain draped on a pole for this purpose. The work room should never be opened to the public-and above all to favored customers; it should be kept sacred to the "secrets of the trade." It is here that stamping, embroidery and finishing articles should be carried on, and the worker should not be subject to the interruptions of the idle and curious. This partition should be made of good matched stuff and stained, and will offer a good surface for the display of such articles as will not be injured by exposure. The wall case should occupy one of the sides of the room. In front of it should be a couple of chairs or footstools and a small stand. On the opposite side of the room should be arranged shelves, in front of which place two of the show cases. Across the end of the room, in front of the partition, place another case; then the room will be covered on three sides with show-case display, and the front windows will be reserved for street display. 
This arrangement is especially desirable when the stock to be carried is limited, and a good appearance is to be made with the smallest possible outlay of money. The store fittings decided upon, the next question is the one of

\section{Stock or Goods to be Carried.}

This is a question that must be decided by each person for herself, as the same rule will not apply to hardly any two localities that could be named. The goods that will find readiest sale and yield best profits depends entirely on the class of people to be catered to.

If the location has a good farming country tributary to it, then such goods must be carried as will appeal to that class, and ideas and styles that are a little old in the cities will find readiest sale. If, however, you intend to depend upon town trade, almost exclusively, then your stock must contain the latest novelties in work and material; but happy is the dealer whose trade combines both these classes.

There are certain things that an art store should always be supplied with, as follows: embroidery needles, hoops or frames, embroidery silks, all sizes and shades, embroidery linen, embroidery cotton, knitting silk, wools, gold threads, couching cords, German cord, stamping patterns and chenilles and arrasenes if the trade calls for them. These articles are staple, and are as necessary to the art supply dealer as flour and sugar are to the stock of a grocer.

In putting in a stock of silk or linen threads select the make that you wish to carry, and put in a complete line of that manufacture, selecting the different sizes, and a good variety of shades in complete lines. Thus, if you buy rope silk in pink, put in the entire line of pink shades; this rule applies to the rope silks, filo floss and royal floss. In etching and heavier twist silks close shading is not so necessary, and only such shades should be carried in stock as are commonly called for, as yellow (different shades), red, pink, blue, brown, green and white. The same applies to linen threads. For a description of these materials see chapter on "Materials." Never allow your stock of embroidery threads, of whatever kind, to become depleted; keep the shades full and complete. Never allow a customer to ask for a shade or color that you cannot supply, and at once, without waiting to order from your jobber. And above all things never mix your stock 
of silks and linens by adding to it from the stock of other silk and linen manufacturers than the one you first selected. Their materials may be as good, the colors as fast, but no two manufacturers ever produce the same line of shades, and it is fatal to mix them. If for any reason you become dissatisfied with the goods you are handling, close them all out before you make a change, and when you are ready put in a full and complete line of the new goods.

\section{Art Fabrics.}

Art fabrics can be purchased from most of the jobbers in quantities as small as half a yard. Where the capital will allow, it is a good plan to purchase Io yards good fine linen, 40 inches wide; Io yards same quality, I 8 inches wide; 2 yards satin damask, 72 inches wide; 2 yards silk bolting; $21 / 2$ yards No. 6 and the same amount No. 4 Bargarren art cloth, in cream white; $21 / 2$ yards cotton tapestry; $1 \mathrm{I} / 2$ yards ecru art linen; 2 yards linen lawn for doyleys; one table cover; I $1 / 2$ yards square, mail cloth; one table cover, same size, of double-faced sateen. As these are high-priced fabrics, they will give an air of elegance to the stock. The above materials should be in addition to the commenced or made-up samples.

Ask your dealer to supply you with a sample book of art fabrics, from which your customers can select, when the goods may be ordered after the sale is made.

We now come to the dressing of the store-to the samples of work; and here lies the key-note of success or failure.

\section{Sample and Display Work.}

The samples should be completed before the store is rented, or stock ordered; and this is the more important if you must, from reasons of economy, do all the work yourself. You should not have the worry of them on your mind while getting ready for the "opening." As an assistance in selecting samples that will make the best display with the least outlay, we append the following list of articles, with their approximate prices.

This list must not be accepted as absolute and arbitrary, as individual cases may, nay undoubtedly will, require modification; but it will, at least, be a guide for all and a rule for many cases.

In giving estimates of cost it is assumed that the prospective dealer will herself do the work of embroidering and making up the various arti- 
cles, and that her purchases for display samples will have been made at wholesale:

\section{List of Desirable Samples.}

One table cover, 54 inches square.

One table scarf, 72 inches long.

One bed spread, 2 I $/ 2$ yards long, 72 inches wide.

One dressing case scarf.

One pair pillow shams.

One embroidered guest towel.

One-half dozen doyleys.

Three center cloths-one square, one round and one long.

Three plate doyleys.

Butter, corn, egg, muffin and fish doyleys-one of each.

One five-o'clock tea cloth.

Three drapes, one toilet set, including bottles, mats, etc.

Six different-shaped toilet cushions.

Three chair rests.

Three sofa cushions.

Two or three mat-covers for small stands, and, if possible, a few madeup novelties in the shape of calendars, photo frames, pen wipers, hair-pin receivers, etc.

\section{Materials for Made-Up Samples.}

This is a question that must be decided according to the fabrics that are newest on the market at the time the samples are required. For the next five or six years the following fabrics may safely be employed, as they are, and will continue, staple: Linen fabrics are always the correct thing for table and bedroom sets, and know no "change of style." For table scarf and cover, either sateen, mail cloth or Russian tapestry may be used. For one sofa cushion, plush, for another, faille silk, and for a third, art linen, in colors. For the pillow sham, good domestic linen; for the bedspread, No. 6 Bargarren art cloth, homespun linen or art tapestry; for the toilet cushions, fine linens and China silk; for the doyleys and center pieces, good, firm linen of a fine quality; for the tea cloth, satin damask. Chair rests may be made from soft silk and linen lawn. Dressing case 
scarfs should be of hollywood drapery, linens, or bolting silk. Drapes are prettiest made of soft China or India silk.

In making up the table cover, stamp the corners with a floral conventional design, as these are more popular than the geometric patterns. Work one corner complete in short and long stitch with rope silk, being careful to shade properly-according to directions for SHADING given in Chapter VII. If there is time, and the cost of the extra silk no inconvenience, this piece should be finished and lined, when it could be displayed on a table or hung on the back of the wall case in such manner as to show all the work.

The table scarf may be made of Bargarren art cloth, plush or basket silk. Both ends should be stamped. Work one end in a floral design, with conventional shading in solid Kensington stitch, the other end may be treated with a conventional design, outlined, and filled over the surface with fancy filling stitches.-See chapters on "Stitches" and "Application of Stitches."

Bed furnishings are subjects in which almost every, woman is interested, therefore the sample bedspread and pillow shams should be undertaken with much thought and care. We might suggest a number of artistic and beautiful combinations, both as to foundation fabric and embroidery threads, that would make a brilliant display, but there is also the question of utility to be considered, so that one cannot do much better than to select a cream-white Bargarren cloth, No. 6 mesh, for the spread, or a heavy Roman twilled linen, if preferred-either fabric improves with repeated launderings. Stamp a conventional design over the entire spread. No patterns with many and intricate details should be used, but branching sprays, with large petaled flowers and generous foliage. Work a good portion of the spread-at least one-quarter-with Bargarren art thread, in outline stitch, or short and long stitches; or, if the twilled linen is selected for the spread, the design can be worked out with rope linen. Finish the side edges of the spread with a handsome crocheted edge, made of Scotch crochet linen thread No. 50-figure 336, on page 393, in the chapter on "Knitting and Crochet," will furnish a beautiful design for this purpose. Such an edge will be the means of selling crochet threads of various kinds, in which there is always a good margin of profit. The shams should be stamped and sampled to match. If possible, an additional pair of shams 
should be stamped with Roman embroidery design for the edge, and the center with Dresden effect, and sampled with linen floss. The material should be. linen. These are not necessary, but make a good show case article. Dressing case scarfs may be made of fine linen; with hemstiiched hem, and have a few scattered flowers over the surface, worked solid; or, one may be made of bolting silk, with a scattered design of chrysanthemums over the entire surface, worked in outline, with one strand filo floss in pale yellow, white or rose-pink. The edges should be finished with fine, soft lace, and the scarf should be displayed over a quilted or tufted mat, made to just fit the top of dressing case. The mat should be made of China silk, the same color as the threads used to embroider with.

A toilet set of bottles and mats should be made, and for a model for this purpose see page 84, chapter on "Art Embroidery." Ideas for center piece, doyleys, mats, cushions, see chapters on "Dainty Trifles" and "Ar: Embroidery." The doyleys and mats should be completed, as they do not show to advantage sampled. The following is an approximate estimate of the

\section{Cost of Commenced Samples.}

Table cover, 54 inches square, mail cloth............\$3 00

Silks for sampling on . corner.................. 40

Silk for completing embroidery $\ldots \ldots \ldots \ldots \ldots \ldots \ldots \ldots \ldots$ I 60

Lining and cord for edge finish $\ldots \ldots \ldots \ldots \ldots \ldots \ldots \ldots \ldots$ I 55

Cost, complete...........................\$6 55

Table scarf, 72 inches long, Bargarren art cloth.........\$ 80 Rope silk, for working both ends short and long stitch and

fancy filling stitches...................... I 20

$\$ 200$

Bedspread, Bargarren art cloth.................. \$2 50

Bargarren art linen, for sampling.................... 60

Crochet thread, for edge...................... 50

$\$ 360$

Shams to match......................... 75

Linen thread, for sampling $\ldots \ldots \ldots \ldots \ldots \ldots \ldots \ldots \ldots, 30$

$\$ 105$ 
One Dressing case scarf of linen, with edge and ends hemstitched ............................. 75

Silk, for sampling $\ldots \ldots \ldots \ldots \ldots \ldots \ldots \ldots \ldots \ldots \ldots \ldots, \quad 25$

Dressing case scarf, silk bolting, cut to fit top of dresser.....\$ $\overline{\text { I } 25}$

Silk, to finish.............................. 50

Lace, to edge........................... I 25

$\mathrm{Pad}$, to place under the scarf..................... I oo

$\$ 400$

Guest towel, with linens for embroidery ............\$I

Material for toilet sets and mats of fine. white linens and wash

Silk embroidery threads.......................\$ 75

Bottles, per pair............................ I

$\$ 275$

Materials for six toilet cushions...............\$2 00

Materials for three chair rests of linen lawn, China silk and domestic linens, with wash silk, to sample.......... 300

Cushions (three), plush, art linen and silk, with material to

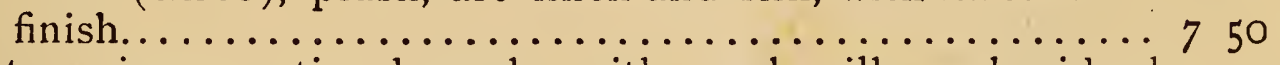

Center pieces, satin damask, with wash silk, embroiderd threads, to complete...................... 250

$\$ 1500$

Materials for working table mats and doyleys, one dozen ...\$ 75

Embroidery silk for working designs................ I 00

Total cost of samples....................\$37 70

This estimate includes sufficient material for finishing complete. For ideas as to stitches and designs, see chapters on "Art Embroidery" and "Stitches."

If it can be afforded, there should be in addition one or two picture throws daintily outlined, and several pieces of work commenced with Roman embroidery, which would swell the total to possibly $\$ 45.00$.

\section{Window Display.}

In the estimate given for samples, there has been nothing listed that will not find ready sale, but it is well to have a show piece-that is, a novelty for 
show window; also to serve as an indication of the skill of the needleworker. For this purpose there is nothing that will so well serve the purpose as a handsome piece of raised work. A sofa cushion of dark-blue plush, with a spray of blush roses (double) in ribbon, and foliage of chenille, if properly made, will attract crowds of ladies; or, a panel of dark red plush, on which is grouped two or three calla lilies, will prove equally irresistible. There is something about the craze for raised embroidery that is infectious-no matter how much out of date it may be there will always be scores of admirers for any well-made sample of it. It would be well to have two or three different samples, so as to change the window attraction occasionally. Lessons for making all kinds of raised work will be found in the chapter on "Raised Embroidery," with amount of material required for any given design. The samples having been disposed of, the next question is that of

\section{STOCK:}

The question of the quality of stock to be carried must depend upon the class of customers, to some extent, though a clever dealer will be able to sell almost anything upon which she sets the seal of her approval. The amount of stock to be carrieb will depend upon the requirements of the trade, the amount of capital at her.command or the "credit" she may be able to obtain.

It is better to put in a small line of materials first, and add to them as the demand is made. A full and complete line of embroidery linen threads, and the same of embroidery silks, should be carried, and the assortment of shades should be kept complete. This part of the stock is necessarily expensive at the start, but it is to an art bazaar what flour is to the grocer, and must be carried. There is, however, a fair margin of profit on it, and decorative needlework cannot be done without it.

A line of wash silks, including etching, rope, filo floss and royal floss should be selected with a reference to the probable uses of each kind. Etching silk is used in Roman embroidery, satin stitch on flannel and cashmere, and for outline work principally, therefore the shadings need not be so close as in silks used for solid effects. A good line of four shades of wood-brown, the same of yellow, pink, old blue, terra cotta, olive green, sage green, white, old rose, baby blue and heliotrope, will most likely be 
sufficient for a start. These silks are put up in $1 / 2$ oz. bunches to a color, each $1 / 2$ oz. containing 22 skeins of etching silks. Thus it will be necessary to purchase 24 ounces of etching silks to secure four shades each of the colors mentioned, this, at a rough estimate, will cost \$17.20.

Filo floss is used for solid Kensington, long and short stitch, etc., and moreshades will be necessary. The following colors and shades are desirable: Yellow, from pale canary to deep orange, twelve shades in all, $1 / 2$ oz. of each; old rose, five shades; olive green, six shades; sage green, five shades; golden brown, seven shades; wood brown, six shades; heliotrope, five shades; lavender, four shades; baby blue, nine shades; rose pink, three shades; cardinal red, ten shades; white, three ounces. The outlay for the stock of filo floss will amount to $\$ 38.00$, approximately.

Rope silk will stand next in importance, though the shading need not be quite so finely graded. The colors should include yellow, from canary to orange, twelve shades; olive green, six shades; golden brown, seven shades; lily green, five shades; old rose, five shades; old blue, five shades; baby blue, four shades; rose pink, three shades; red, ten shades; wood brown, five shades; white. The stock will cost about $\$ 25.60$.

If it is decided to carry a line of linen embroidery threads, it will be necessary to put in a stock of the four sizes, viz.: Bargarren art thread, rope linen, linen floss No.4, and linen floss No. 8 (for description of various embroidery threads see chapter on "Materials"). The cost of a supply of linen of all sizes will be about as follows, $\$ 48.00$. Having selected the embroidery threads, the most expensive part of the stock will have been secured. There should be added gold thread of two sizes, both in skeins and on spools, crochet linen thread, knitting silks, yarns, crochet needles, knitting needles, embroidery needles, an assortment of stuffed cushions of various sizes, and if arrasene and chenille are being used, a limited supply may safely be carried. An assortment sufficient for the last ten mentioned articles can be purchased for about $\$ 60.00$.

If it can be afforded, there should be carried in stock several qualities of white linen of different widths, a few yards of each, a square of basket silk, a few yards of huck-a-buck, a few pieces of drapery silks, in I $1 / 2$ yard lengths, to the extent of at least $\$ 30.00$. The estimate up to this point stands as follows: 
Store furnishings........................ IOU

Commenced samples........................... 38

Stock of embroidery silks................... 80

Stock of embroidery linens................... 48

Stock of knitting silks, crochet linens, various needles, stuffed cushions, gold threads, etc.................. 60

Stock of piece goods, linens, etc.................. 30

Total

Properly disposed, this stock will make a most imposing display. Impty boxes should fill the top of the case of shelves lining one wall, while on the lower shelves the yarns, crochet threads, piece goods, etc., may be displayed. One show case should be attractively filled with the various kinds of embroidery silks, one with the linen threads, while the wall case and one show case should be reserved for commenced samples and made-up trifles.

The windows should be dressed with such articles as can stand the light, and that can easily be cleaned from dust. If it is decided to carry a stock of materials for decorative painting, an attractive display can be made from that stock; stamped linens, such as shams, tea cloth, doyleys, towels, etc., will always attract attention, and can be procured at a very small outlay, while a pretty jardinere of artificial paper flowers will lend a bit of needed color to the display, and, as mentioned above, a piece of raised embroidery is a potent factor in attracting attention to the store.

In giving a list of materials for stock, only staple articles have been included, such as will sell at any season of the year, and every year. There are, however, novelties brought out each season, by the different manufacturers, and it is owing to the introduction of these that interest in fancy work is kept alive, so one cannot afford to ignore the "novelties," though they must be handled with caution, as a left-over stock of out-of-date novelties is the saddest sight a dealer can behold. These "introductions" may be divided into two classes, viz.: Novelties that are destined to have a run of a few weeks only, and those that will retain their popularity for a season or two. How to distinguish between the two classes is a question that vexes often the veteran dealer; how, then, shall the beginner protect herself ? The surest and, indeed, only safe way is to buy sparingly of any novelty, no matter how desirable it may seem. It "ill be found much 
easier to order more, than to dispose of it after the "run" is over. In general, such things as paper flowers, painted novelties, embroidery threads, that are not in themselves artistic or adapted for artistic work, should be purchased with caution, though in the matter of paper flowers justice compels us to admit they have obtained such a hold upon the public, that the materials for making them are carried by all first-class houses. Each dealer must decide for herself what she can best carry in stock, and she can decide only by experimenting with her trade, therefore, the best the writer can do for the beginner is to utter the warning words, which should be used as a motto when the ever-persuasive salesman is abroad in the land: "Caution! Buy Sparingly!" Indeed, it would not be a bad idea to have the quotation printed in large letters and placed in the office desk, where the eye of the dealer will be sure to rest upon it when making out orders.

On the other hand, do not be afraid to put in novelties as they come out, even if only one or two of a kind. It is necessary to keep up with the times. It is of vital importance that your customers find in your stock whatever novelty of the day is being written up by the art journals, taught by traveling teachers, or displayed in the leading stores of your nearest city. It is not only important that you have the article or articles, but that you let people know you have them, and to this end consider that judicious advertising is capital so well invested that it is sure to bring you in the largest profits. The writer calls to mind an energetic little lady in a far western town who started a modest art bazaar with limited means, and still more limited experience. As a matter of course, she stocked up beyond the capacity of the home trade, and, in consequence, found herself, as she naively expressed it, "stuck with a lot of stuff, and not a cent to turn around with." If she had not money, she soon demonstrated the fact that she had plenty of "mother wit." She had an interview with the publisher of the local paper, and the result was that each week appeared two columns of matter on "Fancy Work," describing the most ravishing toilet sets, daintiest of doyleys, pillow shams, and numberless odds and ends, until every woman in the county was fired with the determination to do fancy work, or die in the attempt; but the business came in here-each issue contained a local notice to the effect that Mrs. C—— was prepared to fur- 
nish materials for making each and every article described in the article on "Fancy Work," besides many new and beautiful designs not described; also work stamped and commenced, with proper shades of silks selected, and mailed to any address. The result? In three months her stock was reduced, she had working capital and, better than all else, was known in every corner of her own county, and in many surrounding towns. All the foregoing apropos to advertising. Of course, in the limits of the present article, the writer cannot follow the dealer through all the vicissitudes of a first venture; the most that can be done will be to put up warning signs along the way, wherever pitfalls are likely to trip the unwary.

\section{Stamping Patterns.}

This part of the outfit was purposely left out of the list of stock articles, as we wished to discuss it by itself. Stamping patterns are the most important part of the equipment of an art bazaar or fancy goods store. There must be a supply sufficient for a large variety of work, but here must go up another "warning post"- "Do not purchase too many patterns in a season." The foundation stock must include designs for doyleys, five-o'clock tea cloths, tray cloth, caraffe mats, table mats, lunch sets, shams, bedspreads, curtains, cushions, large and small; dressing case and side board scarfs, and table covers, but do not purchase for a certain purpose more than three different patterns. There must be at least three sizes of alphabets and one monogram, with an assortment of designs for satin stitch on flannel, cashmere, etc., including several scallops with corners. Such a supply of designs can be purchased for $\$ 20$, together with a large blank book of strong manila paper. On the pages of this book stamp all the designs and number them in consecutive order, marking the same number on the pattern. This book is for your customers to select designs from. They should never be allowed to handle the pattern itself. In fact, the pattern should be kept in the work room, spread out flat, without creasing, in a drawer or box. The patterns should be added to, a few each season. As soon as received, they too should be added to the design book and numbered. Remember that new designs are always a good investment; also remember that a few at a time and often will do more good than many and far apart in the season of their purchase. Nearly all large patterns for bedspreads curtains, table covers, etc., come in sections, and as many ladies have not 
the power of imagining how it will look when put together, it will be found a very excellent plan to paste sheets of manila paper together, until a piece the size of a large table cover has been obtained. On this stamp the design cleariy, and display, either in the window or wall case. Do not display too many at one time, but change them frequently. In fact, this advice may be applied to all the display articles-overcrowding of a window or show case is never desirable. A few articles, frequently changed, will be much more effective; also one or two strong pieces in a show window-something that can be seen at a glance, that will stand out, strong and distinct-will be far more attractive than a window crowded with a miscellaneous assortment of small novelties, no matter how pretty they may be in themselves. The window should be changed at least once each week, carefully putting out of sight for a couple of weeks the articles that have been used, when they may be again brought out.

One of the first problems that will confront the woman who intends opening a fancy goods bazaar will be the question: Where to obtain the stock. As a rule women out of business know very little about such things and other women in the business are apt to be rather non-communicative on the subject. We cannot name any firms in the pages of this book, but the manager of almost any large retail dry goods stores will be perfectly willing to give the needed information. It is better to buy of the manufacturers when possible, though for some reasons it is more convenient to purchase of a general jobber who carries all the different articles needed. Nearly all wholesale houses have catalogues, which they will send upon request. Much information can be obtained from them, though it must be understood that the lowest prices and best terms are never printed in them.

The "getting ready" to begin business should not be rushed through. Plenty of time should be allowed for getting samples ready and stock selected, and the venture should be made at the beginning, not at the end, of a season. The best season for this trade is from the middle of September to the middle of January, and from the Ist of April to the last of June. The time between the seasons can be profitably filled in by making up fresh samples, novelties for holiday sales, and the formation of classes in embroidery. There need not be a week, nor a day, when the dealer shall not make a margin of profit, if the right kind of energy is given to the business. 


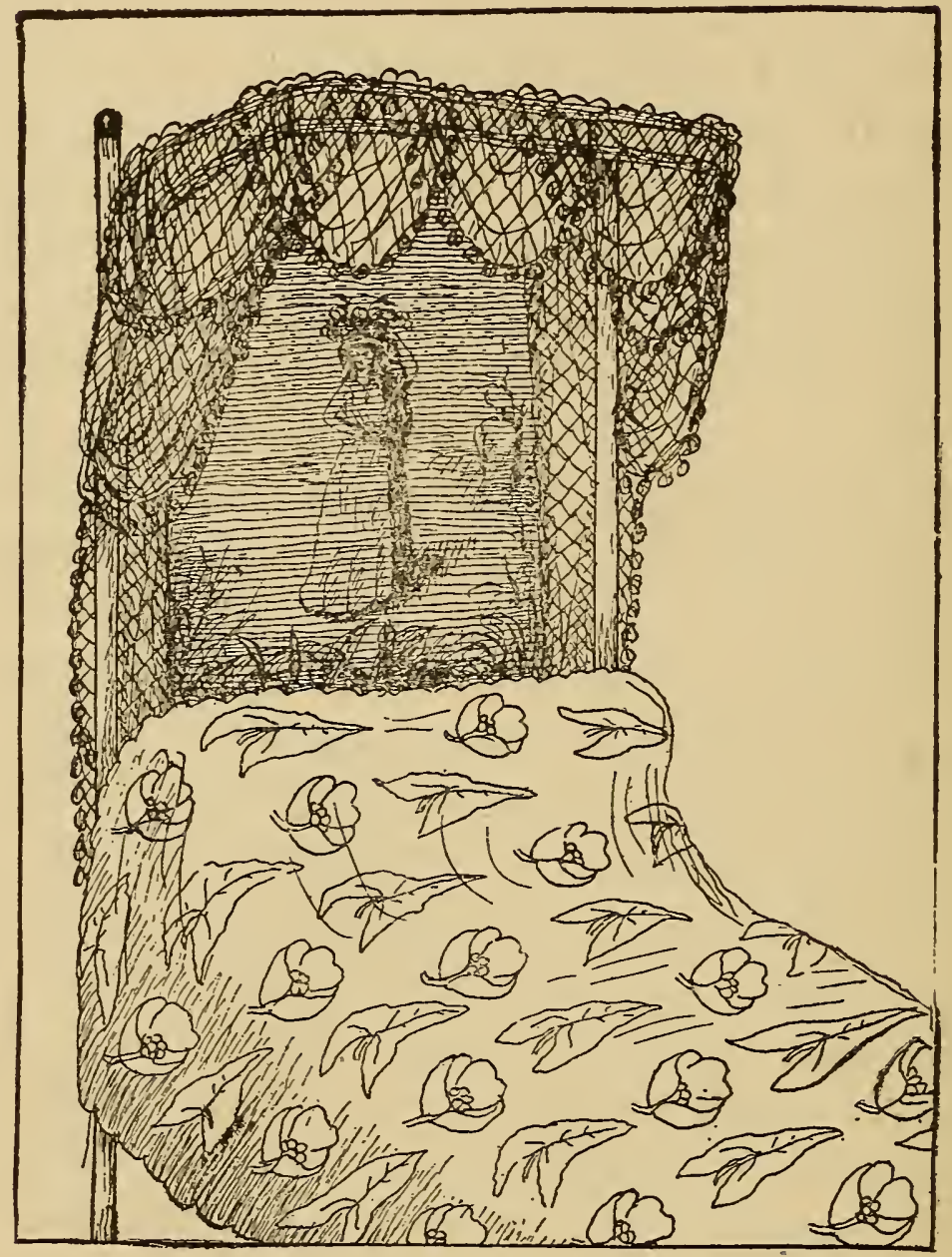

Bed draped with five yards china blue Waban netting. The spread, a section of which is shown, is made of Roman twill linen, the design is a r.owslip blossom resting on a leaf of its own foliage scattered over the sur‘ce. Scotch linen floss in three shades of green and three shades of yellow will be required. Use short and long stitch for the leaves and solid Kensıngton for the flowers. Finish edge of spread with reticella crochet lace made of Real Scotch linen crochet thread, No. 36. 
The pages of this book contain all the various lessons for making all kinds of decorative articles needed in a general art store, and any lady who wishes to open a bazaar for decorative needlework need not spend a dollar for lessons, nor waste time and money in further experiments, if she will but study carefully what we with great care have prepared for her guidance.

For the benefit of that class of women who need actual illustrations of every step to be taken, we show on pages 257 to 308 several articles that may be made up to add to window and show case attractions. They will se found ready sellers at any season and afford also a pleasing variety. Following are the descriptions and numbers of the several articles.

\section{Toilet Set.}

Figure 27I illustrates a beautiful toilet set that is easy of construction and decoration, and not expensive. The cushion is about twelve inches across, square in shape, and stuffed quite high. The cushion should first be covered with a plain covering of pale blue satin, or China silk. The four corners should be finished with large, loose bows of the same colored silk, fringed at the ends. As the entire effect of the cushion depends upon the arrangement of these bows, care should be taken to follow the illustration as closely as possible. The bows should almost meet on the four sides of cushion. For the center, cut a square of bolting silk to just fit the top of cushion, and over the entire surface paint conventional daisies, as shown in illustration, painting with gold paint the outlines of the design. The inner, star-shaped portion of each flower should be painted with two or three shades of blue in water colors, while the exact center should be of deep yellow. The same idea should be carried out on the bottles, which are first covered with the silk and afterward with the painted bolting silk.

\section{Glassine Handkerchief Sachet.}

Figure 272 illustrates a beautiful handkerchief case, made of glassine and silk. A square of glassine, $28 \times 28$ inches, will be required. Slash in the four corners, so as to leave a square in the center 14 XI 4 inches; fold over the slashed corners and fasten in the center with a large ribbon bow. A spider web and floral arrangement is carried out in oil colors in pink, green and gold, but any other design could be substituted. The four corners are finished by small ribbon bows, and the inside of the case is lined with loosely tufted pink China silk, delicately scented. 


\section{Glass Panel Screen.}

Figure 273 shows a dainty little three-panel screen, made of giass with beveled edges. The design starts from the left-hand panel, and extends across the three. The subjects for decorating these glass novelties should be a simple floral arrangement. The model was treated with wild roses in oil colors. The panels are tied together with ribbon bows. These screens are used on the drawing room or parlor tables, placed before the lamp.

\section{Tobacco Pouch.}

Figure 274 shows a tobacco pouch made of chamois skin. The pouch is four inches wide and eight inches long. The side seam is decorated with three ribbon bows and the bottom is embroidered with a fringe made by slashing a piece of the chamois into one-quarter inch strips. To prepare the fringe, select a smooth piece of chamois three inches deep and as wide as the pouch. Paint it all over with liquid gold, and then slash, as previously described, to a depth of two and one-half inches. This will leave a half-inch of material for a heading. The neck of the pouch should have a draw string of one-inch ribbon. The design in illustration is a conventional arrangement, which will look well carried out in brown, with a glint of gold and dash of crimson, relieved with touches of deep, cool green, worked out with oil colors.

\section{Glassine Toilet Set.}

Figure 275 illustrates a unique toilet set. The cushion is square. The top is covered with a puffing of China silk in clover pink, sometimes called dead rose shade. This top cover must be arranged very carefully, for while puffed quite full an appearance of clumsiness must be avoided. Each corner has a narrow scarf of bolting silk, with edges feather stitched in olive green wash filo floss, gathered exactly on the point of the corner. Over this is arranged another scarf of China silk, of the same clover pink shade as is used on the top, tied in a loose bow and ends. Through the bow is run a cord and tassel of a lighter shade than the silk. Over the four corners is now placed a half circle of glassine, having the edges picked out in gold paint, and clover heads painted over the surface in oil colors in natural shades. The effect is wonderfully stylish. 
The bottles are first covered with silk in clover pink shades, and over the bottom portion of each bottle is placed a cover of glassine, painted with a design of clovers to match the cushion pieces. The neck of each bottle is finished with a cord and tassel.

\section{Parchment Parasol Catch-All.}

Figure 276 shows a novel device for holding letters, cards and other small articles of a like nature. To make the parasol, any material may be employed that can be folded or creased without breaking. Parchment is, of course, the best for the purpose, but glassine or silk may be substituted. In case the latter is used it must be lined and also have an inter-lining of stiffening-crinoline will answer admirably.

For the handle select a small-sized bamboo rod, or the handle of an old parasol will answer as well, provided it is of the short style. At the end bore a hole, and another about half way down the stick, and a third at the top. Cut the panels or gores out of the parchment, using as a pattern a section ripped from a discarded parasol. On each panel paint an edge design with a floratesign above, either in oil or water colors, following directions given under those heads for working them. When the panels are thoroughly dry they are ready to be attached to the handle.

Gather the top of the panels around the top of the handle, and fasten them securely to it by sewing with a strong darning needle, threaded with hair wire, both through the panels and the hole in the handle; cover this fastening with a bow of soft faille ribbon of harmonizing color. Now, when the parasol is suspended, bottom up, the parchment cover will fall straight out; therefore, it must be secured in position. The illustration shows the parasol half open. To secure this effect punch a hole through the edge of each gore, and run through from one gore to another a heavy silk cord draw it up until the desired effect is produced, and then pass the ends of the cord through the hole previously bored in the middle of the handle. Add another ribbon bow at this point. A cord and tassel finishes the bottom of the handle. Parchment is of a rich, old ivory color, and therefore a most exquisite background for painting.

\section{Traveling Roll.}

Figure 277 illustrates a most convenient traveling roll. The roll is made of ecru satin-finished linen. A piece of material twenty-seven 
inches long and twenty-four inches wide, was used to make the model, thus making the roll twenty-seven inches long and twenty-four inches around. When sewed together the circular ends must be filled in with a little fullness, and they should also be lined with heavy linen canvas or other stiffening. The edges, where the ends are set in, look best finished with a rich brown cord sewed on. A handle can be purchased ready made, or one can be manufactured at home by covering a piece of stiffening with soft, brown leather or chamois skin. The design of birds in flight is particularly appropriate, and they should be painted in sepia and warm sepia--see directions for painting birch bark.

\section{Duster Case.}

Figure 278 shows a convenient duster case made of brown linen. To make like the model will require a piece of linen twenty-seven inches long and eighteen inches wide. Fold the linen up from the bottom edge, to form the pocket, seventeen inches, and fasten down both side edges. Fold over the top for the lap ten inches. Across the top put a row of heavy machine stitch. ing, but do not sew down the edges of the flap. Bind the entire case with wide silk braid, matching the linen as nearly as possible. The word "Duster" on the flap should be painted with sepia, and the design on the pocket should also be painted with sepia and warm sepia, using the colors lighter and darker. This will give a harmony in browns very pleasing to the eye. Some might prefer colors for the design, but for the purpose of decorating a traveling roll, the quiet, one-toned coloring is far more elegant and correct. Materials ready stamped for these articles always find sale.

\section{Calendar.}

Figure 279 shows an easy way of making a novelty calendar. The materials required are three strips of ribbon, each one yard long, for the calendar numbers, one yard ribbon for bow, and one and a half yards cord and two tassels to suspend by. The celluloid is cut in the shape of a shamrock, and inside each oval a square slit is cut out, leaving an open space. through which to run the ribbon, having the days of the week, names of month and date. These are painted on the respective ribbons with liquid gold paint. The celluloid ovals are outlined with a broad band of gold paint, a:a d springing from the center is a straggling design of conventionalized buitercu?c, painted in oil colors. 
CHAPTER XVIII.

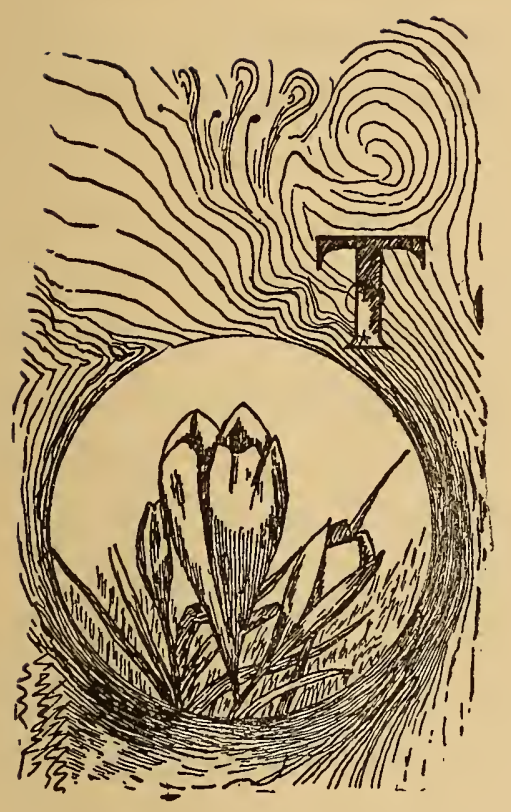

DESCRIPTION OF FULL PAGE ILLUSTRATIONS.

HROUGHOUT the pages of "Dainty Work" will be found full page illustrations of various household articles, ornamented and otherwise. In some cases they bear direct reference to the subject treated of in the article they illustrate, and will need no further description here, while in others they are used merely to give larger scope to individual fancies-to show in the concrete what has been treated of in the abstract.

On page 58 a number of cushions and head rests are shown which are artistic and inexpensive. The roll shown at lower left hand corner of page can be used for head rest on large chair. To make, will require a roll made of stout cotton cloth, stuffed with cotton or sea moss. The size of roll is a matter of taste. The cotton roll in the model was covered with old rose china silk, sewed on plain and gathered in a full frill at each end, the edges of which have been fringed out. The center of the roll has a band of golden brown satin placed over the silk: This center cover comes to within three inches of each end, and is decorated with a conventional vine worked out in several shades of golden brown and rose pink; the ribbon by which it was suspended to the chair is a pale old rose.

The model for the cushion at the lower right hand corner was a square of gas green velvet, folded to the center to meet a pale sea-shell pink puff of India silk. On one of the sections of the square was embroidered a small floral design, in royal floss silk.

Above this cushion is shown another roll for chair back. The roll in the model was stuffed loosely with sea moss and covered with cream col- 
ored India silk, tied at the ends with pale lavender ribbons. The design is a cluster of pansies looped with ribbon. The pansies were worked out with pansy purples in filo floss, and the ribbon outlined in a shade of pale, ulive green royal floss.

Over the roll head rest first mentioned is an infant's pillow covered with a slip of dainty white linen lawn edged with soft snowflake lace, and decorated in one corner with a single spray of lily of the valley; a dainty conception.

The next cushion in the group is a leaf shaped head rest, made of cotton cloth and lightly filled with cotton wool and then covered with light golden brown India silk. The center is veined with pale pink, and the edges finished with short and long stitch in a deep, golden brown with royal floss.

Page II 4 shows four beautiful and inexpensive drapes, suitable for mantel or picture frames. They may be made of any soft clinging fabric, but China silk is at once most satisfactory and artistic.

Page 138 illustrates two drapes and a table doyley. The nirst figure shows a doyley made of white satin damask. A circle of pansies are stamped around the edge. The outer edge of petals are worked in short and long buttonhole stitch, with pale lavender linen floss. The inside of petals are filled with the different fancy stitches which are so easy to do, and so effective when well and evenly worked; the filling stitches should be of the linen floss, of a darker shade of purple than the edges; at least three shades should be employed.

The drape at the left hand side of page is made of India silk-pale cream color. The design is one of leaves which are first tinted a pale green with water colors or Paris tints, and then outlined in short and long stitch with several shades of olive green filo floss, two strands. No two leaves should be alike. The cloth should be cut away on the ends and each leaf finished with a tassel of green filo floss.

The second drape is made of pale blue India silk, on each end is a border of silk bolting, nine inches deep, on which is embroidered in daisy and outline stitch, a dainty design; for this use pale blue filo floss, one strand. The edge is then cut out and each point ornamented with a tasiel made of blue and white filo floss. 
Page I4I illustrates four beautiful drapes. The drape shown on the left hand, upper corner, is of white India silk stamped with a powdered design of roses and buds with broken bits of foliage. This design should be very delicately tinted with water colors in pinks, greens and stem browns, and each part of design lightly outlined with one strand of filo floss. The stitches must be daintily set and very even; match the silk against the tinting carefully. Three skeins of filo floss will work the entire design. The edge should be outlined and finished with green, pink and brown silk tassels.

The drape on the upper right hand corner is of pale yellow surah silk of good quality. The design on the ends is a Roman embroidery pattern worked out with four deeper shades of yellow etching silk; see chapter on "Drawn Work" and "Lace Stitches" for method of filling in. The edge is embellished with tassels, made of several shades of yellow royal floss.

The drape at the lower left hand corner is made of pale green India silk, bordered with a ten inch band of silk bolting, on which is worked a dainty design of jonquils with foliage wrought with filo floss in white, yellow and palest greens. The edge is scalloped, finished with buttonhole stitch in pale green and ornamented with white, yellow and green silk tassels.

At the lower right hand corner is illustrated a drape of silver green surah silk, bordered with a band of silk bolting feather-stitched on. This band is ornamented with a begonia design which is worked out in four shades of silver green filo floss; finished with tassels of white and silver green royal floss.

Page I 48 illustrates different methods of draping beds with spreads and shams, four of which are described as follows:

Figure No. I shows a bed draped in Scotch Homespun, which is a fabric made of pure hand spun flax, in its natural color. The spread in the model has a border of poppies, slightly conventionalized, worked out with linen floss, in two shades of ecru and a rich wood brown. Solid Kensington stitch is employed for the petals and French knots for the center. The monogram in the center is worked in the same shades in satin stitch, raised by working over a padding. The shams are worked in the same manner. The flowers could be worked out in the natural shades of poppy 
reds with equally good effect. This material offers a beautiful background for Roman embroidery, and spread and shams decorated in this style of work are not only very handsome but serviceable.

Figure No. 2 shows a bed also draped in Scotch Homespun. The pattern is a Dresden design of carnations which should be worked with solid Kensington stitch in Real Scotch linen floss, size 8. The pillows are covered with scarf of the Homespun, decorated to match spread. The ends of the scarf may be further embellished with a border of Roman embroidery or drawn work.

Figure 3 is draped with cream-colored Roman twill. The design is a border of ribbon enmeshed roses. The ribbon is outlined and filled with darning stitch in lily green rope silk; the roses are outlined with creamwhite rope silk, the centers filled with pale yellow French knots and the under portion and lower part of petals with a few darning stitches in the palest shade of lily green. The effect is very light and dainty. The roses could be done in pale yellow, or pale rose pink, and the ribbon in wood brown.

Figure No. 4 shows spread and shams of Devonshire art cloth with design of conventional clematis worked out in solid Kensington with heliotrope shades of rope silk. Spread and shams are of one piece.

Page I 54 illustrates a beautiful cover for a library or dining table. The material of which it is made is a square of Devonshire Art cloth in real flax color. The edge is ornamented with an ivy vine worked in outline stitch with wood brown Bargarren art linen thread.

The outline stitch should be made very heavy, with a cord-like appearance. Over the surface of the cloth a conventional design is powdered, as shown in illustration. The leaves of this powdered design are worked in short and long stitch with rope linen in a lighter shade of wood brown than that used for the ivy vine. The stem of leaf is made with the darkest shade of wood brown, in outline stitch. The petals of the design are worked in short and long stitch, for which three shades of wood brown rope linen are used. The edge is treated in a novel and beautiful manner. A border is crocheted from linen crochet thread, No. I8; in pure flax color. After the shell design has been finished tiny tassels of the same linen are tied into the edge, forming a heavy fringe. 
Page 174 illustrates a caraffe mat which is a combination of crochet work and embroidery. The center of the mat is cut out of firm white linen in the shape of an oak leaf. The natural leaf will furnish a good pattern, a little enlarged. Finish the edge with a row of short and long buttonhole stitch over German cord No. 0; for the buttonhole stitch a pale, green linen floss may be used; also for the veining in the center of the leaf. The veining should be done in cord outline stitch. After this part of the work has been finished the edge should be added. Use cream white linen crochet thread: start the design in the edge of leaf as a foundation, and work from the leaf outward, in any design fancied. The wheels may be crocheted separately and added afterward if preferred. Often several designs may be combined in a single edge with excellent effect, the chapter on "Knitting and Crocheting" contains many désigns that can be adapted for this purpose.

Page 294 illustrates a beautiful window drapery. It consists of Waban netting draped over white lace curtains. The material for this drapery is cut in three different pieces; the two lengths that form the long drapery and the length that is draped across the window, which should be disposed as lightly as possible. The netting can be had in all the art shades, or an old fish net maybe pressed into service, though not as artistic as the Waban.

Page 298 shows an entire room draped with the same material. The portiere in the foreground will afford an excellent study for drapery, while the small door and window in the distance show still other effects. This drapery material is inexpensive, and gives an idea of airy elegance to the plainest room. It needs no decoration, and will collect no dust.

Page 302 shows a fire place draped with Waban: this material is the most appropriate for this purpose for several reasons. If one has a handsome mantel which it would be a pity to cover from sight, and yet needs a little drapery to ease the room from an effect of stiffness, Waban will answer excellently for it; as it will drape gracefully, without concealing all the beauty of the mantel. On the other hand if the mantel is an eyesore it will kindly drape and conceal many defects, and take from it the worst feature of its ugliness. Four yards will be required to drape a mantel as shown in the illustration; and it must be cut in three lengths. Page 306 illustrates still another use of this material. For a bamboo or brass easel no prettier drapery could be conceived. It drapes but does not conceal and 


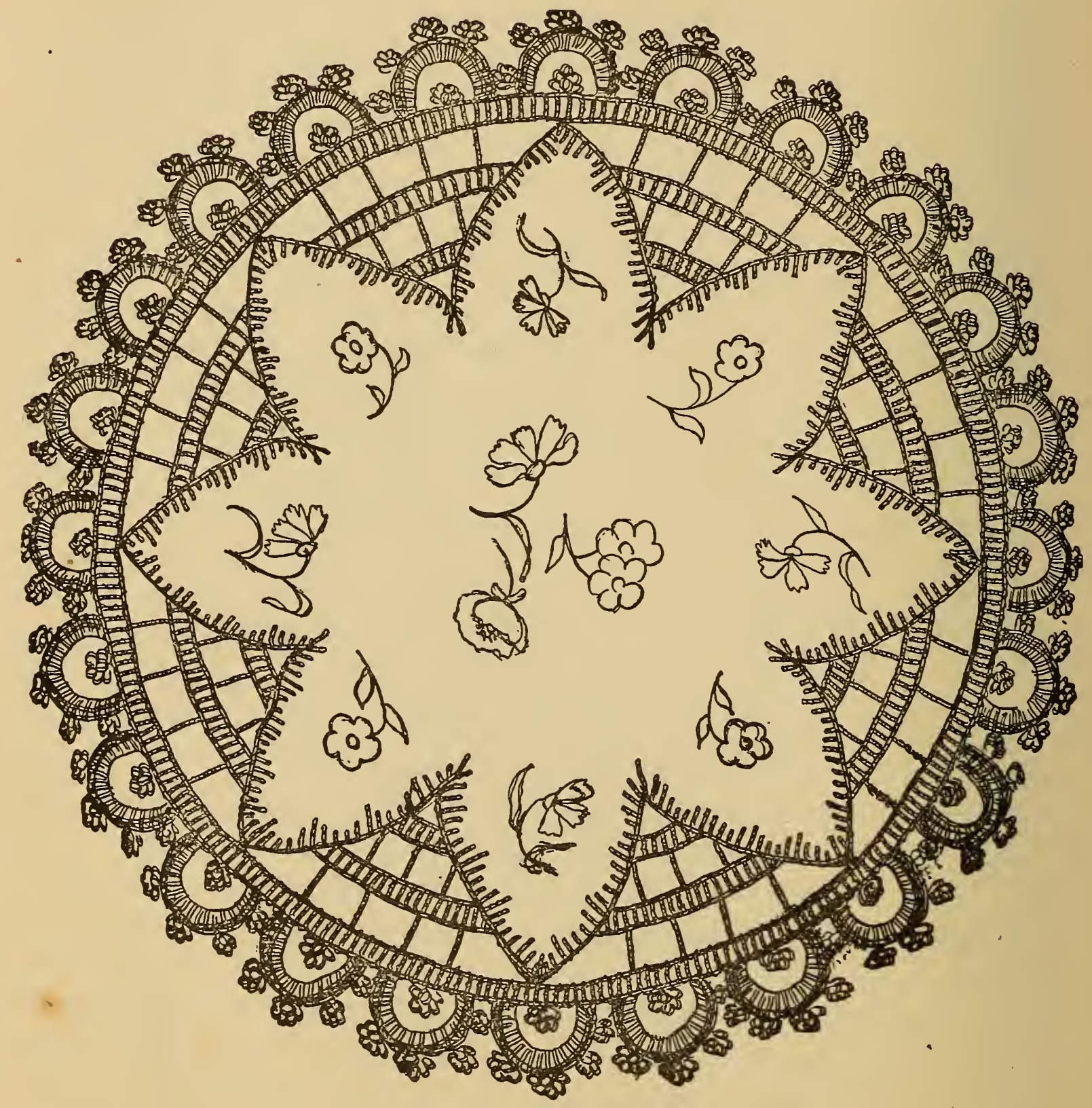

DRESDEN PLATE DOYLEY. 
therefore perfectly fulfills the mission of drapery fabrics; three yards will be necessary to duplicate the drapery shown.

Throughout the pages of "Dainty Work" attention has been given largely to the decorative necessities and consequently bed linen and table linen have received a large share of attention. On page 324 is shown one of a series of table doyleys. These articles of table luxury offer pretty and easy work for many an idle moment, and will prove no tax on either the ingenuity or eyesight of the worker. The doyley in question is called the "Magnolia" The center is made on fine linen huckabuck; the background is darned through each mesh with a single strand of pale green royal floss. The flower is outlined lightly with royal floss silk in a creamy white with a dash of old pink toward the center of petals. The stem is in green worked solid. The edge is crocheted with pale yellow linen floss, size 4. Any design for the crochet may be selected that one fancies, but it should not be of a character too heavy. '

Page 322 illustrates the "Maple Leaf" which is exquisitely dainty. The leaf is stamped on plain white linen, and outlined around the edges with a buttonhole short and long stitch over a German cord No. o, in pale green linen floss. The veins should be outlined with the same material, and the edge should be crocheted with crochet linen thread No. 50 in cream white. The design shown is appropriate and is composed of two patterns shown in the chapter on "Knitting and Crochet."

Page 430 shows the "Dresden" doyley which is stamped on satin damask. The edges are outlined over German cord with white linen floss. The Dresden flowers are worked in solid Kensington stitch, with pale blue and pink linen floss with a bit of brown and green for stems and foliage. The lace is crocheted into the edge of doyley-following the indentationswith pale, blue linen floss No. 4. The picots forming the very edge of design will be more effective if another shade of blue, one tone deeper than that for the inside part of design, be used, or, a combination of blue and white, or pink and white would be artistic, while pure white is always in good taste.

\section{COLORED PLATES.}

Several colored plates have been prepared to further illustrate the subject or color work, and below are the palettes for painting them in oil col- 


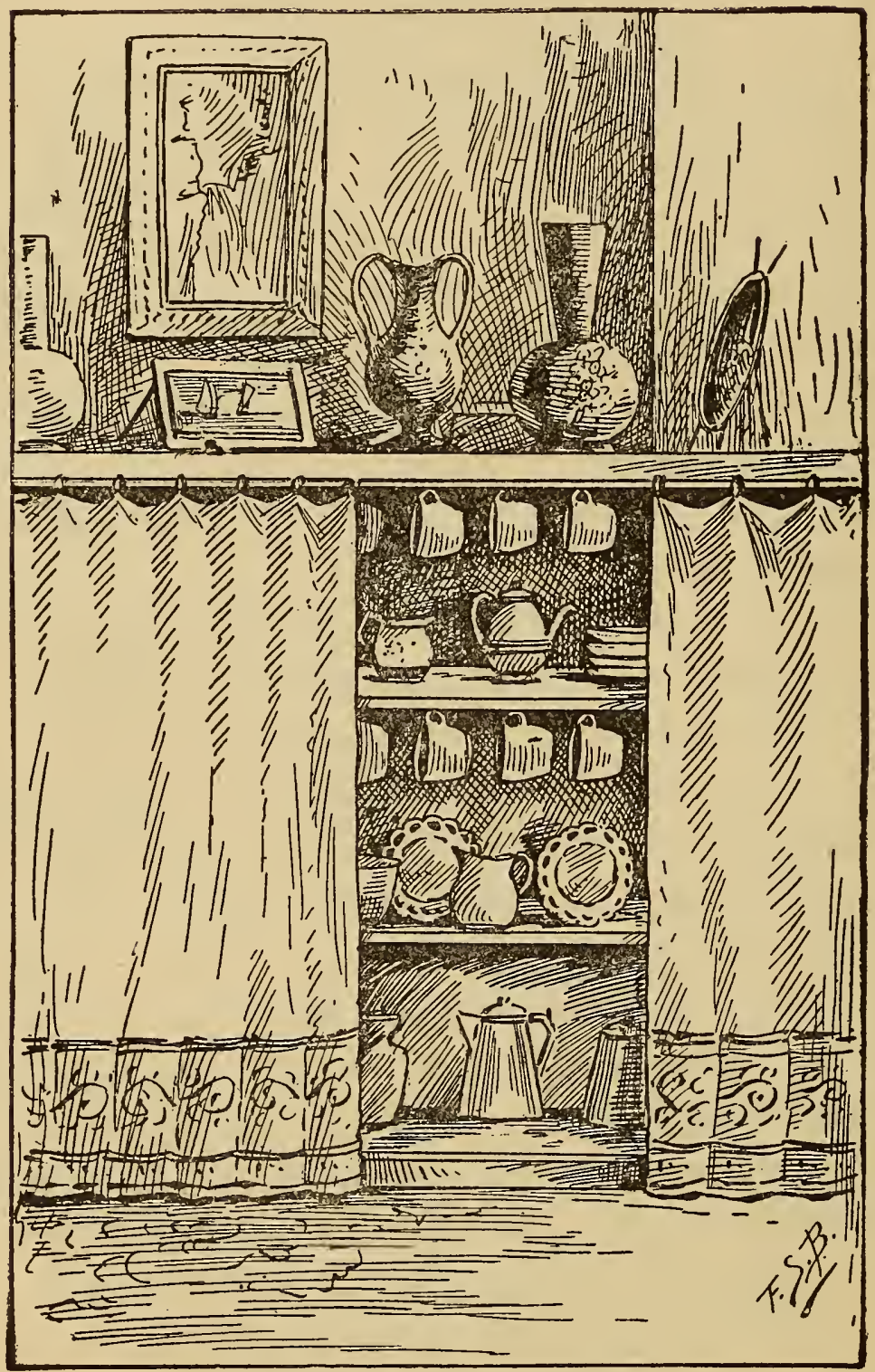

DRAPED CUPBOARD. 
ors. The plates will serve as "studies," and they may be enlarged or not as desired. The author is indebted to an artist friend for the following palettes'

\section{Palettes for Painting in Oil.}

The ground for flower painting should be of a creany white tint, otherwise most of the colors must be made opaque by the admixture of white, to cover the tintbeneath; and with the loss of transparency, from the use of opaque colors throughout the darks and shadows, comes also that of depth and richness in tone. This is as true of a background as of a flower.

For backgrounds make gray by combining a little of each, rose madder or vermilion, cobalt blue and yellow ocher with a good deal of white and a very little black. By varying the proportions of colors your gray will be bluish, greenish, yellowish, or of whatever tone you wish, and either light or dark, as you choose.

For greens, make with blue and yellow, or use any green you have, modifying with raw umber, burnt sienna, black, red, or white, as needed. Lay the tints where they belong, then blend softly to unite their edges. Let them dry well before putting in the final markings, for which use a fine pointed brush, held upright. This rule applies to all the crisp, delicate lines or dots in leaves, stems or flowers.

FLEUR DE LIS.-Mix rose madder or madder lake and cobalt blue for purple. If you have a creamy white ground to paint on lay in all the darker tints, some a little reddish, some bluish, and others darkened with a touch of black, before using any white. Then mix white with any of the tints already made, using much cr little as needed. Match the tints in the copy, laying them side by side, each in its place, and leave them untouched until you have finished a flower. Then blend slightly with a dry bristle brush, just enough to soften the edges of tints together and to break the lines of brush-marks.

Lay in the white flower and bud with a gray made by adding a little black to white, using a tint with more black in to shade with. For the highlights make a creamy tint by adding a little yellow ocher to white.

Make background as illustrated; or one of light yellowish gray for the upper part, shading down into dark reddish brown at the bottom, is very effective. 
Oranges.-Draw in with burnt sienna. Pale, deep and orange camium, with any of the browns mentioned above, may be used; or any bright yellow with vermilion added in the orange parts and white in the highlights, will give the desired orange tints, to be shaded with brown.

Poppies.- These can be painted at once with vermilion, rose madder, white and brown. But much more transparency and beauty of coloring will be secured by following the method given for the lilies. First, then, a bright yellow over all except the white or pale pink parts of the red poppies, and when thoroughly dry paint in the reds and browns. Paint the white poppy with gray of black and white, modeling with the same, as for fleur de lis, then touch in the other tints.

To darken the lower part of the background add a little brown to your background gray.

Oak Leaves, Sumac and Golden Rod.-Paint the reds in this plate of autumn glory as described for lilies and poppies. Add a touch of burnt sienna or brown, and white if needed, to gray for grasses and stems of golden rod. For golden rod use any bright yellow and white, with toucn of black: when dry, dot in the delicate touches of color. The strong finai markings in the sumac "beds" and oak leaves will require bone brown.

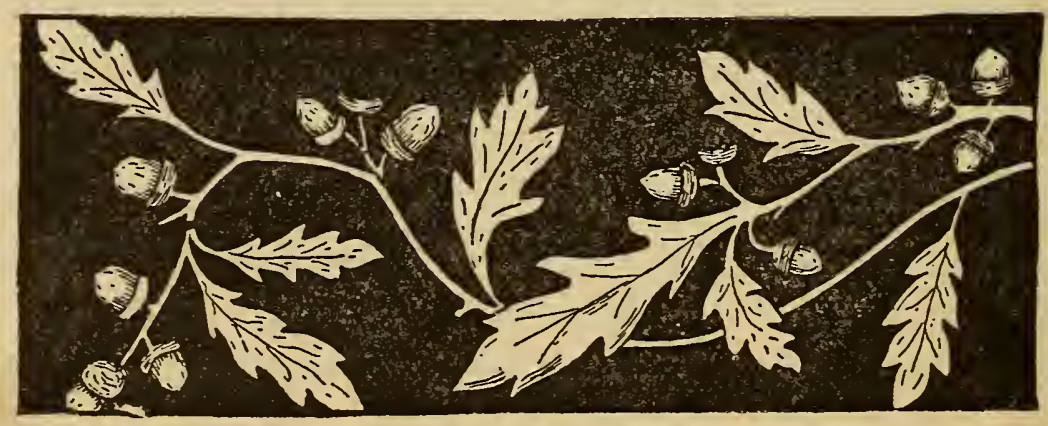


CHAPTER XIX.

SPECIAL ILLUSTRATIONS.

THE FOLLOWING pages show a few dainty household novelties that will prove a veritable gold mine to the teacher or dealer in decorative work if properly followed.
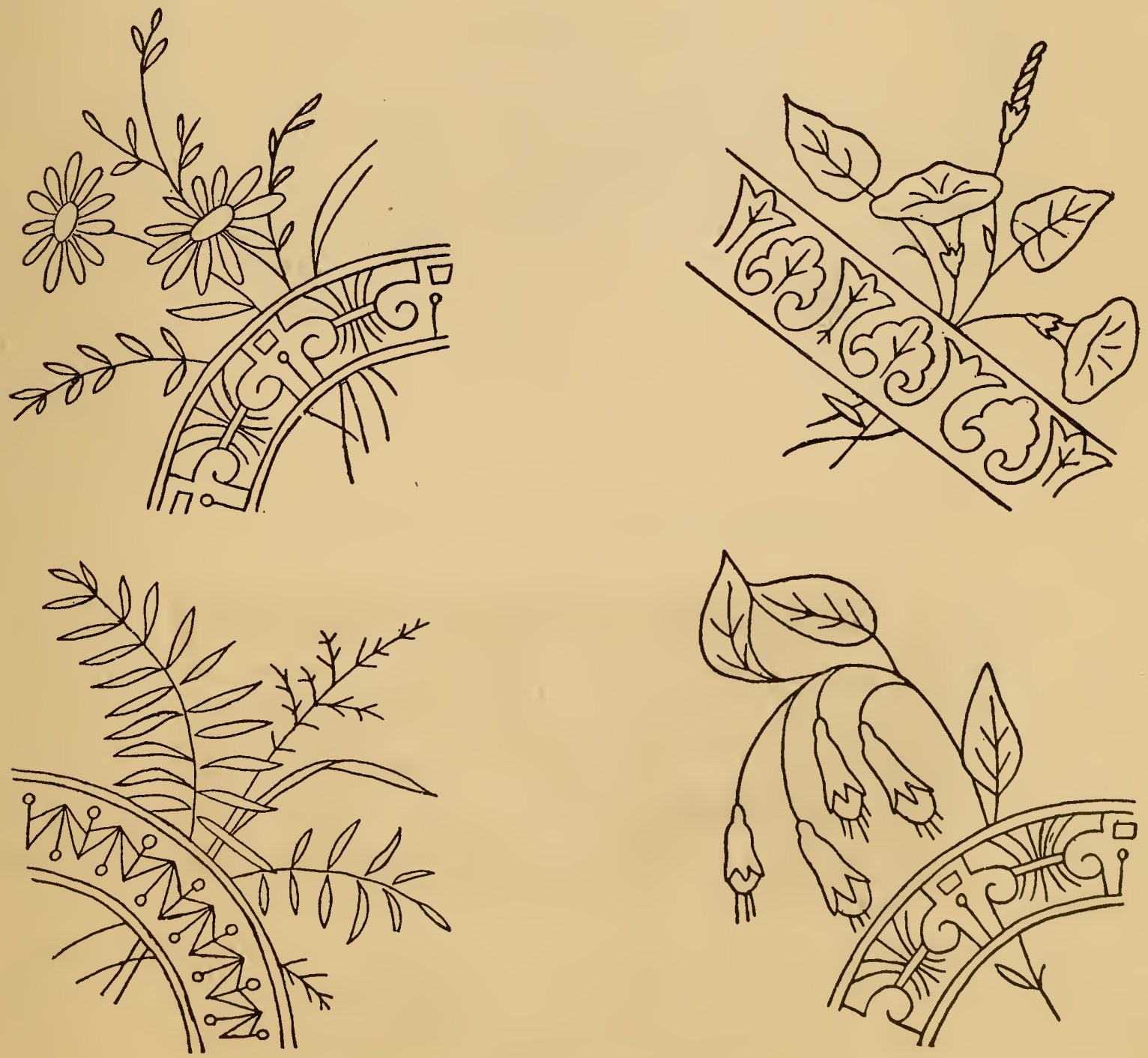

C.ORNERS FOR BREAKFAST DOYLEYS. 


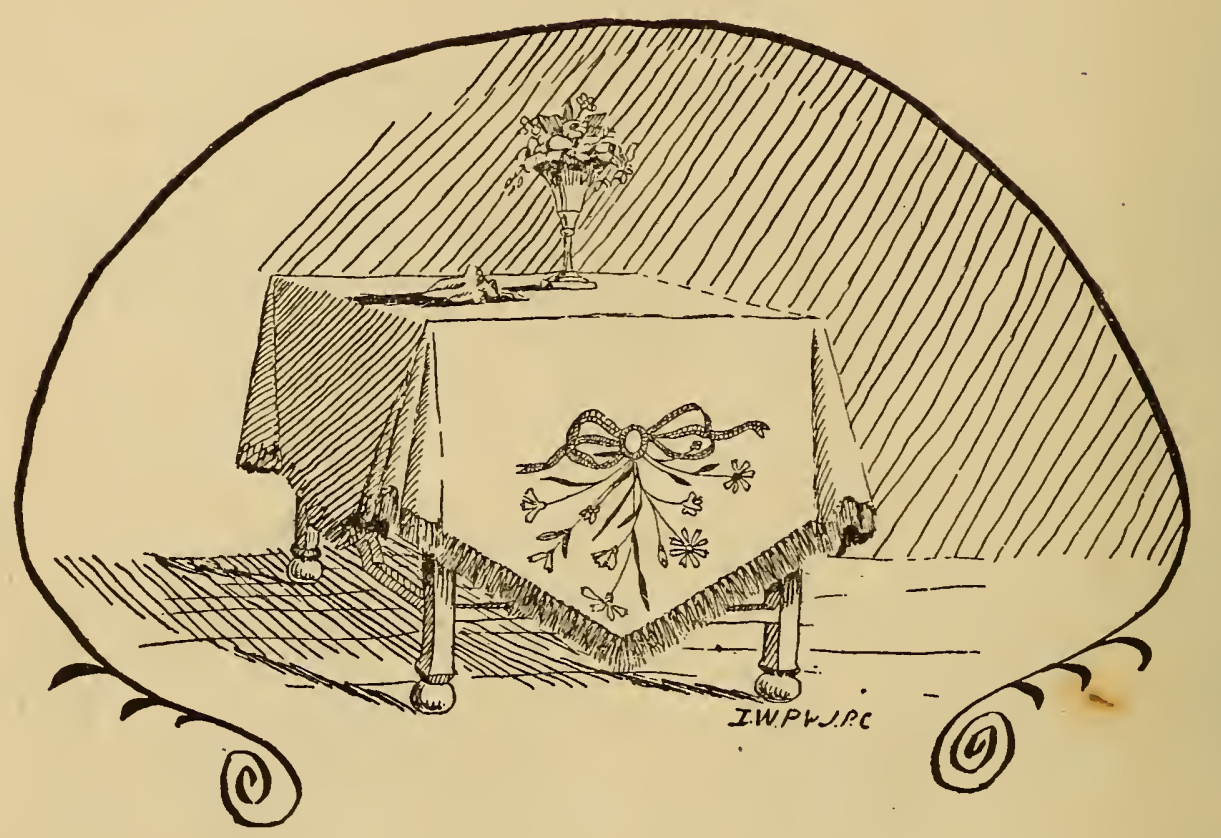

FIVE-O-CLOCK TEA CLOTHS.

The Tea Cloth shown here is made of fine round-thread linen. The edge is finished with a fringe, and each of the four corners should have the design shown repeated. The arrangement shown in the illustration may be reversed, if desired; that is, the bow of ribbon may be at the bottom instead of at the top. It is a matter of choice. The stitch used should be solid Kensington for the flowers and foliage, outline for the stems, and buttonhole point stitch for the ribbon, which may be green or white.

The figures shown on page 435 are suitable for corners of breakfast napkins or doyleys, and will look well worked out with outline, short and long stitch, or solid stitch; or a combination of the three would be quite effective. 


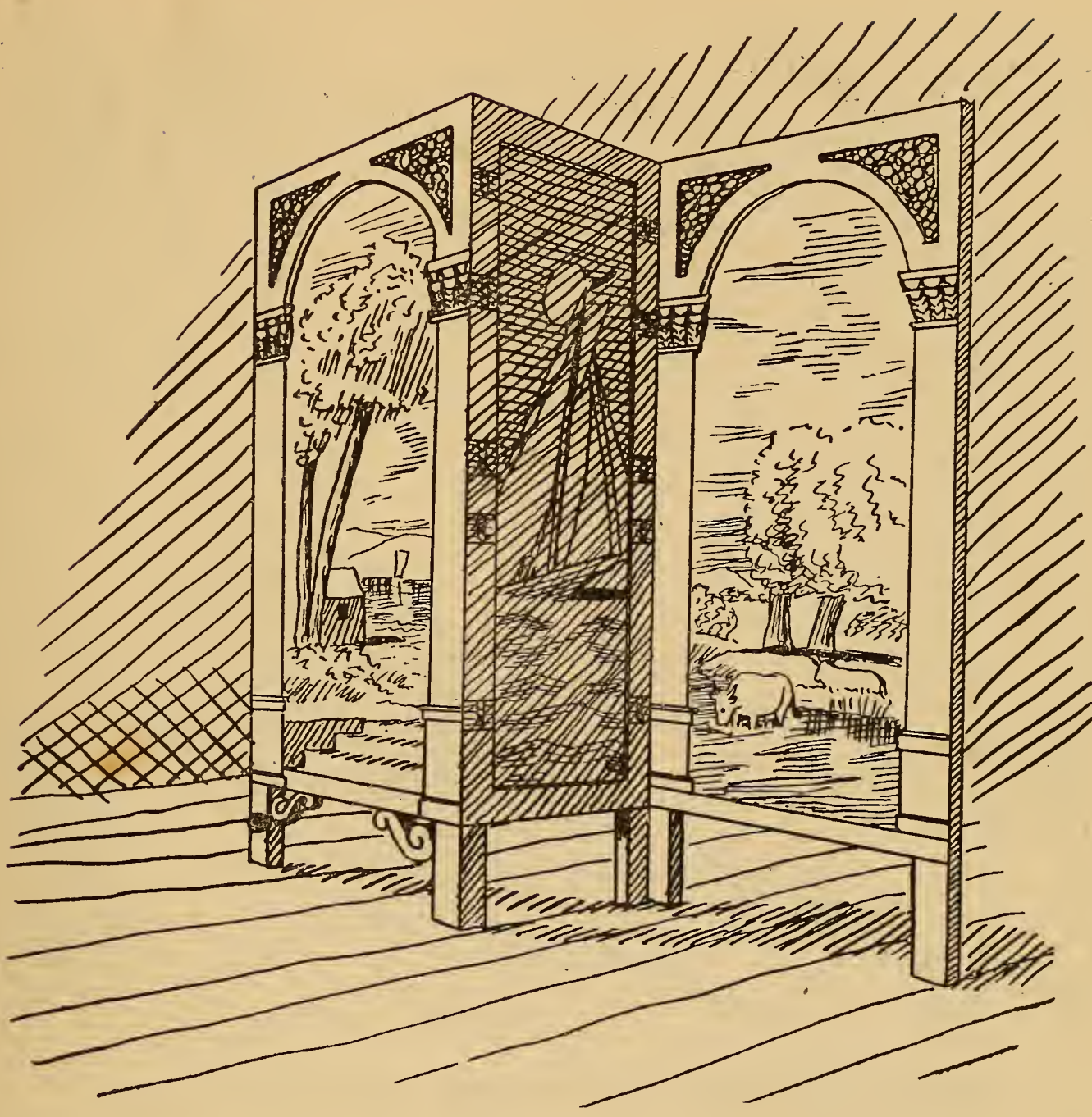

THREE-PANEL SCREEN.

This screen is decorated with a design especially adapted for painting. The frame offers a good suggestion. Any ordinary carpenter can make it, and common pine, free from knots and blemishes, will answer. The ornaments shown near the top can be purchased for a trifling sum from any wood-working shop. After the frame has been put together treat with two coats of ivory-white English enamel. The panels in the top should have a thick coating of copal varnish; while wet sprinkle thickly with broken bits of colored glass, and treat the whole, when dry. with a coat of varnish. The panels may be painted on sea-grass matting or on canvas. 


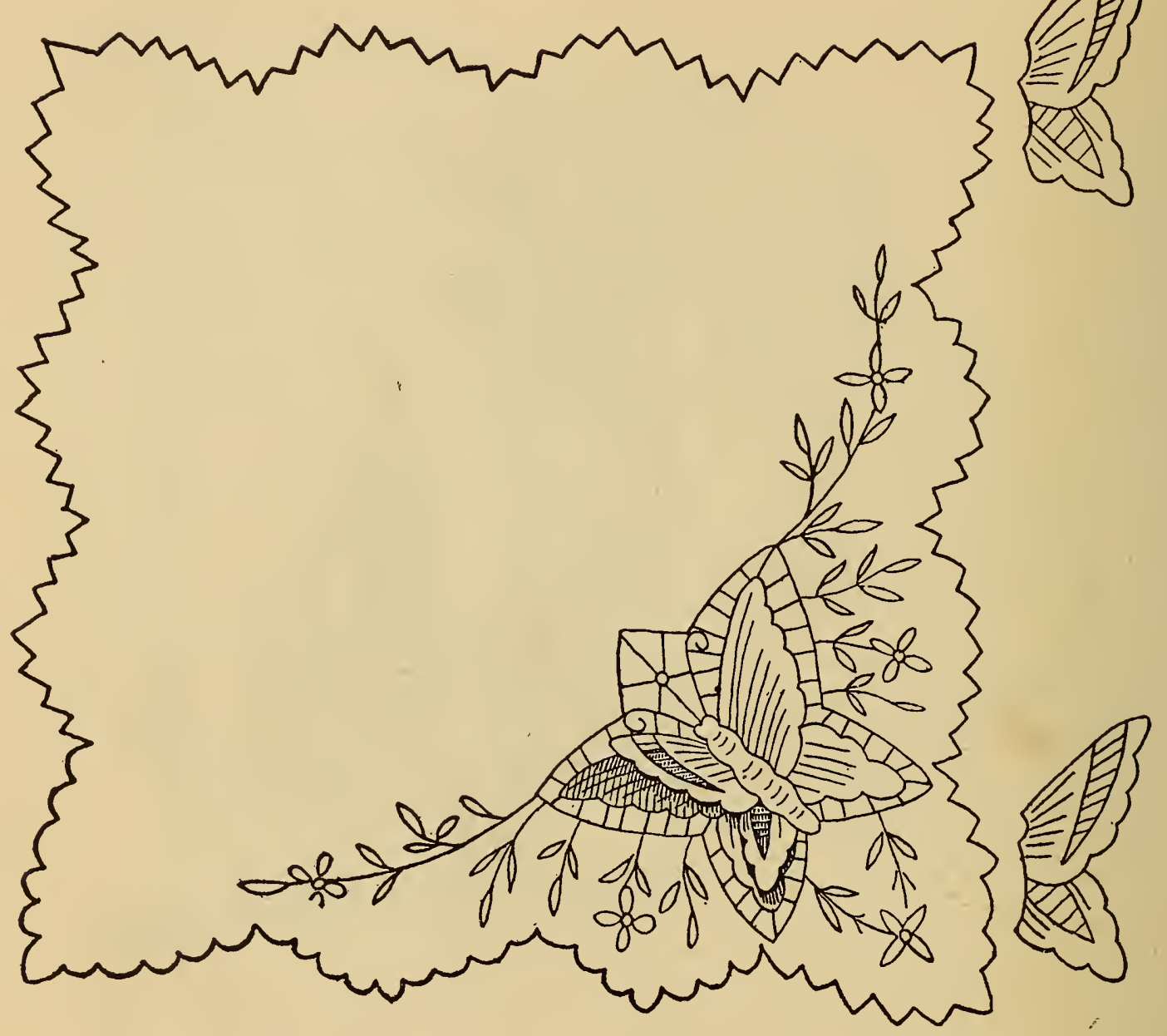

BUTTERFLY CENTER CLOTH AND DOYLEYS.

Raised butterfly decorations are much used on center cloths and doyleys. The above illustration should have the edges scalloped and worked in buttonhole stitch. The spray on which rests the butterfly should be worked in one color, in solid satin and outline stitches. The butterfly should then be worked on the flat in seed, outline and dot stitches, with the cloth cut away around the edges of the wing, after the manner of Roman embroidery. The two wings shown on the side of illustration should then be worked on a separate piece of linen, cut out and attatched to the body with an overcast stitch. The body of the butterfly should be worked last, in a heavy satin stitch. The two applied wings will stand up from the cloth, giving the appearance of a butterfly just alighting. These arrangements may be varied to suit individual taste. A flight of the pretty little insects, of different sizes, may be arranged with pleasing effect. 


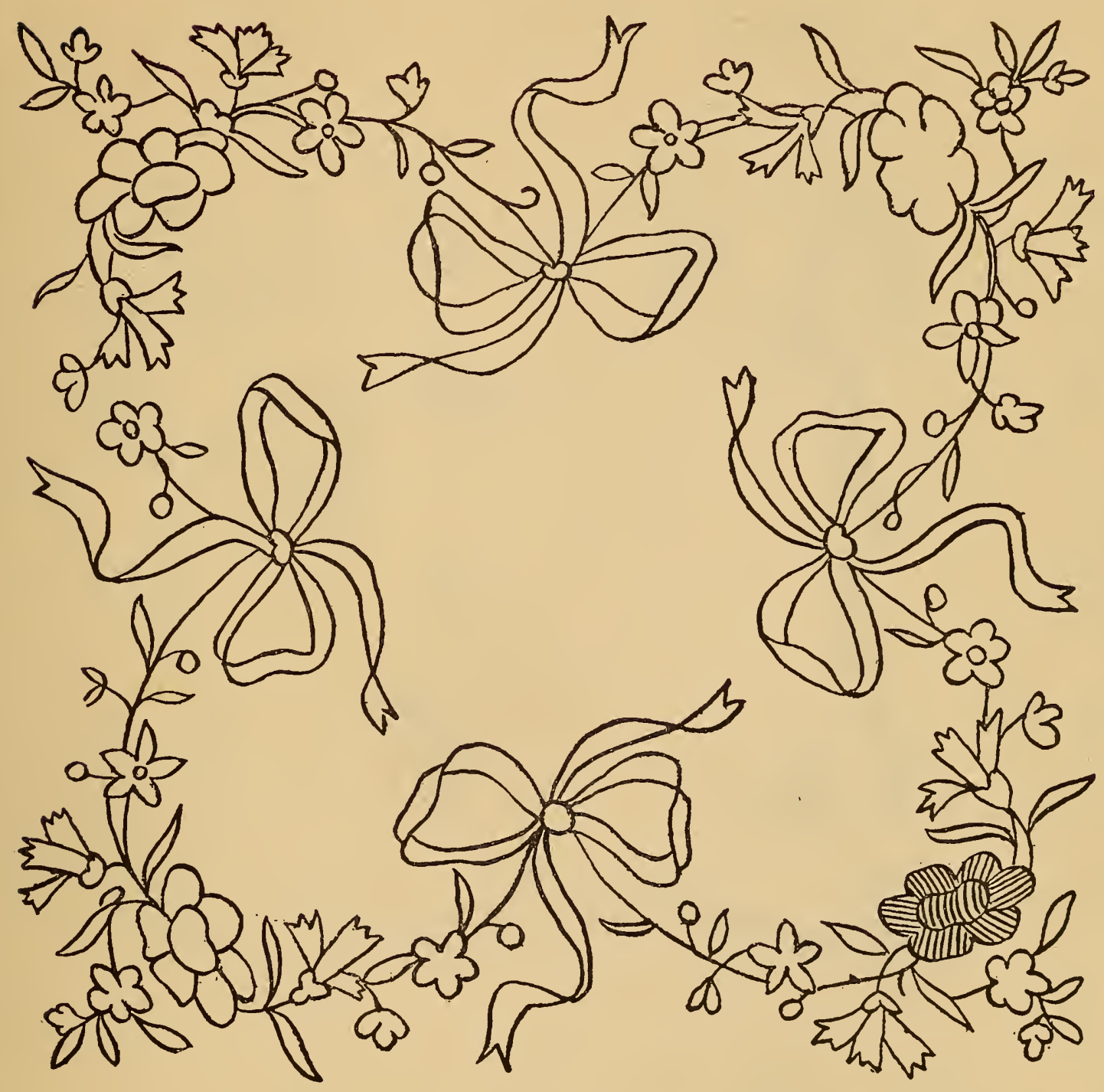

\section{LOUIS XIV DESIGN.}

These designs are having a great revival of popular favor. They are exquisitely dainty, and may be applied to almost any article. The illustration above is a border for doyley or center cloth. For center cloth it should be enlarged five times, while the size illustrated is correct for the doyley. The work should be solid except the ribbon, which may be done in any fancy stitch preferred. 


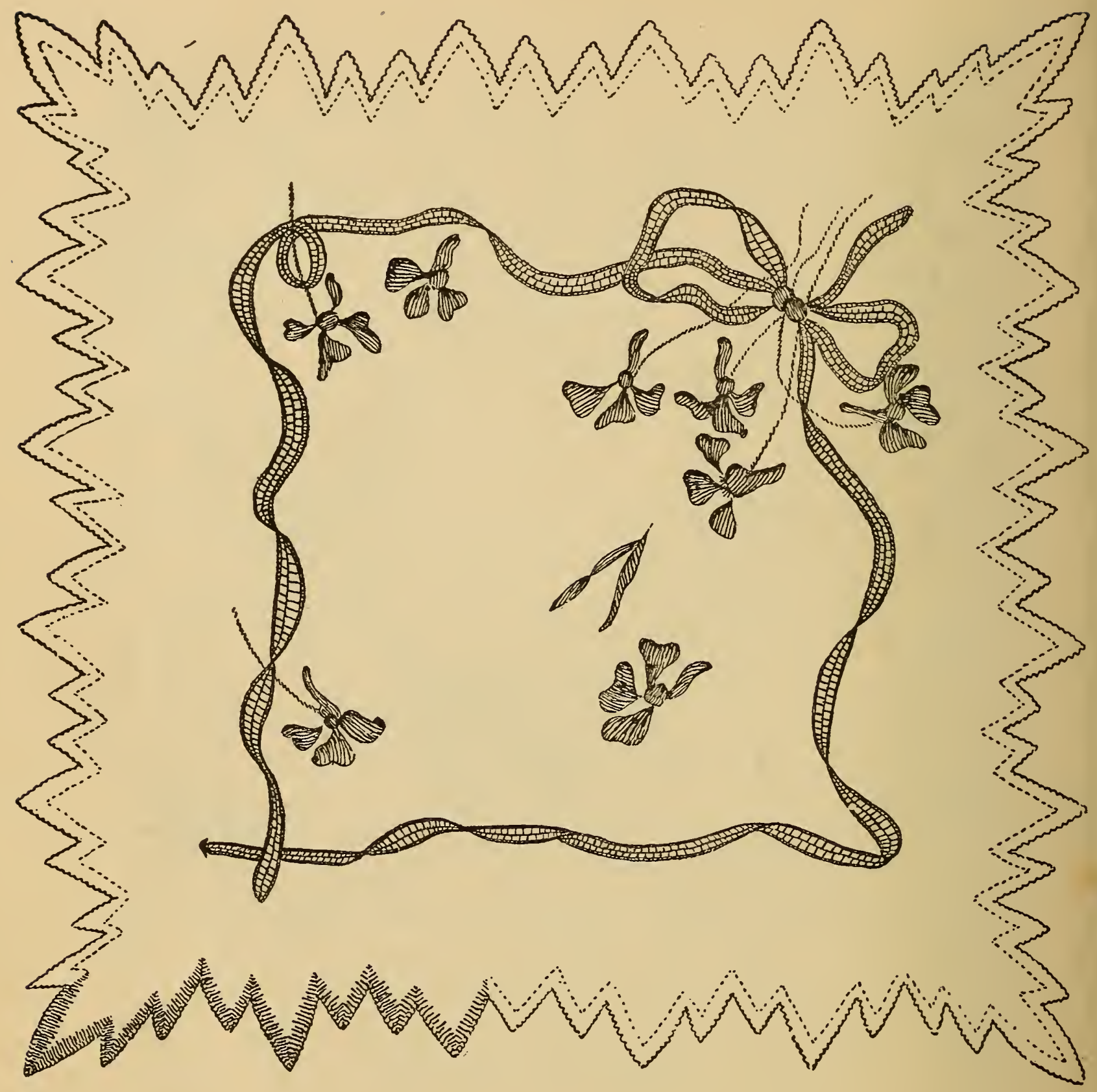

\section{CENTER CLOTH.}

The design shown here is very effective, and simple to work. The material should be fine linen. The pointed scallops look best worked very heavy in buttonhole stitch, with white linen floss. The ribbon should be worked with a delicate lily green, in buttonhole point stitch; the violets in three shades of purple linen floss, size 8 , in Kensington or satin stitcl., 


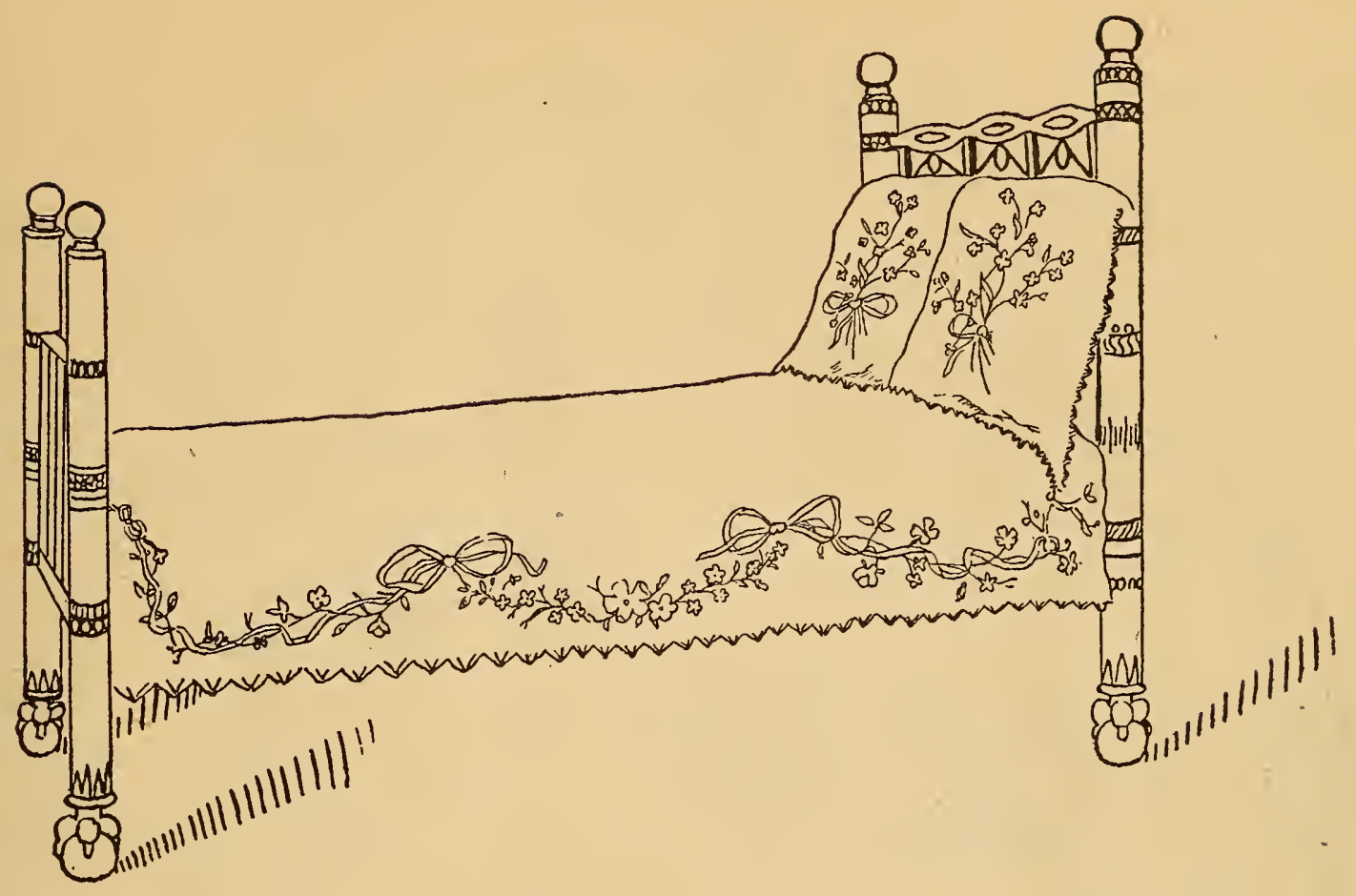

BED SPREADS.-FIG. 1.

The beds shown on this and succeeding page are covered with spreads made of Roman twill linen, decorated with designs that should be worked in outline and solid embroidery. Figure I has the extreme edges worked in scallops with heavy buttonhole stitch, with white linen floss. The design in the center is also outlined with a scalloped edge, worked in the same manner. The scroll above the border edge is effective worked in white rope linen, in a heavy cording outline stitch. The ferns in the corner should be worked solid in two shades of green liner floss, size 8 . The ferns in the center should also be worked in the same manner, and the knots of ribbon should be outlined with white. The same treatment should be carried out on shams.

Figure 2 shows a bed spread with a spread having a garland of tiny flowers arranged as an all-round border. The edge of spread should be worked with buttonhole stitch in scallops, and the garlands in natural color of linen floss size 8; for the small flowers and foliage use satin stitch; for the ribbon, simple outline stitch, filling in with a row of darning stitches if desired. The designs shown on this spread are very popular, and are being applied to many uses. 


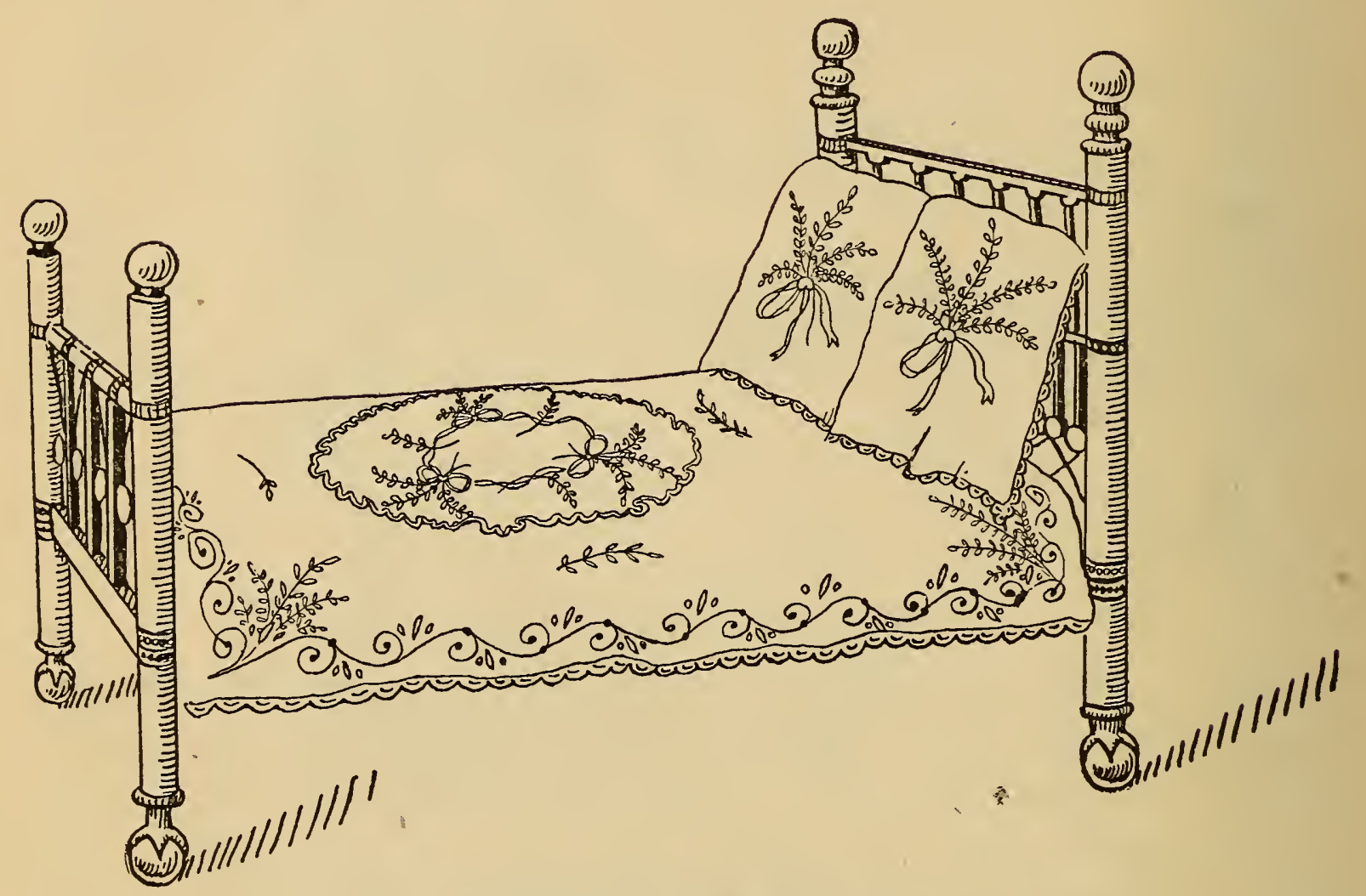

BEDSPREAD.-FIG. 2.

There are many different methods of decorating bed spreads. The two given will, however, serve as a guide and stimulus to invention. The work must be done with exceeding neatness, and the design and coloring selected with the utmost care. Very good effects may be obtained with outline stitch alone, when, of course, the work is reduced to a simple problem of exactness in taking the stitches. For outline work the design should be selected with reference to the treatment; flowing lines and bold outlines being necessary to get a truly artistic effect. 


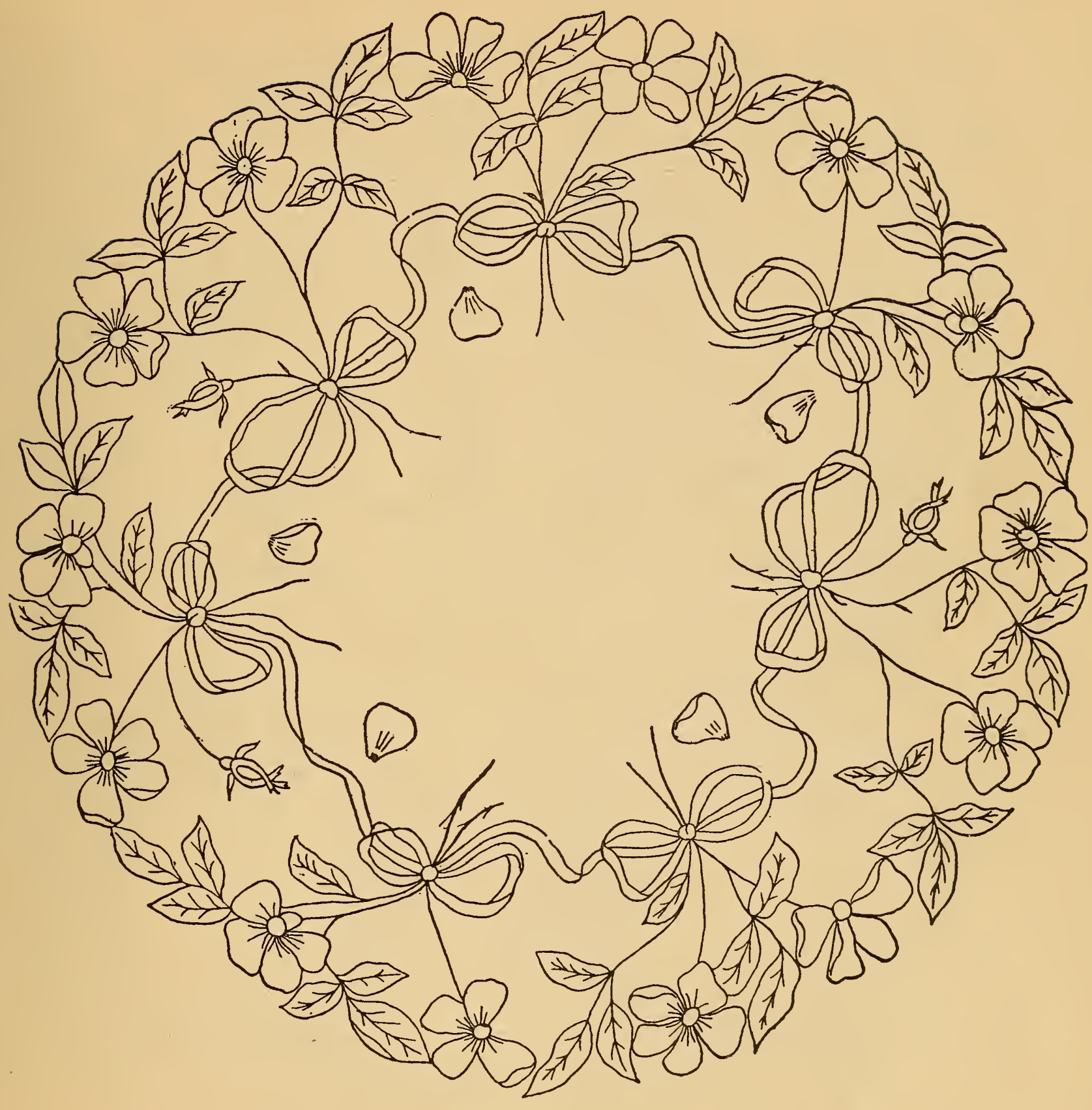

ROSE CENTER CLOTH.

The beautiful design shown above is for center cloth, and should be wrought out in pale pinks, three shades, one shade yellow for center, three shades green for leaves and stems; cither pink, green, white or yellow may be used for the ribbons. Solid Kensington and outline stitches look best on this design. Enlarge four times for correct working size. 


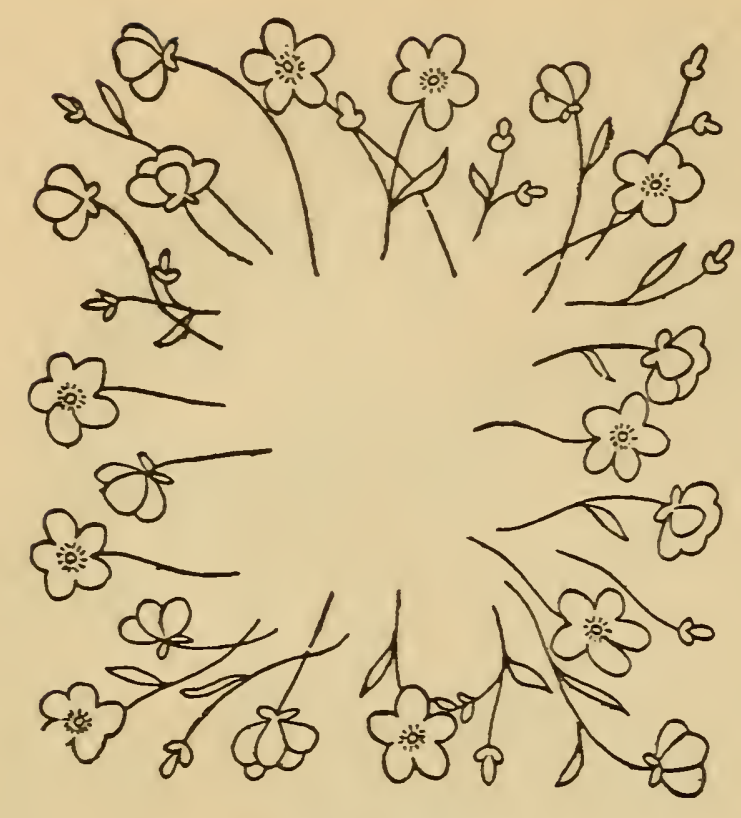

BUTTERCUPS.

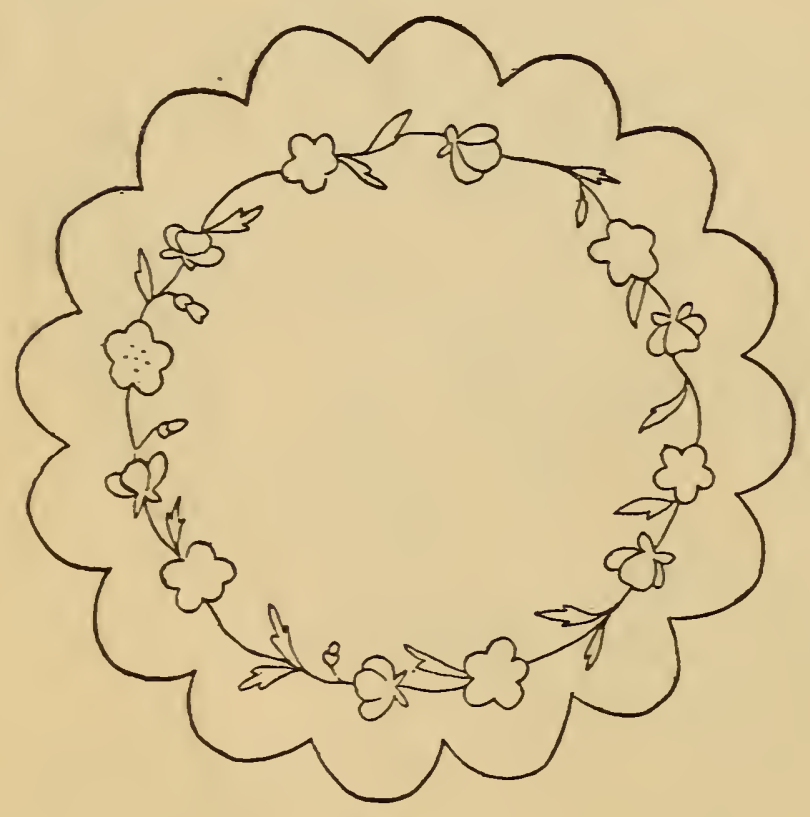

DAISIES.

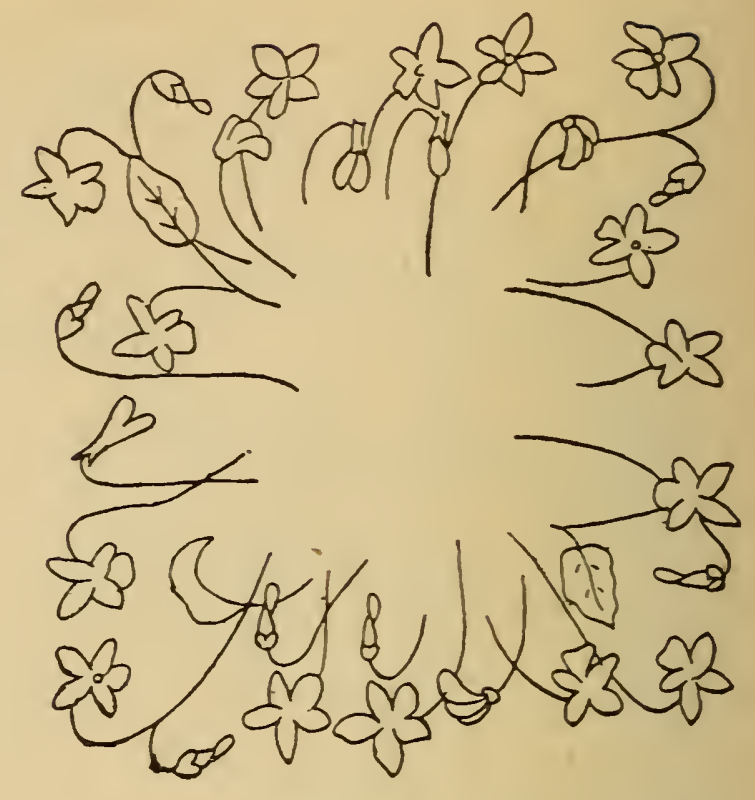

VIOLETS.

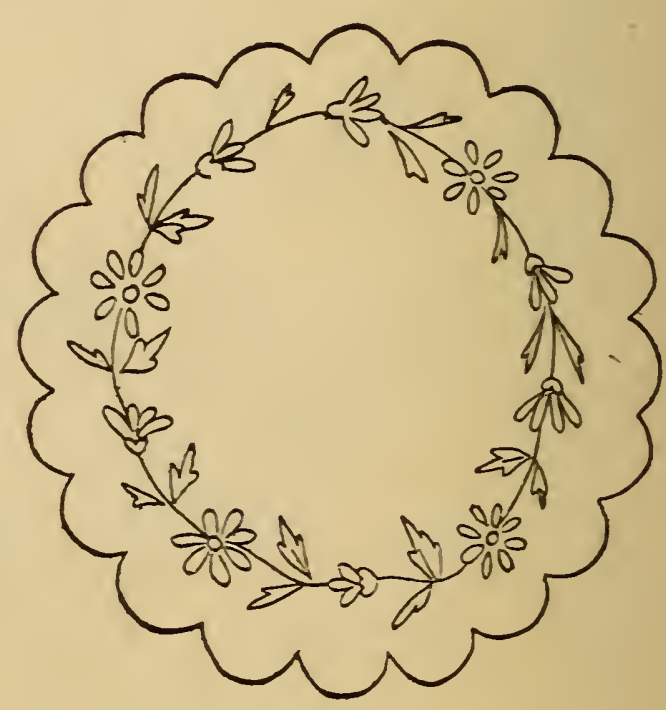

FORGET-ME-NOTS.

DOYLEYS.

The four pretty designs for doyleys shown here are one-half the correct working size. The doyleys should be made on fine white linen, and the work done in solid Kensington and outline stitches. The effect is also good when silk bolting is used in place of the linen. 


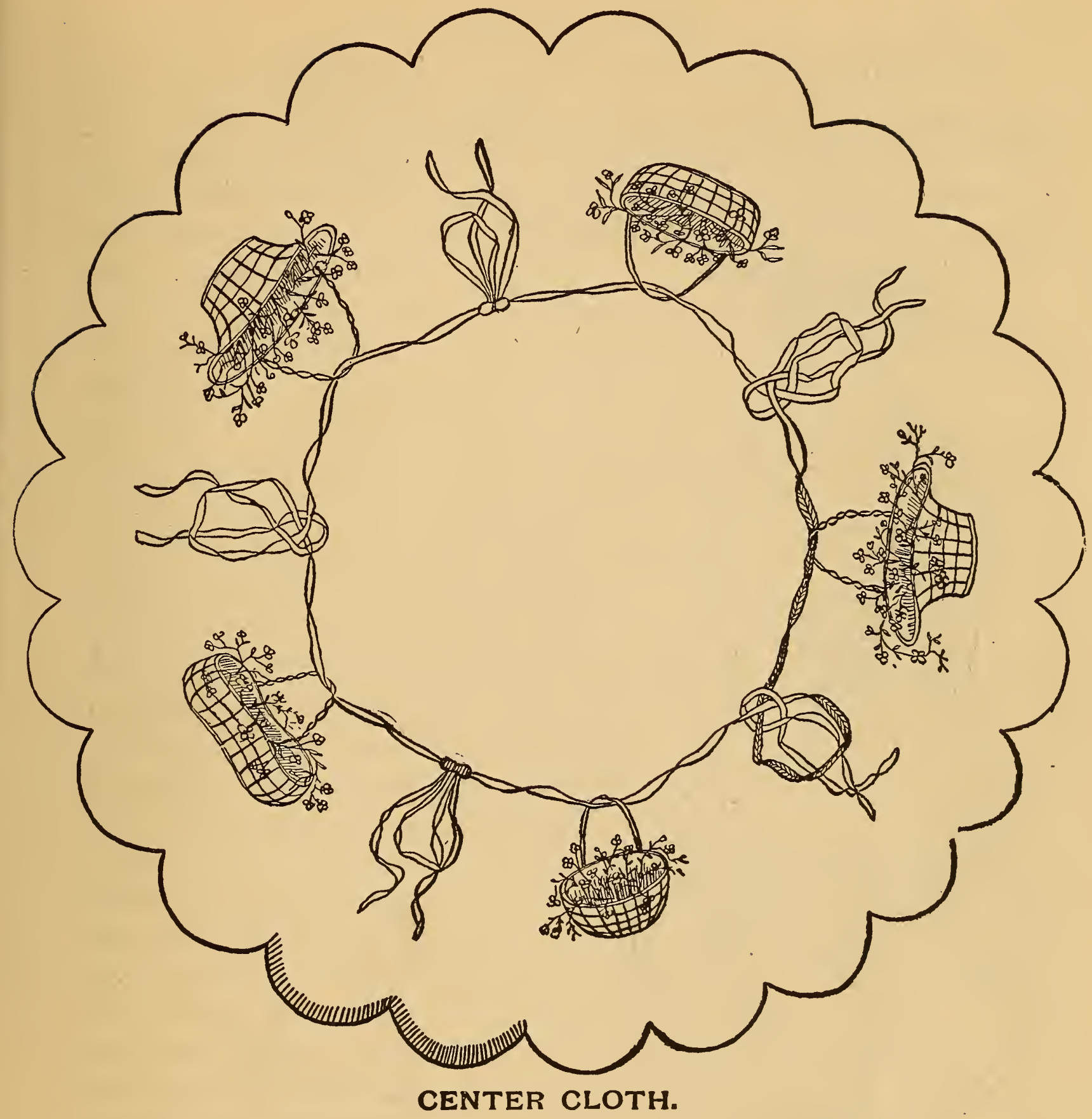

The design shown above is especially suitable for a white linen or lawn center-piece. As will be seen, it is designed on a circle. The edge is finished with broad shallow scallops which are to be worked with deep, close buttonhole stitch with white star floss. The ribbon on which the tiny baskets are suspended is wrought out in pale green etching silk with outline stitch for the edge and a row of feather stitch in the center. 


\section{SPONGE BAGS.}

These little articles are very convenient for use in the bath room or the toilet table, and for traveling bags are almost indispensable. They are so easily made, and the materials for their construction so inexpensive that they will be eagerly sought for Christmas gifts, and will always find a ready sale, cut and stamped ready for working. The sketch shows back and

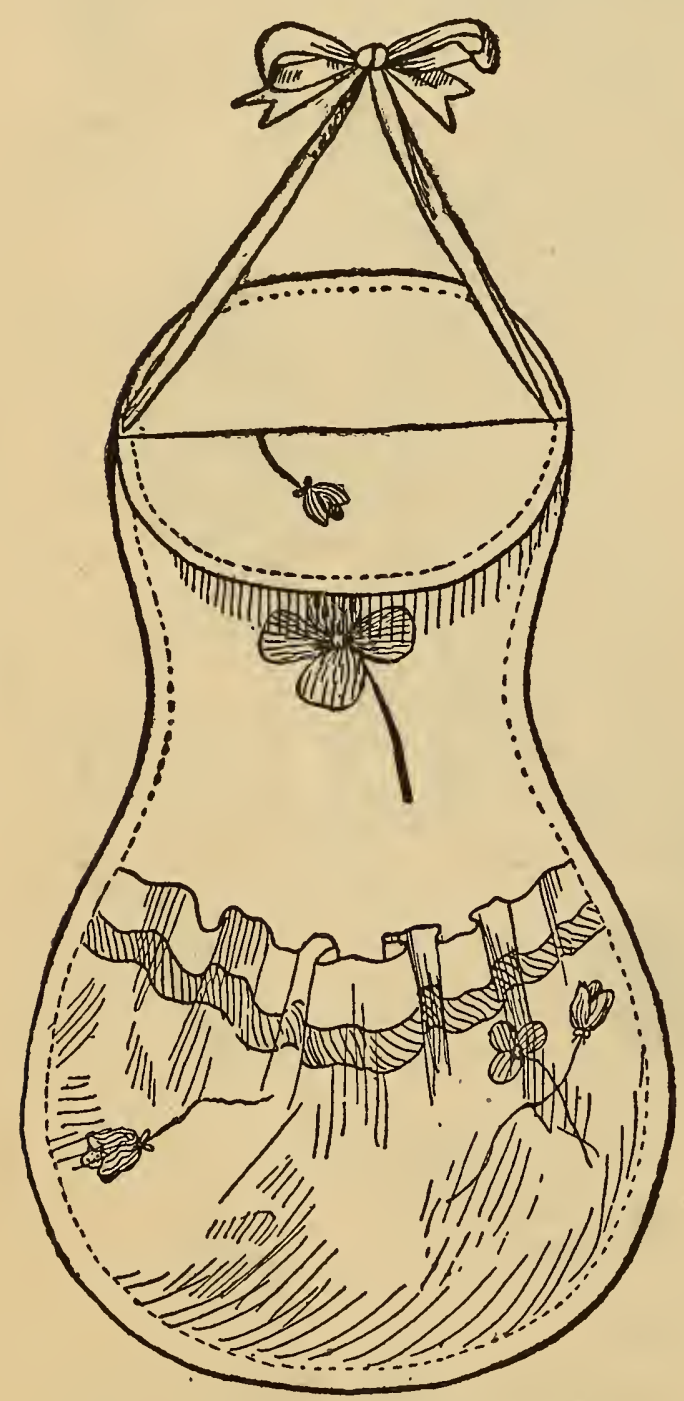

SPONGE BAG. front of bag when completed.

The material may be linen crash, duck, or other wash fabric. The two main sections are first cut

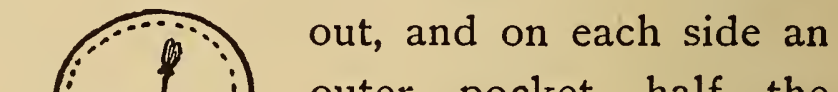
outer pocket, half the depth of the entire bag, is attached. The whole is then bound with silk braid, and loops to hang by are added. The inner and deeper pocket is for brush and comb. One outer pocket is lined with oil silk; this is for the sponge. The remaining outer pockets may be used for any other toilet articles desired. The decoration on the model is a design of pink clover and leaves, scattered over the surface.

The clover is worked in crown stitch with filo floss in three shades of pink. Another dainty sponge bag may be made from thin oiled silk. Cut a strip of oil silk about fourteen inches long and four inches wide; join the two ends together with a pretty ruching of pink satteen, and attach narrow 
ribbon hangers to suspend by. This arrangement leaves the sides of the bag open and allows a circulation of air which dries the sponge quickly and prevents it from becoming musty.

The sides of the oil silk bag may be decorated with some simple design painted with oil colors.

\section{TRAVELING CASE.}

Traveling cases for holding towels, brush, comb, soap, etc., are useful articles and may be made as ornamental as one desires. The model shows a very pretty case of this kind. To make, select a strip of gray or ecru linen canvas, 20 inches wide by 36 inches long. Turn over the canvas at one end to form a pocket nine or ten inches deep-large enough for the night-robe-above this pocket stitch on a lap-over about $3 \frac{1}{2}$ inches wide, fasten down to the pocket by means of buttons and buttonholes. In the center arrange two square pockets, one at each side, fastened also with a lapover. Above these two side pockets, in the center of the width, arrange a strap into compartments for brushes, and above that, about six inches from the end sew on a pocket that will extend from edge to edge. Bind all the pockets with brown silk braid and orna-

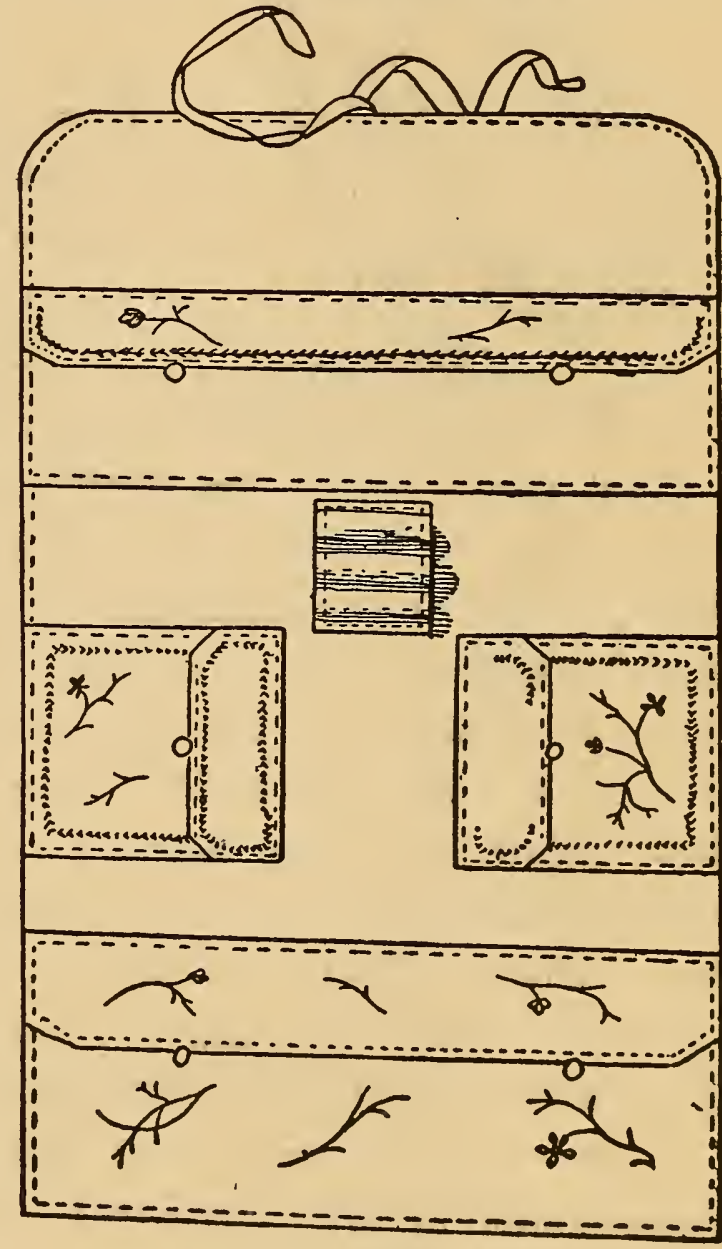

TRAVELING CASE.

ment the hems and edges with a row of coral stitch done in dark brown etching silk. Scatter over the pockets broken sprays of flowers and work in outline stitch with two or three shades of brown etching silk. When in use roll up from the end and fasten with brown satin ribbon.

Pretty traveling medicine cases are made from these materials and in the sáme manner, except that the ends and sides lap over and tie in the center. Inside there is a strip arranged in compartments to hold six medicine bottles. 


\section{PICTURE FRAMES.}

In this day of many photographs and the banishment of the family album, picture frames have become a necessity, and where the frames have to be purchased ready-made the expense becomes an item worth consider-

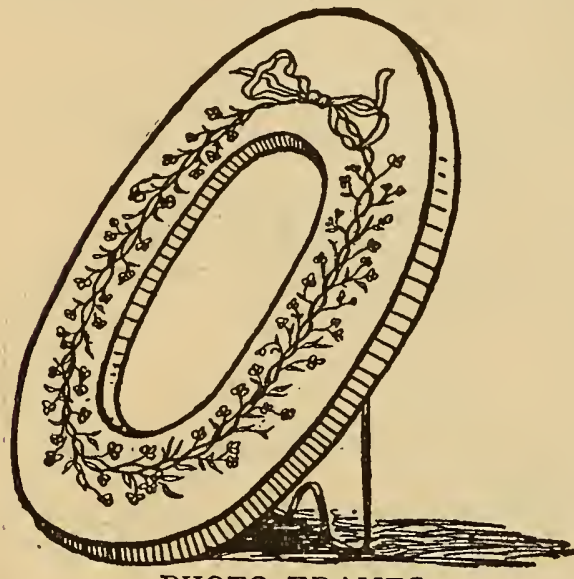

PHOTO FRAMES. ing. There are many pretty styles of frames which can easily be made at home. Some are square with oval openings, or the opening may be square. There are the round frames, the frames in two panels, and again in three. The favorite size is $9 \times 7 \frac{1}{2}$ for the single panel square frame, and 8 inches for the oval and round frame.

To make any of these frames there will be required, of very stiff card-board, two piecesa front piece, having the opening truly cut out, and a plain back piece. These two pieces are to be neatly covered separately, the picture tacked into position on the back piece, and the two pieces must then be firmly glued together. The outer cover is usually made of fine white or ecru linen, embroidered in Dresden design in either silk or linen, in solid stitch. If the frame is covered with leather, mole skin or silk, the design will look best painted. Where the picture is to be used on a table or bracket a standard should be attached to the back. The front and back boards can be purchased for 15 or 25 cents, without the standard, and with, for from 25 to 50 . Where one has not the patience or ingenuity to prepare the foundation this will be found an accommodation.

The model shows an oval, eight inches across, covered with white linen embroidered in forget-me-nots in satin stitch.

Dealers will find a stock of prepared foundations, with and without standards, a paying investment. In connection with these should be carried in stock the front and back covers, cut to fit, having stamped on them suitable designs with the work commenced; the necessary material for finishing should be attached to each in a little paper bag or envelope. Where several covers are thus prepared just the necessary amount of embroidery thread, and no more, need be purchased as one skein of a color may be divided between several covers. 


\section{SOILED HANDKERCHIEF BAG.}

This dainty little trifle is intended for use near the dressing case, and is designed to hold soiled handkerchiefs. The bag is made of plain white linen and is seven inches wide by nine inches long. The outer side of bag is decorated with a spray of buttercups, worked solid with yellow filo floss. The stems in brown filo floss. The top is hemmed over a stiffening, and across one corner is draped a piece of white fish net, with edge finished with tassels. This net can easily be tied from white Real Scotch linen crochet thread, No. I2. A yellow ribbon hanger is attached and the bag is complete. This simple, inexpensive article will find friends everywhere.

These articles will be found ready sellers at bazaars or at

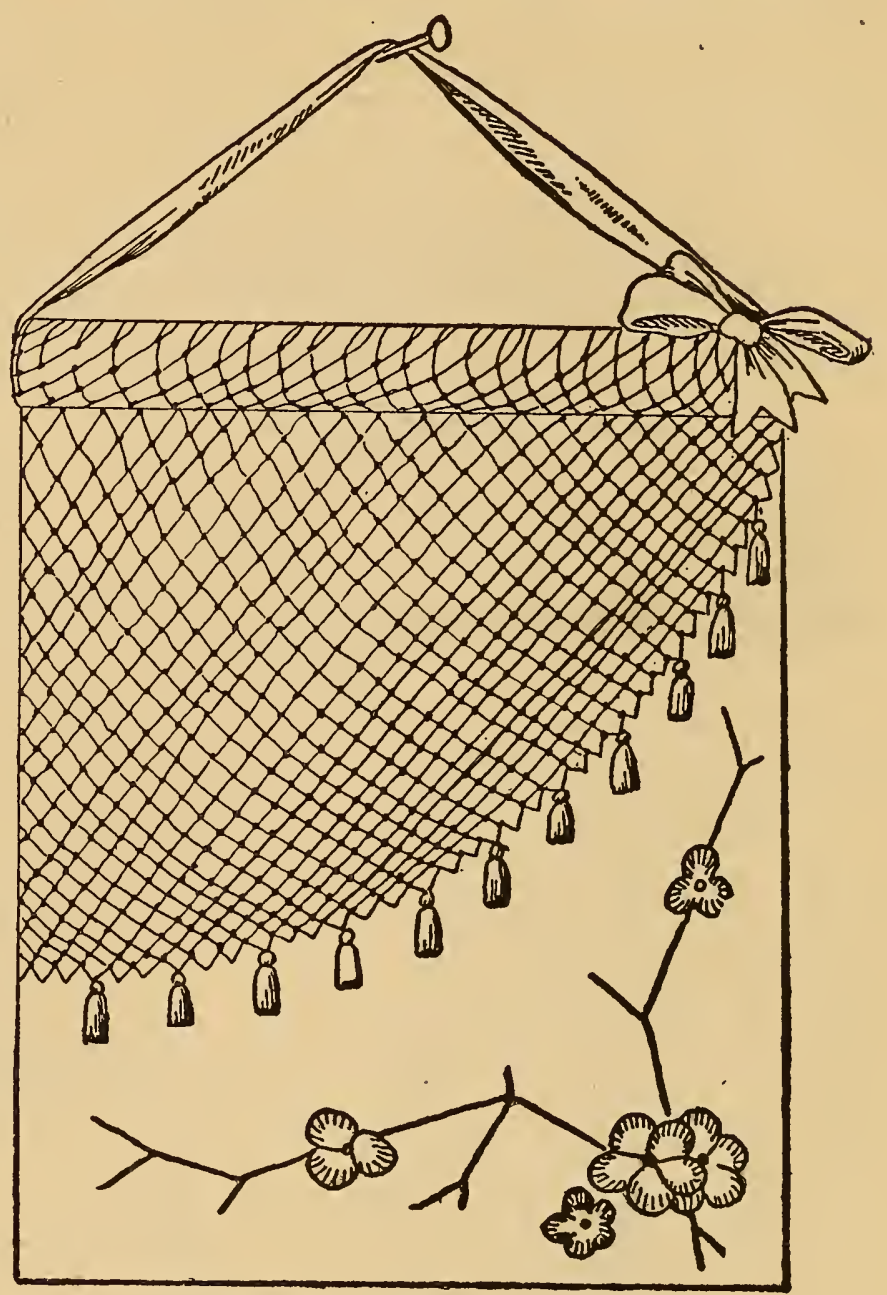

SOILED HANDKERCHIEF BAG.

Christmas time, and a fair profit may be made by the sale of the material cut into shape having the design ready stamped, and with sufficient embroidery thread attached to work the pattern. Where several are to be prepared in this way it is a good idea to have several designs. We suggest for this purpose a small spray of eglantine with flowers, vine, leaves and tendrils. This design may be arranged either in one corner or it may start from the top of bag and droop gracefully downward. Another appropriate pattern could be arranged by grouping small sprays of forget-me-nots over the lower right-hand corner and scattering a few upward over the surface toward the top of bag. 


\section{GLOVE AND VEIL CASES.}

A society girl's dressing case of the present day resembles a bazaar of fancy work, so many are the dainty trifles considered necessary for actual use. Among the daintiest and most useful may be mentioned glove and veil cases. There are many different methods of making these articles,

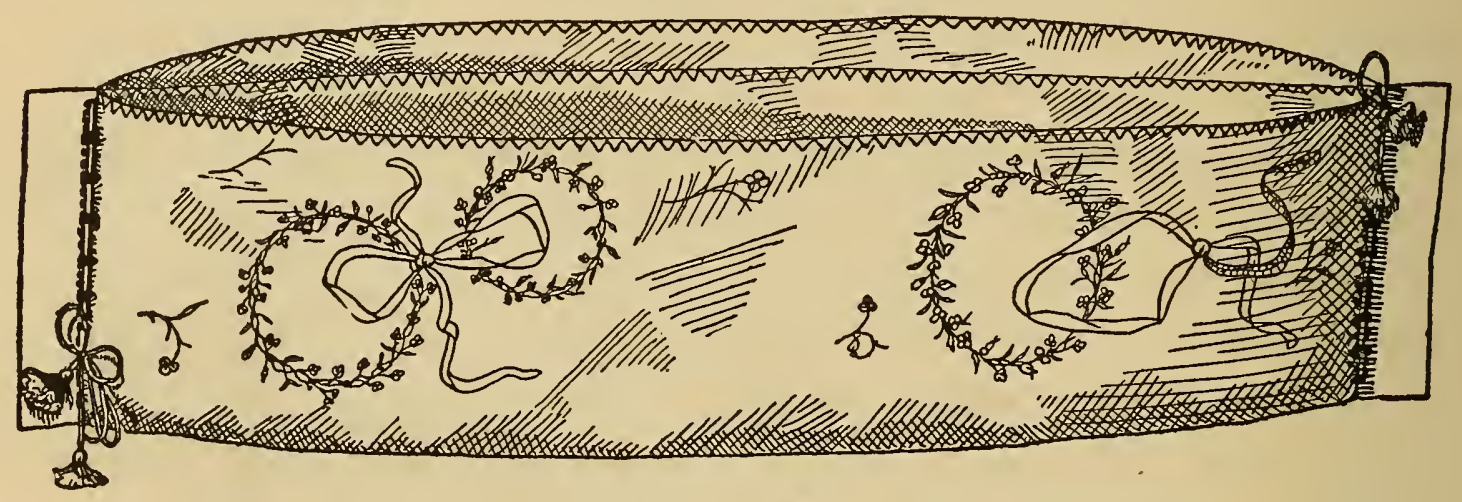

GLOVE CASE.

but models shown here are among the most practical as well as the latest. To make the glove case, select a piece of fine yet heavy white linen, $27 \times 36$ inches; hem the sides and buttonhole stitch the ends; fold the linen backwards and forwards into four even folds, after manner of creasing paper for a fan. The folds will be 9 inches deep; the inside of each will form a pocket for a pair of gloves. The ends are secured by running into cut slits a cord and tassels. The design is Louis XIV., as indicated, worked out with two shades of blue, fine, Real Scotch flourish-

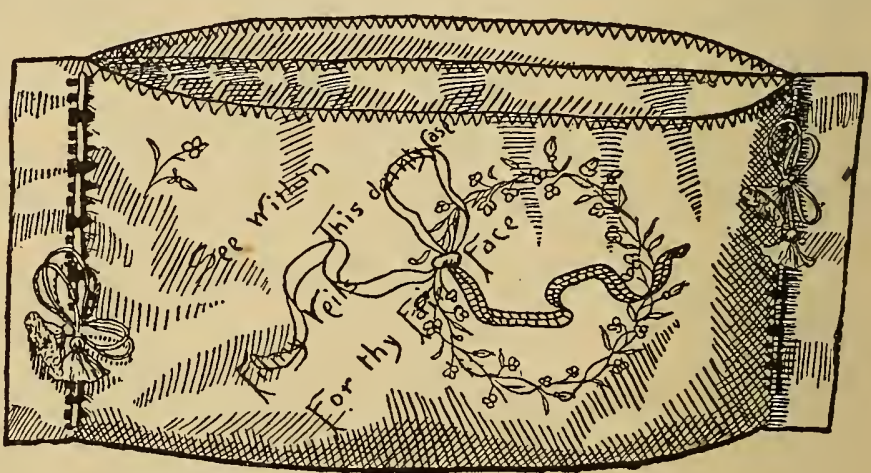

VEIL CASE. ing thread. The ribbon is worked in buttonhole stitch, with linen floss.

A veil case to match the glove case is made in exactly the same manner and with the same materials, except that the dimensions are smaller, being I8XI4 inches. The motto, "See within this dainty case filmy veils for thy fair face," is worked in simple outline stitch in bright gold; the ribbon is pale yellow and the flowers are worked in delicate pinks and blues with Scotch flourishing thread, No. 8. 


\section{INFANT'S FLANNEL ROBE.}

Considerable attention has been paid in previous chapters to decorative needlework for articles of the wardrobe, for if all other styles of embroidery should be doomed by a decree of Dame Fashion, that for embroiciering baby clothes would still be with us. French embroidery, commonly called satin stitch, is usually used for this class of work. Ladies who wish to earn money by the use of the needle will find a paying field of labor in making infant's trosseaux, and as pretty flannel wrappers are considered an indispensable part of an infant's outfit we show one in this connection, made of fine white flannel. The yoke is embroidered with tiny daisies in cream white etching silk. The petals of the daisies are to be worked in satin stitch, and the centers filled with tiny yellow French knots. The yoke and skirt are joined with a row of fine feather stitching done with paleyellow etching silk. A row of the same should be carried down the front, around the bottom and on the sleeves. Any other small flower may be substituted for the daisies. A row of daisies down the front adds to the beauty of the wrapper. The model shown illustrates but one of many dainty and pretty articles for baby's use. Short jackets made of fine flannel, having the edges finished with fancy scallops, buttonholed with etching silk, and spot stitch scattered over the entire jacket, worked with some dainty colored filo floss,

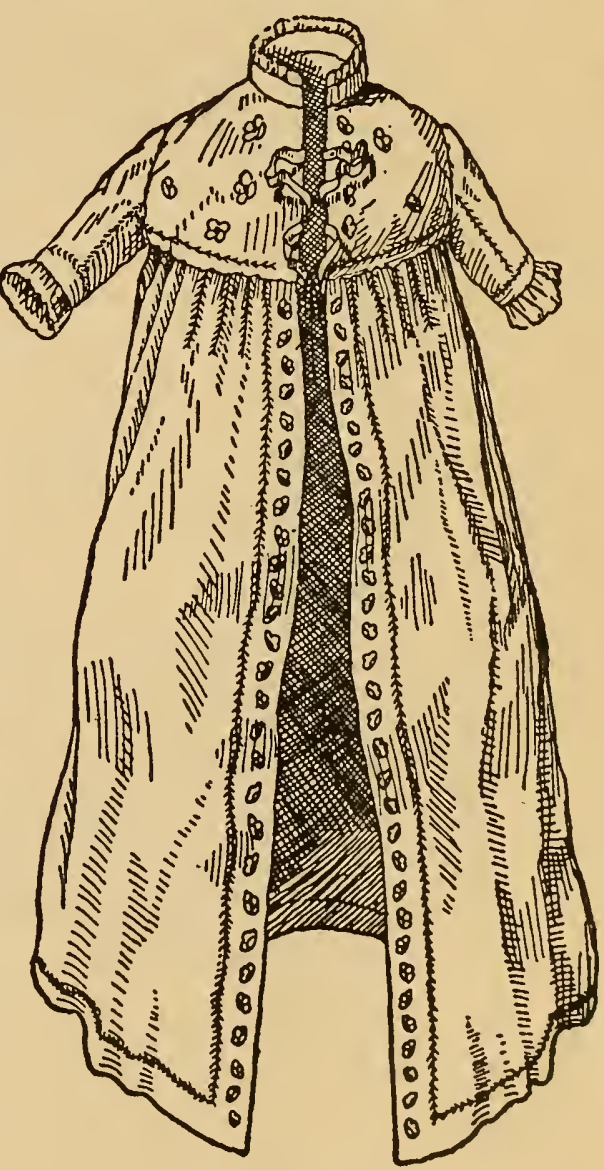

INFANT'S ROBE. are useful as well as pretty additions to baby's toilet. Very pretty little caps may be made from scraps of white cashmere. Cut a crown the desired size. Scallop the edge, work with buttonhole stitch with etching silk; cover the crown with spot stitches worked close together: cut a bias frill for the bonnet, and scallop the edge, same as for the crown, and cover the surface with smaller spot stitches than those used on crown. 


\section{SOFA AND HAMMOCK PILLOW.}

Sofa and hammock pillows are always in demand; and as they really are useful as well as ornamental, they will wear out, thus making room for newer favorites from time to time. Pillows and cushions should always be practical, and should look cozy and comfortable, and above all as if intended for use first and ornament afterward. The model here shown is made of blue denim, the wrong side is used as the right side for the cushion cover.

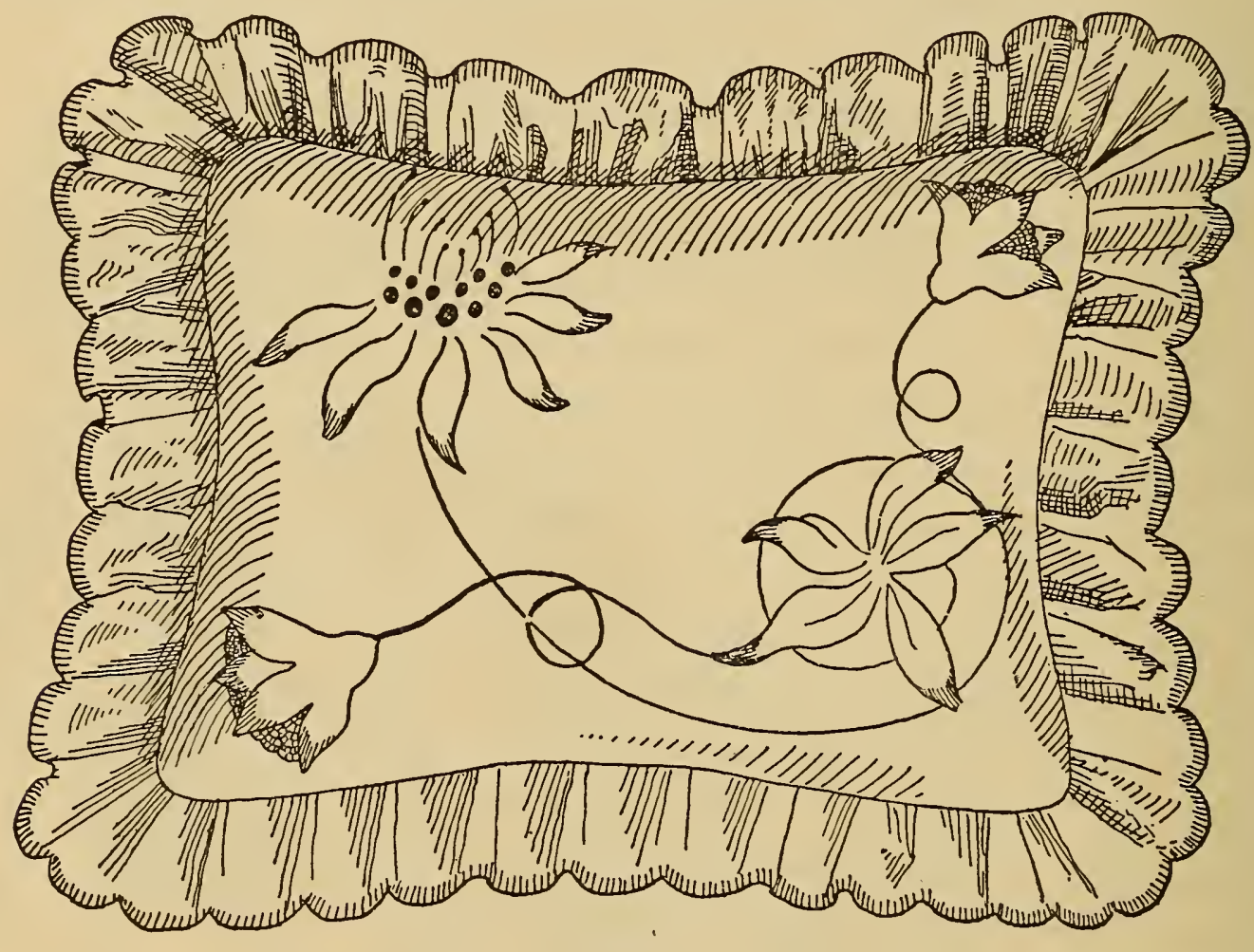

SOFA OR HAMMOCK CUSHION.

The design is strictly conventional. The entire design should be worked out in Real Scotch rope linen in pure white, not cream white. Work the stems with a heavy outline stitch. The star-shaped flower in lower right-hand corner should be worked in short and long stitch, heavier at the tips of petals. The vein in each petal looks best worked with feather stitch, and a cluster of three or four French knots should fill the center. The two buds in opposite corners should be worked alike, i.e., short and long stitch for the outer petals, and French knots massed close together for the center, which is indicated by heavy shading in the illustration. The full-blown flower in upper left-hand corner should 
be treated as follows: work the petals in short and long stitch, heavier at the tips. Cover the dots with French knots, large and small as indicated. The stamens work with fine outline stitch and finish the top of each stamen with a French knot. This treatment is simple and effective. The cushion is finished with a frill of the denim made up on the right side. The edge is scalloped and worked in buttonhole stitch with star floss. These covers stand innumerable launderings and will wear until one heartily wishes a change.

\section{IDEAL HONITON.}

Ideal Honiton has assumed an importance which bids fair to rival drawn work. It is equally as dainty in appearance as the finest drawn work, and has the advantage of being more quickly done and with less strain upon the eyes.

The designs for work of this class are limited; geometric forms only allowing the use of the Honiton braids.

Ideal Honiton decorations may be applied wherever drawn work would be suitable. The materials necessary are ¿wo or three sizes of the Ideal Honiton braid and fine etching silk. Illustrations of this braid may be

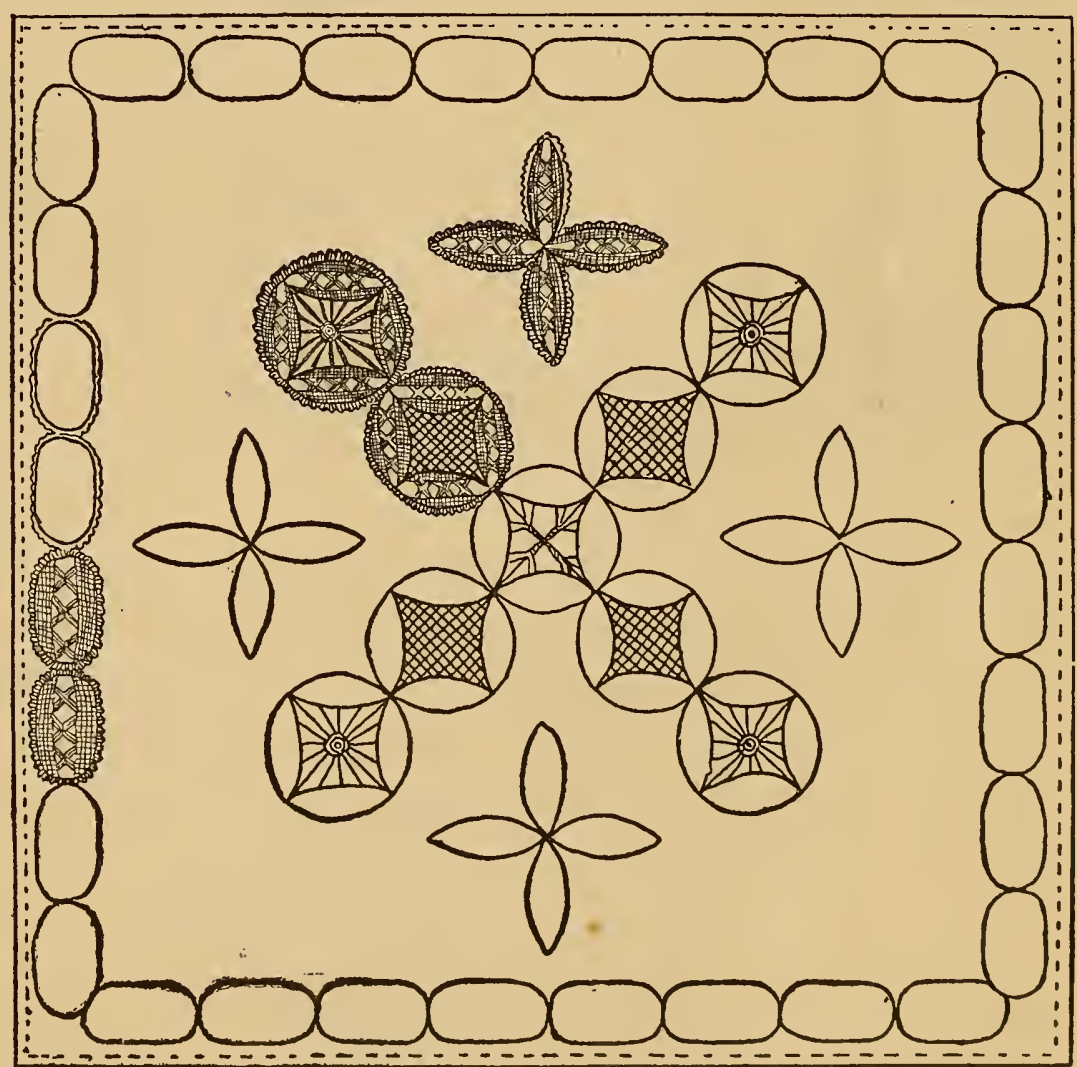

FIG. I. CENTER-PIECE.

found on page $3 \mathrm{IO}$, and by referring to the center-piece shown here, the particular braid more generally used can readily be seen. To use the braid, baste it around the outlines of the design, then buttonhole each edge to the foundation; make the buttonhole stitches close and firm. Where the 
braid is used around the edge of any article, like a doyley etc., the material should be cut away close to the buttonhole stitching. Where patterns are formed of the braid on the surface of any article which leaves an open space, the space should be filled with some lace stitch, after which the material back of this part of the work should be cut away. This will give a light and dainty effect not to be obtained otherwise.

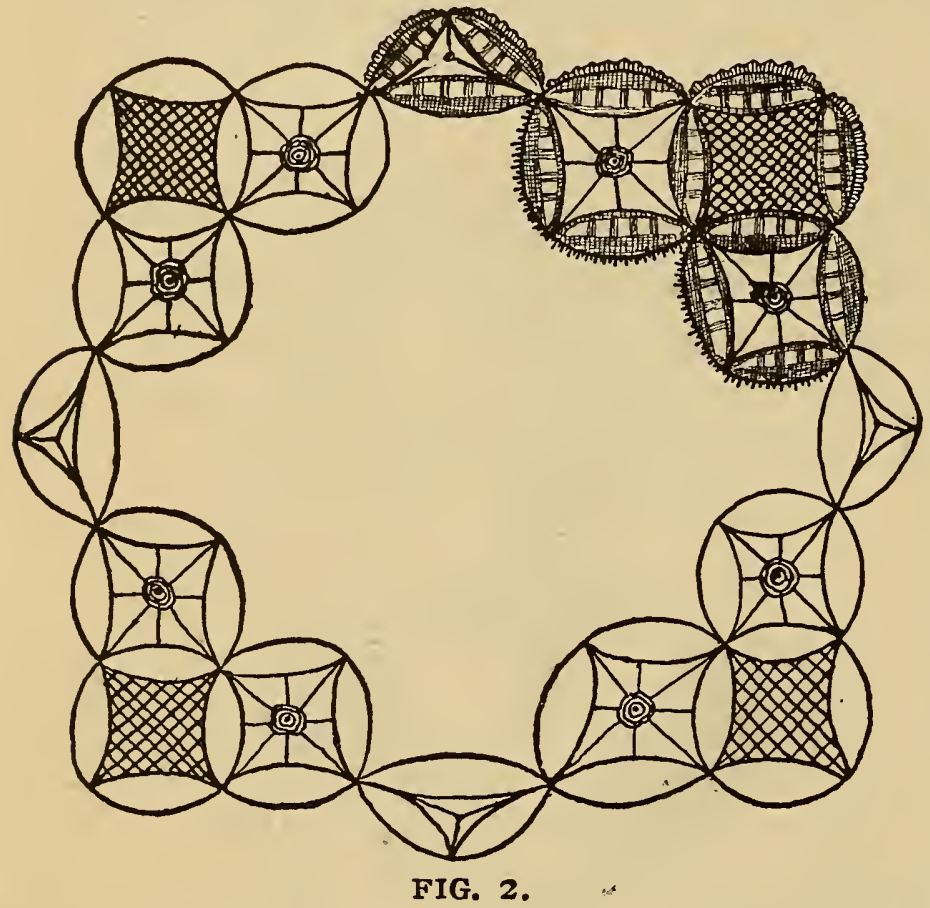

The center piece, figure I will give a very good idea of the different effects to be obtained with Honiton lace oraid. First a row is buttonholed around the edge and the material cut close to the outer edge. The braid is then placed in the form of a cross; as will be seen by studying the design the braid is not sewn in a straight line, as on the border, but is carried around an imaginary square, leaving an open space in the center. In each arm of the cross a four-pointed star is made with the braidwhenever the braid is used remember that both edges must in all cases be buttonholed to the foundation. The spaces left by forming the pattern are to be filled with lace stitches. For spaces shown here the most suitable are English wheel, spider web, Brussels point, all of which are shown on pages 3I2, 3I4, 3I6, and the method is described in "Chapter on Lace Making."

Figure 2, shows a fine linen doyley finished with Ideal Honiton. The reader will have no difficulty in sewing the braid to simulate the pattern here shown as the size of each figure is determined by the length of the loop in the braid-as will be seen by referring to the corner that is shaded in-each pattern that forms the outside of an open space requires just four loops of the braid-no more and no less will answer as a loop may not be divided-on each side there is a pattern formed of three loops only. 
Figure 3 shows a small doyley for bread and butter plate. The arrangment of the braid is quite simple. It is carried around the edge, to simulate a scallop, and for the purpose a braid with small, short loops should be selected. At intervals around the edge, inside the scallop, a single loop is buttonholed to the material, and the different figures in the four corners are made each with four loops of the braid. In following the different designs given for this article be careful to observe that portion of the pattern which is shown completed, as this part

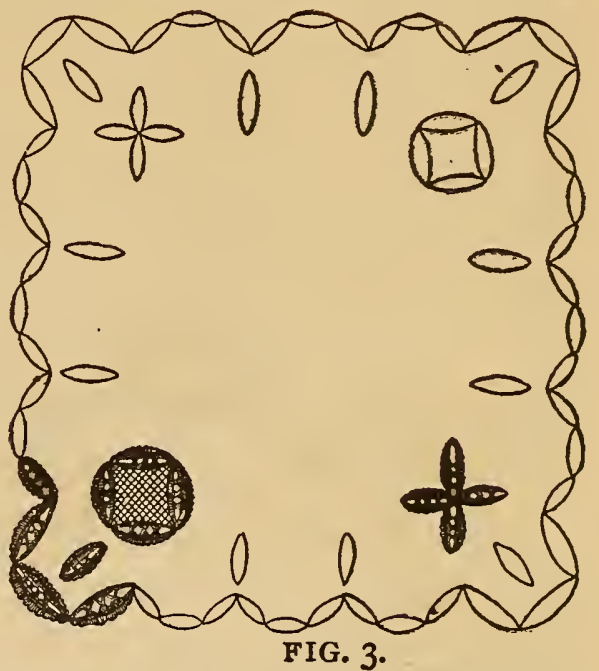
of illustration indicates exactly how the braid should be put into position, as well as the effect of the completed work. An ingenious worker will be able to vary designs shown here, adding a loop or braid here and there.

Figure 4 shows a plate doyley. This treatment is at once effective and

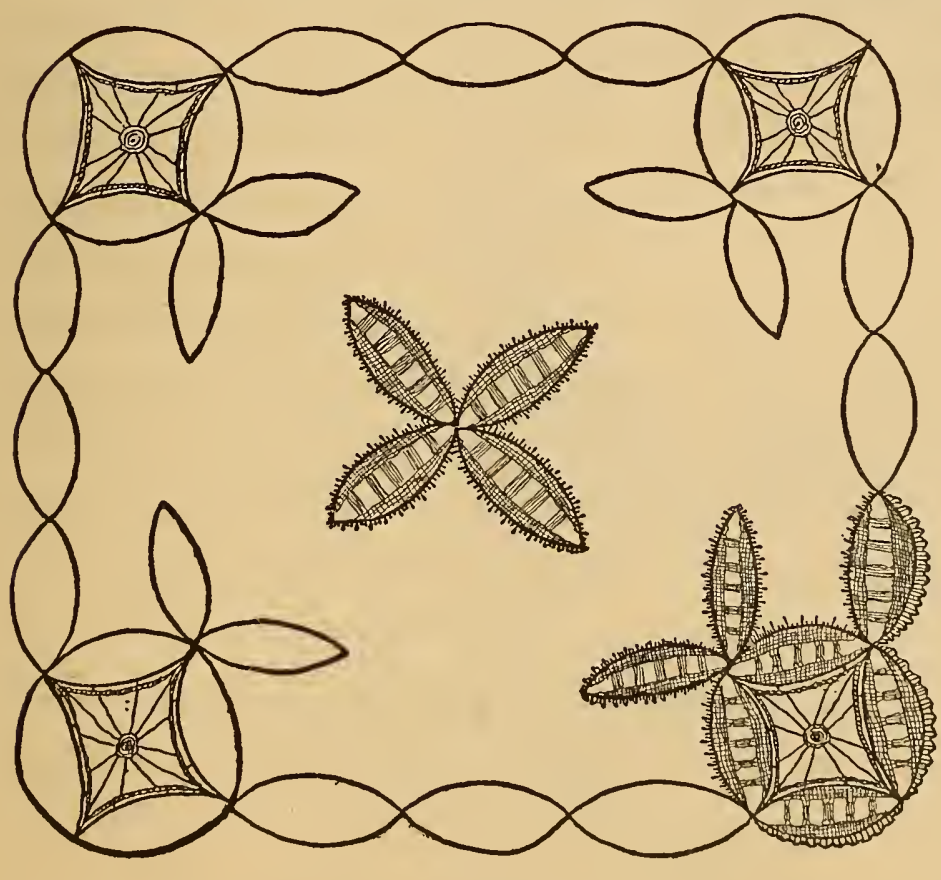

FIG. 4 . simple. In arranging mark off the space in each corner that will be required to allow the four loops of the braid to be put in; then measure carefully to see if there will be room enough between the corners for any numbers of complete loops of the braid, then add the star shape in the center and two loops of braid at the top of each corner piece. The best filling for a design when only the corners are to Be so ornamented is the English wheel shown in the chapter on Drawn Work, as it is more solid than the spider web and gives a better bit of color where colored silk is used to fill in with. 
In arranging designs in Honiton around a border accurate measurements with the braid must be made so that there shall be no mistake about its "coming out" all right. A pretty finish for this design would be to buttonhole all the edges, close and firm, with pale green etching silk and then fill each of the open spaces in the corner with a different stitch, say spike stitch-shown on each end of figure 265 on page 252 for one space, spider web, shown in center of same figure on page 252 for a second space and for third space Brussels point or Spanish point-filling each corner differently, using pale green etching silk.

Some ladies prefer to do the work with pure white silk, and wherever a color is used it should be only the daintiest and lightest shade of the color chosen-yellow, pink, blue, green, violet are all effective.

Figure 5 illustrates a pillow cover for infant's crib, decorated with the

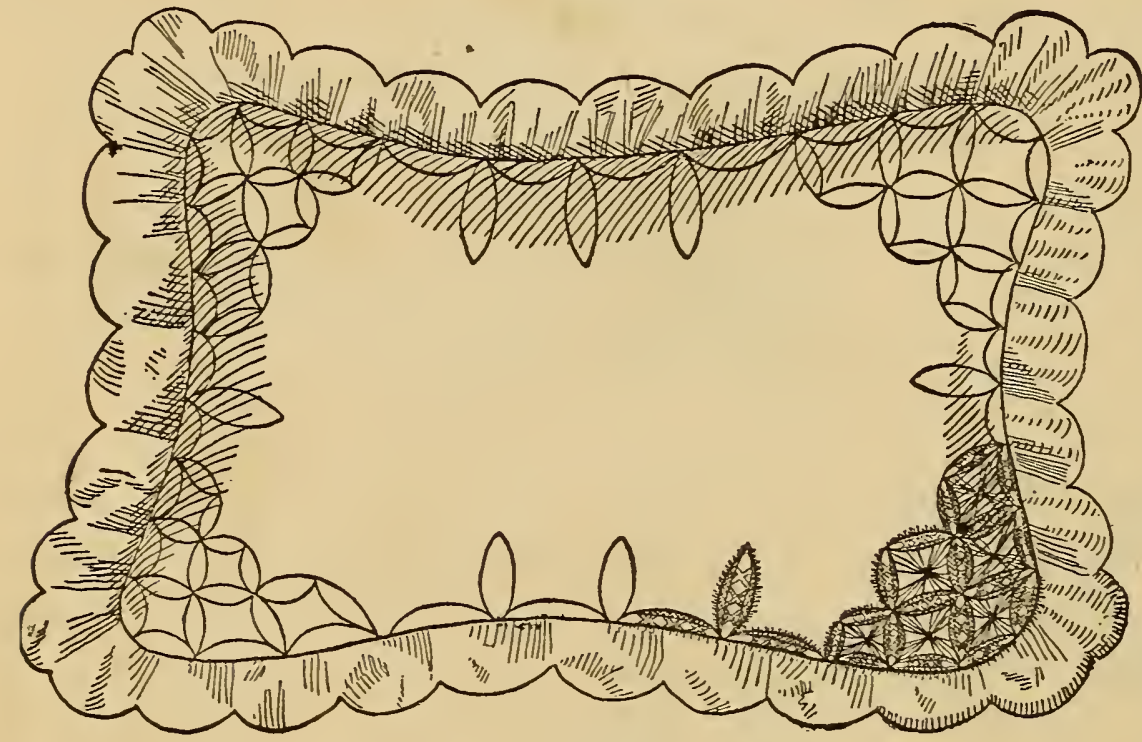

FIG. 5 . ideal Honiton, arranged first as four-pointed stars in the corner. Around the edges a row of the braid is placed with a single loop added between each loop of the border row. The space in the corner where the star shapes are joined should be filled either with spike stitches or spider web. In this case if the foundation is cut away from back of the corner the pillow should have an under lining of plain India or surah silk, or satteen would answer equally well.

We have given sufficient examples to show the beauty and utility of this braid work, and the ingenious worker will be enabled to arrange other designs. The amount of braid required for the various designs shown here will vary from three to six yards. 


\section{INFANT'S CRIB COVER AND PILLOW.}

Dainty crib covers for the wee mites are always in order and will find a ready sale at bazaars or in art parlors, and enterprising dealers can make no mistake in having a few constantly on hand, ready stamped and a portion of the work commenced. These articles all ready embroidered will not be apt to find so quick a market for mothers usually prefer to make these dainty accessories of baby's trosseau with their own hands. The design here given is a Louis XIV and is to be carried out either on fine white lawn or china linen, fine as cob web and soft as silk. The designs of forget-me-nots arranged in wreaths, looped with ribbon, are worked out with one strand of filo floss, in two shades of old blue, in satin stitch, with a tiny seed stitch of yellow floss in the center of each flower. Work

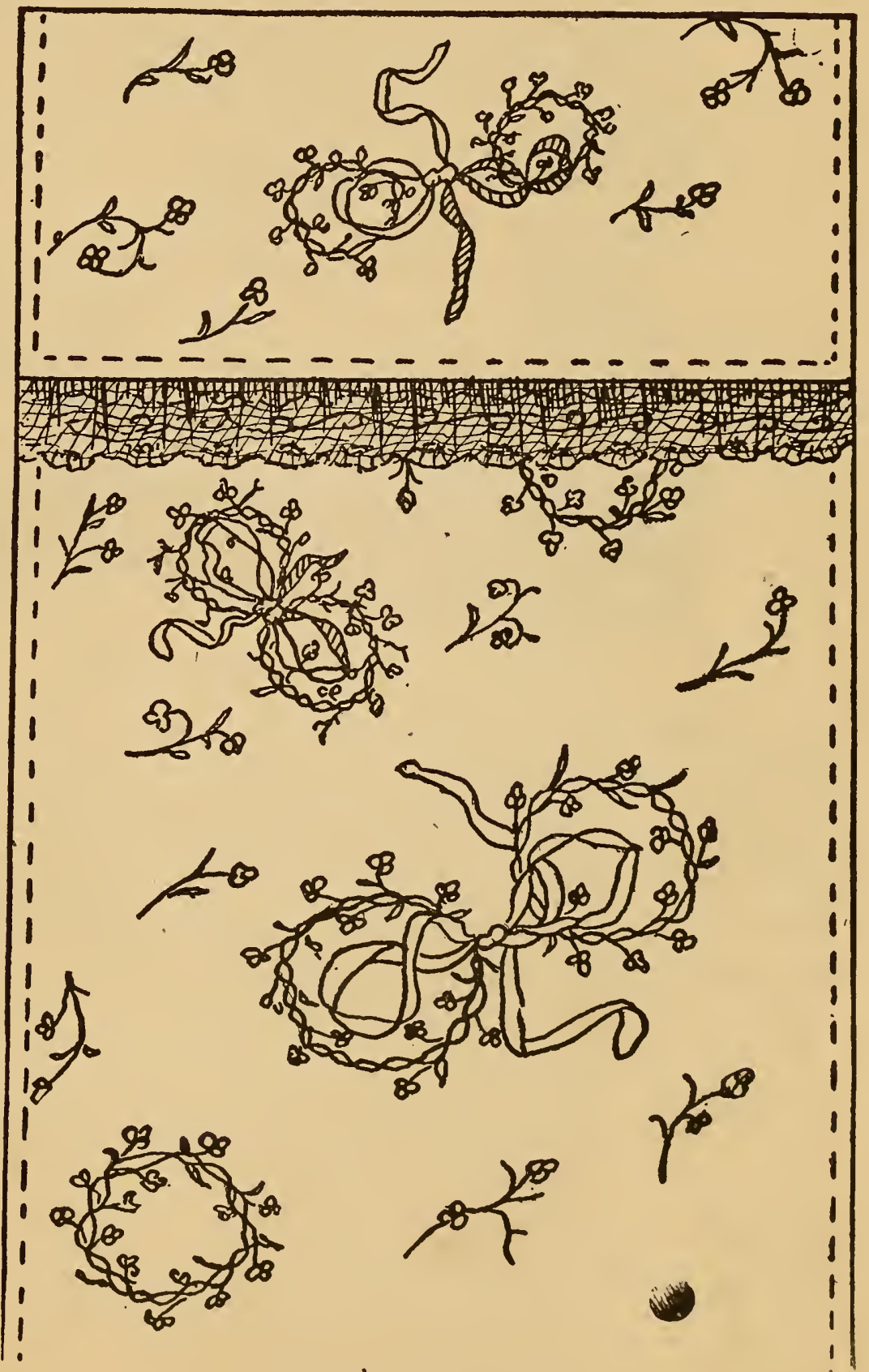

INFANT'S CRIB COVER.

out the stems and foliage in outline and satin stitch in two shades of light olive green filo floss. The ribbon bows and loops work in outline stitch 
with palest yellow filo floss. The cover should be 44 inches long by 36 inches wide. The upper portion should be folded over to a depth of 12 inches to form a sham, and edged with fine white lace. The sides and

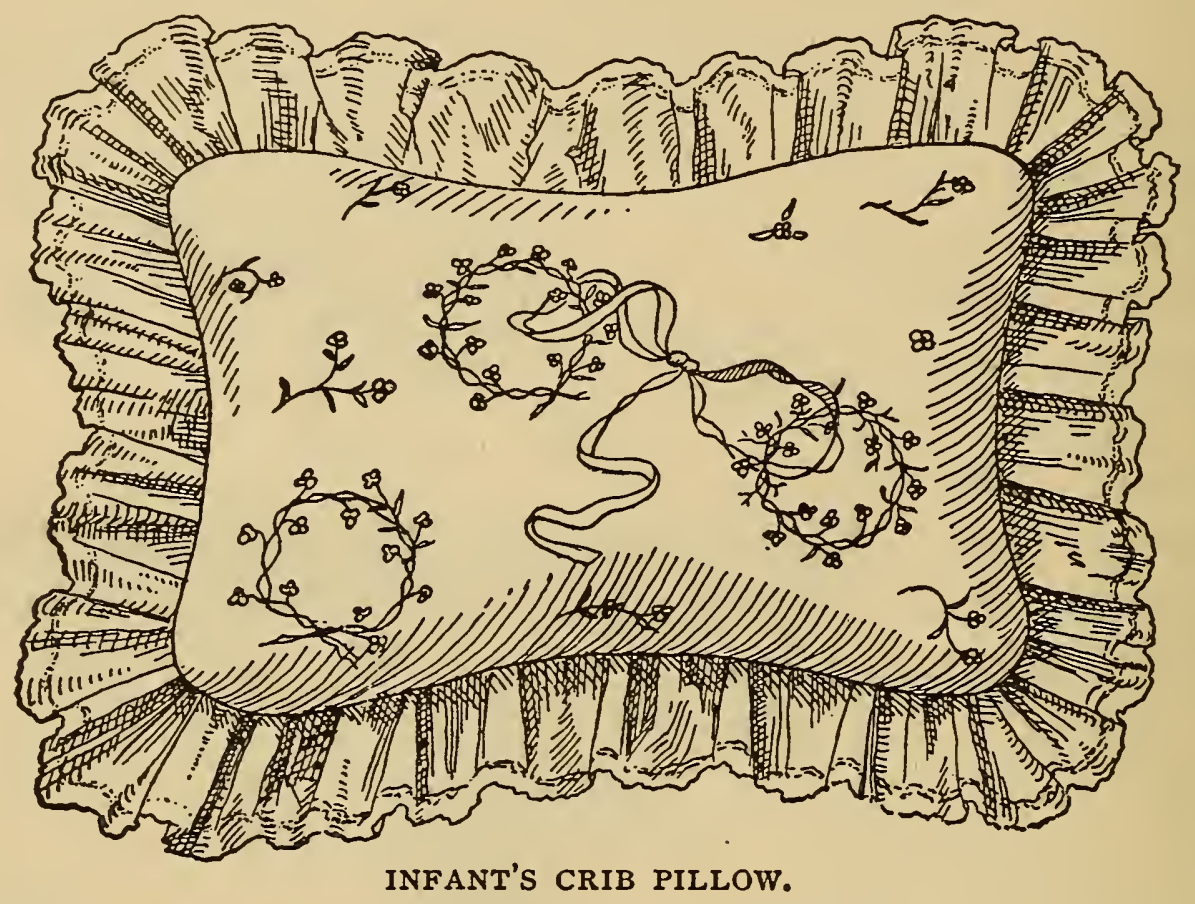

bottom edge are to be finished with a hem-stitched hem. This is a specially choice design. The little pillow completes the crib set. The pillow slip in model is made of the same material as the crib cover. The same design is carried out upon it in exactly the same manner. The frill is three inches deep, very full and finished at the edge with small scallops worked in close and heavy buttonhole stitch. 


\section{TABLE OF CONTENTS.}

\section{CHAPTER I.}

Introduction-Harmony of Interior Decorations-Beautiful Surroundings-Economy in Decorating-Dainty Linen-Appropriate Ornamentation-Use of Homely Materials in Decorative Work...............6

CHAPTER II.

Materials for Decorative Purposes-Linen Crash-Bleached LinensDomestic Linen-Bolton Sheeting-Colored Art Linen-Huck-a-buckArt Satin-Butcher's Linen-Scrim-Morris Cloth-Frieze-Concordia Canvas-Egyptian Cloth-Turkish Crepe-Satin Damask-Gobelin ClothHollywood Drapery-Russian Crash-Devonshire Art Cloth-Bargarren Art Cloth-Bolting Silk-Silks-Velvets and Plushes-Felt-Satin-Banner Silk-Sateen-Russian Tapestry-Mail Cloth-India Silk-Double

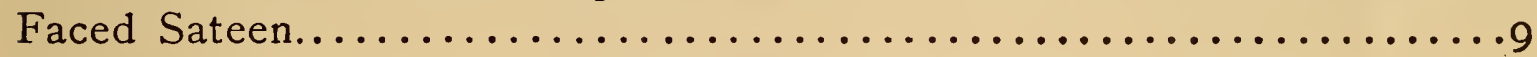

CHAPTER III.

Implements and Embroidery Threads-Embroidery Frames Embroidery Hoops-Needles-Scissors-Stilletto-Embroidery ApronPreparing Material for Embroidery-Placing Material in Hoops or Frames -Crewels-Tapestry Wool-Arrasene-Chenilles-Smyrnasene-Ribbosene-Featherasene-Embroidery Silk-Embroidery Linen...........

\section{CHAPTER IV}

Transferring and Stamping Designs-Tracing Paper-How to usePerforated Stamping Pattern-How to make-How to use-Care of Patterns - How to Clean-Stamping Liquids-How to make-How to use-Pantagraph for Enlarging or Reducing Designs-Conventional Designs......2 I

CHAPTER V.

Embroidery Stitches-Stem or Outline Stitches-Kensington StitchShort and Long Stitch-Satin Stitch-Buttonhole Stitch-Spot StitchAntique Stitch--Persian Stitch-French Knot-Couching Stitches-Hemstitching--Spanish Laid Work--Plush Stitch-Filling Stitches--Honeycomb Stitch-Brick Stitch-Novelty Stitches-Daisy-Star-DarningCrowsfeet-Group-Janina-Double Basket-Single Basket-Close Basket -D'Alencon Bar-Spider Wheel-Point d'Reprise-Rosette.........27 


\section{TABLE OF CONTENTS.}

CHAPTER VI.

Application of Stitches-Darning Stitch for Background-Dado or Frieze-Conventional Design Worked with Filling Stitch-Couching and Satin Stitch Combined-Infant's Trosseaux-French Embroidery-Kensington and Darning Combined-Combination of Spot and Single Daisy Loop-Leaf Border-Grape Vine Border-Application of Kensington Stitch-Short and Long Stitch-Shading Conventional Designs........49

CHAPTER VII.

Shading-System of Shading-Selecting Shades of Embroidery Threads-Table of Colors-How to Purchase-How to Use-Proper Method Shading Specifically Applied-Natural Shading-Conventional Shading-Shading of Stalks, Leaves, Stems and Petals-Where to Put High Lights-Where to Place Shadows-How to Blend Different Shades.

CHAPTER VIII.

Art or Flat Embroidery-Classification of Embroideries-Roman Embroidery-Applique Embroidery-Outline-Wash Fabrics Embroidered-Table Cloths-Lunch Sets-Side Board Scarfs-Doyleys-Carving Cloths-Vegetable Mats-Tray Cloths-Pillow Shams-Scarfs-Bedspreads-Bureau and Commode Scarfs-Splashers-Tidies-Toilet SetsStitches to be Used-Materials to be Employed-Suitable Designs-Articles for Use in Sleeping Room--Pansy Lunch Set-Scroll Border for Center Cloth-Round Cushion-Table Cover-Curtains and Portieres-Screens -Embroideries for Ladies Dresses, Sacques, Collars, Handkerchiefs, Fans, Parasols, Ruffles, Etc._Embroideries for Children's Wardrobe........67

CHAPTER IX.

Church and Bullion Embroideries-Materials for-Stitches Used in Ecclesiastical Embroideries-Couching Stitches-Diamond DraperyPlain Shell-Spider-Vandyke-Wheel-Wavy-Manner of WorkingColors to be used in Ecclesiastical Embroideries-Designs for Gold and Silver Work-Cardboard Foundations...................... I 5

\section{CHAPTER X.}

Berlin Embroidery-Uses of Stitches for-Materials for Berlin WorkDesigns-Colors-Blending of Shades-Combination of Stitches.......139 CHAPTER XI.

Raised Embroidery-Ribbon Work-Louis XVI Designs-Single Ribbon Roses-Double Ribbon Roses-Calla Lily-Tiger and Japan Lilies- 


\section{TABLE OF CONTENTS.}

Water Lilies-Golden Rod-Cattail-Coxcomb-Sumac-Thistles-Snow Balls-Pansies-Raised Fruit-Cherries-Oranges-Strawberries-Grapes -Cotton Plant-Designs and Instructions................... I 49

CHAPTER XII.

Dainty Trifles-How to Make-What to Use-Economic DecorationArticles for Church Fairs and Bazaar Sales-Memorandum Case-Bouquet Holder-Writing Board for Invalids-Foot Stool-Paper Stand-Shoe Box Sunflower Cushion-Work Basket-Hair Pin Holder-Toilet CushionChair Back and Seat-Lamp Shade-Wall Pocket-Bolster and PillowHandkerchief Holder-Photograph Case-Calendar-Night Dress SachetPansy Sachet-Puff Cushion for Head Rest_-Decorated Egg Shell-Mending Bag-Holders-Paper Holder-Handkerchief Sachet-Fancy TablePenwipe-Sofa Cushion-Fancy Work Basket-Foot Rest-Ottoman Cushion-Shaving Paper Case-Laundry Bag-Pumpkin Cushion-Photograph Frame-Photograph Envelope-Pin Trays-Glass Handkerchief Box Eiffel Tower Scrap Bag-Star Quilt-Handy Bag-Toilet Cushion-Emery Cushion-Butterflies-Music Stand-Scissors Case-Crawling Rugs-For the Drawing Room-Doyleys-Mats--Center Cloths-Table Runners-Shoe Box-Burnt Match Holder-Toilet Cushion-Footstool-Child's ChairCatch-all-Head Rest-Hanging Pin Cushion-Fancy Pin CushionsInfant's Crib Coverlet-Table Cover in Cross Stitch-Chicken PenwipeBranch of Fruit-Dust Cap-Sachet Bags-Tobacco Pouches-Leaf Penwipes-Traveling Case-Child's Chair-Mat for Water Pitcher-Needle Case-Cover for Cushion-Nasturtiums-Cushion-Infant's Toilet CaseImportance of a Scrap Bag............................ 75

CHAPTER XIII.

Drawn Work-Materials for-Stitches for-Frames for-Uses ofDesigns for-Corners-Borders-Set Patterns-Wheels-Scrolls-Squares -Preparing the Work-Foundation Stitches-Drawing Threads-Basket Insertion-Lunch Cloth-Center Cloth with Border-Antique Corner and Border-Combination of Drawn Work and Embroidery............24 I

\section{CHAPTER XIV.}

Brush and Palette-Designs for Painting-Painting in Oils on Fabrics - Materials for Painting-To Make Blues-To Make Browns, Buffs and Yellows-To Make Greys-To Paint Daisies, Honeysuckle, Apple, Peach Blossoms, Tea Roses, Feathery Clematis, Violets, White and Purple Lilacs, Pansies, etc.-Palettes for-Morning Glories-Sky Effects-Back GroundsSpecial Palettes-Strawberry Spray-Butterflies-Grapes on Black Velvet 


\section{TABLE OF CONTENTS.}

-Pansies-Purple Thistles-Pond Lilies-Purple Clematis-Oxhart Cherries-Leaves and Berries of Virginia Creeper-Peacocks-Golden Rod Asters-Panel of Hollyhocks-Washable Painting-Medium-How to Use -Water Color Painting-Roses-White Flowers-The Primrose-Blue Flowers-The Convolvulus-Figures in Water Colors-Fabric TintingParis Tinting-Painting on Celluloid or Ivorine-Lilacs in Oil on Celluloid -Lilacs in Water Colors on Celluloid-Luster Painting-Materials forBrushes for-Medium for-Colors for-Method of Using-Kensington Painting-Parrots-Crayon Painting-Portraits-Painting on Birch BarkScorch Painting-Tapestry Painting-Canvas for-Dyes-Brushes-Mixing Colors-Flesh Colors-Flesh Painting-Palette for Sky-Colors for Draperies-Hair and Drapery-Decorative Painting for Profit..........257

\section{CHAPTER XV.}

Point Lace-Honiton Lace-Novelty Lace-Point Lace Braid-Lace Thread-Lace Stitches-Tracing Cloth-Foundation Cloth-or LeatherDesigns for Lace Work-Outlining Design with Braid-Method WorkingLaying Braid-Stitches-Proper or Point-Finishing Edge-Connecting Bars-Wheels-Rosettes-Ground Stitches-Brussels Point-Venice Point -Little Venice Point-Spanish Point-Grecian Point-Valenciennes Stitch -Point d'Alencon-Turkish Point-Net Groundwork Stitch-Mechlin Lace Stitch-Venetian Bars-Dotted Venice Point-Raleigh Bars-Point Lace-Handkerchief-Fischu-Border-Cushion - Cover-Handkerchief

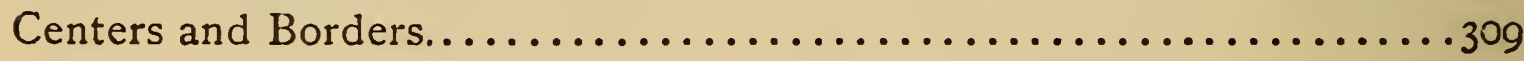

\section{CHAPTER XVI.}

Knitting and Crocheting-Terms Used in Crocheting-Explanation of Abbreviations Used-Child's Dress-Purse-Infant's Sacque-Baby's Shirt -Lady's Vest_Ladies' Basket Pattern Vest-Baby's Hood-Fancy Bag Fringe-Pine Apple Edge-Insertion-Border Crochet-Trimming Crochet -Design for Bed Spread-Wheels for ToiletSet-Edging-Diamond Edge -Fern Edge-Crochet Trimming-Narrow Edging-Broad EdgingCoral Edging-Reticella Crochet-Table Cover-Child's Petticoat-Cro chet Towel-Daisy Crochet Lace-Crocheted Guimpure Lace-Crocheted Table Cover-Skirt Edge, Crochet and Braid-Infant's Knitted JacketMoss Edge-Fern Edge-French Edge-Serpentine Edge-Crochet and Fancy Braid-Shell Edge-Palm Edge-Knitted Hood for Child-Crocheted Hood for Child-Crocheted Pillow Shams-Table Mats-Border to Mat-Crocheted Doll-Torchon Lace-Comfort for Lounge-Porcupine 
TABLE OF CONTENTS.

Stitch--Wheels-Petticoat For Child-Border and Corners for Table Cover-Crocheted Skirt-Infant's Vest-Crocheted Mop Duster-Cro-

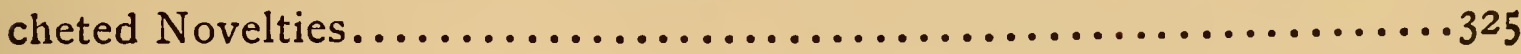

CHAPTER XVII.

Dainty Work for Profit-Work for the Stay-at-homes-The First Start - Commenced Samples-Cost of-Materials for-Designs for Order Work-How to Solicit-Orders for Embroidery-The first Days' WorkTeaching Embroidery-What to Charge-How to Collect-How to Open an Art Bazaar or Fancy Goods Store-How to Conduct-Selection of Location-Store Fittings-How to divide Store-The Work Room-The Show Window-How to Dress-What Stock to Carry-How to Buy-Art Fabrics-Sample and Display Work-List of Desirable Samples-Materials for Made-up Samples-Cost of Commenced Samples-Window DisplayStock-Cost of-Quantity Necessary-How to Buy and Sell NoveltiesDescription of Articles for Stock-Toilet Set-Glasseine Handkerchief Sachet-Glass Panel Screen-Tobacco Pouch-Glasseine Toilet SetParasol Catch-all-Traveling Roll-Duster Case-Calendar.........400

\section{CHAPTER XVIII.}

Description of Full Page Illustrations-Cushions-Head Rests--Roman Embroidery-Star Quilt_-Drapes-Doyleys-Carafe Mat-Table CoversEasel Drapes-Portieres-Mantel Drapes-Window Drape-Magnolia Doyley-Maple Leaf Doyley-Dresden Plate Doyley-Draped BedsColored Plates-Fleur de lis-Poppies-Oranges-Oak Leaves-SumacGolden Rod......................................426

\section{CHAPTER XIX.}

Doyley Designs-Five o'clock Tea Cloths-Screens-Butterfly Center Cloth-Louis XIV Design-Violet Center Cloth-Bed Spreads-Rose Center Cloths-Flower Doyleys-Violet Basket Center Cloth-Sponge Bags-Traveling Cases-Picture Frames-Handkerchief Bag-Glove and Veil Case-Infant's Flannel Robe-Hammock Cushion-Ideal Honiton Lace Work-Dresden Crib Cover and Pillow...............435-458 



\section{ILLUSTRATIONS.}

Devonshire cloth. $\ldots \ldots \ldots \ldots \ldots \ldots \ldots$
Embroidery frame. $\ldots \ldots \ldots \ldots \ldots \ldots \ldots$

Embroidery hoop................ 4- 47

Embroidery scissors............... 5- 18

Work apron................... 6- 18

Pantagraph.................... $7-23$

Stem stitch................... 8- 88

Three outline stitches............. 9- 28

Fancy chain.................... 10-20

Loop outline.................. 11-29

Kensington stitch, detail........... 12-30

Kensington stitch................ 13-30

short and long stitch............. 14- 31

Satin stitch, detail............... 15- 32

Satin stitch.................. 16- 32

Satin stitch.................. 17- 32

Buttonhole stitch................. 18- 33

Buttonhole edge............... . 19- 33

Buttonhole and spot stitch. ......... 20-33

Antique embroidery.............. 21- 34

Persian embroidery.............. 22- 34

French knot.................... 23- 34

Couching outline................24- 35

Couching groups................. 25- 35

Hem stitching................. 26- 36

Spanish laid work............... 27-37

Diamond couching............... 28- 38

Fancy couching ............... 29- 38

Fancy couching.................. 30-38

Brick stitch.................. $31-40$

Honey combstitch............... 32- 40

Honey comb applied............. . 33- 40

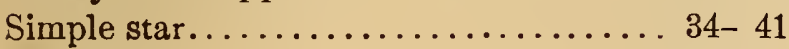

Daisy star................... 35- 41

Crows' feet. ................... 36- 41

Grouped star stitch.............. '37- 41

Grouped star stitch No. 2.......... 38-41

Darning stitch. ................. 39- 42

Cushion........................ 40-42

Janina stitch.................... 41- 42

Janina stitch close................ 42- 43

Single basket stitch............. 43-44

Double and close basket stitch ....... 44- 44

D'Alencon bars................. 45-45

Spider wheel.................. 46-46
Grape vine applique..... . . . . . FIG. $51-58$

Design in Kensington stitch......... 52-54

Flower head rest................ 53-55

Branch of cherries, filling stitches..... 5 54- 56

Poppy correctly shaded No. 1........ 55-63

Popry correctly shaded No. 2......... 56-63

Poppy correctly shaded No. 3........ 57-64

Roman embroidery design........... 58-69

detail........... $59-69$

Lunch cloth....................61- 74

Napkin ...................... 62- 75

Doyley...................... 63-75

Carving cloth.................. 64- 75

Tray cloth $\ldots \ldots \ldots \ldots \ldots \ldots \ldots \ldots \ldots 66-76$

Daisy center cloth ..............66- 77

Geranium center cloth .............67-78

doyley ..............6 68-78

Cruel mat, leaf shape ............6 69-79

Set vegetable mats............... $70-79$

Coffee cozy.................... $71-80$

Caraffe mat ................... 72- 80

Sideboard scarf................. 73-81

Dresser scarf, Roman emb. design..... 74- 83

Toilet bottle.................. 75- 84

$76-84$

Toilet cushions................. $77-84$

Poppy for pillow sham ........... 78- 85

$79-85$

Leaf for pillow shams............. 80-86

Splashers ...................... 81-87

Leaf-bordered lunch cloth .......... 82-88

Dresser scarfs ................. 83- 89

$84-90$

Clover doyley ................. 85- 91

Snowdrop doyley $\ldots . \ldots . \ldots . \ldots . . .86-92$

Dresden doyleys... ............. 87-94

Grape vine center cloth ............. 88- 96

Cherry center cloth .............. 89- 98

Scroll border .................... 90-98

Floral center cloth................ 91-99

Dresden book cover .............. 92-100

Lonventional book cover........... 93-100

Round cushion ................ 94-101

Cross stitch design.............. 95-102

Table cover.................... 96-103

Stand cover ................... 87-104 
Roset:e stitch $\ldots . \ldots \ldots \ldots \ldots+48$

Spot and daisy loop............. 49-51

Combination leaf border............ 50-52

Elandkerchief corner ...............102-109

Butterfly design ................. 103-109

Edge for collar and cuffs ...........104-110

Scallop edge...................105-111

Corner of scallop .................106-111

Daisy design .................107-111

Scallop corner ..................108-112

Deep scallop .....................109-112

Flannel scallop..................110-112

Infant's shawl. ..................111-113

Cross for altar front...............112-116

Jeweled border ...................113-118

Raised basket couching ............. 114-119

Raised basket couching .............115-119

Wavy couching.................116-120

Battlemented couching ............117-120

Brick flat couching ..............118-120

Plain brick couching ............119-121

Broad couching ................120-121

Diamond couching ...............12I-121

Plain flat couching...............122-121

Plain flat couching.............. .123-122

Bullion knot. ..................124-122

Detail of couched design...........125-123

Detail of couched design............126-124

Detail of long stitch design...........127-125

Conventional fleur de lis.............128-126

Design for chausable............. . 129-127

Ecclesiastical design..............130-128

Triangle for ecclesiastical work........131-130

Circles for ecclesiastical work..........132-130

Latin cross and anchor work. ..........133-131

Greek cross.....................134-121

Bullion monograms................135-132

Letters... ...................136-132

Banner.........................137-133

Border, jeweled.................138-133

Easter banner....................139-134

Easter banner.......................140-135

Bleeding heart, The...............141-136

Passion flowers. . ................ 142-137

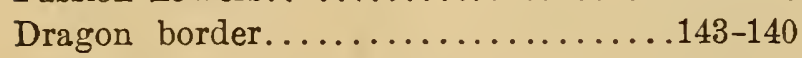

Easy chair.................... 144-141

Cross stitch design................145-142

Cross stitch design................146-143

Long cross stitch.................. 147-143

Persian cross...................148-144

German stitch....................149-144

Tapestry stitch................ 150-144

Double Leviathan stitch.... . . . . . 1F1-145
IIG. FY.

Darning stitch ............... . 9s-104

Darned background.............. 99-105

Scre $n s$................... .100-106

Applique on net................101-108

Trent stitch.....................152-146

Satin stitch................... 153-147

Cross and long stitch..............154-147

Raised Calla lil $5 . . . . . . . . . . . . . .155-159$

Raised Japan lily...................156-160

Raised coxcomb and sumac............157-164

Rais d this"les and golden rod.........158-166

Flat thistles for cover...............159-166

Raised snow balls..................160-167

Raised cotton plant................161-171

Pattern for cotton plant.............162-172

Memorandum card case .............163-176

Bouquet holder...................164-177

Invalid's writing board...............165-178

Foot stool.......................166-178

i pper stand....................167-179

Pattern for decoration of shoe box.......168-180

Covered shoe box...................169-180

Sunflower pin cushion................170-181

Folding work basket..............171-182

Hair-pin holder. . . . . . . . . . . . . . 172-182

Puff toilet cushion. .................. 1 ' $3-183$

Round toilet cushion...............174-183

Chair seat... . ..................175-184

Chair back. .....................176-184

Lamp shade. . . . . . . . . . . . . . . . . 177-185

Wall pocket......................178-185

Bolster, covered.....................179-186

Infant's pillow. .................180-186

Detail of handkerchief case...........18i-187

Handkerchief case. . . . . . . . . . . . . 182-187

Double photograph case.............183-188

Single photograph case..............184-188

Night-dress sachet.................185-189

Pansy sachet.....................186-189.

Puff head.rest....................... 187-190

Decorated egg.shell............... 188-190

Pansy sachet.....................189-191

Mending bag.......................190-191

Ornamental holders.................191-192

Paper holders..................... 192-193

Handkerchief sachets...............193-194

Fancy table.....................194-194

Sunflower pen-wipe............... 195-195

Sofa cushion......................196-195

Linen covered cushion..............197-196

Forget-me-not toilet cushion........... 198-196

Fancy work basket. ............. 199-197

Spanish foot-rest..................200-197

Ottoman cushion................201-198 
FTG. PP.

uttoman cushion. ............... 202-198

Owl shaving case.................203-199

Laundry bag .................204-200

FIG. PP.

Prepared corner... .............253-242

Prepared corner ................ .254-242

Pumpkin toilet cushion.............205-201

Photograph frame................206-202

Photograph envelope..............207-203

Pin trays. ...................208-203

Glass handkerchief box............209-204

Eiffel Tower scrap bag.............210-205

Handy bag.....................211-207

Square toilet cushion............. 212-210

Emery cushion.................213-211

Artificial butterfles...............214-212

Music stand....................215-213

Scissors case ..................216-214

Crawling rug. ..................217-215

Rose doyley...................218-216

Magnolia doyley.................219-217

Center mat.....................220-218

Center piece....................221-219

Fruit plate doyley...............222-220

Shoe box....................223-221

Burnt match holder................224-222

Pilgrim toilet cushion..............225-223

New England footstool............ 226-223

Child's chair...................227-224

Catch-all......................228-224

Head rest.....................229-225

Hanging pin cushion..............230-226

Fruit pin cushion. ................231-226

Fruit pin cushion...............232-227

Infant's crib coverlet..............233-228

Cross stitch table cover...... .......234-228

Detail cross stitch................235-228

Chicken pen-wipe...............236-230

Branch of fruit.................237-231

Ladies' dust cap..................238-231

Sachet bags-crepe paper. ..........239-232

Tobacco pouch.................240-232

Tobacco pouch .................241-233

Leaf pen-wipes................242-233

Traveling case.................243-234

Child's chair..................244-234

Water lily caraffe mat.............245-235

Needle case ....................246-23ฮั

Needle case, closed................247-236

Pocket needle case.................248-236

Kensington cushion cover... ........249-237

Nasturtium cushion. . . . . . . . . . . 250-238

Infant's toilet case.............. 251-239

Four corners prepared........... . .255-243

Hemstitching. .................256-243

Duck's tail stitch ................257-244

Hemstitching, No. 2 . . . . . . . . . . . . . 258-244

Hemstitching, No. 3 . . . . . . . . . . . 2 259-244

Block insertion . . . . . . . . . . . . . . . .260-248

Detail a. b. of $260-248$

Insertion of border...............261-249

Detail a. b. c. d. e. f. of $261,250-251$

Cross bordtr...................262-250

Basket insertion ..............263-247

Lunch cloth with corner and border. . . .264-246

Details of 264-a. b. c. d. e. . . . . 246-247

Block border ..................265-252

Cut-in corner..................266-253

Pattern to fill cut-in corner..........267-246

All-over design for drawn work. ... . . 268-253

Detail of 268-253

Brussels point filling.............269-254

Antique border and corner..........270-255

Toilet set, cushion and botties.........271-261

Details of a. and b of $271-261$

Handkerchief sachet, glasseine. ........272-263

Glass panel screen..............273-273

Chamois tobacco pouch..............274-287

Glasseine toilet set. . . . . . . . . . . 275-290

Details of a. and b. of $275-290$

Parchment parasol catch...........276-293

Traveling bag................277-307

Duster case...................278-307

Calendar .....................279-308

Plate 1, . lace braid................ $\quad 310$

Plate 2 , lace stitches.............. $\quad 312$

Plate 3 , lace stitches............... $\quad 314$

Plate 4, lace stitches............. $\quad 316$

Handkerchief center............... 313

Handkerchief square............. 313

Lace collar ................... $\quad 319$

Lace insertion................. $\quad 320$

Detail of crocheted dress for child......280-328

Detail of crocheted dress f. $\mathrm{r}$ ( hild. . . . . 281-328

Detail ( f crocheted dress for child... . . .282-329

Croche ed dress for child . . . . . . . . .282-4-330

Kuitted p!rse...................285-330

Baby"s sacque..................286-331

Lady's vest. . ..................287-334

Baby's cricheted hood ............ 288-338 
FIG. PP.

Fancy spool bay.................289-339

Fringe......................290-340

Pine apple edge. ..................291-341

Insertion, crochet.... .............292-342

Border, crochet....... ............ 293-343

Trimming crochet.. ...............294-344

Square for counterpane..............298-345

Whecls, crochet..................296-347

Toilet mat....................297-348

Edging, crochet...................298-349

Diamond edging.................299-351

Fern edging.................... 300-353

Crochet trimming. ................301-354

Narrow edging, crochet.............302-355

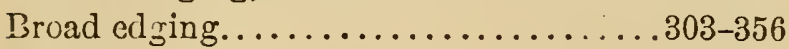

Narrow coral edging.............. . 304-357

Tab.e cuver crocheted insertion ........305-360

Reticule crochet..................306-358

Croc'eted petticoat, for child.........307-361

Crocheted petticoat, for child........ . 3 308-361

Crocheted petticoat, for child detail. . 309-362

Crochct tassels detail. ... . . . . . . . . . 310-363

Dilisy crochet lace ................311-365

Guipure crocheted lace.............312-366

Detail crocheted table cover..........313-368

Edge $f \circ r$ table cover. . . . . . . . . . . . . 314-369

Croch.t.d table cover..............315-370

Ladie s skirt. .................. $316-372$

Crochet braid trimming............317-373

Infunt's knitted jacket. ...........318-375

Moss edging. . .................. . . . .

Fern edging..................... $320-376$

French edging.................. $321-376$

Serpentine braid edge........... . .322-376

Fancy crotchet edge, No. 1..... . . . 325-377

Edging, No. 2.................... 324-377

Shell trimming................. 325-377

Palm irimming................... 326-378

Knitted hood....................327-379

Knitted hood, detail of... . . . . . . . . 328-380

Knitted hood detail of ... . . . . . .329-381

Knitted hood for child........... . . .330-381

Kuittcd hood, detail of . . . . . . . . . . . 331-382

Pillow sham, crocheted........... 332-383

Crocheted table mats.............. . 333-385

Crischeted wheels .............. . 334-390

Child's petticoat................ 335-392

Crocheted border................. . 336-393

Liidies crocheted skirt. ...... . . . . . . 337-394

Infart's knitted bodice............. 338-396

Crocheted dust mop.......... . .. 339-397

Group of head rests............... $\quad 58$

Roman embroidery............. $\quad 70$
Corner mantel................... 114

Table doyley and drape............. 138

Picture and easel drape............ 141

Bedspreads and pillow shams......... 148

Louis XIV design................ 150

Cover for library table............. $\quad 154$

Calla lily working design............ 158

Caraffe mat.................... 174

Point lace braids, plate $1 \ldots \ldots \ldots \ldots \ldots . \quad 210$

Point lace stitches, plate $2 \ldots \ldots \ldots \ldots . \quad 212$

Point lace stitches, plate $3 \ldots \ldots \ldots \ldots \ldots . \quad 214$

Point lace stitches, plate $4 \ldots \ldots \ldots \ldots . . .216$

Drawn work.................. $\quad 246$

Drawn work.................. 247

Window drapery................ 294

Portierres...................... 298

Mantel drapery................. 302

Easel drape................... 306

Maple leaf doyley................ 322

Magnolia doyley................ $\quad 324$

Dresden doyley................... $\quad 430$

Fancy cup board............................ 490

Four doyley designs............................. 435

Five-o'clock tea cloth .................... 436

'Three-panel screen,....................... 437

Butterfly center cloth.................... 438

Louis XIV. center cloth design........ 439

Violet center cloth........................ 440

Bed spreads................................. 441

Bed spreads................................. 442

Rose center cloth ......................... 443

Buttercup, daisy, violet, and forgetme-not doyleys.......................... $\quad 444$

Violet basket center cloth.............. 445

Sponge bags................................. 446

Traveling case.............................. 447

Picture Frames ............................ 448

Handkerchief bag........................... 449

Glove and veil case...................... 450

Infant's flannel robe...................... 451

Hammock cushion....................... $\quad 452$

Ideal Honiton center cloth............... $\quad 453$

Plate doyley............................... 454

Cup doyley and center cloth............ $\quad 455$

Crib pillow.................................. 456

Crib cover................................... 457

Infant's Dresden pillow.................... $\quad 458$

Fleur de lis COLORED PLATES.

Oranges.

Poppies..

Oak leavis suma................ 


\section{INDEX.}

fntroduction.................. MATERIALs.

Linen crash..................

Bleached linen.................

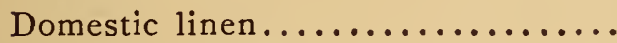

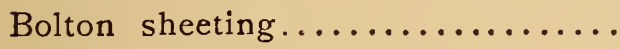

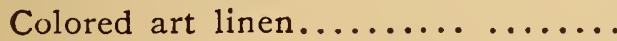

Huck-a-buck ...................

Linen Bolton sheeting.............

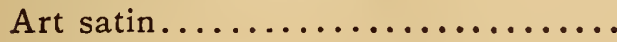

Butcher's linen...............

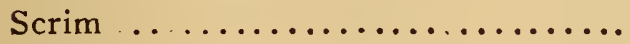

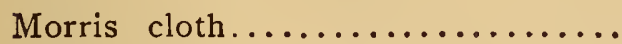

Frieze........................

Concordia canvas ...............

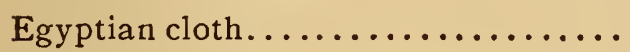

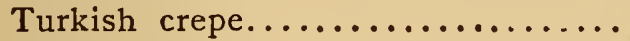

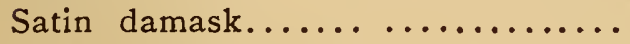

Gobelin cloth...................

Russian crash...................

Hollywood drapery..............

Devonshire art cloth.............

Bolting silk...................

Silks.....................

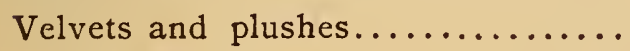

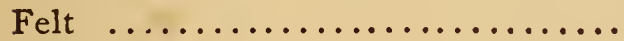

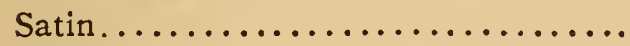

Banner Silk

Upholsterer's plush and satin........

Sateen........................

Russian tapestry...............

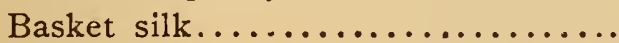

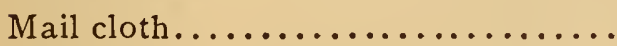

India Silk

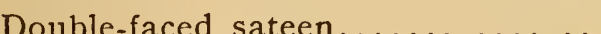

IMPLEMENTS AND EMBROIDERY THREADS

Crewels

Zephyr.

Tapestry wool

Arrasenes

Chenille.
PAGE.

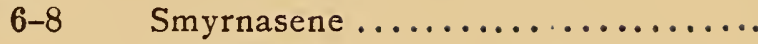

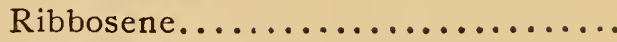

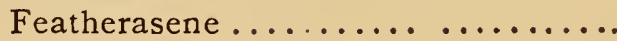

Embroidery cotton..............

Embroidery wash silks... ........

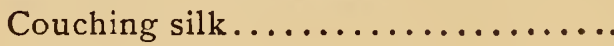

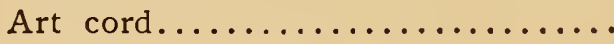

Real Scotch threads .............

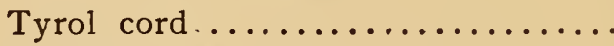

German cord................ $15-26$

Transferring and Stamping Designs. 21

Tracing paper ................ 22

Perforated patterns ........... 23

Materials for stamping........... 24

Directions for stamping.......... 24

Conventional Designs............ 25

Embroidery Stitches............. 27

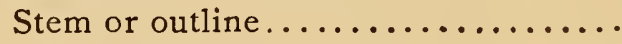

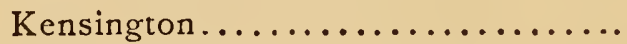

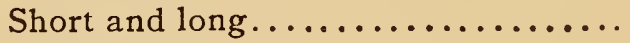

Satin.

Buttonhole...................

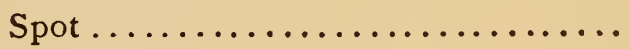

Antique.....................

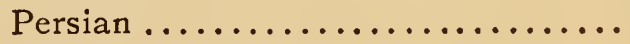

French knot.................

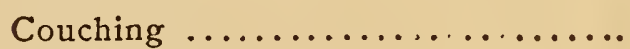

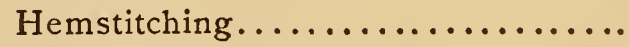

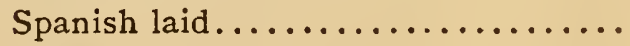

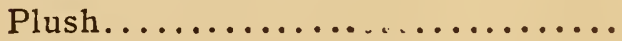

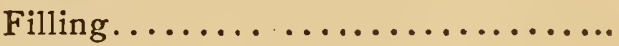

Honeycomb...............

Brick .....................

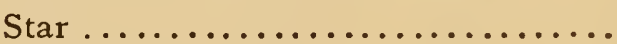

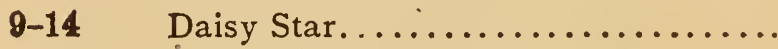

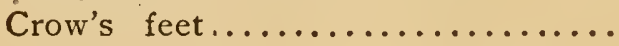

Grouped star..................

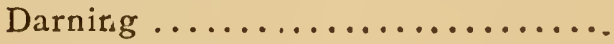

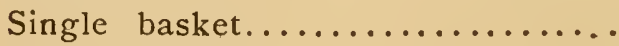

Double basket .................

Close basket...................
PAGR.

27


D’álencon bars.

Spider wheel...

Point de reprise.

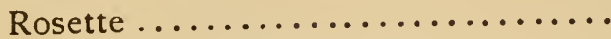

ApPlication of STITCHES............

Combination of spot and single Daisy

Loop.........................

Leafi border....................

Grapevine, applique..............

Kensington stitch applied..........

Short and long stitch.............

Designs, conventional shading......

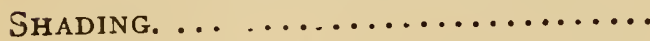

Selecting shades.................

Table of colors .................

Proper method of shading... ......

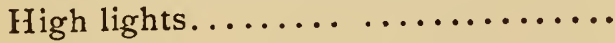

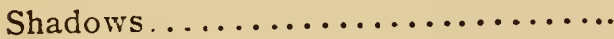

Conventional shading.............

Natural shading.................

Massing color for effect............

Art or Flat EMbroidery............

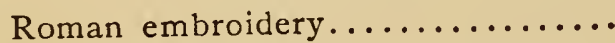

Practical embroidery...............

Use simple designs and stitches......

List of table linen...............

Table cloth...................

Lunch cloth...................

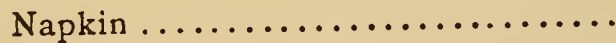

Doyley ........................

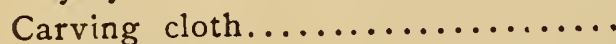

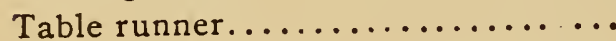

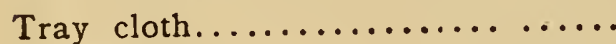

Center cloth.....................

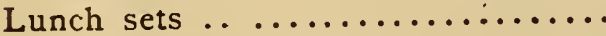

Cruet mats....................

Vegetable mats... ..............

Tea or coffee cozey..............

Mat for water service.............

Sideboard scarf.................

Bedroom furnishings. . ...........

ARTICLES FOR USE IN SLEEPING ROOMS.

Pillow shams................

Pillow scarfs...$\ldots \ldots \ldots \ldots \ldots \ldots$.

Commode scarf..................

Splashers...$\ldots \ldots \ldots \ldots \ldots \ldots$

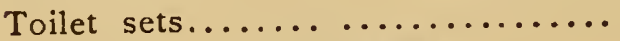

Combination embroideries..........

Scarf three....................

PAGE.

Daisy scarf

$27-48$

49

51

52

53

54

$54-55$

$56-57$

$27-48$
49
51
52
53
54
$54-55$
$56-57$

Doyleys, Clover

\section{Fern.}

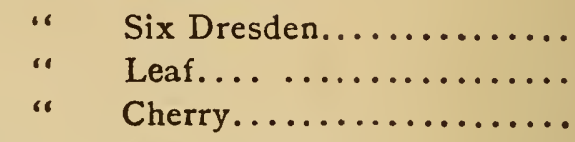

Scroll-bordered center cloth

Floral center cloth.

Book covers.

Dresden...

Conventional

Round cushions

Table covers, cross stitch

Table covers, darned.

Curtains and portieres.

Screens

Applique embroideries.

EMBROIDERED ARTICLES OF DRESS.

Handkerchiefs, corner.

Parasol.

Dresses.

Collar and cuffs

Dressing jacket.

Corner crepe lise ruffle.

Edge of scallops for crepe lise ruffle.

Embroideries for children's Wardrobe.

Scallop for Flannel.

Infant's shawl...

Skirts.

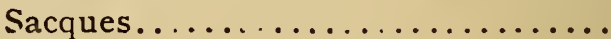

Church and Bullion Embroideries.

Materials:

Gold thread.

Passing.

Filo floss

Purse silk.

Knitting silk

Sewing silk...

Silver thread.

Art cords and spangles.

Frames.

Needles.

$115-117$

STITChes for ECClesiastical Work.

Couching:

Basket

Battlemented

Brick.

Broad.

Diagonal. 
Diamond

Diaper.......................

Plain.

Shell.

Spider.

Vandyke.

Wheel

Wavy

Long. .

Bullion knot.

Chain.

Outline.

Method of using stitches.

Conventional fleur de lis.

Method of working.

Chasuble.

Altar frontal, method of working.

Cardboard foundations.

Mottoes.

Jeweled borders.

Bullion embroidery.

Passion flower.

Colors for Ecclesiastical work.

The bleeding heart.................

BERLIN EMBROIDERY.

Method of working.

Materials

Furniture covers.

Canvas

Stitches.

Back.........................

Cross..........................

Long cross.

Persian cross.

Cushion

Damask.

German.

Tapestry.

Irish

Leviathan

Double Leviathan

Treble Leviathan

Long .

Tent.

Raised.

Satin.

Dragon border.

$143-147$

140

RAISED EMBROIDERY.

149-151

Ribbon work....

117-123

118-126

126

126--127

127

127-128

130-133

131--134

133-134

134-137

136-137

133

136

139

139-147

140-142

141-142

142
PAGE.

(1)

Chan

Louis XVI. design............... ${ }_{151-152}^{\text {PAGR. }}$

Single ribbon roses............. 152-15€

Double ribbon roses............ 156-157

Calla Lily................... 157-160

Tiger and Japan lilies............ 160-161

Water lily.................. 161-162

Golden $\operatorname{rod} . \ldots \ldots \ldots \ldots \ldots \ldots \ldots \ldots$ 162-163

Cattail..................... 163-164

Coxcomb.................... 164-165

Sumac...................... 165

Thistles..................... 165-167

Snowballs.......................167-169

Pansies........................168-168

Fruit....................... 169

Cherries...................... 169

Oranges........................169-176

Strawberries................... 170

Grapes.......................170-171

Cotton plant..................170-172

Designs and Instructions............172-173

Dainty trifles......................... 175

Memorandum card case..............176

Hanging bouquet holders............177

Writing board for invalids...........177-178

Foot stool.....................178-159

Paper stand...................179

Shoe box..................... 180

Sunflower cushion.............. 180-181

Work basket....................181-182

Hair pin holder. . . . . . . . . . . . . . 182-183

Small cushions..... .............188-184

Chair back and seat.................184

Lamp shade......................185

Wall pocket................... . 185-186

Bolster and pillow...............186

Handkerchief holder...............186-187

Photograph or calendar case......... 188-189

Night dress satchet................189

Pansy satchet.................. 189-190

Puff Cushion.....................190

Decorated Eggshell................190

Pansy Satchet....................191

Mending bag.....................192-193

Holders.........................193

Paper holders.....................194

Handkerchief holder................194

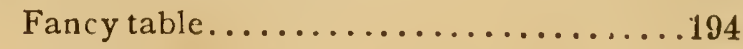

Sunflower pen wipe................ 195

151 Sofacushions................195-196 
vIII.

INDEX.

Fancy work basiet...............196-197

Foot cushion .......................

Ottoman cushion. .................199-199

Shaving paper case...............199

Laundry bag...................200

Pumpkin cushion.................201

Photograph frame.. ..............202

Photograph envelope.............203

Pin trays ....................203-204

Glass handkerchief box.............204

Eiffel tower scrap bag. . . . . . . . . . . 205-206

Star quilt. ....................206-209

Handy bag....................... 209-210

Toilet cushion..................210

Emery cushion...................211

Butterflies.....................211-212

Music stand .....................212-213

Scissors case...................214

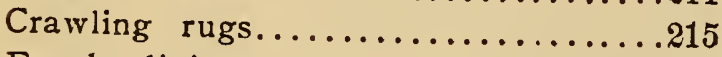

For the dining room.............216-220

Shoe box .. ...................221

Burnt match holder...............222

Toilet cushion . . . . . . . . . . . . . 222-223

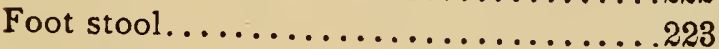

Child's chair.....................223-224

Catch-all......................224-225

Head rest. . . . . . . . . . . . . . . 225

Hanging pin cushion...............225-226

Fancy pin cushions..............226-227

Infant's crib coverlet. . . . . . . . . . . .227-228

Table cover.................. 228-229

Chicken pen wipe............... 229-.230

Branch of fruit................. 230-231

Dust cap.................... 231

Satchet bags.................. . 231-232

Tobacco pouches................ 232-233

Leaf penwipes ................. 233-234

Traveling case................... 234

Child's chair ................... 234

Mat for water pitcher.............. 235

Needle cases.................. 235-236

Kensington cover tor cushions........ 236-238

Nasturtium cushion.............. 238-232

Infant's toilet case................. 239

Keep a scrap bag . ............ 239-240

Drawn Work. . ................. 241

Preparing the work............ 242

Foundation stitches............. 242-244

Basket insertion................ 245
Lunch cloth with border and corner.. $245-251$

Border for center cloth........... 251-25?

Corners...................... 253-254

Antique border and corner........ 255-256

BRUsh and Palette.............. 257

Designs for painting ........... 258

Painting in oils on fabrics........ 258

Materials for painting .......... 258-259

Mixing colors...............

Blues, browns, buffs, yellows, grays, etc............... 258-260

Palettes for...................

Daisies.

Honeysuckle...............

Apple blossoms..............

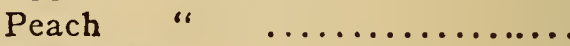

Tea roses..................

Feathery clematis.............

Violets.

White and purple lilacs

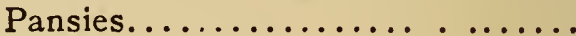

Morning glories.............. 261-262

Sky effects for backgrounds........ 262-263

Special palettes................. 263

Strawberry spray............. 263

Geranium spray.............. 264-265

Butterflies ................... 265

Grapes on black velvets....... .. 265

Pansies....................266

Purple thistles................ 266-267

Pond lilies................... 267

Purple clematis................ 2667

Oxheart cherries .............. 267-268

Virginia creeper, leaves and berries.. 268

Peacocks ...................... 268-269

Golden rod and astors............ 269-270

Hollyhock screen panel.. ........ 270-271

Washable painting............ 271

Medium................... 271-272

Water color painting............ 272-274

Roses....................... 274

White flowers............... 274-275

Primroses.................... 275

Blue flowers................. 275

Convolvulus................. . 276

Figures in water colors......... 276-277

Fabric tinting. ............. 277-279

Paris tinting................. 279-280

Painting on celluloid............ 280 
Lilacs on celluioid in oil........... 280-281

Lilacs in water color on celluloid......, 281-282

Lustre painting. ................... 282-284

Kensington painting............. 284-285

Kensington parrots... .......... 285-286

Crayon painting, portraits.......... 286-289

Painting on birch bark............ 289-290

Scorch painting . . . . . . . . . . . . . . 290-292

Tapestry painting................ 292-293

Canvas for.................. 293-295

Dyes .................... 295-296

Brushes...................... 296

Mixing dyes.................. 296-297

Flesh colors .................. 297-298

Flesh painting................ 297

Hair and drapery............... 298-301

Palettes for draperies, sky. marble, stone, etc. ................ 301-308

Painting for profit .............. 304-308

Point Lace Materials..............

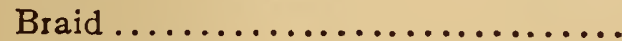

Lace thread $\ldots . \ldots \ldots \ldots \ldots \ldots . . .$.

Tracing cloth .................

Ground cloth ...................

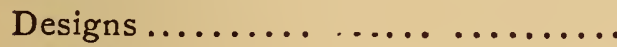

Needles......................

Preparing the work...............

Outlining designs with braid...........

Stitches.......................

Stitches proper or points...........

Brussels point................

Venice point.....................

Spanish point.................

Close Spanish point .............

Treble Spanish point..............

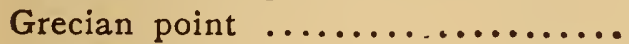

Valenciennes ...................

Point d'Alencon ................

Turkish stitch....................

Net ground stitch $. . . \ldots \ldots \ldots \ldots . . .$.

Point de tulle...................

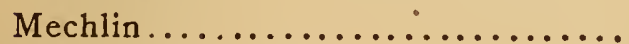

Wheels, rosettes, bars .............

Sorrento bars....................

Point d'Alencon bars................

Venetian bars.....................

Edged Venetian bars

Dotted Venice point.

Dotted Sorrento bars.
Greek Point.....................

PAas.

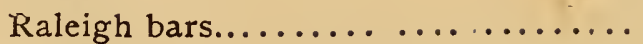

Point d'Alencon ...................

Point lace border . ................

Handkerc ief border................

Handkerchief Centers................

Collarette..................... 309-321

Knitting And Crocheting......... 325-326

Terms used................. 327

Abbreviations ................. 327

Crocheted dress for child.......... 328-330

Purse......................... 330-331

Baby's Sacque................. 331-332

Baby's shirt. ................. 332-333

Lady's vest.................. 334-335

Lady's vest, Basket pattern. ........ 335-337

Baby's hood ................... 337-338

Fancy bag.................... 338-339

Fringe . .................. 339-340

Pineapple edge................ 341

Insertion crochet.............242

Border Crochet.................. 342--343

Trimming crochet.............. 343--344

Knitted square for counterpane...... 345-347

Wheels for toilet set............. 347-348

Edging....................... 348-350

Diamond edge............... 350-352

Fern t:imming................. 352-354

Crochet trimming .............. 354-355

Narrow edging...................... 355

Broad edge.................. 355-356

Narrow coral edging............. 356-357

Reticella crochet............... 357-360

Petticoat for child ............... 361-362

Crochet tassel................... 363-364

Daisy crochet lace................ 364-366

Guipure lace, crocheted........... 366-368

Crocheted table cover............... 368-371

Petticoat . ................... 371-372

Trimming, crochet and braid........ 372-374

Infant's knitted jacket............. 374-375

Moss edging, crochet and braid...... 376

Fern edging................. 376

French edging.................. 376

Edging serpentine............... 376

Fancy braid edge, No. r........... 377

Edging, No. 2................ 377

Shell trimming ............... 377-378

Palm trimming............... 378 
PAGE.

Hood for child.................... 379-380

Hood for child................... 381-382

Pillow shams.................... 383-384

Table mats...................... 385

Border to mat.................... 385

Crocheted doll................... 386-388

Torchon lace.................... 388

Comfort for lounge................ 389

Porcupine stitch............... 389

Directions for wheel.............. 389-391

Petticoat for child............... 391-992

Border and corner............... 392-394

Crocheted skirt.................. 394-395

Infant's boot................... 395-397

Mop for dusting pictures........... 397-398

Dainty work for profit.............. 399

Work for stay-at-homes.......... . 399-400

The first start................ 400

Commenced Samples............. 401-402

Designs for order work............ 402

Soliciting orders for embroidery...... 403-404

Teaching embroidery............ 404

What to charge and how to collect.... 404-405

To start an art store or bazaar...... 405-406

Location of store............... 406

Store fittings................ 406

Arrangement of store.............4 407

Stock of goods to be carried......... 408-409

Art fabrics.................... 409

Samples and display work......... 409-410

List of desirable samples.......... 410

Materials for made-up samples....... . 410-412

Cost of commenced samples.......... 412-413

Window display................ 413-414

Cost of starting a business......... 414-416

Quality and kind of stock for different

localities ...................... 416-418

Stamping patterns............. 418-426

Descriptiors of articles for stock.

Toilet set......................

Glasseine handkerchief sachet......

Glass panel screen................

Tobacco pouch................

Glasseine toilet set...............

Parasol Catch-all..................
PAGE.

Traveling roll...............

Duster case ..................

Calendar................... 420-424

DESCRIPTION OF FULL PAGE ILLUSTRATIONS.

Cushions, head rests..............

Roman embroidery..............

Star quilt...................

Drapes, doyleys................

Carafe mat.....................

Table covers..................

Easel drapes...................

Portieres.......................

Mantel drapes.................

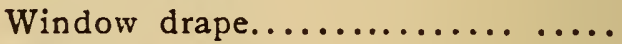

Magnolia doyley.................

Maple leaf doyley. ...............

Dresden plate doyley..............

Draped beds...................

Colored plates..................

Fleur de lis.

Poppies.

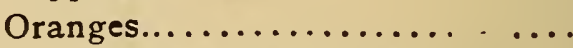

Oak leaves, sumac, golden rod.. . . 4?5-436

Descriptions of Special Illustrations.

Doyley designs.

Five-o'clock tea cloth

Three-panel screen.

Butterfly center cloth.

Louis XIV. design.

Violet center cloth

Bed spread.

Bed spread.

Rose center cloth

Four doyleys.

Violet basket center cloth

Sponge bag

Traveling case.

Picture frame...

Handkerchief bag.

Glove and veil case.

Infant's flannel robe

Hammock cushion

Ideal Honiton lace work

Dresden crib cover and pillow.......435-458 


\section{INDEX OF ILLUSTRA TIONS.}

\section{ALPHABETICALLY ARRANGED.}

$\begin{array}{llll} & \text { FIG. PP. } \\ \text { All-over design for drawn work } \ldots . . & 268-253\end{array}$

Detail of ............... 268-253

Antique burder and corner... ..... 270-255

Antique Embroidery............ 21- 34

Applique on net.............. 101-108

Artificial butterflies.............. 214-212

Baby's crocheted hood............ . 288-338

Baby's sacque................ 286-331

Banner ...................... 137-133

Basket insertion............... 263-247

Battlemented couching......... 117-120

Bedspreads and pillow shams....... 148

Bleeding heart, The............ 141-136

Block border................. 265-252

Block insertion.... . .......... 260-248

Detail a. b. of ............. 260-248

Bolster, covered............... 179-186

Border, crochet............. 293-343

Border, jeweled ............... 138-133

Bouquet holder............... 164-177

Branch of Cherries ............ 54-56

Branch of fruit............... 237-231

Brick stitch................. $\quad 31-40$

Brick flat couching ............ 118-120

Broad couching.............. 120-121

Broad edging ................. 303-356

Brussels point filling........... 269-254

Bullion knot..... ............ 124-122

Bullion monograms............ 135-132

Burnt match holder ............ 224-222

Buttonhole and spot stitch ........ 20-23

Buttonhole edge............... 19-33

Buttonhole stitch............... 18-33

Butterfly design ................ 103-109

Calendar ................... 279-308

Calla lily working design.......... 158

Carafe mat ................. 174
FIG. PP.

Carafe mat................. 72-80

Carving cloth................ 64-75

Catch-all ..................... 228-224

Center mat................... 220-218

" piece .................. 221-219

Chair back................. 176-184

Chair seat..................... 175-184

Chamois tobacco pouch.......... 274-287

Cherry center cloth.............. 89

Chicken pen-wipe. ............ 236-230

Child's chair................. 244-234

" "

“ petticoat .............. 339-392

Circles for ecclesiastical work....... 132-130

Clover doyley................. 89-91

Coffee cozy................. $\quad \mathbf{7 1 - 8 0}$

Combination leaf border ......... 50-52

Conventional book cover.......... 93-100

“ fleur de lis.......... 128-126

Corner mantel................ 114

Corner of scallop............... 106-111

Couching stitches............... 35

" outline.............. 24-35

Covered shoe-box. ............. 169-180

Cover for library table........... 154

Crawling rug................ 217-215

Crocheted border.............. 336-393

Crochet braid trimming.......... 317-373

Crocheted dress for child......... .283-4-330

“ dust mop........... 389-397

، petticoat for child...... 307-361

" " " "

" " " detail.. 309-362

، table cover........... 315-370

“ table mats ........... 333-385

Crochet tassels, detail........... 310-363

Crochet trimming............ 301-354 
FIG. PP.

Crocheted wheels.............. 334-390

Cross and long stitch. . . . . . . . . . . 154-147

“ border............... 262-250

. for altar front. . . . . . . . . . . 112-116

“ stitch design............. 95-102

" " "

“ “ . . . . . ..... 146-143

“ “ table cover......... 234-228

Crows' feet................... 36-41

Cruet mat, lenf shape............ 69-79

Cushion.................... 40-42

Cut in corner. ............... 266-253

D'Alencon bars................ 45-45

Daisy center cloth.............. 66-77

Daisy crochet lace ............. 311-365

“ design ................. 107-111

“ star..................... 35-41

Darned background ............ 99-105

“ stitch................ 39-42

Darning stitch . . . . . . . . . . . . . . . . 98-104

Decorated egg-shell. .............. 188-190

Deep scallop................... 109-112

Design for chasuble... . . . . . . . . . 129-127

Design in Kensington stitch........ 52- 54

Detail crocheted table cover. . . . . . . 313-368

Detail cross stitch... . . . . . . . . . 235-229

Detail of couched design.......... 125-123

" " " ......... 126-124

" crocheted dress for child... . 280-328

Detail of crocheted dress for child ... 281-328

“ " " “ $\quad \ldots .282-329$

“ handkerchief case ....... 181-187

“ long stitch design........ 127-125

Devonshire cloth.............. 1-12

Diamond couching............. 28-38

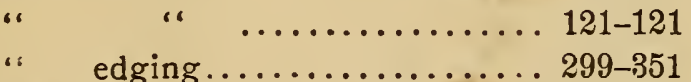

Double and close basket stitch. .... 44-44

Double leviathan stitch........... 151-145

Double photograph case.......... 183-188

Doyley...................... 63-75

Dragon border.................. 143-140

Drawn work................. 246

“ " ................. 247

Drawn work in frame........... 252-241

Dresden book cover ............. 92-10u

. doyley, full page.......... 430

“ doyleys............... 87-94
FIG. PP.

74-83

$83-89$

$257-244$

278-307

Easel drape................. 306

Easter banner. ............... 139-134

“ “ ............... 140-135

Easy chair................... 144-141

Ecclesiastical design............ 130-128

Edge for collar and cuffs . . . . . . . . . 104-110

“ " table cover........... 314-369

Edging, crochet . . . . . . . . . . . . . 29 298-349

“ No. 2............... . 324-377

Eiffel tower scrap bag ..... ..... 210-205

Embroidery frame ............. 2-15

" "

“ harp ............... $4-17$

" scissors.............. 5-18

Emery cushion................ 213-211

Fancy chain................ 10-20

“ couching.............. 29-38

" "

" crochet edge, No. 1......... 325-377

“ spool bag................ 289-339

“ table ................... 194-194

“ work basket.............. 199-197

Fern edging................ 300-353

" “ $\quad \ldots \ldots \ldots \ldots \ldots \ldots \ldots \ldots$. 320-376

Flannel scallop................ 110-112

Flat thistles for cover............ 159-166

Fleur de lis, colored plate...........

Floral center cloth.............. 91-99

Flower head rest.............. 53-55

Folding work basket............ 171-182

Foot stool ................... 166-178

Forget-me-not toilet cushion. . . . . . 198-196

Four corners prepared........... 255-243

French edging.............. 321-376

French knot................ 23-34

Fringe . . . . . . . . . . . . . . . 2 290-340

Fruit pin cushion............. 231-226

" " $"$ "........... 232-227

“ plate doyley............ 222-220

Ceranium center cloth........... 67-78

doyley............. 68-78

German stitch......... . . . . . . . 249-144

Glasseine toilet set............ 275-290)

Details of a. and b. of...... 275-290 
FIG. PP.

Glass handkerchief box... . . . . . . . 202-940

Glass panel screen............. 273-273

Grape vine applique .......... 51-53

“ center cloth......... 88-96

Greek cross.................. 134-131

Grouped star stitch ............. 37-41 " " "No. $2 \ldots \ldots \ldots \ldots$..... 38-41

Group of head rests, full page...... $\quad 58$

Guipure crocheted lace.......... . 312-36

Hairpin holder............... 172-182

Handkerchief case............. 182-187

Handkerchief center ............ 313

Handkerchief corner............ 102-109

Handkerchief square............ 313

Handkerchief sachet, glasseine..... 272-263

“ achets........... 193-194

Handy bag. . ............... 211-207

Hanging pincushion............. 230-226

Head rest ................ 229-225

Hemstitching ... ............ 26-36

Hemstitching ............... 256-243

" $\quad$ No. $2 \ldots \ldots$. . . . . . 258-244

No. 3. ............. 259-244

Honeycomb applied ........... 33-40

" " stitch........... $32-40$

Infant's crib coverlet............. 233-228

. knitted bodice........... 338-396

“ " jacket........... 318-375

“ pillow............... 180-186

"6 shawl .............. 111-113

“ toilet case.............. 251-239

Insertion, crochet. . . . . . . . . . . 292-342

“ of border.............. 261-249

Detail a. b. c. d. e. f. of 261 ..... 250-251

Invalid's writing board.......... 165-178

Janina stitch.................. 41-42

. " close.............. 42-43

Jeweled border ... ............113-118

Kensington cushion cover. . . . . . . . 249-237

Kensington stitch.............. 13-30

Kensington stitch, detail......... 12-30

Knitted hood................. 327-379

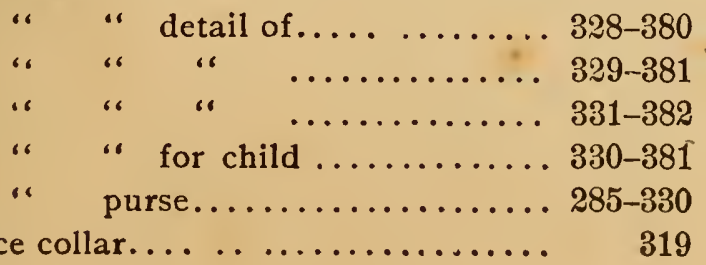

FIG. PR.

Ladies' crocheted skirt. . . . . . . . . . . 337-394

"r dust cap................ 238-231

“ skirt .............. 316-372

“ vest................. 287-334

Lamp shade. . . . . . . . . . . . . . . 177-185

Latin cross and anchor work ...... 133-131

Laundry bag ......... . . . . . . 204-240

Leaf-bordered lunch cloth......... 82-88

Leaf for pillow sham.......... . 80-86

“ penwipes ................ 242-233

Letter. . . . . . . . . . . . . . . . 136-13:

Linen covered cushion.......... 197-196

Long cross stitch............. 147-143

Loop outline................. 11-29

Louis XIV. design, full page....... 150

Lunch cloth.................. 61-74

“ cloth with corner and border. 264-246

Details of 264-a. b. c. d. e..... 246-247

Magnolia doyley, full page ........ 324

“ " ............. 219-217

IMantel drapery, full page.......... 302

Maple leaf doyley, full page . ...... 322

Memorandum card case.......... 163-176

Mending bag.................... 190-191

Moss edging.................. 319-376

Music stand ................. 215-213

Napkin................... 62-75

Narrow coral edging. ............ 304-357

“ edging crochet........... 302-355

Nasturtium cushion............. 250-238

Needle case................... 246-235

“ “ closed............. 247-236

New England footstool........... 226-223

Night dress sachet. . . . . . . . . . . . . 185-189

Oak leaves, Sumac and Golden Rod and

Oranger -Colored Plates.

Ornamental holders ......... .. 191-192

Ottoman cushion.............. 201-198

“ « .............. 202-198

Owl shaving case.............. 203-199

Palm trimming................ 326-378

Pansy sachet................... 186-189

“ « ................. 189-191

Pantagraph ................ 7-23

Paper holders... . . . . . . . . . . . . . 192-193

Paper stand................. 167-179 
FIG. PP.

Parchment parasol catch-all......... 276-293

Passion flowers................ 142-137

Pattern for cotton plant.... . . . . . 162-172

. " decoration of shoe box... 168-180

“ to fill cut-in corner.......... 267-246

Persian cross................. 148-144

“ embroidery.............. 22-34

photograph frame............. 206-202

“ envelope............. 207-203

Picture and easel drape.......... 141

Pilgrim toilet cushion............ 225-222

Pillow sham, crocheted.. .. ... ... 332-383

Pin trays.................. 208-203

Pineapple edge................ 291-341

Plain orick couching............ 119-121

Plain flat couching............. 122-121

" " " $. . \ldots \ldots \ldots \ldots \ldots . . \ldots 123-122$

Plaie 1, lace braid.............. 310

" 2, " stitches ............. 312

“ 3, “ "

“ 4, “ " $\quad$ "........... 316

Pocket needle case............. 248-236

Point de reprise... ........... $4 \dot{7}-46$

". lace braids, piate $1 . . . \ldots \ldots \ldots . \quad 210$

" " stitches, " 2.......... 212

" " " " $"$ " $3 \ldots \ldots \ldots \ldots .214$

“ " “ " $44 \ldots \ldots \ldots \ldots .216$

Poppies, colored plate............

Poppy correctly shaded, No. 1.......

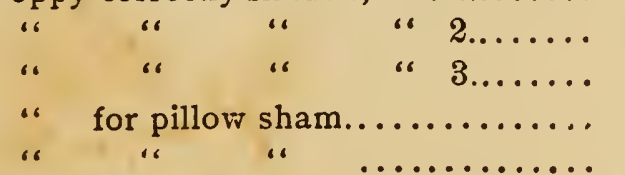

Prepared corner... ............ 253-242

$55-63$

$56-63$

$57-64$

$78-85$

$79-85$

254-242

Pumpkin toilet cushion.......... 205-201

Puff head rest................ 187-190

Raised basket couching........... 114-119

“ “ “ $\ldots \ldots \ldots \ldots \ldots 115-119$

Raised calla lily............... 155-159

" cotton plant............ 161-171

. coxcomb and sumach....... 157-164

“ Japan lily............... 156-160

‘ snow balls.............. 160-167

. thistles and golden rod....... 158-166

Reticella crochet............... 306-358

Roman embroidery........... 58-69
FIG. PP.

59.69

218-216

$48-47$.

Round cushion.................. 9 94-101

Round cushion.................. 9 94-101

Satin stitch................ 16-32

$17-32$

" "

“ " detail............... 15-32

Sachet bags................. 239-232

Scallop corner................ 108-112

edge................. 105-111

Scissors case ... ............. 216-214

Screens .................... 100-106

Scroll border................. 90-98

Serpentine braid edge.......... 322-376

Set vegetable mats............ . 70-79

Shell trimming................ 325-377

Sho box................... 223-221

Short and long stitch........... 14-31

Sideboard scarf............... 73-81

Simple star..... ............ 34-41

Single basket stitch............. 43-44

“ photograys. case........... 184-188

Snowdrop doyicy.............. 86-92

Sofa cushion................... 196-195

Spanish foot rest............... 200-197

“ laid work............... 27-37

Spider wheel................. 46-46

Splashers.. ................. 81-87

Spot and daisy loop........... 49-51

Square for counterpane........... 298-345

“ toilet cushion............ 212-210

Stand cover................. 97-104

Stem stitch.................. 8-28

Sunflower pen-wipe.............. 195-195

pincushion............ 170-181

Table cover.................. 96-103

“ " full page............ 432

" “ crocheted insertion..... 365-360

“ doyley and drape, full page.. 138

Tapestry stitch................ 156 144

Tent stitch ................ 152-146

Three outline stitches........... 9-28

Tobacco pouch............... 240-232

" " ................ 241-233

Toilet bottle................. 75-84

$76-84$ 
FIG. P2.

$77-84$

Tioilet cushions.

Toilet mat. Details of a. and b. of. "raveling bag.

. case.

case

Trzy cloth

Trim n
297-348

271-261

271-261

277-307

243-234

65-76

131-130
Trimming crochet............. 294-344

Wall pocket................ 178-'85

Water lily carafe mat .......... 245-235

Wavy couching.............. 116-120

Wheels crochet .............. 296-347

Window drapery, full page........ 294

Work apron................ 6-18 


\section{L'ENVOI.}

The sum of human happiness lies in the words "Home making, Home keeping." A home well made, well kept, well guarded is as near paradise as any earthly thing may be, and should be the subject of greatest importance in a woman's life from the hour she pronounces the fateful "I will" to the one in which she yields up her charge and joins the "throng innumerable."

The woman who can combine the practical necessary with the decorative necessary, so as to preserve a true balance, is indeed happily endowed, and blessed is the man who calls her "helpmeet." A man is greatly indebted to his home environment and influence for his self-respect or lack of that element. This is also true of children from the hour in which they enter the school room and mix with their kind, to the day when they go out into that larger world to win their place in the plan universal.

The man does not live, however insensible to elegance in the abstract, but feels a thrill of pride, experiences a $f$.ng of importance, an increased self-respect, as he enters the porta! ${ }^{1-}$ of $11, \mathrm{~s}$ home-if that home bears evidence of thrift, fore-handedness, prosperity. It is his money has procured this home for his family - his business sagacity or capacity for earning that renders it possible for his wife to have as good-perhaps better-than other wives in his circle of acquaintance, and he mentally pats himself on the shoulder and says "What a great man am I," perhaps never giving a thought to the ways and means adopted by his domestic partner to produce such satisfactory results, from too often limited resources; but what matters it to the brave little helpmeet. She has done her part in using tc the best advantage what he has labored to secure, and thus each has performed but his and her duty, and neither stands indebted to the other. True it is that a word of loving acknowledgement and sympathy is a sweet morsel to any woman, coming from the lips of the man she has sought to please by her housewifely skill, and to the credit of the Igth century man, be it said, that word is seldom withheld. Firmly imbued with the sentiments here expressed it has been the aim of the writer, in the pages of "Dainty Work," to put within the reach of every woman the knowledge and means of rendering beautiful and elegant her home, in whatever position of life she may find her lot cast; to give voice to the sentiment that "the beautiful is good and the good beautiful", as applied to home making. Whether the task has been well or ill done the author must leave to the judgment of an indulgent public. 



